European

Thyroid Journal

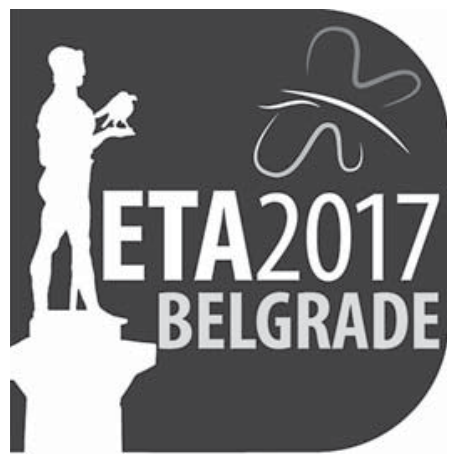

\title{
40th Annual Meeting of the European Thyroid Association
}

\section{Abstracts}

Belgrade, Serbia

September 9-12, 2017

Guest Editors

Pilar Santisteban, Madrid, Spain

Miloš Žarković, Belgrade, Serbia

Abstracts containing errors in spelling, grammar, or scientific content have been published as typed by the author and have not been corrected by the Publisher. 


\section{Saturday, 9th September 2017}

\section{Oral Session 1: Topic Highlights}

\section{OP-01-01 \\ A NOVEL MOUSE MODEL DEMONSTRATES THE PATHOGENIC ROLE OF ACTIVATING ALK MUTATIONS IN POORLY DIFFERENTIATED THYROID CARCINOMA}

Hannah Kohler ${ }^{1}$, Georg Sebastian Hoenes ${ }^{1}$, Soeren Latteyer ${ }^{1}$, Sarah Synoracki ${ }^{2}$, Johannes H Schulte ${ }^{3}$, Xiao Hui Liao ${ }^{4}$, Samuel Refetoff', Kurt Werner Schmid ${ }^{2}$, Dagmar Führer ${ }^{1}$, Lars Christian Möller ${ }^{1}$

${ }^{1}$ Department of Endocrinology and Metabolism, University Hospital Essen, University of Duisburg-Essen, Essen, Germany, ${ }^{2}$ Institute of Pathology, University Hospital Essen, University of Duisburg-Essen, Essen, Germany, ${ }^{3}$ Department of Pediatric Oncology, CharitéUniversitätsmedizin, Berlin, Germany, ${ }^{4}$ Department of Medicine, The University of Chicago, Chicago, IL, USA

Introduction: Radioiodine refractory undifferentiated thyroid cancer is a major clinical challenge. Kinase inhibitors represent potential therapies, if a driving mutation in a kinase is identified. The anaplastic lymphoma kinase (ALK) has been suggested to promote thyroid carcinogenesis. To determine the oncogenic role of ALK in thyroid carcinogenesis, we developed a mouse model for thyrocyte-specific expression of a constitutively active ALK mutant $\left(\mathrm{ALK}^{\mathrm{F} 1147 \mathrm{~L}}\right)$.

Material and Methods: Mice that express tamoxifen-inducible Cre recombinase under control of the thyroglobulin promoter $\left(\mathrm{TgCre}^{\mathrm{ERt}}\right)$ were crossed with lox-stop-lox-ALK ${ }^{\mathrm{F1174}}$ mice, resulting in TgCre $\mathrm{ERt}^{\mathrm{E}} \mathrm{ALK}^{\mathrm{F} 1174 \mathrm{~L}}$ $\left[\mathrm{ALK}^{\mathrm{mut}}\right]$ mice as positive progenitors. TgCre ${ }^{\mathrm{ERt}}$ mice served as controls. Four weeks after birth, thyrocyte-specific ALK ${ }^{\mathrm{F} 1174 \mathrm{~L}}$ expression was induced by i.p. injection of tamoxifen.

Results: ALK $^{\text {mut }}$ mice developed large thyroids and their thyrocytes showed dedifferentiation with loss of thyroglobulin expression and thyroid hormone synthesis. Ultimately, they developed poorly differentiated thyroid cancer (PDTC) with an increased mitotic rate. The thyroid tumors grew invasively into the surrounding muscle and metastasized into the lung. Median survival of ALK ${ }^{\text {mut }}$ mice was significantly reduced to 4 months.

Conclusion: We generated a novel thyroid cancer mouse model that demonstrates the pathogenic role of activating ALK mutations in thyroid carcinogenesis. ALK positive mice developed locally invasive PDTC with lung metastases and severely reduced survival. These data are clinically relevant, because ALK inhibitors exist and could be used in patients with ALK-positive radioiodine refractory thyroid cancer.

\section{OP-01-02 \\ IDENTIFICATION OF A DNA METHYLATION SIGNATURE THAT CAN PREDICT DISTANT METASTASES IN THYROID CANCER}

Helena Rodríguez-Lloveras ${ }^{1}$, Zafon Carles ${ }^{2}$, Nuria Villalmanzo' ${ }^{1}$, Iglesias Carmela ${ }^{3}$, Esther Klein Hesselink ${ }^{4}$, Bettien van Hemel5, Joan Gil' ${ }^{1}$, Cristina Montero ${ }^{6}$, Jordi Lluís Reverter ${ }^{7}$, Dídac Mauricio ${ }^{8}$, Manel Puig-Domingo ${ }^{9}$, Mercedes Robledo ${ }^{10}$, Thera Links ${ }^{11}$, Mireia Jorda ${ }^{12}$ ${ }^{1}$ Program for Predictive and Personalized Medicine of Cancer, Germans Trias I Pujol Research Institute (Pmppc-lgtp), Badalona, Barcelona, Spain, ${ }^{2}$ Hospital Vall D'hebron, on Behalf of the Consortium for the Study of Thyroid Cancer (Cecat), Diabetes and Metabolism Research Unit (Vhir), Barcelona, Spain, ${ }^{3}$ University Hospital Vall D'hebron, Department of Pathology, Barcelona, Spain, ${ }^{4}$ University of Groningen, University Medical Center Groningen, Department of Endocrinology, Groningen, Netherlands, ${ }^{5}$ University of Groningen, University Medical Center Groningen, Department of Pathology, Groningen, Netherlands, ${ }^{6}$ Hereditary Endocrine Cancer Group, Spanish National Cancer Research Centre (Cnio), Madrid, Spain, ${ }^{7}$ Department of Endocrinology and Nutrition, Germans Trias I Pujol Research Institute and University Hospital, Consortium for the Study of Thyroid Cancer (Cecat) of Catalonia, Badalona, Spain, ${ }^{8}$ Department of Endocrinology and Nutrition, Germans Trias I Pujol Research Institute and University Hospital, Center for Biomedical Research on Diabetes and Associated Metabolic Diseases (Ciberdem), Consortium for the Study of Thyroid Cancer (Cecat) of Catalonia, Badalona, Barcelona, Spain, ${ }^{9}$ Department of Endocrinology and Nutrition, Germans Trias I Pujol Research Institute and University Hospital, Isciii Center for Biomedical Research on Rare Diseases (Ciberer), Consortium for the Study of Thyroid Cancer (Cecat) of Catalonia, Badalona, Barcelona, Spain, ${ }^{10}$ Hereditary Endocrine Cancer Group, Spanish National Cancer Research Centre (Cnio), Isciii Center for Biomedical Research on Rare Diseases (Ciberer), Madrid, Spain, ${ }^{11}$ University Medical Center Groningen, Department of Endocrinology, Groningen, Netherlands, ${ }^{12}$ Program for Predictive and Personalized Medicine of Cancer, Germans Trias I Pujol Research Institute (Pmppc-Igtp), Consortium for the Study of Thyroid Cancer (Cecat) of Catalonia, Badalona, Barcelona, Spain

Introduction: Distant metastases occur in up to $10 \%$ of differentiated thyroid carcinomas (DTC) and have an adverse impact on survival. Still no effective methods are available to determine which patients will eventually develop distant metastases. Further insight into the molecular pathogenesis of DTC may be helpful. It is known that genetic and epigenetic alterations play a major role in cancer. However, the involvement of DNA methylation in disease progression of DTC is poorly understood.

Objective: The aim of this study was to investigate whereas DNA methylation plays a role in the metastatic process of DTC and can be used to predict the metastatic risk of primary tumors.

Methods: DNA was extracted from formalin-fixed paraffin-embedded tissues including 30 low-risk/non-metastatic DTC, 35 distant metastatic DTC, 18 paired metastases and 15 matched normal tissues. Genome-wide DNA methylation was profiled using the Illumina Infinium HumanMethylationEPIC platform. Validation of specific loci was carried out by bisulfite-PCR-sequencing.

Results: We found a close relationship between the methylation profile and the histology and driver mutation of the primary tumor, confirming our previous results. Distant metastatic tumors presented a higher number of DNA methylation alterations than non-metastatic ones, and their methylomes were similar to those of the matched metastatic tissues. Most importantly, we identified a $156 \mathrm{CpG}$-signature associated with metastatic DTC independently of histology and mutation, which also distinguished different molecu-

\begin{tabular}{ll}
\hline KARGER & $\begin{array}{l}\text { C } 2017 \text { European Thyroid Association. } \\
\text { Published by S. Karger AG }\end{array}$ \\
$\begin{array}{l}\text { E-Mail karger@karger.com } \\
\text { www.karger.com }\end{array}$ & $\begin{array}{l}\text { Accessible online at: } \\
\text { www.karger.com/etj }\end{array}$
\end{tabular}


lar subgroups of metastatic tumors. These differentially methylated sites were enriched in hypomethylations and over half were located in promoters and enhancers, suggesting their role in gene expression regulation. Further studies are required to investigate their functional implications.

Conclusion: This comprehensive study provides novel insights into the role of DNA methylation in metastatic thyroid cancer as well as potential biomarkers for risk stratification.

\section{OP-01-03}

\section{LOW-NORMAL THYROID FUNCTION} ASSOCIATED WITH INCREASED LIFE EXPECTANCY: THE ROTTERDAM STUDY

Arjola Bano ${ }^{1}$, Klodian Dhana ${ }^{1}$, Layal Chaker ${ }^{1}$, Maryam Kavousi ${ }^{1}$,

M. Arfan Ikram ${ }^{1}$, Francesco Mattace-Raso ${ }^{1}$, Oscar Franco ${ }^{1}$, Robin

Peeters ${ }^{1}$

${ }^{1}$ Erasmus Medical Center, Rotterdam, Netherlands

Background: Variations in thyroid function within reference ranges are associated with an increased risk of cardiovascular disease (CVD) and mortality. However, the qualitative and quantitative impact of thyroid function on an individual's life and health remains unclear.

Objective: To investigate the association of thyroid function with total life expectancy (LE) and the number of years lived with and without CVD among euthyroid subjects.

Design and Setting: The Rotterdam Study, a population-based, prospective cohort study

Participants and Measurements: We included 7785 participants without known thyroid disease and with thyroid-stimulating hormone (TSH) and free thyroxine (FT4) levels within the reference ranges. Multistate life tables were used to calculate total LE and LE with and without CVD among TSH and FT4 tertiles. LE estimates in men and women aged 50 years and over were obtained using prevalence, incidence rates and hazard ratios for three transitions (healthy to CVD, healthy to death and CVD to death), adjusting for sociodemographic and cardiovascular risk factors.

Results: Over a 10 year follow-up, we observed 789 incident CVD events and 1357 deaths. Compared with those in the lowest tertile, men and women in the highest TSH tertile lived $2.0(95 \%$ confidence interval $[\mathrm{CI}]=1.0 ; 2.8)$ and $1.4(\mathrm{CI}=0.2 ; 2.4)$ years longer, respectively; of which $1.5(\mathrm{CI}=0.2 ; 2.6)$ and 0.9 $(\mathrm{CI}=-0.2 ; 2.0)$ years longer without $\mathrm{CVD}$. Compared with those in the lowest tertile, men and women in the highest FT4 tertile lived $3.5(\mathrm{CI}=-5.1 ;-1.7)$ and $3.5(\mathrm{CI}=-5.6 ;-1.5)$ years fewer, respectively; of which $3.3(\mathrm{CI}=-5.3 ;-1.2)$ and $2.5(\mathrm{CI}=-4.4 ;-0.7)$ years fewer without $\mathrm{CVD}$.

Conclusion: At the age of 50 years, subjects with low-normal thyroid function live up to 3.5 years longer overall and up to 3.3 years longer without CVD than subjects with high-normal thyroid function. These findings provide supporting evidence for a reevaluation of the current reference ranges of thyroid function and can help inform preventive and clinical care.

\section{OP-01-04 \\ BMP/FGF-DEPENDENT DIFFERENTIATION OF THYROID CELLS FROM A NOVEL THYROID PROGENITOR CELL POPULATION IN ZEBRAFISH FOREGUT ENDODERM}

Robert Opitz ${ }^{1}$, Benoit Haerlingen ${ }^{2}$, Angelo Molinaro ${ }^{3}$, Isabelle Vandernoot $^{2}$, Achim Trubiroha ${ }^{2}$, Sabine Costagliola ${ }^{2}$

${ }^{1}$ Institute of Experimental Pediatric Endocrinology, Charité Universitätsmedizin Berlin, Berlin, Germany, ${ }^{2}$ Institute of Interdisciplinary Research in Molecular Human Biology (Iribhm), Université Libre de Bruxelles, Brussels, Belgium, ${ }^{3}$ Endocrinology Unit, Department of Internal Medicine, University of Pisa, Pisa, Italy, Endocrine Unit, Massachusetts General Hospital, Harvard Medical School, Boston, MA, USA, Endocrinology and Metabolism, Pisa, Italy

Thyroid gland organogenesis begins with the specification of thyroid precursor cells in the anterior foregut endoderm. However, our understanding of the intrinsic and extrinsic factors orchestrating the earliest stages of thyroid cell differentiation is still limited. Since zebrafish embryos provide an excellent model to study vertebrate organogenesis, our study aimed at a characterization of cellular dynamics during early zebrafish thyroid development, with a special focus on morphogen signaling pathway activities and expression patterns of thyroid transcription factors (pax2a, nkx2.4b). Manipulation of major signaling pathways during early zebrafish development (using small molecule inhibitors and transgenic overexpression models) revealed key roles of BMP and FGF signaling for thyroid precursor cell specification. For example, blocking BMP signaling during early somitogenesis caused thyroid agenesis whereas enhancing BMP signaling at defined stages led to a massive expansion of thyroid marker-expressing cells throughout the entire foregut endoderm. Signaling pathway biosensor models were used to identify when and where BMP and FGF signaling is active in developing foregut endoderm. At $20 \mathrm{hpf}$, FGF signaling became detectable in foregut endoderm and was restricted to a population of endodermal cells expressing the early thyroid marker pax2a. At this stage, endodermal pax $2 \mathrm{a}+$ cells did not co-express other thyroid markers and pax $2 \mathrm{a}+$ cells were initially negative for BMP signaling. During the following 24 hours, BMP signaling became activated in pax $2 \mathrm{a}^{+}$ cells and pax $2 \mathrm{a}+$ cells with active BMP signaling started to co-express the thyroid marker $\mathrm{nk} \times 2.4 \mathrm{~b}$. We also detected a spatial patterning of $\mathrm{nkx} 2.4 \mathrm{~b}$ expression within the pax $2 \mathrm{a}+$ cell population that correlated with the positioning of pax $2 \mathrm{a}+$ cells relative to adjacent cardiac mesoderm. Our data suggest a new model of sequential thyroid cell differentiation in zebrafish including an initial FGF-dependent step to establish a pool of pax2a-expressing thyroid progenitor cells and subsequent BMP-dependent differentiation of thyroid progenitors into committed thyroid precursor cells.

\section{OP-01-05 \\ PROSTAGLANDIN F2-ALPHA EYE DROPS (BIMATOPROST) IN THYROID EYE DISEASE: A RANDOMISED CONTROLLED DOUBLE BLIND CROSSOVER TRIAL (BIMA STUDY)}

Mohd Shazli Draman ${ }^{1}$, Daniel Morris², Sam Evans ${ }^{2}$, Anjana Haridas ${ }^{2}$ Julie Pell', Rosemary Greenwood ${ }^{3}$, Chris Foy ${ }^{4}$, Peter Taylor ${ }^{1}$, Pakinee Pooprasert ${ }^{1}$, Ilaria Muller', Lei Zhang ${ }^{1}$, Carol Lane ${ }^{2}$, Onyebuchi

Okosieme $^{1}$, Marian Ludgate ${ }^{1}$, Colin Dayan ${ }^{1}$

${ }^{1}$ Systems Immunity Research Institute, School of Medicine, Cardiff University, Cardiff, UK, ${ }^{2}$ Department of Ophthalmology, University Hospital of Wales, Cardiff, UK, ${ }^{3}$ University Hospitals Bristol NHS Foundation Trust, Bristol, UK, ${ }^{4}$ Gloucestershire Research Support Service, Gloucestershire Royal Hospital, Gloucester, UK

Background: Reduced eyelid protrusion has been reported as a sideeffect of prostaglandin analogue eye drops (Bimatoprost) in glaucoma treatment. The objective of this study is to determine if bimatoprost is effective at reducing proptosis in inactive thyroid eye disease (TED).

Methods: Following informed consent participants were randomised to receive bimatoprost or placebo for three months after which they underwent a two-month washout, before switching to the opposite treatment. The primary outcome was to compare the change in exophthalmometry readings over the two 3-month treatment periods. This was supported by photographic assessments. Allowing $15 \%$ dropout rate, 31 patients were randomised in order to identify a treatment effect of $1.6 \mathrm{~mm}(\mathrm{p}=0.05$, two-sided, power 0.88 ).

Results: There was female preponderance with 5:1 ratio and mean age of 55 (range 28-74). The median duration of TED was 7.6 (IQR 3.6-12.3) years. The majority were still suffering from diplopia (61.3\%) with bilateral involvement $(61.3 \%)$. Using multilevel modelling adjusted for baseline, phase and carryover, Bimatoprost resulted in $-0.17 \mathrm{~mm}$ exophthalmometry change $(95 \%$ $\mathrm{CI}-0.67$ to +0.32$) \mathrm{p}=0.490$. Subgroup analysis of 12 patients with unilateral proptosis, showed a $-1.17 \mathrm{~mm}$ change $(95 \% \mathrm{CI}-2.62$ to +0.29$)$ compared to the untreated eye of $+1.92 \mathrm{~mm}(95 \% \mathrm{CI}+0.60$ to +3.23$) \mathrm{p}=0.0067$.

Conclusions: Bimatoprost treatment over 3 months does not result in clinically meaningful improvement in proptosis. Results of the subgroup analysis (suggested by an independent reviewer) suggests that those with asymmetry might benefit from this treatment but this will need testing in a further study. Over $60 \%$ of TED patients have diplopia, confirming the unmet clinical need in this patient group. 


\section{OP-01-06}

DIVERSE FUNCTIONAL IMPAIRMENT OF THYROID HORMONE RECEPTOR ALPHA

\section{MUTATIONS}

Karn Wejaphikul', Anja van Gucht', W. Edward Visser ${ }^{1}, K^{1}$ Kishna Chatterjee ${ }^{2}$, Theo J. Visser ${ }^{1}$, Robin P. Peeters ${ }^{1}$, Marcel E. Meima ${ }^{1}$

${ }^{1}$ Academic Center for Thyroid Diseases, Erasmus University Medical Center, Rotterdam, Netherlands, ${ }^{2}$ Wellcome-Mrc Institute of Metabolic

Science, University of Cambridge, Cambridge, UK

Introduction: Mutations in the ligand binding domain of thyroid hormone receptor (TR) ál cause resistance to thyroid hormone alpha (RTHá). Evidence from TRâl studies indicates that the effect of missense mutations on transcriptional activity may not only depend on the reduced affinity for $\mathrm{T} 3$ but also altered interactions with co-factors.

Objective: To evaluate the correlation of T3 binding affinity, transcriptional activity and receptor-cofactor interactions of WT and mutant TRá1.

Methods: Five missense mutations derived from RTHá patients, murine model and interesting TRâ1 mutations were introduced into FLAG-tagged TRá1 constructs. T3 binding affinity of in vitro translated wild-type (WT) and mutant receptors was measured by competitive binding assays. Transcriptional activity of the receptor variants was determined in JEG3 cells co-transfected with luciferase (Luc) reporter constructs containing either direct (DR4), inverted (IR0) or everted (ER6) repeat TRE and stimulated for 24 hours with $0-10,000 \mathrm{nM}$ T3. The T3-dependence of the interaction between TRs and cofactors was determined using a mammalian two-hybrid assay, by co-transfecting VP16-TRá1, GAL4-NCoR1 or SRC1, and the UAS-tkLuc reporter construct in JEG3 cells.

Results: Compared to WT TRá1, the dissociation constant $(\mathrm{Kd})$ of T3 was increased 2.4-2.8 fold for the L287V, P398H, A263S and D211G mutants but only 1.1 fold for T223A. In contrast, the transcriptional activity varied greatly between mutants. The EC50 for T3 stimulation using the DR4-TRE was increased 48 fold for L287V, 28 fold for D211G, 16 fold for P398H, 3 fold for A263S and 1.2 fold for T223A compared to WT. The shifts of EC50s using the other TREs generally followed the same trend for each mutation. The fold increase in IC50 and EC50 for NCoR1 dissociation and SRC1 association, respectively, for mutants versus WT correlated with the changes in EC50 for transcriptional activation (L287V > D211G > P398H > A263S).

Conclusions: The severity of the transcriptional impairment of TRá1 mutants is not only determined by the reduced affinity for T3, but may also be due to altered interactions with cofactors.

\section{Oral Session 2 (Clinical): Clinical Aspects of Autoimmunity}

\section{OP-02-07 \\ GENOTYPE-PHENOTYPE CORRELATIONS IN GRAVES' DISEASE}

Aleksander Kuś1, Konrad Szymañski², Beata Jurecka-Lubieniecka ${ }^{3}$, Edyta Pawlak-Adamska ${ }^{4}$, Dorota Kula ${ }^{3}$, Natalia WawrusiewiczKurylonek ${ }^{5}$, Piotr Miœkiewicz ${ }^{1}$, Joanna Kuœ ${ }^{1}$, Marek Bolanowski ${ }^{6}$, Rafa $^{3} P^{3}$ oski ${ }^{2}$, Artur Bossowski ${ }^{7}$, Jacek Daroszewski ${ }^{8}$, Barbara Jarzab ${ }^{9}$, Tomasz Bednarczuk ${ }^{10}$

${ }^{1}$ Department of Internal Medicine and Endocrinology, Medical University of Warsaw, Warsaw, Poland, ${ }^{2}$ Department of Medical Genetics, Medical University of Warsaw, Warsaw, Poland, ${ }^{3}$ Department of Nuclear Medicine and Endocrine Oncology, Maria SklodowskaCurie Memorial Cancer Center and Institute of Oncology, Gliwice, Poland, ${ }^{4}$ Department of Experimental Therapy, Hirszfeld Institute of Immunology and Experimental Therapy, Polish Academy of Sciences, Wroclaw, Poland, ${ }^{5}$ Department of Endocrinology, Diabetology and Internal Medicine, Medical University of Bialystok, Bialystok, Poland, ${ }^{6}$ Medical University Wrocław, Endocrinology, Wrocław, Poland, ${ }^{7}$ Medical University in Białystok, Dep.of Pediatrics, Endo, Diabeto, Bialystok, Poland, ${ }^{8}$ Dept. Endocrinology, Diabetes, and Isotope Therapy, Wroclaw, Poland, ${ }^{9}$ Msc Memorial Cancer Center and Institute of Oncology, Gliwice Branch, Department of Nuclear Medicine and Endocrine Oncology, Gliwice Branch, Gliwice, Poland, ${ }^{10}$ Warsaw University of Medicine, Nip 52500058 28, Warsaw, Poland

Objectives: Regardless to various clinical manifestations of the disease, most studies on the genetic background of Graves' disease (GD), including genome-wide association studies (GWAS), assess only the susceptibility to the disease in the whole group of studied patients. Here we investigated single nucleotide polymorphisms (SNPs) previously associated with GD and autoimmune thyroid disease (AITD) for association with specific phenotypes of GD in a cohort of well-characterized GD patients.

Materials and Methods: A total of 32 SNPs were genotyped using TaqMan SNP genotyping assays in 1022 GD patients from the Caucasian population. We compared allele frequencies between subgroups of GD patients stratified basing on their clinical characteristics including sex, age of GD onset, presence of Graves' ophthalmopathy (GO), cigarette smoking status and family history of AITD. P-value less than $0.05 / 32=0.0015$ was considered statistically significant and $\mathrm{P}<0.05$ was considered as suggestive association.

Results: We found a significant difference in allele frequencies between older (age of GD onset $\geq 40$ years) and younger (age of GD onset $<40$ years) GD patients for the RNASET2 polymorphism (rs9355610, $\mathrm{OR}=1.39, \mathrm{P}=0.0015$ ), and observed several other suggestive associations for the HCP5 (rs3094228), MAGI3 (rs1230666), PRICKLE1 (rs4768412), PTPN22 (rs2476601) and HLA-DOB/TAP2 (rs1894407) polymorphisms (all $\mathrm{P}<0.05$ ). Also a suggestive difference between male and female GD patients was found for the MMEL1 polymorphism (rs2843403, $\mathrm{OR}=1.31, \mathrm{P}=0.027)$. When we compared GD patients with (NOSPECS class 3-6) and without (NOSPECS class 0-1) GO, suggestive associations were found for the SCGB3A2 (rs1368408), TPO (rs11675434), CTLA4 (rs3087243) and TNF (rs1800629) polymorphisms (all $\mathrm{P}<0.05$ ). No difference was observed between GD patients stratified according to their smoking status or family history of AITD.

Conclusions: Our results showed several novel associations between the studied SNPs and the phenotype of GD. Precise phenotyping and subgroup analysis in association studies may provide a better insight into the genetic background of GD and allow to identify novel loci specifically associated with selected phenotypes of the disease. 


\section{OP-02-08 \\ DOES GRAVES' ORBITOPATHY (GO) EVER DISAPPEAR? ANSWERS TO AN OLD QUESTION}

\section{Elena Sabini', Marenza Leo², Francesca Menconi', Barbara Mazzi',} Ilaria Ionni', Marco Nardi ${ }^{3}$, Paolo Vitti', Claudio Marcocci', Michele Marinò ${ }^{1}$

${ }^{1}$ Department of Clinical and Experimental Medicine, Endocrinology Units, University of Pisa and University Hospital of Pisa, Pisa, Italy, ${ }^{2}$ Endocrinology Unit, University of Pisa, Pisa, Italy, ${ }^{3}$ Department of Surgical, Medical and Molecular Pathology, Ophthalmopathy Unit I, University of Pisa and University Hospital of Pisa, Pisa, Italy

Objectives: There is a general belief that GO is somehow "chronic", namely that patient's eyes do not return the way they were before GO. Here we investigated this issue under the patient's and the physician's point of view.

Methods: We studied 99 consecutive patients (30 men and 69 women, age $58.6 \pm 11.0 \mathrm{yr})$ with a GO duration $\geq 10 \mathrm{yr}$. $(202 \pm 8 \mathrm{mo}$, range $120-585)$ treated for their thyroid disease (Graves' disease $=97$, autoimmune thyroiditis $=2$ ) with radioiodine $(\mathrm{N}=64)$, thyroidectomy $(\mathrm{N}=23)$, total thyroid ablation $(\mathrm{N}=10)$ or L-thyroxine $(\mathrm{N}=2)$, and for $\mathrm{GO}$ with glucocorticoids $(\mathrm{N}=82)$, orbital radiotherapy $(\mathrm{N}=40)$, orbital decompression $(\mathrm{N}=29)$, squint $(\mathrm{N}=12)$ or palpebral $(\mathrm{N}=6)$ surgery, alone or associated. Patients underwent an ophthalmological examination and were asked to fill a questionnaire on self-perception related to GO. GO was considered disappeared when all of the following criteria were fulfilled: objective criteria: i) exophthalmos $\leq 19 \mathrm{~mm}$ in men and $\leq 17 \mathrm{~mm}$ in women; ii) clinical activity score $\leq 1 / 7$ points; iii) absence of diplopia; subjective criteria (questionnaire): i) perception of both eyes identical to the way they were before GO appeared; ii) perception of both eyes as normal; iii) no eye-related limitation in daily activities; iv) no eye-related limitations in social life.

Results: In two patients (2\%) all criteria were fulfilled and they were considered GO-free. In 8 patients (8\%) only objective and in 24 patients $(24.2 \%)$ only subjective criteria were fulfilled. The latter did not correlate with sex, age, GO duration, smoking, thyroid and GO treatment.

Conclusions: GO is a chronic disease in the vast majority of patients. Even after a very long time since its onset complete disappearance is rare, although a minority of patients believe they do not have GO anymore and an even lower proportion do not have relevant GO signs. Our findings have obvious implication in patient management and counseling.

\section{OP-02-09 \\ MONTH OF BIRTH MAY AFFECT THE SUBSEQUENT RISK OF BEING DIAGNOSED WITH AUTOIMMUNE HYPOTHYROIDISM. A NATIONWIDE DANISH REGISTER-BASED STUDY \\ Marianne Thvilum ${ }^{1}$, Frans Brandt ${ }^{2}$, Thomas Brix ${ }^{3}$, Laszlo Hegedüs ${ }^{4}$ \\ ${ }^{1}$ Odense University Hospital, Department of Endocrinology and \\ Metabolism, Odense C, Denmark, ${ }^{2}$ Hospital of Southern Denmark, Sonderborg, Denmark, ${ }^{3}$ Department of Endocirnology, Odense University Hospital, Odense University Hospital, Odense C, Denmark, ${ }^{4}$ Department of Endocrinology and Metabolism, Odense University Hospital, Odense, Denmark}

Objectives: The triggering of autoimmunity in the genetically susceptible remains a conundrum. Infections during gestation and postnatally have proponents, as suggested in diabetes mellitus, with a higher incidence of births during the spring and summer. Whether the development of autoimmune hypothyroidism is influenced by month of birth is less clear, but it has been suggested that subjects born during the autumn have an increased risk of Hashimoto's thyroiditis.

Methods: Observational cohort study. 117,239 individuals diagnosed (from 1996-2012) with a first episode of autoimmune hypothyroidism and non-hypothyroid controls, matched 1:4 according to age and sex, were identified from nationwide Danish health registers. The birth incidence rates for all months and the four seasons in hypothyroid cases and controls were compared by a $\mathrm{Chi}^{2}$-test.

Results: For the winter season (December-February) there was no significant difference in birth incidence rates (BI) between cases and controls (BI cases: 0.243 , controls: $0.241, \mathrm{P}=0.85$ ), likewise for the summer (June-August; BI cases: 0.251 , controls: $0.250, \mathrm{P}=0.41$ ). In the spring (March-May) there was a non-significant trend towards a higher $\mathrm{BI}$ among cases as compared to controls (BI cases: 0.273, controls: $0.270, \mathrm{P}=0.059$ ). In contrast, in the autumn (September-November) the BI among cases was significantly lower as compared to controls (BI cases: 0.233, BI controls: 0.239, $\mathrm{P}<0.001$ ).

Conclusion: In this large scale nation-wide cohort study we found, in contrast to others, a lower risk of autoimmune hypothyroidism when born in the autumn, supporting the hypothesis that seasonal variations in exposures - during gestation and/or early postnatally - may contribute to the development of autoimmune hypothyroidism.

\section{OP-02-10 \\ ASSOCIATION BETWEEN THE TIMESPAN UNTIL RADIOIODINE THERAPY AND THE DURATION OF GRAVES' ORBITOPATHY ACTIVITY}

Simona Gaberscek ${ }^{1}$, Daša Šfiligoj ${ }^{2}$, Katja Zaletel ${ }^{2}$, Edvard Pirnat ${ }^{2}$,

Polona Jaki Mekjavić ${ }^{3}$

${ }^{1}$ Department of Nuclear Medicine, University Medical Centre Ljubljana, Faculty of Medicine, University of Ljubljana, Faculty of Medicine,

University of Ljubljana, Ljubljana, Slovenia, Ljubljana, Slovenia,

${ }^{2}$ Department of Nuclear Medicine, University Medical Centre Ljubljana,

Ljubljana, Slovenia, ${ }^{3}$ University Eye Hospital, University Medical Centre Ljubljana, Faculty of Medicine, University of Ljubljana, Ljubljana,

Slovenia

Objectives: Radioiodine (RAI) is a safe and effective definitive treatment of Graves' disease (GD). In patients with concomitant active Graves' orbitopathy (GO), special caution is usually recommended. Our aim was to retrospectively evaluate the association between the timespan until RAI therapy and the duration of GO activity.

Methods: We reviewed medical records of all patients who were first diagnosed with GD between 2005 and 2009 and later treated with RAI. Out of 724 patients, 154 patients had mild or moderate-to-severe active GO. Immediately after RAI, all but two patients received glucocorticoids in various regimens. The course of GO was followed until GO became inactive.

Results: The median age of 124 women and 30 men with the active GO was 45 years (range, $16-75$ years). The median duration of GO activity was 8 months (range, 2-24 months). The median timespan until RAI was 7 months (range, 1-48 months). The duration of GD activity was significantly shorter in patients who received RAI therapy up to 6 months after GD occurrence than in patients who received RAI later (median, 7 and 10 months, respectively, $\mathrm{p}=0.032$ ). We found a significantly positive correlation between the timespan until RAI and the duration of GO activity $(\mathrm{R}=0.206, \mathrm{p}=0.010)$. Furthermore, with multiple regression analysis, the timespan until RAI was significantly associated with the duration of GO activity $(\mathrm{p}=0.014)$, while other parameters such as thyroid volume, concentration of TSH, free $\mathrm{T}_{4}$, free $\mathrm{T}_{3}$, TSH receptor antibodies, and smoking did not show such association.

Conclusions: Our results reveal the beneficial effect of the timely RAI therapy on the course of GO, since the shorter the time until RAI therapy the shorter the duration of GO activity. It seems that in patients with active GO "the sooner the better" principle as regards RAI therapy should be considered.

\section{OP-02-11 \\ AUTOIMMUNE THYROID DISEASE AND FRACTURE RISK}

Dragana Miljic ${ }^{1}$, Sladjana Zivojinovic ${ }^{2}$, Natasa Milic ${ }^{3}$, Vera Popovic ${ }^{4}$, Dragan Micic ${ }^{5}$

${ }^{1}$ Clinic for Endocrinology, Diabetes and Metabolic Diseases, Clinical Center of Serbia, Belgrade University School of Medicine, Serbia, Clinical Center of Serbia, Belgrade, Serbia, Medical Faculty University of Belgrade, Beograd, Serbia, ${ }^{2}$ Institute for Rheumatology, Belgrade, Serbia, ${ }^{3}$ nsitute for Statisctis and Nmedical Informatics, Medical Faculty University of Belgrade, Belgrade, Serbia, ${ }^{4}$ School of Medicine, University of Belgrade, Belgrade, Serbia, ${ }^{5}$ Beograd-Klinika Za Endokrinologjiu, Dijabetes I Bolesti Metabolezma, Interna B, Belgrade, Serbia

Thyroid hormones have impact on skeletal development, linear growth, cartilage biology and bone turnover and thyroid dysfunction affects the skeleton quality. Autoimmune thyroid disease may be an addition contributing factor to impaired bone quality in these patients. 
Materials and Methods: Measurement of TPOAb, TSH, FT4, DXA were performed in 189 postmenopausal women (110 euthyroid women and 79 women diagnosed with subclinical hypothyroidism) divided into subgroups according to the presence of TPOAb. Fragility fracture risk was estimated by FRAX score calculations.

Results: In multivariate logistic regression analysis only TPOAb were found to be significantly related to fracture, independently of TSH values $(\mathrm{p}=0.018 ; \mathrm{OR}=7.800 ; 95 \% \mathrm{CI} 1.424-42.721)$. Lower bone mineral density and FRAX score for hip and for major osteoporotic fractures were associated with the presence of TPOAb in euthyroid postmenopausal women in an unadjusted logistic regression model, as well as in a model adjusted for age, BMI and smoking. TSH was a stronger predictive factor for fractures in women with subclinical hypothyroidism (FRAX main $\mathrm{p}<0.001 ; 95 \%$ CI for SE $0.858-0.959$ and FRAX hip $\mathrm{p}<0.001 ; 95 \% \mathrm{CI}$ for SE $0.628-0.854$ ) than TPOAb.

Conclusion: In euthyroid postmenopausal women autoimmune thyroid disease is associated with lower BMD and risk for fragility fracture. Presence of TPOAb is a potential marker of higher fracture risk in these patients. However, in subclinical hypothyroidism, TSH is a better indicator of future fragility fractures than TPOAb.

\section{OP-02-12 \\ DISEASE PRESENTATION AND REMISSION RATE IN GRAVES' DISEASE TREATED WITH ANTI-THYROID DRUGS: IS GENDER REALLY A FACTOR? \\ Talia Diker-Cohen ${ }^{1}$, Hadar Duskin-Bitan ${ }^{1}$, Gloria Tsvetov', Ilan \\ Shimon $^{3}$, Amit Akirov' ${ }^{1}$, Dania Hirsch ${ }^{1}$, Eyal Robenshtok ${ }^{1}$ \\ ${ }^{1}$ Endocrinology \& Metabolism Institute, Rabin Medical Center, Beilinson \\ Hospital, Petah-Tikva, Israel, ${ }^{2}$ Endocrinology \& Metabolism Institute, \\ Rabin Medical Center, Beilinson Hospital, Petah Tikwa, Israel, ${ }^{3}$ Rabin \\ Medical Center, Petah-Tiqva, Israel}

Introduction: Male gender has been associated with lower rates and shorter duration of remission of Graves' disease after treatment with anti-thyroid drugs (ATDs). This resulted in early consideration of alternative therapies in men, such as radioiodine ablation and surgery.

Objective: To study disease presentation and treatment outcomes in male and female patients with Graves' disease.

Methods: Patients treated between 2010 and 2015 for Graves' disease at an outpatient endocrinology clinic in a tertiary hospital were included.

Results: Records of two-hundred thirty five patients (64 men, 171 women) were analyzed. The groups did not differ in age at diagnosis $(41.4 \pm 14$ vs $40 \pm 15)$, duration of follow up ( $6.6 \pm 7$ vs $7.7 \pm 6$ years), rate of co-morbid autoimmune diseases ( $13 \%$ vs $12 \%)$, rate of Graves' ophthalmopathy $(31 \%$ vs $28 \%$ ), laboratory values of free $\mathrm{T} 3(16.8 \pm 6$ vs $15.6 \pm 7 \mathrm{nmol} / \mathrm{L})$, or presence of anti-TSH receptor antibodies ( $90 \%$ vs $85 \%)$. Smoking was more prevalent among males $(31 \%$ male vs. $15 \%$ female, $\mathrm{p}=0.009)$. Levels of free $\mathrm{T} 4$ at presentation were slightly higher among males $(46.9 \pm 21.9$ vs. $40.7 \pm 17.5$ $\mathrm{pmol} / \mathrm{L}, \mathrm{p}=0.06$ ). All male patients and 168 of 171 female patients were given ATDs as first line therapy for a median time of 24 and 20 months, respectively $(\mathrm{p}=0.55)$. Remission rates were $47 \%$ in males and $58 \%$ in females $(\mathrm{p}=0.14)$. Males had lower rates of adverse effects ( $9 \%$ vs. $18 \%$ ) and discontinuation of ATD treatment (5\% vs. 16\%). Recurrence of Graves' disease was comparable ( $14 \%$ vs $20 \%, p=0.32)$, as well as requirement of second line treatment, either radioiodine therapy or thyroidectomy ( $26 \%$ vs $29 \%)$.

Conclusion: Disease presentation in male and female patients with Graves' disease is similar. Given the comparable and high rates of remission after ATD among males and females, ATDs are an attractive first line treatment in Graves' disease for both genders.

\section{OP-02-13 \\ GRAVES' ORBITOPATHY IS ASSOCIATED WITH AN INCREASED RISK OF UNNATURAL DEATH}

Thomas Brix ${ }^{1}$, Charlotte Ferløv-Schwensen ${ }^{2}$, Laszlo Hegedüs ${ }^{3}$

${ }^{1}$ Department of Endocrinology, Odense University Hospital, Odense University Hospital, Odense C, Denmark, ${ }^{2}$ Odense Universty Hospital, Odense, Denmark, ${ }^{3}$ Department of Endocrinology and Metabolism,

Odense University Hospital, Odense, Denmark

Objective: Graves' disease is associated with excess mortality, but little is know about manner of death and the specific contribution of Graves' orbitopathy. Here we investigate the risk and manners of unnatural death in Graves' patients with orbitopathy (GO) and without (GD), compared to the background population.

Methods: Observational cohort study covering all adult Danes ( $\geq 18$ years) diagnosed with GD or GO during 1995-2012. Median follow-up time was 7.9 years (range, 0-17.5). Utilizing the Danish Register of Causes of Death and the Danish National Patient Registry, 28,461 subjects with GD, and 3,965 with GO were identified and matched for age and gender with four subjects from the background population. Manner of death was identified and hazard ratios (HR) for mortality due to unnatural death (accident, suicide, violence/homicide, and unknown) were calculated using Cox regression analyses, adjusted for pre-existing morbidity.

Results: After adjustment for pre-existing morbidity, there was no significant difference in overall risk of unnatural death between GD subjects and their controls [HR 1.07 (95\% confidence interval: 0.91-1.25), $\mathrm{p}=0.439$ ]. However, GO cases had a significantly higher risk of suicide [HR 2.73 (1.176.39); $\mathrm{p}=0.020$,]. Comparing manners of unnatural death between GD and $\mathrm{GO}$, showed a higher risk of suicide in GO patients both before and after adjusting for age, gender and pre-existing morbidity [HR 2.09 (1.03-4.24) and 2.02 (0.99-4.12), respectively].

Conclusion: There was a significantly increased mortality of unnatural manner, mainly by suicide, in GO but not in GD-patients. Reasons for this, e.g. use of steroids for therapy, need to be explored.

\section{OP-02-14}

\section{ALEMTUZUMAB-INDUCED THYROID AUTOIMMUNITY: BIOLOGICAL CHARACTERISATION OF AUTOANTIBODIES TO THE THYROTROPIN RECEPTOR, AND POSSIBLE ROLE AS PREDICTIVE MARKER OF DISEASE}

Ilaria Muller ${ }^{1}$, Mark Willis², Sarah Healy², Taha Nasser ${ }^{2}$, Lei Zhang ${ }^{1}$, Mohd Shazli Draman ${ }^{1}$, Peter Taylor ${ }^{1}$, Neil Robertson ${ }^{2}$, Colin Dayan ${ }^{1}$, Marian Ludgate

${ }^{1}$ Thyroid Research Group, Division of Infection \& Immunity, School of Medicine, Cardiff University, Cardiff, UK, ${ }^{2}$ Division of Psychological Medicine and Clinical Neurosciences, School of Medicine, Cardiff University, Cardiff, UK

Objectives: Alemtuzumab (anti-CD52; ALTZ), an effective therapy for relapsing/remitting multiple sclerosis (MS), causes panlymphopenia with subsequent lymphocyte repopulation; $30-40 \%$ of patients develop secondary humoral autoimmunity, mainly thyroidal.

Anti-thyrotropin-receptor (TSHR) autoantibodies (TRAb) can stimulate (TSAb), block (TBAb) or not affect ("neutral") TSHR function, with TSAb causing hyperthyroid Graves' disease (GD), and TBAb hypothyroidism. Lowaffinity neutral TRAb could pre-exist in MS patients, then undergo somatic hypermutation to become high-affinity TSAb/TBAb post-ALTZ, causing thyroid dysfunction

Methods: Sera from MS patients (Welsh Neuroscience Research Tissue Bank), 11 developing post-ALTZ thyroid autoimmunity (TA; 10 GD, 1 hypothyroidism) and 14 not developing it (NO-TA), evaluated at different timepoints: 1) pre-ALTZ, 2) post-ALTZ before disease onset (TA) or latest time post-ALTZ (NO-TA), 3) post-ALTZ during/after thyroid dysfunction onset (TA only).

Flow cytometry (FC) detected any TSHR-binding TRAb. Luciferase bioassays (LB) detected both TRAb presence and bioactivity (neutral/TSAb/ $\mathrm{TBAb}$ ), also deduced from the corresponding thyroid function. TRAb positivity $(\mathrm{TRAb}+)$ was defined as FC and/or LB positivity. 
Results: See table.

\begin{tabular}{llll}
\hline $\begin{array}{l}\text { Time- } \\
\text { points }\end{array}$ & TRAb+ & $\begin{array}{l}\text { Fisher Exact } \\
\text { T-test }\end{array}$ \\
\cline { 2 - 4 } & TA & NO-TA & \\
\hline 1 & $3 / 11(27.3 \%)$ & $0 / 14(0 \%)$ & $\mathrm{p}=0.07$ \\
$1+2$ & $5 / 11(45.5 \%)$ & $4 / 14(28.6 \%)$ & $\mathrm{p}=0.43$ \\
3 & $8 / 11(72.7 \%)$ & NA & NA \\
\hline
\end{tabular}

$\mathrm{NA}=$ Not Applicable

Among overall TRAb+ cases (all time-points considered), TBAb were $2 / 7$ $(28.6 \%)$ in GD, $1 / 1(100 \%)$ in hypothyroidism, and 3/4 (75\%) in NO-TA.

Conclusions: A) Patients who developed TA tended to be TRAb+ prior to ALTZ. Thus TRAb could provide a predictive marker of future TA. B) TRAb+ patients were euthyroid at time-points 1-2, suggesting low-affinity antibodies unable to affect thyroid function. C) Post-ALTZ TBAb subtype is common.

\section{Oral Session 3 (Basic): Cancer - Scientific Aspects}

\section{OP-03-15 \\ ANALYSIS OF THE BIOLOGICAL MECHANISMS DE-REGULATED AFTER PHARMACOLOGICAL BET INHIBITION IN ANAPLASTIC THYROID CANCER Cells}

$\underline{\text { Catia Mio }}^{1}$, Federica Baldan ${ }^{2}$, Lorenzo Allegri ${ }^{1}$, Diego Russo ${ }^{3}$, Jelena Milasin ${ }^{4}$, Milos Lazic ${ }^{4}$, Giuseppe Damante

${ }^{1}$ Department of Medical Area, University of Udine, Udine, Italy, ${ }^{2}$ Department of Internal Medicine and Medical Specialties, University 'Sapienza', Rome, Italy, ${ }^{3}$ Department of Health Sciences, University of Catanzaro "Magna Graecia", Catanzaro, Italy, ${ }^{4}$ Department of Human Genetics, School of Dental Medicine, University of Belgrade, Serbia

BET inhibitors (BETi) are an innovative class of epigenetic anti-cancer drugs, targeting the Bromodomain and Extra-Terminal (BET) proteins. The latter bind to specific histone acetyl groups modulating gene transcription. The aim of this study is to delineate the chief pathways underlying the biological effects derived from BET inhibition, in order to better understand their antineoplastic potential. BET inhibition effects were evaluated in terms of different biological outputs, in diverse anaplastic thyroid carcinoma (ATC)-derived cell lines, used as a model of highly aggressive cancer subtype. The treatment with three BETi (JQ1, I-BET762 and I-BET151) decreased cell viability, increased the proportion of cells stacked in G0-G1 phases and determined an increase in cell death phenomena. In order to find BETi effectors, a RNA-seq was performed, highlighting that a significant portion of the deregulated genes belongs to cell cycle regulators. Among them, MCM5 was down-regulated at both mRNA and protein levels. MCM5 silencing reduced cell proliferation, underlining its involvement in the block of proliferation induced by BETi. Nowadays, many reports showed that miRNA deregulation could contribute to several diseases, including cancer. Hitherto, data concerning the relationship between BET inhibition and miRNA expression are very scanty. Therefore, a second goal of this study was to highlight a possible JQ1-derived miRNA regulation. JQ1 treatment altered the expression of several miRNAs, 7 of which turned out to be commonly deregulated in ATC cells after treatment. Among them, has-miR-4516, already described as involved in apoptotic phenomena, turned out to be strikingly upregulated in JQ1 treated ATC cells. Thus, these data hypothesize that modulation of miRNA expression is one of the multiple mechanisms of BETi action in thyroid cancer cells. Taken together, these data highlighted the multi-target output due to BET inhibition. Moreover, they suggest a possible usage of BET inhibitors in the management of anaplastic thyroid cancer.

\section{OP-03-16 \\ INVESTIGATION OF THE ROLE OF \\ OSTEOPONTIN IN CALCIFICATION AND MATRIX DEPOSITION IN THYROID CANCER}

Luciana Ferreira ${ }^{1}$, Raquel T. Lima ${ }^{2}$, Andreia Machado Silva ${ }^{3}$, Ana Pestana ${ }^{4}$, Catarina Tavares ${ }^{4}$, Elisabete Rios ${ }^{5}$, Catarina Eloy ${ }^{6}$, Manuel Sobrinho-Simões ${ }^{7}$, Etel Gimba ${ }^{8}$, Paula Soares ${ }^{2}$

${ }^{1}$ Instituto Nacional de Câncer - Inca, Institute of Molecular Pathology and Immunology of the University of Porto (Ipatimup), Rio de Janeiro, Brazil, ${ }^{2}$ I3s-Instituto de Investigação e Inovação Em Saúde, Institute of Molecular Pathology and Immunology of the University of Porto (Ipatimup), Medical Faculty, University of Porto, Porto, Portugal, ${ }^{3} / 3 s$ Instituto de Investigação e Inovação Em Saúde, Ineb - Instituto de Engenharia Biomédica, Icbas - Instituto de Ciências Biomédicas Abel Salazar Da Universidade Do Porto, Porto, Portugal, ${ }^{4} \mid 3 s-I n s t i t u t o ~ d e$ Investigação e Inovação Em Saúde, Institute of Molecular Pathology and Immunology of the University of Porto (Ipatimup), Porto, Portugal, ${ }^{5}$ Institute of Molecular Pathology and Immunology of the University of Porto (Ipatimup), Medical Faculty, University of Porto, Department of Pathology, Hospital de S. João, Porto, Portugal, ${ }^{6}$ Institute of Molecular Pathology and Immunology of the University of Porto

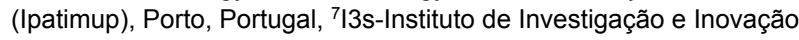
Em Saúde, Universidade Do Porto, Institute of Molecular Pathology and Immunology of the University of Porto (Ipatimup), Department of Pathology, Hospital de S. João, Porto, Portugal, ${ }^{8}$ Instituto Nacional de Câncer-Inca, Universidade Federal Fluminense-Uff, Rio de Janeiro, Brazil

Background: Osteopontin (OPN) and its three spliced variants (OPN-SV: $\mathrm{OPNa}, \mathrm{OPNb}$ and $\mathrm{OPNc}$ ) are overexpressed in several tumors and frequently associated with cancer progression. This holds true for papillary thyroid carcinoma (PTC) which is the most common variety of thyroid cancer (TC) being the histologic type which often presents desmoplasia (collagen deposition) and dystrophic calcification, including a fairly typical feature, the psammoma bodies (PB). The aim of this study was to investigate the role of OPN-SV expression in the calcification and in classical variant of PTC (cPTC).

Methods: Total OPN and OPN-SV expression was analyzed by immunohistochemistry and real time PCR in a series of $48 \mathrm{cPTC}$ cases and three diffuse sclerosing PTCs. The association of OPN expression and the presence of PB as well as between PB in cPTC and the clinicopathological features of the tumors were evaluated. TPC-1 and c643 TC cell lines overexpressing OPN-SV were tested for the ability to promote calcification and to synthesize collagen in vitro.

Results: Overexpression of OPNa transcripts was significantly associated with the presence of PB in cPTC samples. The presence of PB in $\mathrm{cPTC}$ was associated with younger patients and lymph node metastasis. Moreover, OPNa overexpression displayed a strong capacity to promote calcification and substantial collagen synthesis in thyroid cancer cell lines.

Conclusion: Our data suggest that OPNa plays a role in the formation of calcification often associated with cPTC. Basic research on the interactions between OPNa overexpression by tumor cells and the surrounding microenvironment can give clues for a better understanding of cPTC biology and phenotype. 


\section{OP-03-17 \\ EGFR-TARGETED NONVIRAL NIS GENE TRANSFER FOR BIOIMAGING AND THERAPY OF DISSEMINATED COLON CANCER METASTASES}

Sarah Urnauer ${ }^{1}$, Andrea Müller ${ }^{1}$, Christina Schug ${ }^{1}$, Kathrin SchmohP, Mariella Tutter ${ }^{2}$, Nathalie Schwenk ${ }^{2}$, Wolfgang Röd ${ }^{3}$, Stephan Morys ${ }^{3}$, Michael Ingrisch ${ }^{4}$, Jens Bertram ${ }^{5}$, Peter Bartenstein ${ }^{6}$, Dirk-André Clevert ${ }^{7}$, Ernst Wagner ${ }^{3}$, Christine Spitzweg ${ }^{8}$

${ }^{1}$ Department of Internal Medicine II, University Hospital of Munich; Medizinische Klinik und Poliklinik II, Munich, Germany, ${ }^{2}$ Medical Policlinic IV, University Hospital of Munich, LMU Munich, Germany; Medical Policlinic IV, Munich, Germany, ${ }^{3}$ Department of Pharmacy, Center of Drug Research, Pharmaceutical Biotechnology; LudwigMaximilians-University, Munich, Germany, ${ }^{4}$ Department of Clinical Radiology; University Hospital, Munich, Germany, ${ }^{5}$ Department of Nuclear Medicine, Radiopharmacy, Klinikum Rechts der Isar der Technischen Universität, Munich, Germany, ${ }^{6}$ Ludwig-MaximiliansUniversität; Universitätsklinikum München; Klinik und Poliklinik für Nuklearmedizin, Munich, Germany, ${ }^{7}$ Institut für Klinische Radiologie, Munich, Germany, ${ }^{8}$ Department of Internal Medicine II, University Hospital of Munich, Germany; Medizinische Klinik und Poliklinik li; Medizinische Klinik und Poliklinik II, Munich, Germany

Liver metastases present a serious problem in the therapy of advanced colorectal cancer (CRC), as more than $20 \%$ of patients have distant metastases at the time of diagnosis with less than $5 \%$ of patients being cured. Consequently, new curative approaches are of major need together with highresolution imaging methods that allow detection of small metastasis in a high specific manner.

The unique combination of reporter and therapy gene function of the sodium iodide symporter (NIS) constitutes an outstanding tool to target different cancer cells allowing non-invasive imaging of functional NIS expression and therapeutic application of ${ }^{131} \mathrm{I}$ and may represent a promising theranostic strategy for CRC metastases.

For targeted NIS gene transfer, polymers containing linear polyethylenimine (LPEI), polyethylene glycol (PEG) and the synthetic peptide GE11 as epidermal growth factor receptor (EGFR)-specific ligand were complexed with human NIS plasmid DNA (LPEI-PEG-GE11/NIS). Tumor specificity and transduction efficiency were first examined in high EGFR-expressing LS174T metastases by non-invasive diagnostic imaging using ${ }^{18} \mathrm{~F}$-tetrafluoroborate (TFB) as novel NIS PET tracer. Mice that were injected intravenously with LPEI-PEG-GE11/NIS $48 \mathrm{~h}$ before ${ }^{18} \mathrm{~F}$-TFB application showed high tumoral levels $(4.8 \pm 0.6 \%$ of injected dose (ID)) of NIS-mediated radionuclide uptake in comparison to low levels detected in mice that received untargeted control NIS polyplexes. Three cycles of i.v. injection of EGFR-targeted NIS polyplexes followed by therapeutic application of $55.5 \mathrm{MBq}{ }^{131} \mathrm{I} 48 \mathrm{~h}$ later resulted in a marked delay in metastases spread in mice compared to mice that received saline, as determined by contrast-enhanced sonography. This was associated with improved survival.

In conclusion, our preclinical data confirm the enormous potential of EGFR-targeted synthetic polymers for systemic NIS gene delivery in an advanced multifocal colon cancer liver metastases model and open the exciting prospect of NIS-mediated radionuclide therapy in metastatic disease.

\section{OP-03-18 \\ MASSARRAY TECHNOLOGY FOR THE MUTATIONAL PROFILING OF PAPILLARY THYROID CANCER}

Marina Muzza' ${ }^{1}$, Carla Colombo ${ }^{2}$, Chiara Pesenti ${ }^{3}$, Maria Carla Proverbio $^{4}$, Silvia Tabano ${ }^{5}$, Laura Fugazzola ${ }^{6}$

${ }^{1}$ Endocrine Unit, Fondazione Irccs Ca' Granda-Ospedale Maggiore Policlinico, Milan, Italy, ${ }^{2}$ Division of Endocrine and Metabolic Diseases, Istituto Auxologico Italiano Irccs, Milan, Italy, Milan, Italy, ${ }^{3}$ Unit of Pathology, Fondazione Irccs Ca' Granda-Ospedale Maggiore Policlinico, Milan, Italy, ${ }^{4}$ Department of Pathophysiology and Transplantation, University of Milan, Milan, Italy, ${ }^{5}$ Department of Pathophysiology and Transplantation, University of Milan, Unit of Pathology, Fondazione Irccs Ca' Granda-Ospedale Maggiore Policlinico, Milan, Italy, ${ }^{6}$ Department of Pathophysiology and Transplantation, University of Milan, Division of Endocrine and Metabolic Diseases, Irccs Istituto Auxologico Italiano, Milan, Italy

Different oncogenic events have been described in papillary thyroid cancer (PTC), and some of them are considered hallmarks of the disease and are targeted in clinical trials. The Sequenom Mass ARRAY (SMA) technology is a mass spectrometry technique able to detect multiple mutations in the same assay. We aimed to investigate the mutational profile of a large series of 146 PTCs by a custom SMA set up to identify the presence of the most common somatic events. Three different assays were designed to interrogate 15 point mutations in 8 genes (BRAF, H-RAS, N-RAS, K-RAS, EIF1AX, AKT1, PIK3CA, TERTp) in DNA and 6 rearrangements (RET-PTC1, 2 and 3, TRK, TRKT1 and TRKT3) in cDNA. At last one variant was identified in 109/146 $(74.6 \%)$ PTCs. In particular, BRAF ${ }^{\mathrm{V} 600 \mathrm{E}}$ was detected in $45 \%$, TERTp in $25 \%$, codon $61 \mathrm{H}$ - and N-RAS in $6.8 \%$, RET/PTC in $15 \%$ and TRK in $2.7 \%$ of cases. Two or more mutations were found in $19.8 \%$ of PTCs. In patients with $\mathrm{BRAF}^{\mathrm{V} 600 \mathrm{E}}+$ TERTp mutations the stage at diagnosis was significantly higher than in wild-type tumors $(\mathrm{P}=0.003)$. Consistent with findings of the Thyroid Cancer Genome Atlas, the existence of a BRAF-like and a RAS-like population was found, being the latter mostly constituted by follicular variant PTC with a high differentiation and probability of remission. The allelic frequency of each mutation was also evaluated: the most frequent mutations were mostly subclonal (mean cancer cell fraction with mutation: 53\% for BRAF, $63 \%$ for TERT and $63 \%$ for RAS). In conclusion, our custom Sequenom Mass ARRAY allows to genotype PTCs in a fast and economic (around 30 Euros/patient) way. The evaluation of the mutation profile has a major impact in selecting the best first treatment, in predicting the prognosis and the efficacy of target drugs, in view of a personalized therapy. 


\section{OP-03-19 \\ UNREVEALING METASTATIC DORMANCY IN THYROID CANCER: DIFFERENCES IN THE GENOTYPE BETWEEN SYNCHRONOUS AND METACHRONOUS METASTASES}

Adriana Gaspar da Rocha ${ }^{1}$, Miguel Melo ${ }^{2}$, Rui Batista ${ }^{3}$, João Vinagre ${ }^{4}$, José Manuel Cameselle-Teijeiro ${ }^{5}$, Valeriano Leite ${ }^{6}$, Francisco Carrilho ${ }^{7}$ Manuel Sobrinho-Simões ${ }^{8}$, Paula Soares ${ }^{9}$

${ }^{1}$ Instituto de Investigação e Inovação Em Saúde (I3s), Institute of Pathology and Immunology of the University of Porto (Ipatimup), Public Health Unit, Aces Baixo Mondego, Coimbra, Portugal, ${ }^{2}$ Instituto de Investigação e Inovação Em Saúde (I3s), Institute of Pathology and Immunology of the University of Porto (Ipatimup), Department of Endocrinology, Diabetes and Metabolism, Centro Hospitalar e Universitário de Coimbra, Coimbra, Portugal, ${ }^{3}$ nstituto de Investigação e Inovação Em Saúde (I3s), Institute of Pathology and Immunology of the University of Porto (Ipatimup), Medical Faculty, University of Porto, Porto, Portugal, ${ }^{4}$ Instituto de Investigação e Inovação Em Saúde (I3s), Institute of Pathology and Immunology of the University of Porto (Ipatimup), Porto, Portugal, ${ }^{5}$ Department of Pathology, Clinical University Hospital, Sergas, Medical Faculty, University of Santiago de Compostela, Santiago de Compostela, Spain, ${ }^{6}$ Unit for Investigation of Molecular Pathobiology, Portuguese Institute of Oncology - Lisbon Center, Department of Endocrinology, Portuguese Institute of Oncology - Lisbon Center, Department of Endocrinology, Portuguese Institute of Oncology - Lisbon Center, Lisbon, Portugal, ${ }^{7}$ Department of Endocrinology, Diabetes and Metabolism, Centro Hospitalar e Universitário de Coimbra, Coimbra, Portugal, ${ }^{8}$ Department of Pathology and Oncology, Medical Faculty, University of Porto, Department of Pathology, Hospital S. João, Institute of Pathology and Immunology of the University of Porto (Ipatimup), Porto, Portugal, IInstituto de Investigação e Inovação Em Saúde (I3s), Institute of Pathology and Immunology of the University of Porto (Ipatimup), Department of Pathology and Oncology, Medical Faculty, University of Porto, Porto, Portugal

Introduction: Thyroid cancer is a model in which metastatic dormancy is a frequent phenomenon. The concordance of genotype between primary tumours and metastases may be influenced by the time elapsed between the initial diagnosis and the clinical emergence of metastatic lesions.

Objective: We aimed to evaluate the concordance of genotype between primary thyroid tumours, local and distant metastases, according to the time of detection of the metastatic lesion (synchronous or metachronous).

Material and Methods: We studied 230 pairs of primary thyroid tumours and metastatic lesions, including 190 local and 40 distant metastases. BRAF and TERT promoter (TERTp) mutation analysis was performed in both thyroid tumours and metastatic lesions.

Results: We found the following mutation frequency in primary thyroid tumours, local metastases and distant metastases, respectively: $B R A F 42.5 \%$, $38.5 \%$, and $25.0 \%$; TERTp $11.3 \%, 7.3 \%$, and $37.5 \%$. For $B R A F$, the concordance of genotype between primary tumours and metastatic tissue was higher for synchronous lesions (kappa $=0.379 ; \mathrm{p}<0.001$ ) than for metachronous lesions $(\mathrm{kappa}=0.117 ; \mathrm{p}=0.463)$. The latter holds true whether the analysis was performed only in local metastases (synchronous lesions: kappa $=0.379$; $\mathrm{p}<0.001$; metachronous lesions: kappa $=-0.055 ; \mathrm{p}=0.820$ ) or in distant metastases (synchronous lesions: kappa $=0.64 ; \mathrm{p}=0.004$; metachronous lesions: kappa $=-0.112 ; \mathrm{p}=0.540$ ). For TERT $\mathrm{p}$, the concordance of genotype between primary tumours and metastatic tissue was similar and statistically significant for synchronous $(\mathrm{kappa}=0.195 ; \mathrm{p}<0.001)$ and for metachronous lesions $(\mathrm{kappa}=0.159 ; \mathrm{p}=0.016)$. However, we found heterogeneity between local metastases (higher concordance in synchronous lesions) and distant metastases (higher concordance in metachronous lesions).

Conclusions: In the natural history of metastatic thyroid tumours, $B R A F$ and TERTp mutations seem to play different roles. BRAF may be involved in more frequent local and synchronous metastases. TERTp mutations may be one of the major factors involved in distant dissemination and metastatic dormancy.

\section{OP-03-20 \\ AN INVESTIGATION INTO SODIUM-IODIDE SYMPORTER (NIS) DIMERISATION AND ITS IMPACT ON RADIOIODIDE UPTAKE IN THYROID CANCER}

Rebecca Thompson ${ }^{1}$, Alice Fletcher ${ }^{1}$, Hannah Nieto ${ }^{1}$, Mohammed Alshahrani', Katie Baker', Jonathan W. Mueller ${ }^{1}$, Nicholas H.F. Fine ${ }^{1}$ David J. Hodson ${ }^{1}$, Martin L. Read' ${ }^{1}$, Kristien Boelaert ${ }^{1}$, Vicki E. Smith ${ }^{1}$, Christopher J. McCabe ${ }^{1}$

${ }^{1}$ Institute of Metabolism and Systems Research, University of Birmingham, Birmingham, UK

The ability of the thyroid to accumulate iodide via the sodium-iodide symporter (NIS) can be utilised to successfully treat the majority of thyroid cancers with radioiodide. However, approximately $25 \%$ of thyroid cancers lose this functional NIS activity and become unresponsive to radioiodide therapy, resulting in a poorer prognosis. Our knowledge of NIS regulation is limited, but as dimerisation of NIS has been proposed, we sought to investigate NIS dimerisation and its impact on radioiodide uptake. Dimerisation of wild-type NIS was confirmed using proximity ligation assays (PLA) in both a thyroid (SW1736) and non-thyroid (HeLa) cell line. To further assess NIS dimerisation using Förster resonance energy transfer (FRET), novel constructs conjugated to one of the fluorescent proteins cerulean or citrine were created. FRET was increased in the presence of both fluorophore-conjugated NIS constructs compared to single expression ( $1.52 \pm 0.10$ vs $1.08 \pm 0.18, \mathrm{P}<0.0001)$, further validating NIS dimerisation. In order to identify residues potentially involved in dimerisation, a homology model of NIS structure was built based on that of the bacterial protein vSGLT using the modelling platform Phyre2. Using site-directed mutagenesis, we then mutated five residues identified from our homology model (D237A, Y242A, T243A, Q471 A and A525F), and two putative dimerisation motifs identified in the literature (a glycine zipper motif in transmembrane domain (TMD) 12, with key glycine residues mutated to valine, and a leucine zipper motif in TMD6, with key leucine residues mutated to alanine). PLA suggested that all mutants still retained the ability to dimerise, indicating that dimerisation involves multiple, or as yet undiscovered, residues. In summary, NIS dimerisation has been conclusively demonstrated using two discreet methodologies. Further work is ongoing to determine the critical residues, cellular localisation and regulation of NIS dimerisation and its impact on radioiodide uptake.

\section{OP-03-21 \\ PD-L1 AND PD-L2 EXPRESSION IS INCREASED IN THYROID CARCINOMAS}

Sonia Moretti ${ }^{1}$, Nicole Nucci ${ }^{1}$, Elisa Menicali ${ }^{1}$, Silvia Morelli ${ }^{1}$, Efisio

$\underline{\text { Puxeddu }}^{1}$

${ }^{1}$ University of Perugia, Department of Medicine, Perugia, Italy

PD-1 is expressed by activated T lymphocytes (TL) and, when it interacts with its ligands (PD-L1 and PD-L2), causes TL exhaustion characterized by loss of functions, such as IL-2 secretion and cytolytic activity, and impaired proliferative capacity. Different types of human cancer express PD-L1 and the blockade of this pathway is a milestone in cancer immune therapy.

PD-L1 and PD-L2 mRNA expression levels were evaluated by Q-PCR, in 90 papillary thyroid carcinomas (PTC), 11 medullary thyroid carcinomas (MTC), 5 anaplastic thyroid carcinomas (ATC) and in 6 human thyroid cancer cell lines (BcPap, TPC1, FTC133, 8505c, C643, Cal62). PD-L1 protein expression was analysed by IHC in a subgroup of PTC and ATC and in the cancer cell lines using an anti-PD-L1 antibody. To confirm the functional effect of PD-1 ligands expressed by tumor cells, 400,000 cells (BcPap, C643, Hek293) were seeded and co-coltured with 50,000 Jurkat cells (immortalized TL), activated with PHA and PMA. After 48h of co-colture, IL-2 was measured in cellular supernatants by ELISA assay.

PD-L1 mRNA expression resulted significantly higher in PTC (mean \pm SD: $2.30 \pm 1.70 ; \mathrm{p}<0.0001)$ and ATC (mean \pm SD: $3.37 \pm 2.65 ; \mathrm{p}=0.045$ ) compared to normal thyroid; while PD-L2 mRNA was significantly more expressed in $\mathrm{ATC}$ (mean \pm SD: 10.52 $\pm 7.32 ; \mathrm{p}=0.0036$ ). Consistently, when analysed by IHC, PD-L1 revealed a moderate immunostaining in PTC and a higher one in ATC. Evaluation of PD-L1 expression in human cancer cell lines, by Q-PCR and IHC, showed the highest levels in BcPap, C643 and 8505c cells. The co-coltures of Jurkat cells with thyroid cancer cell lines expressing PD-L1 
provoked a significant reduction of IL-2 production compared to co-cultures of Jurkat cells with Hek293 cells that did not express PD-L1 (BcPap -45.7\% $\mathrm{p}=0.0046 ;$ C643 $-54.7 \% \mathrm{p}=0.0096$ ). When Hek293 cells were transfected with PD-L1 and co-coltured with Jurkat cells, a significant reduction of IL-2 in cellular supernatant was observed $(-64.5 \% \mathrm{p}=0.011)$, confirming that the drop in IL-2 secretion was specifically mediated by PD-L1.

In conclusion, these data indicate that thyroid carcinomas (ATC $>$ PTC) express PD-L1 and PD-L2. As shown in the co-culture experiments, these ligands are involved in the functional exhaustion of TL and in the induction of immune tolerance. In particular, this study suggests that monoclonal antibodies, able to block the interaction between PD1 and its ligands, could provide a potential new therapeutic approach useful in advanced differentiated thyroid carcinomas and principally in ATC.

\section{OP-03-98 \\ REGIONAL HYPERTHERMIA FOR OPTIMIZATION OF EFFICACY AND TUMOR SELECTIVITY OF MESENCHYMAL STEM CELL (MSC)-MEDIATED SODIUM IODIDE SYMPORTER (NIS) GENE THERAPY}

Mariella Tutter ${ }^{1}$, Christina Schug ${ }^{2}$, Kathrin Schmohl', Nathalie Schwenk', Wouter Lokerse ${ }^{3}$, Lars H. Lindner ${ }^{4}$, Peter J. Nelson', Christine Spitzweg ${ }^{5}$

${ }^{1}$ Medical Policlinic IV, University Hospital of Munich, Munich, Germany, ${ }^{2}$ Department of Internal Medicine II, University Hospital of Munich, Germany, Medizinische Klinik und Poliklinik II, Munich, Germany, ${ }^{3}$ Medical Policlinic IV, University Hospital of Munich, LMU Munich, Germany, Medical Policlinic IV, Munich, Germany, ${ }^{4}$ Department of Internal Medicine III, University Hospital of Munich, Germany, Medizinische Klinik und Poliklinik III, Munich, Germany, ${ }^{5}$ Medizinische Klinik III, Munich, Germany, ${ }^{6}$ Department of Internal Medicine II, University Hospital of Munich, Germany, Medizinische Klinik und Poliklinik II, Medizinische Klinik und Poliklinik II, Munich, Germany

The tumor homing capacity of mesenchymal stem cells (MSCs) provides the basis for a "Trojan horse" therapy approach in which MSCs act as nonviral gene transfer vectors to transport a therapeutic agent specifically into the tumor stroma. Due to its dual role as reporter and therapy gene, the sodium iodide symporter (NIS) allows detailed molecular imaging of transgene expression and highly effective application of therapeutic radionuclides. To improve the effectiveness and tumor selectivity of MSC-mediated gene therapy, we are investigating the combination of regional hyperthermia with NIS gene therapy

To this end, we established human immortalised MSCs stably expressing the NIS gene under the control of the heat-inducible HSP70B promoter (HSP70B-NIS-MSCs). Functional NIS expression was analysed in vitro using an iodide uptake assay. The HSP70B promoter showed low basal activity and was induced significantly in response to heat. Heat treatment in a water bath at $41^{\circ} \mathrm{C}$ for 60 minutes revealed a nearly 50 -fold increase in 125I uptake compared to untreated HSP70B-NIS-MSCs. Uptake was sensitive to the NIS-specific inhibitor perchlorate, demonstrating NIS dependency. Induction occurred rapidly and reached maximum levels as early as 4 hours after heat shock. Analysis of NIS mRNA by RT-PCR and NIS protein by immunofluorescence staining confirmed heat-induced NIS expression in HSP70B-NIS-MSCs.

In summary, we have established a stable MSC line armed with a heatinducible NIS construct for tumor-specific gene therapy. The combination with regional hyperthermia enables us to control the activation of the therapeutic NIS gene locally as well as temporally. As a next step, we will evaluate potential additive or synergistic therapeutic effects of hyperthermic treatment on NIS-mediated radioiodine therapy in vivo based on its own anticancer effects and its well characterized radio- and chemosensitizing effect.

\section{Oral Session 4 (Clinical): Cancer - Clinical and Diagnostic Aspects}

\section{OP-04-22 \\ 18F-DOPA PET/CT ASSESSMENT OF CERVICAL LYMPH NODE EXTENSION IN PATIENTS WITH MEDULLARY THYROID CARCINOMA}

Lavinia Vija Racaru ${ }^{1}$, Mathilde Bauriaud ${ }^{2}$, Anne-Sophie Salabert ${ }^{3}$, Lawrence O. Dierckx $x^{4}$, Erwan Gabiache ${ }^{5}$, Grunenwald Solange ${ }^{5}$, Pierre Payoux $^{5}$, Philippe Caron ${ }^{7}$, Frederic Courbon ${ }^{5}$, Slimane Zerdoud ${ }^{7}$ ${ }^{1}$ Université Paul Sabatier Toulouse; Chu Toulouse; luct-Oncopole, Toulouse, France, ${ }^{2}$ Institut Claudius Regaud; Radiopharmacy, Toulouse, France, ${ }^{3} \mathrm{Chu}$ Toulouse; Inserm, Toulouse, France, ${ }^{4}$ luctOncopole, Toulouse, France, ${ }^{5} \mathrm{Chu}$ Larrey; Service D' Endocrinologie, Toulouse, France, ${ }^{6} \mathrm{Chu}$ Larrey; 7 eme Etage/Chu Rangueil, Toulouse, France, ${ }^{7}$ Institt Universitaire du Cancer Toulouse Oncopole; Nuclear Medicine, Toulouse, France

Background: Medullary thyroid carcinoma (MTC) is a thyroid neuroendocrine tumor with frequent lymph node extension and recurrence. Patients with post-operative high calcitonin $(\mathrm{Ct})$ levels necessitate lymph node ( $\mathrm{LN})$ extensive staging for adequate cervical surgical management. Conventional neck imaging (ultrasound, CT) can fail to localise cervical disease. 18F-DOPA PET/CT (FDOPAPET) seems to be a useful functional imaging examination for detection of persistent or recurrent cervical disease.

Aim: To assess the utility of FDOPAPET on cervical lymph node metastatic MTC staging.

Material and Methods: Twenty-seven MTC patients (16 men, 11 women, median age 54 years), with increased post-operative $\mathrm{Ct}$ levels (median $270 \mathrm{ng} / \mathrm{ml}$ [28-9100]) were prospectively explored with FDOPAPET $(\mathrm{n}=30$ exams), cervical CT $(\mathrm{n}=17)$ and neck US $(\mathrm{n}=26)$. Pathologic neck lesions were surgically removed, when possible ( $\mathrm{n}=67$ lesions, 31 tumoral LN; $4 \mathrm{pts}$ ). FDOPAPET sensitivity for pathologic lymph node detection was assessed comparatively to histopathological findings.

Results: FDOPAPET presented a good concordance with neck ultrasound $(\mathrm{K}=0.71)$ and cervical $\mathrm{CT}(\mathrm{K}=0.6)$. On patient analysis, FDOPAPET showed higher sensitivity than cervical ultrasound $(52 \%$ vs $46 \%)$ for LN detection. On lesion analysis, compared to histological findings, FDOPAPET was superior to cervical ultrasound for the characterisation of suspect cervical LN (Se: $38.7 \%$ vs $16 \%$ ). On Ct level analysis, for serum Ct levels below $150 \mathrm{pg} /$ $\mathrm{mL}$ (10 patients) FDOPAPET sensitivity was $50 \%$ (exam positive for $\mathrm{Ct} \geq$ $28 \mathrm{pg} / \mathrm{mL})$; between $150-500 \mathrm{pg} / \mathrm{mL}, 89 \%$ (8/9pts), and when $\geq 500 \mathrm{pg} / \mathrm{ml}(8$ patients), $\mathrm{Se}=100 \%$. ROC curve analysis confirmed a specificity of $100 \%$ for metastatic $\mathrm{LN}$ on FDOPAPET for $\mathrm{Ct}$ values $\geq 28 \mathrm{pg} / \mathrm{mL}$. In four patients (16\%), only early cervical acquisitions (10 minutes post injection) detected cervical LN uptake.

Conclusions: FDOPAPET imaging could represent a useful complementary exam for better detection of cervical lymph nodes even for Ct levels $\leq 150$ $\mathrm{pg} / \mathrm{mL}$. Larger studies need to confirm these preliminary results.

\section{OP-04-23 \\ INCREASED INCIDENCE OF SECOND PRIMARY MALIGNANCY IN DIFFERENTIATED THYROID CANCER}

Noemi Fralassi ${ }^{1}$, Maria Grazia Castagna ${ }^{1}$, Elena O'Heir ${ }^{1}$, Fabio Maino ${ }^{1}$, Raffaella Forleo $^{1}$, Filomena Barbato ${ }^{1}$, Furio Pacini ${ }^{1}$

${ }^{1}$ Uoc Endocrinology; Department of Medical, Surgical and Neurological Sciences, University of Siena, Siena, Italy

The occurrence of second primary malignancies (SPM) and the potential association with radioiodine (RAI) treatment are important concerns in patients with differentiated thyroid carcinoma (DTC).

Objectives: to investigate the prevalence of NTPM and its association with RAI treatment in a cohort of 1023 DTC patients.

Methods and Results: overall, 118/1023 patients (11.5\%) had at least another SPM in association with DTC either preceding or following the diag- 
nosis of DTC. Seventy-six/118 (64.4\%) SPM were antecedent or synchronous whereas 42/118 (35.6\%) occurred at least 2 years after DTC with a mean interval of 9.6 years. The most common cancer was breast cancer followed by skin and hematological cancers. The prevalence of SPM in patients with DTC was found to be significantly higher than the prevalence of neoplastic disease observed in the general population $(11,5 \%$ versus $4,3 \%, \mathrm{p}<0.0001$, RR 2.70 , CI al 95\% 2.28-3.20) and was significant for breast cancer $(p<0.0001)$ skin cancers $(p<0.0001)$ renal tumors $(\mathrm{p}=0.0007)$ leukemia $(\mathrm{p}=0.0008)$ and lymphomas $(\mathrm{p}<0.0001)$. However, the prevalence of SPM in DTC patients was not different from the incidence of SPM found in patients with other primary tumors. SPM occurring at least 2 years after DTC, were diagnosed in $4.1 \%$ of RAI treated patients and $3.9 \%$ of non-RAI treated patients $(\mathrm{p}=0.33)$. NTPM rates were $3.4 \%$ and $9.5 \%$ for a cumulative RAI activity of $\leq 11.100 \mathrm{MBq}$ and $>11.100 \mathrm{MBq}$, respectively $(\mathrm{p}=0.009)$. The relative risk of development of SPM in DTC compared with the general population was increased only in patients treated with activity of radioiodine $>11.100 \mathrm{MBq}$ (RR: 2.66 , CI $95 \%$ $1.33-5.30)$.

Conclusions: the overall risk concerning de development of SPM was related to the presence of DTC but not to the exposure to the low activity of RAI. However, an increased risk of SPM was found in patients treated with activity of radioiodine $>11.100 \mathrm{MBq}$.

\section{OP-04-24 \\ LONGITUDINAL ANALYSIS OF QUALITY OF LIFE IN PATIENTS TREATED FOR DIFFERENTIATED THYROID CANCER}

Evert van Velsen ${ }^{1}$, Edward Visser ${ }^{1}$, Boen Kam², Gaston Franssen ${ }^{3}$, Tessa van Ginhoven ${ }^{3}$, Elske Massolt ${ }^{1}$, Robin Peeters ${ }^{4}$

${ }^{1}$ Academic Center for Thyroid Diseases, Department of Endocrinology, Erasmus Medical Center, Rotterdam, Netherlands, ${ }^{2}$ Academic Center for Thyroid Diseases, Department of Nuclear Medicine, Erasmus Medical Center, Rotterdam, Netherlands, ${ }^{3}$ Academic Center for Thyroid Diseases, Department of Surgery, Erasmus Medical Center, Rotterdam, Netherlands, ${ }^{4}$ Academic Center for Thyroid Diseases; Department of Endocrinology, Erasmus, Rotterdam, Netherlands

Objective: Earlier research has shown that patients with differentiated thyroid cancer (DTC) have a significant reduction in Quality of Life (QoL) compared to controls, which seems to be unrelated to thyroid function. However, this is predominantly based on cross-sectional data of patients in which thyroid function before operation was unknown. Therefore, we initiated a longitudinal study to assess the changes in QoL in DTC patients treated with total thyroidectomy and radioactive iodine.

Methods: Patients diagnosed with DTC were recruited from our outpatient clinic. Before surgery and at follow-up, thyroid function (TSH and free-T4) was measured and QoL questionnaires were assessed. The QoL questionnaires consisted of the ThyPRO, RAND-36-item health survey, and Multidimensional fatigue index (MFI)-20. Furthermore, we collected data on patient, tumor, surgery and radioactive iodine characteristics. We used longitudinal analysis comparing QoL at baseline with follow-up, adjusted for thyroid function and several of the aforementioned characteristics.

Results: A total of 55 patients ( $\mathrm{n}=30$ women) with DTC ( $95 \%$ papillary, $5 \%$ follicular) were included, with a mean age of 50 years and median followup of seven (4-36) months. All patients were treated with total thyroidectomy, and radioactive iodine ( $84 \%$ after 3-4 weeks of thyroid hormone withdrawal). Adjusted for age, sex, and thyroid function, the ThyPRO Cognitive Problem, RAND-36 Mental Component, and MFI-20 Mental Fatigue scores showed a significant worsening at follow-up $(\mathrm{p}<0.05)$. The difference between baseline and follow-up QoL was not significantly altered by thyroid function, except for a lower MFI-20 Mental Fatigue score with higher free-T4, and for a higher ThyPRO Emotional Susceptibility score with higher TSH (both $\mathrm{p}<0.05$ ).

Conclusions: In a longitudinal setting, we showed that in patients with DTC treated with total thyroidectomy followed by radioactive iodine mental and cognitive QoL components show worsening compared to baseline at approximately seven months after therapy.

\section{OP-04-25 \\ ChANGE IN CLINICAL PRESENTATION OF PAEDIATRIC DIFFERENTIATED THYROID CANCER - SINGLE CENTRE EXPERIENCE OVER 40 YEARS}

Daria Handkiewicz-Junak', Aleksandra Kropinska ${ }^{1}$, Agnieszka Czarniecka', Tomasz Olczyk ${ }^{1}$, Zbigniew Puch ${ }^{1}$, Aleksandra Ledwon', Ewa Paliczka-Cieslik', Ewa Stobiecka', Józef Roskosz¹, Barbara Jarzab ${ }^{1}$

${ }^{1}$ Msc Memorial Cancer Centre, Gliwice, Poland

Paediatric differentiated thyroid cancer (DTC) is rare and treatable diseases with excellent prognosis. Compared with adults, children with DTC presents with more advanced stage of disease. Studies on adult DTC confirmed changes in clinical presentation, with an increasing number of thyroid microcarcinoma over the last decade. However, there were no studies on paediatric DTC.

The aim of this retrospective study was to evaluated changes in clinical presentation of paediatric DTC diagnosed and treated in one over last 40 years in a single centre.

Material and Methods: Medical histories of 490 children with DTC (age at diagnosis 18 years or younger) were included into this retrospective analysis. Children were divided in two groups, those diagnosed between 1970-1999 (group A, n=197) and 2000-2015 (group B, n=293). Analysed data included age at diagnosis, sex, histopatholgy and stage of the disease. Statistical analysis was performed with Statistica (Statsoft).

Results: The mean age at diagnosis in both groups was 13,9 years and there were no differences between groups. In both groups majority of patients were girls (group A $69 \%$, group B $76 \%(p>0,05) .83 \%$ of patients were diagnosed with papillary thyroid carcinoma and the number of follicular cancer was decreasing $(18 \%$ in group $A$ and $5 \%$ in group $B, p<0,05)$. In $427(87 \%)$ primary tumour diameter was available. There was a statistically significant increase in unifocal cancer less than $1 \mathrm{~cm}(<1 \%$ in group $A$ and $15 \%$ in group B), however $30 \%$ of those patients suffered from lymph nodes metastases. This was not different from $33 \%$ of overall rate of lymph nodes metastases.

Conclusions: The percentage of microcarcinoma in paediatric DTC is increasing. However, in contrast with adults a substantial number of those patients suffer from lymph node metastases.

\section{OP-04-26 \\ A RETROSPECTIVE ANALYSIS OF A COHORT OF 240 PATIENTS TREATED FOR MEDULLARY THYROID CANCER AT A SINGLE TERTIARY CARE CENTER SINCE 1960: FOCUS ON PROGNOSTIC FACTORS FOR SURVIVAL IN 41 PATIENTS WITH METASTATIC DISEASE}

Laurence Barde $^{1}$, Camille Buffet ${ }^{1}$, Jean-Louis Golmard ${ }^{2}$, Nathalie Cherau $^{3}$, Johanna Wassermann ${ }^{4}$, Cécile Ghander ${ }^{1}$, Charlotte LusseyLepoutre $^{1}$, Christophe Trésallet ${ }^{3}$, Fabrice Ménégaux ${ }^{3}$, Laurence Leenhardt ${ }^{1}$

${ }^{1}$ Thyroid and Endocrine Tumors Unit, Institute E3m, Pitié-Salpêtrière Hospital, Paris VI University Cancer Institute, Paris, France,

${ }^{2}$ Biostatistics Department, Pitié-Salpêtrière Hospital, Paris VI University, Paris, France, ${ }^{3}$ Department of Endocrine Surgery, PitiéSalpêtrière Hospital, Paris VI University Cancer Institute, Paris, France, ${ }^{4}$ Department of Medical Oncology, Pitié-Salpêtrière Hospital, Paris VI University Cancer Institute, Paris, France

Objectives: Overall survival of Medullary Thyroid Cancer (MTC) is poorer in comparison with other histological forms of thyroid cancers due to patients with progressive systemic and/or locally aggressive disease. Furthermore, there has been no significant increase in MTC patient's survival during the last decades. The aim of our study was to analyze overall and specific survival and also prognostic factors for death in a cohort of consecutive patients treated in our center for progressive systemic and/or locally aggressive MTC.

Method: Among 240 patients followed for MTC since 1960 in our institution, 41 patients referred to as "metastatic patients" presented with or developed distant metastases and/or had lymph node recurrence. Overall and 
specific survival of "metastatic" and "non metastatic" MTC patients were analyzed according to the Kaplan-Meier method; associated prognostic factors were assessed using Cox's model.

Results: The10-year disease specific survival (DSS) decreased significantly for "metastatic patients" $(66.7 \%)$ compared to "non-metastatic patients" $(93 \%)(\mathrm{P}<0.001)$. Significant prognostic factors for death for "metastatic patients" were: age $>45$ years $(\mathrm{P}<0.09)$, partial or absence of thyroidectomy $(\mathrm{P}=0.0012)$, synchronous vs metachronous metastases $(\mathrm{P}=0.0002)$, lung metastasis vs other initial location $(\mathrm{P}<0.01)$. A threshold of $1800 \mathrm{pg} / \mathrm{mL}$ for calcitonin (CT) and $20 \mu \mathrm{g} / \mathrm{L}$ for anticarcinoembryonic (ACE), determined 3 to 6 months after thyroidectomy was associated with a significant decrease in the DSS of "metastatic patients": $58.3 \%$ at 5 years above $1800 \mathrm{pg} / \mathrm{mL} v \mathrm{~s}$ $95.6 \%$ under $1800(\mathrm{P}=0.010)$ and $73.8 \%$ at 5 years above $20 \mu \mathrm{g} / \mathrm{L} v s 92.8 \%$ under $20(\mathrm{P}=0.03)$, respectively for $\mathrm{CT}$ and ACE.

Conclusions: Patients with progressive systemic disease are responsible for the pejorative prognosis of MTC. The identification of prognostic factors for death in MTC patients presenting with an aggressive disease may improve their management and survival.

\section{OP-04-27 \\ USE OF RECOMBINANT THYROID STIMULATING HORMONE PRIOR TO RADIOACTIVE IODINE ADMINISTRATION AND THYROGLOBULIN TESTING - RESULTS OF A UK SURVEY}

Simon Duke $^{1}$, Tom Roques ${ }^{2}$, Sarah Jefferies ${ }^{1}$

${ }^{1}$ Cambridge University Hospitals, Cambridge, UK, ${ }^{2}$ Norfolk and

Norwich University Hospital, Norwich, UK

Introduction: The administration of recombinant Thyroid Stimulating Hormone (rTSH) for patients with thyroid cancer is used for treatment with radioiodine and when measuring stimulated thyroglobulin as part of dynamic risk stratification (DRS). Use of rTSH prior to radioactive iodine administration has been shown to improve patient quality of life as compared with thyroid hormone withdrawal (THW) for low risk thyroid cancer patients.

The aim of our survey was to quantify the variation of rTSH use in the UK and ascertain the reasons for this.

Methods: A survey was sent out to clinicians via the Thyroid Cancer UK Forum to ascertain the number of patients treated in 2014 who received radioactive iodine ablation, therapeutic radioactive iodine and DRS, and how many of those cases received rTSH. The practicalities of rTSH administration and THW were also surveyed, with respondents giving reasons for their answers.

Results: 19 UK clinicians responded to the survey and geographically these were distributed evenly. These clinicians treated a total of 625 patients with radioactive iodine, $293(47 \%)$ of whom received $\mathrm{rTSH}$ as opposed to THW.

The proportion of patients who received rTSH prior to radioactive iodine administration ranged from $0-100 \%$. The same was true of patients undergoing DRS, although on average the proportion of patients receiving rTSH was higher $(70 \%)$. Some clinicians indicated that they had only recently changed their policy opening up rTSH to all patients.

Conclusions: There was substantial variation in access to rTSH across the UK for both radioiodine treatment and dynamic risk stratification, although a higher proportion of patients undergoing DRS received $\mathrm{rTSH}$.

Finance was the most common barrier to accessing rTSH, although most clinicians had no problems obtaining funding.

It would be valuable to reassess the use of rTSH and THW following the publication of the updated British Thyroid Association guidelines in 2014.

\section{OP-04-28 \\ DOES THE 2015 ATA RISK STRATIFICATION SYSTEM PREDICT THE RISK OF PERSISTENT DISEASE? A PROSPECTIVE STUDY}

Livia Lamartina ${ }^{1}$, Giorgio Grani ${ }^{1}$, Antonio Nicolucci ${ }^{2}$,

Giuseppe Lucisano ${ }^{2}$, Efisio Puxeddu ${ }^{3}$, Emanuela Arvat ${ }^{4}$, Celestino Pio Lombardi ${ }^{5}$, Fabio Orlandi ${ }^{6}$, Alfredo Pontecorvi ${ }^{7}$, Giovanni Tallini ${ }^{8}$, Massimo Torlontano ${ }^{9}$, Maria Chiara Zatelli ${ }^{10}$, Sebastiano Filetti ${ }^{1}$, Cosimo Durante ${ }^{1}$

${ }^{1}$ University of Rome Sapienza, Rome, Italy, ${ }^{2}$ Center for Outcomes Research and Clinical Epidemiology, Pescara, Italy, ${ }^{3}$ University of Perugia, Perugia, Italy, ${ }^{4}$ Univ of Torino - Osp Molinette, DIV. of Endocrinology, Torino, Italy, ${ }^{5}$ Università Cattolica del Sacro Cuore, Roma, Italy, ${ }^{6}$ University of Turin, Dept. of Oncology, Turin, Italy, ${ }^{7}$ Department of Endocrinology and Metabolic Diseases, Catholic University of the Sacred Heart, Rome, Italy, ${ }^{8}$ Department Of Medicine, University of Bologna, Anatomic Pathology, Ospedale Bellaria,

Bologna, Italy, ${ }^{9}$ Uo Endocrinologia, Casa Sollievo Della Sofferenza, San Giovanni Rotondo (Foggia), Italy, ${ }^{10}$ University of Ferrara, Section of Endocrinology, Section of Endocrinology, Ferrara, Italy

Background: Management of differentiated thyroid cancer (DTC) is driven by the likelihood of persistent or recurrent disease. Current practice guidelines are based on risk estimates obtained in studies of small cohorts or retrospective series. To assess their accuracy, we analyzed rates of structural disease 6-18 months after primary treatment in a large prospective cohort of DTC patients.

Methods: We analyzed data from the Italian Thyroid Cancer Observatory's observational, web-based database, which prospectively enrolls newly diagnosed DTC patients in 32 Italian centers (study start 2013). Patients with 6-18 months of follow-up were included in the study. Risk of persistent/ recurrent disease and treatment responses were classified in accordance with 2015 American Thyroid Association (ATA) guidelines. Disease status assessment was based on biochemical (thyroglobulin and anti thyroglobulin antibodies) and morphologic (neck ultrasonography and other imaging studies when needed) findings.

Results: 1398 patients $(75.8 \%$ females, median age at diagnosis: 48 years) were enrolled. Primary surgery consisted of total thyroidectomy in $1380(98.7 \%)$ cases; neck dissection was performed in $575(41 \%)$. Most of the DTCs were papillary $(1303,93 \%) ; 95(7 \%)$ were follicular. $851(61 \%)$ patients underwent radioiodine remnant ablation. Estimated risks of persistent/ recurrent disease were classified as low in $780(55.8 \%)$, intermediate in 532 $(38.1 \%)$, and high in $86(6.1 \%)$. Responses to primary treatment recorded at 6-18 months were as follows: excellent in $864(61.8 \%)$, biochemical incomplete in $66(4.7 \%)$, indeterminate in $381(27.3 \%)$, and structural incomplete in $87(6.2 \%)$. High and intermediate risk statuses were associated with higher rates of structural disease at $6-18$ months $(15 \%$ and $9.2 \%$, respectively, vs. $3.2 \%$ for low risk; odds ratios [95\% confidence intervals]: 3.06 [1.83-5.25] and 5.36 [2.41-11.44], respectively; $\mathrm{p}<0.0001)$.

Conclusion: The 2015 ATA risk stratification system is an effective predictor of persistent/recurrent DTC.

Table. (for Abstract OP-04-27)

\begin{tabular}{|c|c|c|c|}
\hline & Total patients & Received rTSH & $\%$ rTSH \\
\hline Radioiodine ablation & 488 & 226 & 46 \\
\hline Radioiodine therapy & 137 & 67 & 49 \\
\hline $\begin{array}{l}\text { Dynamic Risk Stratification } \\
\text { rTSH was given to: (16 clinicians responded) }\end{array}$ & 410 & 278 & 70 \\
\hline All patients without contraindication & $\begin{array}{l}\text { All except those who have } \\
\text { started THW after surgery }\end{array}$ & Only those with a medical need & No response / N/A \\
\hline $\begin{array}{l}10 \text { clinicians } \\
\text { Access to rTSH affected by: (could tick multiple) }\end{array}$ & 2 & 4 & 3 \\
\hline Finance & Clinician preference & Local Protocol & No problems with funding \\
\hline 7 & 3 & 2 & 11 \\
\hline
\end{tabular}




\section{OP-04-29 \\ NEW POSSIBILITIES FOR IMAGING OF ADVANCED MEDULLARY THYROID CANCER - A CHALLENGE FOR TARGETED THERAPY \\ Bogdan Solnica', Danuta Fedak', Paulina Gawenda', Renata Mikolajczak ${ }^{2}$, Paola A Erba ${ }^{3}$, Clemens Decristoforo ${ }^{4}$, Helmut Maecke, Katja Zalete ${ }^{6}$, Petra Kolenc Peit ${ }^{6}$, Damjan Bergant ${ }^{7}$, Luka Ležaiæ ${ }^{6}$, Theodosia Maina ${ }^{8}$, Bethold Nock ${ }^{8}$, Irene Virgolini ${ }^{9}$, Lorenza Scarpa ${ }^{9}$, Malgorzata Trofimiuk-Muldner', Agata Jabrocka-Hybel', Lideke Froberg $^{10}$, Mariona de Jong ${ }^{10}$, Sebastijan Rep ${ }^{6}$, Mark Konijnenberg ${ }^{10}$ ${ }^{1}$ Jagiellonian University Medical College, Krakow, Poland, \\ ${ }^{2}$ Radioisotope Centre Polatom, Otwock, Poland, ${ }^{3}$ Azienda Ospedaliero Universitaria Pisana, Pisa, Italy, ${ }^{4}$ Innsbruck Medical University, Innsbruck, Austria, ${ }^{5}$ University Hospital Freiburg, Freiburg, Germany, ${ }^{6}$ University Medical Centre Ljubljana, Ljubljana, Slovenia, ${ }^{7}$ Institute of Oncology, Ljubljana, Slovenia, ${ }^{8}$ Inrastes, Ncsr Demokritos, Athens, Greece, ${ }^{9}$ Innsbruck Medical University, Innsbruck, Austria, ${ }^{10}$ Erasmus MC, Rotterdam, Netherlands}

Objectives: The current available therapeutic options in medullary thyroid cancer (MTC) are limited due to often unsuccessful early disease localization which would enable surgical intervention. Although many drugs are under investigation or even tested in a clinical context, there is still no efficient, universally recommended treatment regimen for advanced MTC.

Radiolabelled molecules with high affinity to the gastrin receptors could be effective in MTC patients. Depending on the type of the radionuclide, the radiopharmaceutical can be used either for diagnostics or for Peptide Receptor Radionuclide Therapy (PRRT; theranostic approach).

The aim of the study was to assess the safety of intravenous administration of the gastrin analogue CP04 (DOTA-(DGlu) $)_{6}$-Ala-Tyr-Gly-Trp-Met-AspPhe- $\mathrm{NH}_{2}$ ), in an amount high enough to enable PRRT for the potential future theranostic management of MTC with $\left[{ }^{111} \mathrm{In} /{ }^{177} \mathrm{Lu}\right] \mathrm{CP} 04$.

Material and Methods: Eight patients (age: 30-66 years) with advanced MTC (5 sporadic/3 MEN-related) with positive ${ }^{18} \mathrm{~F}$-FDG PET-CT/CT/MRI or short calcitonin doubling time were enrolled in the study. All patients received ${ }^{111}$ In-labelled $50 \mu \mathrm{g} \mathrm{CP04.} \mathrm{Biodistribution} \mathrm{and} \mathrm{dosimetry} \mathrm{data} \mathrm{were} \mathrm{assessed}$ based on serial planar and SPECT/CT images.

Results: No side effects were observed after ${ }^{111} \mathrm{In}-\mathrm{CP} 04$ injection. In all patients ${ }^{111} \mathrm{In}-\mathrm{CP} 04$ uptake was confirmed in MTC lesions. The dose limiting organ are the kidneys. Effective radiation dose was $5.8 \mathrm{mSv} / 200 \mathrm{MBq}$ and estimated kidney and stomach doses for $50 \mu \mathrm{g}$ of $\mathrm{CP} 04$ for therapeutic isotope ${ }^{177} \mathrm{Lu}$ were 0.32 and $0.13 \mathrm{~Gy} / \mathrm{GBq}$.

Conclusions: ${ }_{-}^{111}$ In-CP04 seems to be promising radiopharmaceutical in identifying MTC lesions. Biodistribution and dosimetry data show that CP04 could be safe for MTC therapy if labeled with ${ }^{177} \mathrm{Lu}$. It is worth stressing that maybe it is the first step in the development of a more efficient therapeutic strategy against this challenging neoplasm.

\section{Oral Session 5 (Basic): Intracellular Metabolism of Thyroid Hormone}

\section{OP-05-30 \\ PROTEOMIC ANALYSIS REVEALS METABOLIC PATHWAYS AFFECTED BY DECREASED EXPRESSION OF TYPE 1 IODOTHYRONINE DEIODINASE IN RENAL CANCER}

Piotr Popawski ${ }^{1}$, Jacek R. Wiœniewski ${ }^{2}$, Beata Rybicka ${ }^{1}$, Agnieszka Piekielko-Witkowska ${ }^{1}$

${ }^{1}$ Centre of Postgraduate Medical Education, Department of Biochemistry and Molecular Biology, Warsaw, Poland, ${ }^{2}$ Max-PlanckInstitute of Biochemistry, Biochemical Proteomics Group, Martinsried, Germany

Objectives: Type 1 iodothyronine deiodinase (DIO1) is one of the key enzymes regulating bioavailability of thyroid hormones. DIO1 expression is severely reduced in renal cancer. In this study we aimed to analyze the global effects of DIO1 re-expression in renal cancer cells by performing proteomic analysis.

Methods: Proteomic analysis was performed on renal cancer cells with (KIJ265T-pcDNA3-DIO1) or without (KIJ265T-pcDNA3) stable ectopic DIO1 expression, following validation (qPCR) on KIJ265T-pcDNA3-DIO1 and KIJ265T-pcDNA3 cells and independent cell lines (KIJ308T-pcDNA3DIO1, KIJ308T-pcDNA3). The expression of DIO1-affected genes was qPCR-analyzed in 30 renal tumors and corresponding non-tumorous controls (approved by the local Bioethical Committee). Survival analysis was performed on independent cohort of 468 renal cancer patients using SurvExpress.

Results: Proteomic analysis revealed 26 downregulated and 59 upregulated proteins $(\mathrm{p}<0.05, \quad \mathrm{FDR}<0.05)$ in KIJ265T-pcDNA3-DIO1 when compared with KIJ265T-pcDNA3 cells. Enrichment analysis showed that UPREGULATED group consisted mainly of proteins involved in metabolic regulation (including reductases, dehydrogenases, oxidoreductases) while DOWNREGULATED group was enriched in proteins involved in adhesion. The key pathways affected by DIO1 expression were metabolism of proteins, nucleobase-containing compounds, and carbohydrates. Top altered proteins included AKR1C2 ( +57 fold), RAP1GAP ( +15.8 fold), AKR1B10 ( +8.2 fold), AFAP1L2 (-31.2 fold), ANPEP (-8.7 fold). qPCR positively verified altered expression of 10 selected genes. The expressions of AKR1C1, S100A2, PLAU, SLC3A2, TBC1D2, and WIZ were decreased, while expressions of TGM2, SLC7A5, and NMI were increased in renal tumors when compared with controls. The expressions of most genes (except for: AKR1C2, SLC7A5) strongly correlated $(p<0.001)$ with DIO1 in tissue samples. Altered expression of most validated genes (except for SLC3A2) correlated with poor survival of renal cancer patients.

Conclusions: Loss of DIO1 expression in renal cancer can affect key metabolic pathways in tumor cells. Disturbed DIO1 expression may contribute to molecular changes that affect patients survival.

Supported by National Science Centre-Poland, grant 2014/13/B/ NZ5/00283.

\section{OP-05-31 \\ OXIDATIVE STRESS INDUCES TYPE 3 DEIODINASE IN MULTIPLE TISSUE AFTER MYOCARDIAL INFARCTION: IMPLICATIONS TO NONTHYROIDAL ILLNESS SYNDROME PATHOPHYSIOLOGY \\ Simone Wajner ${ }^{1}$, Tatiana Lehnen ${ }^{1}$, Ana Luiza Maia ${ }^{1}$ \\ ${ }^{1}$ Ufrgs, Hcpa, Porto Alegre, Brazil}

Dysregulation of type 3 deiodinase (D3) due to imbalances in the redox status alters the metabolism of thyroid hormones in the nonthyroidal illlness syndrome (NTIS). N-acetylcysteine (NAC), a potent antioxidant, corrects the oxidative damage and alterations seen in D3 in the heart in an animal model of 
myocardial infarction (MI) and NTIS. However, the effect of oxidative stress on D3 expression and activity in other tissues are unknown.

Objective: Evaluate the effect of MI-induced oxidative stress in D3 function in the liver, muscle, brown adipose tissue (BAT) and brain in an animal model of myocardial infarction (MI) and NTIS.

Methods: Male Wistar rats submitted to left anterior coronary artery occlusion (MI) received NAC $(10 \mathrm{mg} / \mathrm{kg}, \mathrm{n}=20)$ or placebo $(\mathrm{NaCl} ; \mathrm{n}=20)$. After ten days post-MI the animals were sacrificed and tissues collected. The total carbonyl content and GSH were used as a parameter of intracellular redox balance. D3 expression and activity were measured.

Results: The formation of carbonyls, a marker of oxidative damage to proteins, was increased in liver, muscle, BAT and brain (all $\mathrm{P}<0.001$ ) in the placebo but not in NAC group. GSH levels were also diminished in all tissues of the placebo group $(\mathrm{P}<0.001)$ and maintained in the NAC-treated animals. $\mathrm{GSH} / \mathrm{GSSG}$ ratio was also altered in all tissues. DIO3 expression and activity were increased in the placebo group in liver $(\mathrm{P}=0.002)$, muscle $(\mathrm{P}=0.03)$, BAT $(\mathrm{P}=0.04)$ and brain $(\mathrm{P}=0.01)$ as compared to the NAC group.

Conclusion: MI-induced oxidative stress results in a systemic induction of D3 in MI, contributing to diminishing circulating T3 levels. Antioxidant treatment prevents D3 dysfunction in multiple tissues, which probably contribute to avoiding NTIS.

\section{OP-05-32 \\ INVOLVEMENT OF LAT3 IN THE UNIDIRECTIONAL TRANSPORT OF 3,3'-T2 ACROSS THE CELL MEMBRANE AND FIRST STRUCTURAL INSIGHTS BY HOMOLOGY MODEL GENERATION}

Katrin Manuela Hinz ${ }^{1}$, Carolin Scheffner ${ }^{1}$, Alessandro Volpato ${ }^{1}$, Vidhya

Ravi Madapusi ${ }^{1}$, Gerd Krause ${ }^{1}$

${ }^{1}$ Leibniz-Institut für Molekulare Pharmakologie (Fmp), Berlin, Germany

Thyroid hormone (TH) transporters facilitate or exchange $\mathrm{TH}$ into or out of the cell. L-type amino acid transporters (LAT) transporting among neutral amino acids also thyroid hormones. The exchangers LAT1 and LAT2 mediate $\mathrm{TH}$ across the membrane.

Although the role for facilitators, LAT3 and LAT4 an unidirectional export of TH is assumed, but their role in TH transport is still unclear.

Using Hek293 cells and oocytes of Xenopus laevis expressing individual or co-expressing LAT family members, 3,3'-T2 transport was characterized by functional assays of fluorescence tagged LAT proteins. No import of 3,3'-T2 was detected for LAT3. Subsequently, for export analysis TH carrier proteins into the cell such as LAT2 coupled to CD98 are required. By indirect measurement the export of 3,3'-T2 was confirmed in HEK293 and in oocytes for LAT3.

In order to better understand its structure-function relationships in $\mathrm{TH}$ transport, we report here the first molecular homology model of the human LAT3. Especially two solved crystal structures helped us to model the 12 helices consisting TM domain of LAT3. Based on sequence similarity we used for the first 6-helix bundle (TMH 1-6) of LAT3 the first 6-helix bundle (TMH $1-6)$ of the so called drug efflux protein, YajR (PDB: 3WDO). The second 6TMH bundle of LAT3 (TMH 7-12) suits more to the first 6-helix bundle of the xylose symporter XylE (PDB: 4GBY). For LAT3 a single binding site and transport cavity in the center between the two TMH bundles is proposed.

Follow up in vitro functional analysis with model derived side directed mutagenesis will be carried out to elucidate the structure function analysis of LAT3. Mutants might give hints to obtain relations between transport behavior of TH and specific amino acids positions explaining the TH export as previously were demonstrated for the unidirectional TH import for LAT2.

\section{OP-05-33 \\ THYROID HORMONE AND SKIN CANCER: DYNAMIC CONTROL OF TUMOR FORMATION AND PROGRESSION BY DEIODINASES}

Daniela Di Girolamo', Emery De Cicco', Raffaele Ambrosio², Giuseppina Mancino ${ }^{1}$, Caterina Miro ${ }^{1}$, Maria Angela De Stefano', Carolina Prezioso ${ }^{1}$, Annarita Nappi ${ }^{1}$, Serena Sagliocchi ${ }^{1}$, Domenico Salvatore ${ }^{3}$, Monica Dentice ${ }^{4}$

${ }^{1}$ University of Naples "Federico II", Naples, Italy, ${ }^{2}$ Irccs Sdn, Naples, Italy, ${ }^{3}$ Dipartimento DI Endocrinologia; University of Naples "Federico II"; University of Naples, Federico II, Naples, Italy, ${ }^{4}$ Department of Clinical Medicine and Surgery, University of Naples "federico li;

Endocrinologia + Oncologia, Naples, Italy

Thyroid hormones (THs) mediate pleiotropic cellular processes involved in metabolism, cellular proliferation and differentiation. The intracellular hormonal environment can be tailored by the deiodinase enzymes, D2 and D3, which catalyze $\mathrm{TH}$ activation and inactivation, respectively. In many cellular systems, THs exert well documented stimulatory or inhibitory effects on cell proliferation, however, the molecular mechanisms by which they control rates of cell cycle progression have not yet been entirely clarified. We previously showed that D3-depletion or TH treatment influences the proliferation and survival of basal cell carcinoma (BCC) cells. We also found that BCC cells express not only sustained levels of D3 but also robust levels of D2. The aim of the present study was to dissect the relative contributions of D2 and D3 to TH metabolism in the BCC context. By using the CRISPR/Cas9 technology, we genetically depleted D2 and D3 in BCC cells. Mechanistic investigations revealed that $\mathrm{D} 2$ inactivation accelerates cell cycle progression thereby enhancing the proportion of S-phase cells and cyclin D1 expression. Conversely, D3 mutagenesis drastically suppressed cell proliferation and enhanced apoptosis of BCC cells. Furthermore, the basal apoptotic rate was oppositely regulated in D2- and D3-depleted cells. In conclusion, our results indicate that $\mathrm{BCC}$ cells constitute an example in which the TH signal is finely tuned by the concerted expression of opposite-acting deiodinases. The dual regulation of D2 and D3 expression plays a critical role in cell cycle progression and cell death by influencing cyclin D1-mediated entry into the G1-S phase. These findings reinforce the concept that TH is a potential therapeutic target in human BCC.

\section{OP-05-34}

SELECTIVE ACTIVATION/INACTIVATION OF THE THYROID HORMONE SIGNALING PATHWAY: THE METABOLIC CONSEQUENCES

Karine Gauthier ${ }^{1}$, Guyot Romain ${ }^{2}$, Richard Sabine ${ }^{2}$, Clemmensen Christoffer $^{3}$, Finan Brian ${ }^{3}$, Samarut Jacques ${ }^{2}$, Flamant Frédéric ${ }^{2}$

${ }^{1}$ Ens Lyon, Lyon Cedex 07, France, ${ }^{2}$ Igfl, Ens de Lyon, Lyon, France, ${ }^{3}$ Helmholtz Zentrum Muenchen, Deutsches Forschungszentrum Fuer Gesundheit und Umwelt (Gmbh), Neuherberg, Germany

Introduction: Thyroid hormone (T3) regulates lipid/glucose homeostasis and energy expenditure. Given its ability to decrease cholesterol and body weight, T3 appears as an attractive candidate to fight metabolic disorders such as atherosclerosis and obesity. However T3 can't be use as a safe drug because of its many unwanted side effects especially in bone, muscle and heart. T3 signals via its binding to its nuclear receptors TRs, some transcription factors expressed in most tissues. It was classically accepted that T3 was acting in a given tissue through the locally expressed TR. However recent studies by different groups suggest that at least some of the metabolic peripheral actions of T3 might actually result from its central action. Indeed local injection of $\mathrm{T} 3$ in the brain induces both liver gluconeogenesis and energy expenditure as efficiently as when administered to the whole body.

Objectives: Here we assess the role of endogenous T3 brain signaling under physiological stressors known to be perceived by the hypothalamus and to trigger increase energy expenditure: cold exposure and high fat diet.

Results: Using mice mutants, we showed that neuron selective inactivation of T3 signaling impaired adaptive thermogenesis in response to high fat diet but not cold exposure. Moreover, even though both TR $\alpha 1$ and TR $\beta$ are expressed in the hypothalamus, TR $\alpha 1$ seems to be the only critical to fight diet induced obesity. Conversely neuronal TR $\beta$ seems to be the major player for T3 regulated glucose metabolism in liver. Some new pharmacological approach 
has also been used to selectively deliver $\mathrm{T} 3$ to the brain and study the potential benefits versus side effects of such compounds.

Conclusions: Endogenous neuronal TR signaling is involved in adaptive thermogenesis each TR fulfilling some specific functions.

\section{OP-05-35 \\ NON-GENOMIC EFFECTS OF THYROID HORMONES ON MESENCHYMAL STEM CELLS AND ANGIOGENESIS}

Kathrin Schmohl' ${ }^{1}$, Maike Dohmann ${ }^{1}$, Alexandra Wechselberger ${ }^{1}$, Peter

J. Nelson ${ }^{1}$, Christine Spitzweg ${ }^{2}$

${ }^{1}$ Medical Policlinic IV, University Hospital of Munich, LMU Munich,

Germany, Medical Policlinic IV, Munich, Germany, ${ }^{2}$ Department

of Internal Medicine II, University Hospital of Munich, Germany,

Medizinische Klinik und Poliklinik II, Medizinische Klinik und Poliklinik

II, Munich, Germany

Tumour stroma formation is associated with angiogenesis and requires interactions of various different cell types, including endothelial cells (ECs), pericytes, fibroblasts and mesenchymal stem cells (MSCs). Thyroid hormones (TH) act as non-classical proangiogenic modulators mediated by non-genomic mechanisms via cell surface receptor integrin ávâ3. The aim of this study was to evaluate the stimulatory activity of T3 and T4 versus tetrac, a specific inhibitor of ávâ3-mediated action of TH, on endothelial cell tube formation in concert with the assessment of angiogenic effects of MSCs.

Primary human umbilical vein ECs (HUVECs) were seeded on Matrigel and tube formation was analysed microscopically after $12 \mathrm{~h}$. Compared to untreated HUVECs, treatment with T3 stimulated tube formation, as evidenced by more intricate networks with larger numbers of junctions and meshes. Additional treatment with tetrac reduced tube formation to basal level. Similar, albeit weaker, effects were observed for T4. Further, primary human bone marrow-derived MSC-conditioned medium stimulated tube formation. After additional treatment with $\mathrm{TH}$, an even more pronounced angiogenic effect was observed compared to untreated control cells and tetrac-treated cells. Treatment of MSCs with T3 or T4 \pm tetrac in the presence of tumour cell-conditioned medium resulted in stimulation of expression of genes associated with angiogenesis as determined by qPCR. After $24 \mathrm{~h}$, supernatant from these prestimulated MSCs was added to HUVECs in the tube formation assay, leading to the strongest enhancement of tube formation observed in these experiments and, most strikingly, potent inhibition by tetrac.

Our data suggest that $\mathrm{TH}$, especially $\mathrm{T} 3$, stimulate angiogenesis in HUVECs in an integrin ávâ3-dependent manner, an effect that is enhanced by MSCs, providing further evidence of the critical role of TH in the regulation of angiogenesis and the anti-angiogenic activity of tetrac in the context of tumour stroma formation.

\section{OP-05-36 \\ T3-RECEPTOR ALPHA 1 INTERACTOMES IN HUMAN LIVER AND NEURONAL CELLS}

\section{Marcel Meima $^{1}$, Karn WejaphikuR ${ }^{2}$,W. Edward Visser ${ }^{3}$, Theo. M.} Luider $^{4}$, Theo Visser ${ }^{5}$, Robin Peeters ${ }^{6}$

${ }^{1}$ Academic Center for Thyroid Diseases, Department of Endocrinology, Erasmus Medical Center, Rotterdam, Netherlands, ${ }^{2}$ Academic Center for Thyroid Diseases, Erasmus University Medical Center, Rotterdam, Netherlands, ${ }^{3}$ Erasmus Medical Center, Rotterdam, Netherlands, ${ }^{4}$ Erasmus University Medical Center, Department of Neurology, Rotterdam, Netherlands, ${ }^{5}$ Erasmus University Medical Center, Dept Internal Medicine, Rotterdam, Netherlands, ${ }^{6}$ Academic Center for Thyroid Diseases, Department of Endocrinology, Erasmus, Rotterdam, Netherlands

Objectives: Binding of $\mathrm{T} 3$ to thyroid hormone receptors (TRs) modulates the expression of target genes. Unliganded TRs bind to a complex of co-repressors that are exchanged for co-activators upon binding of T3. The effects of T3 on individual genes can vary depending on the tissue, which may be explained by tissue-specific recruitment of cofactors. To determine the tissue-specificity of cofactor recruitment, we used a tandem-affinity purification protocol to identify the interactome for TRál in human liver and neuronal cell models.
Methods: TRá1 was N-terminally tagged with a FLAG- and HA-epitope (FHTRá1) and stably transduced into HepG2 human hepatocarcinoma and SHSY5Y human neuroblastoma cells. Expression and activity were confirmed by western blotting and a luciferase reporter assay. FHTRá1-expressing or empty vector control cells were incubated for $4 \mathrm{hrs}$ with either vehicle or 100 nM T3. FHTRá1-associated proteins were purified from nuclear extracts using sequential purifications on anti-FLAG and anti-HA resins and identified by LC/MS-MS.

Results: Proteins associated with FHTRál were identified in two independent purifications each from HepG2 and SHSY5Y cells. The profiles clearly differed between unliganded and liganded receptors. There was a general overlap in profiles between the different cell types in the same ligand state for strong hits. In both cell types, retinoid X receptors (RXRs) co-purified with FHTRá1 with or without T3, NCoR1 and TBL1XR1 co-purified with FHTRá1 without T3, whereas the Mediator and the Mi-2/NuRD complex, and the transcription factor Prox1 co-purified with FHTRá1 preferentially in the presence of T3. There were, however, exceptions, most notably the T3-dependent corepressor RIP140, which only co-purified with FHTRá1 in HepG2 cells.

Conclusion: Although the TRá1-interactomes in HepG2 and SHSY5Y cells mostly overlap, we found evidence for potentially specific interactions. Future studies need to determine whether these interactions indeed explain differences in tissue-specific gene regulation.

\section{OP-05-37 \\ MCT8 DEFICIENCY ALTERS NEURONAL GLUCOSE AND ENERGY METABOLISM}

Franziska Meyer ${ }^{1}$, Sebastian Cerdan ${ }^{2}$, Juan Bernaß ${ }^{\beta}$, Ulrich Schweizer ${ }^{4}$, Josef Köhrle ${ }^{5}$, Eva Katrin Wirth ${ }^{6}$

${ }^{1}$ Institut für Experimentelle Endokrinologie, Charite Universitätsmedizin Berlin, Charité Universitätsmedizin Berlin, Berlin, Germany,

${ }^{2}$ Instituto de Investigaciones Biomédicas, Madrid, Spain, ${ }^{3}$ Instituto Investigaciones Biomédicas, Ciberer, Center for Biomedical Research on Rare Diseases, Madrid, Spain, ${ }^{4}$ Rheinische

Friedrich-Wilhelms-Universität Bonn, Institut für Biochemie und Molekularbiologie, Bonn, Germany, ${ }^{5}$ Institut Fuer Experimentelle Endokrinologie, \& Endokrinologisches Forschungs-Centrum Enforcé, Charité-Universitätsmedizin Berlin, Berlin, Germany, ${ }^{6}$ Charité-

Universitätsmedizin Berlin, Institut für Experimentelle Endokrinologie, Berlin, Germany

Monocarboxylate transporter 8 (Mct8)-deficient brains of mice do not show classical signs of hypothyroidism. Yet, MCT8-deficient patients (AllanHerndon-Dudley syndrome) show defective myelination and severe psychomotor retardation. Altered transcriptional regulation and behavioral changes in global Mct8-deficient mice suggest that Mct8 plays a vital role for brain development and function.

In order to specifically assess the role of Mct8 in neurons - independent of blood-brain-barrier effects - we have created neuron-specific Mct8-deficient mice $\left(\mathrm{CamK}-\mathrm{Cre} ; \mathrm{Mct}^{8 \mathrm{fl} / \mathrm{fl}}\right)$.

At the age of 6 months, these mice showed no alterations in serum thyroid hormone concentrations, thyroid hormone regulated genes in the cortex or striatum, and other components of the HPT axis. In vivo analysis of cerebral glucose metabolism revealed abnormalities in CamK-Cre; $M c t 8^{f l / f l}$ mice. $\left(1-{ }^{13} \mathrm{C}\right)$ labeled glucose was infused and its in vivo metabolism followed by NMR measurements of ${ }^{13} \mathrm{C}$ incorporation into cerebral metabolites. Significantly less ${ }^{13} \mathrm{C}$ was incorporated into lactate in neuron-specific Mct8-deficient cortex and striatum compared to control littermates. Phosphofructokinase 1, a rate-limiting enzyme of glycolysis is reduced in CamK-Cre; $M c t 8^{f l / f l}$ brains. In addition, lactate dehydrogenase activities of both astrocytes and neurons were significantly reduced corroborating a reduced demand for energy substrates by Mct8-deficient neurons. Astrocytic transporters for glutamate, glutamine and GABA are down-regulated in neuron-specific Mct8-deficient mice. This finding most likely reflects a reduced demand for reuptake and recycling of neurotransmitters again consistent with lower neuronal activity.

Taken together, our results suggest a reduced activity of neurons in $\mathrm{CamK}$ Cre; Mct $8^{f l / f l}$ brains and shed light on the importance of Mct 8 for neuronal function. Further research needs to delineate the specific role of Mct8 in neurons by either altering cerebral glucose utilization and/or providing thyroid hormones and their metabolites. 
Monday, 11th September 2017

\section{Oral Session 6 (Clinical): Cancer - A Focus on Treatment}

\section{OP-06-38 \\ IMPACT OF MINIMAL EXTRA-THYROIDAL EXTENSION ON OUTCOME IN DIFFERENTIATED THYROID CANCER: SYSTEMATIC REVIEW AND META-ANALYSIS}

Eyal Robenshtok', Dania Hirsch', Carlos Benbassat², Ilan Shimon ${ }^{3}$, Amit Akirov', Talia Diker-Cohen ${ }^{1}$

${ }^{1}$ Endocrinology \& Metabolism Institute, Rabin Medical Center, Beilinson Hospital, Petah-Tikva, Israel, ${ }^{2}$ Endocrine Institute, Asaf Harofe

Hospital, Zerifin, Israel, ${ }^{3}$ Rabin Medical Center, Petah-Tiqva, Israel

Background: Minimal extra-thyroidal extension (mETE) in differentiated thyroid cancer (DTC) patients was defined as an intermediate risk feature in the 2015 ATA guidelines. However, controversy persists as several studies suggested $\mathrm{mETE}$ has little effect on disease outcome.

Aim: To assess the impact of mETE on DTC patients' outcome.

Methods: Meta-analysis of controlled trials comparing DTC patients with and without mETE.

Results: Thirteen studies including 22,472 patients were included, with a median follow-up of 72 months. Data on N0 disease was reported in 6 studies, and N0+N1 in 10 studies ( 3 included both analyses). Studies were heterogeneous in terms of lymph node involvement, mETE definition, type of surgery, and rate of radioiodine ablation. Minimal ETE in patients with N0 disease was associated with increased risk of recurrence (7 studies, OR 1.73, $95 \%$ CI 1.03-2.92). This difference was mostly due to one large outlier study, whereas mETE did not confer increased risk in the other 6 studies (OR 1.37, $95 \%$ CI 0.74-2.53). In patients with mixed N0+N1 disease, mETE resulted in a significantly higher risk of recurrence (8 studies, OR $1.7,95 \% \mathrm{CI} 1.33-2.17$, $\mathrm{I}^{2}=67 \%$ ). Sub-analysis for patients with micro-PTC did not show a significant impact of mETE (OR 1.74, 95\%CI 0.98-3.12). Minimal ETE had no impact on disease related mortality (OR $0.5,95 \% \mathrm{CI} 0.11-2.21, \mathrm{I}^{2}=0 \%$ ).

Conclusion: Current data supports the 2015 ATA guidelines, except for patients with micro-PTC. However, data quality is poor and further studies are required especially in patients with $\mathrm{N} 0$ disease, using standardized mETE definition, and with adjustment for baseline characteristics.

\section{OP-06-39 \\ NEOADJUVANT VEMURAFENIB IN PATIENTS WITH LOCALLY ADVANCED PAPILLARY THYROID CANCER (PTC)}

Maria E Cabanillas ${ }^{1}$, Naifa L. Busaidy ${ }^{1}$, Mark Zafereo ${ }^{1}$, Steven Waguespack ${ }^{1}$, Mimi I Hu ${ }^{1}$, Marie-Claude Hofmann ${ }^{1}$, Erich Sturgis ${ }^{1}$, Steven I. Sherman ${ }^{2}$

${ }^{1}$ The University of Texas MD Anderson Cancer Center, Houston, TX USA, ${ }^{2}$ Department of Endocrine Neoplasia and Hormonal Disorders, Division of Internal Medicine, The University of Texas M. D. Anderson Cancer Center, Houston, TX, USA

Objectives: BRAFV600E mutations are common in PTC. Vemurafenib is an inhibitor of BRAF serine-threonine kinase enzyme that has been studied in a phase 2 trial in BRAF-mutated PTC. Here we report a neoadjuvant (pre-operative chemotherapy) trial with vemurafenib that recently completed enrollment. The primary endpoint is translational (not reported here). Secondary endpoints included response by RECIST (pre-/post- surgery), safety of neoadjuvant vemurafenib, and persistent disease at the surgical site.

Methods: Patients with primary or recurrent BRAFV600E-mutated PTC who were planned for surgical resection were eligible. Patients were treated with vemurafenib $960 \mathrm{mg}$ bid for 56 days followed by surgery and discontinuation of vemurafenib.
Results: From 2/2013-3/2017, 17 patients were enrolled with intent to operate, of which 15 are evaluable (2 currently pending surgery). 11 (73\%) evaluable patients were men; median age was 62 . Responses to vemurafenib: 3 partial responses, 8 stable disease, 1 progression, 3 not evaluable (NE). Nine patients $(60 \%)$ underwent surgery ( 3 primary and 6 revision surgeries), while 6 patients did not due to toxicity to vemurafenib $(\mathrm{n}=4 ; 26 \%)$, progression $(n=1)$, or refused surgery $(n=1)$. Responses in target lesions in 9 operated: 5 complete response, 1 partial response (gross residual), 1 stable disease (gross residual), and 2 NE. Table 1 lists the types of surgeries performed. Three patients underwent post-operative external beam radiation. One patient died 2 weeks post-operatively from a fatal hemorrhage. Another patient had postoperative chest hematoma. All but one operated patient is alive. One in the inoperable group died of progression. Adverse events from vemurafenib will be reported at the meeting.

Conclusions: Neoadjuvant vemurafenib was well tolerated in most patients. Of 9 patients who underwent surgery, over half achieved a postoperative complete response and all but one patient is alive, suggesting that neoadjuvant vemurafenib is a reasonable strategy for patients with extensive, BRAF-mutated PTC, although further studies are required to determine if this approach extends disease-free survival.

Table 1.

\begin{tabular}{lll}
\hline $\begin{array}{l}\text { Surgeries performed } \\
(\mathrm{n}=9 ; 60 \%)\end{array}$ & $\begin{array}{l}\text { Total Thyroid + lateral + central neck }+ \\
\text { laryngectomy (n) } \\
\text { Total Thyroid + lateral + central neck }+ \\
\text { tracheal resection/reconstruction (n) }\end{array}$ & 1 \\
& $\begin{array}{l}\text { Total Thyroid + lateral + central (n) } \\
\text { Lateral + central + laryngectomy (n) }\end{array}$ & 1 \\
& $\begin{array}{l}\text { Lateral + central neck (n) } \\
\text { Central neck only (n) }\end{array}$ & 3 \\
\hline
\end{tabular}

\section{OP-06-40 \\ ROLE OF RADIOACTIVE IODINE IN PATIENTS WITH T1B DIFFERENTIATED THYROID CARCINOMA}

Simone de Leo ${ }^{1}$, Carla Colombo ${ }^{1}$, Michela Perrino ${ }^{1}$, Marina Muzza $^{2}$, Valentina Cirello ${ }^{3}$, Leonardo Vicentini ${ }^{4}$, Laura Fugazzola ${ }^{5}$

${ }^{1}$ Division of Endocrine and Metabolic Diseases, Istituto Auxologico Italiano Irccs, Milan, Italy, Milan, Italy, ${ }^{2}$ Endocrine Unit, Fondazione Irccs Ca' Granda-Ospedale Maggiore Policlinico, Milan, Italy, ${ }^{3}$ Laboratory of Endocrine and Metabolic Research, Istituto Auxologico Italiano Irccs, Milan, Italy, Milan, Italy, ${ }^{4}$ Endocrine Surgery Unit, Fondazione Irccs Cà Granda, Milan, Italy, Milan, Italy, ${ }^{5}$ Dept. of Pathophysiology and Transpl, Istituto Auxologico Italiano Irccs, University of Milan, Milano, Italy

Radioactive iodine remnant ablation is not routinely recommended for ATA low risk patients. However, observational studies reported conflicting data whether RAI improves disease-free survival in patients with T1b differentiated thyroid carcinoma (DTC), i.e. a tumor size of 11-20 mm without extrathyroidal extension and lymph node or distant metastases.

Our study aimed at evaluating whether outcome of T1b patients who were treated with radioactive iodine was different from that of patients not treated with radioactive iodine.

We retrospectively selected 58 patients with a T1b DTC who were treated with radioactive iodine and compared to 79 patients with a T1b DTC not treated with radioactive iodine. The two groups of patients were similar regarding gender (men were $13.8 \%$ and $21.5 \%$, respectively, $\mathrm{p}=\mathrm{NS}$ ), tumor diameter at histology (mean diameter was $15.8 \mathrm{~mm}$ and $14.7 \mathrm{~mm}$, respectively, $\mathrm{p}=\mathrm{NS}$ ), histology (papillary carcinoma were $89.7 \%$ and $92.4 \%$ and follicular carcinoma $10.3 \%$ and $7.6 \%$, respectively, $\mathrm{p}=\mathrm{NS}$ ), papillary variant (classic variant $67.8 \%$ and $71.6 \%$, follicular variant $28.6 \%$ and $24.3 \%$, other variants $3.6 \%$ and $1.4 \%$, respectively, $\mathrm{p}=\mathrm{NS}$ ), multifocal disease $(37.9 \%$ and $35.4 \%$, respectively, $\mathrm{p}=\mathrm{NS}$ ), genetic mutations (no significant differences regarding BRAF, TERT, RET/PTC, RAS and TRK mutations). The two groups differed only regarding patient age at surgery, since patients treated with radioiodine were significantly younger than those not treated with radioiodine (mean age was 39.3 and 50.2 years, respectively, $p<0.0001$ ).

However, the outcome of the two groups was not significantly different: $55 / 58(94.8 \%)$ of patients who were treated with radioactive iodine and 73/79 
(92.4\%) not treated with radioactive iodine were in remission ( $\mathrm{p}=0.57)$, after a mean follow-up of 114 and 68 months, respectively.

Our findings demonstrate that patients with a DTC with a tumor diameter of 11-20 $\mathrm{mm}$ and without extrathyroid extension and lymph node or distant metastases (pT1b) do not benefit from radioactive iodine ablation.

\section{OP-06-41 \\ METASTATIC MEDULLARY THYROID CARCINOMA (MTC): DISEASE COURSE AND TREATMENT MODALITIES}

Katerina Saltiki', Paraskevi Kazakou ${ }^{2}$, George Simeakis ${ }^{3}$, Elli Anagnostou $^{4}$, Eleni Anastasiou ${ }^{5}$, Eva Kassi ${ }^{6}$, Maria Alevizaki ${ }^{7}$ ${ }^{1}$ Clinical Therapeutics, Athens, Athens, Greece, ${ }^{2}$ Endocrine Unit, Dept Clinical Therapeutics, National and Kapodistrian University, School of Medicine, Athens, Greece, ${ }^{3}$ Endocrine Unit, Dept Medical Therapeutics, Alexandra Hospital, Athens University School of Medicine, Endocrinology Dept, Alexandra Hospital, Athens, Athens, Greece, ${ }^{4}$ Endocrine Unit, Dept Medical Therapeutics, Alexandra Hospital, Athens University School of Medicine, Athens, Greece, ${ }^{5}$ Endocrine Unit, Alexandra Hospital, Athens, Greece, ${ }^{6}$ Endocrine Unit, Dept Medical Therapeutics, Athens University School of Medicine, Athens, Greece, ${ }^{7}$ Endocrine Unit, Alexandra Hospital, Endocrine Unit, Athens University, Athens, Greece

Objectives: MTC has varying clinical course. In cases with metastatic MTC (meta-MTC) or rapidly progressive disease further therapeutic modalities are needed. We recorded clinical features and disease progression in metaMTC followed-up in our centre since 1977.

Methods: Of 273 MTC patients 49 showed metastatic disease p. Clinical, biochemical and therapeutic data were recorded.

Results: Meta-MTC was more frequent in men than women $(32.6 \% \mathrm{vs}$ $13.3 \%, \mathrm{p}=0.001)$, likewise in sporadic than familial-MTCs $(29 \% \mathrm{vs} 8.9 \%$, $\mathrm{p}<0.001$ ). Age at diagnosis (range 5-78, median54, IQR25yrs) was higher compared to non meta-MTC (median44, IQR26,p=0.018). Meta-MTC patients had larger, more invasive tumours and unfavorable stage at diagnosis $(\mathrm{p}=0.001)$ as well as shorter calcitonin doubling time (3-36 months, median 8). $11 / 49(22.5 \%)$ had meta-MTC at diagnosis. In those who developed meta-MTC during follow-up the time interval for metastases appearance was 0.6-26years (median 4). The primary metastatic localization was mediastinum $(41.9 \%)$, lungs $(25.8 \%)$, liver $(19.4 \%)$ bones $(12.9 \%)$. Subsequent locoregional treatments were performed such as lymph-node dissection(32\%), liver chemoembolism(24\%), bone external-radiation(16\%), tyrosine-kinase inhibitors (TKI) administration $(12 \%)$ which resulted in biochemical and structural stabilization $(29.2 \%)$, progression $(58.3 \%)$, partial response in $12.5 \%$. New metastatic lesions reappeared in $1-27 \mathrm{yrs}($ median 8$)$ in lungs $(43.3 \%)$, liver $(30 \%)$, bone(13.3\%), mediastinum(6.7\%), and other unusual sites (mammary gland, bartholini gland, pancreas, brain). Further therapeutic intervention such as $\operatorname{TKI}(54.5 \%)$, chemoembolism $(22.7 \%)$, radiation $(9.1 \%)$, surgery $(4.5 \%)$ and multiple interventions $(4.5 \%)$ resulted in stabilization(50.0\%), progres$\operatorname{sion}(35.0 \%)$ or partial response $(15.0 \%)$. In patients with metastases in $\geq 2$ organs the disease progressed more rapidly, affecting mainly bones $(40 \%)$ and lungs(33\%). 25 patients received ÔEEEE, 22/25 Vandetanib (follow-up 0.5-5yrs). During Vandetanib treatment 5/22(23\%) showed partial response, $14 / 22(63.5 \%)$ stabilization and 3/23(13.5\%) progression.

Conclusions: Locoregional treatment modalities may offer disease stabilization in $30.0 \%$ of patients. In cases with further disease progression and new metastases TKIs and especially Vandetanib may be more effective resulting in partial structural response or stabilization in $>80 \%$. This response rate is very promising for patients with aggressive MTC.

\section{OP-06-42 \\ FDG+/RAI+ PATIENTS WITH DISTANT METASTASES FROM DIFFERENTIATED THYROID CANCER CAN BENEFIT FROM RADIOACTIVE IODINE TREATMENT}

Lisa Males ${ }^{1}$, Serena Grimaldi ${ }^{1}$, Marie Terroir ${ }^{1}$, Jean Lumbroso ${ }^{1}$, Desiree Deandreis ${ }^{1}$, Amandine Berdelou ${ }^{2}$, Eric Baudin ${ }^{1}$, Martin Schlumberger ${ }^{1}$, Sophie Leboulleux ${ }^{1}$

${ }^{1}$ Gustave Roussy, Université Paris-Sud, Villejuif, France, Villejuif, France, ${ }^{2}$ Gustave Roussy, Université Paris-Sud, Curie, Saint Cloud, Villejuif, France

Objective: Patients with distant metastases from differentiated thyroid cancer (DTC) with FDG uptake have the same survival rate whether they present or not radioactive iodine (RAI) uptake, suggesting that FDG could indicate resistance to RAI treatment. The purpose of this study was to evaluate tumor response rate following RAI treatment in DTC patients with distant metastases that present both FDG and RAI uptakes (FDG+/RAI+).

Method: A retrospective monocentric study.

Results: Thirty two FDG+/RAI+ DTC patients with distant metastases (16 females, mean age: 60 years) treated with RAI $(100 \mathrm{mCi}$ following thyroid hormone withdrawal every 6 months for 2 years and annually afterwards in case of RAI efficacy) were evaluated. The best scintigraphic response evaluated on post-therapeutic WBS was a complete response (CR) in $1(3 \%)$ case, a partial response (PR) in $25(78 \%)$ cases. The best PERCIST response evaluated on FDG PET was a CR in $5(15 \%)$ cases, a PR in $14(44 \%)$ cases. The best RECIST response among 19 patients with target lesions was PR in 10 (53 $\%$ ) cases and SD in $9(47 \%)$ cases. The mean number of RAI administration to obtain the best response ranged from 1.7 to 2.1 depending on the method used for tumor evaluation. The mean duration of response ranged from 25 to 31 months depending on the method used for tumor evaluation. Thyrogobulin serum levels decreased by more than $50 \%$ in $21(66 \%)$ cases.

Conclusions: FDG+/RAI patients are not cured with RAI but can benefit from RAI administration. Patients should not be excluded from RAI treatment based on FDG uptake.

\section{OP-06-43 \\ V804M RET MUTATION AND VANDETANIB RESPONSE IN METASTATIC MEDULLARY THYROID CANCER}

Laura Valerio ${ }^{1}$, Valeria Bottici ${ }^{1}$, Antonio Matrone ${ }^{1}$, Alessia Tacito ${ }^{1}$ Francesca Casella ${ }^{1}$, Cristina Romei ${ }^{1}$, Paolo Vitti ${ }^{1}$, Rossella Elisei ${ }^{1}$ ${ }^{1}$ Department of Clinical and Experimental Medicine, Endocrine Unit, University of Pisa, Italy

Objective: Vandetanib is the most important treatment for metastatic medullary thyroid cancer (MTC). "In vitro" studies showed that the presence of the V804M RET mutation is associated with a selective resistance to vandetanib. The objective of this study was to evaluate the response to vandetanib in metastatic V804M positive MTC patients.

Methods: Four metastatic MTC patients, treated with vandetanib, were V804M RET positive. In 2 cases this mutation was a germinal mutation, while in other 2 was a somatic mutation. During the follow-up serum calcitonin levels and computed tomography scan were performed.

Results: All cases had multiple metastatic lesions distributed in several organs. In 1 case (n 1) the enrollment was due to the symptom (diarrhea) while in 3 cases (n 2-3-4) to progression of the disease. The patient-1 had a symptomatic familial MTC. During the 55 months of follow up, she showed a stable disease (SD). The patient-2, 3 and 4 had a progressive metastatic sporadic MTC. During follow up, the patient-2 had an initial partial response (PR) and then a SD for 9 months, the patient-3 had an initial PR and then a SD for 14 months, the patient- 4 did not respond to treatment. The most common adverse events were: acneiform rash in all patients, asthenia in patients 1 and 3, nausea, anorexia and weight loss in patient-2.

Conclusions: In our study we observed that patients with ,,resistant“ mutations, such as V804M RET mutation, can respond (3/4) or not respond $(1 / 4)$ to vandetanib. Therefore, also in the presence of this mutation it is indicated to start vandetanib since it can likely act also through mechanisms of action independently from RET inhibition such as VEGF-R inhibition. 


\section{OP-06-44 \\ OUTCOME AFTER LOBECTOMY FOR DIFFERENTIATED THYROID CANCER IN A SERIES OF 291 CONSECUTIVE CASES}

Bosset Matthieu', Bournaud Claire ${ }^{2}$, Lifante Jean-Christophe ${ }^{3}$, Decaussin Myriam $^{4}$, Michel Pugeat ${ }^{5}$, Francoise Borson-Chazot ${ }^{1}$

${ }^{1}$ Hospices Civils de Lyon, Federation D'endocrinologie, Bron, France,

${ }^{2}$ Hospices Civils de Lyon, Centre de Médecine Nucleaire, Bron,

France, ${ }^{3}$ Hospices Civils de Lyon, Service de Chirurgie Endocrinienne, Pierre Benite, France, ${ }^{4}$ Hospices Civils de Lyon, Laboratoire

D'anatomie Pathologique, Pierre Benite, France, ${ }^{5} \mathrm{Hôpital}$ Neuro-

Cardio-Aile A1, Hospices Civils de Lyon, Fédération D'endocrinologie, Bron, France

Context: Recommendations 2015 of the ATA suggest that a lobectomy may be sufficient to treat low risk differentiated thyroid cancer.

Objective: Analyze the outcome of patients after lobectomy for differentiated thyroid cancer

Method: retrospective and monocentric cohort study of 295 patients operated in a tertiary center between 1971 and 2010 (women : 79,3\%, men : $20,7 \%$; median age : 39 years(11-74)). Median follow-up was 19.3 years (3-44). The follow-up was over 5 years for all patients without relapse in the first 5 years.

Results: Histopathology was papillary $(86,8 \%)$, follicular $(12,5 \%)$, or oncocytic $(0,7 \%)$. The ATA risk was considered as low for $91,9 \%$ and intermediate for $8,1 \%$. Total thyroidectomy (followed or not by radio iodine ablation) has been performed in 53 patients $(18 \%)$ for a suspicion of recurrence which was histologically confirmed in $39(13.2 \%)$ : 36 loco-regional recurrences and 3 metastatic recurrences. The initial ATA risk of recurrent patients was low for $87.2 \%$, intermediate for $12.8 \%$. The median time before iterative surgery was 12.2 years (1.4-34.1). Recurrence was diagnosed for $18 \%$ within 5 years, for $28 \%$ between 5 and 10 years and for $54 \%$ more than 10 years after surgery. After a postoperative median follow up of 6.62 years $(0.3-23), 21 / 39$ recurrent patients were in remission, 10 had indeterminate status (no evidence of disease but follow-up $<5$ years after second surgery), 7 had persistent illness $(2.4 \%$ of the entire cohort) and 1 patient died from progression of the disease. Fifty three patients (18\%) with micro nodular disease in the contralateral lobe were regurlaly followed without reoperation since thyroglobulin was low and FNA non suspicious.

Conclusion: the prognosis of differentiated thyroid cancer treated by lobectomy is good but late relapses may occur requiring a very prolonged follow.

\section{OP-06-45 \\ ACTIVE SURVEILLANCE IN PAPILLARY THYROID MICROCARCINOMAS (PMCS): A 2 YEARS FOLLOW UP (FU) AT A SINGLE CENTER}

Letizia Pieruzzi ${ }^{1}$, Antonio Matrone ${ }^{1}$, Laura Agate ${ }^{1}$, Valeria Bottici',

David Viola', Claudio Marcocci' ${ }^{1}$, Paolo Vitti', Rossella Elisei', Eleonora Molinaro ${ }^{1}$

${ }^{1}$ Unit of Endocrinology, Department of Clinical and Experimental

Medicine, University of Pisa, Italy

Objective: an active surveillance can be considered as an alternative to immediate surgery in PMC, however few studies are available at the present. After the approval of our Ethical Committee on November 2014 we started the "active surveillance" in PMC patients (5 yrs for enrollment period and 5 yrs for FU).

Methods: the inclusion criteria were the presence of a single thyroid nodule $<1.3 \mathrm{~cm}$ in the biggest diameter at neck ultrasound (nUS) with a Thy 4 or Thy 5 cytology on FNA, and no evidence of metastatic latero-cervical lymphnodes at nUS. FNAB specimens were also collected for genetic analysis.

Results: we enrolled 66 patients with thyroid nodules with the above mentioned features. They were $15 / 66(23 \%)$ males and 51/66(77\%) females with a mean age of 45 yrs(21-79). Cytology on FNA was Thy4 in 19/66(28,8\%) and Thy5 in $47 / 66(71,2 \%)$ nodules. Patients were followed-up every six months with nUS and thyroid function tests. After a median FU of 24 months(6-28) only $3 / 66(4.5 \%)$ showed a clinical progression with the appearance of metastatic lymph nodes or an enlargement of the nodule (from 10 to 15 months after enrolment). In these 3 patients radioiodine ablation was also performed after surgical treatment, according to the histological features $(2 / 3 \mathrm{pT} 3 \mathrm{~N} 1 ; 1 / 3$ pT3). To date these patients are cured (no structural or biochemical persistent disease). During the first 12 months of FU 7/66(10.6\%) patients withdrew the study for personal reasons and opted for surgery, genetic analysis are ongoing.

Conclusions: an active surveillance appears to be a suitable and safe approach in patients with PMC. Less than 5\% of PMC required to be surgically treated during the first 2 years of observation and their delayed treatment did not impact on the final outcome. It's worth to note that $10 \%$ of patients dropped out for personal reasons.

\section{Oral Session 7 (Basic): Mechanisms of Thyroid Hormone Action in Brain and Thyroid}

\author{
OP-07-46 \\ SELENO-METHIONINE EXERTS PROTECTIVE \\ EFFECTS AGAINST H2O2 INDUCED OXIDATIVE \\ STRESS IN THYROCYTES AND FIBROBLASTS \\ CELLS \\ Rosaria Ruggeri', Teresa Manuela Vicchio', Angela D'Ascola ${ }^{2}$, \\ Salvatore Campo ${ }^{2}$, Fiorenza Giani ${ }^{3}$, Michele Scuruchi', Alfredo \\ Campenni ${ }^{5}$, Francesco Frasca ${ }^{6}$, Francesco Trimarchi ${ }^{7}$ \\ ${ }^{1}$ Department of Clinical-Experimental Medicine and Pharmacology, \\ Division of Endocrinology, University of Messina, Aou Policlinico DI \\ Messina, Messina, Italy, ${ }^{2}$ Dep. of Biomedical and Dental Sciences, and \\ Morfo-Functional Images, University of Messina, Messina, Italy, ${ }^{3}$ Dep. \\ of Clinical and Molecular Bio-Medicine, Endocrinology Unit, Univ. of \\ Catania, Catania, Italy, ${ }^{4}$ Dep. of Clinical and Experimental Medicine, \\ Internal Medicine Unit; Univ. of Messina, Messina, Italy, ${ }^{5} \mathrm{Dep}$. of \\ Biomedical and Dental Sciences, and Morfo-Functional Images, \\ Nuclear Medicine Unit, University of Messina, Aou Policlinico G. \\ Martino Messina, Messina, Italy, ${ }^{6}$ Endocrinology, Dept. of Clinical and \\ Experimental Medicine, Garibaldi-Nesima Medical Center, University \\ of Catania, Italy, Catania, Italy, ${ }^{7}$ University of Messina, Accademia \\ Peloritana Dei Pericolanti, Messina, Italy
}

Background: Selenium ( $\mathrm{Se}$ ) is an essential antioxidant nutrient involved in thyroid hormone metabolism and incorporated into antioxidant selenoproteins thereby protecting thyrocytes from $\mathrm{H}_{2} \mathrm{O}_{2}$ generated by thyroid hormone synthesis. Oxidative stress has been implicated in the pathogenesis of several disorders including autoimmune thyroid diseases (AITD). Since previous studies have evaluated the protective role of selenium supplementation in AIDT, we studied the in vitro effect of Seleno-Methionine (Se-Met) on oxidative damage induced by $\mathrm{H}_{2} \mathrm{O}_{2}$ in both human thyrocytes and fibroblasts.

Methods: Primary cells were obtained from patients subjected to surgery for benign thyroid diseases. Preliminary tests of sensitivity to hydrogen peroxide indicated respectively that $750 \mu \mathrm{M}$ and $50 \mu \mathrm{M} \mathrm{H}_{2} \mathrm{O}_{2}$ were able to induce cell death in $50 \%$ thyrocytes and fibroblasts. Cultured cells were exposed to $\mathrm{H}_{2} \mathrm{O}_{2}$ in the presence or the absence of Se-Met $(5-10-20 \mu \mathrm{M})$ for 24 hours. The cell survival rate was evaluated by MTT assay and genotoxyc stress by DNA fragmentation assay. MTT results were expressed as \% of untreated cells. Statistical analysis was performed by two-tailed ANOVA followed by post hoc Newman-Keuls' test.

Results: The treatment with Se-Met exerted a protective effect in a dosedependent manner. Thyrocytes exposed to $\mathrm{H}_{2} \mathrm{O}_{2}$ and treated with Se-Met $5 \mu \mathrm{M}$, $10 \mu \mathrm{M}$ and $20 \mu \mathrm{M}$ showed a significant increase in cell viability in a dose dependent manner compared to cells incubated with $\mathrm{H}_{2} \mathrm{O}_{2}$ alone $(\mathrm{p}<0,05$; $\mathrm{p}<0,0001 ; \mathrm{p}<0,0001$ respectively). A similar significant protective effect of Se-Met was observed in fibroblasts. A greater extent of fragmented DNA strands were observed in thyrocytes and fibroblasts treated with $\mathrm{H}_{2} \mathrm{O}_{2}$ compared to untreated cells. In contrast, only a small amount of DNA damage was observed in cells treated with both $\mathrm{H}_{2} \mathrm{O}_{2}$ and $20 \mu \mathrm{M}$ Se-met.

Conclusions: These results indicate that in human thyrocytes and fibroblast Se-Met prevents the effect $\mathrm{H}_{2} \mathrm{O}_{2}$ on both cell viability and DNA damage. 


\section{OP-07-47 \\ THE CHICKEN OPTIC TECTUM AS A CONVENIENT MODEL TO STUDY MCT8- DEFICIENT CORTICOGENESIS}

Pieter Vancamp ${ }^{1}$, Michiel Remmerie ${ }^{1}$, Marie-Anne Deprez ${ }^{2}$, Veerle Darras $^{1}$

${ }^{1}$ Laboratory Comparative Endocrinology, Biology Department, Ku Leuven, Biology Department, Ku Leuven, Leuven, Belgium, ${ }^{2}$ Laboratory Functional Biology, Biology Department, Ku Leuven, Leuven, Belgium

Objectives: Thyroid hormones (THs) are essential for the assembly of a layered cortex, even prior to the onset of foetal thyroid function. The relatively high expression of the TH transporter MCT8 during this process suggests an important role in facilitating local TH uptake. Not surprisingly, cortical deficits are prominent in patients lacking functional MCT8 (Allan-Herndon-Dudley syndrome), although a mechanistic explanation is still lacking.

Methods: We used the chicken optic tectum $(\mathrm{TeO})$ as a model to study MCT8-deficient corticogenesis. Neurogenesis in the $\mathrm{TeO}$ commences at embryonic day 3 (E3) and the formation of its layered structure is completed by E12. The neuro-epithelium was electroporated at E3 with an MCT8-RNAi vector to knock down MCT8 expression, or with an empty vector as control. Co-expression of RFP allowed visualisation of transfected cells.

Results: The slower increase in tectal thickness following MCT8 knockdown resulted in a thinner cortex at E12. Cell counts revealed a lower tectal cell number, which is a direct consequence of a significantly smaller progeny of the transfected population. EdU-labelling at E4 and E5 confirmed the reduced progenitor cell pool expansion. Additionally, MCT8-RNAi-transfected cells were mostly retained in the inner layers indicating arrested cell migration. This is consistent with reduced reelin expression and disorganised alignment of radial glial cell fibres found at E6. Finally, the number of BRN3a-expressing glutamatergic cells was significantly reduced at E12. This could be a side effect of the cell loss, as the fraction of differentiating cells in the transfected population was unaltered. The reduction in LHX1/5-expressing GABAergic cells is however also a consequence of reduced differentiation.

Conclusions: Our data imply that MCT8 deficiency impairs the processes of cell proliferation, migration and differentiation that underlie early corticogenesis, and could help to understand the mechanisms underlying brain lesions in the Allan-Herndon-Dudley syndrome.

\section{OP-07-48 \\ ALTERED DEVELOPMENT OF THE CEREBRAL COMMISSURES IN TRANSIENT EARLY POSTNATAL HYPOTHYROID RATS. CORRELATION BETWEEN MRI AND EM}

Federico Lucia-Curt', Jesús Pacheco-Torres², Susana GonzálezGranero $^{3}$, Santiago Canals ${ }^{4}$, María Jesús Obregón ${ }^{5}$, Jose Manuel Garcia-Verdugo ${ }^{3}$, Pere Berbe/ ${ }^{6}$

${ }^{1}$ Departamento de Anatomía e Histología, Universidad Miguel Hernández, Sant Joan D'alacant (Alicante), Spain, ${ }^{2}$ Instituto de Neurociencias Universidad Miguel Hernández-Csic, Sant Joan D'alacant, Alicante, Spain, ${ }^{3}$ Laboratorio de Neurobiología Comparada. Instituto Cavanilles, Universidad de Valencia, Ciberned, Valencia, Spain, ${ }^{4}$ Instituto de Neurociencias Universidad Miguel Hernández-Csic, Sant Joan D'alacant, Alicante, Spain, ${ }^{5}$ Instituto de Investigaciones Biomédicas "alberto Sols", Consejo Superior de Investigaciones Científicas Y Universidad Autónoma de Madrid, Madrid, Spain, ${ }^{6}$ University Miguel Hernández, Histology and Anatomy, Sant Joan D'alacant (Alicante), Spain

The early postnatal age is a critical period for the interhemispheric commissure development of mammals. Recent MRI studies in humans show altered cognitive functions involving interhemispheric connections following transient postnatal hypothyroidism. We aimed to correlate MRI and corpus callosum (CC) and anterior commissure (AC) ultrastructural data of transient postnatal hypothyroid rats, to study alterations in commissural development and recovery after delayed transitory L-thyroxin treatment (T4).

Hypothyroid dams and pups received $0.02 \%$ methimazole and $1 \% \mathrm{KClO}_{4}$ treatment in the drinking water (MMI). The experimental groups were: $\mathrm{MMI}_{\mathrm{P} 0-}$ ${ }_{21}$ (MMI from postnatal day 0 (P0) to $\mathrm{P} 21$ ), $\mathrm{MMI}_{\mathrm{P} 0-21}+\mathrm{T}_{\mathrm{P} 15-21}\left(\mathrm{MMI}_{\mathrm{P} 0-21}\right.$ plus
T4 from P15-P21 and $\mathrm{MMI}_{\mathrm{P} 0}$ (MMI from P0 onwards). Results were compared with control (C) and $\mathrm{MMI}_{\mathrm{E} 10}$ (MMI from embryonic day 10 (E10) onwards). At P150, axon number, percentage and diameter (myelinated and unmyelinated), myelin thickness and g-ratio were quantified from EM images of the $\mathrm{AC}$ (anterior and posterior limbs) and the CC (genu, body and splenium).

Mean $\mathrm{T}_{2}$ ratios $\left(\mathrm{T}_{2} \mathrm{r}\right)$ from the $\mathrm{AC}$ and $\mathrm{CC}$ were obtained at ages from P8-P365 (6-11 pups/group). In the AC and CC from P40 onwards, we observed significant differences between postnatal $\mathrm{MMI}$ and both $\mathrm{C}$ and $\mathrm{MMI}_{\mathrm{E} 10}$ groups. No differences were found between $\mathrm{MMI}_{\mathrm{P} 0-21}$ and $\mathrm{MMI}_{\mathrm{P} 0-21}+\mathrm{T}_{\mathrm{P} 15-21}$ groups in the $\mathrm{AC}$ and $\mathrm{CC}$ body. Regression functions for myelinated axon density, axon diameter (myelinated and unmyelinated) and cell number were calculated from the $\mathrm{T}_{2} \mathrm{r}$ of $\mathrm{C}$ rats. The expected morphometric values obtained from these regression functions in the $\mathrm{AC}$ were similar to quantitative $\mathrm{EM}$ data of MMI rats.

Our data show that a transient early postnatal $\mathrm{TH}$ deficit, including lactation, alters the maturation of $\mathrm{AC}$ and $\mathrm{CC}$ in pups; no recovery was seen after delayed T4 treatment. Morphometric ultrastructural values of the AC can be estimated from $\mathrm{T}_{2} \mathrm{r}$ values using regression functions.

ALICIA KOPLOWITZ Foundation, MINECO-SAF2014-58256-R and GV-PROMETEOII/2014/075.

\section{OP-07-49 \\ EFFECTS OF 3-IODOTHYRONAMINE (T1AM) ON SIGNALING PATHWAYS INVOLVED IN SYNAPTIC PLASTICITY AND NEUROPROTECTION}

Ginevra Sacripanti ${ }^{1}$, Maria Pia Fogliaro ${ }^{1}$, Giulia Giannini ${ }^{1}$, Leonardo

Lorenzini ${ }^{2}$, Riccardo Zucchi ${ }^{3}$, Sandra Ghelardoni ${ }^{1}$

${ }^{1}$ University of Pisa, Pisa, Italy, ${ }^{2}$ Dipartimento DI Patologia Chirurgica,

Pisa, Italy, ${ }^{3}$ University of Pisa, Dept. of Pathology, Pisa, Italy

Background: In mouse, the administration of 3-iodothyronamine (T1 AM) had an antiamnestic and prolearning effect. In addition, T1AM rescued long term potentiation (LTP) in enthorinal cortex exposed to the toxic effect of â-amyloid. In the present paper we used a neuronal cell line to evaluate the effect of T1AM on the expression calcium calmodulin dependent kinase II (CamKII) and protein kinase C (PKC), two key players involved in LTP. The effects on sirtuin 1 (SIRT1), allegedly responsible for the neuroprotective effect of resveratrol, were also investigated.

Methods: A hybrid line of cancer cells of mouse neuroblastoma and rat glioma (NG108-15) was used and treated with $100 \mathrm{nM}$ to $10 \mu \mathrm{M}$ T1AM for $24 \mathrm{~h}$, alone or in combination with $10 \mu \mathrm{M}$ resveratrol and/or $10 \mu \mathrm{M}$ amyloid â peptide (25-35). Protein expression and post-translational modifications were investigated by Western blot technique. Glucose consumption was also measured upon $4 \mathrm{~h}$ treatment.

Results: NG108-15 expressed TAAR1, the putative T1AM receptor, as well as AMPA and NMDA receptors, that are involved in the signaling cascade responsible of LTP. T1AM $(10 \mu \mathrm{M})$ induced CaMKII phosphorylation $(\mathrm{p}<0.001)$, but had no direct effect on PKC and SIRT1 expression. However, in the presence of â-amyloid, T1AM increased PKC and SIRT 1 expression at $1 \mu \mathrm{M}$ and $10 \mu \mathrm{M}$ concentration, respectively, although â-amyloid alone had no significant effect. The response to resveratrol was also modified by T1AM, since resveratrol inhibited PKC expression while the association of resveratrol and T1AM $(100 \mathrm{nM})$ produced a remarkable increase vs the baseline $(\mathrm{P}<0.05)$. Furthermore an increase in glucose consumption was observed at the highest T1AM concentration $(\mathrm{p}<0.01)$.

Conclusions: In NG108-15 cells T1AM activates CaMKII and has complex effects on the response to â-amyloid and resveratrol.

\section{OP-07-50 \\ LOCAL CONTROL OF THYROID HORMONE AVAILABILITY IN THE ADULT NEURAL STEM NICHE \\ Cristina Luongo ${ }^{1}$, Sylvie Remaud ${ }^{2}$, Jean-David Gothié2, Karine Le Blay $^{1}$, Anthony Sebillot ${ }^{1}$, Barbara Demeneix ${ }^{3}$ \\ ${ }^{1}$ Umr Cnrs 7221, Paris, France, ${ }^{2}$ Muséum National D'histoire Naturelle, Paris, France, ${ }^{3}$ Umr7221 Cnrs / Mnhn, Paris, France}

Thyroid hormones (THs) are essential for brain function throughout life, from early development to ageing. How neurons and oligodendrocytes cells are generated from adult neural stem cells (NSCs) is not fully understood. 
Deciphering how endocrine signals affect cell fate decision between neurons and glia is essential for understanding the physiology of niche plasticity and homeostasis.

Our hypothesis is that in murine adult Subventricular Zone (SVZ), neuron-glia lineage fate decisions involve a tightly regulation of THs availability, which is determined locally notably by cell specific expression of TH transporters (THT) and deiodinases.

Using immunofluorescence both in vivo and in vitro (neurosphere assays) on the adult mice SVZ we show that the two THTs, MCT8 and OATP1C1, are highly expressed in both NSCs and neuronal progenitors (NPCs), but not in oligodendrocyte progenitors (OPCs). In contrast, OPCs, but not NPCs, express high levels of the T3-inactivating deiodinase, D3, thus, protecting glial progenitors from the neutralizing effects of $\mathrm{T} 3$.

To address the effects of modulations of TH availability on cell fate, neurosphere cultures were treated with increasing doses of $\mathrm{T} 3$ or $\mathrm{T} 4$. We showed that a T4 administration strongly increases NPCs, without affecting OPCs, especially at the beginning of cell differentiation. Furthermore, T3 increases NPCs genesis at the expense of OPCs, especially at day 7 of differentiation.

Taken together, these data show that TH signalling is required for NSC commitment toward NPCs. In NPCs, high intracellular TH availability is facilitated by the expression of THTs. In contrast, in OPCs the expression of Dio3 reduces $\mathrm{T} 3$ availability promoting glial determination that is impaired by $\mathrm{T} 3$ neuralizing effects.

A better understanding of the mechanisms of glia-neuron cell-fate switch governed by TH is crucial to developing stem cell therapies for treating neurodegenerative diseases.

\section{OP-07-51 \\ POLYPHENOLS FROM CITRUS FRUITS INDUCE STRUCTURAL AND IMMUNOHISTOCHEMICAL CHANGES IN THYROIDS OF OLD-AGED MALE WISTAR RATS}

Marko Miler $^{1}$, Vladimir Ajdžanoviæ ${ }^{1}$, Ivana Jariæ ${ }^{1}$, Jasmina Živanoviæ ${ }^{1}$, Nevena Kardum ${ }^{2}$, Dragan Milenkoviæ ${ }^{3}$, Verica Miloševiæ ${ }^{1}$, Branka Šošiæ-Jurjeviæ

${ }^{1}$ Department of Cytology, Institute for Biological Research "siniša Stanković", University of Belgrade, Belgrade, Serbia, ${ }^{2}$ Centre of Research Excellence in Nutrition and Metabolism, Institute for Medical Research, University of Belgrade, Belgrade, Serbia, ${ }^{3}$ Inra, Umr 1019, Unh, Crnh Auvergne, Clermont Université, Université D'auvergne, Unité de Nutrition Humaine, Clermont-Ferrand, France

Polyphenols are class of plant secondary metabolites that exert various beneficial effects. Namely, citrus polyphenols, naringenin (NAR) and hesperetin (HES) are potent antioxidants that may contribute to maintenance of health at old age by improving redox and metabolic status. However, polyphenols also interfere with thyroid peroxidase (TPO) activity and might affect thyroid structure and hormone production. Keeping in mind impaired thyroid function at older age, in this study we tested weather NAR or HES administration could induce structural and immunohistochemical changes in male 24-monthold Wistar rats. NAR or HES were administrated orally $(15 \mathrm{mg} / \mathrm{kg})$ during 4 weeks, while control groups received vehicle, sunflower oil. Stereological analyses (absolute and relative volume of epithelium, colloid and interstitium) and qualitative immunohistochemical examination of TPO and vascular endothelial growth factor (VEGF) proteins were performed. Results showed that thyroid structure of both flavanone-treated groups was characterized by lower $(\mathrm{p}<0.05)$ absolute and relative volume of luminal colloid, accompanied by elevated $(\mathrm{p}<0.05)$ relative volume of interstitium in comparison with the controls. These changes were more prominent after NAR even no hypertrophy or absolute thyroid volume change was detected. TPO expression was localized in the cytoplasm and on the apical membrane of the active follicles, showing stronger immunostaining in both flavanone treated group in comparison with the controls. VEGF immunohistochemical expression was localized in the thyrocytes's cytoplasm of thyrocytes and C cells, being stronger in flavanone-treated animals. In conclusion, both citrus flavanones induced structural alteration of thyroid tissue. Depleted colloid and stronger apical TPO immunostaining in the thyrocytes indicate higher thyroid hormone secretion and production. In line with this, interstitium volume increment, and higher VEGF expression in thyroid parenchyma might be due to a better blood supply and the expansion of microcirculation around active follicles in the thyroid of old-aged males.

\section{OP-07-52}

\section{A NEW SMALL MOLECULE TSH-RECEPTOR SELECTIVE ANTAGONIST TOWARDS GRAVES' ORBITOPATHY}

Patrick Marcinkowski ${ }^{1}$, Hoyer Inna', Specker Edgar ${ }^{3}$, Jens Furkert ${ }^{3}$, Utta Berchner-Pfannschmidt', Ralf Schuelein ${ }^{3}$, Anja Eckstein ${ }^{5}$, Gerd Krause ${ }^{6}$

${ }^{1}$ Leibniz-Institut für Molekulare Pharmakologie Berlin, Berlin, Germany, ${ }^{2}$ Leibnitz Instutut Fuer Molekulare Pharmakologie (Fmp), Berlin, Germany, ${ }^{3}$ Leibniz Institut für Molekulare Pharmakologie (Fmp), Berlin, Germany, ${ }^{4}$ Univ.-Augenklinik, Molekulare Ophthalmologie, Essen, Germany, ${ }^{5}$ Univ.-Augenklinik, Essen, Germany, ${ }^{6}$ Leibniz-Institut für Molekulare Pharmakologie (Fmp), Berlin, Germany

Functional autoimmune antibodies against the thyrotropin receptor (TSHR) are the main trigger of Graves' Disease (GD) and Graves' Orbitopathy (GO). Since the autoimmune autoantibodies bind on the same site as TSH, the big extracellular portion of the receptor serves as an antigen for TSHRactivating immunoglobulins. In case of GD it results in hyperthyroidism. GO is characterized by the pathological activation of TSHR in retroorbital fibroblasts leading to exophthalmos. Severe GO is considered as a dilemma due to a clinically therapeutic gap.

This motivates us to develop a TSHR antagonist based on a high-throughput screening and subsequent structure-activity relationship studies (SAR by catalog) that included small molecules with several chiral stereo centers. Stereo-selective synthesis led to the racemic molecule S37 exhibiting micromolar inhibition of TSH-induced cAMP accumulation in HEK cells expressing hTSHR. S37 was separated into the two enantiomers S37a and S37b by reversed-phase chiral HPLC. S37a showed a $20 \%$ stronger inhibition than S37, while S37b had only a very weak effect. The absolute configuration of the enantiomers could be assigned by comparing measured and predicted circular dichroism (CD) spectra by the time-dependent DFT method. Testing S37a on beta arrestin1 recruitment on U2OS cells shows less undesirable inhibition. The enantiopure structure of S37a is completely different from other known small molecule TSHR inhibitors. Due to its rigidity and particular shape S37a is highly selective for TSHR and did not at all affect the closely homologous lutropin (LH) and follitropin (FSH-) receptors. Our compound S37a inhibits the monoclonal TSHR-activating antibody M22 and TSH with similar efficacy. Moreover, S37a is notably also able to suppress significantly TSHR-activating sera from GO patients. We demonstrate new data about a TSHR-selective inhibitor, which is promising for in vivo application in mice and has a potential for further development towards GO.

\section{OP-07-53 \\ 3-IODOTHYRONAMINE RESCUES BETA- AMYLOID-DEPENDENT INHIBITION OF LONG-TERM POTENTIATION IN VITRO AND RESTORES RECOGNITION MEMORY IN A MOUSE MODEL OF ALZHERIMER'S DISEASE}

Alice Accorroni ${ }^{1}$, Chiara Criscuolo ${ }^{2}$, Martina Stazi ${ }^{3}$, Martina Sabatini ${ }^{4}$, Nicola Origlia ${ }^{2}$, Riccardo Zucchi ${ }^{5}$

${ }^{1}$ Scuola Superiore Sant'anna, Pisa, Italy, ${ }^{2} \mathrm{Cnr}$ Neuroscience Institute, Pisa, Italy, ${ }^{3}$ University of Pisa, Pisa, ${ }^{4}$ Dept. of Pathology, University of Pisa, Pisa, Italy, ${ }^{5}$ University of Pisa, Dept. of Pathology, Pisa, Italy

3-iodothyronamine (T1AM) acts as a memory enhancer as it improves learning and memory in mice. We investigated T1 AM effects on long-term potentiation (LTP) in vitro and on recognition memory in a mouse model of Alzheimer's disease (AD) after intra-cerebro-ventricular (i.c.v.) administration. We employed entorhinal cortex (EC) slices from WT mice exposed to â-Amyloid (Aâ) and T1AM and a mouse model of AD (mhAPP) to assess recognition memory in the Novel-Object Recognition Test (NORT).

Field potentials were evoked in EC layer II after stimulation of the same layer and LTP was elicited by high frequency stimulation (HFS). T1AM (5 $\mu \mathrm{M})$ and/or Aâ $(200 \mathrm{nM})$ were administered for 10 minutes, starting 5 minutes before HFS. In NORT, after T1AM i.c.v administration, the mouse was presented with two similar objects during the sample session, and then to a new object (NO) during the trial session. The amount of time spent to explore the NO represents an indirect index of recognition memory. Exploration times 
were used to calculate a discrimination index (DI) [exploration of $\mathrm{NO}$ - exploration of old object] / [total time exploring both objects].

Exposure to Aâ inhibited LTP ( $-2 \pm 6 \%$ of the baseline), but T1AM perfusion restored it $(+23 \pm 10 \%$ of the baseline, $\mathrm{P}<0.05)$. In EC from APPJ 20 mice, LTP could not be elicited $(-10 \pm 7 \%$ of the baseline), but it was rescued by T1AM $(+20 \pm 9 \%$ of the baseline, $\mathrm{P}<0.05)$. Recognition memory was impaired in mhAPP mice vs WT (DI:0.34 \pm 0.07 vs $0.68 \pm 0.04 \mathrm{p}=0.01$ ). However, DIs were not significantly different between WT and mhAPP mice treated with $\mathrm{T} 1 \mathrm{AM}(0.68 \pm 0.04$ vs. $0.72 \pm 0.1)$

Our results suggest that T1AM counteracts the effects of Aâ on LTP and restores recognition memory in mhAPP mouse. A more thorough understanding of the memory enhancing properties of T1AM and of the underlying mechanisms might open new perspectives in the treatment of $\mathrm{AD}$.

\section{Oral Session 8 (Clinical): Clinical Aspects of Pregnancy and Childhood}

\section{OP-08-54 \\ MATERNAL AND PLACENTAL RESPONSES PRIOR TO PRETERM DELIVERY: COMPENSATORY ADAPTATIONS TO INCREASE THYROID HORMONE AVAILABILITY FOR THE PRETERM NEWBORN? \\ An Eerdekens ${ }^{1}$, Lies Langouche ${ }^{2}$, Fabian Güiza ${ }^{2}$, Johan Verhaeghe ${ }^{3}$, \\ Gunnar Naulaers ${ }^{1}$, Christine Vanhole ${ }^{1}$, Greet Van den Berghe ${ }^{2}$ \\ ${ }^{1}$ Department of Neonatology, University Hospitals Leuven, Catholic University Leuven, Leuven, Belgium, ${ }^{2}$ Clinical Division and Laboratory of Intensive Care Medicine, Department of Cellular and Molecular Medicine, Catholic University Leuven, Leuven, Belgium, ${ }^{3}$ Department of Obstetrics \& Gynecology, University Hospitals Leuven, Catholic University Leuven, Leuven, Belgium}

Background: During pregnancy, maternal thyroid hormone (TH) supply is crucial for fetal development. Elevated maternal TSH, interpreted as subclinical maternal hypothyroidism, has been associated with pregnancyassociated vascular complications and preterm delivery. Preterm infants often present with hypothyroxinemia. We hypothesized that these entities are linked and that in conditions of pregnancy-associated vascular complications, maternal and placental compensatory mechanisms are activated to increase $\mathrm{TH}$ transfer.

Materials and Methods: Observational case-control study in motherinfant-dyads with complicated pregnancies ending in spontaneous (SPB) $(n=31)$ or indicated preterm birth due to vascular complications (IPB) $(n=45)$ and normal pregnancies (controls) $(\mathrm{n}=41)$. At delivery, maternal and cord blood and placenta samples were collected.

Plasma concentrations of TSH, total T4, fT4/FTI, total T3, TBG, and in mothers TPO-antibodies, were measured. Placental maturity was histologically evaluated and protein and/or gene expression levels of TH deiodinases (DiO) 1, 2 and 3 and transporters (MCT8, MCT10 and OATP1c1) were quantified.

Results: In IPB and SBP, cord plasma T4 concentrations were lower than in controls $(P<0.001)$, whereas T3 was only decreased in SPB $(P<0.001)$. Compared with SPB and controls, IPB was characterized by higher maternal plasma TSH $(P \leq 0.05)$, earlier placenta maturation, higher placental $\mathrm{DiO} 2$ gene and MCT10 protein expression and lower DiO3 gene expression (all $P \leq 0.01)$. OATP1c1 mRNA was not detected in human placenta, DiO1 mRNA expression was just above detection limit in all placenta.

Conclusions: Low T4 was observed in preterm infants irrespective of the cause, but only in IPB maternal (TSH) and placental (Dio2, Dio3 and MCT10) compensatory responses were activated, which may have mediated the normal fetal T3 availability in IPB but not SPB infants.

\section{OP-08-55 \\ UNIVERSAL SALT IODINATION IN CROATIA MEETS IODINE REQUIREMENTS OF THE POPULATION - THE RESULT OFSIMPLIFY STUDY}

Tomislav Jukic ${ }^{1}$, Maria Andersson ${ }^{2}$, Susanne Dold ${ }^{2}$, Michael Zimmermann ${ }^{2}$, Valentina Vidranski ${ }^{3}$, Ivan Jakšiæ ${ }^{3}$, Roko Graniæ ${ }^{3}$, Josip Stanièiæ ${ }^{3}$, Zlatko Topaloviæ ${ }^{4}$, Maja Franceschi', Zvonko Kusiæ ${ }^{5}$ ${ }^{1}$ University Department of Oncology and Nuclear Medicine, Sestre Milosrdnice University Hospital Centre, Zagreb, Croatia, Faculty of Medicine, University Josip Juraj Strossmayer Osijek, Osijek, Croatia, University of Zagreb, School of Medicine, Zagreb, Croatia, ${ }^{2}$ Swiss Federal Inst. of Tech. Zurich, Lab. for Human Nutrition, Bld. Lfv E19, Zürich, Switzerland, ${ }^{3}$ University Department of Oncology and Nuclear Medicine, Sestre Milosrdnice University Hospital Centre, Zagreb,

Croatia, ${ }^{4}$ Primary Health Care Clinic "centar", Zagreb, Croatia,

${ }^{5}$ University of Zagreb, School of Medicine, Zagreb, Croatia

Objectives: Due to universal well-established salt iodization (USI) program, Croatia, China and Philippines were selected to participate in international SIMPLIFY study: Salt Iodization: Meeting the needs of Pregnancy, Lactation and InFancY, funded by Global Alliance for Improved Nutrition, Geneva Switzerland, UNICEF, ETH Zürich and with support of Croatian Science Foundation IP-2014-09-6499 and EU Horizon 2020 project EUthyroid under grant agreement 634453. Data for Croatia are presented.

The aim of the study was to test whether USI in Croatia can meet iodine requirements of the population.

Methods: The study enrolled 200 schoolchildren (SAC) (median age $9 y$ ), 227 women of reproductive age (WRA), (median age 31y), 202 pregnant women (PW) (median age 32y), 145 lactating women (LW) (median age 32y), 111 breastfed infants (BFI) (median age 12 weeks) and 51 toddlers (median age 10 months) as mother-child pairs from the region of Zagreb, capital of Croatia. Urinary iodine concentration (UIC) in spot samples, Tg, TSH, T4 on dried blood spots (DBS) and breast milk iodine content (BMIC) were measured. Supplemet use, salt and iodine rich food frequency questionnaire were assessed. Salt, water and milk samples from households of SAC were collected and iodine content (IC) was measured in 195 salt, 19 milk and 10 water samples. DBS- $\mathrm{Tg} \geq 44 \mu \mathrm{g} / \mathrm{L}$ was considered elevated.

Results: Median UIC and DBS-Tg in $\mu \mathrm{g} / \mathrm{L}$ are 240 and 13.0 in SAC, 166 and 13.8 in WRA, 129 and 12.9 in PW, 67 and 4.8 in LW, 232 and 13.8 in BFI, 249 and 28 in toddlers. All subjects were euthyroid except one WRA with subclinical hypothyroidism. Elevated DBS-Tg was recorded in $1 \%$ SAC, $2.2 \%$ WRA, $3 \%$ PW, $1.4 \%$ LW, $17.2 \%$ BFI and $4.2 \%$ toddlers. Median BMIC was $125 \mu \mathrm{g} / \mathrm{kg}$, water IC $2 \mu \mathrm{g} / \mathrm{L}$ and household milk IC $274 \mu \mathrm{g} / \mathrm{kg}$. Median KI $/ \mathrm{kg}$ salt in $\mathrm{mg} / \mathrm{kg}$ was 23.8 with $3.6 \%<15$ and $0.5 \%$ samples $>40$.

Conclusions: USI in Croatia meets iodine requirements of the population. Thyroglobulin is a sensitive indicator of iodine intake. IC in salt is in compliance with Croatian law (20-30 mg KI/Kg salt). High IC was recorded in household milk samples. BMIC is more accurate biomarker of iodine status than UIC in exclusively breastfeeding women.

\section{OP-08-56 \\ LIVE BIRTH RATE ACCORDING TO THYROXINE REPLACEMENT THERAPY IN THYROID PEROXIDASE ANTIBODY-POSITIVE WOMEN WITH RECURRENT PREGNANCY LOSS}

Sofie Bliddal', Henriette Svarre Nielsen ${ }^{2}$, Åse Krogh Rasmussen ${ }^{1}$, Astrid Maria Kolte ${ }^{2}$, Ole Bjarne Christiansen ${ }^{2}$, Claus Henrik Nielsen ${ }^{3}$, Ulla Feldt-Rasmussen ${ }^{1}$

${ }^{1}$ Department of Medical Endocrinology, Copenhagen University Hospital (Rigshospitalet), Copenhagen, Denmark, ${ }^{2}$ Repeated Pregnancy Loss Unit, Copenhagen University Hospital (Rigshospitalet), Copenhagen, Denmark, ${ }^{3}$ Institute of Inflammatory Research, Copenhagen University Hospital (Rigshospitalet), Copenhagen, Denmark

Objectives: Thyroid antibody-positivity has been associated with pregnancy loss. This study investigated if thyroid peroxidase antibodies (TPOAbs) were associated with pregnancy outcome in women with recurrent pregnancy loss (RPL) and, if so, if this was ameliorated by thyroxine replacement therapy. 
Methods: Prospective cohort study of 900 women with RPL referred to the Copenhagen University Hospital from 2011-2016. Upon first visit, all women were screened for TSH (Roche Modular E170 electrochemiluminescense immunoassays) and TPOAbs (automated Kryptor immunoflourescent assay). TPOAb-positivity $>60 \mathrm{kU} / \mathrm{L}$. RPL $\geq 3$ losses. Test for trends by chi-square or independent t-tests. Regression analyses with covariates: maternal age at referral, TSH (log-transformed), number of losses, and thyroxine replacement. National Data Protection Agency approval was obtained (2017-41-5005)

Results: We included 900 women with a total of 5219 pregnancies. $119(13.2 \%)$ women were TPOAb-positive. TPOAb-positivity was neither associated with number of losses $(\mathrm{p}=0.87$, aOR 0.004(-0.3-0.3), $\mathrm{p}=0.98)$, nor number of pregnancies $(\mathrm{p}=0.62$, aOR 1.02(0.92-1-11), $\mathrm{p}=0.76)$. Among TPOAb-positive women, TPOAb-concentration was not associated with number of losses $(\mathrm{B}=0.75(-0.12-0.27), \mathrm{p}=0.45)$.

In their first pregnancy after referral, 72 of $557(12.9 \%)$ women were TPOAb-positive. Excluding women with extrauterine pregnancies and induced abortions $(\mathrm{n}=65)$, TPOAb-positivity was not associated with live birth rate $(54.4 \%$ vs $60.6 \%, \mathrm{p}=0.35$, aOR $0.98(0.5-1.9) \mathrm{p}=0.94)$. Only total number of losses predicted live birth rate (aOR $0.54(0.45-0.66) \mathrm{p}=0.000)$. Thyroxine replacement therapy was administered in $30(6.6 \%)$ women. Live birth rate was not associated with thyroxine replacement $(63.3 \%$ vs $59.5 \%$, $\mathrm{p}=0.85$, aOR 1.69 (0.67-4.30), $\mathrm{p}=0.27)$. However, among TPOAb-positive women, live birth rate after treatment with thyroxine replacement was $68.4 \%$ vs. $49.0 \%$ in non-treated women $(\mathrm{p}=0.18$, aOR 3.2(0.89-11.55) $\mathrm{p}=0.08)$.

Conclusions: TPOAbs were not associated with pregnancy outcome in women with RPL. However, thyroxine replacement therapy caused a trend towards a significant increase of live birth rate among TPOAb-positive women. Results from multicenter intervention studies are awaited.

\section{OP-08-57 \\ SYSTEMATIC NGS ANALYSIS OF NEWBORNS WITH CONGENITAL HYPOTHYROIDISM RELATED TO DYSHORMONOGENESIS IN A FRENCH COHORT \\ Frederique Savagner $^{1}$}

${ }^{1}$ Insermumr1048, Molecular Laboratory Chu, Toulouse, France

Congenital hypothyroidism $(\mathrm{CH})$ is referred to dyshormonogenesis for 15 to $30 \%$. Homozygous mutations associated have been demonstrated in DUOX2, TPO, TG, SLC5A5, SLC26A4, DUOXA2, and IYD genes.

Previous studies focusing on one or few thyroid-specific genes have proved not to be comprehensive enough for understanding physiopathological mechanisms of $\mathrm{CH}$ with dyshormonogenesis. Emerging diagnostic tools as next generation sequencing (NGS) led to efficiently study some targeted genes involved in a specific disease.

Using this approach, we have studied 48 newborns with $\mathrm{CH}$ and eutopic gland (CHEG) for 18 candidate genes (CDS and UTR) to explore causative genes for permanent or transitory thyroid dyshormonogenesis.

Among the 48 infants, $28(58.3 \%)$ presented mutation in one or more genes of the thyroid hormone synthesis axis. For 9 infants we have identified homozygous or composite heterozygous mutation for TG, DUOX2, TPO or SLC5A5 genes whereas 6 presented heterozygous mutations in two different genes of the thyroid hormonogenesis pathway, TG and DUOX2 or TG and TPO. Exploring the thyroid hormone synthesis pathway by NGS has revealed that $9 / 28$ newborns (32\%) presented composite heterozygous or heterozygous mutation in two genes involved in the hormone synthesis process. Thyroglobulin is the gene with greatest number of pathogenic variant in our population. In all cases the correlation between biological and imaging studies and molecular status was examined.

The systematic exploration of genes involved in the thyroid hormone synthesis by NGS showed high level diagnostic relevance for CHEG. Furthermore, significant percentage of them occurred when two steps in the hormone synthesis process were impaired. Heterozygous composite mutations could be related to the phenotypic heterogeneity observed for $\mathrm{CH}$ with dyshormonogenesis.

\section{OP-08-58 \\ IMPACT OF FIRST TRIMESTER OF PREGNANCY MATERNAL TSH IN OBSTETRICAL AND FETAL COMPLICATIONS: USEFULNESS OF 2.5 MUI/ML CUT-OFF VALUE}

Ines Velasco ${ }^{1}$, Marta Hernández ${ }^{2}$, Carolina López ${ }^{2}$, Berta Soldevila ${ }^{3}$, Dídac Mauricio ${ }^{3}$, Manel Puig-Domingo ${ }^{3}$

${ }^{1}$ Hospital de Riotinto, Obstetrics and Gynecology, Minas de Riotinto, Spain, ${ }^{2}$ Endocrinology and Nutrition Department, Hospital Universitari Arnau de Vilanova., Lleida, Spain, ${ }^{3}$ Endocrinology and Nutrition Department. Hospital Universitari Germans Trias I Pujol., Ciber de Enfermedades Raras (Ciberer) and Ciber de Diabetes $Y$ Enfermedades Metabólicas (Ciberdem), Instituto de Salud Carlos III, Institut D'investigació Germans Trias I Pujol, Campus Can Ruti, Badalona, Universitat Autònoma de Barcelona, Badalona, Spain

Objective: To evaluate the association of fetal-maternal complications with first trimester maternal TSH values.

Methods: A retrospective study in a single tertiary care hospital was performed. TSH universal screening for thyroid dysfunction was performed between weeks 9-12 of gestation. Outcomes included maternal complications and newborn health parameters.

Results: A total of 1981 women studied during 2012 were analyzed (mean age $30.1 \pm 5.7$ years); mean TSH was $1.99 \pm 1.62 \mathrm{mIU} / \mathrm{mL}$. The incidence of perinatal loss was $7,2 \%$ (the miscarriage rate was $5,9 \%$, and $1.1 \%$ for stillbirth, respectively). Mean TSH of women with and without perinatal loss was 2.40 \pm 1.55 vs. $1.95 \pm 1.57 \mathrm{mIU} / \mathrm{mL}$, respectively $(\mathrm{p}=0.009)$. Incidence of preeclampsia was $3.2 \%$; TSH in pregnant women who developed preeclampsia was $2.45 \pm 2.69$ vs. $1.98 \pm 1.58 \mathrm{mIU} / \mathrm{mL}$ in those who did not develop this condition $(p=0.027)$. In women with dystocia in labour, THS was $2.02 \pm 1.7$ vs. $1.93 \pm 1.68 \mathrm{mIU} / \mathrm{mL}$ in those who gave birth with normal progression $(\mathrm{p}=$ $0.044)$. Women with TSH $2.5-5.0 \mathrm{mIU} / \mathrm{mL}$ had higher risk of perinatal loss at any moment of gestation (OR 1.519 (1.040-2.219)) and higher risk of miscarriage at first trimester (OR $1.631(1.082-2.458)$.

Table 1. Association of feto-maternal complications and TSH groups

\begin{tabular}{lll}
\hline Complications & $\begin{array}{l}\text { TSH } 2.5-5.0 \mathrm{mIU} / \mathrm{mL} \\
\text { OR }(95 \% \text { C.I. })\end{array}$ & $\begin{array}{l}\text { TSH }>5.0 \mathrm{mIU} / \mathrm{mL} \text { treated } \\
\text { OR }(95 \% \text { C.I. })\end{array}$ \\
\hline Preeclampsia & $1.243(0.701-2.201)$ & $1.972(0.687-5.657)$ \\
Dystocic labour & $0.864(0.688-1.086)$ & $1.288(0.786-2.109)$ \\
Perinatal loss & $1.519(1.040-2.219)$ & $1.645(0.732-3.694)$ \\
$\quad$ Miscarriage & $1.631(1.082-2.458)$ & $1.765(0.740-4.209)$ \\
$\quad$ Abortion & $0.779(0.087-6.988)$ & $5.23(0.577-47.402)$ \\
$\quad$ Stillbirth & $1.040(0.376-2.877)$ & $1.384(0.180-10.626)$ \\
Premature birth & $1.342(0.988-1.823)$ & $1.334(0.669-2.659)$ \\
\hline
\end{tabular}

Conclusions: There is a positive association between maternal TSH in the first trimester of pregnancy and the incidence of spontaneous miscarriage and perinatal loss. The TSH cut-off value of $2.5 \mathrm{mIU} / \mathrm{mL}$ identified women with higher risk for adverse pregnancy outcomes.

\section{OP-08-59 \\ THE ASSOCIATION OF THYROID FUNCTION WITH MATERNAL AND NEONATAL HOMOCYSTEINE CONCENTRATIONS}

Mirjana Barjaktarovic ${ }^{1}$, Tim Korevaar ${ }^{2}$, Vincent Jaddoe ${ }^{1}$, Yolanda de Rijke $^{1}$, Theo Visser ${ }^{1}$, Eric Steegers ${ }^{1}$, Robin Peeters ${ }^{3}$

${ }^{1}$ Erasmus Medical Center, Rotterdam, Netherlands, ${ }^{2}$ Erasmus MC,Rotterdam, The Netherlands, Endocrinology, Rotterdam, Netherlands, ${ }^{3}$ Academic Center for Thyroid Diseases, Department of Endocrinology, Erasmus, Rotterdam, Netherlands

Objectives: High homocysteine (Hcy) concentrations are associated with adverse cardiovascular, neurological, pregnancy-specific outcomes and neonatal morbidity. Thyroid hormones may interfere with Hcy metabolism and abnormal Hcy concentrations have been observed in patients with thyroid dysfunction. Because gestational thyroid dysfunction is associated with same adverse pregnancy outcomes as high Hcy, we aimed to investigate effects of 
thyroid function on Hcy metabolism during pregnancy and in the neonatal period.

Methods: In a population-based prospective cohort of 5683 women, we measured early pregnancy thyroid stimulating hormone (TSH) and free thyroxine (FT4) $(<18$ weeks) from which 1094 women did not take folic acid supplements during pregnancy. Hcy, folate and vitamin B12 concentrations were determined at the same time point. The same measurements were performed in cord blood of 4475 newborns. The associations of maternal and newborn TSH and FT4 with Hcy, folate and vitamin B12 concentrations were investigated using multiple linear regression analyses.

Results: In mothers, there was a positive association of FT4 with Hcy $(\mathrm{P}=0.026)$ and no association of FT4 with folate or vitamin B12 ( $\mathrm{P}$ for both $>0.05$ ). In newborns, there was a positive association of FT4 with Hcy and an inverse association of TSH with Hcy; the latter associations attenuated after adjustment for folate and vitamin B12 (beta estimate decrease of $45 \%, \mathrm{P}$ value change from $\mathrm{P}<0.0001$ to $\mathrm{P}=0.015$ for FT4 and beta estimate decrease of $48 \%$, $\mathrm{P}$ value change from $\mathrm{P}=0.003$ to $\mathrm{P}=0.11$ for $\mathrm{TSH}$, respectively).

Conclusion: Higher thyroid hormone is associated with higher Hcy concentration in pregnant women and in newborns. In newborns, the underlying mechanism might involve depleting effects of higher thyroid function on folate and vitamin B12. These data provide new insights into the effects of thyroid hormone on folate and vitamin B12-dependent biochemical processes during early growth and development.

\section{OP-08-60}

\section{A DE NOVO DICER1 GERMLINE MUTATION IN A} CHILD WITH MULTINODULAR GOITER

\section{Marek Niedziela ${ }^{1}$, Nelly Sabbaghian ${ }^{2}$, William D Foulkes ${ }^{3}$}

${ }^{1}$ Department of Pediatric Endocrinology and Rheumatology, Karol Jonscher's Clinical Hospital, Poznan University of Medical Sciences,

Poznan, Poland, '2Lady Davis Institute, Segal Cancer Centre, Jewish

General Hospital, Department of Human Genetics, Mcgill University,

Montreal, Canada, ${ }^{3}$ Lady Davis Institute, Segal Cancer Centre, Jewish

General Hospital, Department of Human Genetics, Mcgill University,

Research Institute of the Mcgill University Health Centre, Montreal,

Canada

Objective: Little is known about genetic susceptibility to non-toxic multinodular goiter (MNG). Several hereditary tumor syndromes such as Carney complex, PTEN hamartoma tumor syndrome, Werner syndrome and $A P C$-associated polyposis can be associated with MNG. Germline mutations in DICER1, which are known to predispose to pleuropulmonary blastoma, Sertoli-Leydig cell tumours, cystic nephromas, cervical embryonal rhabdomyosarcomas and other rare paediatric and/or adolescent onset tumours that comprise the DICER1 syndrome, are also known to predispose to MNG.

The aim of the study was to investigate whether DICER 1 , the new potent gene predisposing to familial MNG, plays a pathogenic role in a 9-year-old child with an isolated MNG.

Methods: Three family members, the proband and her parents, were examined. Family history was remarkable for chronic lymphoblastic leukemia and lymphoma in two grandmothers. The patient was euthyroid, had negative thyroid autoantibodies and manifested a nodular goiter of the right lobe. She underwent left lobectomy with isthmus (at 7 years of age) due to a large cystic/solid lesion. A nodular colloid goiter with pseudopapillary areas was diagnosed postoperatively. Thyroid ultrasound showed multiple solid, solid/cystic and cystic lesions with no vascular flow on PD/CD. FNAB showed a colloid nodule. Her mother had autoimmune thyroiditis and her father had small solid incidentalomas. Germline DNA from the proband was screened for mutations in DICER1.

Results: The genetic testing of DICERI in MNG patient identified a de novo germline predicted-truncating DICERI mutation in exon 23 (c.4309_4312delGACT; p.D1437Mfs*16). Her parents did not carry the mutation.

Conclusions: Our work shows that DICER1 is involved in the pathogenesis of MNG in this patient. A careful follow-up particularly in terms of genitourinary tract and ovaries is obligatory. Genetic counseling and testing should be offered to the proband's children in the future.

\section{OP-08-61 \\ PREVIOUS CHILDBIRTH AND INDUCED \\ ABORTIONS MAY PRECEDE LATER DEVELOPMENT OF HYPERTHYROIDISM, BUT ONLY IN GRAVES' DISEASE}

Allan Carle $^{1}$, Stine Linding Andersen ${ }^{2}$, Nils Knudsen ${ }^{3}$, Hans Perrild ${ }^{3}$, Lars Ovesen ${ }^{4}$, Lone Bank Rasmussen ${ }^{5}$, Inge Bülow Pedersen ${ }^{1}$

${ }^{1}$ Department of Endocrinology, Aalborg University Hospital, Aalborg, Denmark, ${ }^{2}$ Department of Clinical Biochemistry, Aalborg University Hospital, Aalborg, Denmark, ${ }^{3}$ Endocrine Unit, Medical Clinic I, Bispebjerg Hospital, Copenhagen, Denmark, ${ }^{4}$ Department of Internal Medicine, Slagelse Hospital, Slagelse, Denmark, ${ }^{5}$ National Food Institute, Technical University of Denmark, Copenhagen, Denmark

Objectives: Reproductive factors are associated with the risk of many autoimmune diseases, but their role in development of various subtypes of hyperthyroidism has never been studied.

Methods: From the Danish population, we included incident thyrotoxic women participating in a case-control study, and for each case we recruited four euthyroid age-sex-region-matched controls from the same population. The nosological subtypes of hyperthyroidism were: Graves' disease, $n=272$ cases / 1088 controls; multinodular toxic goitre, $n=105 / 420$; solitary toxic adenoma, $n=21 / 84$. In a conditional Cox regression model, we analyzed the association between development of hyperthyroidism and reproductive factors such as age of menarche/menopause, total years of menstruations, number of pregnancies/abortions/childbirths, age at first childbirth, investigations for infertility, years on oral contraceptives, and years on postmenopausal hormone replacement therapy also taking possible confounders into account (alcohol intake, smoking, co-morbidity, and education).

Results: In multivariate model, Graves' patients had given births more often than control subjects (no births $=$ reference: odds ratios $(95 \%$-confidence intervals) for $1 / 2 / 3+$ births were $0.69(0.41-1.17) / 1.55(0.97-2.48) / 1.76$ $(1.03-3.03), p$-trend $=0.003$. Similarly, Graves' patients had induced abortions performed a little more often than their controls (no events $=$ reference: odds ratios $(95 \%$-confidence intervals) for $1 / 2+$ induced abortions were $0.79(0.53$ $1.19) / 1.67$ (0.996-2.79), $p$-trend $=0.051)$. However, findings were recaptured in patients diagnosed with Graves' disease before the age of $40 \mathrm{y}$, not among patients aged $40+$ years. No associations were observed between any reproductive factor and the development of multinodular toxic goitre or solitary toxic adenoma.

Conclusions: Childbirths and induced abortions may be risk factors for the development of Graves' hyperthyroidism, which is in line with our previous findings for autoimmune hypothyroidism. However, the very strong association between development of autoimmune hypothyroidism and repeated exposures to birth giving and induced abortions was observed to a lesser extent in Graves' hyperthyroidism. Thus, different autoimmune mechanisms may be responsible for development of hypothyroidism vs. Graves' hyperthyroidism. 


\section{Oral Session 9 (Basic): Cancer - Scientific Aspects 2}

\section{OP-09-62 \\ COMBINED DETERMINATION OF CAVEOLIN-1, BRAF V600E AND EGFR IN FNAB SAMPLES DISCRIMINATES PATIENTS WITH HIGH AND LOW RISK OF AGGRESSIVE THYROID CANCER} Jelena Jankovic ${ }^{1}$, Svetislav Tatic ${ }^{2}$, Vesna Bozic ${ }^{3}$, Vladan Zivaljevic ${ }^{4}$, Dubravka Cvejic ${ }^{1}$, Svetlana Paskas ${ }^{5}$

${ }^{1}$ Institute for the Application of Nuclear Energy - Inep, Department for Endocrinology and Radioimmunology, University of Belgrade, Belgrade, Serbia, ${ }^{2}$ Institute of Pathology, Medical Faculty, University of Belgrade, Belgrade, Serbia, ${ }^{3}$ Department of Endocrine and Cardiovascular Pathology, Clinical Center of Serbia, Belgrade, Serbia, ${ }^{4}$ Center for Endocrine Surgery, Institute of Endocrinology, Diabetes and Diseases of Metabolism, Clinical Center of Serbia, Belgrade, Serbia, 5Institute for Biological Research "Sinisa Stankovic", University of Belgrade, Belgrade, Serbia

Background: Cytological analysis of FNA samples is a reliable method for evaluating thyroid nodules, although it has its drawbacks. The widespread use of FNA biopsy has led to a phenomenon entitled overdiagnosis which is defined as the diagnosis of indolent, asymptomatic carcinomas that would never have caused symptoms during a patient's lifetime. It is argued that total thyroidectomy followed by radioiodine therapy is an overly aggressive treatment for these patients. Therefore, research on thyroid cancer biomarkers is also directed towards the elucidation of the molecular markers that can aid in risk stratification of thyroid cancer patients.

Objective: We investigated the immunocytochemical expression of caveolin-1, the presence of BRAF V600E mutation by AS-PCR and gene expression of EGFR by means of qPCR in 186 FNA samples, and determined their potential in aggressive thyroid cancer risk stratification.

Results: High protein expression of caveolin-1 and $B R A F$ V600E mutation were simultaneously detected in a group of patients with more aggressive thyroid cancer characteristics. Next, we divided the patients with malignant lesions according to the expression of caveolin- 1 and BRAF mutation status, and determined the expression of EGFR in these groups. The highest EGFR expression was detected in a group of patients with low caveolin-1 expression and negative BRAF status. When we compared the groups for aggressiveness, patients with high caveolin-1 expression, positive BRAF status and low EGFR expression had a higher degree of neoplastic infiltration and a higher $\mathrm{pT}$ status than patients with low caveolin-1 expression, negative BRAF status and high EGFR expression.

Conclusion: Using the combination of caveolin-1 and BRAF V600E with EGFR can be useful for early risk stratification of patients with thyroid cancer which may assist in designing optimal surgical and postsurgical management of these patients.

\section{OP-09-63 \\ INHIBITION OF ERK DIMERIZATION AS A THERAPY FOR THYROID CANCER \\ Miguel Zaballos ${ }^{1}$, Adrían Acuña ${ }^{2}$, Garcilaso Riesco-Eizaguirre ${ }^{3}$, Piero Crespo $^{4}$, Pilar Santisteban 5 \\ ${ }^{1}$ Instituto de Investigaciones Biomédicas Alberto Sols, Ciberonc, Madrid, Spain, ${ }^{2}$ Instituto de Investigaciones Biomédicas, Ciberonc, Instituto de Salud Carlos III, Madrid, Spain, ${ }^{3}$ Hospital Universitario de Móstoles, Ciberonc, Instituto de Salud Carlos III, Ciberonc Instituto de Salud Carlos III, Móstoles, Spain, ${ }^{4}$ Santander, ${ }^{5}$ Instituto de Investigaciones Biomédicas, Universidad Autónoma de Madrid, Ciberonc, Instituto de Salud Carlos III, Madrid, Spain}

Mutations in the RAS-ERK pathway, a key node for proliferation and differentiation, are the most frequent genetic alterations in thyroid carcinomas. The small molecule ERK dimerization inhibitor, DEL-22379, has been shown to have anti-tumorigenic effects in human melanoma and colorectal cancer-derived cells. We sought to test the therapeutic effects of DEL-22379 in thyroid cancer using a panel of human thyroid tumor cell lines with different genetic alterations in RAS-ERK signaling and an in vivo orthotopic mouse model.

We found that DEL-22379 impaired ERK dimerization in $B R A F$ mutant but not in $R A S$ mutant cells. Accordingly, ERK-inhibited $B R A F$ mutant cells showed repressed phosphorylation of p90 ribosomal S6 kinase, an ERK cytoplasmic effector, and proliferated less, likely due in part to decreased cyclin D1 expression. Migration and invasion were also affected by DEL-22379 treatment and were presumably caused by alterations in actin cytoskeleton. Consistent with this, we observed a partial reversion of the mesenchymal phenotype in tumor cells. In vivo application of DEL-22379 retarded tumor growth in the thyroid of nude mice and decreased the number of lung metastases. Finally, we observed higher levels of ERK dimers in human papillary thyroid carcinoma cells than in matched healthy tissue.

Inhibition of the kinase activity of different components of RAS-ERK signaling is currently the only alternative to the classical therapies for the treatment of advance thyroid tumors. Our results suggest that therapies directed to impair protein-protein interactions of RAS-ERK signaling are feasible approaches to block thyroid tumor progression.

\section{OP-09-64 \\ VANDETANIB IS ACTIVE AGAINST PRIMARY ANAPLASTIC THYROID CANCER IN VITRO AND IN VIVO \\ Alessandro Antonelli ${ }^{1}$, Guido Bocci ${ }^{1}$, Poupak Fallahi', Silvia Martina \\ Ferrari', Teresa Di Desidero $^{1}$, Alessandro Corti ${ }^{2}$, Simona Piaggi ${ }^{2}$, \\ Gabriele Materazzi ${ }^{3}$, Romano Danesi ${ }^{1}$, Paolo Miccoli ${ }^{3}$ \\ ${ }^{1}$ University of Pisa, Department of Clinical and Experimental \\ Medicine, Pisa, Italy, ${ }^{2}$ University of Pisa, Department of Translational \\ Research and of New Technologies in Medicine and Surgery, Pisa, \\ Italy, ${ }^{3}$ University of Pisa, Department of Surgical, Medical, Molecular \\ Pathology and Critical Area, Pisa, Italy}

Objective: We have studied the antitumor activity of vandetanib, a multiple signal transduction tyrosine kinase inhibitor (TKI), in primary anaplastic thyroid cancer (ATC) cells, in the human cell line 8305C (undifferentiated thyroid cancer) and in an ATC-cell line (AF).

Methods: Vandetanib was tested: in primary ATC cells, in $8305 \mathrm{C}$ cells, and in $\mathrm{AF}$ cells, at the concentrations of $1 \mathrm{nM}, 100 \mathrm{nM}, 1 \mu \mathrm{M}, 10 \mu \mathrm{M}, 25 \mu \mathrm{M}$, $50 \mu \mathrm{M}$; in $8305 \mathrm{C}$ cells in CD nu/nu mice.

Results: Vandetanib significantly inhibited proliferation of $8305 \mathrm{C}$ and $\mathrm{AF}$ cells, inducing also apoptosis. A significant reduction of proliferation with vandetanib in ATC cells $(P<0.01$, ANOVA) was shown. Vandetanib increased the percentage of apoptotic ATC cells dose-dependently $(P<0.001$, ANOVA) and inhibited migration $(P<0.01)$ and invasion $(P<0.001)$. 8305C cells were injected sc in $\mathrm{CD} \mathrm{nu/nu} \mathrm{mice} \mathrm{and} \mathrm{the} \mathrm{treatment} \mathrm{with} \mathrm{vandetanib} \mathrm{(25} \mathrm{or} 12.5$ $\mathrm{mg} / \mathrm{kg}$ /day i.p. for 29 days) was initiated after 30 days from cell inoculation, when the average tumor volume was around $100 \mathrm{~mm}^{3}$. Vandetanib $(25 \mathrm{mg} / \mathrm{kg} /$ day) inhibited significantly tumor growth. Moreover, vandetanib significantly decreased the VEGF-A expression and microvessel density in $8305 \mathrm{C}$ tumor tissues.

Conclusions: The antitumor and antiangiogenic activity of vandetanib is very promising in anaplastic thyroid cancer.

\section{OP-09-65 \\ DISSECTING THE ROLE OF THYROTROPIN IN DNA DAMAGE RESPONSE OF HUMAN THYROCYTES IN PRIMARY CULTURES AFTER RADIOIODINE OR Y-RADIATION}

\section{Aglaia Kyrilli' ${ }^{1}$, Jacques Dumont ${ }^{2}$, Francoise Miot ${ }^{3}$, Bernard Corvilain ${ }^{4}$}

${ }^{1}$ Hôpital Erasme Ulb, Endocrinology Department, Iribhm Ulb,

Brussels, Belgium, ${ }^{2}$ University Brussels, Iribhm, Lasne, Belgium,

${ }^{3}$ Université Libre de Bruxelles, Universite Libre de Bruxelles - Ulb;

Iribhm, Brussels, Belgium, Iribhm, Bruxelles, Belgium, ${ }^{4}$ Ulb-Iribhm and

Department of Endocrinology, Hopital Erasme, Brussels

Background: Thyroid is well known to be susceptile to radiation induced carcinogenesis. We study the role of thyrotropin (TSH) in the modulation of DNA damage response of human thyrocytes after radioiodine $\left({ }^{131} \mathrm{I}\right), \gamma$-radiation and $\mathrm{H}_{2} \mathrm{O}_{2}$. 
Methods: We used human thyrocytes in primary cultures with or without $\mathrm{TSH}$ in the medium. After either $3 \mathrm{~Gy} \gamma$ - radiation or $24 \mathrm{~h}$ incubation with $50 \mathrm{iCi}$ ${ }^{131} \mathrm{I}$, corresponding to an absorbed dose of 6Gy and after a $15 \mathrm{~min}$ exposure to $0.2 \mathrm{mM} \mathrm{H}_{2} \mathrm{O}_{2}$, we evaluated DNA damage (double strand DNA breaks), cell survival and cell proliferation, cell cycle regulation key protein p21 and we performed RNA seq (NGS Illumina HiSeq platform) to study gene expression modulation.

Results: TSH increased basal proliferation rate of human thyrocytes in our culture conditions from $0.86 \%$ to $3 \%$, induced an upregulation of NIS (Na-I symporter) mRNA and increased the amount of DNA double strand DNA breaks induced by $24 \mathrm{~h}{ }^{131} \mathrm{I}$ radiation at basis and till after $48 \mathrm{~h}$. Presence of NIS inhibitors did not completely abolish ${ }^{131} \mathrm{I}$ effects. TSH did not influence DNA damage induced by $\gamma$-radiation or $\mathrm{H}_{2} \mathrm{O}_{2}$ Proliferative agents such as forskolin, EGF, or EGF plus 10\% fetal bovine serum did not influence DNA damage after $\gamma{ }^{131} \mathrm{I}$ radiation or $\mathrm{H}_{2} \mathrm{O}_{2}$. Preliminary RNA sequencing results showed that after $24 \mathrm{~h}^{131} \mathrm{I}$ radiation, genes involved in p53 pathway, cell cycle regulation, DNA replication, mismatch repair are modulated. We confirmed that human cultured thyrocytes present an arrest in $\mathrm{G} 1 / \mathrm{S}$ cell cycle phase up to $48 \mathrm{~h}$ after ${ }^{131} \mathrm{I}$ radiation with a concomitant increase in the amount of $\mathrm{p} 21$, most evident in presence of TSH.

Conclusions: TSH modulates ${ }^{131} \mathrm{I}$ induced DNA damage response of human thyrocytes in vitro but does not seem to influence thyrocyte susceptibility to $\gamma$-radiation or $\mathrm{H} 2 \mathrm{O} 2$.

\section{OP-09-66 \\ IDENTIFICATION OF NOVEL SODIUM IODIDE SYMPORTER (NIS) INTERACTORS WHICH MODULATE IODIDE UPTAKE}

Alice Fletcher ${ }^{1}$, Vikki Poole ${ }^{1}$, Bhavika Modasia ${ }^{1}$, Waraporn

Imruetaicharoenchoke ${ }^{1}$, Rebecca Thompson ${ }^{1}$, Neil Sharma ${ }^{1}$, Hannah

Nieto ${ }^{1}$, Martin Read ${ }^{1}$, Andy TurnelR ${ }^{2}$, Kristien Boelaert', Vicki Smith ${ }^{1}$,

Chris McCabe ${ }^{1}$

${ }^{1}$ Institute of Metabolism and Systems Research, University of

Birmingham, Birmingham, UK, ${ }^{2}$ Institute of Cancer and Genomic

Sciences, University of Birmingham, Birmingham, UK

By exploiting the canonical function of the sodium iodide symporter (NIS), ablative radioiodide therapy is an effective treatment for thyroid cancer and has been hypothesised as a viable treatment for breast cancer. However, over $25 \%$ of patients are unable to accumulate sufficient radioiodide for effective treatment due to decreased expression and/or reduced plasma membrane localisation of NIS. Currently, the regulation of NIS trafficking and its localisation at the plasma membrane is ill-defined. To identify novel NIS-interactors and aid understanding of NIS trafficking, mass spectrometry was performed on proteins co-immunoprecipitating with lentivirally expressed NIS in cell plasma membrane extracts. siRNA knockdown of the top shortlisted interactors was followed by radioiodide uptake assays to determine the impact on NIS function. NIS activity was significantly altered by ADP-ribosylation factor 4 (ARF4) and valosin containing protein (VCP) in thyroid and breast cancer cells lentivirally-expressing NIS. ARF4 downregulation significantly decreased radioiodide uptake by $75 \%$ and $44 \%$, and VCP downregulation increased radioiodide uptake by $71 \%$ and $56 \%$, in the thyroid and breast cells, respectively. Transient overexpression of each gene reversed the effects of siRNA treatment: ARF4 significantly increased radioiodide uptake by $89 \%$ and $43 \%$, and VCP significantly decreased radioiodide uptake by $52 \%$ and $38 \%$, in thyroid and breast cells. Co-immunoprecipitation and proximity ligation assays (PLA) confirmed that NIS interacts with ARF4 and VCP. We additionally identified Ras-related protein Rab-18 (RAB18) as a further NIS-interactor, which differentially altered radioiodide uptake in thyroid and breast cells, with PLA demonstrating RAB18-NIS interaction at the membrane. Analysis of TCGA data from N=58 matched papillary thyroid cancers revealed ARF4 to be significantly repressed and VCP highly upregulated in thyroid cancer, providing a putative explanation for repressed NIS function. These studies elucidating the regulation of NIS localisation have identified three novel potential therapeutic targets for enhancing radioiodide uptake in radioiodide-refractory patients.

\section{OP-09-67 \\ KIF5B/RET REARRANGEMENT IN DIFFUSE SCLEROSING VARIANT OF PAPILLARY THYROID CANCER (DSV-PTC)}

Carlotta Giani ${ }^{1}$, David Viola ${ }^{1}$, Raffaele Ciampi', Cristina Romei ${ }^{1}$, Clara Ugolini' ${ }^{2}$ Eleonora Molinario', Laura Agate ${ }^{1}$, Salvatore Mazzeo ${ }^{3}$, Antonio Chella ${ }^{4}$, Gabriella Fontanini ${ }^{2}$, Fulvio Basolo ${ }^{2}$, Rossella Elisei ${ }^{1}$ ${ }^{1}$ Endocrine Unit, Department of Clinical and Experimental Medicine, Pisa, Italy, ${ }^{2}$ Pathology Unit, Department of Surgical, Medical, Molecular Pathology and Critical Area, University Hospital of Pisa, Pisa, Italy, ${ }^{3}$ Diagnostic and Interventional Radiology, Department of Translational Research and New Technologies in Medicine and Surgery, University Hospital of Pisa, Pisa, Italy, ${ }^{4}$ Unit of Pneumology, University Hospital of Pisa, Pisa, Italy

Objectives: The diffuse sclerosing variant of papillary thyroid cancer (DSV-PTC) is a rare variant of papillary thyroid cancer (PTC). DSV-PTC has peculiar clinicopathological features compared to conventional PTC. It is more frequent in younger patients and characterized by a global enlargement of the thyroid gland without distinct nodular lesions, an advanced stage at diagnosis and a high titers of anti-thyroglobulin antibodies.

Methods: we studied 19 consecutive DSV-PTC cases for the presence of KIF 5B/RET rearrangement and we reviewed their clinical charts for the clinical, biochemical and pathological data.

Results: All DSV-PTC cases resulted negative for the presence of KIF5B/ $R E T$ rearrangement except one case. This was a 39 -year-old man referred to our Institute for thyroid remnant ablation. The post-therapeutic whole body scan showed only an uptake in the central neck, the serum thyroglobulin on L-thyroxine therapy was undetectable. The computerized tomography showed multiple latero-cervical and mediastinum lymph node metastases, a single and spiculated lung lesion and multiple bilateral cerebellum metastases. The patient died after only six months from the initial diagnosis, instead the other 18 DSV-PTC were alive after a median follow-up of 7 years. The histological revision of the thyroid tumor of this fatal case confirmed the diagnosis of DVS-PTC. The immunohistochemistry was positive for thyroid transcription factor-1 but negative for thyroglobulin.

Conclusions: This is the first and unique case of DSV-PTC positive for $K I F 5 B / R E T$ rearrangement. Considering the aggressiveness of the disease, the molecular alteration, described only in lung adenocarcinoma and in none of the other DSV-PTC, and the presence of a lung nodule compatible with a primary tumor, we cannot completely rule out the possibility that this was a fatal case of a lung tumor mimicking DSV-PTC.

\section{OP-09-68 \\ COMPARISON OF GENE EXPRESSION PROFILE OF MEDULLARY THYROID TUMORS WITH RET AND RAS MUTATIONS \\ Malgorzata Oczko-Wojciechowska ${ }^{1}$, Michal Swierniak ${ }^{2}$, Aleksandra Pfeifer $^{3}$, Malgorzata Kowalska ${ }^{3}$, Tomasz Tyszkiewicz ${ }^{3}$, Agnieszka Pawlaczek ${ }^{3}$, Agnieszka Czarniecka ${ }^{4}$, Dagmara Rusinek ${ }^{3}$, Ewa Chmielik ${ }^{5}$, Michal Jarzab ${ }^{6}$, Sylwia Szpak-Ulczok ${ }^{3}$, Tomasz Gawlik ${ }^{3}$, Jolanta Krajewska ${ }^{3}$, Barbara Jarzab ${ }^{3}$ \\ ${ }^{1}$ Department of Nuclear Medicine and Endocrine Oncology, Maria Sklodowska-Curie Memorial Cancer Center and Institute of Oncology, Gliwice Branch, Giwice, Poland, ${ }^{2}$ Department of Nuclear Medicine and Endocrine Oncology, Maria Sklodowska-Curie Memorial Cancer Center and Institute of Oncology, Gliwice Branch, Genomic Medicine, Medical University of Warsaw, Poland, Gliwice, Poland, ${ }^{3}$ Department of Nuclear Medicine and Endocrine Oncology, Maria Sklodowska-Curie Memorial Cancer Center and Institute of Oncology, Gliwice Branch, Gliwice, Poland, ${ }^{4}$ The Oncology and Reconstructive Surgery Clinic, Maria Sklodowska-Curie Memorial Cancer Center and Institute of Oncology, Gliwice Branch, Gliwice, Poland, ${ }^{5}$ Tumour Pathology Department, Maria Sklodowska-Curie Memorial Cancer Center and Institute of Oncology, Gliwice Branch, Gliwice, Poland, 'III Radiotherapy Clinic, Maria Sklodowska-Curie Memorial Cancer Center and Institute of Oncology, Gliwice Branch, Gliwice, Poland}

Objective: Germline mutations of RET protooncogene are associated with hereditary medullary thyroid cancer (MTC). Somatic RET mutations 
are detected in about $50 \%$ of sporadic cases of MTC. Somatic $R A S$ mutations are mutually exclusive with $R E T$ somatic mutations and are also frequently detected in sporadic MTC cases. The aim of our study was to evaluate the differences in gene expression profile between MTC samples with RET and $R A S$ mutations.

Methods: Fresh-frozen tumor samples from 39 MTC patients (28 RET and 11 RAS positive) were collected. RET germline mutation screening was performed according to standard diagnostic algorithm. 21 exons of RET gene were sequenced in sporadic MTC cases. Gene expression profile was analyzed using Gene Chip 1.0 ST Arrays (Affymetrix). An independent set of 33 MTC samples (14 RAS and 19 RET positive) was used for QPCR validation.

Results: Supervised analysis of microarray data revealed 222 genes with FDR $<0.05$ which expression differed tumor samples with $R E T$ and $R A S$ mutation. For validation 79 genes were selected with FDR $<0.05$ and fold change $>2$ or $<0.5$ and the mean probe set level 100 . For 61 selected genes we were able to design proper QPCR amplicons and all of those genes were analyzed. However, verification on separate group of samples showed a statistical significance (p-value $<0.05$ ) only for 11 genes. Interestingly when the site of $R E T$ gene mutation was taken into consideration (extra- or intracellular domain) in comparison with $R A S$ gene mutation we noticed a slight differences in the set of differentiating genes between RAS and RET with extracellular and RET intracellular mutations sample. VGF gene showed different expression in all analyzed subgroups.

Conclusion: The gene expression profile of RET and $R A S$ mutated MTC samples is rather similar however show slight differences. This research was supported by NCBiR [MILESTONE]: STRATEGMED2/267398 /4/ NCBR/2015.

\section{OP-09-69 \\ THE COMBINATION OF VEMURAFENIB AND TRAIL (TNF-RELATED APOPTOSIS INDUCING LIGAND) INDUCES EFFECTIVELY APOPTOSIS IN THE ANAPLASTIC THYROID CANCER CELLS Tania Pilli', Silvia Cantara ${ }^{1}$, Carlotta Marzocchi', Sandro Cardinale ${ }^{1}$, Bellur S. Prabhakar ${ }^{2}$, Furio Pacini \\ ${ }^{1}$ University of Siena, Department of Medical\&surgical Sciences and Neurosciences, Siena, Italy, ${ }^{2}$ University of Illinois at Chicago, Department of Microbiology and Immunology, Chicago, IL, USA}

Introduction: Anaplastic thyroid cancer (ATC) has a poor prognosis and is refractory to conventional treatment. TRAIL induces selectively apoptosis in cancer cells but in some cases it can trigger pro-survival signaling pathways which confer resistance to the drug. This effect may be limited by the use of specific tyrosine kinase inhibitors (TKI).

Purpose: The aim of our study was to evaluate, in vitro and in vivo, the cytotoxicity induced by TRAIL, alone or in combination with TKI targeting selectively BRAF (vemurafenib) or Akt (MK2206), in cell lines derived from ATC.

Methods: We used 4 cell lines derived from ATC: 3 out of them (C643, CAL62 and HTh7) with an activating mutation of RAS and Akt copy gain (HTh7) and one (8505C) with an activating mutation of BRAF (V600E). Apoptosis was measured assessing the expression of the active form of caspase-3 by ELISA. The pro-apoptotic effect of TRAIL was also evaluated in two xenograft mouse models of ATC (HTh7 and $8505 \mathrm{C})$ and a similar experiment is being conducted using the combination of TRAIL and TKI.

Results: The in vitro experiments showed that 3 cell lines were sensitive to TRAIL induced-apoptosis (C643, CAL62 and HTh7) while the 8505C cells were highly resistant. This effect was confirmed "in vivo" in particular we observed in a mouse model of HTh7, one week after the first administration of TRAIL, a significant reduction of tumor growth, compared to controls treated with placebo while the tumor development was not affected in a mouse model of $8505 \mathrm{C}$ cells. Later on we determined, in $8505 \mathrm{C}$ and $\mathrm{HTh} 7$ cells, the minimum dose of vemurafenib and MK2206 necessary to inhibit the specific therapeutic targets, pERK and pAkt respectively. Vemurafenib (used at $1 \mathrm{uM}$ in the $8505 \mathrm{C}$ cells and $5 \mathrm{uM}$ in HTh7 cells) in combination with TRAIL was able to increase significantly the TRAIL induced-apoptosis in $8505 \mathrm{C}$ cells $(+$ $70 \%)$ and in HTh7 $(+25 \%, \mathrm{p}=0.029)$ while MK226 (used at $5 \mathrm{uM}$ in both cell lines) did not show any additive effect in any cell.

Conclusions: Vemurafenib was able to enhance the TRAIL cytotoxic effect in both ATC cells susceptible and resistant.

\section{Tuesday, 12th September 2017}

\section{Oral Session 10: \\ Young Investigators I \\ Clinical + Translational}

\section{OP-10-70 \\ MATERNAL THYROID FUNCTION \\ IN EARLY PREGNANCY AND CHILD NEURODEVELOPMENTAL DISORDERS: A DANISH NATIONWIDE CASE-COHORT STUDY OF 9,650 CHILDREN}

Stine Linding Andersen ${ }^{1}$, Jørn Olsen ${ }^{2}$

${ }^{1}$ Department of Clinical Biochemistry, Aalborg University Hospital, Aalborg, Denmark, ${ }^{2}$ Aarhus University Hospital, Aarhus, Denmark

Objectives: The hypothesis of fetal programming by maternal thyroid disease is biologically plausible, but more studies in humans on child neurodevelopmental disorders are needed to expand the concept.

Methods: Case-cohort study within the Danish National Birth Cohort (established 1997-2003). The study included a randomly selected $12 \%$ sub cohort $(n=7,624)$ and all cases $(n=2,276)$ in the entire cohort with a diagnosis of epilepsy, febrile seizure, specific developmental disorder (SDD), autism spectrum disorder (ASD) and/or attention deficit hyperactivity disorder (ADHD) up to December 31, 2010. All women included had a blood sample drawn in early pregnancy (median week 9) which was stored in the Danish National Biobank and used for measurement of free T4 (fT4) and TSH. Method- and week-specific reference ranges (2.5-/97.5-percentiles) were used for classification of maternal thyroid function. Weighted Cox proportional hazards model was used to estimate adjusted hazard ratio (aHR) with $95 \%$ confidence interval $(95 \% \mathrm{CI})$ for child neurodevelopmental disorders.

Results: Overall, abnormal maternal thyroid function was more frequent among cases $(14.2 \%)$ than in the sub cohort $(12.5 \%), p=0.021$. Maternal hypothyroidism (aHR 1.75 (95\% CI 1.12-2.73) and overt hyperthyroidism (2.18

Table. (for Abstract OP-10-70)

\begin{tabular}{|c|c|c|c|c|c|c|c|c|}
\hline & \multicolumn{2}{|c|}{ Sub cohort } & \multicolumn{3}{|c|}{ Autism spectrum disorder } & \multicolumn{3}{|c|}{ Attention deficit hyperactivity disorder } \\
\hline \multicolumn{9}{|l|}{$\overline{\text { Girls }}$} \\
\hline No maternal thyroid dysfunction & 3,297 & 87.2 & 49 & 76.6 & 1.00 (ref.) & 192 & 83.8 & 1.00 (ref.) \\
\hline Isolated low fT4, normal TSH & 79 & 2.1 & 6 & 9.4 & $4.92(2.03-11.9)$ & 12 & 5.2 & $2.28(1.21-4.29)$ \\
\hline \multicolumn{9}{|l|}{ Boys } \\
\hline All & 3,844 & & 238 & & & 914 & & \\
\hline
\end{tabular}


(1.08-4.39)) was associated with ASD in the child. For epilepsy, unknown maternal hypothyroidism (TSH above $10 \mathrm{mIU} / \mathrm{l}$ ) was a risk factor (aHR 3.45 (1.19-10.0)) as was unknown overt hyperthyroidism for cases diagnosed within the first year of life (aHR 2.95 (1.03-8.44)). Isolated low fT4 (with normal TSH) was associated with ASD and ADHD, and this association was modified by child gender (Table). No associations were observed for outcomes of SDD and febrile seizure.

Conclusions: Abnormal maternal thyroid function in early pregnancy was significantly associated with epilepsy, ASD and ADHD in the child, but not with the less pervasive disorders. Associations differed by subtype of exposure and by child age and gender.

OP-10-71

LENVATINIB FOR THE TREATMENT OF RADIOIODINE REFRACTORY THYROID CANCER IN REAL-LIFE

Amandine Berdelou ${ }^{1}$, Borget Isabelle ${ }^{2}$, Godbert Yann ${ }^{3}$, Nguyen Thierry ${ }^{4}$, Garcia Marie-Eve ${ }^{5}$, Cecile Chougnet ${ }^{6}$, Aurélie Ferru ${ }^{7}$, Laurence Leenhardt ${ }^{8}$, Chabre Olivier ${ }^{9}$, Olivier Huillard ${ }^{10}$, Sophie Leboulleux ${ }^{11}$, Martin Schlumberger ${ }^{12}$

${ }^{1}$ Gustave Roussy, Université Paris-Sud, Curie, Saint Cloud, Villejuif, France, ${ }^{2}$ Gustave Roussy, Université Paris-Sud, Villejuif, France, ${ }^{3}$ Department of Nuclear Medicine, Institut Bergonié, Bordeaux, France, ${ }^{4}$ Department of Medical Oncology, Chru Minjoz, Besançon, France, ${ }^{5}$ Department of Multidisciplinary Oncology and Therapeutic Innovations, Assistance Publique-Hôpitaux de Marseille, Marseille, France, ${ }^{6}$ Saint Louis, Paris, France, ${ }^{7}$ Department of Medical Oncology, Chu de Poitiers, Poitiers, France, ${ }^{8}$ La Pitie Salpetriere Hospital, Thyroid and Endocrine Tumors Unit, Paris, France, ${ }^{9} \mathrm{Grenoble}$ Hospital, Dept of Endocrinology, La Tronche, ${ }^{10}$ Department of Medical Oncology, Hopital Cochin, Assistance Publique-Hôpitaux de Paris, Université Paris Descartes, Paris, France, ${ }^{11}$ Gustave Roussy, Université Paris-Sud, Villejuif, France, Villejuif, France, ${ }^{12}$ Department of Nuclear Medicine and Endocrine Oncology, Gustave Roussy and Université Paris-Sud, Villejuif, France, Gustave Roussy, Université Paris-Sud, Villejuif,

France, Villejuif, France

Objectives: In the SELECT phase III trial on radio-iodine refractory differentiated thyroid cancer (rDTC), lenvatinib improved median progression free survival (PFS) over placebo. The present study evaluates the efficacy and toxicity of lenvatinib treatment in real-life.

Methods: Clinical charts of 88 patients treated with lenvatinib from July 2015 to June 2016 in 24 French centers were retrospectively reviewed. Patients treated for other type of cancer $(\mathrm{n}=11)$ or previously treated with lenvatinib within a trial $(n=2)$ were excluded.

Results: Among the 75 rDTC analyzed ( 33 female, median age: 65 years [35-88]), 12 had ECOG performance status $>1$. They received lenvatinib as a first line systemic treatment in 24 cases. Forty seven $(63 \%)$ patients had documented progressive disease prior initial treatment. Distant metastases were located in lung, bone and lymph nodes in $89 \%, 60 \%$ and $69 \%$, respec- tively. The mean follow-up was 7 months, with a median duration of treatment of 6 months [0.3-15]. Median PFS was 10 months [0.1-14.8]. In the intention to treat population, best tumor response was a partial response in 23 patients $(30.6 \%)$ and stable disease in $38(50.6 \%)$. Eleven patients $(14.7 \%)$ discontinued lenvatinib for disease progression. Forty-four (59\%) and 23 (31\%) patients experienced dose reduction or discontinuation of lenvatinib for adverse events (AE). The last lenvatinib dose was $24 \mathrm{mg} /$ day for twenty patients (27\%). Median dose was $20 \mathrm{mg} /$ day. The most frequent AEs were fatigue, weight loss, hypertension and diarrhea. Eleven deaths occurred during the study (one considered to be drug related). Pneumothorax occurred in 2 patients with lung metastases.

Conclusions: Lenvatinib is an effective drug for patients with rDTC in real-life. AEs are frequent and should be closely monitored.

\section{OP-10-72 \\ CHANGES INPRESCRIPTION PRACTICES WHEN TREATING HYPOTHYROIDISM BETWEEN 2000 AND 2015 - A REGISTERBASED STUDY FROM THE COPENHAGEN PRIMARY CARE LABORATORY (COPLAB) DATABASE}

Bjarke Borregaard Medici', BIrte Nygaard², Jeppe Lerche la Cour ${ }^{3}$, Mia Klinten Grand ${ }^{4}$, Bent Struer Lind ${ }^{5}$, Niels De Fine Olivarius ${ }^{4}$, Volkert Siersma ${ }^{6}$, Håkon Sandholdt ${ }^{4}$, Christen Lykkegaard Andersen ${ }^{4}$

${ }^{1}$ Department of Endocrinology, Herlev University Hospital, Department of Endocrinology, Gentofte University Hospital, Copenhagen, Denmark, ${ }^{2}$ Department of Endocrinology, Herlev University Hospital, University of Copenhagen, Faculty of Health and Medical Sciences, University of Copenhagen, Copenhagen, Denmark, ${ }^{3} \mathrm{H} v i d o v r e$, Denmark, ${ }^{4}$ Research Unit for General Practice, Copenhagen, ${ }^{5}$ Department of Clinical

Biochemistry, Hvidovre Hospital, Copenhagen, ${ }^{6}$ Research Unit for General Practice, Copenhagen, Denmark

Background: Within the last decade increasing attention has been drawn to health- and quality of life studies. This has led to intensified screening for various medical conditions including hypothyroidism, in order to initiate early treatment. A falling Thyroid Stimulating Hormone (TSH) threshold upon initiation of Levothyroxine (T4) treatment has been reported in the UK between 2001- 2009 (Taylor et al. JAMA, 2014). Such changes in clinical practice may lead to less benefit from therapy and risk of overtreatment.

Objective: To investigate changes in clinical practice among general practitioners in the Copenhagen area when treating hypothyroidism

Methods: Retrospective study of all CopLab TSH measurements $(\mathrm{n}=3,5 \mathrm{mio})$ performed between November 2000 and December 2015 merged with the Danish Prescription Database. All TSH analyses were assessed on Centaur/CentaurXP (Bayer, Siemens) and subjected to regular external control of assay reliability.

Results: Preliminary data of persons without a previous T4 prescription is shown in Table 1. A falling threshold was observed for T4 initiation in all age

Table. (for Abstract OP-10-72)

\begin{tabular}{|c|c|c|c|c|c|}
\hline Age groups / & Year & $\begin{array}{l}\text { First time T4 } \\
\text { prescriptions }\end{array}$ & $\begin{array}{l}\text { Number of TSH } \\
\text { measurements }\end{array}$ & Persons examined & $\mathrm{TSH}$ at $\mathrm{T} 4$ initiation $(\mathrm{mIU} / \mathrm{l})^{*}$ \\
\hline \multirow[t]{3}{*}{ All } & 2001 & 633 & 110.886 & 99.259 & $13.0(6.5-38.0)$ \\
\hline & 2005 & 810 & 159.902 & 138.275 & $12.0(7.1-30.0)$ \\
\hline & 2015 & 1591 & 292.911 & 244.503 & $7.3(5.4-12.0)$ \\
\hline \multirow[t]{2}{*}{$30-40$} & 2001 & 75 & 14.701 & 13.541 & $27.0(11.0-69.0)$ \\
\hline & 2005 & 116 & 21.229 & 19.053 & $13.0(6.1-49.2)$ \\
\hline \multirow[t]{4}{*}{$50-60$} & 2001 & 124 & 19.837 & 17.987 & $10.0(6.1-24.0)$ \\
\hline & 2005 & 150 & 28.745 & 25.119 & $12.0(7.5-27.0)$ \\
\hline & 2010 & 291 & 34.567 & 29.477 & $8.3(6.2-14.0)$ \\
\hline & 2015 & 275 & 46.167 & 39.053 & $7.4(5.6-12.0)$ \\
\hline \multirow[t]{3}{*}{$>80$} & 2001 & 82 & 13.064 & 11.078 & $14.0(7.0-35.7)$ \\
\hline & 2005 & 86 & 17.752 & 14.303 & $12.0(7.1-25.7)$ \\
\hline & 2010 & 125 & 20.124 & 15.754 & $8.2(6.6-12.0)$ \\
\hline
\end{tabular}


group. Furthermore a $36 \%$ increase in TSH measurements from 2010 to 2015 did not lead to an overall corresponding increase in T4 prescription.

Conclusion: This study performed on a vast primary care population demonstrates a significant fall in the median TSH level between 2000 and 2015 when initiating levothyroxine therapy in hypothyroid patients. The study also reveals that intensified TSH screening from the 2010 level to the 2015 level did not lead to any further discoveries of hypothyroidism. The eldest age group had a fall in median TSH level similar to the 50-60 years group, which might point to a risk of overtreatment. More to be presented at ETA.

\section{OP-10-73 \\ ASSOCIATION BETWEEN MATERNAL THYROID FUNCTION IN EARLY PREGNANCY AND OFFSPRING AUTISTIC TRAITS: AN INDIVIDUAL LEVEL META-ANALYSIS OF THREE PROSPECTIVE BIRTH COHORTS}

Deborah Levie ${ }^{1}$, Robin Peeters ${ }^{2}$, Tim Korevaar ${ }^{3}$, Sarah Bath ${ }^{4}$, Albert Dalmau-Bueno ${ }^{1}$, Mario Murcia ${ }^{5}$, Mercedes Espada ${ }^{6}$, Jordi Sunyer ${ }^{7}$, Henning Tiemeier ${ }^{8}$, Marisa Rebagliato ${ }^{5}$, Margaret Rayman ${ }^{9}$, Mònica Guxens $^{7}$, Jesús Ibarluzea ${ }^{10}$

${ }^{1}$ Barcelona Institute for Global Health (Isglobal), Barcelona, Spain, ${ }^{2}$ Academic Center for Thyroid Diseases, Department of Endocrinology, Erasmus, Rotterdam, Netherlands, ${ }^{3}$ Erasmus MC,Rotterdam, The Netherlands, Endocrinology, Rotterdam, Netherlands, ${ }^{4}$ University of Surrey, Faculty of Health and Medical Sciences, Department of Nutritional Sciences, Guildford, UK, ${ }^{5}$ Epidemiology and Environmental Health Joint Research Unit, Fisabio-Universitat Jaume I-Universitat de València, Valencia, Spain, ${ }^{6}$ Departamento de Sanidad Gobierno Vasco, Laboratorio Normativo de Salud Pública, Bilbao, Spain, ${ }^{7}$ Barcelona Institute for Global Health (Isglobal), Barcelona, Spain, ${ }^{8}$ Erasmus University Medical Center, Rotterdam, The Netherlands, Rotterdam, Netherlands, ${ }^{9}$ Universary of Surrey, Guildford, UK, ${ }^{10}$ Departamento de Sanidad Gobierno Vasco, Subdirección de Salud Pública de Guipúzcoa, San Sebastián, Spain

Objective: Thyroid hormone (TH) is an important regulator of early brain development. During the first 18-20 weeks of pregnancy, the fetus predominantly depends on the placental transfer of maternal THs. During this period, TH-dependent brain development processes, such as neuronal cell proliferation and migration, take place. These processes have been implicated in the pathophysiology of autism. Therefore, we investigated the association between maternal thyroid function in early pregnancy and offspring autistic traits.

Methods: We included data from three large European, population-based birth cohorts: INMA (Spain), Generation R (The Netherlands) and ALSPAC (United Kingdom). TSH, FT4 and TPOAbs were measured in the first half of pregnancy. Autistic traits were assessed at age 4-7 years using validated parental questionnaires. Cohort-specific effect estimates were combined using random-effects individual data meta-analysis after adjusting for potential confounding variables.

Results: The final study population comprised 7,778 mother-child pairs of which 1.4 to $7.5 \%$ of children across cohorts had autistic traits within the clinical range. Maternal FT4 concentrations below the $5^{\text {th }}$ percentile were associated with a 1.5 -fold higher risk of offspring autistic traits $(95 \%$ CI 1.0 to 2.4$)$. Similarly, maternal hypothyroxinemia was associated with a 1.9 -fold higher risk of autistic traits $(95 \% \mathrm{CI} 1.2$ to 3.0$)$. In addition, FT4 concentrations above the $97.5^{\text {th }}$ percentile were associated with a 1.9 -fold higher risk of autistic traits $(95 \% \mathrm{CI} 1.0$ to 3.5$)$. Neither maternal TSH concentrations continuously, nor subclinical hypothyroidism were associated with offspring autistic traits.

Conclusion: Both low and high maternal FT4 concentrations in early pregnancy are associated with a higher risk of offspring autistic traits. This cross-cohort study indicates the presence of an optimum range for maternal thyroid hormone concentration and suggests that treatment with high dose levothyroxine may have adverse fetal effects.

\section{OP-10-74 \\ EXCESS MORTALITY IN HYPOTHYROID INDIVIDUALS IS AGGRAVATED BY OVERTREATMENT. DATA FROM A LONG-TERM POPULATION-BASED COHORT STUDY}

Mads Lillevang-Johansen ${ }^{1}$, Bo Abrahamsen ${ }^{2}$, Henrik Løvendahl Jørgensen ${ }^{3}$, Laszlo Hegedüs ${ }^{4}$, Thomas Brix ${ }^{5}$

${ }^{1}$ Department of Endocrinology, Odense University Hospital, University of Southern Denmark, Odense C, Denmark, ${ }^{2}$ Holbæk Hospital, Department of Medicine, Open, University of Southern Denmark, Odense, Holbæk, Denmark, ${ }^{3}$ Department of Clinical Biochemistry, Bispebjerg Hospital, Copenhagen, ${ }^{4}$ Department of Endocrinology and Metabolism, Odense University Hospital, Odense, Denmark, ${ }^{5}$ Department of Endocrinology, Odense University Hospital, Odense University Hospital, Odense C, Denmark

Objectives: Excess mortality in hypothyroid individuals has been suggested in prior studies. However, the consequences of treatment with T4 on mortality, in this setting, remain unclarified. We investigated the association between hypothyroidism and mortality in both treated and untreated hypothyroid patients, as well as the consequences of induced low TSH in treated hypothyroid individuals.

Methods: Register- and population-based cohort study of 239.768 individuals who had at least one serum TSH-measurement in the period 19952011 (median follow-up 7.3 years). Mortality rates were calculated using multivariate Cox-regression analyses, adjusting for age, sex and comorbidities. Cumulative periods of decreased and increased TSH were analyzed as time-dependent covariates.

Results: Mortality was increased in untreated hypothyroid individuals $(\mathrm{n}=673)$ [HR $1.46(95 \%$ Confidence Interval: $1.26-1.69, \mathrm{p}<0.001)]$, whereas individuals who received treatment $(\mathrm{n}=2235)$ experienced increased survival [HR 0.83 (95\% CI: 0.75-0.92, $\mathrm{p}<0.001)$ ]. The results were effectively the same when subdividing according to mild (TSH 4-10 mIU/L) and severe hypothyroidism ( $\mathrm{TSH} \geq 10 \mathrm{mIU} / \mathrm{L})$ at presentation. HR for mortality per 6 months with increased TSH was 1.05 (95\% CI: 1.02-1.07, p $<0.0001)$ in both treated and untreated hypothyroid individuals. HR for mortality per 6 months of decreased TSH in patients who received T4 was 1.18 (95\% CI: 1.15-1.21, p $<0.0001)$. Subdividing according to disease severity did not change this result. When subdividing according to age $<65$ years and $\geq 65$ years, HR for mortality per 6 months of decreased TSH was 1.26 (95\% CI: $1.19-1.34, \mathrm{p}<0.0001)$ and 1.16 (95\% CI: $1.13-1.19, \mathrm{p}<0.0001)$, respectively.

Conclusion: Mortality is increased in both mild and severe untreated hypothyroidism. In hypothyroid patients who receive treatment with $\mathrm{T} 4$, the duration of hyperthyroidism seems to play a larger role than duration of hypothyroidism, with regard to mortality, irrespective of age and severity of disease. Physicians should be cautious of over-treatment of hypothyroidism and aim to maintain euthyroidism.

\section{OP-10-75 \\ INCREASED SERUM MICRORNAS ARE CLOSELY ASSOCIATE WITH THE PRESENCE OF RECURRENT / PERSISTENT DISEASE IN THYROID CANCER}

León Wert-Lamas ${ }^{1}$, María Augusta Guillén ${ }^{2}$, Mercedes Robledo ${ }^{3}$, Pilar Santisteban ${ }^{4}$, Garcilaso Riesco-Eizaguirre ${ }^{5}$

${ }^{1}$ Biomedical Research Institute (libm); Autonomous University Madrid (Uam);, Ciberonc, Instituto de Salud Carlos III, Madrid, Spain, ${ }^{2} \mathrm{Hospital}$ Universitario de Móstoles, Móstoles- Madrid, Spain, ${ }^{3}$ Hereditary Endocrine Cancer Group, Spanish National Cancer Research Centre (Cnio), Isciii Center for Biomedical Research on Rare Diseases (Ciberer), Madrid, Spain, ${ }^{4}$ Instituto de Investigaciones Biomédicas, Universidad Autónoma de Madrid, Ciberonc, Instituto de Salud Carlos III, Madrid, Spain, ${ }^{5}$ Hospital Universitario de Móstoles, Ciberonc, Instituto de Salud Carlos III, Ciberonc Instituto de Salud Carlos III, Móstoles, Spain

Objective: Circulating microRNAs (miRNAs) are emerging biomarkers for thyroid cancer. This study sought to identify the profile of circulating miRNAs in thyroid cancer and its response to human recombinant TSH during follow up. 
Methods: we obtained serum samples from 26 patients, 14 with recurrent/ persistent disease (13 PTC: papillary thyroid cancer and 1 FTC: Folicular thyroid cancer) and 12 with complete remission (10 PTC and 2 FTC). The levels of 20 miRNAs were initially analyzed using quantitative reverse-transcription PCR (RT-qPCR). These miRNAs were selected based on its differential expression in primary tumors by next generation sequencing.

Results: Two miRNAs were significantly upregulated in patients with recurrent/persistent disease including miR-221 0.0126 vs. $0.0021, \mathrm{p}=0.013$ and miR-34: 0.0081 vs. $0.0011, \mathrm{p}=0.03$, but not miR-146b as previously shown. Moreover, the levels of x miR-221 significantly correlated with TSH levels. Preliminary results in three patients showed that that miR-221 upregulated in patients with persistent disease after human recombinant TSH whereas it was downregulated complete remission patients.

Conclusions: The two serum miRNAs identified in our study hold potential as auxiliary biomarkers for recurrent/persistent disease in thyroid cancer and their potential relevance in responsiveness to $\mathrm{TSH}$.

\section{Oral Session 11: Young Investigators / Basic}

\section{OP.11-76 \\ DICER1 DOWNREGULATION INDUCES CELL TRANSFORMATION IN THYROID CANCER}

Julia A. Ramirez-Moya ${ }^{1}$, León Wert-Lamas², Pilar Santisteban ${ }^{1}$

${ }^{1}$ Biomedical Research Institute (Csic-Uam), Ciberonc From Health

Institute Carlos III, Madrid, Spain, ${ }^{2}$ Biomedical Research Institute (Csic-

Uam), Ciberonc From Health Institute Carlos III, University Hospital

Mostoles, Madrid, Spain

Background: microRNAs (miRs) have important functions in thyroid cancer; however, little is known about the role played by the machinery involved in their biogenesis. Maturation of most miRs requires processing by the type III ribonuclease DICER. Low levels or mutations in DICER1 have been described in thyroid cancer. The aim of this work was therefore to study the function and regulation of DICER1 in this pathology.

Methods: DICER1 and NKX2.1 mRNA levels were evaluated in PTC patients. DICER1 was overexpressed (SW1736) and silenced (Cal62 and TPC1) in tumour thyroid cells. ECR browser was used to identify transcription factors binding to the DICER1 promoter and miRanda was used to predict associations between miRs and DICER1 3'UTR. Functional assays were used to test binding of transcription factors and miRs.

Results: DICER1 expression is downregulated in PTC patients, confirming that low mRNA levels are associated with thyroid cancer. Loss of DICER1 function provokes global downregulation of miRs, which induces an aggressive behaviour in thyroid cells: (i) increasing proliferation, migration, invasion and EMT markers, and (ii) decreasing expression of thyroid differentiation genes PAX8 and FOXE1. Gain of DICER1 function reduces this behaviour. DICER1 downregulation could be due, at least in part, to the low NKX2.1 levels found in the PTC patients, as we show that this factor bindsDICER1 promoter and increases its transcriptional activity. Interestingly, in silico analysis indicated that DICER1 is a target of the most up-regulated miRs in thyroid cancer. Among them, miR-146b, the most abundant miR in these tumours, targets DICER1 at its 3'UTR, decreasing its expression. Functionally, miR$146 \mathrm{~b}$ promotes transformation of thyroid cells, which is rescued by DICER 1 overexpression.

Conclusions: DICER1 is an important tumour suppressor tightly regulated in thyroid cancer, and its downregulation contributes to cancer progression. DICER1could therefore be used as a prognostic marker.

\section{OP-11-77 \\ REGULATION OF INTRACELLULAR T3 AVAILABILITY AND ACTION IS ESSENTIAL FOR OPTIMAL MACROPHAGE FUNCTION}

Anne van der Spek ${ }^{1}$, Olga Surovtseva ${ }^{1}$, Kin Ki Jim², Adri van Oudenaren $^{3}$, Matthijs Christian Brouwer ${ }^{4}$, Pieter Leenen ${ }^{5}$, Arturo Hernandez ${ }^{6}$, Diederik Van de Beek ${ }^{7}$, Eric Fliers ${ }^{8}$, Anita Boelen ${ }^{9}$ ${ }^{1}$ Academic Medical Center, Amsterdam, Netherlands, ${ }^{2}$ Academic Medical Centre Amsterdam, Neurology, Amsterdam, Netherlands, ${ }^{3}$ Erasmus University Medical Center, Rotterdam, ${ }^{4}$ Academic Medical Center Amsterdam, Department of Neurology, Center of Infection and Immunity Amsterdam (Cinima), Amsterdam, Netherlands, ${ }^{5}$ Erasmus University Medical Center, Immunology, Rotterdam, Netherlands, ${ }^{6}$ Maine Medical Research Center, Scarborough,ME, USA, ${ }^{7}$ Academic Medical Centre Amsterdam, Department of Neurology, Center of Infection and Immunity Amsterdam (Cinima), Department of Neurology, Amsterdam, Netherlands, ${ }^{8} \mathrm{Amc}$, University of Amsterdam, Amsterdam, Netherlands, ${ }^{9}$ Academic Medical Centre, Amsterdam, Netherlands

Innate immune cells have recently been identified as novel thyroid hormone target cells. Macrophages are important phagocytic innate immune cells that play essential roles in tissue homeostasis and immunity. Macrophage dysfunction has been linked to a large number of pathophysiological conditions including cancer, diabetes and atherosclerosis. Macrophages can adopt a wide range of phenotypes in a process known as polarization. These phenotypes range from pro-inflammatory (M1) macrophages, involved in bacterial killing, to anti-inflammatory (M2) macrophages that are important for tissue remodeling and repair. A previous study has shown that type 2 deiodinase (D2), the main triiodothyronine (T3)-generating enzyme, is essential for the pro-inflammatory response in the RAW264.7 macrophage cell line. We hypothesized that intracellular T3 action affects macrophage function, possibly by regulating polarization.

We determined the effect of D2 knockdown on mortality during infection in vivo using a zebrafish embryo bacterial meningitis model. In addition, we analyzed macrophage function in cells derived from D2KO and WT mice. Finally, to further elucidate the role of intracellular T3 action in macrophage polarization we determined the effects of siRNA knockdown of TH receptor á (TRá), the main receptor in macrophages, on polarization in a macrophage cell line.

A lack of D2 in zebrafish embryos increased mortality during pneumococcal meningitis. Primary D2KO macrophages exhibited impaired phagocytosis but normal cytokine responses to stimulation with bacterial endotoxin (LPS). TRá knockdown impaired M1 polarization and amplified M2 polarization in a macrophage cell line.

In summary, a lack of D2, which is expected to reduce intracellular T3 levels, results in an impaired innate immune response in vivo and an impaired pro-inflammatory macrophage response in vitro. A lack of TRá shifts macrophage polarization towards an anti-inflammatory phenotype. These results suggest that proper regulation of intracellular T3 availability and action is essential for optimal macrophage function.

\section{OP-11-78 \\ ANTI-HTERT SIRNA NANOPARTICLES BLOCK THE GROWTH OF ANAPLASTIC THYROID CANCER CELLS IN VITRO AND IN VIVO \\ Giovanni Enrico Lombardo ${ }^{1}$, Valentina Maggisano ${ }^{1}$, Donato Cosco ${ }^{1}$, Chiara Mignogna ${ }^{1}$, Gabriella Cardillo ${ }^{1}$, Marilena Celano ${ }^{1}$, Saverio \\ Massimo Lepore ${ }^{1}$, Sonia Moretti ${ }^{2}$, Efisio Puxeddu ${ }^{3}$, Giuseppe Damante $^{4}$, Massimo Fresta ${ }^{1}$, Diego Russo ${ }^{1}$, Stefania Bulotta \\ 1"Magna Graecia" University of Catanzaro, Catanzaro, Italy, ${ }^{2}$ University of Perugia, Department of Medicine, Perugia, Italy, ${ }^{3}$ University of \\ Perugia, Perugia, Italy, ${ }^{4}$ University of Udine, Udine, Italy}

Mutations in the $h T E R T$ promoter are the most frequent genetic alteration detected in anaplastic thyroid cancer (ATC), and expression of $h T E R T$ mRNA is often increased in ATCs compared to normal thyroid tissues. Moreover, silencing of $h T E R T$ has been demonstrated to inhibit the proliferation and migration of ATC cells.

In this study we encapsulated an $h T E R T$ specific siRNA within nanoparticles made up of poly(D,L-lactide-co-glycolide) (PLGA) and chitosan in order 
to deliver the genetic material into thyroid cancer cells and investigated its anticancer efficacy in vitro against two human ATC cell lines and in vivo on a xenograft model of thyroid cancer induced in NOD/SCID mice.

Nanoparticles were prepared following the nanoprecipitation method of the pre-formed PLGA in an acidified aqueous solution containing chitosan, siRNA antiTERT and poloxamer 188 as a stabilizer.

The nanoplexes were able to reduce TERT expression in CAL-62 and $8505 \mathrm{C}$ cell lines after $48 \mathrm{~h}$ of incubation. The growth of both cell lines was reduced of about $40 \%$ vs. untreated cells $(\mathrm{p}<0.01)$ and similar results were observed in the migration assay. Moreover, in a xenograft tumor obtained by flank injection of CAL62 cells, we observed a great reduction (about 50\%) in the growth and vascularization of the tumor after 14-days of i.v. administration of siTERT nanoparticle. Reduced levels of TERT and ki67 protein were also detected. No signs of toxicity appeared after this treatment. These findings demonstrate the potential suitability of this nanoformulation as novel tool for ATC treatment.

\section{OP-11-79 \\ THE LONG NON-CODING RNA SPTYD1-AS1 IMPAIRS THYROID TUMORIGENESIS BY REPRESSING ONCOMIR ACTIVITY}

León Wert-Lamas ${ }^{1}$, Alice Fletcher ${ }^{2}$, Laura Ruiz Canas $^{3}$, Adrían Acuña ${ }^{4}$ Carlos Wert-Carvajal ${ }^{5}$, Garcilaso Riesco-Eizaguirre ${ }^{6}$, Pilar Santisteban ${ }^{7}$ ${ }^{1}$ Biomedical Research Institute (libm); Autonomous University Madrid (Uam);, Ciberonc, Instituto de Salud Carlos III, Madrid, Spain, ${ }^{2}$ Institute of Metabolism and Systems Research, Level 2 Institute of Biomedical Research (Ibr), University of Birmingham, Birmingham, UK, ${ }^{3}$ Biomedical Research Institute (libm); Autonomous University Madrid (Uam);, Madrid, Spain, ${ }^{4}$ Instituto de Investigaciones Biomédicas, Ciberonc, Instituto de Salud Carlos III, Madrid, Spain, ${ }^{5}$ Carlos III Madrid University (Uc3m), Madrid, Spain, ${ }^{6}$ Hospital Universitario de Móstoles, Ciberonc, Instituto de Salud Carlos III, Ciberonc Instituto de Salud Carlos III, Móstoles, Spain, ${ }^{7}$ Instituto de Investigaciones Biomédicas, Universidad Autónoma de Madrid, Ciberonc, Instituto de Salud Carlos III, Madrid, Spain

Long-noncoding RNAs (LNCRNAs) have been shown to modulate specific pri-miRNA processing and to have a functional role in several tumours, including thyroid carcinomas (TC). Using NGS, we identified the LNCRNA $S P T Y 2 D 1-A S 1$ as silenced in the papillary TC subtype. Using different homology-based algorithms, SPTY2D1-ASI was predicted to interact with pri-miR-146b, -miR-21, -miR-31, and -miR-221, broadly considered as thyroid oncomiRs. We sought to decipher SPTY2D1-AS1 regulation in TC, and to determine the effects of this LNCRNA on the activity of the above oncomiRs and its consequent role in thyroid tumourigenesis.

qPCR analysis of human TC cell lines revealed a reduction in SPTY2D1-AS1 expression associated with hyperactivated PI3K signalling. Further analysis indicated that PTEN induces $S P T Y 2 D 1-A S 1$ promoter activity and its expression.

We then studied the levels of primary, precursor, and mature forms of the above oncomiRs in Cal62 TC cells. Whereas SPTY2D1-AS1 induced the accumulation of pri-miRs, it reduced the levels of pre- and mature miRs, suggesting an impairment at the microprocessor stage. Additionally, luciferase reporter assays of miR-146b activity confirmed that SPTY2D1-AS1 dampens miR-146b action.

We analysed the TCGA-consortium database, observing that decreased $S P T Y 2 D 1-A S 1$ copy number results in higher risk and poorer survival. Thus, we performed an in vivo tumour progression assay in mice. Strikingly, $S P T Y 2 D 1-A S 1$ overexpression not only impaired tumour progression but also reduced tumour size over time.

Finally, as the PI3K signalling pathway is a well-established oncogenic driver in TC and is tightly regulated by the specified oncomiRs, according to miRNA:target prediction algorithms, we evaluated its activation in the generated tumours by western-blotting and immunohistochemistry. We found that SPTY2D1-AS1 dramatically reduced PI3K output, as measured by decreased pAKT and pFOXO1 expression.

In conclusion, we show that SPTY2D1-AS1 quenches the activity of oncomiRs in TC by interfering with their biogenesis, resulting in decreased PI3K output and proliferation, which impairs tumour growth.

\section{OP-11-80 \\ THYROID HORMONE TRANSPORT BY OATP1A2 - CHARACTERISTICS AND GENETIC VARIATION}

Stefania Farina ${ }^{1}$, Wendy M. van Der Deure ${ }^{1}$, Edith C.H. Friesema ${ }^{1}$ Anja L. van Gucht ${ }^{1}$, Chantal Zevenbergen ${ }^{1}$, Elaine C. Lima De Souza ${ }^{1}$, Robin P. Peeters ${ }^{1}$, Theo J. Visser ${ }^{1}$

${ }^{1}$ Erasmus Medical Center, Rotterdam, Netherlands

Introduction: Human organic anion transporting polypeptide 1A2 (OATP1A2) is expressed in different tissues such as brain, liver, kidney and intestine. It is known to transport thyroid hormone $(\mathrm{TH})$ into cells but little is known about the characteristics of this process and about possible consequences of genetic variation.

Objectives: 1) Characterize TH transport by OATP1A2 regarding effects of $\mathrm{pH}$ and substrate concentration. 2) Study the effects of the E172D polymorphism (allele frequency $4.5 \%$ ) on TH transport by OATP1A2.

Materials and Methods: We generated a N-terminal Flag-tagged OATP1A2 construct (F1A2) in the pSG5 expression vector. Subsequently, we introduced the E172D variant as well as the artificial E172A mutation by sitedirected mutagenesis and the correctness of the constructs was confirmed by sequencing. Mammalian COS1 cells were transfected with wild-type (WT) or mutant F1A2 After 2 days, the cells were incubated for 30 minutes with 0.1-100 $\mu \mathrm{M}^{125} \mathrm{I}$-labeled T4 or T3 and, after removal of medium and washing, cell-associated radioactivity was determined.

Results: Uptake of both T4 and T3 were strongly dependent on $\mathrm{pH}$ and substrate concentration. In general, F1A2 mediated transport was greater for T3 than for T4 and increased with decreasing $\mathrm{pH}$ between $\mathrm{pH} 7.3$ and 5.3. Remarkably, the fractional F1A2-mediated TH uptake increased with the substrate concentration between 0.1 and $10 \mu \mathrm{M}$ to become saturated with a further increase to $100 \mu \mathrm{M}$ T3 or T4. Thus, relative to empty vector-transfected control cells, expression of F1A2 resulted in a 5.5-fold increase in T3 uptake at 10 $\mu \mathrm{M}$ substrate and $\mathrm{pH} 5.3$ compared with a 4-fold increase at $\mathrm{pH} 7.3$. Kinetic studies indicated an apparent $\mathrm{Km}$ value of $30 \mu \mathrm{M}$ for T3 at $\mathrm{pH} 7.3$. The E172D polymorphism and the E172A mutation resulted in significant $22 \%$ and $35 \%$ decreases in TH uptake, respectively. Further studies are done to determine if these mutations affect the cellular trafficking or the intrinsic transport activity of OATP1A2

Conclusions: The present study demonstrates that the transport activity of OATP1A2 is stimulated by an acidic extracellular environment. This may well be important for the apical transport of $\mathrm{TH}$ in the intestine and kidney tubules. Further studies are required to determine what the effect is of the E172D polymorphism on TH transport and metabolism in vivo.

\section{OP.11-81 \\ KNOCKDOWN OF THE SELENOCYSTEINE INSERTION SEQUENCE-BINDING PROTEIN 2 (SECISBP2) GENE LEADS TO A MULTISYSTEM DISORDER IN ZEBRAFISH}

Federica Marelli' ${ }^{1}$, Silvia Carra², Giuditta Rurale ${ }^{1}$, Luca Persani ${ }^{3}$

${ }^{1}$ University of Milan, Milan, Italy, ${ }^{2}$ Irccs Istituto Auxologico Italiano, Milan, ${ }^{3}$ University of Milan, Irccs Istituto Auxologico Italiano, Ospedale San Luca, Milan, Italy

Selenium, a trace element that is fundamental to health of almost all species, is incorporated into some proteins as selenocysteine, generating a family of selenoproteins (SPs). Sec incorporation is mediated by a multiprotein complex that includes Sec insertion sequence-binding protein 2 (SECISBP2 or SBP2). Individuals with homozygous or compound heterozygous SBP2 mutations presented characteristic thyroid tests abnormalities, high serum T4, low T3, high rT3 and normal TSH, consistent with the deficiency in deiodinases activity. The overall phenotype includes delayed growth and bone maturation, delayed motor and intellectual milestones, infertility, progressive congenital myopathy and sensorineural hearing loss.

In the present work, we knocked down (KD) the expression of the zebrafish SBP2 by the microinjection of a splice-blocking morpholino. At 5 days post-fertilization (dpf), the defective embryos display low T4 and high T3 levels, in presence of normal thyroid development and TSHb expression. The enzymatic activity of Dio2 is significantly reduced but the mRNA levels are unchanged in the SBP2 deficient embryos at 5dpf. Furthermore, defects in 
other SPs like SEPP, GPX, and SEPW are detected, reflecting a global deficiency in SPs synthesis. The phenotypic manifestation of the SBP2 deficient embryos also includes delayed cartilage and bone development and a progressive disorganization of the muscle fibers that affects their locomotor behaviour. Structural and molecular changes of brain development also indicate the presence of neurological disorders in the SBP2 deficient embryos.

In conclusion, we characterized for the first time the role of SBP2 in the zebrafish. The SBP2-KD results in complex and multisystem phenotype likely reflecting the diminished activity of the SPs and their hierarchy in conditions of Se depletion. Moreover, changes of thyroid hormone metabolism closely resemble those described in human and mice models, providing evidence that zebrafish is a useful model to gain insights on the SBP2 pathology.

\section{Oral Session 12 (Clinical): Clinical Thyroidology}

\section{OP-12-82 \\ ASSOCIATION BETWEEN SERUM 3-IODOTHYRONAMINE (T1AM) LEVELS AND LEFT VENTRICULAR PERFORMANCE IN CHRONIC HEART FAILURE (CHF)}

Jeppe Lerche la Cour ${ }^{1}$, Heidi M Christensen ${ }^{2}$, Josef Köhrle ${ }^{3}$, Ina

LehmphuP $^{\beta}$, Caroline Kistorp ${ }^{1}$, Blrte Nygaard ${ }^{4}$, Jens Faber ${ }^{5}$

${ }^{1}$ Department of Endocrinology, Herlev University Hospital, Herlev,

Denmark, Hvidovre, Denmark, ${ }^{2}$ Department of Gynecology and Obstetrics, Herlev University Hospital, Denmark, Department of Endocrinology, Herlev University Hospital, Herlev, Denmark, Herlev, Denmark, ${ }^{3}$ Institut Fuer Experimentelle Endokrinologie, \& Endokrinologisches Forschungs-Centrum Enforcé, CharitéUniversitätsmedizin Berlin, Berlin, Germany, ${ }^{4}$ Department of Endocrinology, Herlev University Hospital, Herlev, Denmark, Herlev, Denmark, ${ }^{5}$ Department of Endocrinology, Herlev University Hospital, University of Copenhagen, Faculty of Health and Medical Sciences, University of Copenhagen, Copenhagen, Denmark, ${ }^{6} \mathrm{Herlev}$ University Hospital, Dept. of Endocrinology, Faculty of Health and Medical

Sciences, University of Copenhagen, Herlev, Denmark

Thyroid hormone metabolites might affect the heart. Thus the aminergic metabolite 3-Iodothyronamine (T1AM) seems to reduce Left Ventricular Ejection Fraction (LVEF) in rodents and 3,5-diiodothyronine (T2) might have biological effects as well.

Aim: To investigate serum levels of T1 AM and T2 in CHF without thyroid disease, including patients with the worst prognosis, having cardiac cachexia (definition: a non-oedematous weight loss $>5 \%$ ).

Methods: Cross sectional study. CHF was characterized by LVEF $<45$ $\%$ (echocardiography). The following age, sex, and kidney function matched groups were studied ( $\mathrm{n}=19$ in each group): patients with cachexia (CAC), or without (NON-CAC), and controls with prior myocardial infarction and LVEF $>45 \%$ (C). T1AM and T2 concentrations were measured using monoclonal antibody based immunoassays.

Results: LVEF: CAC: $32 \% \pm 9$ (SD); NON-CAC: $38 \% \pm 8$ and C: $60 \% \pm 8$, $(\mathrm{p}<0.0001)$. Serum TSH and T4 were normal whereas T3 was low-normal reflecting chronic illness (no difference between groups, $\mathrm{p}=0.15$ ). Serum T2 did not differ between groups (the 3 groups taken together: median $0.34 \mathrm{nmol} / 1$ (IQR 0.26-0.47)).

Serum T1AM (nmol/1) levels were higher in CHF: CAC: (mean \pm SD) $12.4 \pm 6.6$, NON-CAC: $9.1 \pm 5$ and $\mathrm{C}: 7.3 \pm 2.9)($ ANOVA, $p=0.001)$. T1 AM concentrations were not correlated to TSH, T4, T3 nor T2 ( $>0.09$ for all, Spearman). In univariate regression analysis a negative association between T1 AM and LVEF was found (all 3 groups taken together (coeff. $=-0.80(\mathrm{p}=$ $0.02)$ ). After adjusting for sex, age, estimated GFR the finding remained significant (coeff. $=-0.81(\mathrm{p}=0.03)$.

Conclusion: Increasing T1 AM concentrations were independently associated with reduced LV function in patients with CHF, and T1 AM concentrations were especially high in those patients with the clinical syndrome cardiac cachexia. T1AM might have a negative inotropic effect on the human heart. Whether this is good or bad is unknown at present.

Oral Presentations

\section{OP-12-83 \\ ORAL THYROXINE AND THE SPECTRUM OF GASTRIC PH: THE IN VIVO STUDY}

Camilla Virili' ${ }^{1}$, Giovanni Bruno ${ }^{2}$, Maria Giulia Santaguida ${ }^{1}$, Barbara Porowska $^{3}$, Corrado De Vito ${ }^{4}$, Lucilla Gargano ${ }^{5}$, Carola Severi ${ }^{2}$, Marco

Centanni ${ }^{1}$

${ }^{1}$ Sapienza, University of Rome, Department of Medico-Surgical Sciences and Biotechnologies, Latina, Italy, ${ }^{2}$ Gastroenterology Unit, Department of Internal Medicine and Medical Specialties, "Sapienza" University of Rome, Rome, Italy, ${ }^{3}$ Policlinico Umberto I ${ }^{\circ}$ Rome, Gastroenterology and Clinical Surgery, Rome, Italy, ${ }^{4}$ Department of Public Health and Infectious Diseases, "sapienza" University of Rome Rome, Italy, ${ }^{5}$ Ausl Latina, Latina, Italy

Objective: In vitro evidence supports the notion that the variations of gastric $\mathrm{pH}$ may interfere with $\mathrm{T} 4$ requirement. An increased need for thyroxine has been observed in patients with impaired gastric $\mathrm{pH}$. Here we present the relationship between the minimal effective dose of $\mathrm{T} 4$ and the intragastric $\mathrm{pH}$, directly measured during endoscopy.

Patients: A total of 40 hypothyroid patients $(32 \mathrm{~W} / 8 \mathrm{M})$ were treated with the same brand of oral levothyroxine in tablet and have pledged to take thyroxine under fasting conditions, abstaining from eating or drinking for one hour. The theoretical and the actual dose of thyroxine required to obtain the therapeutic goal were compared with those obtained in normal patients similarly treated. All patients underwent endoscopy, either for dyspeptic symptoms or for an increased T4 need, and multiple biopsy specimens and gastric juice were collected for $\mathrm{pH}$ evaluation.

Results: Thyroxine dose progressively increased along with the increase of intragastric $\mathrm{pH}(\mathrm{r}=0.3224 ; \mathrm{p}<0.0484)$. Therefore, the excess of T4 dose (ED) was plotted with gastric acidity, confirming the inverse relationship between the two variables $(\mathrm{p}<0.0001)$. A multivariate analysis revealed that $\mathrm{pH}$ act as an independent variable in determining the dose of $\mathrm{T} 4(\mathrm{p}<0.047)$. Based on their $\mathrm{pH}$, patients were then subdivided in two groups using $\mathrm{pH} 2.4$ as cutoff (group $\mathrm{A}$ median $\mathrm{pH}=1.52$ and group $\mathrm{B} \mathrm{pH}=6.47$ ). Only 6 out of 24 patients $(25 \%)$ in group A needed a high T4 dose vs 14 out of $16(87.5 \% ; \mathrm{p}<0.0001)$ in group B. Overall, the median T4 dose was increased by $15 \%$ in group A and by $48 \%$ in group B. The ED of T4 was also differently distributed in patients with atrophic gastritis (90\%), with pangastritis $(73 \%)$ and with antritis $(40 \%)$.

Conclusions: These findings have shown, for the first time in vivo, the direct effect of $\mathrm{pH}$ on the daily $\mathrm{T} 4$ requirement.

\section{OP.12-84 \\ TRENDS IN COSTS OF THYROID DISEASE TREATMENT IN DENMARK DURING 1995-2015: THE EFFECT OF IODINE FORTIFICATION}

Line Tang Moellehave ${ }^{1}$, Allan Linneberg ${ }^{2}$, Tea Skaaby ${ }^{3}$, Nils Knudsen ${ }^{4}$ Lars Ehlers ${ }^{5}$, Torben Jørgensen ${ }^{6}$, Betina Thuesen ${ }^{7}$

${ }^{1}$ Research Centre for Prevention and Health, Centre for Health, The Capital Region of Denmark, Glostrup, Denmark, ${ }^{2}$ Research Centre for Prevention and Health, Centre for Health, The Capital Region of Denmark, Department of Clinical Experimental Research, Rigshospitalet, Glostrup, Denmark, Department of Clinical Medicine, Faculty of Health and Medical Sciences, University of Copenhagen, Denmark, Glostrup, Denmark, ${ }^{3}$ Research Centre for Prevention and Health, Centre for Health, The Capital Region of Denmark, Glostrup, ${ }^{4}$ Department of Endocrinology, Bispebejrg University Hospital, Copenhagen, Denmark, Faculty of Health and Medical Sciences, University of Copenhagen, Denmark, Copenhagen, Denmark, ${ }^{5}$ Aalborg University, Department of Business and Management, Aalborg $\varnothing$, Denmark, ${ }^{6}$ Research Centre for Prevention and Health, The Capital Region of Denmark, Glostrup, Denmark, Faculty of Medicin, Aalborg University, Aalborg, Denmark, Faculty of Health Sciences, Copenhagen, Denmark, Glostrup, Denmark, ${ }^{7}$ Research Centre for Prevention and Health, Centre for Health, The Capital Region of Denmark, Glostrup, Denmark, Glostrup, Denmark

Objective: Iodine fortification (IF) prompts changes in the costs of thyroid disease treatment. IF decreases deficiency-associated disorders, but may slightly increase excess-associated disorders in a population. However, it is unknown how this shift in disease pattern impacts the total treatment costs. 
The objective of this study was to assess the trends in annual costs of thyroid disease treatment during 1995-2015 in Denmark, i.e. before and after the implementation of mandatory IF in 2000.

Methods: Information on treatments for thyroid disease (antithyroid medication, thyroid hormone therapy and thyroid surgery) was derived from nationwide registers including all Danish citizens treated during 1995-2015. All health care costs were valued in 2015-prices using sales prices for medicine (including user payments and excluding VAT) and Danish DRG tariffs of surgeries. Results were adjusted for changes in age- and sex distribution.

Preliminary Results: The total costs of thyroid disease treatment increased almost linearly from $€ 8.39$ million in 1995 to $€ 12.96$ million in 2015 . This was mainly due to increased costs of thyroid hormone therapy from $€ 1.70$ million to $€ 4.59$ million during 1995-2015. Costs of thyroid surgery were constant around $€ 5.44$ million until 2008 followed by an increase to $€ 7.04$ million in 2015 . Costs of antithyroid treatment increased transiently after IF to $€ 1.96$ million, but fell below the cost at IF in 2009 and decreased to $€ 1.32$ million in 2015 . The trends in costs of thyroid hormone treatment and thyroid surgery were approximately parallel in regions with previous mild and moderate iodine deficiency. The transient increase in costs of antithyroid treatment was mainly observed in the region with previous moderate iodine deficiency.

Conclusion: IF was not accompanied by lower costs of thyroid disease treatment in Denmark. This may be due to factors other than IF, e.g. changes in treatment practices.

\section{OP-12-85 \\ FIVE- AND TEN-YEAR FOLLOW-UP OF PATIENTS WITH THYROID NODULES IN AN IODINE- SUFFICIENT AREA - A PILOT STUDY \\ Katica Bajuk Studen ${ }^{1}$, Simona Gaberšček ${ }^{1}$, Edvard Pirnat ${ }^{1}$, \\ Katja Zalete/ ${ }^{1}$ \\ ${ }^{1}$ University Medical Centre Ljubljana, Department of Nuclear Medicine, \\ Ljubljana, Slovenia}

Background: Few longitudinal data on the natural course of thyroid nodules exist, especially in areas with sufficient iodine supply. Therefore, our aim was to follow the number and size of thyroid nodules five and ten years after the first evaluation.

Methods: Patients diagnosed with unsuspicious thyroid nodules at first evaluation in 2006 and 2011 were invited for repeat thyroid ultrasound in 2016. Number of thyroid nodules, maximal diameter and nodule volume were recorded.

Results: From the year 2006 (Group 1), 41 patients with 61 nodules, and from the year 2011 (Group 2), 42 patients with 62 nodules were included in this pilot study. In 2016, 36 and 29 new nodules were found in Groups 1 and 2 , respectively. However, in 2016, 14/61 nodules from Group 1 and 6/62 nodules from Group 2 were no longer present. Maximal diameter significantly increased in both groups, and nodule volume only in Group 2 (Table 1). The increase in nodule size was highly significant for 14 nodules from 2006 and 11 nodules from 2011. Therefore, a complete clinical re-evaluation was indicated.

Conclusions: Our preliminary results suggest significant changes in the number and size of thyroid nodules over time. More data regarding the natural course of thyroid nodules is needed to establish the necessity and regimen of the follow-up of thyroid nodules.

Table 1. Five- and ten-year follow up of the thyroid nodule size

\begin{tabular}{lcc}
\hline $\begin{array}{l}\text { Parameters at first } \\
\text { evaluation }\end{array}$ & 2006 (Group 1) & 2011 (Group 2) \\
\hline $\begin{array}{l}\text { Maximal diameter }[\mathrm{mm}] \\
\begin{array}{l}\text { Mean } \pm \text { SD (range) } \\
\text { Nodule volume }[\mathrm{mL}]\end{array}\end{array}$ & $11.4 \pm 7.4(3-36)^{\mathrm{a}}$ & $12.8 \pm 8.6(3-36)^{\mathrm{a}}$ \\
Mean \pm SD (range) & $3.6 \pm 3.5(0.1-7.3)$ & $2.0 \pm 3.4(0.01-14.3)^{\mathrm{a}}$ \\
\hline
\end{tabular}

\begin{tabular}{lcc}
\hline $\begin{array}{l}\text { Parameters at follow-up } \\
\text { evaluation }\end{array}$ & \multicolumn{2}{l}{2016} \\
\hline $\begin{array}{l}\text { Maximal diameter }[\mathrm{mm}] \\
\text { Mean } \pm \text { SD (range) }\end{array}$ & $14.3 \pm 11.1(3-56)$ & $14.8 \pm 9.2(3-38)$ \\
$\begin{array}{l}\text { Nodule volume }[\mathrm{mL}] \\
\text { Mean } \pm \text { SD (range) }\end{array}$ & $2.7 \pm 7.2(0.01-41.7)$ & $2.3 \pm 3.7(0.01-15.5)$ \\
\hline
\end{tabular}

${ }^{\mathrm{a}} \mathrm{p}<0.05$ when compared with 2016.

\section{OP-12-86 \\ IODINE FORTIFICATION ONLY INCREASED THE INCIDENCE OF OVERT HYPOTHYROIDISM MODESTLY - A 16 YEAR DANISH PROSPECTIVE POPULATION STUDY}

Mads Petersen ${ }^{1}$, Allan Carlé ${ }^{1}$, Nils Jakob Knudsen ${ }^{2}$, Lars Ovesen ${ }^{3}$, Lone Banke Rasmussen ${ }^{2}$, Torben Jørgensen ${ }^{4}$, Hans Perrild ${ }^{2}$,

Inge Bülow Pedersen ${ }^{1}$

${ }^{1}$ Aalborg University Hospital, Aalborg, Denmark, ${ }^{2}$ Copenhagen,

Denmark, ${ }^{3}$ Slagelse, Denmark, ${ }^{4}$ Glostrup, Denmark

Objective: Iodine fortification is widespread, but the long-term consequence for development of hypothyroidism is unknown. We performed the first population based prospective monitoring of the incidence rate of overt hypothyroidism starting before iodine fortification (IF) of salt, in an area of Denmark with moderate iodine deficiency. Mandatory IF was initiated in the year 2000. The mandatory iodization of household salt and salt used for production of bread was implemented at a level of $13 \mathrm{ppm}$.

Methods: In an open cohort $(n=309,434)$ including Aalborg city (moderate iodine deficiency prior to IF), cases of overt hypothyroidism were prospectively identified from 1997 to 2015 by applying a diagnostic algorithm to all thyroid function testing performed in the area. Incident cases were verified by contacting the requesting physician.

Results: Standardized incidence rate (SIR) of hypothyroidism was $32.9 / 100.000 /$ year at baseline (1997-1998). SIR increased markedly during the first years of mandatory IF and peaked 40\% above baseline in 2001-2002 (SIRR to baseline: $1.40 ; 95 \%$ CI: $1.15-1.72$ ). The Incidence rate remained significantly elevated at around $40 \%$ above baseline level forward to the years 2005-2006. In 2007-2008 a slight decline was observed rendering the SIR non-significantly elevated at around $20 \%$ above baseline. The SIR became stable at this level for the remainder of the study period ending in 2011-2012 (SIRR to baseline $1.20 ; 95 \%$ CI: $0.96-1.49$ ). The increase was present among all age groups and both genders though most markedly for the younger age group and among men. The decline observed from 2007 and beyond was cause by a decline in incidence rate among the elderly with no such decline observed among the young.

Conclusion: The cautious initiation of the IF program led to an insignificant overall $20 \%$ increase in hypothyroidism incidence after $14-15$ years with a relatively greater increase among men.

\section{OP-12-87 \\ RELATIONSHIP BETWEEN AUTONOMIC NERVOUS FUNCTION AND CIRCADIAN BLOOD PRESSURE RHYTHM IN PATIENTS WITH HYPOTHYROIDISM}

Gen Yasuda ${ }^{1}$, Sanae Saka ${ }^{1}$, Akira Fujiwara ${ }^{1}$, Nobuhito Hirawa ${ }^{1}$

${ }^{1}$ Yokohama City University Medical Center, Yokohama, Japan

Objectives: Interactions between thyroid functions and autonomic nervous activities have not yet fully been understood. Patients with hypothyroidism frequently show the loss of nocturnal blood pressure (BP) fall and impaired autonomic nervous function. However, it is unknown that how autonomic nervous dysfunctions influence circadian BP rhythms in patients with hypothyroidism. We analyzed 24-hour BP in such patients using ambulatory BP monitoring devices. Power spectral analysis of the heart rate was performed for the high frequency (HF) components and low frequency (LF) components, as an index of the parasympathetic nervous activity and the sympathovagal balance, respectively.

Methods: Patients with hypothyroidism (hypothyroidism group; $\mathrm{n}=24$; men/women, 10/14; $49 \pm 9$ years old), who showed thyroid stimulating hormone $(\mathrm{TSH})>5 \mu \mathrm{IU} / \mathrm{mL}, 2.5 \mathrm{pg} / \mathrm{mL}>$ free triiodothyronine, and $0.9 \mathrm{ng} / \mathrm{dL}>$ free thyroxine, participated in the study. Age- and sex-matched healthy subjects (control group; $\mathrm{n}=25$; men/women, $11 / 14 ; 48 \pm 16$ years old), were also examined. Sleeping/waking BP, HF and LF ratios were calculated to evaluate the circadian BP rhythm and autonomic nervous activities.

Results: Mean waking systolic/diastolic BP were $131 \pm 17$ (SD)/76 \pm 10 $\mathrm{mmHg}$ in the hypothyroidism group, which were lower $(\mathrm{p}<0.05)$ than $137 \pm 18 / 81 \pm 8 \mathrm{mmHg}$ in the control group. There were no differences $(\mathrm{p}>0.05)$ in sleeping BP between the hypothyroidism group $(120 \pm 19 / 72 \pm 10 \mathrm{mmHg})$ and the control group $(119 \pm 14 / 69 \pm 8 \mathrm{mmHg})$. The ratios of sleeping/waking 
systolic and diastolic BP in the hypothyroidism group were $0.91 \pm 0.56$ and $0.93 \pm 0.44$, which were higher $(\mathrm{P}<0.05)$ than $0.86 \pm 0.41$ and $0.84 \pm 0.49$ in the control group. The ratios of sleeping/waking HF components were $0.86 \pm 0.33$ in the hypothyroidism group, which were higher $(\mathrm{P}=0.03)$ than $0.72 \pm 0.29$ in the control group. The ratios of sleeping/waking LF components in the hypothyroidism group showed $1.21 \pm 0.56$, which were lower $(\mathrm{p}=0.04)$ than $1.54 \pm 1.44$ in the control group.

Conclusions: These findings indicate the close relationship between the loss of nocturnal BP fall and imbalance of 24-hour autonomic nervous activities in patients with hypothyroidism.

\section{OP-12-88 \\ THE HYPOTHYROID STATE IS ASSOCIATED WITH A REDUCED BRAIN VOLUME}

Till Ittermann ${ }^{1}$, Wittfeld Katharina ${ }^{2}$, Grabe Hans ${ }^{2}$, Henry Völzke ${ }^{3}$

${ }^{1}$ University Medicine Greifswald, Institut für Community Medicine Ship/Klinisch-Epidemiologische Forschung, Greifswald, Germany,

${ }^{2}$ Greifswald, Germany, ${ }^{3}$ Ernst-Moritz-Arndt Universität Greifswald, Institut Für Community Medicine, Ship/Klinisch-Epidemiologische

Forschung, Greifswald, Germany

Background: Previous studies suggest that thyroid dysfunction affect volumes of particular regions of the brain. So far population-based data on this topic is lacking. Thus, our aim was to investigate associations of serum levels of thyroid-stimulating hormone (TSH), free triiodothyronine (fT3) and free thyroxine (fT4) with total brain volume (TBV), grey matter volume (GMV), white matter volume (WMV) and hippocampal volume (HV) in a populationbased study.

Material and Methods: We pooled data of 2557 individuals from two independent population-based surveys of the Study of Health in Pomerania conducted in Northeast Germany. Brain volumes were determined from images derived from 1.5T magnetic resonance imaging by the SPM8 and VBM8 toolboxes for GM and WM and by FreeSurfer 5.1 for HV. TBV was calculated as GM + WM. High and low TSH were defined using the cut-offs $0.40 \mathrm{mIU} / \mathrm{L}$ and $3.29 \mathrm{mIU} / \mathrm{L}$. Associations between thyroid hormone tests and brain volumes were analysed by linear regression models and by voxel-based morphometry (VBM) both adjusted for confounders.

Results: Individuals with high TSH had significantly lower TBV (â = $-26.8 ; 95 \%$ confidence interval $[\mathrm{CI}]=-48.1 ;-5.4)$, WMV $(\hat{\mathrm{a}}=-18.2 ; 95 \%$-CI $=-31.7 ;-4.8)$ and $\mathrm{HV}(\hat{\mathrm{a}}=-222.7 ; 95 \%-\mathrm{CI}=-395.4 ;-50.1)$ than individuals with normal TSH, while low TSH was not significantly associated with any of the brain volumes. We observed no significant associations of serum fT3 or fT4 levels with any of the brain volumes. Likewise we detected no significant associations in VBM analyses after correction for multiple testing.

Conclusion: The results of our study indicate that the hypothyroid state may lead to a reduced brain volume affecting particularly WMV and HV. Longitudinal studies are needed to verify our findings.

\section{OP-12-89 \\ EXCESS MORTALITY IN TREATED AND UNTREATED HYPERTHYROIDISM IS RELATED TO CUMULATIVE PERIODS OF LOW TSH}

Bo Abrahamsen ${ }^{1}$, Mads Lillevang-Johansen ${ }^{2}$, Henrik Løvendahl Jørgensen ${ }^{3}$, Thomas Brix ${ }^{4}$, Laszlo Hegedüs ${ }^{5}$

${ }^{1}$ Holbæk Hospital, Department of Medicine, Open, University of Southern Denmark, Odense, Holbæk, Denmark, ${ }^{2}$ Department of Endocrinology, Odense University Hospital, University of Southern Denmark, Odense C, Denmark, ${ }^{3}$ Department of Clinical Biochemistry, Bispebjerg Hospital, Copenhagen, ${ }^{4}$ Department of Endocirnology, Odense University Hospital, Odense University Hospital, Odense C, Denmark, ${ }^{5}$ Department of Endocrinology and Metabolism, Odense University Hospital, Odense, Denmark

Objectives: Excess mortality in hyperthyroid patients has been suggested in prior studies. However, the effect of anti-thyroid treatment on mortality, especially in subclinical hyperthyroidism, remains unclarified. We investigated the association between biochemically verified hyperthyroidism and mortality in both treated and untreated hyperthyroid individuals.

Patients and Methods: Register- and population-based cohort study of 239,768 individuals who had at least one serum-TSH measurement in the period 1995-2011 (median follow-up 7.3 years). Hyperthyroidism was defined as at least 2 measurements of low serum TSH within a period of 6 months with an interval of at least 14 days. Mortality rates for treated and untreated hyperthyroid subjects compared to euthyroid controls were calculated using multivariate Cox-regression analyses, adjusting for age, sex and comorbidities. Cumulative time with low serum TSH was analyzed as a time-dependent covariate.

Results: Hazard ratio (HR) for mortality was increased in untreated [1.23 (95\% CI: $1.12-1.37 ; \mathrm{p}<0.001)$ ], but not in treated hyperthyroid patients. When including cumulative periods of TSH in the Cox-regression analyses, HR for mortality per every 6 months of decreased TSH was 1.11 (95\% CI: 1.09 $1.13 ; \mathrm{p}<0.0001)$ in untreated hyperthyroid patients $(\mathrm{n}=1137)$, and $1.13(95 \%$ CI: $1.11-1.15 ; \mathrm{p}<0.0001)$ in treated patients $(\mathrm{n}=1656)$. This corresponds to a $184 \%$ and a $239 \%$ increase in mortality after 5 years of decreased TSH in untreated and treated hyperthyroidism, respectively. When subdividing with regard to disease severity, mortality was increased in both overt and subclinical hyperthyroidism.

Conclusion: Mortality is increased in untreated hyperthyroidism, irrespective of age and severity of disease. Cumulative periods of decreased TSH increased mortality in both treated and untreated hyperthyroidism, implying that excess mortality may not be driven by lack of therapy, but rather inability to keep patients euthyroid. Meticulous follow-up during treatment, in order to maintain biochemical euthyroidism, may be warranted.

\section{Oral Session 13 (Basic): Actions of Thyroid Hormone and its Metabolites}

\section{OP-13-90 \\ EFFECTS OF 3,5-DIIODO-L-THYRONINE ON THE CARDIAC TISSUE}

Minh Nguyen ${ }^{1}$, Leonardo Lorenzini ${ }^{2}$, Ginevra Sacripanti', Sabina

Frascarelli ${ }^{1}$, Alessandro Saba ${ }^{1}$, Riccardo Zucchi ${ }^{3}$, Sandra Ghelardoni ${ }^{1}$

${ }^{1}$ University of Pisa, Pisa, Italy, ${ }^{2}$ Dipartimento DI Patologia Chirurgica,

Pisa, Italy, ${ }^{3}$ University of Pisa, Dept. of Pathology, Pisa, Italy

Background: 3,5-diiodo-L-thyronine (T2) is an endogenous derivative of thyroid hormone. Its physiological role is unclear, but it has been suggested to regulate energy expenditure, resting metabolic rate and oxygen consumption. Thyromimetic effects on the myocardial tissue have also been reported. In this study we evaluate $\mathrm{T} 2$ cardiac effects using both in vitro and ex-vivo models.

Methods: To investigate T2 effects on cellular metabolism, MTT test and glucose consumption assay were performed on cultured rat cardiomyoblast (H9c2) cells. T2 cellular uptake was also evaluated using High Performance Liquid Chromatography- tandem Mass Spectrometry (HPLC-MS/MS). To assess cardiac functional effects, isolated working rat hearts were perfused with T2 $(0.1-10 \mathrm{iM})$ using glucose as energy source and hemodynamic parameters were evaluated for $40 \mathrm{~min}$.

Results: MTT test results showed that T2 $(5 \mathrm{nM}-10 \mu \mathrm{M})$ induced a significant increase in cell metabolism $(\mathrm{p}<0.0001)$. Glucose consumption was also significantly affected $(\mathrm{p}<0.01)$ since we observed an increase in the range of $15 \%(100 \mathrm{nM})$ to $18 \%(1 \mathrm{iM})$ compared to control group. HPLC-MS/MS results showed that in the incubation medium T2 $(100 \mathrm{nM}$ or $1 \mathrm{iM})$ concentration remained nearly constant over time while in cell lysate $\mathrm{T} 2$ increased, reaching a steady state after about $60 \mathrm{~min}(0.5 \mathrm{nM}$, with T2 $100 \mathrm{nM})$ or $240 \mathrm{~min}(15 \mathrm{nM}$ with T2 1iM) with a recovery of about $90 \%$. Notably, T2 did not produce any significant change in cardiac output nor in heart rate.

Conclusions: Our findings indicate that $\mathrm{T} 2$ is taken up by cardiomyoblasts and it may modulate cardiac energy metabolism, increasing glucose consumption without affecting the contractile performance. 


\section{OP-13-91 \\ INDUCTION OF HYPOTHYROIDISM AND INFLAMMATION IN A NEW ANIMAL MODEL OVEREXPRESSING INTERLEUKIN-4 IN THE THYROID}

Eskalli Zineb ${ }^{1}$, Miot Françoise ${ }^{1}$, Dumont J.E. ${ }^{1}$, Xavier De Deken ${ }^{1}$

${ }^{1}$ Universite Libre de Bruxelles - Ulb; Iribhm, Brussels, Belgium

Although the role of IL-4 in autoimmune thyroid diseases (AITD) has been studied extensively, the effects of IL-4 on thyroid physiology remain largely unknown. Transgenic mice with thyroid-targeted expression of murine IL-4 (Thyr-IL-4) were generated. Thyr-IL-4 animals had T4 and TSH serum levels in the normal range. However, the Nis gene was downregulated resulting in impaired iodide uptake. RNA profiling of Thyr-IL-4 thyroids revealed modulation of genes involved in inflammation, while no major leukocyte infiltration could be detected. In the present study, we characterized the thyroid phenotype of these transgenic mice after goitrogen treatment or immunization against the human TSH receptor (hTSHr).

Ten-month-old male transgenic animals treated with low iodine diet (LID) $+1 \%$ perchlorate showed an accelerated increase of serum TSH level, already after 7 days. But, the difference between Thyr-IL-4 and WT mice was very weak even after 3 weeks of treatment $(50.4 \pm 1.6$ vs $44.7 \pm 2.2$ pmoles cAMP $/ 50,000$ cells). Young animals were fed with LID only just after weaning. Before the treatment, 4-week-old Thyr-IL-4 males presented a weak, but significant increase of basal TSH levels that were augmented after 4 and 6 weeks on LID compared to WT animals. TSH levels were also significantly increased in transgenic female mice after 6 weeks on LID compared to WT females. Thyroids showed histological characteristics of the goitrogen treatment and expression of Nis and Tpo mRNA were also induced confirming the thyrotropin action. However, Nis mRNA expression remained lower in Thr-IL-4 mice than in WT. After genetic immunization against the hTSHr, $50 \%$ of WT animals developed anti-hTSHr antibodies compared to $64 \%$ of Thyr-IL-4 mice. Thyroid histological inspection showed in some immunized mice an alteration of the thyroid morphology with a loss of the follicular structure, but maintaining T4 staining. No increase of serum T4 could be detected either in WT or transgenic mice after immunization. However, increased CD45 staining was observed in immunized Thyr-IL-4 mice compared to WT animals.

We have demonstrated that Thyr-IL-4 mice are more susceptible to hypothyroidism induced by low iodine diet, most probably caused by the low level of Nis expression. Thyroid overexpression of the IL-4 cytokine seems to potentiate thyroid leukocyte infiltration after mouse immunization against the hTSH receptor. These data suggest that Thyr-IL-4 mice could be used to investigate the role of IL-4 in AITD when appropriate immunization protocols are used and after crossing with other AITD permissive mice.

\section{OP-13-92 \\ ASSAY OF ENDOGENOUS 3,5-DIIODO-L- THYRONINE (3,5-T2) AND 3,3'-DIIODO-L- THYRONINE $\left(3,3^{\prime}-\right.$ T2 $^{\prime}$ IN HUMAN SERUM}

Leonardo Lorenzini ${ }^{1}$, Minh Nguyen ${ }^{2}$, Ginevra Sacripanti ${ }^{2}$, Enrico Serni ${ }^{2}$, Alessandro Saba ${ }^{2}$, Sandra Ghelardoni ${ }^{2}$, Riccardo Zucchi ${ }^{3}$

${ }^{1}$ Dipartimento DI Patologia Chirurgica, Pisa, Italy, ${ }^{2}$ University of Pisa,

Pisa, Italy, ${ }^{3}$ University of Pisa, Dept. of Pathology, Pisa, Italy

Background: 3,5-diiodo-L-thyronine (3,5-T2) is an endogenous derivative of thyroid hormone with potential metabolic effects. It has been occasionally detect in human blood using High Performance Liquid Chromatography- tandem Mass Spectrometry (HPLC-MS/MS), but the results have been quite variable and quality control data were often missing. In this study we used an ad-hoc optimized Solid Phase Extraction (SPE)-based extraction method which allowed us to quantitate 3,5-T2 and its isomer 3,3'-T2 in human serum.

Methods: Serum samples were obtained from 28 patients $(8$ healthy volunteers and 20 women undergoing endocrinological screening and found to be euthyroid). Two $\mathrm{ml}$ of serum were deproteinized with acetonitrile and then extracted using an SPE based process. To lower background noise, after extraction the samples were furtherly cleaned by hexane washing and acetonitrile precipitation of residual proteins. 3,5-T2 and 3,3'-T2 were analyzed by a Sciex API4000 mass spectrometer coupled to an Agilent Infinity 1290 LC system using isotope dilution method. In 20 patients total $\mathrm{T} 3$ and $\mathrm{T} 4$ were also assayed by HPLC-MS/MS.

Results: Accuracy and precision for 3,5-T2 assay were $88-104 \%$ and $95-97 \%$, respectively. Recovery, matrix effect and process efficiency averaged $78 \%, 108 \%$, and $84 \%$ respectively. 3,5-T2 was detected in all samples and its concentration averaged (mean \pm SEM) $41 \pm 5 \mathrm{pg} / \mathrm{ml}$, i.e. $78 \pm 9 \mathrm{pmol} / \mathrm{l}$. In the same samples the concentration of 3,3'-T2 averaged $133 \pm 15 \mathrm{pg} / \mathrm{ml}$, i.e. $253 \pm 29 \mathrm{pmol} / 1.3,5-\mathrm{T} 2$ concentration was significantly related to $3,3^{\prime}-\mathrm{T} 2$ concentration $(\mathrm{r}=0.540, \mathrm{P}<0.01)$, while no significant correlation was observed with either T3 or T4 in the subset of patients in which these hormones were assayed.

Conclusion: Our optimized extraction method is able to quantify 3,5-T2 and 3,3'-T2 in human serum. Their concentrations are in the subnanomolar range, and a significant correlation was detected between these two metabolites in healthy individuals.

\section{OP-13-93}

\section{A NOVEL ROLE FOR THE HIPPO PATHWAY IN THYROID DIFFERENTIATION}

\section{Celia Fernández Méndez ${ }^{1}$, Pilar Santisteban ${ }^{2}$}

${ }^{1}$ Instituto de Investigaciones Biomédicas (Csic-Uam), Ciberonc, Instituto de Salud Carlos III, Madrid, Spain, ${ }^{2}$ Instituto de Investigaciones Biomédicas, Universidad Autónoma de Madrid, Ciberonc, Instituto de Salud Carlos III, Madrid, Spain

The Hippo signalling pathway plays key role in the control of cell proliferation and its deregulation has been associated with tumourigenesis. However, little is known about the regulatory mechanisms of the Hippo pathway in the thyroid gland. TAZ, a transcriptional coactivator in Hippo signalling, has been described to interact with Pax8 and Nkx2.1, key drivers of thyroid differentiation. The aim of this work was to study the role of the Hippo pathway and its mediator TAZ in thyroid differentiation.

We assessed the levels and the location of Hippo pathway components in thyroid follicular cells by qRT-PCR, western blotting and Immunofluorescence. We observed that TSH activated Hippo signalling, leading to TAZ translocation from the nucleus to the cytoplasm. TAZ has been described to act as coactivator of Pax8 on the thyroglobulin promoter. Since Pax8 is also the main positive regulator of the sodium iodide symporter (NIS), we evaluated the involvement of TAZ in NIS regulation using luciferase reporter assays in HeLa cells. Surprisingly, we found that TAZ repressed Pax8 transcriptional activation of the NIS promoter. Pax8 has also been reported to interact with Smad3, triggering NIS transcriptional repression mediated by TGFâ, and we found that treatment with this cytokine further promoted TAZ nuclear localization, thus reinforcing this interaction. Also, TAZ silencing by RNAi in thyroid follicular cells strongly increased NIS protein and mRNA levels, partially impairing TGFâ-induced NIS repression. According to the above data, human thyroid tumor-derived cells, which are known to lose NIS expression and overproduce TGFâ, present increased levels of active TAZ.

In conclusion, these data establish a novel role of the Hippo pathway, and particularly the cofactor TAZ, in the regulation of NIS expression in thyroid cells, repressing Pax8 activity and modulating thyroid differentiation. 


\section{OP.13-94 \\ 3,5 DIIODO-L-THYRONINE (T2) TARGETS DIFFERENT PATHWAYS TO INDUCE WHITE ADIPOSE TISSUE BROWNING}

Rosalba Senese ${ }^{1}$, Federica Cioffi ${ }^{2}$, Pieter de Lange ${ }^{3}$, Cristina Leanza ${ }^{4}$, Liliana Felicia lannucci ${ }^{4}$, Fernando Goglia ${ }^{5}$, Antonia Lanni ${ }^{1}$

${ }^{1}$ Università Degli Studi Della Campania "Luigi Vanvitelli", Caserta, Italy, ${ }^{2}$ University of Sannio, Department of Science e Technology,

Beneveto, Italy, ${ }^{3}$ Università Degli Studi Della Campani, Department of Environmental, Biologi, Caserta, Italy, ${ }^{4}$ Caserta, Italy, ${ }^{5}$ Università Degli Studi del Sannio, Benevento, Italy

Objectives: The conversion of fat-accumulating white adipose cells into energy-dissipating brown adipose cells is known as browning. Many molecular factors involved in this processes have been identified, such as, among the others, miRNA and irisin. As it has been shown that the 3,5-diiodo-L-thyronine (T2), an endogenous metabolite of thyroid hormones, stimulates energy expenditure, the aim of this study is to verify whether $\mathrm{T} 2$ could induce browning and to investigate the underlying mechanism.

Methods: Three groups of rats were used: i) receiving a standard diet (N); ii) receiving a high-fat diet (HFD) for 14 weeks, and iii) receiving a HFD for 10 weeks and subsequently treated for 4 weeks contemporary with HFD and T2 (50ig/100g BW) (HFD-T2). miRNA were extracted from subcutaneous white adipose tissue (SAT) and their expression was quantified by RT-qPCR. Serum irisin was assayed by ELISA. Protein levels were detected by Western Blotting.

Results: In SAT of HFD-T2 rat, we observed an increase of the uncoupling protein 1 expression and a modulation of the expression of miR-133a and miR196a, when compared to N and HFD animals. Associated to reduction of miR-133a there was an increase of Prdm16 expression, a critical regulator of brown adipocyte development, and associated to an increase to miR-196a there was a reduction of Hoxc8, a repressor of adipogenic marker C/EBPâ, which resulted increased. Furthermore, T2 increased serum levels of irisin, a myokine acting via the stimulation of ERK pathway and that regulates positively white-to-brown adipocytes conversion.

Conclusion: The present data demonstrate that $\mathrm{T} 2$ is able significantly to affect important pathways underlying the browning processes such as, among others, miR133a, miR196a and irisin. These effects may constitute part of the mechanism by which T2 exerts the well-known stimulatory effect on metabolism.

\section{OP-13-95 \\ THE OBESITY-RESISTANT WSB/EIJ MOUSE MAINTAINS METABOLIC HOMEOSTASIS DURING AGING DESPITE LOW CIRCULATING THYROID HORMONE LEVELS}

Marie-Stéphanie Clerget-Froidevaux ${ }^{1}$, Jeremy Terrien ${ }^{2}$, Stéphanie

Decherf ${ }^{3}$, James Bowers ${ }^{3}$, Isabelle Seugnet ${ }^{3}$, Raphaël Denis ${ }^{4}$, Serge

Luquet $^{4}$, Emmely de Vries ${ }^{5}$, Chantal Zevenbergen ${ }^{6}$, Theo Visser ${ }^{7}$, Anita

Boelen $^{8}$, Barbara Demeneix ${ }^{9}$

${ }^{1} \mathrm{Mnhn} / \mathrm{Cnrs}$ Umr7221, Paris, France, ${ }^{2}$ Team Bioadapt Mnhn/ Cnrs

Umr 7179, Brunoy, France, ${ }^{3} \mathrm{Mnhn} / \mathrm{Cnrs}$ Umr 7221, Paris, France,

${ }^{4}$ Team C2offee, Unit of Functional and Adaptive Biology (Bfa), Cnrs Umr 8251, Université Paris Diderot, Sorbonne Paris Cité, Paris,

France, ${ }^{5}$ Academic Medical Center, Departement of Endocrinology and Metabolism, Amsterdam, Netherlands, ${ }^{6}$ Erasmus University

Medical Center, Rotterdam, The Netherlands, Internal Medicine,

Rotterdam, Netherlands, ${ }^{7}$ Erasmus University Medical Center, Dept

Internal Medicine, Rotterdam, Netherlands, ${ }^{8}$ Academic Medical Centre, Amsterdam, Netherlands, ${ }^{9}$ Umr7221 Cnrs / Mnhn, Paris, France

Previous studies have demonstrated that small mammals with lower circulating thyroid hormones (TH) exhibit a longer lifespan. This observation is echoed by studies in humans, whereby nonagenarians and their offspring have lower circulating total thyroxin (tT4) and thyroid-stimulating hormone (TSH) concentrations. The wild-derived mouse strain, WSB/EiJ, is long-lived and although euthyroid, has lower than average serum tT4. Furthermore, these mice are resistant to diet-induced obesity (DIO). Given the role of TH in metabolism, we tested the hypothesis that these phenotypic characteristics are inter-dependent. Endocrine and metabolic responses were measured in 3-month and 18-month old male C57BL/6J and WSB/EiJ mice on different diets: control (10\% Kcal from fat), short-term (3-day) or long-term (8-week) high fat diet (HFD) (45\% Kcal from fat). Data on body weight, glucose tolerance and hormone profiles during the 8-week HFD treatment confirmed resistance to DIO despite lower circulating thyroid hormone levels in WSB/EiJ compared to $\mathrm{C} 57 \mathrm{BL} / 6 \mathrm{~J}$ mice, and this was maintained with age. Indirect calorimetry showed that control fed WSB/EiJ mice displayed a strong circadian substrate-switching capacity that was dampened but maintained, under HFD. Moreover, the WSB/EiJ brown adipose tissue displayed a greater thermogenic capacity, with a higher BAT temperature when compared to C57BL/6J, correlated with an increased deiodinase 2 activity under HFD. All these metabolic features were conserved in aged WSB/EiJ mice.

Thus, the higher metabolism in WSB/EiJ mice, even under HFD, induces a 'fasting' message (lower leptin) at the level of the hypothalamus, resulting in a constant low level of circulating THs, even in aged mice. However, knowing the role played by $\mathrm{TH}$ in energy expenditure, there is a discrepancy between the WSB/EiJ low circulating THs and their high levels of metabolism. Local regulation of TH (centrally or peripherally) is required to bypass the low circulating levels. Current research is addressing this question.

\section{OP-13-96}

\section{A SENSITIVE LC-MS/MS METHOD} FOR THE DETERMINATION OF TRACE DI-IODOTHYRONINES IN HUMAN BLOOD

\section{SERUM}

Richards Keith H. ${ }^{1}$, Rathmann Daniel', Raymond Monk ${ }^{1}$, Josef Köhrle ${ }^{2}$

${ }^{1}$ Berlin, ${ }^{2}$ Institut fuer Experimentelle Endokrinologie \&

Endokrinologisches Forschungs-Centrum EnForCé, Charité-

Universitätsmedizin Berlin, Germany

There has been much recent interest in the thyroid hormone metabolites (THM) 3,5-diiodothyronine (3,5-T2) and 3- iodothyronamine 3-T1AM. Although there have been reports of the determination of endogenous 3-T1AM in human blood by isotope dilution liquid chromatography electrospray tandem mass spectrometry (ID-LC-MS/MS), most of these did not include quantification or were even unable to detect this THM endogenously The same issue holds true for 3,5-T2. Although 3,5-T2 has been quantified in human blood serum at endogenous concentrations using chemiluminesence immunoassay (CLIA), until now there have been no confirmatory reports of the endogenous detection/quantification of this T2 isomer by LC-MS/MS while 3,3'T2 has been determined in human blood samples with correlations to clinical conditions We report here an ID-LC-MS/MS method that incorporates a third dimension of MS (ID-LC-MS/MS/MS or ID-LC-MS3) using a linear ion trap facility to unambiguously detect and quantify endogenousT2 isomers (both 3,5- and 3,3'-T2) in human blood serum. MS3 displays a clear advantage over traditional tandem MS for determining analyte concentrations close to method detection limits. The technique delivers an extra dimension of analyte selectivity with significantly reduced chemical noise, ensuring the unambiguous identification and quantification of trace compounds in complex biological matrices. The method was validated for linearity, limits of quantification and detection (LLOQ and LLOD respectively), accuracy, precision, process efficiency (PE), matrix effect (ME) and relative recovery (RE). We applied our method to the analysis of blood sera from 15 healthy individuals. Concentrations of 3,5-T2 were close to the LLOQ of the method, in the low picomolar range, significantly below those reported by CLIA; 3,3'-T2 concentrations were higher, in the range $25-100$ picomolar, indicating that in healthy individuals 3,5-T2 is a minor metabolite derived from T3, compared to 3,3'$\mathrm{T} 2$ which may originate from both $\mathrm{T} 3$ and $\mathrm{rT} 3$. 


\section{OP-13-97}

THE DEGREE OF FATTY ACID SATURATION

INFLUENCES THE EFFECTS OF T3 AND 3,5-T2

ON INSULIN SENSITIVITY IN MUSCLE CELLS

Antonia Giacco ${ }^{1}$, Giuseppe delli Paoli ${ }^{2}$, Elena Silvestri ${ }^{3}$, Rosalba

Senese ${ }^{4}$, Federica Cioff ${ }^{5}$, Maria Moreno ${ }^{6}$, Antonia Lanni ${ }^{4}$, Fernando

Goglia ${ }^{7}$, Pieter de Lange ${ }^{8}$

${ }^{1}$ Benevento, Italy, ${ }^{2}$ Caserta, Italy, ${ }^{3}$ University of Sannio, Benevento,

Dep. Science and Technology, Benevento, Italy, ${ }^{4}$ Università Degli Studi

Della Campania “Luigi Vanvitelli", Caserta, Italy, ${ }^{5}$ University of Sannio,

Department of Science e Technology, Beneveto, Italy, ${ }^{6}$ Universitá

Sannio, Benevento, Italy, ${ }^{7}$ Università Degli Studi del Sannio,

Benevento, Italy, ${ }^{8}$ Università Degli Studi Della Campani, Department of

Environmental, Biologi, Caserta, Italy

Objectives: $\mathrm{T} 3$ and its metabolite 3,5-T2 both increase skeletal muscle insulin sensitivity but is unknown if they act directly on muscle or only indirectly, preventing muscular fatty acid (FA) uptake through increased hepatic FA oxidation. Furthermore, the metabolic effects of thyroid hormones in animal models vary with the diet's FA composition or the animal model. To clarify these issues, we studied whether T3 and 3,5-T2 prevent insulin resistance in myotubes induced by FAs with different saturation degrees.
Methods: Rat L6 myotubes were treated with palmitate, oleate or linoleate and $100 \mathrm{nM} 3,5-\mathrm{T} 2$ or T3. Cells treated with 0.5 or $0.75 \mathrm{mM}$ FAs were lysed for RNA isolation after $16 \mathrm{~h}$, or serum-starved for $2 \mathrm{~h}$, with a subset being treated with insulin $(20 \mathrm{nM})$ for $15 \mathrm{~min}$. Lysates were assayed for protein kinase B (PKB)/Akt and AMP-activated protein kinase (AMPK) phosphorylation, and for caspase 3 cleaveage to assess apoptosis. Gene expression analysis of relevant genes was performed using real-time PCR.

Results: In the presence of insulin, reduced Akt phosphorylation in response to palmitate was fully abolished by 3,5-T2 conferming data obtained in skeletal muscle of rats treated with diets based on saturated FAs, and only partially by T3. Oleate- mediated enhanced insulin sensitivity was normalized by 3,5-T2 and T3, instead linoleate-mediated enhanced insulin sensitivity was further enhanced by 3,5-T2 and T3. AMPK phosphorylation inversely correlated with increased FA saturation, and T3 further increased AMPK phosphorylation in presence of oleate. PGC-1alpha expression reflected insulin-induced Akt phosphorylation caused by the FAs and thyroid hormones. Caspase 3 cleaveage was significantly enhanced only by $\mathrm{T} 3$.

Conclusions: These results show that 3,5-T2 and T3 can act directly on muscle cells by differentially modulating FA- induced insulin resistance, and allow to predict that dietary backgrounds profoundly alter the effects of thyroid hormones on muscle insulin sensitivity in vivo. 


\section{Poster Plan}

\section{European}

Thyroid Journal

Saturday, 9th September, 16.00-17.00

\begin{tabular}{|l|l|l|l|}
\hline Topic & Posters & Room & Information \\
\hline Autoimmunity 1 & P1-01-01-P1-01-10 & Room Aegean & P1-01-09 withdrawn \\
\hline Cancer Diagnosis & P1-02-11-P1-02-21 & Room Mediterranean & \\
\hline Cancer Prognostic Factors & P1-03-22-P1-03-27 & Room Tisa & P1-04-28 withdrawn \\
\hline Goitre and Nodules 1 & P1-04-28-P1-04-37 & Room Adriatic & \\
\hline $\begin{array}{l}\text { Hyper- and Hypothyroidism } \\
\text { Molecular Mechanisms of Thyroid }\end{array}$ & P1-05-38-P1-05-47 & Room Baltic & Room Danube \\
Hormone Action & P1-06-48-P1-06-54 & &
\end{tabular}

Sunday, 10th September, 12.00-13.00

\begin{tabular}{|l|l|l|l|}
\hline Topic & Posters & Room & Information \\
\hline Autoimmunity 2 & P2-01-55-P2-01-62 & Room Tisa & P2-02-67 is now \\
\hline Cancer Basic & P2-02-63-P2-02-72 & Room Danube & OP8 \\
\hline Cancer Miscellaneous & P2-03-73-P2-03-82 & Room Baltic & Room Mediterranean \\
\hline Case Reports & P2-04-83-P2-04-93 & Room Adriatic & \\
\hline Goitre and Nodules 2 & P2-05-94-P2-05-103 & Room Aegean & \\
\hline Hypothyroidism & P2-06-104-P2-06-113 & &
\end{tabular}

Monday, 11th September, 12.00-13.00

\begin{tabular}{|c|c|c|c|}
\hline Topic & Posters & Room & Information \\
\hline Autoimmunity 3 & P3-01-114 - P3-01-123 & Room Adriatic & \\
\hline Cancer Case Reports & P3-02-124 - P3-02-130 & Room Tisa & \\
\hline Cancer Treatment & P3-03-131 - P3-03-138 & Room Aegean & \\
\hline Case Reports, Goitre and Nodules 3 & P3-04-139 - P3-04-146 & Room Danube & \\
\hline Clinical Thyroidology & P3-05-147 - P3-05-157 & Room Mediterranean & \\
\hline lodine and Reproduction & P3-06-158 - P3-06-167 & Room Baltic & \\
\hline
\end{tabular}




\section{P1-01 Autoimmunity 1}

\section{P1-01-01 \\ USE OF LOW-DOSE RADIOIODINE ABLATION FOR GRAVES' ORBITOPATHY (GO): RESULTS OF A PROSPECTIVE CLINICAL STUDY}

Michele Marino ${ }^{1}$, Marenza Leo ${ }^{2}$, Elena Sabini ${ }^{3}$, Ilaria Ionni', barbara mazzi $^{5}$, Francesca Menconi $i^{5}$, Eleonora Molinaro ${ }^{6}$, Francesca Bianchi $i^{5}$, Federica Brozzi $i^{5}$, Pierina Santini ${ }^{2}$, Rossella Elisei ${ }^{7}$, Paolo Vitti ${ }^{8}$, Claudio Marcocci ${ }^{9}$

${ }^{1}$ University of Pisa, Endocrinology Unit, Pisa, Italy, ${ }^{2}$ Endocrinology Unit, University of Pisa, Pisa, Italy, ${ }^{3}$ Department of Clinical and Experimental Medicine, Endocrinology Units, University of Pisa and University

Hospital of Pisa, Pisa, Italy, ${ }^{4}$ Department Of Clinical And Experimental Medicine, Endocrinology, University of Pisa, Pisa, Italy, ${ }^{5}$ Endocrinology Unit, University of Pisa, Pisa, ${ }^{6}$ University of Pisa, Endocrinology Unit, Department of Endocrinology, Pisa, Italy, ${ }^{7}$ Endocrine Unit, Dept of Clin and Exp Medicine, University Pisa, Pisa, Italy, ${ }^{8}$ Endocrinology, Pisa, Italy, ${ }^{9}$ Department of Clinical and Experimen, University of Pisa, Pisa, Italy

Objective: Total thyroid ablation (TTA), namely thyroidectomy followed by radioiodine (RI), may be beneficial for GO. TTA is usually performed with $30 \mathrm{mCi}$ of 131-I, which, according to Italian legislation, must be followed by a $24-\mathrm{h}$ protected hospitalization, implying long waiting lists. A $15 \mathrm{mCi}$ radioiodine dose can instead be given without hospitalization and with much lower costs. GO patients usually have serum TSH-receptor stimulating antibodies (TRAb), therefore increasing RI uptake, possibly resulting in the need for a lower RI dose, which we investigated here.

Methods: 12 serum anti-thyroglobulin $(\mathrm{Tg})$ antibodies-negative thyroidectomized patients with Graves' hyperthyroidism and GO (8 women, 4 men, age $50.5 \pm 13 \mathrm{yr}$ ) were treated with either 15 or $30 \mathrm{mCi}$ of RI, given after 2 recombinant human TSH (rhTSH) injections. The primary outcome was rhTSH-stimulated Tg at 6 months.

Results: The 2 groups were similar at ablation for age, gender, TRAb, baseline Tg, TSH and Tg after rhTSH, 24-h RI uptake. 6 months after ablation baseline $\mathrm{Tg}$ was $0.1 \pm 0.1 \mathrm{ng} / \mathrm{ml}$ in the $15 \mathrm{mCi}$ and $0.3 \pm 0.6 \mathrm{ng} / \mathrm{ml}$ in the 30 $\mathrm{mCi}$ group, respectively, with no difference between the two groups $(\mathrm{P}=\mathrm{NS})$. rhTSH-stimulated Tg was also similar in the two groups $(15 \mathrm{mCi}: 0.0 \pm 0.0 \mathrm{ng} /$ $\mathrm{ml} ; 30 \mathrm{mCi} 0.5 \pm 1.0 \mathrm{ng} / \mathrm{ml} ; \mathrm{P}=\mathrm{NS}$ ), as were TRAb levels at 6 months.

Conclusions: A $15 \mathrm{mCi}$ RI dose seems to be as effective as a $30 \mathrm{mCi}$ dose in ablating patients with Graves' hyperthyroidism. Routine use of $15 \mathrm{mCi} \mathrm{RI}$ ablation in thyroidectomized patients with GO may therefore be used with remarkable reduction of the waiting time and of the social costs.

\section{P1-01-02 \\ THE EFFECT OF OBESITY AND DIETARY HABITS ON OXIDATIVE STRESS IN HASHIMOTO'S THYROIDITIS}

Maria Giannakou ${ }^{1}$, Katerina Saltiki ${ }^{2}$, Emily Mantzou ${ }^{3}$, Eleni Loukari ${ }^{3}$, Georgios Philippou ${ }^{3}$, Konstantinos Terzidis ${ }^{3}$, Charalampos Stavrianos ${ }^{3}$, Miltiades Kyprianou ${ }^{1}$, Theodora Psaltopoulou ${ }^{4}$, Maria Alevizaki ${ }^{2}$ ${ }^{1}$ Endocrine Unit, Dept Med Therapeutics Alexadra Hospital, Athens University School of Medicine, Athens, Greece, ${ }^{2}$ Endocrine Unit, Dept Med Therapeutics Alexadra Hospital, Athens University School of Medicine, Dept of Endocrinology and Metabolism, Athens University School of Medicine, Athens, Greece, ${ }^{3}$ Dept of Endocrinology and Metabolism, Athens University School of Medicine, Athens, Greece, ${ }^{4}$ Department of Hygiene, Epidemiology and Medical Statistics, School of Medicine, University of Athens, Athens, Greece

Objective - Aim: We have previously observed an association between oxidative stress and the presence and severity of Hashimoto's thyroiditis. We investigated whether oxidative stress is further influenced by obesity and dietary habits.

Methods: 218 consecutive euthyroid women (age 46.0 $\pm 12.7,19-69$ years) with Hashimoto's thyroiditis were studied and separated in two groups; 102 with $\mathrm{T}_{4}$ replacement (treatment group) and 114 without (notreatment group). For the evaluation of oxidative stress, total lipid peroxide levels in serum (TOS) were measured and recoded as high TOS $(\geq 590 \mu \mathrm{mol} / \mathrm{L})$ versus medium or low TOS $(<590 \mu \mathrm{mol} / \mathrm{L})$. The type of food and consumption frequency was recorded. Two binary variables were considered: normal fruit consumption (at least two servings per day) versus low fruit consumption (less than two servings) and daily versus sporadic vegetables consumption.

Results: High TOS was more frequent in the treatment group (31.4\%vs $14.7 \%$, OR $=2.795 \% \mathrm{CI}: 1.4-5.2)$. ROC curve analysis revealed that BMI had significant discrimination ability for high TOS levels $(\mathrm{AUC}=0.678$ 95\%CI: $0.590-0.765$ ) with the best cutoff value coinciding the one discriminating normal BMI values from overweight and obese $\left(25 \mathrm{Kg} / \mathrm{m}^{2}\right)$. Furthermore, the prevalence of high TOS levels was higher among overweight and obese women compared to women with normal BMI $(30.4 \%$ vs $10.5 \%$, OR=3.1 $95 \%$ CI: 1.5-6.4). Low fruit consumption was associated with increased high TOS prevalence (30.6\%vs $10.9 \%$ OR=3.0 95\% CI: 1.4-6.2). Sporadic vegetable consumption was associated with increased high TOS prevalence compared to daily consumption (29.9\%vs $13.5 \%$ OR=2.7 95\%CI: 1.3-5.7). Stepwise logistic regression proved that all four factors (treatment, BMI, fruit and vegetables consumption) were significant independent predictors of high TOS levels.

Conclusions: Oxidative stress is increased in women with Hashimoto's thyroiditis under treatment; nevertheless, behaviors such as maintaining a normal BMI and consuming fruit and vegetables all contribute in maintaining oxidative stress at low levels.

\section{KARGER (C) 2017 European Thyroid Association. Published by S. Karger AG}




\section{P1-01-03 \\ ASSOCIATION BETWEEN THE EFFICACY OF RADIOIODINE THERAPY AND THE OCCURENCE OF GRAVES‘ ORBITOPATHY AFTER RADIOIODINE}

Edvard Pirnat ${ }^{1}$, Daša Šfiligoj', Katja Zaletel', Polona Jaki Mekjavić2 Simona Gaberscek ${ }^{3}$

${ }^{1}$ Department of Nuclear Medicine, University Medical Centre Ljubljana, Ljubljana, Slovenia, ${ }^{2}$ Faculty of Medicine, University of Ljubljana,

University Eye Hospital, University Medical Centre Ljubljana, Ljubljana, Slovenia, ${ }^{3}$ Department of Nuclear Medicine, University Medical Centre Ljubljana, Faculty of Medicine, University of Ljubljana, Ljubljana,

Slovenia

Objectives: Graves' orbitopathy (GO) may occur or worsen after radioiodine (RAI) therapy of patients with Graves' disease (GD). The aim of our study was to retrospectively establish factors associated with the occurrence of GO after RAI in patients without GO before RAI.

Methods: We reviewed medical records of all patients who were first diagnosed with GD between 2005 and 2009 and later treated with RAI. Out of 724 patients, 552 patients ( 427 women and $125 \mathrm{men}$ ) did not have GO immediately before RAI. The state of GO was followed for 12 months after RAI therapy.

Results: The first dose of RAI was effective in 504 (91.3\%) GD patients, while the second and the third dose of RAI were required in $48(8.7 \%)$ and 3 $(0.5 \%)$ patients respectively. In the 12-months period after RAI, 529 (95.8\%) patients remained without GO, while GO occurred in $23(4.2 \%)$ patients. In patients treated with the second and third dose of RAI, GO occurred significantly more frequently than in patients treated with a single dose of RAI $(p=0.008$ and $p<0.001$, respectively). With respect to age, patients without GO after RAI did not differ from patients with GO after RAI (median, 46 and 43 years, respectively, $\mathrm{p}=0.113$ ). Furthermore, there were no statistically significant differences between patients without GO after RAI and patients with GO after RAI with respect to sex, smoking, duration of treatment with antithyroid drugs, and level of TSH receptor antibodies $(\mathrm{p}=0.261, \mathrm{p}=0.380, \mathrm{p}=0.379$, $\mathrm{p}=0.875$, respectively).

Conclusions: Our results reveal the importance of a sufficiently high first dose of RAI in patients with GD, since additional doses of RAI increase the likelihood of the occurrence of GO.

\section{P1-01-04 \\ Clinical performance Of A fully AUTOMATED ASSAY FOR THE MEASUREMENT OF ANTI-TSH-R AUTOANTIBODIES \\ Christian Konrad $^{1}$, Sigrid Sjölander ${ }^{2}$, Matthias Kast ${ }^{1}$ \\ ${ }^{1}$ Thermo Fisher Scientific, Freiburg, Germany, ${ }^{2}$ Thermo Fisher \\ Scientific, Uppsala, Sweden}

Background: Graves' disease (GD), a major cause of hyperthyroidism in humans, is characterized by the presence of autoantibodies against the thyroidstimulating hormone receptor (TSH-R). The detection of anti-TSH-R autoantibodies in patient samples represent a diagnostic criterion for this autoimmune disease. Immunoassays measuring anti-TSH-R autoantibodies aid in the diagnosis of GD.

Aim: In this study, the clinical performance of a fully automated competitive enzyme immunoassay for quantitative measurement of serum antibodies specific for TSH-R using human recombinant TSH-R as antigen and a thyroid stimulating monoclonal antibody as conjugate was evaluated.

Methods: A serum collective comprising 100 untreated and treated GD patients as well as 300 disease controls (100 Hashimoto's thyroiditis patients and 200 patients diagnosed with other autoimmune and non-autoimmune diseases) were analyzed using EliA $^{\mathrm{TM}}$ anti-TSH-R. For comparison, a fully automated anti-TSH-R assay from a different manufacturer was included in this study. Additionally, the agreement with a competitive radioimmunoassay (RIA) was determined.

Results: EliA anti-TSH-R found 79 GD patient samples positive (sensitivity $79 \%$ ) with a specificity of $98 \%$. Four GD samples were found within the equivocal range. The other manufacturer's assay had a sensitivity of $84 \%$ and a specificity of $94 \%$. Both assays had an agreement of $95 \%$ with the RIA, and the correlation coefficient between EliA anti-TSH-R and the RIA was $>$
0.9. The correlation coefficient between the RIA and the other manufacturer's assay was not determined as 20 of the positive GD patient samples and six samples of the disease controls showed values above the latter's measuring range.

Conclusions: The clinical performance of EliA anti-TSH-R was found to aid in the diagnosis of GD patients and its very high specificity allows a better differentiation from patients with other disorders. The lower level of false positives that need additional testing reduces the costs for the health care system.

\section{P1-01-05 \\ EFFECTS OF RITUXIMAB (RTX) AND INTRAVENOUS METHYLPREDNISOLONE (IVMP) ON PRO-INFLAMMATORY CITOKINES IN PATIENTS WITH ACTIVE GRAVES'ORBITOPATHY (GO)}

Guia Vannucchi $i^{1}$, Irene Campi ${ }^{2}$, Danila Covelli ${ }^{3}$, Poupak Fallahi', Nicola Curró ${ }^{5}$, Davide Dazzi ${ }^{6}$, Alessandro Antonelli ${ }^{4}$, Luca Persani ${ }^{7}$, Mario Salvi

11department of Endocrine and Metabolic Diseases, Irccs Istituto Auxologico Italiano, Milan, Irccs Istituto Auxologico Italiano, Milano, Italy, ${ }^{2}$ Irccs, Istituto Auxologico Italiano, Division of Endocrine and Metabolic Diseases, Department of Pathophysiology and Transplantation, University of Milan, Milan, Italy, ${ }^{3} 1$ graves' Orbitopathy Unit, Endocrinology, Fondazione Ca' Granda Irccs, University of Milan, Italy, Medical Sciences, Milano, Italy, ${ }^{4}$ University of Pisa, Department of Clinical and Experimental Medicine, Pisa, Italy, ${ }^{5}$ Ophthalmology, Fondazione Irccs Cà Granda, Milan, Italy, ${ }^{6}$ Internal Medicine, Ospedale DI Fidenza, Fidenza, Italy, ${ }^{7}$ University of Milan, Irccs Istituto Auxologico Italiano, Ospedale San Luca, Milan, Italy, ${ }^{8}$ Dipartimento Scienze Mediche, Endocrinologia, Endocrine Unit, Fondazione Irccs Cà Granda, Milano, Italy

In active GO, proinflammatory cytokines predominate. Circulating TSHreceptor antibodies (TRAb) have been correlated with GO clinical activity and severity. In a double blind, randomized, controlled study (EUDRACT 2007003910-33) in which patients with active moderate-severe GO were treated with either RTX $(n=16)$ or IVMP $(n=16)$, we measured serum TRAb levels, TNFalpha, CXCL-10, MCP-1, IL-10, IL-17 and IL-28A at baseline, 8 and 24 weeks after treatment. GO activity was assessed by the clinical activity score (CAS). We observed a progressive, significant decrease of serum TRAb levels from $18.1 \pm 21.6$ and $10.7 \pm 9.1$ to $17.5 \pm 24.3$ and $4.0 \pm 4.5$ at 24 weeks after IVMP and RTX ( $\mathrm{P}=0.05$ and $\mathrm{P}=0.005$, respectively). IL- 10 and IL- 17 did not significantly change in relation to treatment. Mean basal \pm SE serum CXCL10 concentrations were $178.2 \pm 22.4 \mathrm{pg} / \mathrm{ml}$ and significantly decreased to $75.4 \pm 18.6$ at 24 weeks after IVMP $(\mathrm{P}=0.001)$, but not after RTX, as we observed a slight, not significant, increase of CXCL-10 from $135.5 \pm 14.5 \mathrm{pg} / \mathrm{ml}$ to $139.1 \pm 12$ at 24 weeks $(\mathrm{P}=0.9)$. TNF $\alpha$ was significantly associated with CAS in IVMP patients and decreased with the reduction of CAS $(\mathrm{P}=0.008)$. Serum TRAb levels significantly correlated with IL-28A $(\mathrm{P}=0.007)$ in patients treated with IVMP and with MCP-1 in those of the RTX arm $(\mathrm{P}=0.005)$. In conclusion, patients with active GO have a different cytokine pattern whether they are treated with IVGC or RTX, probably due to the effects of RTX-induced B cell lysis, which interferes with the pro-inflammatory cytokine milieu.

\section{P1-01-06 \\ MONTH OR SEASON OF BIRTH IS NOT ASSOCIATED WITH THE DIAGNOSIS OF GRAVES' DISEASE. A NATIONWIDE COHORT STUDY \\ Frans Brandt Kristensen ${ }^{1}$, M. Thvilum ${ }^{2}$ S. Sivalingam ${ }^{1}$. Thomas Brix ${ }^{2}$, Laszlo Hegedüs ${ }^{2}$ \\ ${ }^{1}$ Hospital of Southern Jutland; Department of Internal Medicine, Sønderborg, Denmark, ${ }^{2}$ Odense University Hospital; Department of Endocrinology and Metabolism, Odense, Denmark}

Introduction: The etiology of Graves' disease (GD) remains an enigma. As for other autoimmune diseases, intricate interactions between genetic and environmental triggers are thought to be the culprit. Infections, viral and/or 
bacterial, with a seasonal overload and potential perinatal effects have been postulated to influence susceptibility to autoimmune disease later in life. Studies in diabetes mellitus have described higher birth incidence rates in the spring and the summer. Previous limited-scale studies in GD have been inconclusive.

Method: Nationwide, register-based, cohort study of 28,461 adult Danes diagnosed with GD between 1995 and 2012, each matched with four nonhyperthyroid control subjects for age and gender. The birth incidence rates for all four seasons and all twelve months in GD cases and controls were compared by a Chi2-test.

Results: Seasonal birth incidence rates in the GD and the control population are shown below:

\begin{tabular}{llll}
\hline Season of year & $\begin{array}{l}\text { Birth incidence rates } \\
\text { Graves' disease }\end{array}$ & $\begin{array}{l}\text { Birth incidence rates } \\
\text { Controls }\end{array}$ & P-value \\
\hline Spring & 0.265 & 0.269 & 0.111 \\
Summer & 0.252 & 0.249 & 0.357 \\
Autumn & 0.239 & 0.240 & 0.703 \\
Winter & 0.245 & 0.242 & 0.272 \\
\hline
\end{tabular}

Neither season (Table) nor month (data not shown) of birth differed significantly between cases and controls.

Conclusion: In a nationwide and large-scale investigation we were unable to associate, significantly, the month, or season, of birth with the subsequent development of Graves' disease.

\section{P1-01-07 \\ ASSOSIATION OF THE IL-6 AND IL-13 GENES POLYMORPHISMS WITH THE RELAPSE RATE OF GRAVES“ DESEASE IN RUSSIAN POPULATION \\ Natalia Peikrishvili ${ }^{1}$ Anna Volkova ${ }^{1}$, Svetlana Dora ${ }^{1}$ \\ ${ }^{1}$ Saint Petersburg, Russian Federation}

Objectives: Graves' disease (GD) is an organ-specific autoimmune disorder, which occurs due to loss of immune tolerance resulting in production of the thyroid stimulating hormone receptor antibody (TRAb). Relapse rate of GD estimates $\sim 70 \%$ after antithyroid therapy withdrawal. The aim of this case-control study was to evaluate the association between single nucleotide

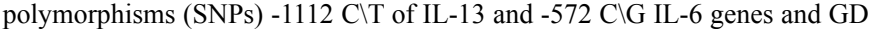
recurrence risk in Russian population.

Methods: 270 patients with GD (210 women, 60 men) and 200 healthy adults were examined. The mean age of GD onset was $41 \pm 13.6$ years. Levels of TSH, free T4, T3, TRAb were measured. Genotypes of -1112 C\T IL-13 and -572 C\G IL-6 genes were detected using direct sequencing and polymerase chain reaction-restriction fragment length polymorphism method. Alleles and genotypes frequencies were compared between groups using the Chi-square or Fisher's exact probability test.

Results: There were no significant differences in the distribution of alleles and genotypes between GD patients and control group. Among patients with intractable GD the IL-6 -572G allele (CG and GG genotypes) and IL-13 $-1112 \mathrm{C}$ allele (CC and CT genotypes) carriers were significantly more frequent $(\mathrm{p}-0.03, \mathrm{OR}=1.8)$ than in patient with GD in remission. The differences in goiter size, high levels of free T4 and T3 at time of onset, higher levels of TRAb also were significantly more frequent among IL-6 -572G allele and IL-12 -1112C allele carrier $(\mathrm{p}-0.04, \mathrm{OR}=1.8)$.

Conclusion: Our results suggest that SNPs at positions $-572 \mathrm{C} \backslash \mathrm{G}$ Il-6 and $-1112 \mathrm{C} \backslash \mathrm{T}$ IL-13 are associated with the severity of hyperthyroidism and influence the clinical course of GD. At first time in Russian population with GD genotypes of IL-6 and IL-13 of were identified.

\section{P1-01-08 \\ THYROID DYSFUNCTION AND AUTOIMMUNITY IN PATIENTS WITH CHRONIC MYELOID \\ LEUKAEMIA TREATED WITH TYROSINE KINASE INHIBITORS: PRELIMINARY RESULTS FROM A SINGLE CENTRE COHORT STUDY}

Fabiana Pani ${ }^{1}$, Olga Mulas ${ }^{2}$, Giovanni Caocci ${ }^{2}$, Alessandra Casti ${ }^{1}$,

Giorgio La Nasa², Stefano Mariotti ${ }^{1}$

${ }^{1}$ Endocrinology Unit, Department of Medical Sciences and Public

Health, Cagliari, Italy, ${ }^{2}$ Hematology, Department of Medical Sciences

and Public Health, Cagliari, Italy

Objective: Thyroid dysfunction (TD) is a well-known side effect of tyrosine kinase inhibitors (TKIs). Imatinib [I], Nilotinib [N] and Dasatinib [D] are TKIs employed in chronic myeloid leukaemia (CML), but their thyroid toxicity has been rarely investigated.

Methods: We assessed TSH, thyroid autoantibodies (ATA: TgAb and TPOAb) and thyroid ultrasound in 39 CML patients treated with TKIs (20 with I, 13 with $\mathrm{N}$ and 6 with $\mathrm{D}$ ). Patients were studied 1-14 years after beginning of therapy. Complete molecular remission (CMR) was defined as undetectable BCR-ABL transcript by RT-PCR with an assay sensitivity of $\mathrm{MR}^{4.0}$. Sixteen patients $(41 \%)$ were in CMR, while the remaining $23(59 \%)$ showed major $\mathrm{MR}^{3.0}$ or less.

Results: Fourteen (35.8\%) patients displayed TD ranging from isolated ATA-positivity to autoimmune hypothyroidism with thyroiditis-like ultrasound pattern (Table).

\begin{tabular}{|c|c|c|c|c|c|c|}
\hline \multirow[t]{2}{*}{ TKI } & \multirow{2}{*}{$\begin{array}{l}\text { Treatment } \\
\text { duration: years } \\
\text { (range,median) }\end{array}$} & \multicolumn{4}{|c|}{$\mathrm{TD}$} & \multirow[t]{2}{*}{ No TD } \\
\hline & & $\mathrm{a}$ & $\mathrm{b}$ & $\mathrm{c}$ & Total (\%) & \\
\hline $\mathrm{I}(\mathrm{n}=20)$ & $1-14 ; 5$ & 1 & 2 & 1 & $4(20 \%)$ & 16 \\
\hline$N(n=13)$ & $1-12 ; 3$ & 2 & 2 & 3 & $7(53.8 \%)$ & 6 \\
\hline $\mathrm{D}(\mathrm{n}=6)$ & $1-8 ; 3$ & 1 & 2 & 0 & $3(50 \%)$ & 3 \\
\hline Total $(n=39)$ & $1-14 ; 4$ & 4 & 6 & 6 & $14(35.8 \%)$ & 25 \\
\hline
\end{tabular}

a: Hypothyroidism, ATA+, thyroiditis-like ultrasound

b: Euthyroidism, ATA + , thyroiditis-like ultrasound

c: Euthyroidism, ATA+, normal ultrasound

The presence of TD was observed in most of CMR patients $(14 / 16=87.5 \%)$ and only in a minority $(1 / 25=4 \%)$ of patients with detectable BCR-ABL. ( $\mathrm{p}<0.0001$ by $?^{2}$ test).

Conclusions: High prevalence of autoimmune TD was detected in a cohort of CML patients undergoing TKIs treatment. TD was more frequently observed with second generation TKIs (N and D) and was almost exclusively found in patients with complete molecular remission. TD could therefore represent a biomarker of molecular response and a predictor of TKI improved efficacy in CML patients, but prospective controlled studies are needed to confirm these preliminary results.

\section{P1-01-09 WITHDRAWN}

\section{P1-01-10 \\ TH17 AND REGULATORY B CELLS PROFILE IN PATIENTS WITH HASHIMOTO'S THYROIDITIS ASSOCIATED WITH SYSTEMIC SCLEROSIS}

Maria Giulia Santaguida1, Giorgio Mangino¹, Ilaria Stramazzo¹, Poupak Fallahi ${ }^{2}$, Alessandro Antonelli ${ }^{2}$, Nunzia Brusca ${ }^{1}$, Miriam Cellini ${ }^{1}$, Camilla Virili ${ }^{1}$, Marco Centanni ${ }^{1}$

${ }^{1}$ Sapienza, University of Rome, Department of Medico-Surgical Sciences and Biotechnologies, Latina, Italy, ${ }^{2}$ University of Pisa, Department of Clinical and Experimental Medicine, Pisa, Italy

Objectives: Hashimoto's thyroiditis (HT) is highly prevalent in women with systemic sclerosis (SSc). HT is characterized by a Th17/Th1 polarization and an increased number of B regulatory (Breg) cells. Previous studies 
described an increased amount of naïve B cells with a decrease of memory B cells in isolated SSc. The aim of the study has been to characterize Th17 and Breg cell subsets in patients with HT associated with SSc.

Methods: A total of 49 patients $(44 \mathrm{~W} / 5 \mathrm{M}$; mean age: 48 years) were selected: of these, $19(17 \mathrm{~W} / 2 \mathrm{M})$ had isolated HT and 12 patients $(11 \mathrm{~W} / 1 \mathrm{M})$ had SSc; seven of these latter also had Hashimoto's thyroiditis (SSc+HT). Eighteen healthy donors (HD) $(16 \mathrm{~W} / 2 \mathrm{M})$ represented the reference group. Freshly PBMCs were assessed by FACS to characterize Th17 and Breg lymphocytes phenotypes. Moreover, activated Breg (IL-10 ${ }^{+}$) have been measured upon stimulation with $\mathrm{CpG}$ oligonucleotide.

Result: Mean percentage of Th $17^{+}$lymphocytes was $2.4 \pm 1.7 \%$ in isolated $\mathrm{HT}$ and significantly lower when associated with $\mathrm{SSc}(0.9 \pm 0.9 \% ; \mathrm{p}=0.0109)$. Breg cells (CD24 $\left.4^{\text {hi }} \mathrm{CD} 38^{\text {hi }}\right)$ were increased in patients affected by SSc, both isolated $(4.6 \pm 2.9 \%)$ or associated with HT $(4.6 \pm 3.2 \%)$, as compared to both patients with isolated HT $(2.4 \pm 0.9 \%)$ and $\mathrm{HD}(2.0 \pm 0.7 \%$; $\mathrm{p}=0.033)$. Following stimulation, the percentage of Breg cells was significantly higher in patients with SSc $(13.6 \pm 5.6 \%)$ and SSc+HT $(10.4 \% \pm 4.9)$ than in $\mathrm{HD}(\mathrm{p}<0.001$ and $\mathrm{p}=0.0002)$ and isolated HT $(\mathrm{p}=0.0027$ and $\mathrm{p}=0.0008)$. However, despite this increase, the cells producing IL-10 were lower in patients with SSc and $\mathrm{SSc}+\mathrm{HT}(0.5 \pm 0.4 \%$ and $0.8 \pm 1.2 \%)$ than in $\mathrm{HD}(2.4 \pm 1.1 \% \mathrm{p}=0.0231$ and $\mathrm{p}=0.0015)$ and in isolated HT $(3.9 \pm 1.8 \% ; \mathrm{p}=0.012$ and $\mathrm{p}=0.0008)$.

Conclusions: In patients with HT and SSc there was a reduction of Th17 and an increased percentage of Breg cells, but a clear reduction of IL- $10^{+}$Breg, as compared with those in isolated HT patients.

\section{P1-02 Cancer Diagnosis}

\section{P1-02-11 \\ NUCLEAR MEDICINE IMAGING IN PATIENTS WITH METASTATIC HUERTHLE CELL THYROID CARCINOMA - A SINGLE INSTITUTIONAL EXPERIENCE}

Nikola Besic ${ }^{1}$, Jerica Novak', Jan Zmuc ${ }^{1}$, Barbara Vidergar Kralj',

Andreja Antonija Schwarzbartl Pevec ${ }^{1}$, Ivana Žagar

${ }^{1}$ Institute of Oncology Ljubljana, Ljubljana, Slovenia

Background: Hürthle cell thyroid carcinoma (HCTC) is a rare disease, so there are only limited data about treatment of patients with metastatic HCTC. Our aim was to find out the usefulness of different nuclear medicine imaging modalities in detection of metastatic Hürthle cell thyroid carcinoma.

Methods: Altogether 133 patients were treated for HCTC from 1972 to 2014 in our tertiary center and 35 patients ( 21 females, 14 males; median age 64.5 years) had either initially proven metastatic disease $(\mathrm{N}=14)$ or distant progression after initial treatment $(\mathrm{N}=21)$. Data about imaging with FDGPET/ $\mathrm{CT}$, radioiodine and ${ }^{99 \mathrm{~m}} \mathrm{Tc}$-sestamibi (MIBI) were retrospectively collected and analyzed. At least one nuclear medicine imaging investigation was used in 30 patients with metastases (three imaging modalities in 2 patients, two in 14 patients, only one imaging modality in 14 patients).

Results: Uptake of isotope was found in all 11 patients in whom FDG PET/CT was used. Uptake of MIBI was found in 13/15 patients in whom MIBI was used. Radioiodine uptake was present in 18/22 patients after radioiodine therapy. They received from 1 to 10 radioiodine therapies (median 4). In 9 of patients the uptake of radioiodine was $0.5 \%$ or larger. Among 7 patients who had uptake of FDG PET/CT and therapy with radioiodine, uptake of RAI was present in $5 / 7$ patients.

Conclusions: Uptake of isotopes in metastatic HCTC is heterogeneous. Scintigraphy with FDG PET/CT and ${ }^{99 \mathrm{~m}} \mathrm{Tc}$-sestamibi showed uptake of isotope in $11 / 11$ and $13 / 15$ patients, respectively. Radioiodine uptake in metastases was present in 18/33 (54\%) patients with metastatic HCTC.

\section{P1-02-12}

CLINICAL SIGNIFICANCE OF FOLLOW-UP I-131 DIAGNOSTIC SCINTIGRAPHY AFTER RADIOACTIVE IODINE ABLATION THERAPY ON WELL-DIFFERENTIATED THYROID CANCER

Ha Kyoung Park ${ }^{1}$, Heesung Song 2

${ }^{1}$ Department of Surgery, Busan Paik Hospital, College of Medicine, Inje University, Busan, Korea, Rep. of South, ${ }^{2}$ Department of Nuclear Medicine, School of Medicine, Jeju National University, Jeju-Si, Korea, Rep. of South

Objective: This retrospective study researched for clinical significance of routine follow-up (F/U) I-131 diagnostic scintigraphy (Dx-scan) after radioactive iodine therapy (RI-Tx) on well-differentiated thyroid cancer.

Methods: Three hundreds eighteen patients were enrolled. All underwent total thyroidectomy due to well-differentiated thyroid cancer and high dose RI-Tx to ablate remnant thyroid tissue. The definition of complete ablation was below 2.0 of F/U stimulated thyroglobulin (sTg) plus negative finding of F/U Dx-scan after RI-Tx. The rest except complete ablation was regard as incomplete ablation. Re-treatment performed when pathologic results of recurrence or clinical findings of highly suspicious recurrence.

Results: Five of complete ablation patients $(n=185)$ were taken the retreatment $(2.7 \%)$. Main diagnostic modalities affecting the re-treatment were Dx-scan $(n=2)$, contrast CT $(n=1)$, ultrasonography $(n=1)$ and $\mathrm{sTg}(\mathrm{n}=2) .29$ of incomplete ablation patients $(\mathrm{n}=133)$ performed the re-treatment $(21.8 \%)$. Chief diagnostic tests affecting the re-treatment were FDG PET/CT $(n=14)$, contrast CT $(n=5)$, ultrasonography $(n=9)$, Dx-scan $(n=2)$, and $\operatorname{sTg}(n=2)$. Dx-scan influenced in $1.1 \%$ of complete ablation patients and $1.5 \%$ of incomplete ablation patients for the re-treatment.

Conclusion: F/U Dx-scan after RI-Tx is not useful for detecting recurrence especially in incomplete ablation patients, considering difficulties of the preparation of I-131 diagnostic scan compared to FDG PET/CT, ultrasonography and contrast CT.

\section{P1-02-13 \\ Clinical PERFORMANCE OF UNSTIMULATED HIGH SENSITIVE THYROGLOBULIN MEASUREMENT IN WELL DIFFERENTIATED THYROID CANCER IN COMPARISON WITH A FIRST GENERATION ASSAY}

Ana Saavedra ${ }^{1}$, Elisabete Rodrigues ${ }^{1}$, Pedro Souteiro ${ }^{1}$, Luísa RafaeR João Tiago Guimarães ${ }^{2}$, Davide Carvalho

${ }^{1}$ Department of Endocrinology Diabetes and Metabolism, Centro Hospitalar S. João, Faculty of Medicine, University of Porto, Instituto de Investigação e Inovação Em Saúde, University of Porto, Porto, Portugal, ${ }^{2}$ Department of Clinical Pathology, Centro Hospitalar S. João, Porto, Portugal

Introduction: Serum thyroglobulin (TGB) is the primary biochemical tumour marker of well differentiated thyroid cancer (WDTC).

Objective: To compare TGB levels measured with two commercial assays: conventional/first generation (cTGB) vs. high-sensitive/second generation (hsTBG) in patients with WDTC.

Methods: During 3 consecutive months, serum of adult patients collected during the usual WDTC follow-up were evaluated using two TGB assays: cTGB - Siemens (Immulite) ${ }^{\circledR}$ and hsTGB - Roche Elecsys Tg II ${ }^{\circledR}$.

Results: We included 85 patients $[90,6 \%$ female; age at diagnosis $50.5( \pm 15.2)$ years; follow-up $7( \pm 6)$ years]. All patients underwent total thyroidectomy; 10 performed cervical lymphadenectomy. AJCC/TNM staging system ( $7^{\text {th }}$ edition): stage I $67.1 \%$ patients, stage II $19.6 \%$, stage III $16.5 \%$ stage IVa $5.9 \%$. According to initial stratification for recurrence: $56.5 \%$ were low, $36.5 \%$ intermediate and $6,1 \%$ high risk. $72.9 \%$ of patients received ${ }^{131}$ Iodine therapy. TGB was detectable in $14,1 \%$ of patients with cTGB assay vs. $24.7 \%$ with hsTGB assay. Comparison of average TGB levels within subgroups of recurrence risk didn't showed any significant statistical difference. In 72 of 85 patients, TGB results were concordant between two assays (positive correlation $\mathrm{r}=0.56 ; p<0.001$ ). Eleven patients with undetectable cTGB had measurable values in hsTGB assay. Seven out of 67 patients reclassified as 
"Excellent Response to therapy" and with undetectable levels in cTGB had measurable values in hsTGB assay (average $0.16 \pm 0.01 \mathrm{ng} / \mathrm{mL}$ ).

Conclusions: In accordance with published literature, hsTGB assays seem to be more useful in the follow-up of WDTC as they permit to document the TGB evolution in patients in which TGB would be undetectable if followed with a first generation assay.

\section{P1-02-14}

\section{Clinical EVALUATION OF THE NEW HIGHLY SENSITIVE COBAS THYROGLOBULIN II ASSAY IN PATIENTS WITH DIFFERENTIATED THYROID CARCINOMA: A MULTICENTRIC STUDY}

\section{Pierpaolo Trimboli ${ }^{1}$, Arnoldo Piccardo ${ }^{2}$, Alfredo Campenni ${ }^{3}$,}

Fabio Orlandi ${ }^{4}$, Luca Giovanella ${ }^{5}$

${ }^{1}$ Thyroid Center, Oncology Institute of Southern Switzerland, Lugano, Switzerland, ${ }^{2}$ Department of Nuclear Medicine, Ospedale Galliera, Genova, Italy ${ }^{3}$ Departments of Nuclear Medicine, University of Messina, Messina, Italy, ${ }^{4}$ Department of Internal Medicine and Endocrinology, University of Torino, Torino, Italy, ${ }^{5}$ Department of Nuclear Medicine and Thyroid Centre, Oncology Institute of Southern Switzerland, Bellinzona, Switzerland

Objective: High sensitive thyroglobulin (hsTg) has significantly improved the management of differentiated thyroid carcinoma (DTC). Recently, a new fully automated hsTg (Cobas ${ }^{\circledR}$ Tg II, Roche Diagnostics, Penzberg, Germany) has become available but it has not been tested in a clinical cohort. The aim was to evaluate the performance of Cobas ${ }^{\circledR} \mathrm{Tg}$ II and compare it with the well-established Access $\odot$ Tg in a series of DTC.

Methods: This was a prospective study on a multicenter series. All data of DTC cases diagnosed, treated and followed-up since January 2005 at four institutions were screened and selected by several criteria. Samples had been stored at $-80^{\circ} \mathrm{C}$. For this study $\mathrm{Tg}$ was simultaneously measured by $\mathrm{Tg}$ II Cobas $₫$ and $\operatorname{Tg}$ Access $\odot$ assays.

Results: Two-hundred-four DTC were finally included. Of these, a $10.8 \%$ had structural recurrence (sREC) and $81.4 \%$ showed no evidence of disease (NED). Basal and rhTSH Tg values of Cobas ${ }^{\circledR}$ and Access $\complement$ were significantly correlated. At ROC curve analysis the most accurate cut-off of basal Tg to detect sREC was $>0.41 \mathrm{ng} / \mathrm{mL}$ for Cobas $\odot$ and $>0.36 \mathrm{ng} / \mathrm{mL}$ for Access $\subseteq$, providing $100 \%$ sensitivity and $96 \%$ specificity for both assays. The optimal thresholds of rhTSH Tg were $>1.82 \mathrm{ng} / \mathrm{mL}$ for Cobas $\odot$ and $>1.62$ for Access $\odot$, with $100 \%$ sensitivity and $95 \%$ specificity for both. The multivariate analysis showed significant performance $(\mathrm{p}<0.0001)$ of Cobas $\subseteq$ and Access $($ (Table 1). Also, having basal or rhTSH Tg above these thresholds reduced significantly the percentage of event-free cases.

Conclusions: Tg II Cobas ${ }^{\circledR}$ showed optimal performance to detect DTC relapse with no significant difference from $\mathrm{Tg}$ Access $\odot$.

\section{P1-02-15 \\ EXOGENOUS THYROGLOBULIN (TG) EQUILIBRATION WITH SERUM TG AUTOANTIBODY (TGAB) IS TIME (DAYS), TG DOSE, TG SOURCE AND TGAB DEPENDENT - SIGNIFICANCE FOR TG RECOVERY PROTOCOLS Ivana Petrovic ${ }^{1}$, Shireen Fatemi ${ }^{2}$, Carole Spencer ${ }^{3}$ \\ ${ }^{1}$ Usc Endocrine Laboratory, University of Southern California, Pasadena, CA, USA, ${ }^{2}$ Southern California Permanente Medical Group, Panorama City, CA, USA, ${ }^{3}$ Usc Endocrine Laboratory, University of Southern California, Tech Director, Usc Endocrine Labs, Pasadena, CA, USA}

Objectives: Exogenous Tg recoveries from sera are used to screen for $\mathrm{TgAb}$ interference - a persistent problem with Immunometric assay (IMA) methodology that causes falsely low/undetectable Tg-IMA values by epitope masking. Tg recoveries can be influenced by $\mathrm{Tg}$ dose, $\mathrm{Tg}$ heterogeneity and the equilibration time needed for $\mathrm{Tg}-\mathrm{TgAb}$ complex formation. Currently $>70 \%$ Tg-IMA recovery of glandular Tg from a serum after $<24$-hour incubation suggests no interference. This study explored the influence of $\mathrm{Tg}$ dose, $\mathrm{Tg}$ source, and equilibration time on $\mathrm{Tg}$ recovered from sera with different $\mathrm{TgAb}$ concentrations.

Methods: Glandular Tg (CRM-457) at final concentrations (FC) 2.3 and $5.5 \mu \mathrm{g} / \mathrm{L}$, or metastatic DTC-Tg $(\mathrm{FC} \sim 2$ and $\sim 4 \mu \mathrm{g} / \mathrm{L}$,) was spiked into three concentrations of a TgAb+ DTC serum $(\mathrm{TgAb}-\mathrm{H}=450, \mathrm{TgAb}-\mathrm{M}=45$ and $\mathrm{TgAb}-\mathrm{L}=4.5 \mathrm{kIU} / \mathrm{L}$ ). All dilutions were made in a $\mathrm{Tg}-\mathrm{TgAb}-\mathrm{DTC}$ serum pool. Percent Tg recovered/expected after 0.5hours to 6days@ambient temperature incubations were evaluated. A stable Tg-IMA nadir likely represented maximal epitope-masking by $\mathrm{Tg}-\mathrm{TgAb}$ complex formation. Assays were Beckman Tg-IMA (functional sensitivity $(\mathrm{FS})=0.10 \mu \mathrm{g} / \mathrm{L}$ ) and Beckman TgAb-IMA (FS=1.0 kIU/L).

Results:

Table 1. \% Recovery (R) vs. Time (hours/h or days/d)

\begin{tabular}{|c|c|c|c|c|c|c|c|c|}
\hline & $\begin{array}{l}0.5 \mathrm{~h} \\
\% \mathrm{R}\end{array}$ & $\begin{array}{l}4 \mathrm{~h} \\
\% \mathrm{R}\end{array}$ & $\begin{array}{l}1 \mathrm{~d} \\
\% \mathrm{R}\end{array}$ & $\begin{array}{l}2 \mathrm{~d} \\
\% \mathrm{R}\end{array}$ & $\begin{array}{l}3 \mathrm{~d} \\
\% \mathrm{R}\end{array}$ & $\begin{array}{l}4 \mathrm{~d} \\
\% \mathrm{R}\end{array}$ & $\begin{array}{l}5 \mathrm{~d} \\
\% \mathrm{R}\end{array}$ & $\begin{array}{l}6 \mathrm{~d} \\
\% \mathrm{R}\end{array}$ \\
\hline \multicolumn{9}{|c|}{$T g-C R M-457 F C=5.5 \mathrm{ug} / \mathrm{L}$} \\
\hline added to $\mathrm{TgAb}-\mathrm{H}$ & 23.6 & $<4$ & $<4$ & $<4$ & $<4$ & $<4$ & $<4$ & $<4$ \\
\hline added to TgAb-M & 61.8 & 22 & 4.2 & $<4$ & $<4$ & $<4$ & $<4$ & $<4$ \\
\hline added to TgAb-L & 92.7 & 56 & 36.4 & 23.6 & 17.8 & 10.9 & 7.3 & 5.3 \\
\hline \multicolumn{9}{|c|}{$T g-C R M-457 F C=2.3 \mathrm{ug} / \mathrm{L}$} \\
\hline added to TgAb-H & $25.7^{\circ}$ & $<4$ & $<4$ & $<4$ & $<4$ & $<4$ & $<4$ & $<4$ \\
\hline added to TgAb-M & 65.2 & 22 & $<4$ & $<4$ & $<4$ & $<4$ & $<4$ & $<4$ \\
\hline added to TgAb-L & 100 & 56 & 35.7 & 23.9 & 15.2 & 8.7 & 5.2 & $<4$ \\
\hline
\end{tabular}

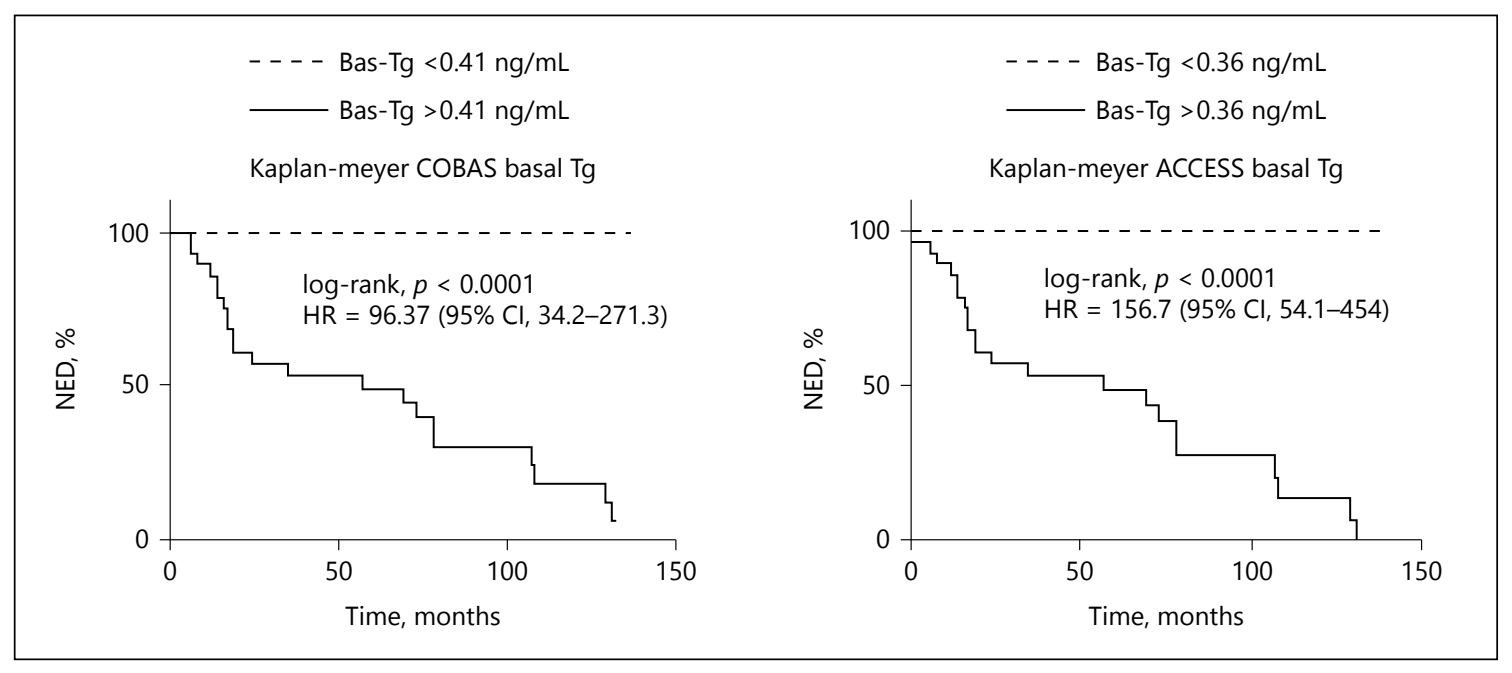

Fig. 1. (for of Abstract P1-02-14). 
4-day recoveries of tumor Tgs from $\mathrm{TgAb}-\mathrm{L}$ differed among patients (5-17\%) and from CRM-457 (12\%).

Conclusions:

1. $\mathrm{Tg}$ dose, $\mathrm{Tg}$ source, $\mathrm{TgAb}$ concentration and incubation time all affect recovery percentages.

2. The typical $<24 \mathrm{~h}$ incubation employed for recovery protocols is inadequate. Most $\mathrm{TgAb}+\mathrm{DTC}$ patients have low $\mathrm{TgAb}$ necessitating $>6$ days incubation for $\mathrm{Tg}-\mathrm{TgAb}$-complex equilibration.

3. Recoveries of glandular $\mathrm{Tg}$ added to some patient sera may not represent endogenous $\mathrm{Tg}-\mathrm{TgAb}$ complex formation, because both $\mathrm{Tg}$ and $\mathrm{TgAb}$ epitope specificities are patient-specific.

\section{P1-02-16 \\ THE CLINICAL APPLICABILITY OF LOW LEVELS OF THYROGLOBULIN AUTOANTIBODIES (TGABS) AS CUT-OFF POINT FOR TGAB POSITIVITY}

Bernadette Dekker ${ }^{1}$, Anouk van der Horst-Schrivers ${ }^{2}$, W.J. Sluiter ${ }^{2}$, Eef Lentjes $^{3}$, Annemieke Heijboer ${ }^{4}$, Anneke Muller Kobold ${ }^{5}$, Thera Links ${ }^{2}$ ${ }^{1}$ University Medical Center Groningen; Department of Endocrinology, Groningen, Netherlands, ${ }^{2}$ University Medical Center Groningen, Department of Endocrinology, Groningen, Netherlands, ${ }^{3}$ University Medical Center Utrecht, Department of Clinical Chemistry and Hematology, Laboratory of Endocrinology, Utrecht, Netherlands, ${ }^{4}$ Vu University Medical Center, Department of Clinical Chemistry, Endocrine Laboratory, Amsterdam, Netherlands, ${ }^{5}$ University Medical Center Groningen, Department of Laboratory Medicine, Groningen, Netherlands

Objectives: Thyroglobulin (Tg) is an established tumor marker in differentiated thyroid carcinoma (DTC). However, Tg assays can be subject to interference with autoantibodies against $\operatorname{Tg}$ (TgAbs). No consensus exists on the cut-off value of $\mathrm{TgAb}$ positivity and the relation with $\mathrm{Tg}$ assay interference. Several cut-off values, with increasing levels, are proposed; limit of detection (LoD), limit of quantification (LoQ), manufacturer cut-off (MCO) and institutional cut-off (ICO). Our aim was to investigate the most applicable cut-off value for $\mathrm{TgAb}$ positivity and to evaluate whether tumor characteristics differ between $\operatorname{TgAb}$ positive and negative patients during ablation therapy using the $\mathrm{MCO}$ and ICO.

Methods: This single-center retrospective cohort study, included 230 DTC patients, diagnosed between January 2006 and December 2014. Serum $\mathrm{Tg}$ and $\mathrm{TgAbs}$ were measured with the Tg-IRMA (Thermo Fischer) and ARCHITECT Anti-Tg assay (Abbott Laboratories). Patients were divided in $\mathrm{TgAb}$ negative and positive based on $\mathrm{LoD}(\geq 0.07 \mathrm{U} / \mathrm{ml}), \mathrm{LoQ}(\geq 0.31 \mathrm{U} / \mathrm{ml})$, $\mathrm{MCO}(\geq 4.11 \mathrm{U} / \mathrm{ml})$ and ICO $(\geq 10 \mathrm{U} / \mathrm{ml})$.

Results: All patients were $\mathrm{TgAb}$ positive based on the LoD; 1 patient was negative on LoQ (see table). Histology, presence of multifocality, TNM, ATA risk stratification and disease state at the end of follow-up were not different comparing $\mathrm{TgAb}$ negative and positive patients using MCO and ICO during ablation.

Conclusions: This study supports the use of a higher cut-off value instead of the $\mathrm{LoQ}$ for $\mathrm{TgAb}$ positivity in clinical setting. The $\mathrm{LoD}$ and $\mathrm{LoQ}$ are too sensitive to discriminate $\mathrm{TgAb}$ positivity and negativity in DTC patients during ablation therapy. Secondly, presence of TgAbs during ablation is not related with tumor characteristics and risk profile, supporting that $\mathrm{TgAb}$ positivity cannot be considered as separate risk factor.

\begin{tabular}{llcl}
\hline & Total $(\mathrm{N})$ & TgAb negative $(\mathrm{N})$ & TgAb positive $(\mathrm{N})$ \\
\hline LoD $(0.07 \mathrm{U} / \mathrm{ml})$ & 230 & 0 & 230 \\
LoQ $(0.31 \mathrm{U} / \mathrm{ml})$ & 230 & 1 & 229 \\
MCO $(4.11 \mathrm{U} / \mathrm{ml})$ & 230 & 175 & 55 \\
ICO $(10.0 \mathrm{U} / \mathrm{ml})$ & 230 & 196 & 34 \\
\hline
\end{tabular}

Poster Presentations

\section{P1-02-17 \\ THYROGLOBULIN ANTIBODIES (TGAB) TITER SPONTANEOUSLY DECREASE UNTIL THE NEGATIVIZATION OR STABILIZATION IN MOST OF CASES OF PAPILLARY THYROID MICROCARCINOMA (MPTC) TREATED WITH TOTAL THYROIDECTOMY BUT NOT ABLATED WITH 131 I}

Antonio Matrone $^{1}$, Eleonora Molinaro ${ }^{1}$, Laura Agate $^{1}$, Valeria Bottici ${ }^{1}$, David Viola', Laura Valerio ${ }^{1}$, Loredana Lorusso ${ }^{1}$, Alessio Faranda ${ }^{1}$, Paolo Piaggi ${ }^{2}$, Paolo Vitti ${ }^{1}$, Rossella Elisei ${ }^{1}$

${ }^{1}$ University of Pisa, Department of Clinical and Experimental Medicine, Endocrine Unit, Pisa, Italy, ${ }^{2}$ Phoenix Epidemiology and Clinical Research Branch National Institute of Diabetes and Digestive and Kidney Disease - National Institutes of Health - Phoenix, Arizona, USA, Phoenix, AZ, USA

Background: Thyroglobulin antibodies (TgAb) are present in about $25 \%$ of patient affected by Differentiated Thyroid Cancer (DTC). In these patients, after total thyroidectomy and radioiodine ablation (RAI), TgAb trend becomes a "surrogate" marker to assess the persistent/recurrent disease. Little is known about the clinical significance of $\mathrm{TgAb}$ trend in patients not submitted to RAI (No-RAI), as in case of microcarcinomas (mPTC). The aim of our study was to verify the $\mathrm{TgAb}$ trend during the follow-up of No-RAI cases.

Methods: Among all No-RAI mPTC patients $(n=383)$ followed at our Department from 2005 to 2012, we selected pts who had at least 3 determinations of serum $\mathrm{TgAb}$. The epidemiological, clinico-pathological and neck ultrasound data have been collected.

Results: 107/383 (27.9\%) pts were TgAb positive ( $\geq 8 \mathrm{mU} / \mathrm{L})$. At the first post-surgical evaluation (3-6 months), $35.5 \%$ (38/107) pts had TgAb $8 \leq 20$ $\mathrm{mU} / \mathrm{L}, 23.4 \%(25 / 107)$ pts $21 \leq 50 \mathrm{mU} / \mathrm{L}, 24.3 \%(26 / 107) \mathrm{pts} 51 \leq 100 \mathrm{mU} / \mathrm{L}$ and $16.8 \%(18 / 107) \mathrm{pts}>100 \mathrm{mU} / \mathrm{L}$. At the end of the follow up (mean $5.9 \mathrm{yrs}$; median $5.75 \mathrm{yrs}), 62.6 \%(67 / 107)$ pts had $\mathrm{TgAb}<8 \mathrm{mU} / \mathrm{L}, 21.5 \%(23 / 107)$ pts $8 \leq 20 \mathrm{U} / \mathrm{ml}, 9.3 \%(10 / 107)$ pts $21 \leq 50 \mathrm{mU} / \mathrm{L}, 2.8 \%(3 / 107)$ pts $51 \leq 100$ and only $3.7 \%(4 / 107)$ had $\mathrm{TgAb}>100 \mathrm{mU} / \mathrm{L}[\mathrm{p}<0.01]$. In all but one case the titer of $\operatorname{TgAb}$ was increasing and neck US became positive for local thyroid disease. Neck US was negative for local a/o lymph nodes metastatic disease in all patients at every time of the follow up.

Conclusions: 1) After 6 years of follow up, in the majority of cases the $\mathrm{TgAb}$ titer was decreasing and became negative in $62.6 \% \mathrm{pts} ; 2$ ) The only case of increasing $\mathrm{TgAb}$ showed the reappearance of thyroid tissue at neck US during the follow up

\section{P1-02-18}

\section{LYMPH NODE METASTASIS OF MEDULLARY THYROID CANCER: ROLE OF CALCITONIN IN THE WASHOUT FLUID OF FINE-NEEDLE ASPIRATION BIOPSIES}

Bernardo Marques ${ }^{1}$, Raquel Martins ${ }^{1}$, Nuno Cunha ${ }^{1}$, Joana Couto ${ }^{1}$ Jacinta Santos ${ }^{1}$, Teresa Martins ${ }^{1}$, Ana Paula Moniz', Olga Ilhéu',

Frederico Valido ${ }^{1}$, Paulo Figueiredo ${ }^{1}$, Fernando Rodrigues ${ }^{1}$

${ }^{1}$ Portuguese Institute of Oncology of Coimbra Fg, Epe, Coimbra, Portugal

Introduction: The diagnostic value of calcitonin (CT) measurement in fine-needle aspiration biopsies (FNAB-CT) for medullary thyroid cancer (MTC) lymph node (LN) metastasis remains to be determined. It may increase the diagnostic sensitivity, along with FNA cytology, but, unlike the well-established method of thyroglobulin measurement in FNA washout, the data on this subject is sparse. Our study aimed to evaluate the utility of FNA-CT in the diagnosis of LN metastasis of MTC.

Methods/Design: This was a retrospective study of 72 consecutive cytologies of FNA from 45 patients identified from our institutional database, who underwent FNA citology and CT measurement in needle washout for suspicious LN, between 2012 and 2016.

Results: From the total of 72 FNA cases, $26(36.1 \%)$ had personal history of MTC. MTC was detected in 18 FNA cytologies $(25 \%)$ and CT was detectable in needle washout in 21 cases (median was $4653 \mathrm{ng} / \mathrm{mL}$ (interquartile range: $214.5-27668 \mathrm{ng} / \mathrm{mL}$ ). $\mathrm{LN}$ ressection was performed in 34 
cases $(47.2 \%)$, based on the combined results of FNA-CT and FNA cytology. Histology reported LN metastasis of MTC in 21 lesions (62\%).

Regarding the diagnosis of MTC LN metastasis, FNA citology showed sensitivity $100 \%$ and specificity $86 \%$ and FNAB-CT values above $0.2 \mathrm{ng} / \mathrm{mL}$ showed sensitivity $96 \%$ and $95 \%$ specificity. Combining both diagnostic strategies showed superior diagnostic power than using either strategy alone (sensitivity $100 \%$ and specificity $100 \%$ ).

We evaluated the optimal cutoff value of FNAB-CT in determining LN metastasis of through ROC analysis and the optimal cutoff value was $8.85 \mathrm{ng} /$ $\mathrm{mL}$ (sensitivity $95 \%$; specificity $100 \%$ ).

Conclusion: FNAB-CT may be a valuable diagnostic tool for detection of MTC LN metastasis, along with FNA citology and it should be included in the clinical work-up of lateral neck adenopathies when suspected.

\section{P1-02-19 \\ USEFULNESS OF CALCITONIN CALCIUM STIMULATION TEST AS A PREDICTOR OF MEDULLARY THYROID CANCER}

Cristina Gheorghiu ${ }^{1}$, Andra Caragheorgheopo ${ }^{2}$, Cristina Corneci ${ }^{1}$,

Ruxandra Dobrescu ${ }^{1}$, Corin Badiu ${ }^{3}$

${ }^{1}$ National Institute of Endocrinology, Bucharest, Romania, ${ }^{2}$ Bucharest,

Romania, ${ }^{3 " C}$.I.Parhon" National Institute of Endocrinology, „C.Davila“

University of Medicine, „C. Davila“ University of Medicine and

Pharmacy, Bucharest, Romania

Background: Medullary thyroid cancer (MTC) is a highly aggressive neuroendocrine tumour originating in the thyroid parafollicular cells. Calcitonin measurement and RET gene ascertainment have been used as markers for early diagnosis of MTC. Since pentagastrin is not available, calcium stimulation test helps to preclude "gray zone" values of basal calcitonin, diagnose minimal residual disease and help the early diagnosis of $\mathrm{C}$ cell hyperplasia.

Aim: To establish a gender specific threshold for positive calcium stimulation test as well as indication for thyroidectomy and therefore prevention of MTC through early diagnosis.

Material and Methods: We conducted the calcium stimulation test on a series of patients with either nodular goiter, single nodule, RET positive or history of MTC associated with elevation of basal calcitonin values. Calcium gluconate $25 \mathrm{mg} / \mathrm{kg}$ adapted on the ideal BMI was administered over 3 minutes under ECG monitoring. Blood was collected before, at 2; 5 and 10 minutes after calcium infusion. Calcitonin was measured using LIAISON XL assay.

Results: The ongoing test has been performed on 17 patients, aged (23-67), $11 \mathrm{M}, 6$ F. Mean calcitonin values were: basal=19.98 pg/ml (normal range: $1-11.8 \mathrm{pg} / \mathrm{ml}$ ); at $2 \mathrm{~min}=209.159 \mathrm{pg} / \mathrm{ml}$; at $5 \mathrm{~min}=143.619 \mathrm{pg} /$ $\mathrm{ml}$, at $10 \mathrm{~min}=100.557 \mathrm{pg} / \mathrm{ml} ; 10$ subjects had a significant $2 \mathrm{~min}$ stimulated calcitonin $(>100 \mathrm{pg} / \mathrm{ml})$ and were operated, 3 subjects had an intermediate response $(53.31-81.7 \mathrm{pg} / \mathrm{ml})$ and 4 patients had low stimulated values $(<54 \mathrm{pg} /$ $\mathrm{ml}$ ) therefore they are to be followed-up. $\mathrm{C}$ cell hyperplasia $(\mathrm{CCH})$ and MTC was confirmed histologically in 4 out of 10 patients (2 $\mathrm{CCH}$ and $2 \mathrm{MTC}$ ); 2 subjects had benign nodular goiter and 2 papillary thyroid microcarcinomas. More results are expected.

Conclusions: Stimulated calcitonin may be useful in the early diagnosis and follow-up of MTC and could reduce false negative rate of basal calcitonin measurement.

\section{P1-02-20 \\ IN VIVO IMAGING OF TUMOUR HETEROGENEITY DRIVES THERAPY OF METASTATIC CARCINOMA}

Mara Ventura ${ }^{1}$, Miguel Melo ${ }^{1}$, Gracinda Costa ${ }^{1}$, Cristina Ribeiro ${ }^{1}$, Luís Cardoso $^{1}$, Nuno Vicente ${ }^{1}$, Diana Martins ${ }^{1}$, Diana Oliveira ${ }^{1}$, Adriana Lages $^{1}$, Nelson Cunha ${ }^{1}$, Francisco Carrilho ${ }^{1}$

${ }^{1}$ Hospitais Da Universidade de Coimbra, Coimbra, Portugal

Introduction: Several options for imaging differentiated thyroid cancer (DTC) in nuclear medicine have been proposed in the last years. Theranostic radioiodine molecular imaging, which combines diagnostic testing and therapeutic imaging modalities, has been a useful tool on this issue.

Case Report: A 55-year-old female presented with lower back pain for the past six months. A Computed Tomography (CT) showed a lytic bone lesion in the left sacrum with $7,5 \times 4,8 \times 4,1 \mathrm{~cm}$. The biopsy was consistent with a metastasis of a carcinoma with probable origin in the thyroid. The patient was submitted to total thyroidectomy, and the histological exam showed a 1,7 $\mathrm{cm}$ minimally invasive and angioinvasive follicular carcinoma $\left(\mathrm{pT}_{1} \mathrm{NxM}_{1}\right)$. Positron-emission tomography (PET)/CT with 2-[(18)F]fluoro-2-deoxy-dglucose (18F-FDG) was performed and a single-photon emission computed tomography (SPECT)/CT was obtained after radioiodine (131I) therapy. For therapeutic decision, all imaging procedures were fused in one single image. The two functional imaging used, representing iodine and glucose metabolism, showed different uptake patterns within the mass. 131I uptake was present in the lower and upper poles, while most of the lesion only demonstrated glucose uptake, indicating a less differentiated area. The following treatment procedure was radiotherapy, because the mass was predominant $18 \mathrm{~F}-\mathrm{FDG}$ positive and 131I negative.

Conclusions: In Oncology, the combination of diagnostic and treatment imaging procedures (Theranostic) is growing and promotes a tailor based therapy strategy. If multiple diagnostic procedures are used, a method that allows the integration of all information, in a single image, may enable a more accurate in vivo picture of the tumour biology. Gathering information about the distribution, within a tumour mass, of the metabolism and the state of cell differentiation, help us to predict treatment response and give us a real-time prognostic tool.

\section{P1-02-21 \\ VALUE OF CALCIUM STIMULATION TEST IN DIFFERENTIAL DIAGNOSIS OF HYPERCALCITONINEMIA}

Bojana Popovic ${ }^{1}$, Djuro Macut ${ }^{2}$, Valentina Elezovic-Kovacevic ${ }^{2}$, Tatjana Isailovic ${ }^{2}$, Ivana Bozic-Antic ${ }^{2}$, Tamara Bogavac ${ }^{2}$, Dusan llic ${ }^{2}$, Sanja

Ognjanovic ${ }^{2}$, Gordana Rodic ${ }^{2}$, Milan Petakov'2, Svetozar Damjanovic ${ }^{2}$

${ }^{1}$ Clinic for Endocrinology, Diabetes and Metabolic Diseases, Belgrade,

Serbia, ${ }^{2}$ Belgrade, Serbia

Introduction: Interpretation of hypercalcitoninemia can be challenging, since it can be observed not only in patients with medullary thyroid cancer (MTC), but also in patients with benign thyroid pathology, and with calcitonin secreting neuroendocrine tumors (NET). Potentially useful ancillary tool could be calcium stimulation test, but it is still not standardized.

Patients and Methods: We analyzed 4 groups of patients: 1) 30 patients with MTC, 2) 15 patients with calcitonin secreting NET, 3) 21 patients with benign thyroid nodules and/or autoimmune thyroid disease (controls), 4) 7 patients with confirmed mutation in RET protooncogene, but without confirmed thyroid pathology at the point of investigation. Beside standard diagnostic procedures, all patients underwent calcium stimulation test with calcitonin measurement. Statistical analysis was done with SPSS software.

Results: Patients with NET and MTC had similar basal calcitonin levels $(p=0.847)$, but significantly higher when compared to controls $(p<0.01$ for both), and patients with RET mutation but without thyroid pathology $(\mathrm{p}<0.01$ for both). After calcium stimulation, patients with NET achieved significantly lower calcitonin levels $(\mathrm{p}=0.01)$, but no different compared to controls $(\mathrm{p}=0.102)$, and patients with RET mutation $(\mathrm{p}=0.056)$. Patients with MTC also achieved significantly higher levels compared to both other groups ( $p<0.01$ for both), though the degree of response was similar in all 3 groups (mean increase was $19.5 \pm 19.8$ times in MTC, $14.8 \pm 12.9$ times in controls, and $32.5 \pm 41.5$ times in patients with RET mutation; $\mathrm{p}=0.421$ and $\mathrm{p}=0.608$ ). Majority (55.6\%) of patients with NET had concomitant goiter and $26.3 \%$ had concomitant TPOAb, but there was no significant correlation with basal calcitonin levels in these patients $(\mathrm{p}=0.816$ and $\mathrm{p}=0.670)$.

Conclusion: Calcitonin response to calcium stimulation can be used as discriminatory in differential diagnosis of patients with hypercalcitoninemia, especially between those with different types of neuroendocrine tumors (MTC vs. NET). Cut off values are still to be determined 


\section{P1-03 Cancer Prognostic Factors}

\section{P1-03-22 \\ PREDICTIVE VALUE OF THE 8TH EDITION AJCC/UICC TNM CLASSIFICATION FOR DIFFERENTIATED THYROID CARCINOMA: COMPARISON WITH THE 7TH EDITION}

Kenichi Matsuzu ${ }^{1}$, Kiminori Sugino ${ }^{2}$, Tadatoshi Osaku', Tomoaki

Tanaka', Yuna Ogimi', Chie Masaki', Junko Akaishi', Kiyomi Hames

Yamada', Chisato Tomoda ${ }^{1}$, Akifumi Suzuki ${ }^{1}$, Takashi Uruno ${ }^{1}$, Keiko

Ohkuwa $^{1}$, Wataru Kitagawa ${ }^{3}$, Mitsuji Nagahama', Hiroshi Takami ${ }^{3}$,

Koichi Ito ${ }^{1}$

${ }^{1}$ Ito Hospital, Tokyo, Japan, ${ }^{2}$ Ito Hospital, Department of Surgery,

Tokyo, Japan, ${ }^{3}$ Ito Hospital, Tokyo

Objective: The purpose of this study was to evaluate the 8th edition AJCC/UICC TNM classification (8th) for differentiated thyroid cancer (DTC).

Methods: Both the 7th edition AJCC/UICC TNM AJCC/UICC TNM classification (7th) for DTC and the 8th were applied in a retrospective analysis of 1369 DTC patients who underwent initial surgery at Ito Hospital between 1986 and 1995. Cause-specific survival rates (CSS) were assessed using Kaplan-Meier methods and compared using the log-rank test. Effects of TNM stage on CSS were evaluated by univariate analysis using the Cox proportional hazards models.

Results: According to the revision from 7 th to 8 th, the number of patients with Stage I DTC increased from $776(56.7 \%)$ to $1145(83.6 \%)$. On the other hand, numbers of patients in Stages II, III, and IV decreased from $174(12.7 \%)$ to $141(10.4 \%)$, from $274(20.0 \%)$ to $71(5.2 \%)$, and from $145(10.6 \%)$ to 10 $(0.7 \%)$, respectively. A total of 776 patients classified as Stage I in 7 th stayed at the same stage in 8th, but the other 593 patients classified as Stages II, III, IVA, and IVC in 7th were all downstaged in 8th. CSS rates at 20 years under 7 th for Stages I, II, III, IVA, and IVC were $99.7 \%, 100 \%, 94.6 \%, 81.6 \%$, and $50.6 \%(\mathrm{p}<0.0001)$. In 7 th, Stages III, IVA, and IVC were related to reduced CSS, but CSS of Stages I and II showed no difference. CSS rates under 8th for Stages I, II, III, and IVB were $98.9 \%, 90.4 \%, 73.2 \%$, and $13.0 \%$, respectively $(\mathrm{p}<0.0001)$. In 8th, hazard ratios for Stages II, III, and IVB were 7.8, 26.4, and 313.3, respectively.

Conclusion: Generally, patients are more likely to be classified into an earlier stage under 8th. Clinical outcomes for Stages I and II in 7th showed no significant difference, but CSS decreased stepwise in 8th.

\section{P1-03-23 \\ ASSOCIATION BETWEEN BODY MASS INDEX AND SEVERITY OF PAPILLARY THYROID MICROCARCINOMA}

Songl Yang ${ }^{1}$, Jiyoung Yoo ${ }^{2}$, KwangKuk Park ${ }^{3}$, Jeong Hoon Kim ${ }^{4}$ ${ }^{1}$ Kosin University College of Medicine, Department of Surgery, Seo-Gu, Busan, Korea, Rep. of South, ${ }^{2}$ Department of Surgery, Kosin University College of Medicine, Busan, Korea, Rep. of South, ${ }^{3} \mathrm{Hub}-\mathrm{Hu}$ Hospital, Department of Surgery, Sahagu, Busan, Korea, Rep. of South, ${ }^{4}$ Kosin University Gospel Hospital, Busan, Korea, Rep. of South

Purpose: Obesity is associated with aggressive pathological features and poor clinical outcomes in breast and prostate cancers. However, the associations between excess weight and prognostic factors for thyroid cancer are uncertain. This study aimed to evaluate the associations between body mass index (BMI) and severity of papillary thyroid microcarcinoma.

Methods: Retrospective analysis of 2579 patients with PTMC was performed. Patients were grouped according to BMI (underweight, normal weight, overweight and obesity)-based World Health Organization standardized categories. Clinicopathological factors were analyzed and compared between normal and other groups.

Results: According to the results, 2205 patients were women (85.5\%) and mean age was 47.15 years. There were no significant associations between BMI quartiles and Multifocality, cervical lymph node metastasis, or distant metastasis. Higher BMI were significantly associated with extrathyroidal extension of PTMC $(\mathrm{P}<0.001)$. And higher BMI were significantly associated with advanced TNM stage. $(\mathrm{P}=0.005)$
Conclusion: Increased BMI might elevate the risks of aggressive clinicopathological features of PTMC, such as extrathyroidal invasion and advanced TNM stage. To confirm this result, further studies with long-term follow-up and more patients are required.

Key Words: Obesity, Body mass index, Papillary thyroid microcarcinoma

\section{P1-03-24 \\ PREDICTION OF MALIGNANCY IN THYROID NODULES WITH MICROCALCIFICATION BY ULTRASONOGRAPHY}

Yoko Omi ${ }^{1}$, Momoko Sakurai ${ }^{1}$, Erin Nagai ${ }^{1}$, Hiroki Tokumitsu ${ }^{1}$, Yusaku Yoshida $^{1}$, Kiyomi Horiuchi', Takahiro Okamoto' ${ }^{1}$, Yoji Nagashima ${ }^{2}$ ${ }^{1}$ Department of Breast and Endocrine Surgery, Tokyo Women's Medical University, Tokyo, Japan, ${ }^{2}$ Department of Surgical Pathology,

Tokyo Women's Medical University, Tokyo, Japan

Introduction: Microcalcification is a feature of papillary thyroid carcinoma. However, the association between macrocalcification and malignancy remains unclear. The purpose of this study is to clarify findings of the ultrasonography (US) that predicts malignancy in thyroid nodules with macrocalcification.

Materials and Methods: We retrospectively reviewed US findings of 1172 patients who underwent US and fine-needle aspiration (FNA). Macrocalcifications were classified as i) irregular peripheral calcification (IP); ii) intrathyroidal macrocalcification ( $>=1 \mathrm{~mm}$ ) (I); iii) egg-shell like calcification (E). For IP and E, the presence of interruption of the calcification and soft tissue outside calcification rim were also evaluated.Malignancy rate was calculated for each group and compared with microcalcification $(<1 \mathrm{~mm})(\mathrm{MI})$ or non-calcified thyroid nodule (non-CTN). Results of FNA were set as the gold standard.

Results: Numbers of nodules were 106 for IP, 167 for I, 81 for E, 144 for MI and 897 for non-CTN. Malignancy rates of IP, I, E and MI were $45.6 \%$, $48.7 \%, 22.7 \%, 59.7 \%$, respectively and significantly higher than that of nonCTN $12.4 \%(p<0.05)$. There were no significant difference in malignancy rates between MI and IP or I but that of MI was higher than that of E $(p<0.01)$. Malignancy rates of IP with interruption or soft tissue rim or both were $33.8 \%$, $40.0 \%, 39.5 \%$, respectively, and were significantly higher than that of IP without interruption and soft tissue rim $14.8 \%(p<0.05)$. Malignancy rates of E with interruption or soft tissue rim or both were $13.5 \%, 13.0 \%, 12.5 \%$, respectively, and were not significantly higher than that of $\mathrm{E}$ without interruption and soft tissue rim $15.3 \%$.

Conclusion: Thyroid nodules with macrocalcification such as IP, especially IP with interruption or soft tissue rim or both and I in thyroid nodules should be examined carefully may need more further investigation as they are likely to be malignant than non-CTN.

\section{P1-03-25 \\ VARIATIONS IN BASAL THYROGLOBULIN ALLOW PREDICTION OF DISEASE PROGRESSION IN THYROID CANCER PATIENTS WITH INDETERMINATE RESPONSE TO INITIAL THERAPY}

Pasqualino Malandrino ${ }^{1}$, Dario Tumino ${ }^{1}$, Marco Russo ${ }^{2}$, Martina

Tavarelli1, Giulia Sapuppo ${ }^{1}$, Sebastiano Squatrito ${ }^{1}$, Gabriella Pellegriti ${ }^{1}$, Francesco Frasca $^{1}$

${ }^{1}$ Endocrinology, Dept. of Clinical and Experimental Medicine, GaribaldiNesima Medical Center, University of Catania, Italy, Catania, Italy, ${ }^{2}$ Endocrinology, Dept. of Clic. and Experim. Medicine, Univ. of Messina, G. Martino Policlinic, Messina, Italy, Messina, Italy

Background and Aim: Thyroid cancer (TC) patients after initial treatment (surgery $+/-{ }^{131} I$ ) are considered with either "Excellent response" (Exc-r) or "Incomplete response" (Inc-r) on the basis of the absence or presence of residual disease (American Thyroid Association, 2015). However, in the presence of nonspecific biochemical or structural clues of residual disease, TC patients are considered with "Indeterminate response" (Ind-r). To date, disease evolution in Ind-r patients is not known and the management of this category still controversial. 
Aim of the study is to evaluate the trend of thyroglobulin (Tg) during LT4 therapy (basal-Tg) in patients with Ind-r.

Methods: We analyzed data of consecutive TC patients after surgery and ${ }^{131} \mathrm{I}$, who performed $\mathrm{Tg}$ measurement after rhTSH stimulation in the period 2004-2015. We selected patients with stimulated $\mathrm{Tg}$ between 1 and $5 \mathrm{ng} / \mathrm{ml}$, negative imaging for specific disease and no further treatments. After the rhTSH test all patients were followed-up by annual basal-Tg (2nd generation assay, functional sensitivity $=0.1 \mathrm{ng} / \mathrm{ml}$ ) and neck ultrasound. Patients with stable nonspecific findings and who spontaneously resolved were named Group A. Patients who progressed were named Group B. Trend of basal-Tg in the two groups was compared by multilevel linear regression analysis.

Results: We selected 148 patients with Ind-r (38M, 110F, mean age

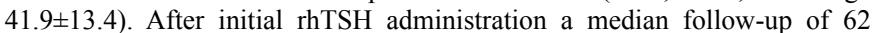
months was performed. During this follow up, 137 patients $(92.6 \%)$ were included into Group A: 73 (53.3\%) with Exc-r and 64 (46.7\%) with Ind-r. The remaining 11 patients $(7.4 \%)$ were included into Group B: $4(36.4 \%)$ with biochemical and 7 (63.4\%) with structural Inc-r. The trend of basal-Tg was significantly different between the two groups $(\mathrm{p}<0.001)$. Basal-Tg slightly decreased (mean percent change $=-4.1 \%$ ) in Group $\mathrm{A}$, whereas it increased (mean percent change $=+33.5 \%$ ) in Group B.

Conclusions: Most of patients with Ind-r (approximately 90\%) did not display any progressive disease. Trend of basal-Tg is reliable to predict disease progression after initial therapies thus allowing for a better selection of patients that require further investigations.

\section{P1-03-26 \\ DELAYED RISK STRATIFICATION OF PATIENTS WITH THYROID CANCER IN A SINGLE CLINICAL CENTRE}

Agata Baldys-Waligorska' ${ }^{1}$,Maciej Kolodziej², Monika Buziak-Bereza², Michal Koziara ${ }^{2}$, Andrzej Sokolowsk ${ }^{3}$

${ }^{1}$ Chair and Clinic of Endocrinology, Jagiellonian University,

Medical College, Endocrinology, Krakow, Poland, ${ }^{2}$ Department of

Endocrinology, Jagiellonian University Medical College, Krakow,

Poland, ${ }^{3}$ Department of Statistics, Cracow University of Economics,

Krakow, Poland

Purpose: To evaluate the risk of progression, analysis of the staging of thyroid cancer at the time of its diagnosis, prior to 131-I radioiodine treatment and one year later were undertaken, at a single clinical centre.

Materials and Methods: A total of 1008 patients were treated and followed up over the years 2000-2016 at our Clinic. For this preliminary survey,

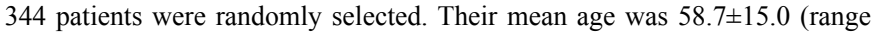
$20-88$ years), of whom $303(88.1 \%)$ were diagnosed with papillary cancer, $36(10.5 \%)$ - with follicular cancer and $4(4.6 \%)$ - with poorly differentiated cancer. All patients were treated with $3657 \mathrm{MBq}$ of 131-I after endogenous or rhTSH stimulation.

Results: The risk of progression was evaluated at the beginning of treatment, basing on the TNM classification system (UICC2010). In the investigated group, $77.1 \%$ of patients were classified as low risk, and $22.9 \%$ - as high risk. Prior to administration of radioiodine, $38.6 \%$ of patients had stimulated concentration of thyroglobulin (Tg) below $1.0 \mathrm{ng} / \mathrm{ml}$, while $61.4 \%$ of patients had $\mathrm{Tg} \geq 1.0 \mathrm{ng} / \mathrm{ml}$ (median $[\mathrm{IQR}]=6.1[10.1] \mathrm{ng} / \mathrm{ml}$ ). In the whole body scan (WBS) $98.7 \%$ of patients accumulated 131-I only in the thyroid bed, while only 4 patients $(1.3 \%)$ accumulated it elsewhere. Twelve months later all patients underwent control of efficacy of primary treatment. Excellent response (stimulated Tg $<1.0 \mathrm{ng} / \mathrm{ml}$ ) was stated in $82.4 \%$ of patients, incomplete response (stimulated $\mathrm{Tg} \geq 10 \mathrm{ng} / \mathrm{ml}$ ) $-3.6 \%$, and indeterminate response (stimulated Tg 1-10.0 ng/ml) - 14\%. Evidence of structural disease, based on WBS, was stated in $0.6 \%$ of patients.

Conclusion: One-year delayed risk stratification of DTC patients, together with their known response to primary treatment, enables better individual evaluation of further risk of disease progression.
P1-03-27

THE FACTORS FOR PREDICTING THE PATTERN

OF LYMPHATIC METASTASIS IN PAPILLARY

THYROID CANCER: PROSPECTIVE STUDY

Min Sik Kim ${ }^{1}$, Kwang-Jae Cho ${ }^{1}$, Jun-Ook Park ${ }^{1}$, Young-Hoon Joo ${ }^{1}$

${ }^{1}$ The Catholic University of Korea, Department of Otolaryngology-Hns,

Seoul, Korea, Rep. of South

Objective: To evaluated the predictive factors and the distribution of lymphatic metastasis to explore the surgical managing modality for papillary thyroid carcinoma.

Methods: Prospectively evaluated the clinical and pathological data of 265 patients (56 male and 209 female) with receiving bilateral paratracheal lymph node(LN) dissection, and patients with radiological positive cervical node received selective neck dissection(I-IV) of involved side. Analysis of central LN involvement and clinical and molecular factors for predictive of nodal metastasis were performed.

Results: For the central compartment, $81 / 265(30.6 \%)$ of cases showed metastatic disease at the ipsilateral paratracheal nodal site, and 11(4.2\%) at the contralateral paratracheal site. Only ipsilateral paratracheal node metastasis was significantly correlated with contralateral paratracheal node metastasis $(\mathrm{p}=0.02)$. For the lateral compartment, 65 patients who were clinical N1b received selective neck dissection, and 30 patients bilaterally. 25 patients(38.4\%) showed multilevel disease, and 20(7.5\%) showed skip lateral metastasis. Sex(male) and extracapsular invasion were significantily correlated with $\mathrm{N}$ stage $(\mathrm{p}=0.02,0.03)$.

Conclusions: Ipsilateral central LN metastasis is present, the possibility of contralateral central LN involvement is high. Therefore, complete bilateral central LN dissection would be a proper option. Lateral cervical metastasis frequently occurs in patients of male sex, with extracapsular invasion, and with ipsilateral central LN metastasis. More precise pre-operative evaluation should be needed in those patients for lateral LN metastasis.

\section{P1-04 Goitre and Nodules 1}

\author{
P1-04-28 \\ WITHDRAWN
}




\section{P1-04-29 \\ DIAGNOSTIC PERFORMANCE OF ULTRASOUND- BASED FINE NEEDLE ASPIRATION BIOPSY CRITERIA FOR THYROID MALIGNANCY: A COMPARATIVE STUDY BASED ON SIX INTERNATIONAL SOCIETY GUIDELINE}

\section{Eun Ju Ha${ }^{1}$, Donggyu $\mathrm{Na}^{2}$}

${ }^{1}$ Ajou University School of Medicine, Department of Radiology, Suwon, Korea, Rep. of South, ${ }^{2}$ Human Medical Imaging \& Intervention Center, Seoul, Korea, Rep. of South

Purpose: To investigate the diagnostic performances of ultrasound (US)based fine needle aspiration (FNA) criteria for detecting thyroid cancer and to compare the unnecessary FNA rates based on six international society guidelines (ATA, AACE/AME/ETA, FSE, KTA/ KSThR, NCCN, and SRU)

Materials and Methods: From January 2010 to May 2011, a total of consecutive 2000 thyroid nodules $(\geq 1 \mathrm{~cm})$ with final diagnoses in 1802 patients were included in this study. US features of the thyroid nodules were retrospectively reviewed and classified according to the categories defined by the six international society guidelines. The diagnostic performance of US-based FNA criteria for detecting thyroid cancer and unnecessary FNA rate was calculated and compared by using the generalized estimating equation method.

Results: Of the 2000 thyroid nodules, 1546 (78.3\%) were benign and 454 $(22.7 \%)$ were malignant. Sensitivity and negative predictive value (NPV) for detecting thyroid cancer was the highest with $\mathrm{KTA} / \mathrm{KSThR}$ guideline $(94.5 \%$ and $94.2 \%, \mathrm{P}<0.001)$ followed by NCCN $(92.5 \%$ and $93.2 \%)$, ATA $(87.6 \%$ and $91.6 \%$ ), AACE/AME/ETA ( $80.4 \%$ and $91.0 \%)$, FSE $(72.7 \%$ and $88.6 \%$ ), and SRU guidelines $(70.9 \%$ and $82.9 \%)$. The unnecessary FNA rates was the lowest in FSE $(29.1 \%, \mathrm{P}<0.001)$ followed by AACE/AME/ETA $(32.5 \%)$, SRU (45.2\%), ATA (51.7\%), NCCN (54.0\%), and KTA/KSThR guidelines $(56.9 \%)$.

Conclusion: Since the diagnostic performance of US-based FNA criteria are different based on the international society guidelines, we have to be aware of its strength and weakness in the management of thyroid nodules.

\section{P1-04-30 \\ THYROIDECTOMY IMPROVES TRACHEAL ANATOMY AND AIRFLOW IN PATIENTS WITH NODULAR GOITER. A PROSPECTIVE COHORT STUDY}

Jesper Roed Sørensen ${ }^{1}$, Jeppe Faurholdt Lauridsen², Helle Døssing ${ }^{3}$, Nina Nguyen ${ }^{4}$, Laszlo Hegedüs ${ }^{5}$, Steen Joop Bonnema ${ }^{6}$, Christian Godballe ${ }^{7}$

${ }^{1}$ Department of Orl Head and Neck Surgery, Odense University Hospital, Denmark, Odense C, Denmark, ${ }^{2}$ Department of Nuclear Medicine, Odense University Hospital, Odense, Denmark, ${ }^{3}$ Odense University Hospital, Department of Orl Head \& Neck Surgery, Department of Orl Head \& Neck Surgery, Odense, Denmark, ${ }^{4}$ Department of Radiology, Odense University Hospital, Odense, Denmark, ${ }^{5}$ Department of Endocrinology and Metabolism, Odense University Hospital, Odense, Denmark, ${ }^{6}$ Department of Endocrinology and Metabolism, Odense University Hospital, Department of Clinical Research, Faculty of Health Sciences, University of Southern Denmark, Odense C, Denmark, ${ }^{7}$ Dept. of Orl - Head \& Neck Surgery, Odense University Hospital, Odense, Denmark

Objectives: A large goiter may cause compression of the trachea and lead to respiratory insufficiency. We aimed at investigating the effects of thyroidectomy on tracheal anatomy and airflow in patients with benign nodular goiter, employing a prospective observational study.

Methods: Magnetic resonance images (MRI) of the neck and respiratory flow-volume curves, including both in- and expiration, were performed prior to and six months following surgery. The evaluated tracheal dimensions included the smallest cross-sectional area of the trachea (SCAT), tracheal narrowing, and tracheal deviation. Effect sizes (ES) were calculated as mean change divided by standard deviation at baseline. ES of 0.2-0.5 were defined as small, $0.5-0.8$ as moderate, and values $>0.8$ as large.

Results: Sixty-five patients completed all examinations. Median goiter volume was $58 \mathrm{~mL}$ (range, 14-642 $\mathrm{mL}$ ) before surgery with surgical removal of median $46 \mathrm{~mL}$ (range, $5-642 \mathrm{~mL})(\mathrm{p}<0.001)$. Six months post-surgery, tracheal narrowing and deviation were diminished by median $26 \%$ ( $\mathrm{ES}=0.67)$, and $33 \%(\mathrm{ES}=0.73)$, respectively, while SCAT increased by $17 \%(\mathrm{ES}=0.61)$ (Table 1). Correspondingly, each $10 \%$ decrease in goiter volume resulted in an increase of $6.0 \%$ in SCAT ( $<<0.001$ ), and a decrease of $1.0 \%$ in tracheal narrowing $(\mathrm{p}<0.001)$. Concomitantly, a small improvement was seen in forced inspiratory flow at $50 \%$ of forced vital capacity $\left(\mathrm{FIF}_{50 \%}\right)(\mathrm{ES}=0.32)$.

Conclusions: In patients with symptomatic benign nodular goiter, thyroidectomy leads to substantial improvement in tracheal compression, but only minor improvements in tracheal airflow. This information is pertinent when counselling patients before choice of treatment.

Table 1 Tracheal and respiratory parameters (median(range) and mean \pm SD) for 65 patients with nodular goiter

\begin{tabular}{lccc}
\hline Parameters & Before surgery & After surgery & $p$-value \\
\hline Tracheal narrowing $(\%)$ & $39(0-90)$ & $29(0-54)$ & $<0.001$ \\
Tracheal deviation $(\mathrm{mm})$ & $12 \pm 7$ & $8 \pm 4$ & $<0.001$ \\
Tracheal volume $(\mathrm{mL})$ & $18.2 \pm 5$ & $20.3 \pm 6$ & $<0.001$ \\
SCAT $\left(\mathrm{cm}^{2}\right)$ & $1.5 \pm 0.5$ & $1.8 \pm 0.5$ & $<0.001$ \\
CCAT $\left(\mathrm{cm}^{2}\right)$ & $2.5 \pm 0.7$ & $2.6 \pm 0.7$ & 0.17 \\
$\mathrm{FIF}_{50 \%}(\mathrm{~L} / \mathrm{min})$ & $4.8 \pm 1.4$ & $5.3 \pm 1.5$ & $<0.001$ \\
$\mathrm{FEF}_{50 \%}(\mathrm{~L} / \mathrm{min})$ & $3.5 \pm 1.4$ & $3.4 \pm 1.3$ & 0.03 \\
$\mathrm{FEF}_{50 \%} / \mathrm{FIF}_{50 \%}$ & $0.8 \pm 0.3$ & $0.7 \pm 0.3$ & 0.001 \\
\hline
\end{tabular}

Comparison of values before and six months after surgery. SCAT: Smallest cross-sectional area of the trachea. CCAT: Cross-sectional area two $\mathrm{cm}$ above the carina.* Tracheal narrowing (degree of upper airway obstruction) is defined as (CCAT-SCAT)/CCAT. FIF $_{50 \%}$ : Forced inspiratory flow at $50 \%$ of forced vital capacity. $\mathrm{FEF}_{50 \%}$ : Forced expiratory flow at $50 \%$ of forced vital capacity.

\section{P1-04-31 \\ EARLY VOICE QUALITY CHANGES FOLLOWING SINGLE-SESSION HIGH INTENSITY FOCUSED ABLATION (HIFU) VERSUS OPEN CERVICAL HEMITHYROIDECTOMY FOR SYMPTOMATIC BENIGN THYROID NODULE: A PROPENSITY- MATCHED ANALYSIS}

Brian Lang ${ }^{1}$, Carlos Wong ${ }^{2}$, Estella Ma $^{3}$

${ }^{1}$ Department of Surgery, University of Hong Kong, Queen Mary Hospital, Hong Kong, Hong Kong, ${ }^{2}$ Department of Family Medicine and Primary Care, University of Hong Kong, Hong Kong, Hong Kong, ${ }^{3}$ Division of Speech and Hearing Sciences, University of Hong Kong,

Hong Kong, Hong Kong

Background: High intensity focused ultrasound (HIFU) is a promising non-surgical treatment for symptomatic benign thyroid nodule. However, its impact on voice quality afterwards has remained unclear. The present study aimed to evaluate changes in acoustic voice parameters and subjective voice quality in the first 6 months following single-session of HIFU ablation and to compare them with a similarly matched cohort who had undergone an open hemithyroidectomy.

Methods: Consecutive patients who underwent one-sided HIFU ablation or unilateral thyroid resection for symptomatic benign thyroid nodule were analyzed. Voice quality was assessed by a computerized multi-dimensional voice program and a Voice Handicap Index (VHI)-30 questionnaire at 1-, 3and 6-months after treatment. Propensity-score matching was used to minimize potential biases. Propensity scores were calculated from patient age, sex and size of dominant nodule. The primary outcome was the 6-month change in the 5 acoustic parameters from baseline and these 5 acoustic parameters were fundamental frequency $\left(\mathrm{F}_{0}\right)$, Jitter, Shimmer, Noise-to-Harmonic ratio and maximum phonation time.

Results: The propensity-score matched cohort comprised 60 HIFUs and 60 hemithyroidectomies. After adjusting for age, sex and size of the dominant nodule, the surgical group was more likely to have a significant deterioration ( $>2 \%$ worsening from baseline) in mean $\mathrm{F}_{0}(\mathrm{OR}=2.49,95 \% \mathrm{CI}=1.33-6.37$, $p=0.001)$, minimum $\mathrm{F}_{0}(\mathrm{OR}=1.78,95 \% \mathrm{CI}=1.42-4.87, \mathrm{p}=0.021)$ and Jitter $(\mathrm{OR}=10.43,95 \% \mathrm{CI}=3.26-20.31, p<0.001)$ than HIFU group in the first 3 
months of treatment. However, the 6-month acoustic parameters and VHI-30 score did not significantly from baseline or between the two groups.

Conclusions: Relative to those who underwent HIFU ablation, patients who underwent an open hemithyroidectomy for a symptomatic benign thyroid nodule had significantly lower pitched voice and roughness of voice in the first 3 months. However, these voice disturbances became perceptually less obvious in the latter 3 months. HIFU ablation. This study has provided a strong case for HIFU ablation as a treatment for symptomatic benign thyroid nodule.

\section{P1-04-32 \\ THYROID NODULES ARE A DISCRETE INTRINSIC DISORDER THAT DOES NOT INVOLVE HYPERPLASIA AT THE WHOLE-GLAND LEVEL}

Giorgio Grani 1 , Giuseppe Lucisano ${ }^{2}$, Rocco Bruno ${ }^{3}$, Giuseppe Costante $^{4}$, Domenico Meringolo ${ }^{5}$, Alessandra Paciaroni ${ }^{1}$, Efisio Puxeddu 6 , Massimo Torlontano ${ }^{7}$, Salvatore Tumino ${ }^{8}$, Marco Attard ${ }^{9}$, Livia Lamartina' ${ }^{1}$, Antonio Nicolucci ${ }^{2}$, Sebastiano Filetti' ${ }^{1}$ Cosimo Durante ${ }^{1}$

${ }^{1}$ University of Rome Sapienza, Rome, Italy, ${ }^{2}$ Center for Outcomes Research and Clinical Epidemiology, Pescara, Italy, ${ }^{3}$ Unità DI Endocrinologia, Ospedale DI Tinchi-Pisticci, Matera, Italy, ${ }^{4}$ Institut Jules Bordet, Université Libre de Bruxelles, Medicine, Brussels, Belgium,

${ }^{5}$ Uosd Endocrinologia, Ospedale Bentivoglio, Bologna, Italy, ${ }^{6}$ University of Perugia, Perugia, Italy, ${ }^{7}$ Uo Endocrinologia, Casa Sollievo Della Sofferenza, San Giovanni Rotondo (Foggia), Italy, ${ }^{8}$ Dip. DI Medicina Clinica e Sperimentale, Università DI Catania, Catania, Italy, ${ }^{9}$ Uo Endocrinologia, Ao Ospedali Riuniti Villa Sofia-Cervello, Palermo, Italy

Background: The term "nodular goiter" is used to refer to the presence of thyroid nodules on the assumption that nodule growth is associated with hyperplasia of the surrounding non-nodular tissue. Our aim was to determine whether this assumption is correct.

Methods: Between January 2006 and January 2008, 8 Italian centers consecutively enrolled 992 euthyroid patients with 1-4 presumably benign nodules and no autoimmune thyroidopathy. Patients were followed annually for 5 years with sonographic measurements of thyroid, dominant thyroid nodule, and thyroid lobe volumes. Goiter was defined as thyroid volumes $>2$ SD of the mean in healthy Italians. Growth trends over time were evaluated using a mixed model for repeated measurements.

Results: In 330 cases, baseline thyroid volumes were classified as goiter; 21 other cases met the definition after 5 years. In the 839 patients whose nodules remained stable or shrank, mean thyroid volume remained unchanged (baseline: $15.0 \mathrm{~mL}$; year 5: $15.1 \mathrm{~mL}$ ). In the 153 participants with significant growth of one or more nodules, mean thyroid volume increased linearly and was significantly greater by year $5(17.4 \mathrm{~mL}$ vs. $15.1 \mathrm{~mL}$ at baseline, $\mathrm{p}<0.001)$. In 76 individuals with unilateral nodules that grew, the mean volume of the unaffected lobe remained stable (baseline: $6.7 \mathrm{~mL}$; year 5: $6.9 \mathrm{~mL}$ ). Mean volume of the nodular lobes increased steadily and was significantly greater by year 5 (baseline: $8.6 \mathrm{~mL}$, year $5: 10.1 \mathrm{~mL}, \mathrm{p}<0.001$ ). If the volume of the largest growing nodule was subtracted from the affected lobe volume, growth of affected lobe volume disappeared.

Conclusions: "Nodular goiter" is a misnomer for the condition described above. Gland enlargement is generally absent. Moreover, thyroid nodule growth is a focal phenomenon and not associated with growth of the surrounding non-nodular tissue. This condition is more appropriately referred to as "nodular thyroid gland".

\section{P1-04-33 \\ NEAR INFRARED AUTOFLUORESCENCE IMAGE GUIDED PARATHYROID GLAND NAVIGATION IN THYROIDECTOMY}

Kang Dae Lee ${ }^{1}$, Sung Won Kim², Hyoung Shin Lee ${ }^{2}$, Chan Woo Park ${ }^{2}$ Chulho Oak ${ }^{3}$, Yikeun Kim ${ }^{4}$, Seong Keun Lee ${ }^{5}$, Yeh-Chan Ahn ${ }^{4}$, Seok Won Jeon ${ }^{2}$

${ }^{1}$ Department of Otolaryngology-Head and Neck Surgery, Kosin University College of Medicine, Busan, Rep. of South Korea, ${ }^{2}$ Department of Otolaryngology-Head and Neck Surgery, Kosin University College of Medicine, Busan, Rep. of South Korea, ${ }^{3}$ Department of Internal Medicine, Kosin University College of Medicine, Busan, Rep. of South Korea, ${ }^{4}$ Department of Biomedical Engineering, Pukyong National University, Busan, Rep. of South Korea, ${ }^{5}$ Department of Endocrinology, Isam Hospital, Busan, Rep. of South Korea

Objectives: Near infrared(NIR) autofluorescence(AF) imaging for parathyroid gland(PG) localization without exogenous dye showed promising outcomes. Current studies demostrated if surgically identified PGs are autofluorescent to NIR. However, it is more important to predict location of PGs before they are surgically exposed by naked eye. We investigated of the feasibility of PG mapping with NIR AF imaging technique to localize unexposed PGs.

Methods: Seventy PGs from 38 thyroidectomy patients were enrolled in this prospective study. NIR imaging was taken at the areas where PGs were predicted to exist. We named the procedure as Parathyroid Navigation. The parathyroid navigation was photographed in three steps. Step P1, taking images at pre-identification by naked eye, step $\mathrm{P} 2$, taking images at post-identification by naked eye, and step P3, as taking images in removed specimens.

Results: Of the 69 PGs identified, 64 (92.8\%) were found at step P1, 4 $(5.8 \%)$ at step P2, and $1(1.4 \%)$ at step P3. Even when the PGs were covered by connective or fat tissues, NIR navigation at step P1 showed the sensitivity ( $92.75 \%)$, specificity (100\%), PPV (100\%), NPV (16.66\%), accuracy ( 92.85 $\%)$. Five PGs of step P1 negative were identified at step P2 and P3 revealing $100 \%$ of total accuracy rate. The average parathyroid/background ratio was 4.78 .

Conclusions: These results suggest the concept of parathyroid navigation is feasible. We believe surgeons can get benefits of preserving parathyroid gland with the use of our NIR imaging method.

\section{P1-04-34 \\ PROSPECTIVE VALIDATION OF ATA ULTRASOUND RISK ASSESSMENT OF THYROID NODULES SELECTED FOR FINE NEEDLE ASPIRATION: PRELIMINARY RESULTS}

Fabio Maino $^{1}$, Maria Grazia Castagna ${ }^{1}$, Martina Martinelli ${ }^{1}$, Raffaella

Forleo $^{1}$, Filomena Barbato ${ }^{1}$, Noemi Fralassi ${ }^{1}$, Furio Pacini ${ }^{1}$

${ }^{1}$ Uoc Endocrinology; Department of Medical, Surgical and Neurological Sciences, University of Siena, Siena, Italy

Fine needle aspiration cytology (FNAC) is the procedure of choice in the evaluation of thyroid nodules. According to the American Thyroid Association (ATA) guidelines, thyroid ultrasound (US) should be used to stratify the risk of malignancy in thyroid nodules, and to aid decision-making about whether FNA is indicated.

Objective: the aim of our study is to validate the ATA US pattern risk based on sonographic pattern of thyroid nodules undergoing FNAC with evaluation of the results based on the Bethesda System for Reporting Thyroid Cytopathology.

Methods and Results: we prospectively evaluated thyroid nodules selected for FNAC and classified according to ATA sonographic pattern risk. There were 218 patients (171 females, 47 males) and 274 thyroid nodules. Nodules were classified as High $(2.9 \% ; n=8)$, Intermediate $(25.2 \% ; n=69)$, Low $(27.7 \% ; \mathrm{n}=76)$, Very Low $(42.7 \% ; \mathrm{n}=117)$ risk of malignancy and Benign $(1.4 \% ; n=4)$. Cytological results were available in $219 / 274$ thyroid nodules $(79.6 \%)$. Thy1 $(16 / 219,7.3 \%)$ nodules were excluded from the statistical analyses. The distribution of Bethesda cytology classification according to ATA sonographic pattern risk is reported below. 


\begin{tabular}{llllll}
\hline \multicolumn{5}{c}{ Cytology } & \multicolumn{1}{l}{ ATA US pattern risk } \\
\cline { 2 - 6 } & $\begin{array}{l}\text { Benign } \\
(\mathrm{n}=4)\end{array}$ & $\begin{array}{l}\text { Very Low } \\
\text { suspicion } \\
(\mathrm{n}=83)\end{array}$ & $\begin{array}{l}\text { Low } \\
\text { suspicion } \\
(\mathrm{n}=65)\end{array}$ & $\begin{array}{l}\text { Intermediate } \\
\text { suspicion } \\
(\mathrm{n}=48)\end{array}$ & $\begin{array}{l}\text { High } \\
\text { suspicion } \\
(\mathrm{n}=3)\end{array}$ \\
\hline Thy2 & $100 \%(4 / 4)$ & $97.6 \%(81 / 83)$ & $93.8 \%(61 / 65)$ & $69.8 \%(35 / 48)$ & $0 \%$ \\
Thy3 & $0 \%$ & $1.2 \%(1 / 83)$ & $4.6 \%(3 / 65)$ & $18.8 \%(9 / 48)$ \\
Thy4-5 & $0 \%$ & $1.2 \%(1 / 83)$ & $1.6 \%(1 / 65)$ & $8.3 \%(4 / 48)$ & $66.7 \%(1 / 3)$ \\
\hline
\end{tabular}

The rate of malignant/suspicious cytology in lower risk category (Benign, Very Low and Low suspicion) was very low, with a negative predictive value of $98,6 \%$

Conclusions: this prospective study validates the new ATA sonographic pattern risk assessment for selection of thyroid nodules for FNAC based on the Bethesda cytology. These results should be validated in surgical series.

\section{P1-04-35 \\ COMPUTER-AIDED DIAGNOSIS SYSTEM FOR THYROID NODULES ON ULTRASOUND: INITIAL EXPERIENCE}

Eun Ju Ha ${ }^{1}$, Miran $\mathrm{Han}^{1}$

${ }^{1}$ Ajou University School of Medicine; Department of Radiology, Suwon,

Rep. of South Korea

Purpose: To prospectively evaluate the diagnostic performance of a computer-aided diagnosis (CAD) system (S-Detect for Thyroid ${ }^{\mathrm{TM}}$ ) for thyroid US in the differential diagnosis of thyroid nodules and to determine interobserver agreement between the $\mathrm{CAD}$ and experienced radiologist.

Materials and Methods: This study was approved by the ethics committee of the institution, and all patients provided written informed consent. We consecutively enrolled patients with thyroid nodules with decisive diagnosis whether benign or malignant between June 2016 and July 2016. An experienced radiologist reviewed US images characteristics of thyroid nodules in a prospective design, and a CAD system additionally provided nodule diagnosis whether benign or malignant. We compared the diagnostic performance of experienced radiologist, CAD system, and CAD system assisted radiologist for thyroid cancer and determined interobserver agreement between the CAD and experienced radiologist.

Results: A total of 117 thyroid nodules from 50 consecutive patients were included. The mean size of nodules was $1.5 \pm 1.1 \mathrm{~cm}$ (range: $0.5-10.0 \mathrm{~cm}$ ) and final diagnoses were $67(57.3 \%)$ benign nodules and $50(42.7 \%)$ malignant nodules. The CAD system showed similar sensitivity and specificity compared with the experienced radiologist (sensitivity: $80.0 \%$ versus $87.0 \%, \mathrm{P}=0.754$; specificity: $88.1 \%$ versus $95.5 \%, \mathrm{P}=0.180$ ). The diagnostic accuracy was not significantly different between the CAD and experienced radiologist $(84.6 \%$ versus $90.6 \%, \mathrm{P}=0.646$ ). The $\mathrm{CAD}$ system assisted radiologist reached the significant higher diagnostic sensitivity when compared to the radiologist without the CAD system $(91.8 \%$ versus $87.0 \%, \mathrm{P}=0.031)$. The interobserver agreement between radiologist and $\mathrm{CAD}$ system were substantial agreement for a final diagnosis (kappa $=0.661$ ).

Conclusion: The diagnostic performance of CAD system for differentiating thyroid nodule was as good as that of the experienced radiologist with a substantial agreement. The CAD system assisted radiologist could achieve the highest diagnostic sensitivity for thyroid cancer.

\section{P1-04-36 \\ ASSESSMENT OF THE EFFICACY OF THE BRITISH THYROID ASSOCIATION'S 2014 GUIDELINES FOR SONOGRAPHIC ASSESSMENT OF THYROID NODULES}

\section{Henry Sheppard ${ }^{1}$, Shayan Ahmed ${ }^{2}$, Osama Omrani', Pauline Rajan ${ }^{2}$}

${ }^{1}$ Barts and The London School of Medicine and Dentistry, Queen Mary University of London, London, UK, ${ }^{2}$ Princess Alexandra Hospital NHS Trust, Harlow, UK

Objectives: We aimed to assess the efficacy of the 2014 British Thyroid Association's guidelines for the sonographic assessment of thyroid nodules using the 'U-grade' scoring system as a predictor of neoplasm.
Methods: A retrospective cohort analysis was performed, including all thyroid and neck ultrasounds which identified thyroid nodules. Results were divided into two groups consisting of samples taken six months pre- and postguideline introduction (10/2014), comparing number of nodules found, FNAs performed and neoplasms identified. For the post-guideline group, U-grades (if provided) were collated and, if FNA was performed, corresponding cytological Thy-grades were recorded. We then assessed whether U-grading was a suitable predictor of neoplasia using Receiver Operating Characteristic (ROC) analysis. We classified a Thy-grade of $\geq 3 \mathrm{~F}$ as neoplastic and $<3 \mathrm{~F}$ as non-neoplastic. A U-grade of $\geq 3$ was classified as a positive result.

Results: In the pre-guideline group, 303 nodules were assessed and 83 (27\%) went to FNA. Of these, $23(28 \%)$ were found to be neoplastic. In the post-guideline group, a total of 264 thyroid nodules were identified with 81 (31\%) receiving an FNA and $19(23 \%)$ demonstrating neoplasia. The table below shows the U-grade and corresponding Thy-grade, where applicable, of each nodule. Eleven patients with U2 nodules received FNA for reasons such as debulking. Thirteen patients with U3-5 nodules were lost to follow up and hence, there is no cytology data available. ROC analysis produced an average area under the curve of $0.724(\mathrm{p}=0.004,95 \%$ CI $0.624-0.823)$.

\begin{tabular}{llcllllllr}
\hline & Thy1 & Thy2 & $\begin{array}{l}\text { Thy } \\
\text { 2C }\end{array}$ & $\begin{array}{l}\text { Thy } \\
3 \mathrm{~A}\end{array}$ & $\begin{array}{l}\text { Thy } \\
3 \mathrm{~F}\end{array}$ & Thy4 & Thy5 & $\begin{array}{l}\text { No } \\
\text { FNA }\end{array}$ & Total \\
\hline U2 & 3 & 6 & 2 & 0 & 0 & 0 & 0 & 40 & 51 \\
U3 & 1 & 39 & 3 & 2 & 4 & 0 & 2 & 12 & 63 \\
U4 & 1 & 2 & 1 & 1 & 6 & 1 & 2 & 1 & 15 \\
U5 & 0 & 0 & 0 & 0 & 0 & 0 & 1 & 0 & 1 \\
Total & 5 & 47 & 6 & 3 & 10 & 1 & 5 & 53 & 130 \\
\hline
\end{tabular}

Conclusions: As recommended by the 2014 BTA guidelines, the use of U-grade classification for assessment of thyroid nodules is a good predictor of neoplasm. There has been little change in the number of FNAs performed or neoplasms identified since introduction of the guidelines.

\section{P1-04-37 \\ RECOVERY OF PARATHYROID AND \\ LARYNGEAL FUNCTION IN PATIENTS WITH DIFFERENTIATED THYROID CANCER AFTER ROBOTIC THYROIDECTOMY COMPARED WITH OPEN THYROIDECTOMY: A PROPENSITY SCORE MATCHING ANALYSIS}

\section{Dongsik Bae ${ }^{1}$, Do Hoon Koo}

${ }^{1}$ Department of Surgery, Haeundae Paik Hospital, Inje University

College of Medicine, Busan, Rep. of South Korea

Background: The aim of this study, from a surgical and oncologic perspective, was to identify whether robotic total thyroidectomy (RTT) has any differences compared with open thyroidectomy (OTT).

Methods: Seven hundred fifty-six patients who were eligible for total thyroidectomy were primarily reviewed in Haeundae-Paik Hospital between July 2010 and December 2015 by three surgeons using both robotic and open Methods: For removing the effects of selection bias, we adjusted the clinicopathologic characteristics (sex, age, BMI, laterality of CND, tumor size, extrathyroidal extension and thyroiditis) using 2:1 propensity score matching.

Results: Propensity-based matching consisted of 123 patients with robot group and 246 patients matched with open group with standardized differences in patient clinicopathologic characteristics of less than $10 \%$, indicating a high degree of similarity in the distributions of covariates. There were no differences in postoperative complication rate and oncologic results of between two groups except mean operation time $(198.39 \pm 37.93$ vs. 123.51 \pm 32.63 (minutes); $P<0.001$ ). But laryngeal nerve function and hypoparathyroidism median recovery time was short in robot group compared with open group ( $81.62 \pm 50.04$ vs. $133.75 \pm 80.83$ (days); $P=0.042$ and $86.49 \pm$ $69.67 \mathrm{vs} .139 .21 \pm 140.65$ (days); $P=0.033$ ), respectively.

Conclusion: Although the difference not measured in the incidence of postoperative complications and results of oncology between RTT and OTT, but the recovery time of parathyroid and laryngeal function was short in RTT. 


\section{P1-05 Hyper- and Hypothyroidism}

P1-05-38

\section{LIPID PARAMETERS IN PATIENTS WITH} AUTOIMMUNE THYROIDITIS OF HASHIMOTO

Mira Valentinova Siderova ${ }^{1}$, Gergana Tosheva ${ }^{1}$, Kiril Hristozov ${ }^{1}$, Yana Bocheva $^{2}$

${ }^{1}$ University Hospital „St. Marina“, Medical University Varna, Department of Endocrinology, Varna, Bulgaria, 'University Hospital „St.Marina“,

Medical University Varna, Central Clinical Laboratory, Varna, Bulgaria

The aim of this retrospective study was to assess the lipid disturbances in patients with autoimmune thyroiditis.

Methods: 1380 patients with autoimmune thyroiditis attending the Endocrinology department of "St. Marina" Hospital - Varna for the period of 2004-2015 year, participated in the study. After excluding conditions influencing lipid profile, 771 patients remained for analysis (36 men and 735 women, mean age 49,81 $\pm 13,98$ years). They were divided in three groups according to TSH values - group A (0,4-4 mU/l), group B (4,01-10 mU/l), group C $(\mathrm{TSH} \geq 10,01 \mathrm{mU} / 1)$. Group B was further divided into two subgroups - B1 patients with negative thyroperoxidase antibodies (TPO-Ab) and B2 - patients with positive TPO-Ab. We evaluated thyroid ultrasound data, laboratory tests of TSH, free $\mathrm{T} 3\left(\mathrm{FT}_{3}\right)$, free $\mathrm{T} 4\left(\mathrm{FT}_{4}\right)$, TPO-Ab, total cholesterol (TC), triglycerides (TG), LDL - and HDL-cholesterol levels.

Results: With the increase of TSH value, we observed elevation in serum triglycerides (group A- TG 1,34 mmol/l, group B - 1,44 mmol/l, group C $1,69 \mathrm{mmol} / \mathrm{l}$ ); as well as in LDL-c values (group A $-3,41 \mathrm{mmol} / \mathrm{l}$; group B$3,53 \mathrm{mmol} / \mathrm{l}$; group C- $4,19 \mathrm{mmol} / \mathrm{l})$. The comparison of the two subgroups B1 and B2 found out increasing levels of TC, TG, LDL-c and a decreasing of HDL-c. A significant difference in TG between patients in group $\mathrm{A}$ and $\mathrm{C}$ was observed $(p=0,001)$. The difference in LDL-c level was statistically significant between groups $A$ and $C(p=0,000)$, groups $B$ and $C(p=0,000)$, and groups $B 2$ and $C(p=0,001) .16,9 \%$ of the patients from group $B$ and $17,1 \%$ from group $C$ had already coronary heart disease and/or cerebrovascular disease.

Conclusions: Autoimmune thyroiditis as one of the main reasons for hypothyroidism is associated with lipid abnormalities and increased cardiovascular risk, which are more pronounced in overt than in subclinical hypothyroidism.

\section{P1-05-39 \\ COMPARATIVE STUDY ON THE EFFICACY OF TWO DIFFERENT DOSIMETRY TECHNIQUES IN PATIENTS WITH GRAVES‘ DISEASE (GD) SUBMITTED TO RADIOIODINE THERAPY (RAI)}

Irene Campi ${ }^{1}$, Danila Covelli ${ }^{2}$, Guia Vannucchi ${ }^{3}$, Virgilio Longari ${ }^{4}$, Cristina Canzi ${ }^{5}$, Laura Fugazzola ${ }^{6}$, Nicola Currò ${ }^{7}$, Mario Salvi ${ }^{8}$ ${ }^{1}$ Irccs, Istituto Auxologico Italiano, Division of Endocrine and Metabolic Diseases, Department of Pathophysiology and Transplantation, University of Milan, Milan, Italy, ${ }^{2} 1$ graves' Orbitopathy Unit, Endocrinology, Fondazione Ca' Granda Irccs, University of Milan, Italy, Medical Sciences, Milano, Italy, ${ }^{3} 1$ department of Endocrine and Metabolic Diseases, Irccs Istituto Auxologico Italiano, Milan, Irccs Istituto Auxologico Italiano, Milano, Italy, ${ }^{4}$ Fondazione Irccs Ca' Granda, Ospedale Maggiore Policlinico, Department of Nuclear Medicine, Milan, Italy, ${ }^{5}$ Fondazione Irccs Ca' Granda, Ospedale Maggiore Policlinico, Department of Nuclear Medicine, Milan, ${ }^{6}$ Dept. of Pathophysiology and Transpl, Istituto Auxologico Italiano Irccs, University of Milan, Milano, Italy, ${ }^{7}$ Ophthalmology, Fondazione Irccs Cà Granda, Milan, Italy, ${ }^{8}$ Dipartimento Scienze Mediche, Endocrinologia, Endocrine Unit, Fondazione Irccs Cà Granda, Milano, Italy

Objective: compare the outcome of RAI performed with fixed or calculated doses as measured by dosimetry in patients with GD.

Methods: 29 patients were randomized in two groups depending on the time of methimazole withdrawal one day (Group 1) or ten days (Group 2) ahead of dosimetry. We measured thyroid function when dosimetry started (T0), at the time of RAI (T1), at 45, 90 and 180 days after RAI. Calculated dose was estimated considering the thyroid volume (TV) and the RAI uptake. All patients received prednisone prophylaxis.
Results: the results are summarized in this table. Data are expressed as mean \pm SD

\begin{tabular}{llll}
\hline & Group 1 & Group 2 & P \\
\hline N & 14 & 15 & \\
Age & $51 \pm 16$ & $44 \pm 14$ & NS \\
activity (MBq) & $483 \pm 135$ & $452 \pm 116$ & NS \\
Delivered dose (MBq) & $317 \pm 79$ & $362 \pm 64$ & NS \\
TRAb (U/l) & $7.8 \pm 13.5$ & $9.7 \pm 16.7$ & NS \\
Baseline TV (ml) & $23 \pm 15$ & $19 \pm 9$ & NS \\
Reduction of TV & $48 \pm 17 \%$ & $62 \pm 12 \%$ & NS \\
FT3 (pg/ml) T0 & $3.6 \pm 0.99$ & $7.9 \pm 7.1$ & 0.008 \\
FT4 (pg/ml) T0 & $12.1 \pm 2.8$ & $23.9 \pm 19.2$ & 0.01 \\
FT3 (pg/ml) T1 & $8.5 \pm 8.4$ & $8.6 \pm 6.9$ & NS \\
FT4 (pg/ml) T1 & $25.4 \pm 18.8$ & $26.8 \pm 18.4$ & NS \\
Response rate (45 d) & $33 \%$ & $36 \%$ & NS \\
Response rate (90 d) & $53 \%$ & $64 \%$ & NS \\
hypothyroidism (180 d) & $73 \%$ & $71.4 \%$ & NS \\
euthyroidism (180 d) & $7 \%$ & $14.3 \%$ & NS \\
hyperthyroidism (180 d) & $20 \%$ & $14.3 \%$ & NS \\
\hline
\end{tabular}

Conclusion: our study suggests that RAI is equally effective whether methimazole withdrawal was 1 or 10 days before dosimetry. The longer duration from withdrawal in Group 2 induces worsening of hyperthyroidism with potentially harmful thyrotoxicosis. Calculated doses allow the administration of significantly lower 131I activity than fixed doses, but the overall costs may be higher considering the need of complex measurements of radioiodine uptake (1) and the indirect costs (e.g. absence from work) of the procedure.

1. de Rooij A. Eur J Endocrinol. 2009;161:771-7

\section{P1-05-40 \\ FAMILIAR NON-AUTOIMMUNE HYPERTHYROIDISM: THE CASE OF A FAMILIY WITH 19 MEMBERS ACROSS FOUR GENERATIONS}

Andrej Malej ${ }^{1}$, Katarina Trebusak Podkrajsek ${ }^{2}$

${ }^{1}$ General Hospital Izola, Izola, Slovenia, ${ }^{2}$ University Medical Centre

Ljubljana, University Children's Hospital Ljubljana, Ljubljana, Slovenia

Familiar non-autoimmune hyperthyroidism (FNAH) is a rare form of hyperthyroidism with largely unknown incidence. This article presents the phenotype, clinical and genetic features, and treatment of family members born between 1923 and 2013. In a group of 12 males and 7 females, 10 males and 4 females developed the clinical features of hyperthyroidism, but did not show characteristics of autoimmune hyperthyroidism (i.e. ophthalmopathy, dermopathy, TPO, TG antibodies, a hypoechoic pattern at ultrasound, lymphocytic infiltration on histology). All affected members shared some physical similarities: they were tall, slim, had long thin fingers, an aquiline nose, and staring eyes. Members of the first generation (born between 1923 and 1942) were diagnosed as hyperthyrotic after they developed cardiac complications (rhythm disorders, thrombembolic events, cardiac insufficiency) at ages $>$ 42 years. Conservative treatment (antithyroid drugs and subtotal thyroidectomy) resulted in frequent relapses. They became long-term euthyrotic after radioiodine administration and permanent substitution. In the next generation (born between 1952 and 1974) hyperthyroidism was revealed earlier (at ages between 17 and 41), since we were aware of the familiar history of FNAH. They were treated accordingly. In contrast to the first generation, they have not developed cardiac complications as yet. In the third generation (born 1984 and 1988), hyperthyroidism was confirmed at ages $<10$ years, paediatric treatment was conventional, but no permanent remission was attained until radioiodine administration. Children in the fourth generation born between 2010 and 2013 are under the control of paediatricians. The hereditary nature of disease was confirmed by TSHR gene sequencing. Affected members had TSHR mutation NP_000360.2:p.Met453Val (NM_000369.2: c.1357A>G). This novel, yet so far not reported mutation was predicted to be causative with in silico models. Our experience with this multi-generation family indicates the need for early diagnosis and appropriate treatment of FNAH to avoid severe forms of hyperthyroidism in children, and to prevent late cardiovascular complications in adults. 


\section{P1-05-41 \\ DOES THYROID HORMONE REPLACEMENT FOR SUBCLINICAL HYPOTHYROIDISM HAVE BENEFICIAL EFFECTS ON PREGNANCY OUTCOMES?}

Tamar Peshkova ${ }^{1}$, Elene Giorgadze ${ }^{2}, K^{\prime}$ Koba Kamashidze ${ }^{3}$

${ }^{1}$ Batumi, Georgia, ${ }^{2}$ National Institute of Endocrinology, Tbilisi, Georgia,

${ }^{3}$ Batumi Maternity House, Batumi, Georgia

Objective: Subclinical hypothyroidism $(\mathrm{SCH})$ in pregnancy is associated with adverse maternal outcomes and perinatal complications but it is unclear whether levothyroxine (LT4) treatment initiated during pregnancy is beneficial. The aim of this study was to assess the potential benefits of LT4 therapy in pregnant women with $\mathrm{SCH}$ initiated in second trimester of pregnancy.

Methods: We analyzed medical records of 2876 pregnant women with an available second trimester thyroid function testing and known pregnancy outcome. SCH was defined (using population based reference interval $-97.5^{\mathrm{th}}$ percentile) as an elevated serum thyroid stimulating hormone (TSH) of $>4.0$ $\mathrm{mIU} / \mathrm{L}$ but $\leq 10 \mathrm{mIU} / \mathrm{L}$. Pregnant women with $\mathrm{SCH}$ were categorized into two groups according whether they were treated or not. The main outcome measures included maternal outcomes and perinatal complications.

Results: Compared with euthyroid pregnant women, untreated women with SCH had higher adjusted odds of gestational hypertension (odds ratio $2.23,95 \%$ confidence interval $1.25-4.03)$, preterm delivery $(1.63,1.13$ to $2.25)$, intrauterine growth restriction $(2.71,1.53$ to 5.11$)$, and low birth weight (1.61, 1.10 to 2.37). There was no significant difference in adverse outcomes of pregnancy found in pregnant women who had been diagnosed and treated with LT4 for SCH at the time of presentation when compared to euthyroid patients.

Conclusion: Thyroid hormone replacement for subclinical hypothyroidism initiated even in the second trimester of pregnancy showed beneficial effects on pregnancy outcomes.

\section{P1-05-42 \\ EFFECTIVENESS OF ORAL L-THYROXINE (L-T4) LIQUID WITH RESPECT TO TABLET FORMULATION, IN PATIENTS SUBMITTED TO TOTAL THYROIDECTOMY (WITHOUT MALABSORPTION)}

Poupak Fallahi', Silvia Martina Ferrari', Francesca Ragusa', Giusy

Elia ${ }^{1}$, Gabriele Materazzi ${ }^{2}$, Paolo Miccoli ${ }^{2}$, Alessandro Antonelli ${ }^{1}$

${ }^{1}$ University of Pisa, Department of Clinical and Experimental Medicine,

Pisa, Italy, ${ }^{2}$ University of Pisa, Department of Surgical, Medical,

Molecular Pathology and Critical Area, Pisa, Italy

Objective: Since the efficacy of levothyroxine (L-T4) liquid formulation in patients who recently underwent a total thyroidectomy is not yet well-known, our purpose was to evaluate the effectiveness of L-T4 liquid formulation with respect to L-T4 tablets. We have enrolled patients recently submitted to total thyroidectomy for euthyroid benign nodular goiter (without malabsorption or drug interference).

Methods: The study involved 105 patients, 53 of whom treated with L-T4 in tablets, and 52 with liquid L-T4 at the same dosage $(1.5 \mathrm{mcg} / \mathrm{kg} / \mathrm{day})$, 30 min before breakfast, starting the day after thyroidectomy. In both groups serum thyrotropic hormone (TSH), free thyroxine (FT4), and free triiodothyronine (FT3) were re-evaluated after 6 weeks (first control), and again after 12 weeks (second control).

Results: TSH values were significantly lower in the liquid L-T4 group, than in the tablet L-T4 group at the first control $(\mathrm{P}<0.05)$, as well as at the second control $(\mathrm{P}<0.01)$, while FT4 and FT3 levels were not significantly different. The prevalence of patients in the hypothyroid range (TSH $>3.6 \mathrm{mcU} /$ $\mathrm{ml}$ ) was significantly higher in the L-T4 tablet group.

Conclusions: Our data suggest a better control of TSH levels in thyroidectomized patients (without malabsorption, gastric disorders, or drug interference) who follow a liquid L-T4 therapy.

\section{P1-05-43 \\ TEN YEAR EXPERIENCE OF RADIOIODINE DOSIMETRY FOR THE TREATMENT OF GRAVES، DISEASE}

Steve Hyer ${ }^{1}$, Brenda Pratt ${ }^{2}$, Matthew Gray $^{3}$, Clive Harmer ${ }^{3}$, Sarah Chittenden $^{4}$, Glen Flux ${ }^{3}$

${ }^{1}$ Endocrine Department; Dept. of Endocrinology \& Diabetes; St Helier Hospital, Carshalton, Surrey, UK, ${ }^{2}$ Royal Marsden Hospital, London,

UK, ${ }^{3}$ Thyroid Unit; Royal Marsden NHS Foundation Trust, Sutton, UK,

${ }^{4}$ Thyroid Clinic; Royal Marsden NHS Foundation Trust, Sutton, UK

Aim: To assess the long term outcome of a radioiodine dosimetry approach for Graves' disease using a planned thyroid absorbed dose of 60Gy to render patients euthyroid.

Methods: A prospective study of patients with Graves' disease receiving 60Gy absorbed dose based on thyroid volume, maximum ${ }^{131} \mathrm{I}$ uptake and effective half-life. Patients with thyrocardiac disease, toxic adenoma or autonomous thyroid nodules were excluded. Clinical outcomes were analysed at yearly intervals for up to 10 years with a median follow-up of 37.5 months.

Results: For a target absorbed dose of $60 \mathrm{~Gy}$, the median effective halflife, thyroid volume and median absorbed dose delivered to patients with Graves' disease $(\mathrm{n}=284)$ was 5.18 days, $16.9 \mathrm{ml}$ and $55.8 \mathrm{~Gy}$ respectively. The median therapeutic radioactivity required to deliver $60 \mathrm{~Gy}$ was $77 \mathrm{MBq}(75-$ $84 \mathrm{MBq})$. The presence of nodules on ultrasonography did not adversely affect treatment outcome. To achieve a $60 \mathrm{~Gy}$ dose, only 5 patients required $400 \mathrm{MBq}$ or more, the standard activity administered in routine cases. In $62 \%$ of the patients, a single radioiodine administration was sufficient to render a patient either euthyroid or hypothyroid for 10 years. The number of patients remaining hyperthyroid defined as requiring antithyroid medication was $158(56 \%)$ and $65(23 \%)$ at 6 and 12 months respectively. Of the 175 patients treated with a single administration, $38 \%, 32 \%$ and $26 \%$ were euthyroid on no specific thyroid medication at 3,5 and 10 years respectively.

Conclusions: A personalised dosimetric approach to radioiodine therapy of Graves' disease designed to deliver 60Gy absorbed dose to the thyroid delayed the long term onset of hypothyroidism in $26 \%$ of patients in this study. Furthermore the administered activities using this methodology are much lower than those currently recommended by the fixed activity strategies.

\section{P1-05-44 \\ EFFECT OF SELENIUM SUPPLEMENTATION ON QUALITY OF LIFE IN PATIENTS WITH PRIMARY HYPOTHYROIDISM}

Marina Nikolic Djurovic ${ }^{1}$, Alberto M Pereira ${ }^{2}$, Johannes Smit ${ }^{3}$, Olga Vasovic $^{4}$, Zvezdana Jemuovic ${ }^{5}$, Svetozar Damjanovic ${ }^{5}$, Milika Asanin ${ }^{6}$, Dragan Pavlovic ${ }^{7}$, Dragana Jankovic ${ }^{5}$, Sandra Pekic Djurdjevic ${ }^{8}$, Dragana Miljic ${ }^{9}$, Marko Stojanovic ${ }^{10}$, Milan Petakov ${ }^{11}$

${ }^{1}$ Clinical Center of Serbia, Internal Medicine, Belgrade, Serbia, ${ }^{2}$ Center for Endocrine Tumors Leiden, Leiden University Medical Center, Leiden, Netherlands, ${ }^{3}$ Department of General Internal Medicine, Radboud University Nijmegen Medical Centre, XXX, Nijmegen, Netherlands, ${ }^{4}$ Department of Endocrinology and Metabolic Diseases, Institute for Gerontology and Palliative Care, Belgrade, Serbia, ${ }^{5} \mathrm{Clinic}$ for Endocrinology, Diabetes and Diseases of Metabolism, University Clinical Centre, Belgrade, Serbia, ${ }^{6}$ Clinic for Cardiology, University Clinical Centre, Belgrade, Serbia, ${ }^{7}$ Institute of Neurology, University Clinical Centre, Belgrade, Serbia, ${ }^{8}$ Clinic for Endocrinology, Diabetes and Diseases of Metabolism, University Clinical Center, School of Medicine, University of Belgrade, Belgrade, Serbia, ${ }^{9} \mathrm{Clinic}$ for Endocrinology, Diabetes and Metabolic Diseases, Clinical Center of Serbia, Belgrade University School of Medicine, Serbia, Clinical Center of Serbia, Belgrade, Serbia, Medical Faculty University of Belgrade, Beograd, Serbia, ${ }^{10} \mathrm{Clinic}$ for Endocrinology, Clinical Centre of Serbia, University of Belgrade, Belgrade, Serbia, ${ }^{11}$ Department of Neuroendocrinology, Clinic for Endocrinology, Clinical Center of Serbia \& School of Medicine, University of Belgrade, Belgrade, Serbia

Introduction: Patients with chronic autoimmune thyroiditis have impaired health-related quality of life. Selenium supplementation in people with Hashimoto thyroiditis(HT) might reduce antibody levels and improve quality of life, most probably due to its role in immunity.

Eur Thyroid J 2017;6(suppl 1):23-118 73 
Aim: to evaluate quality of life in patients on long-term levothyroxine replacement for primary hypothyroidism on selenium supplementation.

Design: cross-sectional, case-control study.

Patients and Methods: Sixty nine patients with HT (mean age $43.52 \pm 14.33 y$ rs ) on long-term levothyroxine replacement and 57 euthyroid healthy controls, matched for age, sex, and educational level, were included. HT patients were divided into 2 groups, 25 pts with ( $\mathrm{Se}+$ ) and 44 pts without selenium supplementation (Se-). Assessment of quality of life was made by SF36 questionnaire. SF-36 is a self-completed questionnaire measuring eight aspects(scales) of health status, which are further interpreted into 2 dimensions: physical and mental health and total SF36 score.

Results: Selenium levels were within the normal range in all groups, but significantly higher in $\mathrm{HT}(\mathrm{Se}+)$ vs controls $(94.88 \pm 20.63$ vs $86.95 \pm 14.95 \mathrm{mcg} / \mathrm{l}$, $\mathrm{p}<0.05)$.

TSH concentrations were higher in patients (both $\mathrm{HT}(\mathrm{Se}+)$ and $\mathrm{HT}(\mathrm{Se}-$ )) when compared to controls (3.01 \pm 1.66 and $3.01 \pm 1.90$ vs $1.77 \pm 0.88 \mathrm{mU} / 1$, $\mathrm{p}<0.000)$, but FT4 concentrations were not different:(12.81 \pm 4.01 vs $12.46 \pm 2.64$ vs $12.93 \pm 2.88 \mathrm{ng} / 1, \mathrm{p}>0.05)$. TPOab concentrations were different, both between $\mathrm{HT}(\mathrm{Se}+)$ and $\mathrm{HT}(\mathrm{Se}-)$, as well as between patients and controls (1399.25 \pm 1968.30 vs $4290.96 \pm 3177.13$ vs $20.48 \pm 30.73 \mathrm{U} / \mathrm{ml}, \mathrm{p}<0.001$ ).

In scales 1,3 and 4 , as well as in dimension $\mathrm{A}$ (physical health), and total SF36 score, there were statistically significant differences among groups / $\mathrm{HT}(\mathrm{Se}+)$ vs HT(Se-) vs controls/ Scale 1: $80.0 \pm 23.0 v 575.0 \pm 23.3 \mathrm{vs} 86.0 \pm$ $16.8, \mathrm{p}<0.05$, Scale $362.9 \pm 21.1 \mathrm{vs} 61.0 \pm 22.8 \mathrm{vs} 71.6 \pm 21.6, \mathrm{p}<0.05$, Scale 4 $62.9 \pm 20.6 v s 63.3 \pm 17.2 v s 71.5 \pm 14.9, \mathrm{p}<0.05$, Dimension A $65.4 \pm 20.6 \mathrm{vs} 63.0 \pm 18$ $.0 \mathrm{vs} 74.0 \pm 16.8, \mathrm{p}<0.05$, Total SF36 65.8 $\pm 21.6 \mathrm{vs} 64.0 \pm 17.8 \mathrm{vs} 73.6 \pm 17.7, \mathrm{p}<0.05$.

Conclusion: patients treated for primary hypothyroidism show persistent impairments in quality of life which were, in certain domains, improved with selenium supplementation. In addition, selenium HT treated patients showed a slight decrease in TPOAb. Future studies are still needed to provide reliable evidence to support selenium supplementation in clinical practice.

\section{P1-05-45 \\ EFFECT OF THYROID DEFICIENCY ON ATHEROGENIC LIPOPROTEINS AND INSULIN RESISTANCE IN OBESITY}

Lachezar Lozanov ${ }^{1}$, Veselina Koleva², Bojan Lozanov ${ }^{3}$, Desislava

Gorcheva 4 , Boyka Kostova ${ }^{5}$, Radoslav Borisov ${ }^{2}$

${ }^{1}$ Tokuda Hospital Sofia, Endocrinology Deprtment, Acibadem Cityclinic, Sofia, Bulgaria, ${ }^{2}$ Tokuda Hospital Sofia, Acibadem Cityclinic, Sofia, Bulgaria, ${ }^{3}$ Acibadem Cityclinic Tokuda Hospital Sofia, Sofia, Bulgaria, ${ }^{4}$ Tokuda Hospital Sofia, Acibadem Cityclinic, Sofia, Bulgaria, ${ }^{5}$ Tokuda Hospital, Acibadem Cityclinic, Acibadem Cityclinic, Sofia, Bulgaria

Introduction: Hypothyroidism and obesity are common diseases known to influence the lipid metabolism associated with atherosclerotic complications (1). Some lipoproteins represent strong risk factors for cardiovascular disease $(1,2)$.

Objective of the study was to estimate the effect of hypothyroidism on serum lipoprotein-a (Lp-a) and apolipoprotein B (ApoB) related to BMI, insulin resistance (IR) and adiponectin.

Material and Methods: 118 patients were assessed divided into four agematched groups (mean age $43 \pm 11$ years): gr.A- hypothyroid obese patients (BMI $36 \mathrm{~kg} / \mathrm{m} 2$ ), gr.B- euthyroid obese (BMI $38 \mathrm{~kg} / \mathrm{m} 2$ ), gr.C - hypothyroid $(\mathrm{BMI}<25 \mathrm{~kg} / \mathrm{m} 2)$, gr.K - euthyroid (BMI $<25 \mathrm{~kg} / \mathrm{m} 2)$. Lp(a), ApoB, insulin, fasting glucose, adiponectin were assessed.

Results: Positive correlations of TSH with Lp(a), ApoB, Total Cholesterol, Adiponectin, and HOMA-IR were established. Lp(a) in both hypothyroid patients groups $(\mathrm{A}, \mathrm{C})$ were significantly increased compared to those of euthyroid groups B and $\mathrm{K}$ (medians of 77.9 and $156.6 \mathrm{mg} / \mathrm{l}$ vs 36,5 and 7,35 $\mathrm{mg} / \mathrm{l}$ respectively, $\mathrm{p}<0,0001$ ). Hypothyroid patients with normal BMI (gr.C) showed much higher adiponectin than obese euthyroid patients (gr.B) and controls (gr.K): mean values of $19.1,7.5$ and $6.0 \mu \mathrm{g} / \mathrm{ml}$ respectively $(\mathrm{p}<0.0001)$.

Conclusion: The data showed that hypothyroidism was associated with extremely increased $\mathrm{Lp}(\mathrm{a})$ and adiponectin independent of BMI. It might be assumed that higher adiponectin level in obese hypothyroid patients compared to that in patients with normal body weight plays a compensatory role or appears the result of adiponectin receptor resistance.

References:

1 Third Report of National Cholesterol Educational Program (NCEP), Circulation,2002,106,3143;
2 Nordestdaard BG et al: Lipoproten (a) as a cardiovascular risk factor: current status. Eur Heart J, 2010, 31(23):2844-53

\section{P1-05-46 \\ ASSOCIATION BETWEEN THYROID VASCULARISATION AND SKIN MICROCIRCULATION IN PATIENTS WITH GRAVES‘ DISEASE}

Nataša Bedernjak Bajuk ${ }^{1}$, Simona Gaberšček ${ }^{2}$, Helena Lenasi ${ }^{3}$

${ }^{1}$ University Medical Centre Ljubljana, Ljubljana, Slovenia, ${ }^{2}$ University

Medical Centre Ljubljana, University of Ljubljana, Ljubljana, Slovenia,

${ }^{3}$ University of Ljubljana, Ljubljana, Slovenia

Objectives: Graves' disease (GD) is the main cause of hyperthyroidism, which is associated with a marked decrease in vascular resistance by affecting the function of vascular endothelial and smooth-muscle cells. In untreated GD, several studies indicate that thyroid blood flow is significantly increased. Less data are available on skin microcirculation which potentially reflects generalized vascular function. Our aim was to evaluate the thyroid vascularisation in untreated and treated GD and its association with skin microcirculation.

Methods: In our prospective case-control study, we included 31 hyperthyroid GD patients (hyperthyroid GD) and 30 age- and sex-matched healthy volunteers (controls). Patients were treated with methimazole and after 5.8 \pm 0.8 months, when euthyroid state was established, 26 GD patients were re-examined (euthyroid GD). Physical examination, thyroid ultrasound including measurements of the peak systolic velocity (PSV) at the level of intrathyroid arteries by the spectral Doppler and baseline laser Doppler flux (LDF) of skin microcirculation on the volar forearm were performed. Thyroid hormones and TSH receptor antibodies (antiTSH-R) were measured.

Results: In hyperthyroid GD patients, PSV at the level of intrathyroid arteries was significantly higher than in the euthyroid GD group and in controls $(\mathrm{p}<0.001$ and $\mathrm{p}<0.001$, respectively). In euthyroid GD patients, PSV was higher than in controls $(\mathrm{p}<0.001)$. In all subjects, the baseline LDF on volar forearm positively correlated with PSV at the level of intrathyroid arteries $(\mathrm{R}=0.450 ; \mathrm{p}<0.001)$ and also with antiTSH-R concentration $(\mathrm{R}=0.353$; $\mathrm{p}=0.008$ ). In the euthyroid GD group with increased levels of antiTSH-R, a significantly positive correlation of LDF and PSV with antiTSH-R concentration was established $(\mathrm{R}=0.821 ; \mathrm{p}<0.05$ and $\mathrm{R}=0.681 ; \mathrm{p}<0.010$, respectively).

Conclusions: GD patients have significantly increased intrathyroid blood flow, which correlates with the baseline LDF on volar forearm. Intrathyroid and skin microcirculation blood flow might also be regulated by antiTSH-R, which is supported by our findings.

\section{P1-05-47 \\ TREATMENT OF AMIODARONE-INDUCED THYROTOXICOSIS RESISTANT TO CONVENTIONAL THERAPY}

Tanja Nisic ${ }^{1}$, Ljubica Jovanovic ${ }^{2}$, Milos Stojanovic ${ }^{2}$, Mirjana Stojkovic $^{2}$, Slavica Savic ${ }^{2}$, Jasmina Ciric ${ }^{2}$, Marija Miletic ${ }^{2}$, Biljana Beleslin-

Nedeljkovic ${ }^{2}$, Milos Zarkovic ${ }^{2}$

${ }^{1}$ Clinic for Endocrinology, Diabetes and Metabolic Diseases, Clinical

Center of Serbia, Belgrade, Belgrade, Serbia, ${ }^{2}$ Belgrade, Serbia

Introduction: Amiodarone as an antiarrhythmic medication, necessary in the prevention and treatment of malignant ventricular arrhythmias, disrupts the function of the thyroid gland by the type of hypothyroidism and thyrotoxicosis, however, $50 \%$ of patients are euthyroid.

Case Report: A 27 year-old female patient, hospitalized at the Clinic for Endocrinology due to amiodarone-induced thyrotoxicosis (FT4 115; FT3 26; TSH <0,005). Anti-Tg, anti-TPO and TSH-r AT were negative, ultrasound examination of the thyroid gland showed no focal lesions, color Doppler signal was not amplified. Patient was receiving amiodarone for two years due to dilated cardiomyopathy (EF $25 \%$, left ventricle $56-57 / 47 \mathrm{~mm}$ ) with mild Ebstein's anomaly and WPW Sy. Patient was implanted with an ICD-VR and prescribed amiodarone due to recorded episodes of "non sustained VT". Treatment with propylthiouracil (PCU) and dexamethasone was initiated prior to hospitalization. Upon admission the patient developed toxic liver lesion induced by PTU, medication discontinued. Thyrosol, which maintains the liver lesion parameters, was introduced. The treatment had no effect on the 
correction of the thyroid status. Perchlorate (Irenat) therapy was introduced, along with glucocorticoids (per os and intrathyroidal). After fifty days of treatment, laboratory signs of thyrotoxicosis were maintained, and eight plasmapheresis sessions were applied in the treatment. Each plasmapheresis resulted is a significant drop in FT4 and a slight drop in FT3. After seventy two days of treatment, an optimal hormonal status of the thyroid gland was established and a total thyroidectomy was performed.

Conclusion: Patient was treated for amiodarone-induced thyrotoxicosis (AIT) type 2, which was resistant to conventional therapy for a long period of time. Successful treatment was accomplished by applying plasmapheresis although the effect of perchlorate and glucocorticoids application cannot be disregarded.

Key words: amiodarone, thyrotoxicosis, plasmapheresis

\section{P1-06 Molecular Mechanisms of Thyroid Hormone Action}

\author{
P1-06-48 \\ 3-IODOTHYROACETIC ACID (TA1), AN END \\ METABOLITE OF THYROID HORMONE, \\ STIMULATES MICE ATTENTION AND \\ VIGILANCE: NEW EVIDENCE FOR THE \\ INVOLVEMENT OF THE HISTAMINE-THYROID \\ AXES \\ Annunziatina Laurino ${ }^{1}$, Elisa Landucci ${ }^{2}$, Francesco Resta ${ }^{3}$, Gaetano \\ De Siena ${ }^{4}$, Laura Raimondi ${ }^{5}$ \\ ${ }^{1}$ University of Florence, Firenze, Italy, ${ }^{2}$ University of Florence, \\ Department of Health Science, Firenze, Italy, ${ }^{3}$ University of Florence, \\ Department of Neurofarba, Firenze, Italy, ${ }^{4}$ University of Florence, \\ Department of Health Science, Firenze, Italy, ${ }^{5}$ University of Florence, \\ Dept. of Pharmacology, Dept. of Pharmacology, Florence, Italy
}

Background and Purpose: We recently reported 3-iodothyroacetic acid (TA1), a metabolite of thyroid hormone, stimulated learning and gave hyperalgesia activating the histaminergic system. We now aim to investigate whether TA1 could induce other typical histamine-dependent effects including increase of attention of vigilance and of wakefulness.

Experimental Approach: CD1 male mice received i.p. TA1 (1.32, 4 and $11 \mu \mathrm{gkg}-1)$ or saline and, after $15 \mathrm{~min}$, spontaneous locomotor activity, motor coordination, and the immobility time were evaluated on the hole board, the rota road and the forced swim test respectively. The effect of TA1 $(1.32,4$ and $11 \mu \mathrm{gkg}-1$ i.p.) on the sleeping time of mice receiving an acute dose of ethanol (3.5 mgkg-1; i.p.) was evaluated. The experiments were repeated in mice pretreated with pyrilamine ( $10 \mathrm{mgkg}-1)$ or zolantidine $(5 \mathrm{mgkg}-1)$.

Hypothalamic levels of p-mTOR and p-GSK-3 beta in mice receiving 4 $\mu \mathrm{gkg}-1 \mathrm{TA} 1$ or saline in the absence or presence of immepip (5 mgkg-1) (a type 3 histamine receptor agonist) were measured by Western blot.

Key Results: TA1 (4 and $11 \mu \mathrm{gkg}-1$; i.p.) increased mice spontaneous locomotor activity, reduced the immobility time in the forced swim test without giving motor incoordination, effects reverted by zolantidine and pyrilamine respectively. TA1 (4 $\mu \mathrm{gkg}-1)$ significantly delayed the onset and shortened the duration of ethanol-induced sleep. At the same dose TA1 increased hypothalamic p-mTOR and p-GSK-3 beta levels, an effect prevented by immepip pre-treatment.

\section{P1-06-49 \\ THYROXINE PROMOTES TUMOR GROWTH IN AN ORTHOTOPIC LUNG CANCER MOUSE MODEL}

Soeren Latteyer ${ }^{1}$, Sandra Christoph ${ }^{2}$, Sarah Synoracki ${ }^{3}$, Xiao Hui Liao $^{4}$, Samuel Refetoff', Kurt Werner Schmid ${ }^{3}$, Dagmar Führer ${ }^{1}$, Lars

Christian Möller'

${ }^{1}$ Department of Endocrinology and Metabolism, University Hospital Essen, University of Duisburg-Essen, Essen, Germany, ${ }^{2}$ Department of Bone Marrow Transplantation, University Hospital Essen, University of Duisburg-Essen, Essen, Germany, ${ }^{3}$ Institute of Pathology, University Hospital Essen, University of Duisburg-Essen, Essen, Germany,

${ }^{4}$ Department of Medicine, The University of Chicago, Chicago, IL, USA

Introduction: Thyroid hormones are important for physiology and homeostasis. Besides the nuclear thyroid hormone receptors, a plasma membrane protein, integrin $\alpha \mathrm{v} \beta 3$, has been shown to be a receptor for both $\mathrm{T} 3$ and T4. Our hypothesis was that thyroid hormone signaling via $\alpha v \beta 3$ promotes tumor growth.

Material and Methods: To test this hypothesis, murine lung carcinoma cells (3LL), stably transfected with luciferase, were injected into mouse lungs. Mice then remained untreated or were rendered hypothyroid and treated with $\mathrm{T} 3$ or T4 with or without the $\alpha \mathrm{v} \beta 3$ inhibitor 3,5,3',5'-tetraiodothyroacetic acid (tetrac). Tumor growth was determined by serial in vivo imaging of bioluminescence emitted from the injected luciferase expressing 3LL cells. Tumor weight was recorded at the end of the experiment. Neoangiogenesis was determined by immunohistochemistry for CD 31 .

Results: Tumor growth was reduced in hypothyroid mice and increased in T4 treated mice. Strikingly, only T4, but not T3, treatment promoted tumor growth. This $\mathrm{T} 4$ effect was completely abrogated by co-treatment with the $\alpha v \beta 3$ inhibitor tetrac. Tumor weight was significantly higher in the T4 treatment group and immunohistochemistry showed significantly increased neoangiogenesis only in tumors of T4 treated mice. Again, the T4 effect on tumor weight and neoangiogenesis was abolished by tetrac.

Conclusion: We demonstrate that thyroid hormone promotes tumor growth in an orthotopic lung cancer mouse model. This tumor promoting effect includes increased neoangiogenesis and is exclusively mediated by T4 via the plasma membrane integrin $\alpha v \beta 3$. Such effects of levo-thyroxine need to be considered in cancer patients on $\mathrm{T} 4$ substitution.

\section{P1-06-50}

\section{ANTICONVULSANT AND NEUROPROTECTIVE ACTIONS OF 3-IODOTHYROACEIC ACID (TA1), A BY- PRODUCT OF THYROID HORMONE METABOLISM IN EPILEPSY MODELS}

\section{Annunziatina Laurino ${ }^{1}$, Elisa Landucci ${ }^{2}$, Francesco Resta ${ }^{3}$, Gaetano} De Siena ${ }^{4}$, Domenico Edoardo Pellegrini-Giampietro ${ }^{2}$, Alessio Masi ${ }^{5}$, Guido Mannaioni ${ }^{6}$, Laura Raimondi ${ }^{7}$

${ }^{1}$ University of Florence, Firenze, Italy, ${ }^{2}$ University of Florence, Department of Health Science, Firenze, Italy, ${ }^{3}$ University of Florence, Department of Neurofarba, Firenze, Italy, ${ }^{4}$ University of Florence, Department of Health Science, Firenze, Italy, ${ }^{5}$ University of Florence, Department of Neurofarba, Firenze, Italy, ${ }^{6}$ University of Florence, Department of Neurofarba, Firenze, Italy, ${ }^{7}$ University of Florence, Dept. of Pharmacology, Dept. of Pharmacology, Florence, Italy

Background and Purpose: Both thyroid and histaminergic system dysfunctions associated with increased risk of seizures. 3-iodothyroacetic acid (TA1) is a by-product of thyroid hormone metabolism suspected to behave as a neuromodulator of the histaminergic system. We aimed to investigate whether TA1 was endowed of anticonvulsant effects in mice.

Experimental Approach: Mice received vehicle or pyrilamine (10 mgkg1 ; s.c; PYR) and after $10 \mathrm{~min}$ TA1 ( 4, 7, 11, $33 \mu \mathrm{gkg}-1$; i.p.) followed, $10 \mathrm{~min}$ later by $90 \mathrm{mgkg}-1$ pentylenetrazole s.c. (scPTZ). The latency of seizure onset and the number of convulsant mice were measured. Hippocampal signals (including PI $3 \mathrm{~K} / \mathrm{AKT} / \mathrm{GSK}-3 \beta$ and $\mathrm{c}$-fos) activated by $7 \mu \mathrm{gkg}-1$ TA1 were measured by Western blot. Organotypic hyppocampal slices were exposed for $5 \mathrm{~min}$ to vehicle or $1 \mathrm{M}$ PYR or $10 \mu \mathrm{M}$ LY 294002, or $10 \mathrm{nM}$ TG-C before adding vehicle or $10 \mu \mathrm{M}$ TA1 followed by $5 \mathrm{M}$ kainate (KA). Toxicity 
was evaluated $24 \mathrm{~h}$ later by measuring propidium iodide (PI) fluorescence. KA-induced current in CA3 neurons was evaluated electrophysiologically.-

Key Results: TA1 (7 and $11 \mu \mathrm{gkg}-1)$ reduced the number of convulsant mice and increased seizure latency. TA1 activated PI3K/AKT/GSK-3 $\beta$ signaling cascade and restored c-fos levels in mice hippocampus. $10 \mu \mathrm{M}$ TA1 reduced KA toxicity, by activating the PI3K/AKT/GSK-3 $\beta$ cascade. TA1 did not modify KA-induced current in CA3 hyppocampal neurons. Both anticonvulsant and neuroprotective effects were abolished by PYR.

Conclusion and Implications: TA1 is anticonvulsant and neuroprotective against excitotoxicity by activating the PI3K/AKT/GSK-3 $\beta$. These findings, indicate the potential of TA1 to be exploited as a novel drug for treating seizures and neurological consequences of epilepsy.

\section{P1-06-51 \\ PHENYLBUTYRATE RESCUES SEVERAL PATHOGENIC MCT8 MUTATIONS - BUT WHAT IS THE MOLECULAR MECHANISM BEHIND?}

\section{Doreen Braun ${ }^{1}$, Ulrich Schweizer ${ }^{2}$}

${ }^{1}$ Institut für Biochemie und Molekularbiologie, Universität Bonn, Bonn, Germany, ${ }^{2}$ Rheinische Friedrich-Wilhelms-Universität Bonn, Institut für Biochemie und Molekularbiologie, Bonn, Germany

The monocarboxylate transporter 8 (MCT8) is a highly specific transporter for thyroid hormones. Mutations in MCT8 lead to a rare genetic disease (Allan-Herndon-Dudley syndrome - AHDS) which is associated with severe mental retardation, muscle weakness, absent of speech and inability to walk. However, some MCT8-deficient patients show milder symptoms suggesting a residual activity of the mutated protein.

Mutations in MCT8 can either affect $\mathrm{T}_{3}$ binding and transport, the translocation of the protein to the cell surface or its protein stability. The two latter effects may lead to functional but reduced MCT8 molecules at the cell surface with the consequence of decreased thyroid hormone transport across plasma membranes. This partial inactivation is probably responsible for the milder phenotype found in some AHDS patients.

We already reported that sodium phenylbutyrate $(\mathrm{NaPB})$ increases the protein stability and function of several partially inactivated MCT8 mutants $\left(\mathrm{MCT}^{\mathrm{delF} 501}, \mathrm{MCT} 8^{\mathrm{S} 194 \mathrm{~F}}, \mathrm{MCT} 8^{\mathrm{S} 290 \mathrm{~F}}, \mathrm{MCT} 8^{\mathrm{L} 434 \mathrm{~W}}, \mathrm{MCT} 8^{\mathrm{R} 445 \mathrm{C}}, \mathrm{MCT} 8^{\mathrm{L} 492 \mathrm{P}}\right.$ and $\mathrm{MCT} 8^{\mathrm{L} 568 \mathrm{P}}$ ) in vitro. However, not much is known about the molecular mechanism behind the action of NaPB. Thus, we started to unravel the mode of action and found that $\mathrm{NaPB}$ is not only able to increase the protein stability but also helps mutated proteins to translocate to the plasma membrane. In addition, we could detect an increase of mRNA levels under NaPB treatment. Until now, it is unclear if $\mathrm{NaPB}$ is able to modulate the concentration of endogenous chaperones in our cell culture model. Modulation of endogenous chaperone concentration was reported before in a model for cystic fibrosis.

\section{P1-06-52}

A RARE MISSENSE VARIANT OF TRACE AMINE ASSOCIATED RECEPTOR 1 IN BIPOLAR

\section{DISORDER}

Grazia Rutigliano ${ }^{1}$, Vittoria Carnicelli², Liliana Dell'Osso ${ }^{3}$, Riccardo Zucchi $^{4}$

${ }^{1}$ Institute of Life Sciences, Scuola Superiore Sant'anna, National Research Council (Cnr), Institute of Clinical Physiology (Ifc), Department of Clinical and Experimental Medicine, University of Pisa, Pisa, Italy, ${ }^{2}$ Department of Pathology, University of Pisa, Pisa, Italy, ${ }^{3}$ Department of Clinical and Experimental Medicine, University of Pisa, Pisa, Italy, ${ }^{4}$ University of Pisa, Dept. of Pathology, Pisa, Italy

Background and Objectives: Trace Amine Associated Receptor 1 (TAAR1) is a G protein-coupled receptor (GPCR) that is stimulated with nanomolar affinity by 3 -iodothyronamine (T1AM), an endogenous messenger thought to derive from thyroid hormone metabolism. Evidence from animal model based pharmacological and functional data supports a role for TAAR1 in neuromodulation. The region of the long branch of chromosome 6 where the human genes for TAAR are located was associated with neuropsychiatric disorders in linkage studies. We screened TAAR1 for single nucleotide polymorphisms (SNPs) and tested their association with Bipolar Disorder.
Materials and Methods: We recruited 36 patients receiving treatment for Bipolar Disorder at the Psychiatry Clinic, University of Pisa, and 40 controls. Consensus diagnoses were assigned using DSM-5 criteria. Genomic DNA was isolated from saliva samples by standard methods and quantified. We screened by Sanger sequencing a 511pb-amplicon spanning the 3' UTR and a fragment of the coding sequence reported to include 5 SNPs that result in sub-functional receptors.

Results: We found 5 SNPs already documented in public database (National Center for Biotechnology Information, dbSNP, http://www.ncbi. nlm.nih.gov/SNP/. All SNPs were in Hardy-Weinberg equilibrium in the sample. Three of them, namely rs9375907, rs8192620, and rs8192619 had MAF > 0.2 and were not significantly associated with Bipolar Disorder. Two different affected patients carried the synonymous SNP rs8192621 and the missense SNP rs142169206. The latter results in the variant Cys263Gly in the receptor sixth transmembrane domain.

Conclusions: In this preliminary analysis of TAAR1 SNPs, we identified a heterozygous missense variant (Cys263Gly) in a single patient with Bipolar Disorder and in no unaffected controls. Since it is localized in a highly conserved motif in the transmembrane receptor, this variant could produce a functional change in TAAR1 and have an etiological role in Bipolar Disorder.

\section{P1-06-53 \\ AGE-RELATED CHANGE OF TSH AND FREE THYROID HORMONES}

\section{Neda Milinković ${ }^{1}$, Svetlana Ignjatović ${ }^{1}$, Milos Zarkovic ${ }^{2}$, Biljana}

Beleslin ${ }^{3}$, Jasmina Ćirić ${ }^{3}$

${ }^{1}$ Clinical Center of Serbia, Faculty of Pharmacy, Belgrade, Serbia,

${ }^{2}$ Clinic of Endocrinology, Diabetes and Metabolic Diseases, Clinical

Center of Serbia, Medical School University of Belgrade, Belgrade,

Serbia, ${ }^{3}$ Belgrade

Objectives: Literature data indicate a pronounced impact of ageing on the change of thyroid function and effect on thyroid function tests. Although there are many thyroid tests, the most commonly used functional assays for the assessment of the thyroid disease are determination of thyroid stimulating hormone (TSH), free thyroxine (fT4) and free triiodothyronine (fT3). The aim of this study was to retrospectively investigate influence of gender and age on distribution and correlation of the free thyroid hormones and TSH.

Methods: Thyroid hormones were determined in 66842 sera, by the same method (CMIA) on Abbot, Architect analyzer Ci8200. We used data from the laboratory database, which is stored in the laboratory information system in Department of polyclinic laboratory diagnostic, Center for Medical Biochemistry, Clinical Center of Serbia in Belgrade.

Results: We found a significant negative correlation between TSH and fT4 $(\mathrm{p}<0.01)$ and significant positive correlation between TSH and fT3/fT4 index $(\mathrm{p}<0.01)$ in every decade of life. There was a significant increase in TSH values in the last two decades of life $(61-70$ and $>70)(p<0.05)$. Also, we found a significant deviation of the values of the all analyzed hormones in the third and fourth quartile of TSH in patients older than 70 years of age $(p<0.05)$. There was no significant impact of gender on the correlation of the analyzed thyroid hormones.

Conclusions: This study suggests that ageing has a great influence on the complex ratio of the free thyroid hormones and TSH. Our data indicates the need for separate reference values for thyroid parameters with respect to the decade of life. This could provide an adequate treatment and monitoring of subclinical thyroid disease. 


\section{P1-06-54 \\ DIFFERENTIAL EFFECTS OF 3,5-DIIODO-L- THYRONINE AND 3,5,3'-TRIIODO-L-THYRONINE ON THE FUNCTION OF MITOCHONDRIAL RESPIRATORY SUPERCOMPLEXES IN LIVER FROM HYPOTHYROID RATS}

Elena Silvestri ${ }^{1}$, Assunta Lombardi ${ }^{2}$, Maria Coppola ${ }^{3}$, Rosa Anna Busiello $^{4}$, Alessandra Gentile ${ }^{4}$, Celia Di Munno ${ }^{5}$, Maria Moreno 6 , Fernando Goglia ${ }^{7}$

${ }^{1}$ University of Sannio, Benevento, Dep. Science and Technology, Benevento, Italy, ${ }^{2}$ University of Naples, Scienze Bilogiche, Napoli, Italy, ${ }^{3}$ University of Sannio, Benevento, ${ }^{4}$ University of Naples Federico II, Napoli, ${ }^{5}$ University of Sannio, Benevento, Benevento, ${ }^{6}$ Universitá Sannio, Benevento, Italy, ${ }^{7}$ Università Degli Studi del Sannio,

Benevento, Italy

Objective: Both 3,5-diiodo-L-thyronine (T2) and 3,5,3'-triiodo-L-tyronine (T3) affect energy metabolism with mitochondria being a major target. The mitochondrial electron transport chain undergoes rearrangements to achieve an optimum performance via a balanced distribution between respiratory complexes and supercomplexes. We evaluated whether T2 or T3 influence liver mitochondrial respiratory pathways by acting on the distribution and activity of respiratory complexes and supercomplexes when injected into hypothyroid rats.

Methods: Hypothyroidism was induced by propylthiouracil and iopanoic acid; T2 and T3 were i.p. administered at 25 and $15 \mu \mathrm{g} / 100 \mathrm{~g}$ bw for 1 week, respectively. Mitochondrial functions were studied by combining respirometry, Blue Native-PAGE and western blot.

Results: Hypothyroidism (vs. euthyroidism) reduced mitochondrial respiration rate which was significantly enhanced by both T2 and T3. Complex-I, complex-II, and glycerol-3 phosphate dehydrogenase (G3PDH)-linked oxygen consumptions were increased by both iodothyronines, with the effect of $\mathrm{T} 3$ being higher than that of T2. In-gel activities of individual respiratory complexes and of complex I-supercomplexes revealed a similar pattern.

The mitochondrial abundance of all respiratory complexes and of G3PDH was increased by T3 while T2 affected only complex V and G3PDH.

Conclusions: Both $\mathrm{T} 2$ and $\mathrm{T} 3$ significantly stimulate mitochondrial respiration rate with the underlying molecular/biochemical pathways being only partially overlapping. Indeed, only the effects exerted by T3 are associated with an increase of all respiratory complexes and supercomplexes content likely indicating differences in the two iodothyronines-cellular signalling mechanisms. These results furnish new information on the functional role of the respiratory complexes and supercomplexes in thyroid-mediated action on energy metabolism.

\section{P2-01 Autoimmunity 2}

\section{P2-01-55 \\ ACTION OF SEVERAL BIOAVIABLE ANTIOXIDANT IN ORBITAL FIBROBLASTS FROM PATIENTS WITH GRAVES‘ ORBITOPATHY (GO): A NEW FRONTIER IN GO TREATMENT? \\ Giovanna Rotondo Dottore ${ }^{1}$, Giamberto Casini ${ }^{2}$, Stefano Sellari- Franceschini ${ }^{3}$, Marco Nardi ${ }^{2}$, Paolo Vitti ${ }^{1}$, Claudio Marcocci ${ }^{1}$, Michele Marinò ${ }^{1}$ \\ ${ }^{1}$ Endocrinology Unit, University of Pisa, Italy, ${ }^{2}$ Ophthalmopathy Unit I, University of Pisa, Italy, ${ }^{3}$ Ent Unit I, University of Pisa, Italy}

Objective: Oxidative stress is involved in the pathogenesis of Graves' Orbitopathy (GO) and an antioxidant approach has been advocated for GO treatment. Here we investigated the action of the following antioxidants in orbital fibroblasts: Vitamin C, N-Acetyl-1-Cysteine, melatonin, retinol, betacarotene, Vitamin E

Methods: Primary cultures of orbital fibroblasts from 6 GO patients and 6 control subjects were established. Cells were treated with $\mathrm{H} 2 \mathrm{O} 2$ to induce oxidative stress. Cell vitality assays tests were performed to determine the non-cytotoxic dose of each antioxidant. The following assays were performed: glutathione disulfide (GSSG), as a measure of oxidative stress; cell proliferation; hyaluronic acid (HA); TNF-alpha; IFN-gamma; IL1-beta.

Results: $\mathrm{H} 2 \mathrm{O} 2$ induced oxidative stress, increased proliferation and cytokine release, but did not affect HA release. All substances, except for retinol, reduced $\mathrm{H} 2 \mathrm{O} 2$-dependent oxidative stress (GSSG). Vitamin C reduced proliferation in GO, but not in control cells. N-Acetyl-1-Cysteine reduced proliferation and IFN-gamma in GO, and IL1-beta in both GO and control cells. Melatonin reduced IL1-beta (GO and controls) and IFN-gamma (GO). Betacarotene reduced proliferation (GO) and IL1-beta (GO and controls). Vitamin E reduced IL1-beta (GO and controls).

Conclusions: Our findings show an antioxidant actions of several new compounds, especially N-Acetyl-1-Cysteine, in orbital fibroblasts. Some of the actions are exclusive to GO fibroblasts. These observation have important clinical implication, in that some of these antioxidants could be tested in patients with GO.

\section{P2-01-56}

\section{EFFECT OF SIMVASTATIN ONCIGARETTE SMOKE INDUCED GENE EXPRESSION IN 3T3-L1 PREADIOPOCYTES}

Bushra Shahida ${ }^{1}$, Tereza Planck ${ }^{2}$, Pernilla Sahlstrand-Johnson ${ }^{3}$, Peter Åsman ${ }^{4}$, Mikael Lantz ${ }^{2}$

${ }^{1}$ Lund University, Department of Clinical Sciences Malmö, Diabetes \& Endocrinology, Malmö, Sweden, ${ }^{2}$ Skåne University Hospital, Department of Endocrinology, Lund University, Department of Clinical Sciences, Diabetes \& Endocrinology, Malmö, Sweden, ${ }^{3}$ Skåne University Hospital, Department of Otorhinolaryngology, Lund University, Department of Clinical Sciences, Malmö, Sweden, ${ }^{4}$ Skåne University Hospital, Department of Opthalmology, Lund University, Department of Clinical Sciences, Diabetes \& Endocrinology, Malmö, Sweden

Objectives: Smoking is a strong risk factor for the development of Graves' ophthalmopathy (GO). Immediate early genes (IEGs) including cysteine-rich angiogenic inducer 1 (CYR61) are overexpressed in patients with active GO compared to healthy controls and their expression is higher in smokers with GO compared to non-smokers. The aim was to investigate the effect of simvastatin on expression of IEGs in 3T3-L1 preadipocytes exposed to cigarette smoke extract (CSE).

Methods: 3T3-L1 preadipocytes were cultured in Dulbecco's modified Eagle's Medium (DMEM) with 10\% fetal bovine serum (FBS). CSE was generated by a validated pumpsystem, for $100 \%$ concentration, 4 cigarettes were smoked through $30 \mathrm{ml}$ DMEM, each cigarette by 10 puffs, each of $35 \mathrm{ml}$. Two days post confluent cells were treated with simvastatin for 24 hours then exposed to CSE. The differentiation cocktail (DC), rosiglitazone, dexamethasone and insulin was added to the cells. Cells were taken at various time points (every 30min for 2 hours) for RNA extraction. Gene expression in cells treated with simvastatin, DC and CSE were compared with cells only exposed to CSE and treated with DC.

Results: The expression of CYR61 and zinc finger protein 36 (ZFP36) were overexpressed in cells treated with CSE or DC with a maximum in 90 minutes and was reduced, with $38 \%$ and $44 \%$ respectively, in cells treated with simvastatin.. Furthermore simvastatin decreased the expression of Stearoyl$\mathrm{CoA}$ desaturase1 (SCD1) and peroxisome proliferator activated receptor gamma (PPARG) with $67 \%$ on differentiation day 6 .

Conclusion: Simvastatin reduced the expression of CYR61, ZFP36 in early adipogenesis, in addition SCD1 and PPARG were reduced in the late adipogenic phase in preadipocytes treated with simvastatin. If these findings can be confirmed in orbital fibroblasts suggests that simvastatin should be investigated in a clinical trial 


\section{P2-01-57 \\ SERUM INTERLEUKIN- 37 (IL-37) LEVELS WERE INCREASED AND CORRELATED WITH OXIDATIVE STRESS PARAMETERS IN HASHIMOTO'S THYROIDITIS (HT) PATIENTS}

Rosaria Ruggeri ${ }^{1}$, Teresa Manuela Vicchio ${ }^{1}$, Maria Teresa Cristani ${ }^{2}$, Angela Alibrandi ${ }^{3}$, Francesco Trimarchi ${ }^{4}$, Sebastiano Gangemi ${ }^{5}$ ${ }^{1}$ Department of Clinical-Experimental Medicine and Pharmacology, Division of Endocrinology, University of Messina, Aou Policlinico DI Messina, Messina, Italy, ${ }^{2}$ Department of Pharmaceutical Sciences and Health Products, University of Messina, Messina, Italy, ${ }^{3}$ Department of Economy Unit of Statistical and Mathematical Sciences, University of Messina, Messina, Italy, ${ }^{4}$ University of Messina, Accademia Peloritana Dei Pericolanti, Messina, Italy, ${ }^{5}$ Division of Clinical Immunology and Allergology; University of Messina, Aou Policlinico DI Messina, Messina, Italy

Background: IL-37, member of the IL-1 family, is a natural suppressor of immune and inflammatory responses. Increased serum IL-37 levels were observed in several autoimmune diseases, including Graves' disease. No data on $\mathrm{TH}$ are available from the literature.

Materials and Methods: We enrolled 45 euthyroid HT patients (5 M e 40 F, mean age $38.5 \pm 12.6$ ) and 50 age- and sex-matched healthy controls. None was on LT-4 therapy. Smokers, subjects with kidney failure, history of cancer or autoimmune, inflammatory and infection comorbidities were excluded. Serum IL-37 levels were measured by ELISA (kit IL-37 DuoSet Elisa, R\&D System, Minneapolis, USA; minimum detectable dose $10 \mathrm{pg} / \mathrm{ml}$ ). Specific serum tests, such as d-ROMs (derived Reactive Oxygen Metabolites) test and BAP (Biological Antioxidant Potential) test [Diacron International, Italy], were performed in all subjects to investigate the changes in oxidative balance, and AGEs (Advanced Glycation End Products) were determined as a specific marker of oxidative stress.

Results: IL-37 levels were significantly higher in TH (mean \pm SD: 999.59 $\pm 1064.93 \mathrm{pg} / \mathrm{ml})$ than in controls $(448.02 \pm 498.96 \mathrm{pg} / \mathrm{ml} ; \mathrm{P}=0.018)$. In both groups, these values correlated negatively with age $(\mathrm{TH}, \mathrm{RS}=-0.377 \mathrm{P}=0.016$; controls, RS=-0.404; $P=0.06$ ) and were tendentially higher in men than in women $(P=0.063)$. Furthermore, there was a positive correlation between serum IL-37 levels and TSH, statistically significant in TH (RS $=0.412$ $\mathrm{P}=0.008$; in controls, $\mathrm{RS}=0.418 ; P=0.053$ ). The regression analysis showed a significant positive association between IL-37 and d-ROMs $(\mathrm{P}=0.029)$ and AGEs ( $\mathrm{P}=0.014)$ : if serum d-ROMs and AGEs levels increased, serum IL-37 levels also increased. A significant direct correlation between serum IL-37 levels and AGEs was also observed $(\mathrm{RS}=0.578 ; \mathrm{P}=0.006)$.

Conclusion: Serum IL-37 levels were significantly increased in $\mathrm{TH}$ patients and correlated with oxidative stress parameters. Given the antiinflammatory properties, IL-37 could be an innovative research strategy for TH pathogenesis and therapy.

\section{P2-01-58}

ANALYSIS OF PREVALANCE OF TSH RECEPTOR ANTIBODIES IN CHILDREN WITH AUTOIMMUNE THYROID DISEASE-MULTICENTER STUDY

Artur Bossowski ${ }^{1}$, Karolina Stozek2, Katarzyna Ziora ${ }^{3}$, Anna

Bossowska ${ }^{4}$, Tanja Diana ${ }^{5}$, George J. Kahaly ${ }^{6}$

${ }^{1}$ Medical University in Białystok, Dep.of Pediatrics, Endo, Diabeto, Bialystok, Poland, ${ }^{2}$ Dep. of Pediatric Endcorinology, Diabetology With Cardiology Unit, Medical University in Bialystok, Bialystok, Poland, ${ }^{3}$ Department of Paediatric Nephrology and Endocrinology, Silesian Medical University, Zabrze, Poland., Zabrze, Poland, ${ }^{4}$ Division of Cardiology, Internal Affairs Ministry Hospital in Białystok, Bialystok, Poland, ${ }^{5}$ Johannes Gutenberg University, Medical Center, Mainz, Germany, ${ }^{6}$ Johannes Gutenberg University Medical Center, Dept. of Medicine I, Mainz, Germany

Introduction: Autoimmune thyroid diseases (AITD) strongly associate with the appearance of functional TSH receptor antibodies (TSHR-Abs). We can distinguish two types of TSHR-Abs: thyroid-stimulating immunoglobulin (TSI) promoting the production of thyroid hormones and thyroid-blocking immunoglobulin (TBI) inhibiting the activity of TSH. Circulating TSIs may attach other organs like orbital tissue leading to thyroid associated orbitopathy (TAO)

The aim of the study was to analysis prevalence of functional TSH receptor antibodies in children with autoimmune thyroid diseases and healthy controls.

A total of 240 serum samples were obtained from 205 pediatric patients with AITD: 32 with GD and 69 with HT, 66 with type 1 diabetes (T1D), 5 with juvenile arthritis (JA), and 33 healthy controls (C). TSI and TBI were measured in bioassays using $\mathrm{CHO}$ cells expressing a chimeric TSH-receptor and a c-AMP response-element-dependent luciferase.

Children with GD were positive for TSI in 47/53 samples (88.7\%) while those with GD-TAO showed TSI posivity in $95.8 \%$ (23/24 samples). Serum TSI levels were SRR\% $319.86 \pm 156.55$ and $416.7 \pm 134.71$ in GD vs. GD-TAO, respectively $(\mathrm{p}=0.02)$. TBI were negative in 41 tested samples of children with GD and positive in one HT child. In comparison, in children with HT, TSI were positive in 4/83 (4.8\%) samples of whom two had HT-TAO. TSI levels were also increased in the children with HT-TAO vs HT (SRR\% 177.3 vs. 50.7; $\mathrm{p}<0.01$ ). All healthy controls, JA and T1D without thyroid involvement were TSI and TBI negative.

Conclusion: TSI is prevalent in children with GD. Occurrence of orbitopathy associates with TSI's presence both in GD and HT. Higher TSI levels in group with vs. without TAO are observed.

\section{P2-01-59 \\ COMPARISON OF IMMUNOHISTOCHEMICAL EXPRESSION OF IL-1BETA AND IL-23 IN AUTOIMMUNE THYROID DISEASE}

Tatjana Zake ${ }^{1}$, Sandra Skuja ${ }^{2}$, leva Kalere ${ }^{3}$, Ilze Konrade ${ }^{1}$, Valerija Groma $^{2}$

${ }^{1}$ Riga Stradins University, Riga East Clinical University Hospital, Riga, Latvia, ${ }^{2}$ Institute of Anatomy and Anthropology, Riga Stradins University, Riga, Latvia, ${ }^{3}$ Riga Stradins University, Riga, Latvia

Objectives: Th17 cells producing mostly IL-17, IL-21 and IL-22 were reported to be involved in the development of thyroid autoimmunity. In the presence of IL-1 $\beta$, IL-6, and IL-23, Th0 cells give rise to Th17 lymphocytes, whereas IL-23 induces conversion of classical Th17 cells into pro-inflammatory pathogenic lymphocytes. Furthermore, IL-1 $\beta$ has been shown to modify thyroid epithelium integrity via junction protein alteration. However, there is limited data on immunoexpression levels of IL-23 and IL- $1 \beta$ in autoimmune thyroid disease. The aim of our study was to estimate the levels of immunoexpression of IL-23 and IL-1 $\beta$ within thyroid tissue of patients with Hashimoto's thyroiditis (HT) and Graves' disease (GD) and compare them with controls.

Methods: 47 adult patients presenting 21 cases of HT, 8 of GD, and 18 cases of ordinary colloidal goiter without autoimmune component, which served as control group, were enrolled in this study. Immunostaining was performed using an anti-IL-23 and IL-1 $\beta$ antibodies. Levels of immunopositivity were defined semiquantitatively.

Results: In HT patients the expression of IL-1 $\beta$ and IL-23 was detected both in the abundant inflammatory lymphocytic infiltrates characteristic of HT and thyrocytes, by contrast, tissues obtained from GD patients demonstrated the weak thyrocytic expression predominantly. Majority of patients in controls showed negative or occasional expression of IL-23 and IL-1 $\beta$. The highest IL-1 $\beta$ and IL-23 immunopositivity was observed in HT patients, furthermore, it was significantly higher compared to the control group ( $<<0.001$; both) and GD $(p<0.001 ; p=0.043$, respectively). No difference was found between the expression of IL-1 $\beta$ and IL-23 in GD patients and the control group ( $\mathrm{p}=0.144$; $\mathrm{p}=0.324$, respectively). All patients showed a moderate positive correlation between IL-1 $\beta$ and IL-23 immunoreactivity $(\mathrm{r}=0.618, \mathrm{p}<0.001)$.

Conclusion: Overexpression of IL-1 $\beta$ and IL-23 might play a role in the pathogenesis of HT, inducing differentiation of Th17 cells. 


\section{P2-01-60 \\ DOES FOKI (RS2228570) VDR POLYMORPHISM CONTRIBUTE TO AUTOIMMUNE THYROID DISEASES SUSCEPTIBILITY? - OWN RESULTS AND A META-ANALYSIS}

Adam Maciejewski ${ }^{1}$, Michał Kowalczyk ${ }^{2}$, Teresa Gasińska ${ }^{3}$, Waldemar Herman $^{4}$, Adam Czyżyk ${ }^{5}$, Jolanta Dorszewska ${ }^{6}$, Ryszard Żaba ${ }^{2}$,

Katarzyna Łącka ${ }^{1}$

${ }^{1}$ Poznan University of Medical Sciences, Department of Endocrinology, Metabolism and Internal Medicine, Poznań, Poland, ²Poznan University of Medical Sciences, Department of Dermatology and Venereology, Poznań, Poland, ${ }^{3}$ Medical University of Silesia, Department of Internal Diseases and Oncological Chemotherapy, Katowice, Poland, ${ }^{4}$ Outpatients Unit of Endocrine Diseases, Wschowa, Poland, ${ }^{5}$ Poznan University of Medical Sciences, Department of Gynecological

Endocrinology, Poznań, Poland, ${ }^{6}$ Poznan University of Medical Sciences, Laboratory of Neurobiology, Department of Neurology, Poznań, Poland

Objectives: Vitamin D is believed to be an important factor in the immune system function and cell proliferation regulation. It is suspected that vitamin $\mathrm{D}$ effectiveness can be modified by vitamin $\mathrm{D}$ receptor gene $(V D R)$ polymorphisms. Among them FokI (rs2228570) SNP has been most consequently reported to be associated with autoimmune thyroid diseases (AITD). Therefore, the aim of this study was to assess the association of FokI SNP with the risk of AITD among the Caucasian-Polish patients. We also conducted a meta-analysis of currently available studies on this association.

Methods: The studied group consisted of 395 subjects diagnosed with AITD (258 with autoimmune thyroiditis (HT) and 137 with Graves` orbitopathy (GO)) and 129 healthy, age and sex matched controls. FokI SNP was studied by PCR-RFLP analysis. The statistical significance of differences in allele or genotype frequencies between AITD vs. controls, as well as between HT or GO vs. controls was calculated by $\chi^{2}$ test. Any p-value of $<0.05$ was considered significant.

Results: $\mathrm{C}$ allele and $\mathrm{CC}$ genotype of the studied polymorphism were more frequent in AITD compared to controls, although the differences were not statistically significant. When HT and GO were analyzed separately, the higher difference in allele frequency was observed between GO patients and the control group $(\mathrm{p}=0.056)$ comparing to HT vs. controls $(\mathrm{p}=0.442)$. After systematic review, we found also fourteen eligible studies, which together with our own results were included in a meta-analysis. It showed that FokI SNP is significantly associated with AITD development (C vs. T: OR=1.29, $95 \% \mathrm{CI}=1.10-1.51$ ), but not in Caucasian subgroup ( $\mathrm{C}$ vs. $\mathrm{T}$ : $\mathrm{OR}=1.14$, $95 \% \mathrm{CI}=0.94-1.39$ ).

Conclusions: There is no significant association between FokI VDR SNP and AITD risk in our study, what is in accordance with other results for Caucasians.

\section{P2-01-61 \\ IMMUNE REACTIVITY TO GLIADIN AND OTHER FOOD ANTIGENS IN GRAVES‘ DISEASE}

Danila Covelli', Giuseppe Colucci' ${ }^{2}$, Ulike Kaiser ${ }^{3}$, Anja Eckstein ${ }^{4}$, Maria Cristina Burlacu ${ }^{5}$, Chantal Daumerie ${ }^{6}$, Gez Richell, Petros Perros $^{8}$, Mohd Shazli Draman ${ }^{9}$, Marian Ludgate ${ }^{10}$, Giulia Masetti ${ }^{11}$, Filippo Biscarini ${ }^{11}$, Mario Salvi ${ }^{12}$

'19raves' Orbitopathy Unit, Endocrinology, Fondazione Ca' Granda Irccs, University of Milan, Italy, Medical Sciences, Milano, Italy, 21graves' Orbitopathy Unit, Endocrinology, Fondazione Ca' Granda Irccs, University of Milan, Italy, Milan, ${ }^{3}$ Univ-Augenklinik, Essen, ${ }^{4}$ Univ.Augenklinik, Essen, Germany, ${ }^{5}$ Cliniques Universitaires Saint-Luc, Bruxelles, ${ }^{6}$ Cliniques Universitaires Saint-Luc, Université Catholique de Louvain, Endocrinologie, Brussels, Belgium, ${ }^{7}$ Royal Victoria Infirmary, Newcastle, ${ }^{8}$ Freeman Hospital, Endocrine Unit, Newcastle-Upon-Tyne, UK, ${ }^{9}$ Division of Infection and Immunity, Cardiff, ${ }^{10}$ Division of Infection \& Immunity, School of Medicine, Cardiff University, Cardiff, UK, ${ }^{11} \mathrm{Ptp}$ Science Park, Lodi, ${ }^{12}$ Dipartimento Scienze Mediche, Endocrinologia, Endocrine Unit, Fondazione Irccs Cà Granda, Milano, Italy

Objectives: The increased rates of autoimmunity reported in urban areas suggest a possible influence of dietary antigens and dysbiosis. We studied the antibody response to a panel of forty food antigens (FA) in GD patients. Since $10 \%$ of celiac patients develop autoimmune thyreoditis we also focused on gliadin (DGP), transglutaminases (tTG)

Methods: 71 sera from INDIGO European centres were tested for IgG to FA and compared to 25 healthy controls. 105 and 108 sera were tested for IgG and IgA to tTG and DGP, respectively and compared to epidemiological data. Commercially available ELISA assays were performed according to the manufacturer's instructions.

Results: Sensitivity against food antigens in GD patients, although not significantly different from healthy control ( $p$-value $=0.3$ ), showed variation in relation to the region of provenience. Interestingly, some antigens (cow's milk, egg white, wheat, yeast) are more frequently positive than others. The distribution of antibodies against TSH receptor (TRAb) values was not different in positive or negative sera.

The prevalence of tTG ( 6 out of 105 sera; $5.7 \%$ ) and that of DGP-IgA (16 out of $107 ; 15 \%)$ and DGP-IgG (7 out of $108 ; 6.5$ was higher compared to the worldwide prevalence of celiac disease $(1 \%$; p-value $<0.001)$. Prevalence of smokers and ocular involvement was not higher in patients with positive antibodies.

Conclusion: GD patients showed a higher prevalence of positive tTG antibodies compared to the worldwide prevalence of celiac disease. Immune reactivity to food antigens showed regional differences in Europe suggesting that diet may contribute to increased sensitivity. More studies are needed to confirm these data.

\section{P2-01-62 \\ POLYMORPHISMS OF RS7093069 AND \\ RS7647305 ON GRAVES‘ DISEASE IN COMPARISON WITH DIABETES TYPE 1 IN CHILDREN}

Artur Bossowski ${ }^{1}$, Joanna Goscik², Natalia Wawrusiewicz-Kurylonek ${ }^{3}$, Wojciech Mlynarski ${ }^{4}$, Adam Kretowski ${ }^{3}$

${ }^{1}$ Medical University in Białystok, Dep.of Pediatrics, Endo,

Diabeto, Bialystok, Poland, ${ }^{2}$ Centre for Experimental Medicine, Medical University in Bialystok, Bialystok, Poland, ${ }^{3}$ Department of Endocrinology and Diabetes With Internal Medicine, Medical University in Bialystok, Bialystok, Poland, ${ }^{4}$ Department of Pediatrics, Oncology,

Hematology With Diabetology, Medical University in Łódź, Poland

Background: The etiology of autoimmune diseases, including Graves' disease (GD) and diabetes type 1 is multifactorial and involves genetic and environmental factors. Family and population studies confirmed the strong genetic influence and inheritability in the development of these diseases. Most papers evaluating the relationship of rs7093069 and rs7647305 polymorphism with lipid metabolism and obesity. Possible differences in overexpression of the IL2RA, SFRS10, ETV5 and DGKG genes polymorphisms on GD and diabetes type 1 remain unclear.

Objective and Hypotheses: To identify the differences between polymorphisms of IL2RA, SFRS10, ETV5 and DGKG genes in patients with Graves' disease and patients with type 1 diabetes.

Method: The study was performed in 142 patients with GD and 94 patients with diabetes type 1 . The two single nucleotide polymorphisms (SNPs): rs7093069 - IL2RA and rs7647305 - SFRS10, ETV5 and DGKG were genotyped by TaqMan SNP genotyping assay using the real-time PCR.

Results: Rs7093069 C alleles were more frequent in GD patients in comparison to patients with diabetes type $1(\mathrm{p}<0.005$ with $\mathrm{OR}=1.9)$. Rs7647305 C alleles were more frequent in GD patients in comparison to patients with type $1(\mathrm{p}<0.005, \mathrm{OR}=3)$.

Conclusion: Rs7093069 C/T and rs7647305 C/T polymorphisms could contribute to development of GD. The main risk factor for 7093069 is $\mathrm{C}$ allele. In case of rs7647305 the main risk factor is also allele $\mathrm{C}$. 


\section{P2-02 Cancer Basic}

\section{P2-02-63 \\ VERY LOW EXPRESSION OF PD-L1 IN MEDULLARY THYROID CARCINOMA AND ITS INFLAMMATORY INFILTRATE}

Massimo Bongiovanni', Caterina Rebecchini', Chiara Saglietti ${ }^{1}$, JeanLuc Bulliard ${ }^{2}$, Laurence de Leval', Gerasimos Sykiotis ${ }^{3}$

${ }^{1}$ Service of Clinical Pathology, Institute of Pathology, Lausanne University Hospital, Lausanne, Switzerland, ${ }^{2}$ Institute of Social and Preventive Medicine, Lausanne, Switzerland, ${ }^{3}$ Service of Endocrinology, Diabetology and Metabolism, Lausanne University Hospital, Lausanne, Switzerland

Monoclonal antibodies that inhibit the interaction between PD1 and PD-L1 are approved for clinical use in several cancer types, and they are also in clinical trials for additional indications, including thyroid carcinomas. A few papers have reported on PD-L1 expression in thyroid carcinomas, including a recent large study using tissue microarrays on differentiated and anaplastic thyroid carcinoma. However, the expression of PD-L1 in medullary thyroid carcinoma (MTC) has not been reported so far, even though ongoing clinical studies aim to test the effectiveness of checkpoint inhibitors in this rare histotype as well. We thus assessed PD-L1 expression in both tumor cells and tumor-infiltrating immune cells in a series of 16 MTC cases at a tertiary center. Tumor cells were positive in only one case, which had $5 \%$ positive cells. Moreover, PD-L1 was present in tumor-infiltrating immune cells in only two cases, where $1 \%$ and $2 \%$ of the inflammatory cells were stained, respectively. No correlation was evident between PD-L1 expression and clinicopathological parameters or survival in our series. Our results are indicative of near uniform absence of PD-L1 expression in MTC and its accompanying inflammatory cells; these results should be replicated on a larger scale in other centers. Definitive answers regarding the utility of PD1/PD-L1 immunophenotyping in MTC and of the use of checkpoint inhibitors in the treatment of this aggressive and rare thyroid cancer must await the results of the respective ongoing clinical trials, which should perform correlations with PD1/PD-L1 expression.

\section{P2-02-64 \\ OVEREXPRESSION OF EGF RECEPTORS IN PAPILLARY THYROID CARCINOMA CORRELATES WITH HIGH FAK EXPRESSION AND ADVERSE CLINICOPATHOLOGICAL PARAMETERS}

Sonja Selemetjev ${ }^{1}$, Aleksandar Bartolome ${ }^{1}$, Tijana Isic Dencic ${ }^{1}$, Ilona Djoric $^{1}$, Ivan Paunovic ${ }^{2}$, Dubravka Cvejic ${ }^{1}$

${ }^{1}$ Institute for the Application of Nuclear Energy - Inep, Department for Endocrinology and Radioimmunology, University of Belgrade, Belgrade, Serbia, ${ }^{2}$ Center for Endocrine Surgery, Institute for

Endocrinology, Diabetes and Diseases of Metabolism,Clinical Center of Serbia, Belgrade, Serbia

Background: Up-regulated epidermal growth factor receptor (EGFR) and abberant EGFR-signaling have been correlated with tumor progression in a wide variety of carcinomas. Focal adhesion kinase (FAK) is a multifunctional cytoplasmic protein tyrosine kinase which integrates integrin and growth factor receptors signaling to modulate cellular functions and to promote cell migration, thus being involved in invasion and metastasis of cancer cells. Since overexpressions of EGFR and its downstream transducer FAK have been associated with tumor aggressiveness, we aimed to investigate the relationship between EGFR and FAK expressions and their correlation with clinical phenotype of papillary thyroid carcinoma (PTC) patients.

Methods: Expression profiles of EGFR and FAK were analyzed in PTC tissue samples $(\mathrm{n}=104)$ using immunohistochemistry and Western blotting. Additionally, EGFR and FAK were immunohistochemically analyzed in 20 primary tumors paired with their metastatic tissue in lymph nodes.

Results: High expressions of EGFR and FAK were found in 55.8\% and $57.7 \%$ cases, respectively, with a strong positive correlation to each other $(p<0.0001$, Spearman's correlation coefficient $=0.844)$. Expression of each molecule and their coexpression significantly correlated with the presence of lymph node metastasis, depth of tumor infiltration, extrathyroid invasion and pT status of the patients. Western blot analysis confirmed the coexpression of high levels of EGFR and FAK in correlation with adverse clinicopathological features. Metastatic tissue in lymph nodes expressed EGFR and FAK at the same high levels as their primary tumors indicating their concordant expression during lymphatic spreading.

Conclusions: High levels of EGFR and FAK, its downstream effector, in association with lymphatic spreading and tumor infiltration, indicate their involvement in PTC progression and suggest that both molecules may predict its aggressive behavior. Furthermore, FAK could be a potential target for anticancer therapy in patients with advanced thyroid cancer.

\section{P2-02-65 \\ THYROID TRANSCRIPTION FACTOR FOXE1 REGULATES THE EPITHELIAL-MESENCHYMAL TRANSITION PROTEIN ZEB1 IN THYROID CANCER CELLS}

Jesús Morillo $^{1}$, Lara P. Fernández ${ }^{2}$, Pilar Santisteban ${ }^{3}$

${ }^{1}$ Biomedical Research Institute (Csic-Uam), Ciberonc, Carlos III Institute, Madrid, Spain, ${ }^{2}$ Imdea Food Institute, Madrid, Spain, ${ }^{3}$ Instituto de Investigaciones Biomédicas, Universidad Autónoma de Madrid,

Ciberonc, Instituto de Salud Carlos III, Madrid, Spain

The thyroid-specific transcription factor FOXE1, is essential for thyroid gland development and for the maintenance of the differentiated state in adults. FOXE1 has been associated with thyroid cancer susceptibility and recent studies have described FOXE1 overexpression in papillary thyroid carcinomas, implicating a possible role for FOXE1 in carcinogenesis and tumour progression. Nevertheless, little is known about the mechanism underlying FOXE1-induced thyroid tumorigenesis. In a preliminary genome-wide expression screening, we identified new genes regulated by FOXE1 in thyroid cells. Among them, ZEB1, a major epithelial to mesenchymal transition (EMT) inducer, emerged as a putative FOXE1 target gene. To explore FOXE1mediated regulation of EMT, we studied the mechanism of ZEB1 control by FOXE1 in thyroid cancer cell lines.

RNAi knockdown of FOXE1 in thyroid cancer cell lines resulted in a decrease in ZEB1 expression analyzed by RT-qPCR and western blotting. Accordingly, we searched for a canonical FOXE1-binding site within the proximal human ZEB1 promoter, finding a FOXE1 binding domain highly conserved across evolution. To determine whether FOXE1 directly binds to the ZEB1 promoter, we performed electrophoretic mobility shift assays with a radioactive labeled probe containing the specific recognition sequence for FOXE1. Our results showed that FOXE1 bound to the probe within the specific sequence of the ZEB1 promoter. Finally, using a promoter luciferase assay in HeLa cells, we found that FOXE1 trans-activates the ZEB1 promoter.

In conclusion, we have identified ZEB1 as a bona fide target of FOXE1 in thyroid cancer cells, providing new insights into the role of FOXE1 in regulating EMT in thyroid cancer. The potential functional role of FOXE1 in thyroid cell migration and invasion is under study. 


\section{P2-02-66 \\ ROLE OF NADPH OXYDASE NOX4 IN THE REDOX REGULATION OF THE SODIUM (NA+)/ IODIDE (I-)SYMPORTER IN PAPILLARY THYROID CANCER \\ Buffet Camille ${ }^{1}$, Juliana Cazarin de Menezes ${ }^{1}$, Laurence Barde ${ }^{2}$, Raphaël Corre ${ }^{1}$, Fabio Hecht', Ruy Andrade Louzada Neto ${ }^{1}$, Abir Al Ghuzlan $^{3}$, Dana Hart ${ }^{3}$, Sophie Leboulleux ${ }^{3}$, Martin Schlumberger ${ }^{4}$, Corinne Dupuy ${ }^{1}$ \\ ${ }^{1}$ Institut Gustave Roussy; Umr 8200 Cnrs, Villejuif, France, ${ }^{2}$ Thyroid and Endocrine Tumors Unit, Institute E3m, Pitié-Salpêtrière Hospital, Paris VI University Cancer Institute, Paris, France, ${ }^{3}$ Gustave Roussy, Université Paris-Sud, Villejuif, France, ${ }^{4}$ Department of Nuclear Medicine and Endocrine Oncology, Gustave Roussy and Université Paris-Sud, Villejuif, France; Gustave Roussy, Université Paris-Sud, Villejuif, France}

BRAFV600E contributes to the aggressiveness of papillary thyroid cancers (PTC) with altered expression of the Sodium/Iodide Symporter (NIS), which is crucial for the treatment of patients with radioiodine. Our recent data highlight that the inactivation of the NADPH oxidase NOX4, a ROS generating system, promotes the reexpression of NIS in BRAFV600E-mutated thyroid cancer cell lines. The reversible nature of this inhibition suggests the existence of epigenetic modifications in the promoter region of the gene SCL5A5 (NIS), controlled by NOX4. Our hypothesis is that the recruitment of a silencing complex containing DNA methyltransferase (DNMT) and Histone Deacetylase (HDAC), following NOX4-mediated DNA damage, over the NIS promoter, may inhibit its transcription.

We show in the human PTC BRAFV600E-mutated cell line BCPAP that treatment with $\mathrm{H}_{2} \mathrm{O}_{2}$ results in enrichment in DNMT1 in the chromatin fraction which decreased after treatment with antioxydants (Diphenyliodonium (DPI), N-Acetyl-L-cystein). NOX4 siRNA inactivation reduced DNMT1 protein both in whole cell lysates and chromatin-enriched fraction. We have previously demonstrated that NOX4 is a key effector of TGF $\beta$. So we tested the effect of TGF $\beta$ treatment in BCPAP cells and also observed an increase in DNMT1 protein levels in chromatin fraction which was reversed by DPI treatment. In addition, we show that a nuclear-specific HDAC inhibitor (MS275) strongly increases NIS expression in the BRAFV600E-mutated cell lines BCPAP and $8505 \mathrm{C}$. Interestingly this is associated with NOX4 decreased mRNA levels in BCPAP cells. The work is now in progress. Further chromatin immunoprecipitation experiments will test the role of DNMT1 and HDAC1 in the regulation of NIS expression under the control of NOX4.

The data obtained should lead to a better understanding of the mechanisms by which NOX4 controls the expression of NIS in thyroid tumor cells and bring functional data for the development of new tools of diagnosis and / or prognosis.

P2-02-67 is now OP-03-98

\section{P2-02-68}

ROLE OF THE SCAFFOLD PROTEINS IQGAP1 AND IQGAP2 IN MAPK PATHWAY AND ITS IMPLICATION IN THYROID CANCER

Adrian Acuña ${ }^{1}$, Miguel Zaballos ${ }^{2}$, Marta Morante ${ }^{3}$, Rocío García Gómez ${ }^{4}$, Garcilaso Riesco-Eizaguirre ${ }^{5}$, Piero Crespo ${ }^{6}$, Pilar

Santisteban ${ }^{7}$

${ }^{1}$ Instituto de Investigaciones Biomédicas, Ciberonc, Instituto de Salud Carlos III, Madrid, Spain, ${ }^{2}$ Instituto de Investigaciones Biomédicas Alberto Sols, Ciberonc, Madrid, Spain, ${ }^{3}$ Insituto de Biomedicina Y Biotecnología de Cantabria, Santander, Spain, ${ }^{4}$ Instituto de Biomedicina Y Biotecnología de Cantabria, Santander, Spain, ${ }^{5} \mathrm{Hospital}$ Universitario de Móstoles, Ciberonc, Instituto de Salud Carlos III, Ciberonc Instituto de Salud Carlos III, Móstoles, Spain, ${ }^{6}$ Instituto de Biomedicina Y Biotecnología de Cantabria, Ciberonc, Instituto de Salud Carlos III, Santander, Spain, ${ }^{7}$ Instituto de Investigaciones Biomédicas, Universidad Autónoma de Madrid, Ciberonc, Instituto de Salud Carlos III, Madrid, Spain

Ras GTPase-activating-like proteins (IQGAPs) function as scaffold proteins and integrate signalling through the ERK/MAPK pathway. IQGAP1 is over-expressed in human thyroid tumours and a role in migration and invasion has been proposed. By contrast, IQGAP2 expression is frequently decreased in human tumours and it may function as a tumour suppressor.

We analysed the expression and function of IQGAPs, particularly regarding cell migration and proliferation. For this purpose, we used human thyroid tumour-derived cells, an orthotopic mouse model and human thyroid tumour samples,

Silencing of IQGAP1 in $8505 \mathrm{c}$ cells decreased ERK dimerization and activated p90RSK, an ERK cytoplasmic effector. We found that IQGAP1 relocalizes to the membranes of migrating cells where it co-localizes with activated p90RSK in actin structures involved in migration. In agreement with this, IQGAP1 appears enriched in the invasive front of thyroid tumours in an orthotopic mouse model and in human tumours. Unexpectedly, depletion of IQGAP1 by CRISPR/CAS9 resulted in increased proliferation of RAS mutant cells. Using an IQGAP1 mutant defective for the ERK binding site, we observed that this suppressive effect on proliferation occurs in an ERKindependent manner.

Additionally, IQGAP family members are differentially expressed in thyroid tumour cell lines. IQGAP1 was expressed in all cell lines tested; however, IQGAP2 expression was detectable only in RAS mutant cells. These data were confirmed analysing the TGCA data base. Furthermore, we also showed increased IQGAP1 expression in human PTC samples, more markedly in the invasive front and in lymph node metastasis.

Our data are consistent with a role for IQGAP1 in the regulation of tumourigenic related processes in an ERK-dependent and independent manner. Finally, our preliminary data on IQGAP2 expression show that it is very low expressed in BRAF mutant cells, pointing to a possible link between IQGAP2 loss of expression and BRAF-mediated tumourigenesis.

\section{P2-02-69 \\ UTILITY OF BRAF VGOOE IMMUNOPEROXIDASE STAIN IN CYTOLOGY PREPARATIONS OF CELL BLOCK AND ASPIRATE SMEARS IN THYROID CANCER \\ Maria E Cabanillas ${ }^{1}$, Amber Smith ${ }^{1}$, Michelle Williams ${ }^{1}$, John Stewart ${ }^{1}$, Wei-Lien Wang ${ }^{1}$, Savitri Krishnamurthy ${ }^{1}$, Sinchita Roy Chowdhuri ${ }^{1}$ ${ }^{1}$ The University of Texas MD Anderson Cancer Center, Houston, TX, USA}

Objectives: BRAF V600E is a common mutation in thyroid cancer. BRAF inhibitors are under investigation in advanced thyroid cancers based on of the presence of the BRAFV600E mutation. In advanced patients, the ability to perform BRAFV600E immunoperoxidase (IPOX) staining on FNA facilitates rapidly triaging patients to treatment options.

Methods: The pathology database was searched for thyroid FNA specimens with available material from pap smears and cell block sections and prior BRAF mutational status by molecular testing. An anti-BRAFV600E (clone VE1, 1:50; Spring Bioscience) was used for immunoperoxidase evaluation (IPOX). Positive IPOX staining was cytoplasmic, and scored as positive 
(strong 2-3+), equivocal (1+), or negative (0). The results of the BRAF IPOX in cytology preparations were compared with mutational analysis of corresponding surgical pathology material and used to calculate the sensitivity and specificity. Cytology preparations with equivocal results were excluded from the statistical analysis.

Results: 23 malignant cases and 2 benign nodules were identified. All 23 carcinoma cases had $B R A F$ mutational analysis by molecular sequencing; 4 cases also had prior BRAF immunohistochemical evaluation on surgical material. 5 of $23(21 \%)$ malignant cases were equivocal or discordant on cytology material. One of 14 (7.1\%) cell blocks were equivocal with corresponding positive molecular assessment, but none were discordant; whereas 2 of 20 $(10 \%)$ smears were equivocal and 3 of $20(15 \%)$ were discordant with positive IPOX on smear and negative molecular. Of cell block cases, sensitivity was $100 \%$ with specificity of $70 \%$ ( 3 of 21 cases), excluding equivocal cases.

Conclusion: BRAFV600E IPOX staining can be performed reliably on cell block cytology preparations. However, false positive results by BRAF IPOX on FNA smears limit their utility for BRAF assessment in thyroid cancer. Our study supports FNA assessment in recurrent thyroid carcinomas with cell block preparations specifically, providing another tool for expedited assessment of $B R A F$

\begin{tabular}{|c|c|}
\hline Total cases & $\mathrm{N}=25$ \\
\hline Classification (n) & $\begin{array}{l}\text { Carcinoma (23) } \\
\text { Benign (2) }\end{array}$ \\
\hline Tumor types (n) & $\begin{array}{l}\text { Papillary thyroid carcinoma (14) } \\
\text { Poorly differentiated thyroid carcinoma (4) } \\
\text { Anaplastic thyroid carcinoma (3) } \\
\text { Mixed carcinoma (2) } \\
\text { Hürthle cell neoplasm (2) }\end{array}$ \\
\hline Material (n) & $\begin{array}{l}\text { Cell block (9) } \\
\text { Smear (13) }\end{array}$ \\
\hline Agreement (n) & $\begin{array}{l}\text { Concordant cases }(13) \\
\text { Discordant/equivocal cases }(7)\end{array}$ \\
\hline $\begin{array}{l}\text { False Positive/Negative Cases } \\
\text { by Material Type Compared to } \\
\text { Molecular Testing (n) }\end{array}$ & $\begin{array}{l}\text { False positive smears }(3) \\
\text { False positive cell blocks }(0) \\
\text { False negative smear }(0) \\
\text { False negative cell block }(0)\end{array}$ \\
\hline
\end{tabular}

\section{P2-02-70 \\ IMMUNOCYTOCHEMICAL DETECTION OF THE BRAF-V600E MUTATION IN PAPILLARY THYROID CARCINOMA - A PILOT STUDY}

Kristine Zøylner Swan ${ }^{1}$, Viveque Egsgaard Nielsen ${ }^{2}$, Steen Joop Bonnema ${ }^{3}$, Marie Louise Jespersen ${ }^{4}$

${ }^{1}$ Department of Otorhinolaryngology, and Head and Neck Surgery, Aarhus University Hospital, Institute for Clincal Medicine, Aarhus University, Aarhus C, Denmark, ${ }^{2}$ Institute for Clinical Medicine, Aarhus University, Aarhus C, Denmark, ${ }^{3}$ Department of Endocrinology and Metabolism, Odense University Hospital, Department of Clinical Research, Faculty of Health Sciences, University of Southern Denmark, Odense C, Denmark, ${ }^{4}$ Department of Pathology, Aarhus University Hospital, Aarhus, Denmark

Objective: The incidence of differentiated thyroid carcinoma (DTC) is increasing. Papillary thyroid carcinoma (PTC) is the most common type of DTC and $40-50 \%$ of PTC harbor the $\mathrm{BRAF}^{\mathrm{V} 600 \mathrm{E}}$ (BRAF) mutation, which has been associated with more aggressive clinicopathological characteristics of PTC. Different methods for preoperative identification of the BRAF mutations exist, but one has not been recommended over the others. The objective of the study was to preoperatively evaluate immunocytochemical (ICC) detection of the BRAF mutation in PTC.

Methods: Prospectively collected preoperative cytological clots from patients suspected for PTC were tested with BRAF-ICC. Histological samples from the same patients were tested with BRAF immunohistochemestry (IHC). The reference standards for diagnosis and mutation status were histopathological examination and the Cobas test (PCR) on histological specimens.

Results: Thirty-seven patients were included in the study [female/male: 29/8; PTC/control (not PTC): 19/18]. ICC and IHC staining as well as the Cobas test were available in 18 patients. Eleven patients were histologically diagnosed with PTC, of whom five (45\%) had a BRAF mutation detected by the Cobas test. There was complete concordance with ICC, and all seven patients without PTC were negative on both tests. Thus, ICC performed with $100 \%$ sensitivity and specificity as compared with the Cobas test. When comparing IHC with the Cobas test (and thus ICC) two samples showed discordant results (one false negative and one false positive), resulting in a sensitively of $80 \%$ and specificity of $92 \%$.

Conclusions: ICC may be useful for preoperative detection of the BRAF mutation, by showing absolute concordance between BRAF-ICC (cytology) and the Cobas test (histology). To confirm these preliminary results ICC accuracy must be investigated in a larger population. Furthermore, optimization of the BRAF-ICC and BRAF-IHC method for PTC is warranted before clinical implementation.

\section{P2-02-71 \\ TRANSCRIPTION FACTOR SOX9 IS DIFFERENTIALLY EXPRESSED IN TUMOUR THYROID CELLS AND HAS A ROLE ON CELL MIGRATION \\ Carlos Carrasco López ${ }^{1}$, Pilar Santisteban ${ }^{2}$ \\ ${ }^{1}$ Instituto de Investigaciones Biomédicas (Csic-Uam), Ciberonc, Instituto de Salud Carlos III, Madrid, Spain, ${ }^{2}$ Instituto de \\ Investigaciones Biomédicas, Universidad Autónoma de Madrid, Ciberonc, Instituto de Salud Carlos III, Madrid, Spain}

The sex-determining region Y (SRY)-box (SOX) family of transcription factors are involved in regulating many developmental processes, including organogenesis and stem cell maintenance. Due to their participation in the regulation of cell differentiation, proliferation and survival, they have been widely studied in carcinogenesis. SOX9, a member of the SOX family, appears to play a role in different types of malignancies, acting either as an oncogene or a tumour suppressor; however, its role on thyroid cancer remains unclear.

SOX9 expression was analysed by RT-qPCR and western blotting in a range of tumour thyroid cell lines. We found that SOX9 expression was lower in PTC cell lines than in FTC cell lines. SOX9 expression in ATC cell lines was variable; whereas Hth83 cells presented the highest levels of SOX9, Cal62 and $8505 \mathrm{c}$ cells had mild expression. These data suggest a wide variability between cell lines with different mutations. To evaluate whether this variability was the result of the different oncogenes harboured by these cells, we analysed Sox9 levels in $\mathrm{PCCl} 3$ cells conditionally expressing BRAF or HRAS. We found that both oncogenes increased SOX9 levels. However, analysing the TCGA data-base, we were unable to find any association between the expression of SOX9 and the aforementioned driver oncogenes. To begin to analyse SOX9 function in ATC cells, we silenced its expression in Hth83 cells by RNAi and we overexpressed it in Cal62 and 8505c cells. Among the tumourigenisis parameters analysed, we observed that SOX9 increased cell migration.

In conclusion, SOX9 is expressed in multiple thyroid cancer cell lines where it plays a role in controlling migration. This regulation could be mediated through control of the epithelial mesenchymal transition pathway and future experiments will explore this possibility.

\section{P2-02-72 \\ NO GERMLINE MUTATIONS OF DICER1 AND HABP2, THE TWO NEW POTENT GENES PREDISPOSING TO FAMILIAL PAPILLARY THYROID CARCINOMA}

Nelly Sabbaghian ${ }^{1}$, Leanne de Kock', William D Foulkes², Marek Niedziela $^{3}$

${ }^{1}$ Lady Davis Institute, Segal Cancer Centre, Jewish General Hospital, Department of Human Genetics, Mcgill University, Montreal, Canada, 2Lady Davis Institute, Segal Cancer Centre, Jewish General Hospital, Department of Human Genetics, Mcgill University, Research Institute of the Mcgill University Health Centre, Montreal, Canada, ${ }^{3}$ Department of Pediatric Endocrinology and Rheumatology, Karol Jonscher's Clinical Hospital, Poznan University of Medical Sciences, Poznan, Poland

Objective: Nonmedullary thyroid cancers of follicular-cell origin (NMTC) comprise more than $95 \%$ of all thyroid cancers. Familial NMTC 
(fNMTC) is inherited in an autosomal dominant fashion and accounts for 3 to $9 \%$ of all NMTC cases. ANMTC may occur in isolated form (nonsyndromic) or may coexist with other cancers or tumors (syndromic form). The vast majority of fNMTC cases have papillary thyroid carcinoma (PTC), which is the most common type of thyroid cancer.

The aim of the study was to investigate whether DICER1 (mutations or large duplications or deletions) and the $H A B P 2$ p.G534E variant, the two new potent genes predisposing to familial PTC, play a pathogenic role in a family with 3 cases of PTC.

Methods: A total of 11 family members were investigated [ 1 teenager with autoimmune thyroiditis and 10 adults among whom 3 had multifocal PTC (grandmother and her 2 sons), 2 had autoimmune thyroiditis, 2 had multinodular goiters and 3 were healthy subjects]. Patients' history, clinical examination, thyroid ultrasound, thyroid function tests, autoimmune profile and anthropometric parameters were evaluated. A DNA sample from the grandmother with PTC was screened for mutations in DICERI by DNA sequencing and by MLPA. DNA samples from all family members were screened for the G534E loss of function mutation in $H A B P 2$.

Results: The genetic testing of DICERI in PTC patient was negative. The family was also not found to carry the known pathogenic p.G534E variant in HABP2.

Conclusions: Our work shows that both genes, DICER1 and HABP2, are not involved in the pathogenesis of fPTC in this family. The results do not rule out of other mutation types in DICERI such as deep intronic mutations, mutation/deletion in other positions of $H A B P 2$ or mutations in other genes.

\section{P2-03 Cancer Miscellaneous}

\section{P2-03-73 \\ VITAMIN D LEVELS: A POTENTIAL MODIFIABLE RISK FACTOR FOR THYROID MALIGNANCY}

Saleh Fahad Aldahri', Mohammed AlEssa ${ }^{1}$, Mohammed Alghammass ${ }^{1}$ Fahad Aldahri ${ }^{1}$

${ }^{1}$ Riyadh, Saudi Arabia

Objective*: The aim of this study is to look at the correlation of thyroid cancer incidence with 25-hydroxy-vitaminD levels based on Institute Of Medicine's reference ranges.

Methodology*: King Abdulaziz University Hospital's thyroid database was used to retrospectively review patients who underwent thyroidectomy between January 2011 and March 2016. Patients' demographics, histopathology, levels of vitamin D, corrected calcium, PTH and TSH were collected for analysis.

Patients were categorized according to their vitamin D levels to: Deficient $<30$, Insufficient $=30-50$ and sufficient $>50$.

Vitamin D levels at admission were then compared to final histopathological diagnosis using Fischer's exact test and logistic regression.

Results*: 783 patients were included of which $79 \%$ were female, with a mean age of 41 years. $43 \%$ of patients were vitD deficient while $23 \%$ were insufficient. $47 \%$ of patients were diagnosed as malignant.

Vitamin D levels didn't show any statistically significant correlation with the incidence of thyroid cancer $(\mathrm{P}$ value $=0.35)$.

A multivariate analysis done on Vitamin D levels, BMI, age and TSH also showed no significant correlation of either with thyroid cancer incidence.

Conclusions: In this study we conclude that our study population did not show any correlation between thyroid cancer incidence and their vitaminD levels upon presentation.

\section{P2-03-74 \\ DIFFERENTIATED THYROID CANCER- ULTRASOUND AND HISTOPATHOLOGICAL FEATURES. A TERTIARY CENTER EXPERIENCE IN ROMANIA}

Carmen Sorina Martin ${ }^{1}$, Oana Budianu ${ }^{2}$, Oana Ion ${ }^{3}$, Andreea Grigore ${ }^{3}$, Anca Sirbu ${ }^{4}$, Alice Albu ${ }^{4}$, Carmen Barbu ${ }^{4}$, Cosmin Giulea ${ }^{5}$, Adrian Miron $^{5}$, Florin Andrei ${ }^{6}$, Simona Fica ${ }^{7}$

${ }^{1}$ Carol Davila University of Medicine and Pharmacy, Endocrinology Department, Elias Hospital, Endocrinology Department, Bucharest, Romania, ${ }^{2}$ Elias Hospital, Endocrinology Department, Bucharest, ${ }^{3}$ Carol Davila University of Medicine and Pharmacy, Bucharest, ${ }^{4} \mathrm{Carol}$ Davila University of Medicine and Pharmacy, Endocrinology Department, Elias Hospital, Endocrinology Department, Bucharest, ${ }^{5}$ Carol Davila University of Medicine and Pharmacy, Surgery Department, Elias Hospital, Surgery Department, Bucharest, ${ }^{6}$ Elias Hospital, Pathology Department, Bucharest, ${ }^{7}$ Carol Davila University of Medicine and Pharmacy, Endocrinology Department, Elias Hospital, Endocrinology Department, Bucharest

Objectives: Differentiated thyroid cancer (DTC) comprises follicular (FTC), a relatively rare form, and papillary (PTC), the most common type of thyroid neoplasia (about $50-90 \%$ of all DTC). The aim of our study was to analyze the ultrasonographic and histopathological features of DTC at diagnostic, in patients undergoing thyroidectomy.

Methods: We retrospectively analyzed the files of 493 patients that underwent thyroidectomy in our surgery department between 01.01.201230.09.2015. Anthropometric, biological and imagistic data, indications for thyroid surgery, surgical procedures and pathology results were recorded.

Results: 86 patients with differentiated thyroid carcinoma were enrolled, 81 (94.18\%) with PTC and 5 (5.82\%) with FTC. We found no significant differences between PTC and FTC patients regarding most ultrasound features of the thyroid nodules: diameter $(\mathrm{p}=0.540)$, halo presence $(\mathrm{p}=0.558)$, microcalcifications $(\mathrm{p}=0.221)$, vascularization $(\mathrm{p}=0.583)$ and lymph nodes involvement $(\mathrm{p}=0.822)$, yet PTC nodules were more often hypoechoic $(75.6 \%$ vs $33.3 \%, p=0.002)$ that FTC nodules. Pathological tumor stages in PTC patients were: $\mathrm{T} 1$ in $31(38.3 \%)$, T2 in $7(8.6 \%), \mathrm{T} 3$ in $40(49.4 \%)$ and $\mathrm{T} 4$ in 3 (3.7\%) patients, whereas in FTC were: T1 in $1(20 \%)$ and T2 in $4(80 \%)(\mathrm{p}<$ $0.001)$ patients. Vascular invasion was present in $17.3 \%$ PTC compared to all FTC patients $(p<0.001)$. Furthermore, there were no significant differences between PTC and FTC patients regarding other histopathological characteristics: tumor diameter $(\mathrm{p}=0.460)$, multifocality $(\mathrm{p}=0.100)$, capsular invasion $(\mathrm{p}=0.846)$ or the presence of local lymph nodes metastasis $(\mathrm{p}=0.378)$ at diagnosis.

Conclusions: Differentiated thyroid carcinoma, the most common primary malignancy of the thyroid gland, is represented primarily by papillary and less frequently by follicular thyroid carcinoma. Our data showed that PTC nodules were more often hypoechoic and that FTC was diagnosed in earlier stages.

\section{P2-03-75 \\ EVALUATION OF PAPILLARY CARCINOMA}

$\underline{R e n a t a ~ M a r k o s y a n}^{1}$

1"Muratsan" University Clinic, Yerevan State Medical University,

Yerevan, Armenia

Objectives: The aim of this study was to investigate hormonal and ultrasound features in patients with papillary carcinoma.

Materials and Methods: We evaluated 23 patients with papillary carcinoma admitted to the "Muratcan"university clinic between June 2015 and January 2017 years. Diagnosis was based on ultrasound investigation (echogenity, structure, vascularization, volume, size and amount of nodules), hormonal assessment (TSH, f T4, anti-TGA, anti-TG, thyroglobulin), and FNAC of thyroid (the Bethesda System ) and post operation hystomorphology.

Results: 16 female $(69,6 \%)$ and 7 male $(30,4 \%)$ was included into this study. The main age at the diagnosis was $32,9 \pm 13.8$ years. There was no difference between female and male ages at diagnosis $(\mathrm{p}<0.05)$. Serum TSH levels were normal in $78,2 \%(18)$ of patients, elevated in $21,8 \%(5)$. Nobody has not decreased level of TSH. Thyroid antibodies were present in $26 \%(6)$ of patients. The mean level of thyroglobulin was elevated in $78,2 \%(18)$. 
Single nodule was present in $73,9 \%(17)$, more nodules are present in $26,1 \%$ (6)patients. The main size of nodules were $1,2 \pm 0,9 \mathrm{~cm}$.Intranodular vascularization was present in $60,8 \%(14)$ patients.69,5\% (16)of of patients had hypoechoic nodules. Lymph node metastasis was present in $13 \%(3)$ patients. $52 \%(12)$ of patients had lymph nodes and $13 \%$ (3)distant metastasis.

Conclusion: There were difference between gender, elevated level of thyroglobulin $(\mathrm{p}<0.05)$. We found correlation between ultrasound features and presents of papillary carcinoma. There was now correlation between serum level of TSH and presents of papillary carcinoma. Further studies are needed.

\section{P2-03-76 \\ CHANGES IN HISTOPATHOLOGIC AGGRESSIVENESS OF PAPILLARY THYROID MICROCARCINOMA OVER A 16-YEAR TIME PERIOD IN CRETE, GREECE}

Vassiliki Daraki ${ }^{1}$, Theodoros Liarigkovinos ${ }^{1}$, Paraskevi Floroskoufi', Konstantinos Kalotinis ${ }^{1}$, Maria Chrysoulaki ${ }^{1}$, Maria Dolapsaki ${ }^{1}$, Theano Roumeliotaki ${ }^{2}$, Garyfallia Tzifa ${ }^{1}$, Emmanuel Prokopakis ${ }^{3}$, Georgios Chalkiadakis $^{4}$, Odysseas Zoras ${ }^{5}$, Evangelia Mamalaki', Stathis Papavasiliou ${ }^{1}$

${ }^{1}$ Department of Endocrinology, Diabetes and Metabolic Diseases, University Hospital of Crete, Heraklion, Crete, Greece, ${ }^{2}$ Department of Social Medicine, Faculty of Medicine, University of Crete, Heraklion, Crete, Greece, ${ }^{3}$ Department of Otolaryngology-Head and Neck Surgery, University Hospital of Crete, Heraklion, Crete, Greece, ${ }^{4}$ Department of General Surgery, University Hospital of Crete, Heraklion, Crete, Greece, ${ }^{5}$ Department of Surgical Oncology, University Hospital of Crete, Heraklion, Crete, Greece

Objectives: Thyroid papillary microcarcinoma (PTMC) is a subtype of papillary thyroid cancer with less than $10 \mathrm{~mm}$ diameter. Although PTMCs are characterized by benign behavior, they show very diverse disease extent with widely varying reported frequencies of aggressive features. Therefore, their clinical significance and optimal management is still controversial. We aimed to investigate possible changes in histopathologic characteristics of PTMCs during the last 16 years in the island of Crete, Greece.

Methods: We retrospectively analyzed all histology records from patients $(n=3253)$ who had undergone thyroid surgery in an academic tertiary medical center (University Hospital of Heraklion, Crete, Greece) from January 2000 to December 2016. Patients' records were entered in a database, which included medical condition, history, and demographics. Data were grouped according to year of surgery: period 1, 2000-2005; period 2, 2006-2011; and period 3, 2012-2016.

Results: Thyroid cancer was confirmed in 1037 patients (32\%). Papillary carcinoma (PTC) represented the most frequent type (947 patients, 91.32\%), with an increased incidence during the 16-year time period, reaching its maximum value in the year 2016 (94 cases, 56\%). Of all PTC cases $69 \%(n=653)$. were microcarcinomas (PTMCs). PTMCs had significantly increased frequency of multifocal disease and co-existence with thyroiditis Hashimoto in the third period ( $p=0.001$ and $p<0.001$, respectively). PTMCs had also significantly increased frequencies of capsular invasion, vascular embolus, tumor extension beyond the thyroid parenchyma and metastatic lymphadenopathy in the third period $(\mathrm{p}<0.001, \mathrm{p}<0.001, \mathrm{p}=0.002, \mathrm{p}=0.039$, respectively), compared with the other two periods.

Conclusions: Our findings suggest a significantly increased frequency of aggressive behavior of PTMCs in the last 5 years in the island of Crete. As recent guidelines recommend a non-aggressive treatment for PTMCs further investigation of the clinical impact of the observed aggressiveness is fundamental for the optimal management of this type of thyroid cancer.

\section{P2-03-77 \\ WELL DIFFERENTIATED THYROID CANCER : A TERTIARY CENTRE EXPERIENCE AMONG AN INDIAN COHORT}

Shiga Rappai ${ }^{1}$, Vasantha Nair

${ }^{1}$ Amrita Institute of Medical Sciences, Kochi, India

Objective: Well-differentiated thyroid cancer (WDTC) is the most common form of thyroid malignancy. This paper aims to describe the experience of a Tertiary Hospital in managing patients with differentiated thyroid cancer.

Method: We performed a retrospective cohort study of 417 patients with WDTC (363 papillary and 54 follicular), seen between 2010 to February 2017 at The Thyroid Cancer Clinic, Department of Endocrinology, Amrita Institute of Medical Sciences, India. This study was undertaken to evaluate the clinicopathologic profile and the treatment modalities received. We also aimed to study the incidence of persistence/recurrence of disease at the end of 1 year.

Results: A total of 417 patients were included in the study, 117(28.1\%) males and 300 (71.9\%) females. Their mean age was $45.10 \pm 13.128$. In our cohort family history was known for 330 subjects, out of which 19 had a positive family history of differentiated thyroid carcinoma. Among the 417, $208(49.9 \%)$ underwent surgery at our institution, $175(42 \%)$ were operated elsewhere and 34(8.2\%) at both institutions. Out of the 417, 212(50.8\%) were classical papillary carcinoma, 91(21.8\%) were follicular variant of papillary cancer, $54(12.9 \%)$ were follicular cancer and rest $60(14.4 \%)$ micropapillary cancer. $18(4.3 \%)$ patients had distant metastasis at diagnosis. Out of 417 patients, 273(65.5\%) underwent total thyroidectomy, 96(23\%) underwent total thyroidectomy along with neck dissection, $48(11.5 \%)$ underwent less than total thyroidectomy. $65(20.4 \%)$ had tumor less than $10 \mathrm{~mm}$ size, $68(21.4 \%)$ between $10-20 \mathrm{~mm}, 118(37.1 \%)$ between $20-40 \mathrm{~mm}$ and $67(21.1 \%)$ more than $40 \mathrm{~mm}$

Conclusion: In the absence of sufficient data on WDTC in this region, the descriptive data from our centre showed classical papillary carcinoma to be the most common WDTC with majority having a tumor size of $20-40 \mathrm{~mm}$.

\section{P2-03-78 \\ DEMOGRAPHIC, ClINICAL, PATHOLOGICAL CHARACTERISTICS AND REMISSION RATES OF 624 DIFFERENTIATED THYROID CANCER (DTC) PATIENTS FROM A SINGLE CENTER}

Sule Canlar ${ }^{1}$, Murat Cinel', Berna İmge Aydoğan ${ }^{2}$, Çağlar Keskin ${ }^{1}$, Sevim Gullu1', Özgür Demir', Rifat Emral', Sevim Gullu ${ }^{3}$, Demet Çorapçıoğlu' ${ }^{1}$, Vedia Tonyukuk Gedik ${ }^{1}$, Nilgün Baskal' ${ }^{1}$, Murat Erdogan ${ }^{4}$ ${ }^{1}$ Ankara University School of Medicine, Department of Endocrinology and Metabolic Diseases; Faculty of Medicine, Ankara, Turkey, ${ }^{2}$ Ankara University School of Medicine, Department of Endocrinology and Metabolic Diseases, Ankara, Turkey, ${ }^{3}$ Ankara University School of Medicine, Department of Endocrinology and Metabolic Diseases; Faculty of Medicine, Ankara, Turkey, ${ }^{4}$ Ibni-I-Sina Hastanesi; Endokrinoloji Ve Met Hst B.D., Ankara, Turkey

Objective: Thyroid cancers constitute approximately $2 \%$ of all malignant tumors. DTC forms the majority of thyroid cancers. Our aim is to reveal the demographic, clinical, pathological features and remission rates of patients with DTC followed up in our single center.

Material-Method: Some characteristics of 624 DTC patients on followup in our department between 1985-2015 were retrospectively analyzed from the thyroid cancer database.

Results: 624 patients $(247 \mathrm{M} / 377 \mathrm{~F})$ had a mean age at diagnosis of $41.9 \pm 12.7$ years and a mean follow-up of $51.3 \pm 42.1$ months. Out of the 624 DTC patients, $95.2 \%$ were diagnosed with PTC and $4.8 \%$ with FTC. $78 \%$ of PTC cases were classical type, $18.5 \%$ follicular, $2.3 \%$ oncocytic and $1.2 \%$ tall cell variant.

Mean tumour size was $16.7 \pm 13 \mathrm{~mm} .42 .2 \%$ of the patients had capsule invasion, $34.8 \%$ of the patients were multicentric, $22 \%$ had lymph node metastasis, $16.7 \%$ had extrathyroidal extension, $6.9 \%$ had vascular invasion and $2.6 \%$ had distant metastases. According to the TNM classification $42.2 \%$ were $\mathrm{T} 1 \mathrm{a}, 32 \% \mathrm{~T} 1 \mathrm{~b}, 20.3 \% \mathrm{~T} 2$ and $5.5 \% \mathrm{~T} 3-\mathrm{T} 4$ tumors. Accordingly complete remission rates were $96.9 \%, 89.1 \%, 85.8 \%$ and $70 \%$ respectively for T1-4 tumors. 101 patients(16.2\%) underwent second surgery due to residual or recurrent disease. 
Conclusions: In the current series from a single center, $74.2 \%$ of patients had T1 low-risk tumors. Complete remission rates were compatible with the results reported in the literature.

\section{P2-03-79 \\ PREVALENCE OF INCIDENTAL THYROID CARCINOMA IN BENIGN THYROID DISEASES - A 13-YEAR SINGLE CENTER EXPERIENCE \\ Dimitrios Askitis ${ }^{1}$, Eleni I. Efremidou ${ }^{1}$, Agis Esebidis ${ }^{1}$, Michail A Karanikas $^{1}$, Alexandra Giatromanolaki ${ }^{2}$, Alexandros Polychronidis ${ }^{1}$, Nikolaos Lyratzopoulos ${ }^{1}$ \\ ${ }^{1}$ First Surgical Department, University Hospital of Alexandroupolis, Faculty of Medicine, Democritus University of Thrace, Alexandroupolis, Greece, ${ }^{2}$ University Pathology Department, University Hospital of Alexandroupolis, Faculty of Medicine, Democritus University of Thrace, Alexandroupolis, Greece}

Objective: Thyroid cancer comprises the most common endocrine malignancy, while the prevalence of incidentally discovered small thyroid carcinomas is continuously rising due to the frequent surgical treatment of otherwise benign thyroid disorders.

Objective of the current retrospective study was the assessment of the prevalence of incidental thyroid carcinoma (ITC) in benign thyroid diseases, subjected to total thyroidectomy (TT) over a period of 13 years in our department.

Patients-Methods: In First Surgical Department between 1.1.200431.12.2016 a total of 793 patients (660 females/ 133 males; mean age 51,1 years) underwent TT after referral for surgical treatment of benign thyroid diseases. Surgical specimens were evaluated in University Pathology Department and the diagnosis of ITC was recorded regarding preoperative diagnosis. Patients with a preoperative cytological diagnosis or suspicion of thyroid cancer were excluded.

Results: 112 patients $(14.1 \%)$ were diagnosed with microcarcinoma (females/males: $85 / 27$ ), while 681 patients $(85.9 \%)$ were free of malignancy. $40 / 124(32.3 \%)$ patients with solitary thyroid nodule (STN) and 68/530 $(12.8 \%)$ subjects with euthyroid multinodular goiter (MNG) were harboring an incidentally detected malignancy. 3 out of 37 subjects with Graves' disease $(8.1 \%)$ and $4 / 87$ patients $(4.6 \%)$ with toxic multinodular goiter featured malignancy. Interestingly, an incidental thyroid malignancy was diagnosed in 2/13 patients $(15.4 \%)$ with a history of surgical intervention of the thyroid gland (recurrences after subtotal thyroidectomy or lobectomy).

Conclusions: Incidental thyroid carcinoma was identified in a significant proportion of patients treated with total thyroidectomy for presumably benign thyroid diseases. The vast majority presented in people with non-toxic disorders, while nearly $1 / 6$ patients with thyroid disease recurrence featured malignancy. Therefore, total thyroidectomy appears as the surgical procedure of choice for patients featuring a strong indication for operative treatment of benign thyroid disease.

\section{P2-03-80 \\ REEVALUATION OF HISTOLOGICAL DIAGNOSES AND ClINICAL OUTCOMES OF PATIENTS PREVIOUSLY DIAGNOSED WITH FOLLICULAR THYROID CANCER}

Kiminori Sugino ${ }^{1}$, Tetsuo Kondo², Tomoaki Tanaka ${ }^{3}$, Yuna Ogimi ${ }^{4}$, Chie Masaki ${ }^{4}$, Akifumi Suzuki ${ }^{4}$, Junko Akaishi', Chisato Tomoda ${ }^{4}, K^{4}$ Kyomi Hames Yamada ${ }^{4}$, Kenichi Matsuzu ${ }^{4}$, Takashi Uruno ${ }^{4}$, Keiko Ohkuwa ${ }^{4}$, Wataru Kitagawa ${ }^{4}$, Mitsuji Nagahama ${ }^{4}$, Koichi Ito ${ }^{4}$

${ }^{1}$ Ito Hospital, Department of Surgery, Tokyo, Japan, ${ }^{2}$ Department of Pathology, University of Yamanashi, Yamasashi, Japan, ${ }^{3}$ Ito Hospital, Tokyo, Japan, ${ }^{4}$ Ito Hospital, Tokyo, Japan

Background: Diagnostic controversy and interobserver variability have rendered a practical diagnosis of follicular thyroid cancer (FTC) difficult. Furthermore, the 2004 WHO classification of Tumors of Endocrine Organs list poorly differentiated thyroid carcinoma (PDTC) as a separate tumor entity. The present study histologically reviews diagnoses of FTC and the long-term outcomes of patients at our hospital.

Patient and Methods: We assessed the records of 109 patients (male, $\mathrm{n}$ $=27$; female, $\mathrm{n}=82$; median age, 54 years) who were initially treated by sur- gery at our hospital under a histological diagnosis of minimally invasive FTC (MIFTC; $\mathrm{n}=92$ ) or widely invasive FTC (WIFTC; $\mathrm{n}=17$ ) between 2000 and 2004. The surgical specimens were all histologically reexamined by the same pathologist (TK) who was blinded to the clinical courses.

Results: Contemporary examination reclassified $5(4.6 \%), 3(2.8 \%), 10$ $(9.2 \%)$ and $4(3.6 \%)$ to papillary thyroid carcinoma (PTC), follicular adenoma, poorly- differentiated thyroid carcinoma (PDTC) and other thyroid diseases, respectively (total, 22 [20.2\%]) reclassifications. Among 87 patients diagnosed with FTC, 73 and 14 diagnoses were reclassified as MIFTC and WIFTC, respectively. Of these, $13(11.9 \%)$ had distant metastasis at initial surgery and $21(19.2 \%)$ developed distant metastasis during follow-up. The 10-year MFTC- and WFTC-specific survival rates before reclassification were $94.5 \%$ and $87.5 \%$, respectively. After reclassification, the 10-year MFTC-, WFTC- and PDTC-specific rates were $100 \%, 84.6 \%$ and $64.3 \%$, respectively. None of the patients with PTC died of cancer or developed recurrence.

Conclusion: The survival data support contemporary methods of histological classification for a differential diagnosis of FTC.

\section{P2-03-81 \\ SHEAR WAVE ELASTOGRAPHY IN THYROID NODULES WITH INDETERMINATE CYTOLOGY: RESULTS OF A PROSPECTIVE BICENTRIC STUDY}

Stephane Bardet ${ }^{1}$, Renaud Ciappuccini ${ }^{2}$, Herve Monpeyssen ${ }^{3}$, Claire Pellot-Barakat ${ }^{4}$, Jean-Jacques Michels ${ }^{5}$, Frederique Tissier ${ }^{6}$ David Blanchard ${ }^{7}$, Fabrice Menegaux 8 , Dominique De Raucourt ${ }^{9}$ Muriel Lefort ${ }^{10}$, Yves Reznik ${ }^{11}$, Agnes Roussel ${ }^{12}$, Natacha Heutte ${ }^{13}$, Frederique Brenac ${ }^{14}$, Alexandra Leconte ${ }^{13}$, Buffet Camille ${ }^{15}$, Bénédicte Clarisse $^{16}$, Laurence Leenhardt ${ }^{17}$

${ }^{1}$ Centre Francois Baclesse, Nuclear Medicine and Thyroid Unit, Caen, France, ${ }^{2}$ Centre Baclesse, Nuclear Medicine Department, Thyroid Unit, Caen, France, ${ }^{3}$ American Hospital of Paris, Neuilly Sur Seine, France, ${ }^{4}$ In Vivo Molecular Imaging, Imiv Laboratory Inserm, Cea, Cnrs, University Paris Sud and Paris Saclay, Orsay, France, ${ }^{5}$ Department of Pathology, Centre F Baclesse, Caen, France, ${ }^{6}$ Department of Pathology, Pitié-Salpétriere Hospital, University Paris VI, Paris, France, ${ }^{7}$ Centre Francois Baclesse, Head and Neck Surgery, Caen, France, ${ }^{8}$ Endocrine Surgery, Pitié Salpetriere Hospital, University Paris VI, Paris, France, ${ }^{9} \mathrm{Head}$ and Neck Surgery, Centre Francois Baclesse, Caen, France, ${ }^{10}$ Biomedical Imaging Laboratory, Lib, Upmc University Paris VI, Inserm, Cnrs, Sorbonne University, Paris, France, ${ }^{11}$ Endocrinology, University Hospital, Caen, France, ${ }^{12}$ Thyoid and Endocrine Tumors Unit, Institute of Endocrinology, Pitié-Salpétrière Hospital, University Paris VI, Paris, France, ${ }^{13}$ Clinical Research, Centre Francois Baclesse, Caen, France, ${ }^{14}$ Department of Radiology, Centre Francois Baclesse, Caen, France, ${ }^{15}$ Institut Gustave Roussy; Umr 8200 Cnrs, Villejuif, France, ${ }^{16} \mathrm{Clinical}$ Research, Centre Francois Baclesse, Caen, France, ${ }^{17} \mathrm{La}$ Pitie Salpetriere Hospital, Thyroid and Endocrine Tumors Unit, Paris, France

Objectives: The clinical management of thyroid nodules with indeterminate cytology (IC) remains challenging. The role of Shear Wave Elastography (SWE) in this setting is controversial. The aim of the study was to assess the performances of SWE in terms of prediction of malignancy, reproducibility, and combined analysis with ultrasound (US) examination in thyroid nodules with IC.

Methods: This prospective study was conducted in two referral centers. Eligible patients had a thyroid nodule $\geq 15 \mathrm{~mm}$ with IC (Bethesda class III to V) for which surgery had been recommended. Patients underwent a standardized US evaluation combined with a SWE exam followed by surgery. SWE parameters included mean (meanEI) and max (maxEI) elasticity values determined in a manually selected region of the nodule, and ratio (meanEI nodule/parenchyma).

Results: 131 nodules (median size, $30 \mathrm{~mm}$ ) in 131 patients were studied. IC was class III in $28 \%$, IV in $64 \%$ and V in $8 \%$ of cases. After surgery, 21 (16\%) nodules were malignant including 9 papillary thyroid cancer (PTC), 3 follicular thyroid cancer with minimal invasion, 3 Hürthle-cell carcinomas, 5 poorly differentiated carcinomas and one large B-cell lymphoma. SWE parameters were similar in benign and malignant nodules, including meanEI (20.2 vs $19.6 \mathrm{kPa}$ ), maxEI ( 34.3 vs $32.5 \mathrm{kPa}$ ) or ratio (1.57 vs 1.38 ). In malignant nodules, meanEI, maxEI and ratio were higher in the classic PTC variants $(n=4)$ than in the other PTC variants $(n=5)(p<0.02)$ or than in non-PTC tumors $(\mathrm{n}=12)(\mathrm{p}<0.005)$. Intra- and inter-observer coefficients of variation 
for meanEI in nodules were $23 \%$ and $26 \%$, respectively. Neither the TI-RADs score nor the ATA US classification were associated with malignancy.

Conclusions: Despite high EI in classic PTC variants, conventional SWE indexes were not found pertinent to discriminate between benign and malignant tumors in thyroid nodules with IC.

\section{P2-03-82 \\ CORRELATIONS BETWEEN TSH LEVEL AND MORPHOLOGICAL FEATURES OF THYROID TUMOURS \\ Oksana Sulaieva ${ }^{1}$, Olena Chernenko ${ }^{1}$ \\ ${ }^{1}$ Ukrainian Research and Practical Center of Endocrine Surgery, Kyiv, Ukraine}

Objectives: It is widely accepted that thyroid stimulating hormone (TSH) plays a role in nodular thyroid disease and risk of thyroid malignancy. In this study we explored the relations between serum TSH and morphological characteristics of thyroid neoplasm.

Methods: The tumor size, extra thyroidal extension (ETE), metastases, histological variant, intrathyroid invasion, Hashimoto's thyroiditis (HT) as well as serum TSH were assessed in 236 patients with thyroid neoplasm, including thyroid adenoma $(n=16)$, follicular carcinoma $(n=14)$ and different types of papillary thyroid carcinoma (PTC; $\mathrm{n}=206$ ). There were 192 females and 44 males among patients aged $46,4 \pm 0,87$ years. 89 of patients with PTC had histologically approved HT. Data assessment was conducted using statistical software (MedCalc Software Inc, Broekstraat, Belgium). X², Fisher's exact test, and one-way analysis of variance (ANOVA) were used when appropriate.

Results: There were no significant differences in THS level between patients with thyroid adenoma and follicular carcinoma, whereas patients with PTC demonstrated higher level of TSH $(p<0,05)$. Tumor size depended on gender $(\mathrm{P}=0,012)$ rather than TSH level. We did not find differences in TSH level among patients in regards with tumor size, age, gender and histological type of PTC. There were no relations between TSH and ETE or metastases among observed cohort. However, TSH was significantly higher in PTC with concurrent HT than in PTC alone $(p<0,001)$. In addition, HT was related with ETE among women with PTC $(\mathrm{p}=0,04)$.

Conclusions: A high proportion of patients with PTC has concurred HT, that was associated with higher TSH level and association with ETE among females.

\section{P2-04 Case Reports}

\section{P2-04-83 \\ BRIDGE OVER TROUBLED WATERS: THERAPEUTIC APHERESIS IN A PATIENT WITH THYROTOXICOSIS WITH SEVERE HEPATIC COMPLICATIONS AND LIVER CIRRHOSIS FROM CHRONIC HEPATITIS B INFECTION}

Everly Faith Ramos ${ }^{1}$, Diane Carla Bernardo ${ }^{2}$

${ }^{1}$ Department of Medicine, Philippine General Hospital, Manila, Philippines, ${ }^{2}$ Section of Endocrinology, Department of Medicine,

Philippine General Hospital, Manila, Philippines

Background: Thyroid storm is an endocrine emergency with high mortality rates of $20-30 \%$. Hepatic dysfunction is commonly observed in patients with thyroid storm. This complicates the treatment with thionamides, which are metabolized in the liver. Plasmapheresis is described in case reports as a treatment option for thyroid storm where conventional treatment failed.

Case: We report a case of a 32 year-old Filipino female admitted to our institution for abdominal pain. She is a known case of Graves' disease and chronic hepatitis B infection. She was managed as a case of thyroid storm and acute liver failure from hyperthyroidism on top of liver cirrhosis from chronic hepatitis B infection. The liver failure and active hepatitis prevented the giving of thionamides. One session of therapeutic plasma exchange with fresh frozen plasma was done which led to rapid clinical improvement in thyrotoxic symptoms, thyroid hormone levels and thyroid antibodies shown below. The ALT decreased by $74 \%$ from 741 to 192 IU/L and the AST by $56 \%$ from 627 to 279 IU/L. The effect lasted biochemically for 24 hours and clinically, for six days. Hepatitis B infection was simultaneously treated with tenofovir.

Discussion and Conclusion: Thyroid dysfunction from hyperthyroidism and chronic liver disease complicates treatment of thyroid storm. Plasma exchange is a viable treatment option to rapidly achieve decline in the thyroid hormones among patients in thyroid storm for which thionamides cannot be administered. It can be used as a bridge to definitive treatment of radioactive iodine or surgery. Further, in patients with concomitant chronic liver disease and Graves' disease, early definitive treatment for Graves' disease should be achieved to reduce exposure to hepatotoxic anti-thyroid medications.

\section{P2-04-84 \\ ETANERCEPT INDUCED DESTRUCTIVE THYREOIDITIS - A CASE REPORT}

Zoltan Hella ${ }^{1}$, Gábor László Kovács ${ }^{2}$, Piroska Turbucz ${ }^{3}$, Laszlo Hodinka ${ }^{4}$

11st Department of Internal Medicine, Flor Ferenc Hospital, Kistarcsa, Hungary, ${ }^{2}$ National Institute of Rheumatology and Physiotherapy,

Budapest, Hungary

65 years old male patient received low dose methylprednisolone baseline therapy and full dose etanercept treatment for 5 years due to rheumatoid arthritis. The patient experienced pain in neck, weakness, fever and dysphagia, and was admitted to our outpatient clinic for investigation and treatment. On the day of reception the patient had normal blood count but accelerated erythrocyte sedimentation rate, elevated C-reactive protein and clinical hyperthyroidism. The autoimmune origin could be excluded because of normal values of antibodies against the thyroidea peroxidase and thyroid stimulating hormone receptor. The ultrasound investigation showed focal hypoechogenic structure and low vascularisation. Based on the laboratory and ultrasound results as well as clinical signs etanercept related destructive/ subacute thyroiditis was supposed. As a therapy we interrupted the etanercept treatment and gave high dose of methylprednisolone for 5 days, then moderate dose for a week. After rapid improvement, the symptoms got worse again so we repeated the administration of high dose methylprednisolone treatment for 5 days, and continued with moderate dose of methylprednisolone for two months. Thyroid functions and the inflammatory markers normalized. We concluded the necessity of monitoring the thyroid function during etanercept treatment because rarely we could encounter side effects like clinical destructive thyroiditis.

Key Words: destructive thyroiditis, etanercept, methylprednisolon treatment

Table (for abstract P2-04-83)

\begin{tabular}{|c|c|c|c|c|c|}
\hline Parameter & Before plasmapheresis & $6 \mathrm{~h}$ after plasmapheresis & $24 \mathrm{~h}$ after plasmapharesis & Unit & Normal value \\
\hline TRAb & 719.95 & 537.99 & 603.7 & $\mathrm{IU} / \mathrm{mL}$ & \\
\hline Anti-TPO & 182.38 & 150.68 & 202.51 & $\mathrm{IU} / \mathrm{mL}$ & $<100$ \\
\hline FT3 & 16.4 & 2.2 & 14.5 & $\mathrm{pM}$ & $2.5-5.8$ \\
\hline FT4 & 72.71 & 70.3 & 77.22 & $\mathrm{pM}$ & $11-24$ \\
\hline TSH & 0.0002 & 0.0251 & 0.0033 & $\mathrm{uU} / \mathrm{mL}$ & $0.5-6$ \\
\hline
\end{tabular}




\section{P2-04-85 \\ DOWN SYNDROME AND HYPERTHYROIDISM: A RARE CASE IN PEDIATRIC PRACTICE}

\section{Lusine Navasardyan}

${ }^{1}$ Yerevan State Medical University, Endocrinology, Yerevan, Armenia

Introduction: Down syndrome (21 trisomy, DS) is a common chromosomal disorder in pediatric practice. Hypothyroidism is revealed in up to $13-25 \%$ of children with DS increasingly with age. Hyperthyroidism in DS is a very rare diagnosis, which is found mostly in adolescents and young.

Case Report: Here a case of hyperthyroidism in a 2.5-year-old girl with DS is described. 12 children with DS aged 0-5 years have been followed, from which in 4 patients $(33.3 \%)$ hypothyroidism was revealed by yearly screening of TSH and T4, and only 1 patient found to have clinically overt hyperthyroidism, and the rest 7 patients $(58.3 \%)$ were euthyroid. Anti-TPO, anti-TG, antiTSH-receptor autoantibodies in girl with hyperthyroidism found to be very high. The child had remarkable signs of hyperthyroidism, which became evident within a month, and included anxiety, nervousness, sweating, tremor and weight loss. The difficulty concerned the appropriate treatment and the absent of evidence-based data of anti-thyroid treatment in pediatric population. The child was given methimazole $0.4 \mathrm{mg} / \mathrm{kg} / \mathrm{day}$, but the clinical signs were persistent during several months. They gradually started to decline after 7 months of treatment with overdoses of methimazole. After 1.5 year of following she is euthyroid and taking $0.2 \mathrm{mg} / \mathrm{kg}$ /day maintenance dose of methimazole.

Conclusion: Even the yearly thyroid funstion screening in patients with DS does not exclude the possibility of missing the thyroid dysfunction. This case of diffuse toxic goiter in a 2.5 year old child shows the difficulties in early revelation and proper treatment of hyperthyroidism in pediatric practice. Further investigations should be done to evaluate the safety, adequacy, and later impact of methimazole on the organism of children under 5 years old.

\section{P2-04-86 \\ SEVERE METHIMAZOLE-INDUCED HEPATOTOXOCITY COMPLICATED BY RENAL FAILURE AND SEPSIS}

\section{Julie Anne Gabat}

${ }^{1}$ Philippine General Hospital, Manila, Philippines

Introduction: Methimazole is associated with fewer adverse reactions compared to Propylthiouracil, therefore it is recommended as the drug of choice for patients with hyperthyroidism. Methimazole-induced cholestatic liver injury resolves, albeit slowly, upon discontinuation of the drug. Severe and even fatal methimazole-induced liver injury can occur particularly in the presence of an underlying liver disease.

Case report: A 54-year old female with Graves' Disease was supposedly for radioactive iodine therapy when she developed panophthalmitis. She was admitted at our institution and intravitreous and parenteral antibiotics were administered. Atrial fibrillation was noted with markedly elevated FT4 and suppressed TSH levels. Methimazole was resumed at $40 \mathrm{mg}$ per day. Septic work-up was negative for systemic and intravitreal infections. Orbital CT scan showed no evidence of thyroid ophthalmopathy. Echocardiography showed concentric left ventricular hypertrophy with preserved systolic function without valvular abnormalities. Panophthalmitis and hyperthyroidism resolved. Two weeks after, she developed fever, epigastric pain, thrombocytopenia and agranulocytosis, hence, methimazole was stopped. Five days after stopping methimazole, she developed encephalopathy, jaundice, coagulopathy and renal failure for which she underwent hemodialysis. Holoabdominal ultrasound showed normal liver with normal bile ducts and gallbladder, normal renal sizes with signs of parenchymal disease and poor corticomedullary differentiation. She developed hospital acquired pneumonia from a multi-drug resistant organism (Acinetobacter baumanii) and eventually succumbed to septic shock. Post-mortem examination of the liver revealed canalicular cholestasis, microvesicular steatosis and portal triaditis consistent with druginduced liver injury. No evidence of vasculitis or allergic interstitial nephritis was noted.

Conclusion: Methimazole can cause severe hepatotoxicity even in the absence of an underlying liver disease and it can occur even after stopping the medication. Factors other than direct liver injury may be responsible for this latency that needs further investigation.

\section{P2-04-87 \\ SERUM CALCITONIN NEGATIVE A LARGE MEDULLARY THYROID CARCINOMA: A CASE REPORT}

Jiyoung Yoo ${ }^{1}$, Song I Yang ${ }^{2}$, Jeong Hoon $\mathrm{Kim}^{2}$

${ }^{1}$ Department of Surgery, Kosin University College of Medicine, Busan, Korea, Rep. of South, ${ }^{2}$ Department of Surgery, Kosin University College of Medicine, Busan, Korea, Rep. of South

Introduction: A serum-calcitonin(CT) level is most sensitive and specific indicators of medullary thyroid carcinoma(MTC). However few cases of CT-negative MTC have been reported. Usually, tumor size and basal CT level has direct correlation. Therefore, CT-negative MTC is often associated with lower tumor burden due to incidentaloma detected by routine screening.

Case Report: We describe here a 55-year-old woman with a large and palpable medullary thyroid carcinoma with normal serum calcitonin level. The patient presented with a palpable neck mass. Initially, ultrasound-guided fine needle aspiration biopsy cytology and MTC was suspected. But serum calcitonin and CEA level were nomal. She underwent bilateral total thyroidectomy with ipsilateral modified radical neck dissection. Histopathologically, The tumor was a $4 \mathrm{~cm}$ sized medullary thyroid carcinoma, well circumscribed lesion and there was no lymph node metastasis. Immunohistochemical staining showed positive for calcitonin and synaptophysin and chromogranin, which are neuroendocrine markers, and negative for thyroglobulin and thyroid transcription factor-1, whitch are thyroid follicular cell markers

Conclusion: We report a case of a large medullary thyroid carcinoma diagnosed in a patient with no elevation of preoperative calcitonin and CEA levels

\section{P2-04-88 \\ AUTONOMIC ALTERATIONS IN A PATIENT WITH ENCEPHALOPATHY ASSOCIATED WITH AUTOIMMUNE THYROID DISEASE}

Maria Tomkins ${ }^{1}$, Maura Connel${ }^{2}$, Emma Stanley ${ }^{3}$, Federica Cavalcoli ${ }^{1}$, Gianluca Tamagno ${ }^{1}$

${ }^{1}$ Mater Misericordiae University Hospital, Department of Endocrinology/ Diabetes Mellitus, Dublin, Ireland, ${ }^{2}$ Mater Misericordiae University Hospital, Department of Clinical Neurophysiology, Dublin, Ireland, ${ }^{3}$ Mater Misericordiae University Hospital, Department of Radiology, Dublin, Ireland

Introduction: Encephalopathy associated with autoimmune thyroid disease (EAATD) is a rare neurological condition that may occur in patients with clinical or sub-clinical autoimmune thyroid disease. The diagnostic criteria include neurological or psychiatric symptoms, high levels of anti-thyroid antibodies, and exclusion of other possible causes of encephalopathy. In the large majority of cases, EAATD patients respond to immunosuppressant therapies.

Case Report: We report the case of a woman with recurrent manifestations of encephalopathy and history of Hashimoto's thyroiditis. On the last presentation, there was marked ataxia, language and cognitive impairment, and neurovegetative/autonomic symptoms (hypothermia, bradycardia, hypotension). The patient was euthyroid with raised anti-thyroid peroxidase antibodies. Following extensive work-up, a diagnosis of EAATD likely affecting the hypothalamus was made and treatment with prednisolone was started with dramatic improvement of the symptoms.

Conclusions: To the best of our knowledge, this is the first reported case of neurovegetative/autonomic alterations in the setting of EAATD. Indeed, EAATD is characterized by a profound variability in the clinical presentation and represent a diagnostic challenge for the physicians. This case report adds to the many presentations that have been previously described. 


\section{P2-04-89 \\ SEVERE THYROID HYPOPLASIA ASSOCIATED WITH THYROTROPIN RECEPTOR GENE MUTATION - CASE PRESENTATION}

\section{Malgorzata Kumorowicz-Czoch ${ }^{1}$, Pia Hermanns ${ }^{2}$, Joahim Pohlenz ${ }^{2}$}

${ }^{1}$ Private Pediatrics and Pediatric Endocrinology Practice, Department of Pediatric and Adolescent Endocrinology, Chair of Pediatrics, PolishAmerican Institute of Pediatrics, Jagiellonian University Medical College, Cracow, Poland, ${ }^{2}$ Department of Pediatrics, Johannes Gutenberg University Medical School, Mainz, Germany

Background: The morphological and biochemical phenotype of TSHR gene mutation in patients with congenital hypothyroidism $(\mathrm{CH})$ is variable. The contribution of mutations in TSHR gene in children with $\mathrm{CH}$ still remains a subject of interest of researchers.

Material and Methods: The patient was part of a study group which included 45 children (32 girls, 13 boys) with $\mathrm{CH}$ and thyroid dysgenesis (TD). The study participants were born in south-eastern Poland in the years 19932012 and selected in neonatal mass screening for CH. DNA was extracted from peripheral blood samples with the use of Master Pure DNA Purification Kit (Epicentre Biotechnologies). TSHR coding sequences were amplified by PCR. Capillary electrophoresis was run on ABI 3500 (Applied Biosystems).

Results and Case Presentation: A heterozygous variation in TSHR gene was revealed in one of 45 children with $\mathrm{CH}$ and TD. The patient was a 12 -year-old boy who was diagnosed to have isolated $\mathrm{CH}$ at neonatal screening with serum TSH of $49.5 \mathrm{mU} / \mathrm{L}(\mathrm{N}: 0.4-9)$ and a fT4 of $2.4 \mathrm{pmol} / \mathrm{L}(\mathrm{N}: 12-$ 26). Thyroglobulin value was $0.5 \mathrm{ng} / \mathrm{mL}(\mathrm{N}<45)$. He had a hypoplastic thyroid in ultrasound $(\mathrm{V}=0.2 \mathrm{~mL})$. The molecular analysis revealed p.A68P change in TSHR gene. At the time of the study the patient was euthyroid under L-thyroxine supplementation. His 10-year-old sister had also $\mathrm{CH}$ and thyroid hypoplasia. The patient's parents were healthy and euthyroid.

Conclusions: We report an occurrence of heterozygous variation in TSHR gene in a boy with $\mathrm{CH}$ and hypoplastic thyroid. Estimation of the contribution of the revealed p.A68P mutation to the etiology of $\mathrm{CH}$ in the boy with hypoplastic thyroid requires further functional analysis.

\section{P2-04-90 \\ RAPID REMISSION OF SEVERE GRAVES DISEASE WITHOUT THIONAMIDES UNDER GLUCOCORTICOID TREATMENT FOR CONCOMITANT AUTOIMMUNE HEPATITIS}

Georgios Papadakis ${ }^{1}$, Faiza Lamine ${ }^{1}$, Monserrat Fraga Christinet ${ }^{2}$, Eleni Moschouri ${ }^{2}$, Alexandra Schneider ${ }^{3}$, Francois-Regis Duss ${ }^{3}$, Amedeo Sciarra ${ }^{4}$, Christine Sempoux ${ }^{4}$, Darius Moradpour ${ }^{2}$, Gerasimos Sykiotis

${ }^{1}$ Service of Endocrinology, Diabetology and Metabolism, Lausanne University Hospital, Lausanne, Switzerland, ${ }^{2}$ Service of Gastroenterology, Lausanne University Hospital, Lausanne, Switzerland, ${ }^{3}$ Service of Internal Medicine, Lausanne University Hospital, Lausanne, Switzerland, ${ }^{4}$ Institute of Pathology, Lausanne

University Hospital, Lausanne, Switzerland

Introduction: Even though thionamides are a first-line treatment for Graves' disease, their potential hepatotoxicity renders their use challenging in cases of concomitant liver disease. For such cases, and if radioactive iodine and surgery cannot be used, alternative medical treatments are not well established.

Case Report: A 28-year-old Caucasian female with unremarkable medical history consulted for progressive jaundice. Laboratory tests showed an alanine aminotransferase of $1437 \mathrm{U} / 1$ and a total bilirubin of $286 \mu \mathrm{mol} / \mathrm{l}$. Investigations revealed type I autoimmune hepatitis, with positive anti-nuclear and anti-smooth muscle autoantibodies, IgG of $15.7 \mathrm{~g} / 1$ and a typical histology. An undetectable TSH prompted a targeted history that revealed recent restlessness and fatigue, rapid heartbeat and increased stool frequency without heat intolerance. The clinical exam showed signs of hyperthyroidism (sinus tachycardia, fine tremor, hyperactive Achilles tendon reflexes). Free T4 and free T3 were increased at $60 \mathrm{pmol} / 1$ (normal range, $12-22 \mathrm{pmol} / 1$ ) and 14.6 $\mathrm{pmol} / \mathrm{l}$ (normal range, 3.1-6.8 pmol/1), respectively. Thyrotropin receptor antibodies (TRAb) were strongly positive (11.1 UI/l; normal range, $<1.75 \mathrm{UI} / 1)$. Ultrasonography revealed a normally sized but heterogeneous thyroid with increased vascularity. Given the hepatic impairment, thionamides were withheld and only propanolol and a low dose of cholestyramine (4 gr per day) were prescribed. Prednisone was started at a dose of $50 \mathrm{mg}$ per day, with rapid improvement of the patient's clinical condition and liver function tests, allowing for the introduction of azathioprine and the progressive tapering of corticosteroids. After 1 month of immunosuppressive treatment liver function tests as well as free T4 and free T3 were normal, and TRAb decreased to $4.2 \mathrm{IU} / 1$.

Conclusions: Glucocorticoids are an alternative medical option for Graves" hyperthyroidism when contraindication to thionamides is present. In addition to the well-established suppression of T4 conversion to T3, reduction of TRAb-mediated thyrocyte stimulation seems to be the main mechanism of action.

\section{P2-04-91 \\ CASE REPORT OF CARDIOTOXICITY WITH TYROSINE KINASE INHIBITORS IN PATIENT WITH ADVANCED PAPILLARY THYROID CANCER}

Elena Shavarova ${ }^{1}$, Ilya Romanov ${ }^{2}$, Zhanna Kobalava ${ }^{3}$

${ }^{1}$ Rudn University, Moscow, Russian Federation, ${ }^{2}$ Rcrc, Moscow,

Russian Federation, ${ }^{3}$ Peoples Friendship University of Russia,

Moscow, Russian Federation

Introduction: tyrosine kinase inhibitors (TKI)-mediated cardiotoxicity is common adverse reaction. Prevalence of arterial hypertension (AH) is up to $68 \%$, systolic dysfunction of left ventricle - up to $19 \%$.

Case Report: 59-year-old male with untreated mild essential hypertension was diagnosed with papillary thyroid cancer T3N1M0 in 2008. After thyroidectomy with lymph node dissection three courses of radioactive iodine therapy were done in 2008, 2012, 2013 (total dose $7 \mathrm{GBq}$ ) following that thyroid cancer was defined as radioactive iodine-refractory. In June of 2016 disease progression was treated with 2-month sorafenib therapy $(600 \mathrm{mg}$, dose was reduced because of hand-foot syndrome grade 3 onset) that was stopped due to lack of efficacy. From Oct 2016 till now lenvatinib $24 \mathrm{mg}$ was prescribed. Target node size diminished from $14.5 \times 8 \times 13$ to $13 \times 5 \times 10 \mathrm{~cm}$, sum of large-sized tumor niduses decreased from 23.3 to $17.4 \mathrm{~cm}$ by PET-CT. Level of thyroglobulin decreased from 311 to $22 \mathrm{ng} / \mathrm{ml}$. Arterial hypertension was observed with maximal blood pressure level up to 205/102 $\mathrm{mm} \mathrm{Hg}$. Antihypertensive therapy with hydrochlorothiazide $25 \mathrm{mg}$ OD, enalapril $10 \mathrm{mg}$ BID, carvedilol $6.25 \mathrm{mg}$ BID was prescribed and blood pressure in self-measurement reports was less 140/90 mm Hg. In 2013 ejection fraction of left ventricle (LVEF) before TKI therapy was $67 \%$ (by Simpson) without local myocardial contractility dysfunction. After 2-month sorafenib administration decreasing of LVEF to $37 \%$ was observed. Second line target therapy with lenvatinib is ongoing during 6 month. LVEF is monitoring closely. No progression of left ventricle systolic dysfunction is revealed. LVEF is $37 \%$ on echocardiography from March 2017.

Conclusions: $\mathrm{AH}$ grade 3 was observed, target blood pressure was achieved on three-component antihypertensive therapy. Cardiac function evaluation should be planed before and during treatment with TKIs. Clinical studies are necessary to understand are this systolic dysfunction idiosyncratic or dependent on pre-existing undiagnosed cardiac comorbidities.

\section{P2-04-92 \\ NIVOLUMAB-ASSOCIATED THYROID DYSFUNCTION}

Rita Silva ${ }^{1}$, Joana Oliveira ${ }^{1}$, César Esteves ${ }^{1}$, Gabriela Fernandes ${ }^{2}$ Cláudia Caeiro ${ }^{3}$, Lúcia Águas ${ }^{3}$, Joana Queirós ${ }^{1}$, Davide Carvalho ${ }^{1}$ ${ }^{1}$ Department of Endocrinology, Diabetes and Metabolism, Centro Hospitalar São João, E.P.E., Porto, Portugal, ${ }^{2}$ Department of Pulmonology, Centro Hospitalar São João, E.P.E., Porto, Portugal, ${ }^{3}$ Department of Medical Oncology, Centro Hospitalar São João, E.P.E., Porto, Portugal, Porto, Portugal

Background: Nivolumab is a monoclonal antibody specific for human programmed cell death protein-1 (PD-1), a checkpoint molecule highly expressed in several malignancies. Besides its benefits as an immunotherapeutic strategy for cancer, it may exhibit endocrine side effects.

Case Reports: We report 3 cases of thyroid dysfunction during nivolumab treatment. Two males ( 70 and 64 years old) and one female ( 56 years old) were 
treated with nivolumab for metastatic clear cell renal carcinoma, lung adenocarcinoma and esophageal melanoma, respectively. No patient had previous thyroid disease or familial thyroid pathology. A few months after starting treatment, the patients developed thyrotoxicosis with suppressed thyroid-stimulating hormone (TSH) and normal (2 patients) or high (1 patient) free thyroxine (FT4) and free triiodothyronine (FT3). All patients had positive thyroglobulin antibody $(\mathrm{TgAb})$ and/or positive peroxidase antibody (TPOAb) and negative TSH-receptor antibodies (TRAb). In one patient treatment with methimazole was instituted for 3.5 months, leading to symptom improvement and with no recurrence after antithyroid drug withdrawal. The other patients had spontaneous remission of thyrotoxicosis within 1-2 months and then developed hypothyroidism, leading to the diagnosis of nivolumab-associated thyroiditis and treatment with levothyroxine. Currently all patient maintain normal thyroid function.

Conclusion: Our cases highlight thyroid dysfunction due to a possible immune-mediated mechanism and the importance to test for thyroid function at baseline and during nivolumab treatment. No patient has discontinued nivolumab owing to thyroid dysfunction, but symptoms, evolution and treatment may differ among patients. Since the number of patients treated with nivolumab is expected to increase, health providers must be aware of endocrine disorders that may be associated with immunomodulatory therapies for a timely diagnosis and correct treatment.

\section{P2-04-93 \\ THYROTOXIC HYPOKALEMIC PERIODIC PARALYSIS AS PRESENTATION OF GRAVES‘ DISEASE IN A CAUCASIAN MALE PATIENT \\ Joao Sergio Neves ${ }^{1}$, Luís Teles ${ }^{2}$, Sofia Castro Oliveira ${ }^{3}$, Pedro Souteiro ${ }^{3}$, Ana Varela ${ }^{3}$, Ana Isabel Oliveira ${ }^{3}$, Celestino Neves ${ }^{3}$, Paula \\ Freitas $^{4}$, Davide Carvalho ${ }^{4}$ \\ ${ }^{1}$ Endocrinology, São João Hospital, Departamento de Fisiologia, \\ Fmup, Porto, Portugal, ${ }^{2}$ Serviço de Medicina Interna. Centro Hospitalar Entre Douro e Vouga, Santa Maria Da Feira, Portugal, ${ }^{3}$ Department of Endocrinology, Diabetes and Metabolism, São João Hospital Center. Faculty of Medicine of Porto University, Porto, Portugal, ${ }^{4}$ Department of Endocrinology, Diabetes and Metabolism, São João Hospital Center. Faculty of Medicine of Porto University, Instituto de Investigação e Inovação Em Saúde Da Universidade Do Porto, Porto, Portugal}

Introduction: Thyrotoxic hypokalemic periodic paralysis is a rare manifestation of Graves' disease. This manifestation presents a significantly higher frequency in Asiatic patients, being rare in Caucasian patients.

Case Description: A 25-year old Caucasian male was evaluated in our emergency department due to decreased muscular strength after waking up with an associated fall. No consciousness loss or muscular pain was observed. The patient reported a similar episode two weeks before that occurred during rest after physical activity. In the two months before, the patient lost $10 \mathrm{~kg}$ and presented frequent palpitations, tremor, hyperhidrosis and insomnia. The history of Graves' disease in mother and sister was the most relevant finding from the personal and family history. On the initial examination, the patient was tachycardic $(110 \mathrm{bpm})$ with normal blood pressure $(136 / 80 \mathrm{mmHg})$, decreased muscular strength without other neurologic abnormalities. At initial analytic evaluation, a severe hypokalemia $(1.9 \mathrm{mEq} / \mathrm{L})$ and a mild hypomagnesemia $(1.35 \mathrm{mEq} / \mathrm{L})$ were shown without other relevant findings. An evaluation of thyroid function revealed a TSH $<0.001 \mu \mathrm{UI} / \mathrm{mL}$ and a free $\mathrm{T} 4$ of $2.75 \mathrm{ng} / \mathrm{dL}$ and a diagnosis of thyrotoxic hypokalemic periodic paralysis was established. The patient was supplement with potassium and magnesium and started treatment with methimazole and bisoprolol. During the first three days of stay in the hospital, two more episodes of sudden decrease of muscular strength were observed. No more episodes of muscular strength loss were observed since then. The presence of positive TRAB antibodies $(4.5 \mathrm{U} / \mathrm{L})$ confirmed the diagnosis of Graves' disease. The patient was discharged with methimazole and propranolol.

Conclusions: The authors presented a case of thyrotoxic hypokalemic periodic paralysis as presentation of Graves' disease in a caucasian male patient. Although is it a rare manifestation in non-asiatic patients, the presence of sudden decreased muscular strength with additional clinical findings suggestive of hyperthyroidism must prompt evaluation of thyroid function.

\section{P2-05 Goitre and Nodules 2}

\section{P2-05-94 \\ ACUTE AND TRANSIENT THYROID SWELLING FOLLOWING FINE-NEEDLE ASPIRATION BIOPSY: CLINICAL FEATURES AND ULTRASONOGRAPHIC FINDINGS}

Tetsuya Mizokami ${ }^{1}$, Katsuhiko Hamada ${ }^{1}$, Tetsushi Maruta ${ }^{1}$, Kiichiro

Higashi $^{1}$, Junichi Tajiri ${ }^{1}$

${ }^{1}$ Tajiri Thyroid Clinic, Kumamoto, Japan

Introduction: Acute diffuse swelling of the thyroid gland in the absence of hematoma formation is a rare complication following fine-needle aspiration biopsy (FNAB) that often resolves spontaneously. Owing to the limited number of cases available for review, the true incidence, as well as etiology and optimal management of this complication, have not been elucidated.

Cases: We performed 16,817 FNABs for 9,596 thyroid nodules under ultrasonographic guidance between January 2011 and December 2015. We identified 10 patients ( 8 women and 2 men) with acute and transient thyroid swelling without significant hematoma. Indications for FNAB included multinodular goiter (likely adenomatous goiter; $n=7$ ), thyroid nodule (including one follicular adenoma; $n=2)$, and papillary carcinoma $(n=1)$. The thyroid glands enlarged by 1.3-4.8 (median 2.7)-fold accompanied with anterior neck pain and/or swelling immediately after FNAB (abrupt onset) in five cases, 5-15 minutes after FNAB in two (sub-abrupt onset), and a few hours after FNAB (delayed onset) in three. Ultrasonography revealed dendritic hypoechoic lesions (hypoechoic "cracks") scattered throughout the swollen thyroid gland in all cases. Some punctured solid nodules were not clearly enlarged, and spared from hypoechoic "cracks". Hypoechoic "cracks" did not show blood flow signal by color-flow Doppler imaging. Two cases were treated with intravenous methylprednisolone (125 mg), and five cases with oral prednisolone (10-30 mg/day for 1-3 days). Three cases with smaller goiter were observed with neck cooling. Swelling of the thyroid gland subsided within several hours after symptom onset.

Conclusion: The incidence of acute and transient thyroid swelling was $0.10 \%$ (10 of 9,596 nodules) in our clinic. Hypoechoic "cracks" throughout the diffusely swollen thyroid gland was the ultrasonographic feature of this FNAB complication despite pathological differences in the punctured nodules and different time intervals between FNAB and onset of acute thyroid swelling.

\section{P2-05-95 \\ RECOVERY OF PRATHYROID AND LARYNGEAL FUNCTION IN PATIENTS WITH DIFFERENTIATED THYROID CANCER AFTER ROBOTIC THYROIDECTOMY COMPARED WITH OPEN THYROIDECTOMY: A PROPENSITY SCORE MATCHING ANALYSIS}

Dongsik Bae ${ }^{1}$, Do Hoon Koo ${ }^{1}$, In Hee Shim ${ }^{2}$

${ }^{1}$ Department of Surgery, Haeundae Paik Hospital, Inje University College of Medicine, Busan, Rep. of South Korea, ${ }^{2}$ Department of Psychiatry, Cancer Center, Dongnam Institute of Radiological \& Medical Sciences, Busan, Rep. of South Korea

Background: The aim of this study, from a surgical and oncologic perspective, was to identify whether robotic total thyroidectomy (RTT) has any differences compared with open thyroidectomy (OTT).

Methods: Seven hundred fifty-six patients who were eligible for total thyroidectomy were primarily reviewed in Haeundae-Paik Hospital between July 2010 and December 2015 by three surgeons using both robotic and open methods. For removing the effects of selection bias, we adjusted the clinicopathologic characteristics (sex, age, BMI, laterality of CND, tumor size, extrathyroidal extension and thyroiditis) using 2:1 propensity score matching.

Results: Propensity-based matching consisted of 123 patients with robot group and 246 patients matched with open group with standardized differences in patient clinicopathologic characteristics of less than $10 \%$, indicating a high degree of similarity in the distributions of covariates. There were no dif- 
ferences in postoperative complication rate and oncologic results of between two groups other than mean operation time $(198.39 \pm 37.93$ vs. $123.51 \pm 32.63$ (minutes); $P<0.001$ ). But laryngeal nerve function and hypoparathyroidism recovery time was short in robot group compared with open group (81.62 \pm 50.04 vs. $133.75 \pm 80.83$ (days); $P=0.042$ and $86.49 \pm 69.67$ vs. $139.21 \pm$ 140.65 (days); $P=0.033$ ), respectively.

Conclusion: Although the difference not measured in the incidence of postoperative complications and results of oncology between RTT and OTT, but the recovery time of parathyroid and laryngeal function was short in RTT.

\section{P2-05-96 \\ SURGEON PERFORMED THYROID ULTRASOUND - PROVIDING UTILITY AND CREDABILITY IN SELECTING PATIENTS FOR FINAL NEEDLE ASPIRATION ACCORDING TO THE AMERICAN THYROID ASSOCIATION GUIDELINES \\ Oded Cohen ${ }^{1}$, Tom Raz Yarkoni', Yonatan Lahav ${ }^{1}$, Offer Azoulay', Doron Halperin ${ }^{1}$, Moshe Yehuda ${ }^{2}$ \\ ${ }^{1}$ Kaplan Medical Center, Rehovot, Israel, ${ }^{2}$ Kaplan Medical Center, Tel- Aviv Sourasky Medical Center, Tel Aviv, Israel}

Background: Ultrasound is a pivotal tool in the assessment of thyroid nodules, though limited by large interobserver variability. Surgeon performed ultrasound (SUS) may provide a practical platform which eliminates this disadvantage.

Methods: The data of all patients who underwent SUS between 7/2009 and 9/2012 was reviewed retrospectively. Exclusion criteria were missing pertinent data and patients whose thyroid nodules were not recommended for FNA according to ATA guidelines. Data included selected demographics, risk factors for thyroid cancer, sonographic features, cytological result and final pathology when surgery was performed.

Results: A total of 553 thyroid nodules in 498 patients were evaluated. Fifty five $(11 \%)$ non diagnostic biopsies were excluded from final data analysis. Four hundred ninety eight nodules were included. Solid texture, irregular margins, hypoechogenicity and intranodular vascularity were significantly associated with non-benign cytology compared to benign, and also when compared benign and malignant pathology. Lack of suspicious features was significantly associated with benign lesions, with negative predictive value of $94 \%$. Except for taller than wider shape, malignancy odds ratio was significantly higher for known suspicious features, reaching 4.81 for irregular borders (CI $2.42-9.55, \mathrm{p}<0.001)$.

Table 1. Adjusted Odds Ratio (OR) Estimates of Thyroid Cancer Risk in Relation to Surgeon Performed Ultrasound Characteristics

\begin{tabular}{llll}
\hline SUS Characteristics & OR & $95 \% \mathrm{CI}$ & P Value \\
\hline Malignant associated features & 2.04 & $1.56-2.66$ & $<0.001$ \\
Irregular borders & 4.81 & $2.42-9.55$ & $<0.001$ \\
Hypoechogenicity & 3.43 & $1.94-6.07$ & $<0.001$ \\
Microcalcifications & 2.33 & $1.27-4.25$ & 0.006 \\
Solid texture & 1.82 & $1.03-3.2$ & 0.038 \\
Taller than wider & NS & NS & NS \\
Intranodular vascularity & 2.4 & $1.26-4.56$ & 0.008 \\
\hline
\end{tabular}

Conclusions: SUS has proven to be a reliable and consistent tool thyroid nodules' risk stratification, which may overcome its greatest flaw of inter-observer variability, Thyroid caregivers, including Surgeons and endocrinologist, should be encouraged to recognize the potential of this tool and its implementation.

\section{P2-05-97 \\ MAY THE ARFI ELASTOGRAPHY INCREASE THE DIAGNOSTIC VALUE OF FNA IN THYROID \\ NODULES}

Armen Varzhapetyan

${ }^{1}$ Yerevan State Medical University; Astghik Medical Center, Yerevan, Armenia

Background: Over the last decade, the scientific medical literature actively discusses the diagnostic value and accuracy of various types of elastography in the diagnosis of thyroid cancer.

Objectives: To analyze the informative value of FNA when combining it with ARFI elastography.

Methods: Analysis of clinical data of 122 patients with thyroid nodules was performed. Patients are divided into 3 groups. In all groups, the results of the preoperative diagnosis were compared with the findings of a post-operative histological examination. The first group $(n=36)$ consisted of patients whose preoperative diagnosis was based on FNA results. The second group is represented by patients $(\mathrm{n}=28)$ who refused FNA, and the operation was performed based on data of ARFI elastography. In the third group $(n=58)$ ARFI elastography was produced and under control of which the targeted FNA was primarily made from the densest sections of the node.

Results: In the first group, the diagnostic accuracy was $77.8 \%$ (28/36). However, in this group the diagnostic specificity of FNA was reduced due to impossibility of differentiation between follicular adenoma and carcinoma. When analyzing the data of the second group, the maximum node density of up to $4 \mathrm{~m} / \mathrm{sec}$ was determined by ARFI elastography in 16 patients out of $28(57 \%)$. The fact of high density in the nodules with thyroiditis Hashimoto sharply reduces the diagnostic accuracy of this group. The diagnostic accuracy in third group was $94.8 \%$.

\begin{tabular}{llcr}
\hline & \multicolumn{2}{l}{ Result } & Total \\
\cline { 2 - 3 } & Positive & Negative & \\
\hline Group 1 & 28 & 8 & 36 \\
Group 2 & 16 & 12 & 28 \\
Group 3 & 55 & 3 & 58 \\
Total & 99 & 23 & 122 \\
\hline
\end{tabular}

$\chi^{2}=9.21, p<0.01$

Conclusions: Thus, the combined use of FNA and ARFI elastography leads to optimization and increase of accuracy and specificity of thyroid nodules diagnosis.

\section{P2-05-98}

\section{D AND 2D VIRTUAL NAVIGATION TECHNOLOGY FOR ULTRASOUND LOCALIZATION OF THYROID LESIONS DETECTED BY SCINTISCAN OR PET-CT. A FEASIBILITY STUDY}

Enrico Papini ${ }^{1}$, Agnese Persichetti ${ }^{2}$, Rinaldo Guglielmi ${ }^{1}$, Filomena Graziano $^{1}$, Antonio Bianchini ${ }^{3}$, Dario Valle ${ }^{3}$, Luca Velari ${ }^{3}$, Giancarlo Bizzarri ${ }^{3}$

${ }^{1}$ Dept. of Endocri. \& Metabol. Disease; Ospedale Regina Apostolorum, Albano Roma, Italy, ${ }^{2}$ Department of Molecuar Medicine, Sapienza University of Rome, Rome, Italy, ${ }^{3}$ Department of Diagnostic of Imaging, Regina Apostolorum Hospital, Albano Laziale, Rome, Italy

Aim of The Study: Ultrasonography (US) is the modality of choice for thyroid examination and for image-guided biopsy of neck lesions but lacks functional information while radioisotope scan and PET-CT provide functional information which are of use for specific thyroid lesions. The clinicallybased ("cognitive") correlation between US features and functional findings may be misleading. We report a feasibility study on the use of 3D and 2D Virtual Navigation (VN) with BMT for the characterization of thyroid and parathyroid lesions. 
Methods: A MyLabTwice US scanner equipped with 2D VN with BodyMark and 3D VN (Esaote., Italy) was used on 20 patients with hyperfunctioning multinodular goiter, cervical lymph node 131-I uptake after thyroidectomy, and Tc/MIBI uptake in hyperparathyroidism). Functional images were loaded on the equipment before US examination. After a standard US scan, US was co-registered with scintiscan or PET-CT using anatomical landmarks (linear probe LA533, 5-13 MHz with magnetic sensor support, CIVCO, USA). Each patient was blindly examined by 3 proficient operators that registered their confidence in localization with cognitive versus VN or BMT technology. The time employed for cognitive and VN or BMT and the consistency of the reports were recorded.

Results: VN and BMT increased the confidence in localization of pathologic uptake in $76 \%$ of cases. Diagnosis were confirmed with image-guided biopsy or surgery. The mean time necessary for cognitive, VN and BMT was 4,2 and 5 minutes, respectively.

Conclusion: 3D VN and 2D Navigation with BMT increased the confidence and consistency in US localization of abnormal neck functional findings on scintiscan and PET-CT when compared to the traditional cognitive approach. Navigation techniques resulted to be time-sparing in the decision process and seem to be promising tools for the improvement of thyroid lesions targeting.

\section{P2-05-99 \\ POSSIBILITIES OF SONOELASTOMETRY OF SHEAR WAVE FOR EVALUATION OF A PROCESS CHARACTER IN THYROID NODUKES}

Elina Gasparyan ${ }^{1}$

${ }^{1}$ Medical Centre; Endocrinology; Medical Academy of Postgraduate

Studies, St. Petersburg, Russian Federation

Aim: To evaluate the possibility of using Acoustic Radiation Forced Impulse (ARFI) technology for determining elasticity of thyroid (TH) nodules and a process character in them.

Material and Methods: We have observed 131 patients (15 males and 116 females) aged $12-78$ (average $44.2 \pm 14.5$ years old), directed to our medical centre for carrying fine needle aspiration biopsy (FNAB). All patients underwent ultrasound assessment of TH nodules with the use of B-regime and Color Doppler, evaluating the nodule size, structure, outlines and vascularization. Elasticity (density) of tissues is known to influence the shear wave velocity (SWV), namely, the more elastic the tissue is, the lower is the velocity index. In this connection, we used ARFI technology of Acuson S2000

(Siemens $\}$ apparatus for evaluation of SWV and estimation of nodule elasticity- elastometry. Up to 10 measurements in $\mathrm{m} / \mathrm{s}$ we carried out with calculations of arithmetical average. Elastometry indexes we retrospectively compared with nodule cytological picture.

Results: The cytological picture of nodules was the following: colloid nodule was found in $39.7 \%$ of cases, Hashimoto's thyroiditis - in $9.9 \%$, follicular tumor - in $38.0 \%$, papillary cancer - in $12.4 \%$ of patients. It turned out that SWV indexes were dependent on the character of the cytological picture of nodules, as well as, on echogenecity, vascularization, presence or absence of degenerative changes and calcificates in them. The higher indexes of elastometry were found in paplllary cancer $(3.2 \pm 1.17 \mathrm{~m} / \mathrm{s})$ and in pseudonodules in Hashimoto's thyroiditis $(2.34 \pm 0.39 \mathrm{~m} / \mathrm{s})$, in comparison with colloid $(1.84 \pm 0.52 \mathrm{~m} / \mathrm{s})$ and follicular tumors $(1.95 \pm 0.72 \mathrm{~m} / \mathrm{s})$, probably due to decrease of elasticity and increase of density in them because of infiltrative and inflammatory processes, respectively. Hypoecogenic avascular nodules of homogeneous structure with the presence of calcificates had a higher SWV index in comparison with isoecogenic nodules with intraperi- and perinodular vascularization and degenerative changes in them.

Conclusions:

- Elastometry of thyroid nodules (ARFI technology) with evaluation of SWV is a noninvasive available study, permitting to predict the character of the process in them within a few minutes.

- Papillary cancer nodules have definitely higher indexes of elastometry

- In patients with multinodule goiter SWV indexes may serve an additional criterion of selection of nodules for FNAB

\section{P2-05-100 \\ HIFU TREATMENT OF BENIGN THYROID NODULES WITH DIFFERENT POWER \\ SCHEDULES}

Pierpaolo Trimboli ${ }^{1}$, Fabiano Bini ${ }^{2}$, Franco Marinozzi ${ }^{2}$, Luca Giovanella ${ }^{3}$ ${ }^{1}$ Thyroid Center, Oncology Institute of Southern Switzerland, Lugano, Switzerland, ${ }^{2}$ Department of Aerospace and Mechanical Engineering, Sapienza University of Rome, Rome, Italy, ${ }^{3}$ Nuclear Medicine and Thyroid Centre, Oncology Institute of Southern Switzerland, Bellinzona, Switzerland

Objectives: In the last decade the thermal ablation treatments of thyroid nodules have gained momentum due to the possibility to avoid surgical complications. As the last technique introduced, the high intensity focused ultrasound (HIFU), allows thermal treatment by energy ultrasound beam inside the targeted zone with no needle nor invasive instruments. Aim of our study was to evaluate HIFU treatment performed by different power schedules (i.e. high and low delivered dose) in terms of clinical results and patient's tolerability of therapy.

Methods: Since 2016, 22 patients with benign thyroid nodules presenting local discomfort and/or mild compressive symptoms were treated by HIFU at our center. For the present study, we prospectively enrolled in a randomized fashion two groups of patients. One group included subjects undergoing HIFU with a fixed energy of $45 \mathrm{~W} /$ site [high dose (HD group)]; the other group comprised patients undergoing HIFU with lower dose (LD group): in these patients the first ultrasound pulse was performed at $30 \mathrm{~W} / \mathrm{site}$, this power was maintained for next pulses only when patient had good comfort, and in case of poor tolerability the power of the subsequent pulses were tailored on the patients tolerability with a range from 19 to $25 \mathrm{~W} / \mathrm{site}$. The patient's comfort was recorded by a questionnaire in a 10-points scale (from 1-poor to 10-optimal). All cases had a follow-up of at least 6 months.

Results: The final series included 18 females and 4 males with age 60.4 yrs and each study group comprised 11 cases. Nodule size of the entire series was $22.5 \mathrm{~mm}(4.2 \mathrm{~mL})$, the treatment was performed with a mean power of $33.8 \mathrm{~W} /$ site and the energy delivered was $2.3 \mathrm{~kJ}$. At last follow-up, nodules were reduced by $28.7 \%$. The characteristics of the two groups are detailed in Table 1 . As a main results, nodules' volume reduced by about $30 \%$ without significant differences between the two groups of patients.

Table 1. Features of the series (mean values)

\begin{tabular}{llll}
\hline & HD group & LD group & $\mathrm{p}$ \\
\hline Nodule' size & $22 \mathrm{~mm} / 4.6 \mathrm{~mL}$ & $23 \mathrm{~mm} / 3.7 \mathrm{~mL}$ & $0.27 / 0.15$ \\
Power/site $(\mathrm{W})$ & 43.4 & 24.1 & $<0.0001$ \\
Energy/nodule $(\mathrm{kJ})$ & 2.6 & 2.0 & 0.88 \\
Dimensional variationr & $30.9 \%$ & $26.4 \%$ & 0.5 \\
Tolerability & 4 & 7 & 0.012 \\
\hline
\end{tabular}

Conclusions: Basing on our preliminary results, the power delivered within the nodules may be reduced by about $50 \%$ of the recommended dose of $45 \mathrm{~W} /$ site without affecting nodules' volume reduction at 6 months. This is especially relevant in patients with reduced tolerability to the treatment. 


\section{P2-05-101 \\ RADIOFREQUENCY THERMAL ABLATION FOR BENIGN THYROID NODULES: PRELIMINARY RESULTS FROM AN ITALIAN MULTICENTER STUDY}

Maurilio Deandrea ${ }^{1}$, Alberto Mormile ${ }^{2}$, Francesca Garino ${ }^{2}$, Roberto Garberoglio $^{3}$, Ruth Rossetto ${ }^{3}$, Nadia Bonelli ${ }^{3}$, Salvatore Monti', Maria Grazia Deiana ${ }^{4}$, Vincenzo Toscano ${ }^{5}$, Stefano Spiezia 6 , Massimo De Santis $^{6}$, Pio Assanti ${ }^{6}$, Chistian Cugini ${ }^{7}$, Pietro Valenti ${ }^{8}$, Alberto Dorigo $^{9}$, Paolo Piero Limone ${ }^{2}$

${ }^{1}$ Department of Endocrinology, Diabetes and Metabolism, Ao Mauriziano, Turin, Italy, ${ }^{2}$ Mauriziano Hospital of Turin, Endocrinology and Metabolism, Turin, Italy, ${ }^{3} \mathrm{Ao}$ Città Della Salute Torino, University Department of Endocrinology, Diabetology and Metabolism, Turin, Italy, ${ }^{4}$ Uoc Endocrinology, Sant'andrea Hospital Rome, Rome, Italy, ${ }^{5}$ Psicology and Medicine Faculty, University La Sapienza Rome, Italy, ${ }^{6}$ Uossd Endocrine Surgery Asl Napoli 1 Centro, Naples, Italy, ${ }^{7}$ Hospital Villa Salus Mestre, Venezia, Italy, ${ }^{8} \mathrm{Hospital} \mathrm{Ca}$ ' Foncello Hospital, Medicina Interna 2, Treviso, Italy, ${ }^{9}$ Department of Diagnostic and Interventional Radiology, Ca' Foncello Hospital Treviso, Treviso, Italy

The purpose of this study is to validate the generalizability of the efficacy and safety of radiofrequency (RF) ablation for treating benign thyroid nodules (BTN) in a large population multicenter study and to find the US-findings that can predict nodules' shrinkage.

Methods: This study included patients with solid cold thyroid nodules from 6 institutions. The age of patient was 28 to $89 \mathrm{yrs}$ (medium 57), 131 were females $(75 \%)$ and 43 were males (25\%). RF ablation was performed using an 18-gauge, internally cooled electrode. Nodule volume and US pattern, thyroid function, symptom/cosmetic scores, and complications were evaluated before treatment and during each follow-up visit according to a specific reference protocol (before treatment, 6 and 12 months).

Results: The median basal nodule volume was $18,9 \mathrm{~mL}$ and significantly decreased after treatment at 6 months $(6,2 \mathrm{ml}, \mathrm{p}<0.001)$; further significant volume reduction was found at 12 months $(5,4 \mathrm{~mL}$, p vs 6 months $=0.027)$. A correlation was tested between nodule's shrinkage and US-structure, vascularity, presence of macrocalcifications and nodule volume at basal, showing significant results for US-structure (pattern 2 spongiform showing a $84 \%$ reduction vs $71 \%$ and $73 \%$ of pattern 1 e 3 respectively, $p<0,001$ ) and macrocalcifications (pattern 1 and 3 showing a $73 \%, 80 \%$ and $79 \%$ respectively reduction vs $50 \%$ of pattern 2 with shell calcifications, $\mathrm{p}<0,05$ ). A slight inverse correlation was found between volume reduction and nodule basal volume (spearman $-0,22$ ), while vascularity seemed not to affect at all the treatment result. The mean symptom and cosmetic scores were significantly reduced at the last follow-up. No major complications were encountered.

Conclusions: This multicenter study validated the efficacy and safety of $\mathrm{RF}$ ablation for treating BTN and showed a positive correlation between final shrinkage and some US-findings easily detectable before treatment. RF ablation can be considered a safe and effective alternative to surgery for cold BTN.

\section{P2-05-102 \\ DYNAMIC OF SERUM BIOMARKERS GAL- 3, MMP-2 AND MMP-9 IN THYROID TUMOR DISEASE IN RELATION TO SURGERY}

Ruxandra Dobrescu ${ }^{1}$, Sorina Schipor ${ }^{2}$, Catalina Picu², Dana Manda ${ }^{2}$, Corin Badiu $^{3}$, Andra Caragheorgheopo ${ }^{2}$

${ }^{1}$ National Institute of Endocrinology, Dpt. Thyroid Disorders, Bucharest,

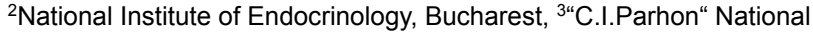
Institute of Endocrinology, "C.Davila“ University of Medicine, „C. Davila“ University of Medicine and Pharmacy, Bucharest, Romania

Background: It is suggested that higher levels of galectin-3 (Gal-3) and metalloproteinases MMP-2 and MMP-9 might be useful in pre-surgical discrimination of patients with differentiated cancer (DTC) $v s$ benign thyroid disease (BD), with higher MMP-9 levels in aggressive carcinomas, but this is still controversial. There are no studies to date evaluating these markers pre- $v s$ post-thyroidectomy.

Aim: We evaluated pre-and post-surgical serum Gal-3,MMP-2 and MMP-9 levels in a series of patients with DTC or BD and to assess their value as potential diagnostic and prognostic assays in DTC.
Patients and Methods: We included 69 patients referred for thyroidectomy, aged $51.32 \pm 12.48$ years, 56 women, 13 men. According to pathology, the patients were with benign or malignant disease: BD $(\mathrm{N}=38)$ and DTC $(\mathrm{N}=30)$. Sera were collected before surgery and 1-2 months after surgery. Gal3, MMP-2 and MMP-9 were measured by Elisa (R\&D Systems). The study was approved by IRB.

Results: Gal-3 was non-significantly higher in DTC $v s$ BD both preand post-surgery, and significantly decreased post-surgery in the BD group $(8.45 \pm 2.92$ vs $7.36 \pm 2.44 \mathrm{ng} / \mathrm{ml}, \mathrm{p}<0.001)$. MMP-2 was significantly higher in DTC $v s$ BD both pre-surgery $(251.8 \mathrm{ng} / \mathrm{ml}$ vs $199.6 \mathrm{ng} / \mathrm{ml})$ and post-surgery $(427.19 \mathrm{ng} / \mathrm{ml}$ vs $291.77 \mathrm{ng} / \mathrm{ml})$, suggesting a possible role as a diagnostic marker for DTC. MMP-2 significantly increased after surgery in both groups $(\mathrm{p}<0.01)$, an apparent paradox which might reflect its multiple pro-homeostatic and anti-inflammatory roles. We found MMP-9 higher in BD vs DTC, both pre-and post-surgery, but significant only post-surgery $(793.91 \mathrm{ng} / \mathrm{ml}$ vs $562.73 \mathrm{ng} / \mathrm{ml})$. MMP-9 decreased post-surgery for both groups, but significant only in DTC patients $(739.03 \mathrm{ng} / \mathrm{ml}$ vs $562.73 \mathrm{ng} / \mathrm{ml} \mathrm{p}<0.05)$.

Conclusion: Gal-3, MMP-2 and MMP9 are disputed markers for thyroid neoplasia both in DTC and BD, and their dynamic in relation to surgery could participate to the global prognosis.

The study was funded by UEFISCDI grant PN-II-PT-PCCA-2011-3.2 no. $135 / 2012$

\section{P2-05-103 \\ PARATHYROID HORMONE WASHOUT (PTHW) IN ULTRASOUND-GUIDED FINE NEEDLE ASPIRATION CYTOLOGY (US-FNA) FOR DIFFERENTIAL DIAGNOSES AND MANAGEMENT OF THYROID AND PARATHYROID NODULES \\ Maria de Lurdes Godinho de Matos ${ }^{1}$, Joanna Prokop ${ }^{1}$, Miguel \\ Vasques $^{1}$, Conceição Godinho ${ }^{2}$, Isaura Rodrigues ${ }^{2}$, Paula Tavares ${ }^{3}$, José Coutinho ${ }^{3}$, Ana Maria Agapito ${ }^{1}$ \\ ${ }^{1}$ Hospital Curry Cabral, Endocrinology, Lisboa, Portugal, ${ }^{2} \mathrm{Hospital}$ Curry Cabral, Laboratory, Lisboa, Portugal, ${ }^{3}$ Hospital Curry Cabral, Surgery, Lisboa, Portugal}

Introduction: To distinguish between thyroid and parathyroid nodules remains difficult in many cases, despite of image or cytological exams. PTH washout in US-FNA of cervical nodules has been performed to differential diagnoses of thyroid and parathyroid nodules and to locate parathyroid nodules in primary hyperparathyroidism proposed to surgery.

Aims: To study the diagnostic value of PTH washout in US-FNA for differential diagnoses and management of thyroid and parathyroid nodules.

Methods: This study was performed between 2012 and 2017, in patients with one or more nodules suspected of parathyroid origin after neck ultrasound. The cohort was characterized for clinical presentation, hormone assay, ultrasonography, Sestamibi scan, 4D CTscan and cytological diagnosis. After US-FNA, the needle used was washed in $1 \mathrm{ml}$ of normal saline and intact PTH was determinate by chemiluminescent immunoassay; PTHw defined as positive if superior to serum PTH. PTHw results were compared with histological diagnoses.

Results: We studied 59 patients $(13,6 \%$ men), mean age $60 \mathrm{yr}(37-86 \mathrm{yr})$, with nodules suspected to be parathyroid origin, mean size $2.1 \mathrm{~cm}(0.98-5 \mathrm{~cm})$. Primary hyperparathyroidism diagnosed in 20 patients (33.9\%). Eighty lesions were submitted to US-FNA. Cytological results: non diagnostic (47), thyroid (30) and parathyroid (3); PTHw positive in 12 cases with a mean value 2542.7 $\mathrm{pg} / \mathrm{ml}(59.2-6000 \mathrm{pg} / \mathrm{ml})$. Surgery was performed in 25 patients $(42.3 \%)$. Histological results: 20 parathyroids (17 adenoma/2 hyperplasia/1 malignant) and 10 thyroids ( 4 benign $/ 1$ microPTC/4 PTC/ 1 microFTC). Positive correlation between PTHw and histology in 9 patients $(45 \%)$, for a specificity of $100 \%$, a sensitivity of $45 \%$, false negative $55 \%$ and no false positive.

Conclusions: These results confirmed the diagnostic value of an elevated. PTHw for differential diagnosis and management of thyroid and parathyroid nodules. There was a strong positive correlation between high levels of PTHw and parathyroid lesions. This diagnostic technique may allow for targeted surgical approach. 


\section{P2-06 Hypothyroidism}

\section{P2-06-104 \\ EFFECTIVENESS OF ORAL L-THYROXINE GEL CAPSULE VS TABLET IN PATIENTS SUBMITTED TO TOTAL THYROIDECTOMY: A PRELIMINARY STUDY}

Poupak Fallahi', Silvia Martina Ferrari', Ilaria Ruffilli', Andrea Bonatti', Gabriele Materazzi ${ }^{2}$, Paolo Miccoli ${ }^{2}$, Alessandro Antonelli ${ }^{1}$

${ }^{1}$ University of Pisa, Department of Clinical and Experimental Medicine, Pisa, Italy, ${ }^{2}$ University of Pisa, Department of Surgical, Medical,

Molecular Pathology and Critical Area, Pisa, Italy

Objective: The effectiveness of levothyroxine (L-T4) soft gel capsule formulation in patients recently submitted to total thyroidectomy for multinodular goiter has not been yet evaluated. The purpose of this study was to compare the efficacy of L-T4 gel capsule, with L-T4 tablets, in patients who recently underwent total thyroidectomy for multinodular goiter (without malabsorption or drug interference).

Methods: Forty-eight patients were consecutively recruited: 24 patients were treated with L-T4 therapy in tablets, while 24 with gel capsule L-T4 at the same dosage $(1.5 \mathrm{mcg} / \mathrm{kg} / \mathrm{day}), 30 \mathrm{~min}$ before breakfast, starting the day after thyroidectomy. Serum thyrotropic hormone (TSH), free thyroxine (FT4), and free triiodothyronine (FT3) were re-evaluated after 6 weeks (first control) and 12 weeks (second control) in both groups.

Results: TSH values were significantly lower in the L-T4 gel capsule group, than in L-T4 tablet group, at the first control $(\mathrm{P}<0.05)$, as well as at the second control $(\mathrm{P}<0.01)$, while FT4 and FT3 levels were not significantly different. The prevalence of patients in the hypothyroid range (TSH $>3.5 \mathrm{mcU} /$ $\mathrm{ml}$ ) was significantly higher in the L-T4 tablet group.

Conclusion: These data suggest a better efficacy of soft L-T4 gel capsule than L-T4 tablets in controlling TSH levels in patients thyroidectomized for thyroid cancer not having malabsorption, gastric disorders, or drug interference. A larger number of studied patients is necessary to obtain a definitive result.

\section{P2-06-105 \\ THE EFFECTS OF T4 MONO REPLACEMENT VERSUS T4/T3 COMBINATION REPLACEMENT ON PSYCHOLOGICAL DISTRESS AFTER TOTAL THYROIDECTOMY IN THYROID CANCER PATIENTS - PROSPECTIVE, RANDOMIZED, DOUBLE-BLIND, T4 COMPARATIVE CLINICAL STUDY}

In Hee Shim ${ }^{1}$, Dong Sik Bae ${ }^{2}$

${ }^{1}$ Cancer Center, Dongnam Inst. of Radiolocial \& Cancer Sciences,

Busan, Rep. of South Korea, ${ }^{2}$ Haeundae Paik Hospital, Busan, Rep. of South Korea

Objectives: Patients undergoing thyroid hormone replacement therapy often suffer from mild to moderate psychological distress even if appropriate thyroid-stimulating hormone (TSH) levels are maintained. Thus, the primary goal of the present study will be to compare the degree of psychological distress experienced by thyroid cancer patients receiving standard thyroxine (T4) replacement monotherapy with that in patients receiving $\mathrm{T} 4 /$ triiodothyronine (T3) combination replacement therapy after a total thyroidectomy.

Methods: The present study will include patients who have undergone a total thyroidectomy for thyroid cancer and who are experiencing symptoms of psychological distress, such as depression, anxiety, and fatigue. All patients will be screened for distress using the Hospital Anxiety and Depression Scale (HADS $\geq 8$ ) for depression and anxiety and the MD Anderson Symptom Inventory-Fatigue (MDASI-F; $\geq 4$ ) for fatigue. The patients will be randomly assigned to T4 monotherapy or T4/T3 combination therapy using a random numbers table. Assessments of psychological distress will be made at baseline and at 4, 12, and 24 weeks. Levels of distress after thyroidectomy will be assessed using the HADS, Beck Depression Inventory (BDI), and Beck

Poster Presentations
Anxiety Inventory (BAI) for depression and anxiety, and the Brief Fatigue Inventory (BFI) for fatigue.

Results: Participants are currently being recruited for this study, which has an estimated completion date of December 2017. For both groups, the primary outcome measure is the change in HADS total score from baseline to 24 weeks and the secondary outcome measure is the change in BFI total score from baseline to 24 weeks.

Conclusions: The present study will compare patients receiving T4 with those receiving T4/T3 in terms of psychological distress, including depression, anxiety, and fatigue. These findings will contribute to decreasing the prevalence of mild to moderate distress and improve quality of life in thyroid cancer patients after a total thyroidectomy.

\section{P2-06-106 \\ PREVALENCE OF THYROID DYSFUNCTION AND AUTOIMMUNITY IN THE OLDER POPULATION AND IMPLICATIONS OF AGE-SPECIFIC REFERENCE RANGES}

Kris Poppe $^{1}$

${ }^{1}$ Chu Saint-Pierre (Ulb), Brussels, Belgium

Objective: To investigate the prevalence of thyroid dysfunction and autoimmunity (TAI) and to determine age specific reference ranges in individuals $<60$ and $\geq 60$-year-old. Furthermore we investigated the impact of the age-specific reference ranges on the prevalence of thyroid dysfunction.

Design: Retrospective analysis of laboratory data collected over six months in 2015, mainly from individuals consulting the outpatient clinic.

Method: Data from 676 individuals were withheld, after having applied strict exclusion criteria to avoid confounders. After exclusion of individuals with TAI (TPO-abs $>60 \mathrm{kIU} / \mathrm{L}$ ) and/or outliers, data of 547 individuals were used to determine age-specific reference ranges. The prevalence of subclinical hypothyroidism ( $\mathrm{SCH}$ ) and subclinical hyperthyroidism (sch) was determined according to the reference ranges from the commercial assay and also according to the calculated age-specific reference ranges from our study population.

Results: From the 676 individuals included $559(83 \%)$ were $<60$ year-old and $117(17 \%) \geq 60$ year-old. The prevalence of sch and TAI was comparable between both groups ( $8.6 \%$ vs. $13.7 \%$ and $15.4 \%$ vs. $20.5 \%$ respectively). The prevalence of SCH was significantly higher in individuals $\geq 60$ years, compared to that in individuals $<60$ years $(14.5 \%$ vs. $5.4 \%$; $\mathrm{p} \mathrm{b} 0.001)$. The calculated 2.5 and 97.5 percentile for the age-specific TSH range was 0.24 and $4.4 \mathrm{mIU} / \mathrm{L}$ in individuals $<60$ years and 0.15 and $8.2 \mathrm{mIU} / \mathrm{L}$ in individuals $\geq 60$ years. When these the prevalence of sch and $\mathrm{SCH}$ was then determined on the basis of the age-specific reference ranges, the prevalence of SCH significantly decreased in individuals $\geq 60$ years $(14.5 \%$ to $5 \% ; \mathrm{p}=0.027)$ and it then became comparable with that in individuals $<60$ years $(5 \%$ vs. $3 \%)$.

Conclusions: The prevalence of $\mathrm{SCH}$ was higher in individuals $\geq 60$ years, compared to that in individuals $<60$ years, but when age-specific TSH reference ranges were used, it was comparable between both study groups. In order to avoid misclassification in older individuals, it is important to use agespecific reference ranges in daily clinical practice.

\section{P2-06-107 \\ CORONARY FLOW RESERVE IN SUBCLINICAL HYPOTHYROIDISM}

Mirjana Stojkovic ${ }^{1}$, Slavica Savic ${ }^{2}$, Biljana Nedeljkovic-Beleslin ${ }^{1}$, Jasmina Ciric ${ }^{1}$, Tanja Nisic ${ }^{2}$, Marija Miletic ${ }^{2}$, Milos Zarkovic ${ }^{1}$

${ }^{1}$ Clinic of Endocrinology, Diabetes and Metabolic Diseases, Clinical Center of Serbia, Faculty of Medicine, University of Belgrade, Belgrade, Serbia, ${ }^{2}$ Clinic of Endocrinology, Diabetes and Metabolic Diseases, Clinical Center of Serbia, Belgrade, Serbia

Objectives: Thyroid hormone is an important regulator of cardiac function and cardiovascular hemodynamics. Triiodothyronine, (T3) is the physiologically active form of thyroid hormones. T3 mediated effects on the systemic vasculature include relaxation of vascular smooth muscle resulting in decreased arterial resistance and diastolic blood pressure. In hypothyroidism, cardiac contractility and cardiac output are decreased and systemic vascular resistance is increased. Many articles show that patients with subclinical hypothyroidism manifest many of the same cardiovascular changes, but to a lesser degree than that which occurs in overt hypothyroidism.

Eur Thyroid J 2017;6(suppl 1):23-118 
Although the level of circulating TSH provides a sensitive and quantitative indicator of thyroid hormone action at the level of the hypothalamicpituitary axis, there are few reliable peripheral or intracellular markers of thyroid hormone action. In the euthyroid person the absolute concentration of free hormone remains constant and correlates with the tissue hormone level and its biologic effect. In subclinical hypothyroidism, reason for compensatory increase of TSH is maintenance the free hormone concentration at normal level, provided the patient is in a steady state.

Coronary flow reserve (CFR) is the maximum increase in blood flow through the coronary arteries above the normal resting volume. CFR can be thought of as the capacity of the coronary circulation to dilate and thus increase flow following an increase in myocardial metabolic demands. It is one of the marker of coronary vascular resistance. There are many factors that alter the vascular resistance. Vascular compliance is determined by the muscle tone which is subject to continual homeostatic changes by hormones and cell signaling molecules that induce vasodilatation and vasoconstriction to keep blood pressure and blood flow within reference ranges.

Methods: we have used CFR measurement by trans-thoracic Doppler echocardiography (TTDE) to determine coronary microvascular function in patients with subclinical hypothyroidism and healthy subjects. The study group consisted of 32 patients with subclinical hypothyroidism and 12 subjects with normal thyroid hormone axis. None of our patients had diabetes or heart disease.

Results: in stepwise multiple regression analysis that includes T4, T3, TSH, HbA1c, HOMA, cholesterol, median arterial pressure (MAP) and waist circumference (WC), we found that T3, cholesterol and HbA1c contributed significantly to the CFR for LAD (p-value for regression 0.0004). There was no significant relation between TSH level and CFR for LAD.

Conclusion: more studies are needed for providing the answer about relations in vascular profile and subclinical hypothyroidism.

\section{P2-06-108 \\ SYSTEMIC SCLEROSIS AND FATIGUE IN HYPOTHYROID PATIENTS}

Silvia Martina Ferrari ${ }^{1}$, Poupak Fallahi ${ }^{1}$, Sabrina Rosaria Paparo ${ }^{1}$, Marco Centanni ${ }^{2}$, Camilla Virili ${ }^{2}$, Michele Colaci ${ }^{3}$, Dilia Giuggioli ${ }^{3}$, Clodoveo Ferri ${ }^{3}$, Alessandro Antonelli ${ }^{1}$

${ }^{1}$ University of Pisa, Department of Clinical and Experimental Medicine, Pisa, Italy, ${ }^{2}$ Sapienza, University of Rome, Department of MedicoSurgical Sciences and Biotechnologies, Latina, Italy, ${ }^{3}$ University of Modena and Reggio Emilia, Department of Medical, Surgical, Maternal, Pediatric and Adult Sciences, Modena, Italy

Objective: Persistent fatigue is defined as a continuing exhaustion, disproportionate to effort, without any relief given by rest. Patients with Systemic sclerosis (SSc), affected by this condition, have a reduced quality of life, with a limited capacity to conduct daily activities, work disability, and impaired physical function. Different studies show a high prevalence of hypothyroidism in SSc patients, and L-thyroxine (L-T4) substititutive therapy can be used to cure hypothyroidism and the associated fatigue symptoms. Our purpose was to evaluate the evolution of fatigue symptoms in SSc hypothyroid patients following a L-T4 substititutive therapy.

Methods: We enrolled twelve clinical hypothyroid and twenty-five subclinical hypothyroid female SSc patients (mean age 54 \pm 11 ), having diffuse scleroderma, who were treated with L-T4 substitutive therapy. Controls were assessed after two months, measuring TSH, FT3, FT4 and adjusting the dosage of the therapy; then patients were re-evaluated at four months.Patients completed the General Fatigue Index (GFI) of the Multidimensional Fatigue Inventory at baseline and at 4 months.

Results: Mean baseline GFI scores in hypothyroid SSc (15.7 \pm 5.1$)$ were significantly higher (greater fatigue; ANOVA, $\mathrm{P}<0.01$ ) compared to the same patients after reaching euthyroidism at 4 months $(9.6 \pm 3.1)$.

Conclusions: We show: 1) the importance of screening female SSc patients for thyroid function, especially in the presence of fatigue symptoms; 2) an appropriate L-T4 substitutive therapy could be helpful to mitigate these symptoms. However, other longitudinal studies (including a larger number of SSc hypothyroid patients) are needed to evaluate the usefulness of L-T4 therapy in alleviating complications of SSc (as skin thickness and pulmonary hypertension).

\section{P2-06-109 \\ HYPOTHYROIDISM IS RELATED WITH \\ AGE,GENER AND LOCATION}

Anila Rrupulli ${ }^{1}$, Elona Mollsi ${ }^{2}$, Lindita Vrushi ${ }^{3}$

${ }^{1}$ Endocrinology, Elbasan, Albania, ${ }^{2}$ University of Elbasan „Aleksander

Xhuvani“, Elbasan, Albania, ${ }^{3}$ Laboratory Lui Paster, Elbasan, Albania

Background and Aim: We aim to study the relation of age, gener and location to study population with Tsh level more than 4.5.

Method: We study a group of population,patients have the symptom of hypothyroidism such as weakness, weight gain,bradicardia, cold. All adult (20-60 years)with these symptom are screening for tsh during a period of 5 years (Janury 2012-Janury 2017

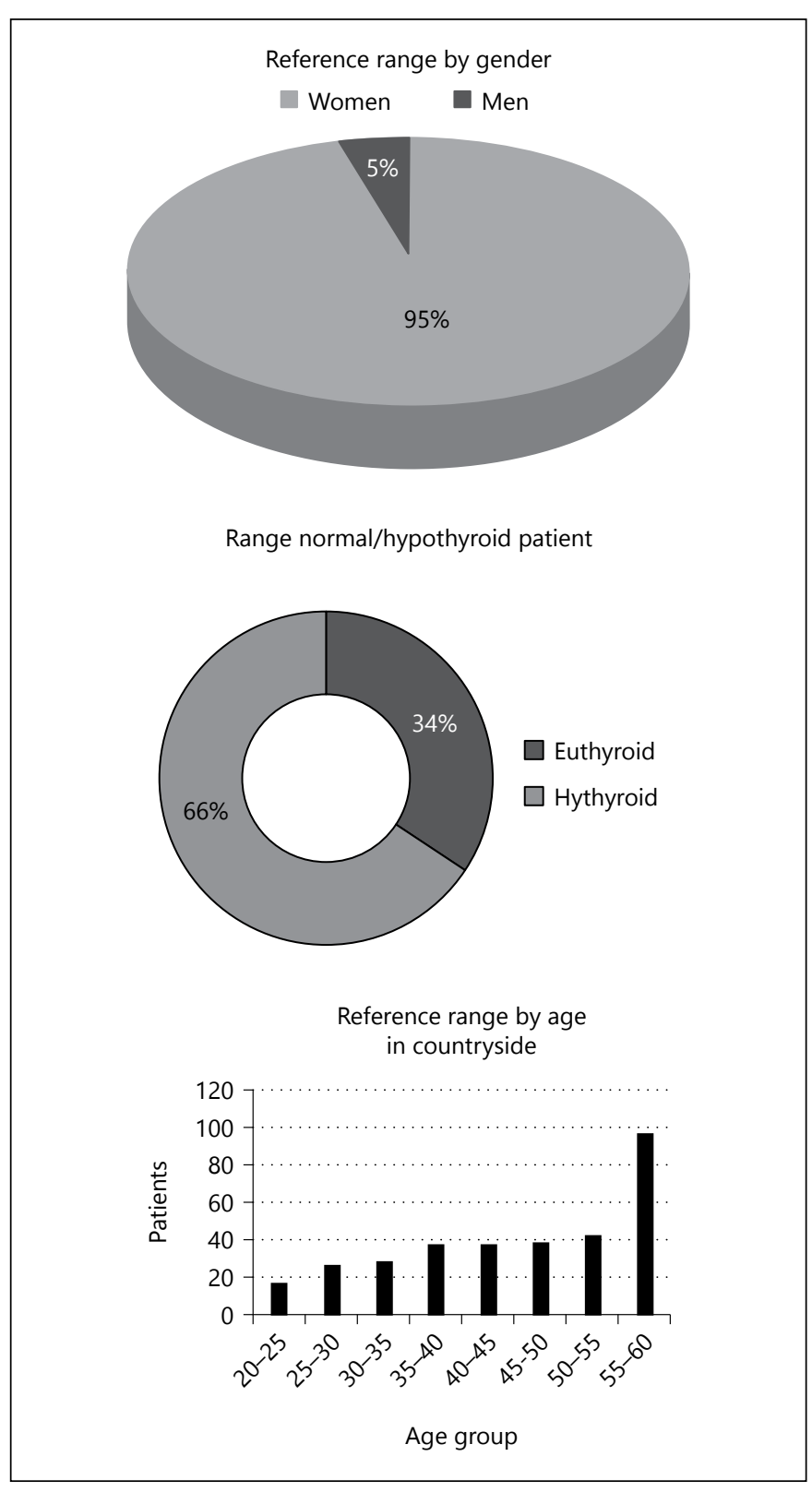

Fig. 1-3.

Result. We study 794 persons which are consulting to us with the sign of hypothyroidism. From these group 521 of persons have elevated Tsh $>4.5 \mu \mathrm{U} /$ 1Level of Tsh is detected by Elisa method..5\% of the group are man and $95 \%$ are women. In the group of patients who live in city we detected elevated Tsh 


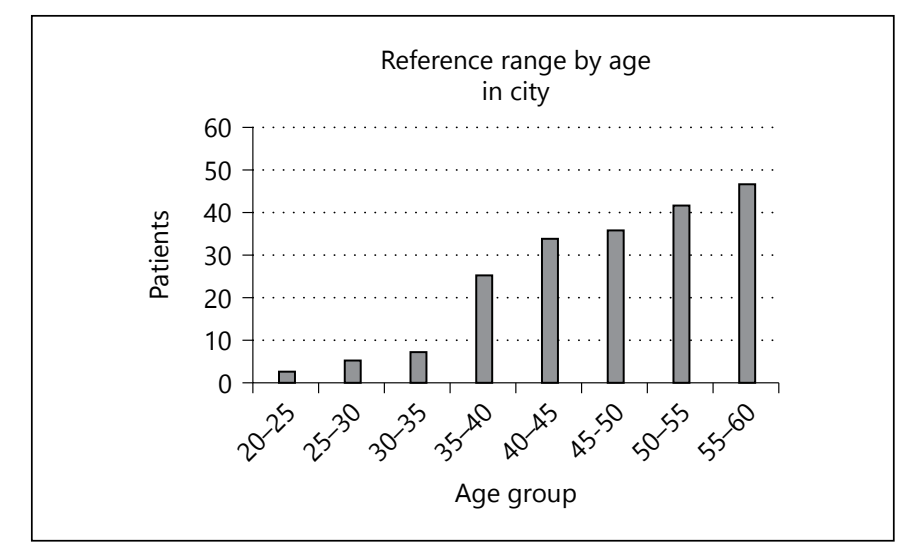

Fig. 4.

value age group $(20-60) 1 \%(20-25) 2 \%(25-30) 3 \%(30-35) 13 \%(35-40) 17 \%(40-$ $45) 18 \%(45-50) 21 \%(50-55) 25 \%(55-60)$.In the group of patients who live in the country we dected 5\%(20-25)8\%(25-30)9\%(3035)11\%(35-40)12\%(40$45) 12 \%(45-50) 13 \%(50-55) 30 \%(55-60-35) 11 \%(35-40) 12 \%(40-45) 12 \%(45-$ $50) 13 \%(50-55) 30 \%(55-60)$

Conclusion: Hypothyroidism is more evident in women than men. By increasing the age the hypothyroidism is more evident. More evident in countryside

\section{P2-06-110 \\ EVALUATION OF A THREE-MONTH TRIAL OF THYROXINE REPLACEMENT IN SUBCLINICAL HYPOTHYROIDISM: THE IMPACT ON CLINICAL PRESENTATION, QUALITY OF LIFE AND ADOPTION OF LONG-TERM THERAPY \\ Milena Pandrc $^{1}$, Andjelka Ristić ${ }^{2}$, Vanja Kostovski ${ }^{2}$, Biljana \\ Nedeljković-Beleslin ${ }^{3}$, Jasmina Ćirić3 \\ ${ }^{1}$ Military Medical Center, Belgrade, Serbia, ${ }^{2}$ Military Medical Academy, Belgrade, Serbia, ${ }^{3}$ Clinic for Endocrinology, Diabetes and Metabolic \\ Diseases, Clinical Center of Serbia, School Of Medicine University of Belgrade, Belgrade, Serbia}

Objective: To examine the effect a 3-month trial of levothyroxine (LT4) treatment on clinical presentation, quality of life and patient's opinion on further treatment of subclinical hypothyroidism $(\mathrm{SCH})$.

Methods: Clinical parameters(disease-specific score) and quality of life (Short Form 36 questionnaire) were measured in 35 patients with persistent $\mathrm{SCH}$ before the intervention (TSH 7.0 $\pm 2.1 \mathrm{mIU} / \mathrm{L}$ ), 3 months after the euthyroid state had been achieved and 3 months after cessation of LT4 substitution.

Results: The median of Zulewski index significantly decreased after the treatment with LT4: 5.0 (4.0-7.0) vs 3.0 (2.0-5.0), representing a reduction of symptoms. LT4 treatment significantly reduced the percentage of patients with symptoms and signs of overt hypothyroidism and increased the number of individuals classified as intermediate and euthyroid. The most common ailments before treatment were dry skin $(71.4 \%)$, periorbital edema $(48.6 \%)$ and cold skin $(46.7 \%)$. After the treatment, there was a significant reduction in the frequency of constipation, dry skin, hoarseness, decreased sweating, and delayed Achilles reflex. Weight gain was reduced, but not significantly. Quality of life was not changed significantly on LT4. Among 35 patients who were asked to stop the treatment and recheck its effects, 17 patients were satisfied with the improvement of symptoms and refused to experience them again. In the group of 18 patients who discontinued treatment, many symptoms and signs reappeared with the increasing of TSH $(6.8 \pm 1.1 \mathrm{mIU} / \mathrm{L})$ : periorbital edema, constipation, weight gain, decreased sweating, slow motion and delayed Achilles reflex. Median of Żulewski index after discontinuation of LT4 was 6.0 (4.0-9.0). Also, there was a statistically significant reduction in general health score, vitality, role emotional and mental health scores.

Conclusions: The effect of thyroxine substitution on quality of life is controversial. The improvement of clinical presentation after achieving euthyroidism supports individually adjusted treatment of patients with $\mathrm{SCH}$.

Key words: thyroxine replacement, subclinical hypothyroidism, quality of life

\section{P2-06-111 \\ HEART FAILURE IN PATIENTS WITH HYPOTHYROIDISM}

\section{Nino Latsabidze ${ }^{1}$}

${ }^{1}$ Department of Endocrinology, Elizabeth Blackwell Hospital,

Department of Cardiology, Tbilisi, Georgia

The cardiovascular system is the main target organ of thyroid hormone. Overt hypothyroidism decreases myocardial contractility, and diminished myocardial contractility reduces thyroid hormone metabolism. Subclinical hypothyroidism also exhibits impairment of left ventricular diastolic function that returns to normal after thyroid hormone replacement.

We investigate 12 with overt hypothyroidism, 15 with subclinical hypothyroidism subjects. The patients were evaluated for cardiac disease by physical examination, electrocardiography, and echocardiography. Serum samples were taken for free thyroxine (fT4), TSH, NT-pro BNP before and after achievement of euthyroidism in patients.

Results: Basal mean NT-pro BNP level of overt hypothyroid group was significantly higher than the subclinical hypothyroid group (98.8 $\pm 63.0 \mathrm{vs}$ $43.40 \pm 23.66 \mathrm{pg} / \mathrm{ml}, \mathrm{P}<0.001)$. Mean NT-pro BNP significantly decreased after achievement of euthyroidism in both group $(\mathrm{P}=0.002)$. Basal NT-pro $\mathrm{BNP}$ level was negatively correlated with heart rate in hypothyroid group ( $\mathrm{r}$ : $-0.6, \mathrm{P}=0.03$ ).

Conclusion: Studies to date suggest that thyroid hormone is one of the important factors that regulate serum NT-pro BNP level. Thus, in patients without severe cardiac dysfunction, elevated NT-pro BNP level may be suggestive for both group - as overt hypothyroid, as subclinical hypothyroidism too.

\section{P2-06-112 \\ THE CORRELATION BETWEEN BODY MASS INDEX AND THYROID-STIMULATING HORMONE IN DIABETIC PATIENTS WITH HYPOTHYROIDISM}

Snezana Vujosevic', Sanja Borozan², Milan Bogojevic', Sreten Kavaric ${ }^{1}$, Olivera Boskovic ${ }^{1}$, Aleksandar Djogo ${ }^{1}$, Djordjije Krnjevic ${ }^{3}$,

Emir Muzurovic ${ }^{1}$

${ }^{1}$ Podgorica, ${ }^{2}$ Podgorica, Montenegro, ${ }^{3} \mathrm{Clinical}$ Center of Montenegro,

Podgorica, Montenegro

Objective: Diabetes mellitus (DM) and dysfunction of the thyroid gland are consider as two most frequent endocrine disorders with increasing prevalence. Recent Meta-analysis revealed a mean frequency of thyroid disease of $11 \%$ in patients with DM. A significant positive association between body mass index (BMI) and thyroid-stimulating hormone (TSH) is observed in euthyroid subjects but in hypothyroid patients results are conflicting. On the other hand, achieving a TSH level within the normal reference range is known to prevent weight gain. Our objective was to determine any possible relationship between BMI and TSH in diabetic patients with primary hypothyroidism $(\mathrm{PH})$.

Methods and Results: An observational study was performed on 25 patients with $\mathrm{PH}$ and DM, out of 297 diabetic patients hospitalized at Clinical Centre of Montenegro from October 2015 to December 2016. Clinical and laboratory evaluation of patients was performed and, furthermore, three groups are formed according to diabetes therapy: patients with metformin, combined therapy and insulin. Statistical evaluation was performed using Pearson correlation coefficient (r).

All of the 25 enrolled patients were female, aged 25 to 77 years (mean $57.56 \pm 14.21)$. In metformin group were 5 patients $(20 \%)$, all of them with BMI above the WHO cut-off point for obesity $(>30 \mathrm{~kg} / \mathrm{m} 2) ; 11(44 \%)$ were treated with both metformin and insulin $(63.6 \%$ of them obese) and $9(36 \%)$ with insulin (11.1\% were obese). The correlation between BMI and TSH in every group was statistically insignificant $(\mathrm{r}=0.245, \mathrm{p}=0.691 ; \mathrm{r}=-0.242$, $\mathrm{p}=0.473 ; \mathrm{r}=-0.051, \mathrm{p}=0.896$ respectively). Analyzing all examined patients, for positive correlation between BMI and TSH, $p$ was low $(p=0.116)$. This indicates that $\mathrm{p}$ could be statistically significant if the sample was larger.

Conclusion: In our study we didn't find any significant relationship between BMI and TSH. Further enlargement of the sample is planned in order to establish new or confirm our findings. 


\section{P2-06-113 \\ CENTRAL HYPOTHYROIDISM IN CHILDHOOD CRANIAL IRRADIATION}

Sandra Pekic Djurdjevic $^{1}$, Mirjana Doknic ${ }^{1}$, Dragana Miljic ${ }^{1}$, Marko Stojanovic ${ }^{1}$, Zvezdana Jemuovic ${ }^{2}$, Marina Nikolic Djurovic ${ }^{1}$, Vera Popovic ${ }^{3}$, Milan Petakov ${ }^{1}$

${ }^{1}$ Clinic for Endocrinology, Diabetes and Diseases of Metabolism, University Clinical Center, School of Medicine, University of Belgrade, Belgrade, Serbia, ${ }^{2}$ Clinic for Endocrinology, Diabetes and Diseases of Metabolism, University Clinical Center, Belgrade, Serbia, ${ }^{3}$ School of Medicine, University of Belgrade, Belgrade, Serbia

Background: Patients treated with cranial radiation are at risk of anterior pituitary hormone deficiencies, among others for central hypothyroidism. We assessed the prevalence of central hypothyroidism in survivors of childhood cranial irradiation.

Procedure: We evaluated anterior pituitary function in 35 childhood cranial irradiated survivors. We investigated 35 adult patients (age, 23.1 1 .0yr; 16 female and 19 male; mean body mass index, $26.2 \pm 1.6 \mathrm{~kg} / \mathrm{m}^{2}$ ) who received cranial radiation for craniopharyngioma $(n=11)$, germinoma $(n=9)$ and other primary brain tumor distant from hypothalamic-pituitary region (medulloblastoma, $n=11$; astrocytoma, $n=3$, acute lymphoblastic leukemia, $n=1$ ). The patients were tested $8.6 \pm 1.2 \mathrm{yr}$ after cranial radiotherapy (range, 1-29 yr). The age at diagnosis and cranial irradiation was $14.4 \pm 1.2 \mathrm{yr}$ (range, 4-24 yr). Patients received, after surgical treatment, a mean radiation dose of 52.8 \pm 1.4 Gy directed at the primary tumor, whereas the estimated dose delivered to the hypothalamus/pituitary ranged from 25-30 Gy. Anterior pituitary function (thyroid, gonadal, adrenal and somatotroph axes, prolactin) was assessed in all patients.

Results: Central hypothyroidism was identified in $26(74 \%)$ of all childhood cranially irradiated patients. All patients with craniopharyngioma (11/11) had central hypothyroidism. Fifteen patients with central nervous system tumors $(15 / 24,62.5 \%)$ had central hypothyroidism. Other anterior hormone deficiencies were present in 30 patients $(86 \%)$ with $\mathrm{GH}$ deficiency, 24 patients $(69 \%)$ with central hypogonadism, 20 patients $(57 \%)$ with central hypocorticism and 5 patients $(9 \%)$ had diabetes insipidus.

Conclusions: The greatest risk for central hypothyroidism after cranial irradiation in childhood were patients with craniopharyngioma. Annual testing for anterior pituitary hormone deficiencies and continued life-long surveillance is mandatory after cranial irradiation in childhood for CNS tumor.

\section{P3-01 Autoimmunity 3}

\section{P3-01-114 \\ THE ROLE OF THYROTROPIN RECEPTOR ANTIBODIES, CALSEQUESTRIN ANTIBODIES AND COLLAGEN XIII IN GRAVE'S OPHTHALMOPATHY \\ Narine Martirosian ${ }^{1}$, Nina Petunina ${ }^{1}$, Liubov Trukhina ${ }^{1}$, Maria Grigoryeva ${ }^{1}$, Svetlana Saakian ${ }^{2}$, Olga Panteleeva ${ }^{2}$ \\ ${ }^{1}$ Sechenov First Moscow State Medical University, Moscow, Russian Federation, ${ }^{2} \mathrm{Helmholtz}$ Moscow Research Institute of Eye Diseases, Moscow, Russian Federation}

Objective: The aim of our study was to evaluate the association between thyrotropin receptor antibody (TRAb), antibodies against calsequestrin (antiCASQ) and collagen XIII level with clinical activity and severity of GO and to compare the sensitivity of two different TRAb assays.

Methods: We have studied 88 patients with Grave's Disease (GD) of which 74 had GO. Activity of GO was measured with the clinical activity score (CAS), we defined active $\mathrm{GO}$ as a $\mathrm{CAS} \geq 3$. We evaluated the prevalence of antiCASQ antibodies and collagen XIII level, serum TRAb level was measured by two different methods, 2nd generation TRAb assay and 3rd generation TRAb assay using human monoclonal thyroid-stimulating antibody M22.

Results: At the time of testing all of patients were euthyroid: $54,1 \%$ had active GO and $45,9 \%$ inactive GO. AntiCASQ were positive in $70,3 \%$ patients with GO and only in $7,1 \%$ with GD $(\mathrm{p}<0,001)$. In patients with GO
antiCASQ were positive in $100 \%$ patients with $\mathrm{CAS}=5-7$, in $84,8 \%$ patients with $\mathrm{CAS}=3-4$ and in $45.9 \%$ with inactive GO. AntiCASQ also depended on the severity of GO and were significantly more frequent in patients with severe GO (100\%), compared with moderate-to-severe (68.1\%) and light GO $(46.2 \%), p=0.004$. Collagen XIII level didn't differ in patients with GD and GO. Sensitivity of the 2nd and 3rd generation TRAb assays were $65.8 \%$ and $87.7 \%(\mathrm{p}<0,001)$. Both 2 nd and 3rd generation TRAb assays correlate with GO activity $(\mathrm{r}-0,391, \mathrm{p}-0,002 ; \mathrm{r}-0,458, \mathrm{p}<0,01)$ and severity $(\mathrm{r}-0,3, \mathrm{p}-0,021$; $\mathrm{r}-0,312, \mathrm{p}-0,016)$.

Conclusion: Our results suggest that antibodies against calsequestrin could be considered as a good marker of GO, associated with disease activity and severity. TRAb level measured both with 2 nd and 3rd generation assay correlate with GO activity and severity and 3rd generation assay shows higher sensitivity.

\section{P3-01-115 \\ SEVERE INFLAMMATORY OPHTHALMOPATHY IN A EUTHYROID PATIENT WITH NON-SMALL LUNG CANCER (NSLC) DURING NIVOLUMAB TREATMENT}

Pauline Campredon ${ }^{1}$, Philippe Imbert ${ }^{2}$, Céline Mouly ${ }^{1}$, Solange Grunenwald $^{3}$, Julien Mazieres ${ }^{4}$, Philippe Caron ${ }^{5}$

${ }^{1}$ Department of Endocrinology and Metabolic Diseases; Chu Larrey, Toulouse, France, ${ }^{2}$ Service D'ophtalmologie; Clinique du Parc, Toulouse, France, ${ }^{3}$ Chu Larrey; Service D' Endocrinologie, Toulouse, France, ${ }^{4}$ Toulouse University Hospital; Department of Pneumology, Toulouse, France, ${ }^{5} \mathrm{Chu}$ Larrey; 7eme Etage/Chu Rangueil, Toulouse, France

Introduction: Nivolumab is a promising treatment in patients with advanced malignancies. Among immune-related adverse events, autoimmune thyroid disorders (thyrotoxicosis, hypothyroidism, inflammatory thyroiditis) are frequently reported.

Case Report: A 61-year-old male patient had no history of familial or personal thyroid disease. In 2012 this heavy-smoker (40-45 pack-years) patient presented a NSLC treated with radiotherapy and chemotherapy. In 2015, lung cancer progressed with cervical compressive symptoms and the patient was treated with nivolumab. After 3 infusions, bilateral eyelid ptosis and bilateral conjunctival redness with chemosis were observed. Ophthalmologic examination revealed severe proptosis $(28 \mathrm{~mm})$ with complete ophthalmoplegia but with normal vision, color test and optic disc. Thyroid function tests were nor$\mathrm{mal}(\mathrm{TSH}=0.65 \mathrm{mU} / \mathrm{L}, \mathrm{fT} 4=12 \mathrm{pg} / \mathrm{ml})$ without anti-thyroperoxidase or antiTSH receptor antibodies. Ultrasonography of the neck showed a normal-sized thyroid gland. CT scan of the orbits confirmed marked bilateral proptosis with expansion of the orbital adipose tissue without significant thickening of extra-ocular muscles. T2-weighted MRI showed inflammation of orbital adipose tissue. Clinical work-up found no evidence for myasthenia gravis, orbital cellulitis or metastases. Nivolumab treatment was withdrawn and the patient received weekly intravenous high-dose methylprednisolone ( $1 \mathrm{~g}$ for 2 weeks, $500 \mathrm{mg}$ for 4 weeks and $250 \mathrm{mg}$ for 5 weeks). After the first 3 cycles, significant improvement of left chemosis was observed whereas bilateral ptosis and ophthalmoplegia were unchanged. The tolerance of steroid therapy was good. The patient was euthyroid without anti-thyroid antibodies one week prior to his death due to massive hemoptysis.

Conclusion: We report severe thyroid-like ophthalmopathy in a euthyroid patient with NSLC during nivolumab therapy. Other immune changes than auto-immunity seem to play a role in the pathogenesis of ophtalmopathy secondary to nivolumab. The occurrence of such ophthalmic adverse events is likely to increase during nivolumab therapy in patients with advanced malignancies. 


\section{P3-01-116 \\ CLINICAL CHARACTERISTICS OF PATIENTS WITH NEWLY DIAGNOSED GRAVES DISEASE AND DIFFERENT ANTIBODY PROFILES}

Ralitsa Mekova ${ }^{1}$, Mihail Boyanov ${ }^{2}$, Deniz Bakalov ${ }^{3}$, Adelina Tsakova ${ }^{1}$ Medical University Sofia, University Hospital Alexandrovska, Clinic of Endocrinology and Metabolism, Department of Internal Medicine, Sofia, Bulgaria, ${ }^{2}$ Medical University Sofia, Internal Medicine, University Hospital Alexandrovska, Clinic of Endocrinology and Metabolism, Department of Internal Medicine, Sofia, Bulgaria, ${ }^{3}$ University Hospital Alexandrovska, Endocrinology Clinic, Medical University Sofia, Department of Internal Diseases, Medical University Sofia, Sofia, Bulgaria, ${ }^{4}$ Medical University Sofia, University Hospital Alexandrovska, Department of Clinical Laboratory and Clinical Immunology, Sofia, Bulgaria

Objectives: To examine the clinical characteristics of patients with newly diagnosed Graves disease (GD) who differ in the number of positive antithyroid antibodies.

Methods: 249 patients (191 women, 58 men) with newly diagnosed GD participated. Levels of TSH-receptor antibodies (TRAb), anti-thyroglobulin antibodies (antiTg) and anti-thyroid peroxidase antibodies (antiTPO) and thyroid hormones (TSH, FT4, FT3) were measured with third generation ECLIA assays. Thyroid ultrasound was performed, thyroid volume was calculated and presence of nodules was noted. Patients were evaluated for the presence of thyroid-associated ophtalmopathy (TAO) and its severity (according to NOSPECS). Patients who had elevated levels of TRAb only were marked as group 1+, those with additionally elevated antiTPO or antiTG were marked as group $2+$ and those who were positive for all the three antibodies - as group $3+$.

Results: Patients were equally distributed among the groups $(1+-34 \%$, $2+-33 \%, 3+-33 \%)$. Patients with more positive antibodies tended to be younger $(\mathrm{p}=0.001, \mathrm{r}-0.232)$. They had higher levels of TRAb $(\mathrm{p}=0.018$, $\mathrm{r}-0.189)$, antiTPO $(\mathrm{p}<0.005, \mathrm{r} 0.234)$ and antiTg $(\mathrm{p}<0.005, \mathrm{r} 0.234)$. Thyroid volume was also rising with the accumulation of positive antibodies $(\mathrm{p}=0.015$, $\mathrm{r} 0.210$ ) and patients with increased volume prevailed in the $3+$ group, while the $1+$ prevailed in those with normal volume $(\mathrm{p}=0.015, \mathrm{r} 0.178)$. The presence of thyroid nodules was negatively correlated with the number of positive antibodies $(\mathrm{p}<0.005, \mathrm{r}-0.278) .63 \%$ of patients with nodules were from the $1+$ group. The presence of TAO was also negatively associated with the number of positive antibodies $(\mathrm{p}=0.002, \mathrm{r}-0.218)$, while its severity didn't differ among the groups.

Conclusion: GD patients may be stratified according to the number of positive antibodies at disease presentation. Those groups would differ in age, levels of FT4 and thyroid antibodies, thyroid volume and the presence of TAO or thyroid nodules.

\section{P3-01-117 \\ LEAD CONCENTRATION CORRELATES WITH TRAB LEVEL IN PATIENTS GRAVES، ORBITOPATHY. PRELIMINARY REPORT}

Iwona Palyga ${ }^{1}$, Danuta Gasior-Perczak ${ }^{1}$, Agnieszka Walczyk ${ }^{1}$, Klaudia Gadawska-Juszczyk ${ }^{1}$, Estera Mikina ${ }^{1}$, Monika Szymonek ${ }^{1}$, Tomasz Trybek', Monika Piwowar', Robert Palyga ${ }^{1}$, Anna Słuszniak', Ryszard Mezyk ${ }^{1}$, Urszula Majewska ${ }^{2}$, Stanislaw Gozdz ${ }^{3}$, Aldona Kowalska ${ }^{3}$

${ }^{1}$ Holycross Cancer Centre, Kielce, Poland, ${ }^{2}$ Jan Kochanowski University, Kielce, Poland, ${ }^{3}$ Holycross Cancer Centre, Jan

Kochanowski University, Kielce, Poland

Introduction: Blood lead $(\mathrm{Pb})$ level is a known exponent of either environment pollution or pollution from smoking- which apart from genetic factors, stress and others - are the risk factors for the development of Graves' orbitopathy (GO).

Objective: To determine the blood lead level (BLL) and determine the dependency of $\mathrm{Pb}$ concentration with known markers of disease exponent: TRAB levels, and clinical activity score (CAS) in patients with GO.

Material: 62 patients ( 49 women, 13 men; average age- 54,5 years) with active, moderate to severe GO who qualified for i.v. metylprednisolone treatment according to the EUGOGO scheme, at a single site in the years 2014 -2016 .
Method: set the average and median concentrations for $\mathrm{Pb}$, TRAB and $\mathrm{CAS}$ in the group of patients and determined the correlation coefficients between serum $\mathrm{Pb}$ and TRAB and CAS.

Results: blood lead level was below reference range for adults $(10 \mu \mathrm{g} / \mathrm{dl})$ in all patients: min. $1,3 \mu \mathrm{g} / \mathrm{dl}$, max- 9,6 $\mu \mathrm{g} / \mathrm{dl}$; median 3,1 $\mu \mathrm{g} / \mathrm{dl}$. Positive correlation between BLL and TRAK level was found $\mathrm{p}=0,0073$; correlation coefficient $-0,338$. BLL was not correlated with CAS.

Conclusion: 1) The impact of environmental pollution or smoking expressed by the concentration of lead in the blood may have an impact on the intensification of autoimmune processes in GO 2) It seems to be crucial to reduce exposure to lead either from smoking or industrial exposition.

\section{P3-01-118 \\ THE IMPLEMENTATION OF NURSE SPECIALIST IN THE MOTIVATION FOR SMOKING CESSATION IN GRAVES ORBITOPATHY}

Jasmina Ciric ${ }^{1}$, Milos Zarkovic ${ }^{1}$, Biljana Nedeljkovic-Beleslin ${ }^{1}$, Maja

Stojiljkovic ${ }^{2}$, Mirjana Stojkovic ${ }^{1}$, Slavica Savic ${ }^{2}$

${ }^{1}$ Clinic of Endocrinology, Diabetes and Metabolic Diseases, Clinical

Center of Serbia, Faculty of Medicine, University of Belgrade,

Belgrade, Serbia, ${ }^{2}$ Clinic of Endocrinology, Diabetes and Metabolic

Diseases, Clinical Center of Serbia, Belgrade, Serbia

Smoking has a great negative impact on Graves' orbitopathy (GO).

Objectives: The aim of this study was to evaluate the contributing role of nurse specialist (GO nurse) in the intensive motivation of GO patients for smoking cessation.

Methods: We investigated patients with moderately severe GO, current smokers at the time of weekly treatment with iv methylprednisolone (MP): group 1 - 25 patients starting therapy, control group 2 - 40 patients treated 3-6 months earlier. A nurse with experience for GO was engaged with the task to motivate patients in group1 to stop smoking. She used leaflets and poster with detailed information on negative effects of smoking on eyes in thyroid disease. During the 3 months iv MP treatment, the GO nurse discussed regularly with every patient in group 1 about the progress and problems. She advised patients on how to stop smoking on their own and tried to persuade them to visit clinics and counselling willing to prioritize the treatment of our patients. Group 2 was informed about the effects of smoking in a general manner before the treatment and remained from time to time.

Results: There were no differences between groups related to the age (48.88 \pm 9.14 vs $54.32 \pm 11.42$ ), years of smoking $(27.04 \pm 10.44$ vs $26.35 \pm 10.40)$ number of cigarettes consumed per day $(26.20 \pm 9.27$ vs $21.62 \pm 10.25)$, nor tobacco exposure expressed as pack years $(38.23 \pm 26.54$ vs $30.87 \pm 22.34)$. According to the questionnaire, there was no differencese between groups in the awareness of negative effects of smoking on GO. The main source of information was specialist, especially in tertiary clinic. Only $15 \%$ in group 1 and $17.5 \%$ in group 2 received information from general practitioners. There was no significant difference in the previous attempts to quit smoking due to eye disease (group 1, 52\%, group 2, 60\%); self-quitters in group $1-9 / 12$ and in group $2-16 / 18$ patients). The overall success in smoking cessation during the treatment of GO was in group $1-20 \%$, in group $2-17.5 \%$. There was a higher success rate in sustained smoking reduction in group $1-80 \%$ than in group $2-40 \%$. Only one patient in group 1 went to the group counselling, but did not stop smoking.

Conclusion: The success rate of dedicated motivation for smoking habits improvement in GO was disappointing regarding cessation. Taking into account a dose-dependent negative effects of smoking, reduction of cigarette number to less than five represents a limited success.

\section{P3-01-119}

APOPTOSIS AND PROLIFERATION OF THYROCYTES IN GRAVES‘ DISEASE PATIENTS

Anna Volkova, Svetlana Dora, Margarita Rybakova, Dmitry Alekseev,

Olga Dygun

Saint Petersburg, Russian Federation

Currently there is absence of some criteria which can make prognosis of Graves' disease duration and outcomes after surgery. Search of such criteria is very important for clinical practice and science. 
Objectives: To study markers of apoptosis and proliferation of thyrocytes in operated Graves; disease patients.

Methods: We included 24 patients with Graves' disease, which was diagnosed from 1970 up to 2010 ( 8 patients with postoperative hypothyroidism (HT), 8 thyrotoxicosis (TT), 8 - euthyroidism (ET). Patients were undergone surgery due to absence of remission. Levels of TSH, fr.T4, fr.T3, TSAb were measured by the immunoassay. Histological slices of thyroid gland samples were taken during surgery. The expression of apoptosis and proliferation proteins in thyrocytes (Ki-67, Bcl-2, Bax, Fas-L) was assessed by immunohistochemical analysis.

Results: In patients with postoperative TT expression of antiapoptotic protein Bcl-2 was $3.13 \pm 0,29$. It was significantly higher compared with expression in patients who had HT $(1.24 \pm 0,29(\mathrm{p}=0.001)$ and who had preserved ET $(1,36 \pm 0,25(\mathrm{p}=0.003)$. Positive correlation between the expression of antiapoptotic protein $\mathrm{Bcl}-2$ and the initial level of antibodies to the TSH receptor $(\mathrm{p}=0.000, \mathrm{R}=0,659)$ was revealed in patients with postoperative TT. This data has indicated greater baseline Grave's disease activity. The ratio of expression of Bcl-2/Bax was significantly higher in patients with TT - of 7.68 $\pm 3,80$, compared with HT group $-1,09 \pm 0,23(\mathrm{p}=0.007)$ and ET $-1,20 \pm 0,10)(\mathrm{p}=0.001)$. The expression of Fas-L was higher in patients with postoperative hypothyroidism $(\mathrm{p}=0.002$ ). The protein expression of proliferation Ki-67 was significantly higher in patients with postoperative TT 2,04 $\pm 0,40$ compared with the rate in patients with HT $0,31 \pm 0,11(\mathrm{p}=0.000)$ and ET $0,07 \pm 0,06(\mathrm{p}=0.001)$.

Conclusion: Clinical and morphological criteria in patients with recurrence of thyrotoxicosis after surgery treatment have showed significantly higher proliferative activity and inhibition of the process of apoptosis. Obtained data can predict postoperative prognosis in Grave's disease patients.

\section{P3-01-120 \\ EFFICACY OF RADIOTHERAPY IN THE TREATMENT OF MODERATE TO SEVERE GRAVES' ORBITOPATHY: A SINGLE CENTER EXPERIENCE}

Paolo Piero Limone ${ }^{1}$, Francesca Garino ${ }^{2}$, Maurilio Deandrea ${ }^{1}$, Annalisa Rossi $^{2}$, Lavinia Bianco ${ }^{2}$, Daniela Nassisi ${ }^{2}$, Marco Mellano ${ }^{2}$, Bruno Oldani ${ }^{2}$, Maria Grazia Ruo Redda ${ }^{2}$

${ }^{1}$ Department of Endocrinology, Diabetes and Metabolism, Ao

Mauriziano, Turin, Italy, ${ }^{2}$ Turin

Radiotherapy is used, alone or in combination with glucocorticoids, for treating moderate to severe Grave's orbitopathy (GO). We report a retrospective analysis of the patients treated at our institution from 2005 to 2013 .

Material and Methods: 69 patients ( 9 with monolateral, 60 with bilateral orbitopathy, median age 54, 24 F $45 \mathrm{M}$ ) were treated by RT. All patients had been previously treated by i.v. glucocorticoids, with incomplete response. All subjects underwent a complete ophtalmological evaluation, including assessment of the activity of the disease by CAS score (seven points scale) and of the severity by NOSPECS score both before RT and during follow-up (median 33 months). Most of patients were treated with a a dose of $19.8 \mathrm{~Gy}$ in 11 fractions with photons generated by a Linear Accelerator of 18 or $6 \mathrm{MeV}$ energy. The effects of the radiation treatment was assessed at the end of treatment and during the follow up visits. For statistical analysis Pearson chi-square test was used.

Results: Radiotherapy significantly improved inflammatory signs (Median basal CAS 4.5, at six months 3.0, p < 0.001). 2 and 4 NOSPECS categories significantly improved at six months $(p<0.003$ for class 2 and $p$ $<0.0001$ for class 4). $29.3 \%$ of the patients didn't report any change in diplopia, $53.1 \%$ a partial response and $17.6 \%$ a complete response; as a whole, $70.7 \%$ of the patients showed an improvement of muscular dysfunction. Proptosis was unchanged. Seven patients developed a cataract in the long-term follow-up. Eight patients showed conjunctivitis, 4 complained of photophobia, 2 of eye pain. The symptoms were controlled by low-dose oral steroids and topical drugs. No retinopathy was observed in the long-term follow-up.

Conclusions: Radiotherapy improves orbital inflammation and eye muscle dysfunction in moderate to severe GO, and could be beneficial in patients with incomplete response to steroids.

\section{P3-01-121 \\ EPIDEMIOLOGY AND LABORATORY FINDINGS \\ OF INFLAMMATORY BOWEL DISEASE AND IRRITABLE BOWEL SYNDROME IN PATIENTS WITH AUTOIMMUNE THYROIDITIS}

Leonidas Duntas ${ }^{1}$, Savvas Tanteles ${ }^{2}$, Sofia Tsaousidou ${ }^{3}$, Athanasios Tsakris $^{3}$

${ }^{1}$ Evgenideion Hospital, Univ.Athens, Unit of Endocrinology, Metabolism and Diabetes, Athens, Greece, ${ }^{2}$ Evgenideion Hospital, Department of Radiology, Ultrasound Section, University of Athens, Athens, Greece, ${ }^{3}$ Evgenideion Hospital, Unit of Medical Biopathology, Department of Microbiology, University of Athens, Athens, Greece

Background: The intestinal commensal microbiota have been associated with the development of autoimmune diseases, including inflammatory bowel disease (IBD), though a link to autoimmune thyroiditis (AIT) has not been established as yet. It was the aim of this study to examine the frequency of IBD and of irritable bowel syndrome (IBS) in patients with AIT.

Methods: From a large number $(\mathrm{n}=722)$ of consecutive patients attending the outpatient thyroid clinic, $89(12.3 \%)$ patients with AIT were selected based on the presence of autoimmune parameters and ultrasound criteria. Among these 89 patients, $16(17.9 \%)$ presented symptoms of IBS with lactose and gluten intolerance, while $5(5.6 \%)$ had a diagnosis of IBD including celiac disease and Crohn's disease. Serum concentrations of TSH, FT4, T3, TPOAb, $\mathrm{TgAb}, 25(\mathrm{OH}) \mathrm{D} 3$ and selenium (Se) were measured. A group of 102 non thyroid individuals served as control. The frequency of IBS/IBD was $12(10.8 \%)$ and $3(2.7 \%)$, respectively.

Results: There was no statistical difference for TSH $(5.3 \pm 1.2$ (Gr1) vs $5.8 \pm 1.2 \mathrm{mU} / \mathrm{L}$ ), thyroid hormones, TPOAb and TgAb between the IBS/IBD $(\mathrm{Gr} 2)$ and the AIT0 (Gr1) groups. Se $(74 \pm 19 \mu \mathrm{g} / \mathrm{L}$ vs. $66 \pm 12 \mu \mathrm{g} / \mathrm{L})$ and $25(\mathrm{OH}) \mathrm{D} 3(23 \pm 3.6 \mathrm{ng} / \mathrm{dl}$ vs. $15 \pm 2.4)$ were found to be lower in Gr2 but only Vit D3 reached the level of significance $(\mathrm{p}<0.05)$.

Conclusions: Both IBS and IBD seem to have a higher prevalence in AIT as compared to controls. The low Se and Vit D3 levels may indicate a pathogenetic substrate possibly aggravated by the inflammatory response to dysbiosis of microbiota.

\section{P3-01-122 \\ CLINICAL ASSOCIATIONS OF ANTITPO ANTIBODIES IN PATIENTS WITH NEWLY DIAGNOSED GRAVES DISEASE}

Mihail Boyanov ${ }^{1}, \underline{\text { Ralitsa Mekova }}{ }^{2}$, Deniz Bakalov ${ }^{3}$, Adelina Tsakova ${ }^{4}$ ${ }^{1}$ Medical University Sofia, Internal Medicine, University Hospital Alexandrovska, Clinic of Endocrinology and Metabolism, Department of Internal Medicine, Sofia, Bulgaria, ${ }^{2}$ Medical University Sofia, University Hospital Alexandrovska, Clinic of Endocrinology and Metabolism, Department of Internal Medicine, Sofia, Bulgaria, ${ }^{3}$ University Hospital Alexandrovska, Endocrinology Clinic, Medical University Sofia, Department of Internal Diseases, Medical University Sofia, Sofia, Bulgaria, ${ }^{4}$ Medical University Sofia, University Hospital Alexandrovska,, Department of Clinical Laboratory and Clinical Immunology, Sofia, Bulgaria

Anti-thyroid peroxidase antibodies (antiTPO) are often elevated in patients with Graves disease (GD) but their clinical significance is unclear.

Objectives: To examine the relationship between antiTPO levels and positivity and thyroid status and thyroid-associated ophthalmopathy (TAO) in patients with newly diagnosed GD.

Methods: 249 patients (191 women, 58 men) with newly diagnosed GD participated. Levels of TRAb, antiTg and antiTPO antibodies and thyroid hormones (TSH, FT4, FT3) were measured with third generation ECLIA assays. Thyroid ultrasound was performed, thyroid volume was calculated and presence of nodules was noted. Patients were evaluated for the presence of thyroidassociated ophtalmopathy (TAO) and its severity (according to NOSPECS). Smoking status, presence of concomittant autoimmune diseases and family history for thyroid disease were reviewed. Patients who had elevated levels of antiTPO were regarded as antiTPO+ and those with elevated levels of antiTG - as antiTg+.

Results: AntiTPO levels were negatively correlated with the patients' age and those who were antiTPO+ were younger ( 40 vs. 46.5 years, $\mathrm{p}=0.004$ ). 
There was a positive association between antiTPO positivity and their levels and the other thyroid antibodies, with highest levels observed in the group where all three antibodies were positive $(\mathrm{p}<0.005)$. AntiTPO+ patients had higher FT4 levels- 46.2 vs. $36.3 \mathrm{mU} / 1$ ( $\mathrm{p}=0.018)$. Thyroid volume correlated positively with antiTPO $(\mathrm{p}=0.004)$. AntiTPO + patients had higher thyroid volume than antiTPO- ( $20 \mathrm{vs} .16 \mathrm{ml}, \mathrm{p}=0.012)$. The prevalence of thyroid nodules was lower in TPO+ patients $(10.6 \%$ vs. $34.9 \%, \mathrm{p}<0.005)$ and it was negatively correlated with antiTPO levels $(\mathrm{p}<0.005)$. The prevalence of TAO was also lower in TPO+ patients $(34.2 \%$ vs. $52.9 \%, \mathrm{p}=0.004)$ and showed a negative association with antiTPO levels $(\mathrm{p}=0.009)$.

Conclusion: AntiTPO levels in newly diagnosed Graves disease were associated with higher thyroid volume, FT4 and antibodies levels and lower prevalence of TAO and thyroid nodules.

\section{P3-01-123 \\ CARDIOVASCULAR RISK FACTORS IN GRAVES، DISEASE ACCORDING TO THYROID FUNCTION AND AUTOIMMUNITY \\ Sofia Castro Oliveira ${ }^{1}$, Celestino Neves ${ }^{1}$, João Sérgio Neves ${ }^{1}$, Helena Greenfield ${ }^{2}$, Oksana Sokhatska ${ }^{3}$, César Esteves ${ }^{1}$, Miguel Pereira ${ }^{1}$, José Luís Medina ${ }^{2}$, Luís Delgado ${ }^{3}$, Davide Carvalho ${ }^{4}$ \\ ${ }^{1}$ Department of Endocrinology, Diabetes and Metabolism, São João \\ Hospital Center. Faculty of Medicine of Porto University, Porto, \\ Portugal, ${ }^{2}$ Faculty of Medicine of Porto University, Porto, Portugal, \\ ${ }^{3}$ Immunology Department, São João Hospital, Faculty of Medicine, University of Porto, Porto, Portugal, ${ }^{4}$ Department of Endocrinology, Diabetes and Metabolism, São João Hospital Center. Faculty of Medicine of Porto University, Institute for Research and Innovation in Health (I3s), Porto, Portugal}

Introduction: Thyroid hormones modulate the metabolism of the lipoproteins and glucose. The objective of our study was to evaluate the relationship between thyroid function, autoimmunity and the cardiovascular risk factors in Graves' disease.

Material and Methods: We performed a retrospective study, which included 126 patients with Graves' disease in their first cycle of treatment with metimazol. We evaluated thyroid function (FT3, FT4, TSH), autoimmunity (TRAB), and the levels of total cholesterol, LDL, HDL, triglycerides, apolipoprotein (apo) A1, apo B, lipoprotein $\mathrm{Lp}$ (a), reactive C protein (CRP), homocysteine, vitamin B12, folic acid, glucose and insulin with OGGT and markers of insulin resistance such as HOMA-IR (Homeostasis Model Assessment for Insulin Resistance). The patients were divided into a TRAB positive and a TRAB negative group and into groups with clinical hyperthyroidism, subclinical hyperthyroidism and normal thyroid functions. For the statistical analysis we employed Spearman correlation, t-tests and Mann-Whitney tests. Data are presented as mean \pm standard deviation or median (25-75th percentile) according to distribution.

Results: In the total group the mean age was $44.9 \pm 15.2$ years, with a predominance of the female sex $(92.9 \%)$. The mean body mass index was $26.0 \pm 4.7 \mathrm{~kg} / \mathrm{m} 2$. We observed a positive correlation between the levels of TSH and apo $\mathrm{B}(\mathrm{r}=0.236 ; \mathrm{p}=0.016)$ and a negative correlation between the levels of TRAB and apo B $(r=-0.211 ; \mathrm{p}=0.030$. The FT3 and FT4 levels were positively correlated with the fasting insulin levels $(\mathrm{r}=0.268 ; \mathrm{p}=0.008$ and $\mathrm{r}=0.226$; $\mathrm{p}=0.025$, respectively) and with HOMA-IR $(\mathrm{r}=0,258 ; \mathrm{p}=0,010$ and $\mathrm{r}=0,259$; $\mathrm{p}=0,010$, respectively). Compared to the normal subgroup, the group with clinical hyperthyroidism had significantly lower apo B levels ( $70.9 \pm 25.8$ vs. $89.8 \pm 24.0 \mathrm{mg} / \mathrm{dL} ; \mathrm{p}=0.007)$, and higher levels of HOMA-IR [2.09 (1.29-4.53) vs. $1.55(0.96-2.13) ; \mathrm{p}=0.023]$ and $\mathrm{CRP}[0.57(0.20-0.93)$ vs. $0.20(0.07-0.38)$ $\mathrm{mg} / \mathrm{L} ; \mathrm{p}=0.005]$. In the clinically hyperthyroid group we observed a negative correlation between FT4 and apo $\mathrm{B}(\mathrm{r}=-0.520 ; \mathrm{p}=0.039)$ and a positive correlation between TSH and HDL cholesterol $(\mathrm{r}=0.618 ; \mathrm{p}=0.004)$. Compared to the subgroup with negative TRAB, the positive TRAB subgroup had significantly lower levels of apo B $(80.3 \pm 23.9$ vs. $89.7 \pm 23.8 \mathrm{mg} / \mathrm{dL} ; \mathrm{p}=0.047)$ and $\mathrm{TSH}$ $[0.180(0.002-1.080)$ vs. $1.020(0.235-2.055) \mu \mathrm{UI} / \mathrm{mL} ; \mathrm{p}<0.001]$. In the TRAB positive group, the levels of FT3 and FT4 were positively correlated with the fasting insulin levels $(r=0.402 ; \mathrm{p}=0.008$ and $\mathrm{r}=0.368 ; \mathrm{p}=0.015$, respectively) and with HOMA-IR ( $r=0.396 ; p=0.009$ and $r=0.388 ; p=0.010$, respectively).

Conclusion: In Graves' disease the relationships between thyroid function, autoimmunity, insulin resistance and lipid profile may contribute to increased cardiovascular risk.

\section{P3-02 Cancer Case Reports}

\section{P3-02-124 \\ ONSET, PROGRESSION AND MANAGEMENT OF GRAVES، ORBITOPATHY WITH COEXISTENT PAPILLARY THYROID CARCINOMA}

Roussanka Kovatcheva ${ }^{1}$, Mariya Stoynova ${ }^{2}$, Jordan Vlahov ${ }^{2}$, Alexander Shinkov ${ }^{2}$, Ralica Ivanova ${ }^{2}$, Radina Ivanova ${ }^{2}$, Tanjo Sechanov', Tatjana Hadjieva $^{3}$

${ }^{1}$ Medical University of Sofia, University Hospital of Endocrinology,

Clinical Center of Endocrinology, Sofia, Bulgaria, ${ }^{2}$ Medical University of Sofia, University Hospital of Endocrinology, Sofia, Bulgaria, ${ }^{3}$ City Clinic

- Sofia, Bulgaria

Introduction: Graves' Orbitopathy (GO) is one of the characteristic features of Graves' disease and it usually develops about the time of diagnosis of hyperthyroidism. The management of its moderate-to-severe forms requires a multidisciplinary approach in order to achieve optimal results. Radioiodinetherapy (RAIT) is a known risk factor for worsening of pre-existing GO or even for new-onset of ocular symptoms.

Papillary thyroid carcinoma (PTC) is the most common thyroid cancer. Its management includes surgery followed by RAIT and levothyroxine suppressive therapy in high-risk cases.

The combination of GO and PTC poses additional therapeutic challenges.

Case Report: We present a case of a 41-year-old female with Graves' disease and concurrent PTC who underwent thyroidectomy. Three months after surgery, RAIT with prior thyroid hormone withdrawal (THW) was performed due to large thyroid remnants. Two months later, moderate-to-severe GO was diagnosed. Administration of intravenous glucocorticoids led to incomplete effect with persistent activity of GO. As a second therapeutic option, orbital radiotherapy was performed with significant improvement of the clinical symptoms and transition of GO into a stable inactive state.

One year after the first RAIT, a second one was considered because of persistent thyroid remnants. It was performed with rhTSH-stimulation instead of THW in order to minimize the risk of worsening of GO. A year after the second RAIT, there was no evidence of 131I-uptake on diagnostic whole-body scan.

GO remained inactive for approximately two years after orbital radiotherapy. In the subsequent years, there were two exacerbations of the ocular symptoms provoked by psychological stress and viral infection, which necessitated an additional glucocorticoid treatment.

Conclusion: The management of GO in the postoperative period of PTC is difficult and needs a complex approach. The use of rhTSH-stimulation is more appropriate than THW in order to prevent an exacerbation of GO symptoms.

\section{P3-02-125 \\ CLINICAL, RADIOLOGICAL AND HISTOLOGICAL CHARACTERISTICS OF SIX ANAPLASTIC THYROID TUMOURS}

Catarina Roque ${ }^{1}$, Catarina Saraiva ${ }^{1}$, Clotilde Limbert ${ }^{1}$, Marlene

Carriço $^{1}$, Carlos Vasconcelos ${ }^{1}$

${ }^{1}$ Endocrinology Department Hem Chlo, Lisbon, Portugal

Objectives: Investigate clinical, radiological and histological findings of anaplastic carcinomas (AC) over a 4-year period.

Methods: All FNAC from 1/01/2013-1/01/2017 were retrospectively investigated. Patients with histological confirmation of AC were included. Clinical files provided age, sex, symptoms, signs, thyroid function, autoimmunity and stage. Ultrasound, tomography scans, histological and surgical reports were evaluated.

Results: Among 4928 FNAC, 6 were histologically proven AC. Femaleto-male ratio was 2:1 and the age range 56-87 years. Hypothyroidism and subclinical-hyperthyroidism were each reported once, the remainder were euthyroid. None had demonstrable autoimmunity. All patients presented a previously undiagnosed goitre with local symptoms, except for one. Stony-hard consistency was described in 4/6, fixation in $2 / 6$ and lymphadenopathy in $1 / 6$. Dysphagia was the commonest complaint (4/6), followed by dysphonia $(2 / 6)$ or pain (2/6) with a progression of 1-4 months. Most glands were multinodular 
(4/6). The aspirated nodes had 47-96mm, were hypoechoic and heterogeneous, 3 had cystic degeneration, 3 were exophytic, 2 showed macrocalcifications. Homolateral adenopathy and extrathyroidal invasion was reported in 3 US. On contrasted-CT all patients had extrathyroidal extension and lymphadenopathy. Compression of respiratory and digestive structures was observed in 3/6 and 4 had displacement of the vascular sheath. All patients had thoracic involvement, by mediastinal lymphadenopathy (3/6) and/or pulmonary nodules (4/6). Stage IVc was common (4/6), followed by IVb (2/6). These 2 patients had poorlydifferentiated components in their tumours. The most dominant subtype was giant-cell, followed by spindle cell. The most extensively disseminated case had a sarcomatoid variant with osteoclast-like cells and immunohistochemistry positivity for desmin+AML+vimentin.

Conclusions: The rare AC may show characteristic clinical and radiological features, enough to suspect the diagnosis even when FNAC result is "malignant" but not suggestive of AC. CT shows clear superiority to US on the detection of local and distant disease, making it indispensable in preoperative evaluation of suspicious cases.

\section{P3-02-126 \\ RECTAL ADENOCARCINOMA METASTASIZING AS A BULKY THYROID TUMOR \\ Gherald Bermudez $^{1}$ \\ ${ }^{1}$ University of the Philippines - Philippine General Hospital, Manila, \\ Philippines}

Metastatic lesions to the thyroid gland are rarely reported. The thyroid gland is an infrequent site of metastasis of primary malignant tumors which represents $2-3 \%$ of all malignant tumors of the thyroid gland. Malignant melanoma, carcinomas of the breast, lung, and kidney frequently metastasize to the thyroid. Rectal adenocarcinomas also metastasize to the thyroid gland although these are rarely seen especially if they present as bulky neck mass lesions.

Here, we present a 53-year old male admitted at our institution because of worsening dyspnea and dysphagia. He has been diagnosed with rectal adenocarcinoma stage IIIB in 2003, underwent colorectal surgery and chemoradiotherapy. A pea-sized anterior neck mass was noted in 2008 and cytology showed a benign process; however, it abruptly increased in size six months prior to admission. Physical examination showed a $16 \times 6 \times 4 \mathrm{~cm}$ anterior neck mass with cervical lymphadenopathies. Neck ultrasound revealed a diffusely enlarged thyroid gland with calcifications. Computed tomography of the neck and chest showed thyroid masses with aggressive features with laryngeal, tracheal, and esophageal invasion, cervical lymphadenopathy, and bilateral pulmonary nodules. Thyroid functions test showed hypothyroidism. Levothyroxine was started. Intubation was initially done to secure airway. Tumor debulking and tracheostomy tube insertion were done subsequently. Histopathology revealed adenocarcinoma metastatic to the thyroid, strap muscles, and trachea which was consistent with a diagnosis of metastasis from the primary rectal adenocarcinoma. He was discharged without complications seven days later.

We presented a rare case of rectal adenocarcinoma metastasizing as a bulky thyroid tumor, a possibility that should be always be considered whenever thyroid changes are seen in a patient with a history of carcinoma elsewhere. In any patient with a known history of previous carcinoma, the appearance of a new thyroid mass should be suspected of potential metastasis until proven otherwise.

\section{P3-02-127 \\ THYROGLOBULIN ANTIBODIES - A TUMOUR MARKER? CLINICAL CASE}

Cristina Ribeiro ${ }^{1}$, Gracinda Costa ${ }^{2}$, Miguel Melo ${ }^{3}$, Joana Saraiva ${ }^{4}$, Diana Catarino ${ }^{2}$, Francisco Carrilho ${ }^{2}$

${ }^{1}$ Endocrinology University Hospital, Coimbra, Portugal, ${ }^{2}$ Coimbra,

Portugal, ${ }^{3}$ Instituto de Investigação e Inovação Em Saúde (I3s),

Institute of Pathology and Immunology of the University of Porto

(Ipatimup), Department of Endocrinology, Diabetes and Metabolism,

Centro Hospitalar e Universitário de Coimbra, Coimbra, Portugal,

${ }^{4}$ University and Hospital Center of Coimbra, Faculty of Medicine,

University of Coimbra, Coimbra, Portugal

Introduction: Thyroglobulin ( $\mathrm{Tg}$ ) is a specific and sensitive tumor marker of differentiated thyroid cancer Thyroglobulin Antibodies (TgAb) negative. The presence of TgAb limits this usefulness of Tg. We'll present a clinical case documenting the utility of $\mathrm{TgAb}$ as a tumor marker in these situations.

Clinical Case: A woman, 59 years, submitted to total thyroidectomy in 2000 with "multicentric papillary cancer, with high cells", followed by I131 ablation. Afterwards, $\mathrm{Tg}$ remained undetectable $(<0,2 \mathrm{ng} / \mathrm{ml})$, but $\mathrm{TgAb}$ positive $(22-743 \mathrm{UI} / \mathrm{ml})$. In 2005, limphnode cervical methastases were excised. From 2005 till 2013, Tg and TgAb became undetectable. After 2013, TgAb levels were progressively higher: $21-48-84 \mathrm{UI} / \mathrm{ml}$ with undetectable $\mathrm{Tg}(<0,04$ $\mathrm{ng} / \mathrm{ml})$. In june 2015, I13 (136mCi) was administrated with "slight cervical fixation". In february 2017 , after $\mathrm{TgAb}-773 \mathrm{UI} / \mathrm{ml}$, she performed 18 FDGPET: "Bilateral hypermetabolic pulmonary methastases".

Discussion: This clinical case demonstrates both the interference of $\mathrm{TgAb}$ in Tg measurement and their clinical utility as a tumor marker in differentiated thyroid carcinoma.

\section{P3-02-128 UNDIFFERENTIATED HIGH GRADE PLEOMORPHIC SARCOMA OF THYROID GLAND - CASE REPORT}

\section{Djordjije Krnjevic $^{1}$, Sanja Borozan ${ }^{1}$, Ljiljana Vuckovic ${ }^{1}$}

${ }^{1}$ Clinical Center of Montenegro, Podgorica, Montenegro

Introduction: Thyroid sarcoma as the most lethal thyroid cancers represents less than $1 \%$ of all thyroid tumors. It's characterized by severe local course, progressive metastatic dissemination and very poor prognosis (mean survival rate beneath 6 months).

Case Report: The patient is a 61 year old female with no prior medical history.

Total thyroidectomy was done in July 2016 due to the presence of suspected malignant, calcified knot in the right lobe of the thyroid gland, size of $23 \times 20 \mathrm{~mm}$. Pathohistological findings indicated most probable Fibrohistiocytoma malignum, moderate degree of atypia, Ng: II, mitotic 8/10HPF (Vimentin+, Actin +/-), Ki67 45\%. Possibility of anaplastic carcinoma has been taken into consideration as differential diagnosis.Substitution treatment $-100 \mathrm{mcg}$ of L-thyroxine. PH revision made in Belgrade, confirmed Undifferentiated high grade pleomorphic sarcoma (Desmin focal+/-, SMA +/-, Vimentin+, Actin focal+, Ki67 75\%). Postoperative CT neck scan showed no signs of local recurrence, except several submandibulary lymphatics size of $11 \mathrm{~mm}$. FNAB of lymphatics and PH examination showed no atypia. Chest $\mathrm{X}$-rays and brain CT scan showed no pathological changes. The patient was presented to Consilium for sarcomas, who decided to perform a post-operative radiotherapy. 50Gy/25 of radiotherapy was made. In February 2017, neck and thorax CT scan showed micronodular changes in pulmonary parenchyma, suspected metastases. Bronchoscopy exam showed no pathological changes. Open lung biopsy is planned.

Conclusion: We presented a case of a high grade undifferentiated thyroid gland sarcoma with probable metastatic appearances in lung tissue where metastases are most often located (80\%).

At the beginning we had to make a clear distinction between sarcoma and anaplastic carcinoma as usually confused with. Although it's a rare kind of tumor, physicians should be aware of this type of thyroid malignancy and understand difficulties in making a definitive diagnosis. 


\section{P3-02-129 \\ BONE METASTASES IN PAPILLARY THYROID MICROCARCINOMA: AN UNUSUAL CASE}

Joana Saraiva ${ }^{1}$, Cristina Ribeiro ${ }^{2}$, Miguel Melo $^{1}$, Lúcia Fadiga ${ }^{2}$, Gracinda Costa ${ }^{1}$, Francisco Carrilho ${ }^{2}$

${ }^{1}$ University and Hospital Center of Coimbra; Faculty of Medicine, University of Coimbra, Portugal, ${ }^{2}$ University and Hospital Center of Coimbra, Portugal

Introduction: Most papillary microcarcinomas have an indolent course with high survival rates. Distant metastases are exceedingly rare $(<1 \%)$. Bone metastases when occur are usually osteolytic and radiodine therapy is rarely curative.

Case Report: We present the case of a 78-year-old woman who underwent total thyroidectomy in 2010 for a bifocal papillary thyroid microcarcinoma (1200micra and 910micra)-T1aNxMx. After surgery: TSH 1.9uUI/ $\mathrm{mL}(0.4-4)$, fT4 0.9ng/dlL (0.8-1.9), thyroglobulin (Tg) $9.2 \mathrm{ng} / \mathrm{mL}$ and negative anti-Tg antibodies (TgAbs). Radioidine was not given. Patient maintain follow-up in her medical assistant during 5 years. In 2016 she was sent to our Thyroid Oncology clinic because of thyroid remnant of $13 \mathrm{~mm}$ in neck ultrasonography, with no cervical adenopathies. Analitically: TSH $2.5 \mathrm{uUI} / \mathrm{mL}$, fT4 $1.5 \mathrm{ng} / \mathrm{dL}, \mathrm{Tg} 2621 \mathrm{ng} / \mathrm{dl}$ and negative TgAbs. She was submitted to fine needle aspiration of hypoecogenic nodular area in the remaining right thyroid lobe with $1.6 \times 0.8 \mathrm{~cm}$ which showed no evidence of papillary carcinoma. In February 2016, after recombinant TSH, she was submitted to 18F-FDG PET/ $\mathrm{CT}$ and radiodine therapy $(71 \mathrm{mCi})$. The post-therapy whole body scan (WBS) and 18F-FDG-PET/CT showed four bone metastases (skull, D6, L5 and left iliac spine) that, despite increased glycolytic metabolism, maintain some iodine uptake. Subsequently she received two more radioiodine treatments in $07 / 2016(191 \mathrm{mCi})$ and $01 / 2017(167 \mathrm{mCi})$. Last post-therapy WBS showed that the bone lesions preserved their radiodine uptake capacity but with less uptake, reduced size and with significant increase in mineral density. Tg levels progressively reduced to $641 \mathrm{ng} / \mathrm{ml}$ in last evaluation.

Conclusion: This case indicated that, despite the excellent prognosis, papillary microcarcinomas need to maintain proper follow-up. Although bone metastases traditionally have a poorer response to radioidine our patient has presented a good response and more treatments will probably be performed.

\section{P3-02-130 \\ MULTIPLE ENDOCRINE NEOPLASIA TYPE 1 ASSOCIATED WITH PAPILLARY THYROID CARCINOMA: A CASE REPORT}

Sanja Borozan ${ }^{1}$, Snezana Vujosevic ${ }^{2}$, Sanja Čejović ${ }^{2}$ Svetozar Damjanović ${ }^{3}$

${ }^{1}$ Podgorica, Montenegro, ${ }^{2}$ Podgorica, ${ }^{3}$ Belgrade

Introduction: Multiple endocrine neoplasia type 1 (MEN1) is a rare, hereditary syndrome characterized by a combination of anterior pituitary, parathyroid and pancreatic islet cell tumors. The protein encoded by the MEN1 gene, menin, acts as a tumor supressor and is ubiquitously expressed in endocrine tissue. Papillary thyroid cancer (PTC) is the most common form of well-differentiated thyroid cancer. Until present, only several cases of PTC combined with MEN1 are reported.

Case Report: A 59-year-old-female was reffered to endocrinologist because of persistent pain in neck, shoulders and lower legs accompanied with fatique and increased palmar sweating. She noticed enlargement of hands and increased shoe size in last year. Her past medical history included partial thyroidectomy with right inferior parathyroidectomy because of $2.5 \mathrm{~cm}$ parathyroid adenoma in 2011. Four years later, total thyroidectomy was performed and histopathology revealed PTC, follicular variant, $1.1 \times 0.8 \mathrm{~cm}$ without spreading into nearby tissue. No prior exposure to radiation was reported. On current admission, the replacement therapy with levothyroxine was adequate (TSH $0.2 \mathrm{mIU} / 1$ ), primary hyperparathyroidism (PHPT) persisted (PTH 12.5 $\mathrm{pmol} / \mathrm{l}$, serum calcium up to $3.0 \mathrm{mmol} / \mathrm{l}$ ) and hyperprolactinemia was detected (prolactine values in daily profile: 788.8..719.8...775.6 mIU/1). Magnetic resonance imaging showed a 17 x $13 \mathrm{~mm}$ sized pituitary mass with infrasellar propagation. According to all findings, the patient underwent transsphenoidal surgery for pituitary adenoma and repeated paratyroid exploration that revealed adenoma at the previously surgically treated site. Genetic testing

Poster Presentations was performed on the MEN1 gene which indicated a mutation of gene which encodes menin but detailed analysis is yet to be fulfilled.

Conclusion: Our case showed a patient with PTC and MEN1. Further examination is needed to clarify whether this is incidental occurrence or there is possible existence of common genetic pathways in tumorogenesis.

\section{P3-03 Cancer Treatment}

\section{P3-03-131 \\ OPTIMAL TIMING OF THE START OF TYROSINE KINASE INHIBITOR TREATMENT FOR RADIOACTIVE IODINE THERAPY REFRACTORY, PROGRESSIVE, RECURRENT DIFFERENTIATED THYROID CANCER PATIENTS}

Kiminori Sugino ${ }^{1}$, Mitsuji Nagahama², Wataru Kitagawa ${ }^{2}$, Keiko Ohkuwa $^{2}$, Takashi Uruno ${ }^{2}$, Kennich Matsuzu², Akifumi Suzuki², Chie Masaki², Chisato Tomoda², Junko Akaishi², Kiyomi Hames ${ }^{2}$, Yuna Ohgimi $^{2}$, Koichi Ito ${ }^{2}$

${ }^{1}$ Ito Hospital, Department of Surgery, Tokyo, Japan, ${ }^{2}$ Ito Hospital, Tokyo, Japan

Background: New insights in thyroid cancer biology propelled the development of targeted therapies for RAI- refractory differentiated thyroid cancer (RF-DTC) as salvage treatment, and the tyrosine kinase inhibitor lenvatinib has become available as a new line of therapy for RF-DTC. The aim of this study was to investigate the optimal timing of the start of lenvatinib treatment.

Methods: Between May 2015 and August 2016, 26 patients with progressive, RF-DTC, 7 males and 19 females, median age 66 years, were treated with lenvatinib and they were the subjects of this study. Fourteen patients were started on lenvatinib before developing any symptom (Group A), and 12 patients were started on lenvatinib after developing a symptom (Group B). Efficacy was evaluated on the basis of RECIST ver. 1.1. Progression free survival (PFS) and overall survival (OS) were calculated and evaluated by Kaplan-Meir methods and the log-rank test. Multivariate analyses to identify risk factors related to PFS and OS were performed by using Cox's proportional hazard models.

Results: Median duration of lenvatinib treatment was 11.3 months and median drug intensity was $13.1 \mathrm{mg}$. To date, a partial response (PR), stable disease (SD), and progressive disease (PD) have been observed in 13 patients $(50 \%), 5$ patients $(19.2 \%)$, and 8 patients $(30.8 \%)$ patients, respectively. Seven patients have died of the disease (DOD). A PR, SD, PD and DOD have been observed in $9(64.3 \%), 4(38.5 \%), 1(7.1 \%)$ and $1(7.1 \%)$, respectively, in Group A, and in $4(33.3 \%), 1(8.3 \%), 7(58.3 \%)$, and $6(50 \%)$, respectively, in Group B. Multivariate analyses showed that the presence of symptom was a significant factor related to PFS and OS

Conclusion: Although the number of subjects was small, we concluded that sufficient clinical benefit will be achieved by starting lenvatinib treatment just before any symptoms appear.

\section{P3-03-132 \\ CANCER IMMUNOTHERAPY, A “CRO'S" PERSPECTIVE}

Jaikrishna Balkissoon ${ }^{1}$, Panteli Theocharous ${ }^{2}$, Austin Combest ${ }^{3}$, Richard Tran ${ }^{4}$, Dirk Reitsma ${ }^{5}$, Nikolay Stoyanov ${ }^{6}$, Binh Nguyen $^{5}$ ${ }^{1}$ Bay Area Tumor Institute, Piedmont, CA, USA, ${ }^{2} \mathrm{Ppd}$, Cambridge, UK, ${ }^{3}$ Ppd, Wilmington, NC, USA, ${ }^{4}$ Wilmington, NC, USA, ${ }^{5}$ Rockville, MD, USA, ${ }^{6}$ Sofia, Bulgaria

One of the advantages of being a large global contract research organization (CRO) that has a major focus in immuno-Oncology (IO) is the unique opportunity to see first hand the numerous IO novel agents being developed as monotherapy or as combination therapy by different sponsors using different trial designs, endpoints and other surrogates to assess clinical benefit. Given PPDs significant experience in the IO space working with numerous sponsors, it is important to share this unique experience. We are currently collaborating with sponsors to create more efficient adaptive trial designs in 
early phase development that study multiple novel agents and increase the efficiency of clinical trials. At PPD we have consolidated our vast IO experience into the PPD IO center of excellence (IOCOE). This focused therapeutic area allows us to evaluate promising IO agents and also understand the difficult challenges that can be associated with developing these IO agents in addition to leveraging all efficiencies associated with their development. Our IO center of excellence has been a good resource for sponsors in developing their IO agents. With 1 IO approval in 2010 to 21 approvals including both US and EU from 2015 to 2017 is clearly an amazing feat. This success was based on better understanding of the tumor microenvironment, mechanisms of resistance, including indications with an unmet need and more active IO agents with increased responses that have been durable. We are also seeing increased breakthrough designations, more priority reviews and more accelerated approvals in the IO space. We are seeing multiple new drug approvals and label expansions across the U.S. and Europe which are benefiting thousands of patients according to currently-approved labels. What more can we expect in 2017 and 2018? We will discuss a "CROs" perspective of immuno-Oncology in 2017 and beyond.

\section{P3-03-133 \\ LENVATINIB-THERAPY FOR PATIENTS WITH RADIOIODINE-REFRACTORY DIFFERENTIATED THYROID CANCER - OUR EXPERIENCES WITH LENVIMA® AT THE DEPARTMENT OF NUCLEAR MEDICINE AND ENDOCRINOLOGY IN KLAGENFURT, AUSTRIA}

Stefan Sorko ${ }^{1}$, Hans-Juergen Gallowitsch ${ }^{1}$, Susanne Kohlfuerst ${ }^{1}$, Sabine Matschnig ${ }^{1}$, Manfred Sorschag ${ }^{1}$, Michael Rieser $^{1}$, Peter Lind $^{1}$ ${ }^{1}$ Klinikum Klagenfurt, Department of Nuclear Medicine and

Endocrinology, Pet/Ct Center, Klagenfurt, Austria

Objectives: For many years therapy-options for patients with radioiodinerefractory differentiated thyroid cancer were limited, but in the last decade enormous progress was made in the management of RAI-refractory DTC.

The improved understanding of the molecular alterations underlying RAIrefractory DTC has boosted the interest in developing new targeted-treatment therapies - e.g. Lenvatinib, a multi-targeted receptor tyrosine kinase inhibitor.

Methods: After the approval of Lenvima ${ }^{\circledR}$ in Austria in summer 2015 we started using this TKI at our department for the treatment of patients with RAI-refractory DTC. Until March 201710 patients [30\% papillary DTC, $50 \%$ follicular DTC and $20 \%$ poorly differentiated thyroid cancer], treated with Lenvima ${ }^{\circledR}$, were followed up for a median period of 12,8 months.

We focused our attention on the frequency and degree [according to the CTCAE] of side effects, the necessity of dose reduction and the variation of the thyroglobulin value under the TKI-therapy. Therefore continuous laboratory checks were performed, including follow up PET/CTs.

Results: Dose reductions because of side-effects caused by Lenvima ${ }^{\circledR}$ were necessary in $100 \%$ of our cases. On average the first reduction was performed after 37,5 days. The final "excepted" dose was reached after 117,7 days.

Grade 3 treatment-related adverse events like hypertension (50\%), appetite loss $(20 \%)$, decreased weight $(20 \%)$ and proteinuria $(10 \%)$ occurred in $80 \%$ of our Lenvima ${ }^{\circledR}$ - recipients. Because of uncontrollable side effects Lenvima ${ }^{\circledR}$-therapy was stopped in one case.

Compared with the baseline value the three months TG-value has dropped on average about $85 \%$ [-96,8\% to $-54 \%]$. This observation correlated with the $\mathrm{PET} / \mathrm{CT}$ findings.

Conclusion: Lenvima ${ }^{\circledR}$ is an effective drug for patients with RAIrefractory DTC, but we noticed severe side effects in $80 \%$ of our patients. In most cases a dose reduction could reduce these side effects.

Patients responding to Lenvima ${ }^{\circledR}$ showed a makeable decline of the TG-value.

\section{P3-03-134 \\ LOW AND INTERMEDIATE RISK DIFFERENTIATED THYROID CARCINOMA TREATED WITH TOTAL THYROIDECTOMY OR LOBECTOMY: EVALUATION OF THE DISEASE OUTCOME}

Carla Colombo ${ }^{1}$, Simone de Leo $^{1}$, michela perrino ${ }^{2}$, Leonardo Vicentini $^{3}$, Laura Fugazzola ${ }^{4}$

${ }^{1}$ Division of Endocrine and Metabolic Diseases, Istituto Auxologico Italiano Irccs, Milan, Italy, Milan, Italy, ${ }^{2}$ Department of Clinical Sciences and Community Health, University of Milan, Endocrine Unit, Fondazione Irccs Ca' Granda, Milan, Italy, Milan, Italy, ${ }^{3}$ Endocrine Surgery Unit, Fondazione Irccs Ca' Granda-Ospedale Maggiore Policlinico, Milan, Italy., Milan, Italy, ${ }^{4}$ Dept. of Pathophysiology and Transpl, Istituto Auxologico Italiano Irccs, University of Milan, Milano, Italy

Background: An individualized risk-based approach to the treatment of differentiated thyroid cancers (DTCs) is being extensively discussed in the recent literature, but controversies remain about the ideal surgical approach of less aggressive cases.

Methods: We evaluated retrospectively 358 patients affected with DTC (mean age 48 yrs, 288 females) and followed up at a single Institution. According to ATA guidelines, 321/358 were in the low, and 37/358 in the intermediate risk classes. The first surgical treatment was not chosen based on the risk class, but on surgeon's and patient's choice: 318 (291 low, and 27 intermediate risk) were treated with total thyroidectomy (TT) and 40 (30 low, and 10 intermediate risk) with lobectomy (LT). Remission or persistent/recurrent disease was defined on the basis of biochemical and/or structural evidence of disease after a median follow-up of 65 months.

Results: Only $2 / 318$ patients treated with TT needed additional therapy, while $15 / 40$ patients treated with LT were submitted to following treatments. As far as the outcome concerns, remission was documented in 298/318 cases $(94 \%)$ and $31 / 40$ cases $(78 \%)$ in the TT and LT groups, respectively $(\mathrm{P}=0.0004)$. In low-risk tumors the prevalence of persistent or recurrent disease was not significantly different in patients submitted to TT or LT ( $\mathrm{P}=0.09)$; conversely intermediate risk tumors submitted to LT had a poorer outcome than cases treated with TT $(\mathrm{P}=0.028)$. The frequency of post-surgical complications (especially the recurrent laryngeal nerve lesion) was significantly higher in patients treated with TT compared to patients treated with LT $(\mathrm{P}=0.0001)$.

Conclusions: Our data show the excellent outcome of low risk DTCs treated by either TT or LT, suggesting that a conservative treatment should be always adopted in these cases. On the other hand, despite the higher frequency of post-surgical complications, TT should be favored in intermediate risk tumors, in order to avoid retreatment.

\section{P3-03-135 \\ TYROKISE KINASE INHIBITORS IN ADVANCED THYROID CANCER - EXPLORATORY ANALYSIS OF A CENTER EXPERIENCE}

Diana Martins ${ }^{1}$, Joana Saraiva ${ }^{1}$, Cristina Ribeiro ${ }^{1}$, Miguel Melo ${ }^{1}$, Diana

Oliveira', Adriana Lages ${ }^{1}$, Mara Ventura ${ }^{1}$, Nelson Cunha ${ }^{1}$, Lúcia

Fadiga $^{1}$, Diana Catarino ${ }^{1}$, Francisco Carrilho ${ }^{1}$

${ }^{1}$ Centro Hospitalar e Universitário de Coimbra, Coimbra, Portugal

Background: Tyrosine kinase inhibitors (TKIs) are an established therapeutic option for patients with advanced thyroid cancer (ATC), with documented efficacy and prolonged PFS in this setting. However, class-related adverse effects may affect quality of life and limit the clinical usage.

Objective: Investigate the efficacy and toxicity of TKIs administered outside clinical trials in patients with ATC.

Methods: Retrospective analysis of a cohort of patients with ATC. Primary endpoints were progression-free survival (PFS) and overall survival (OS) at 2 years of follow-up. Drug withdrawal and dosage reduction caused by drug toxicity were also reported.

Results: We identified a small sample of 8 patients with ATC selected for TKIs therapy in our Center; five women and three men, median age of diagnosis $47 \mathrm{yr}$ (range, $14-70 \mathrm{yr}$ ). Five patients $(62.5 \%)$ presented follicular thyroid 
carcinomas, one papillary and two had medullary thyroid carcinomas. Seven patients were treated with sorafenib and one with vandetanib on first line therapy; one patient initiated lenvatinib on second line therapy. Median time of treatment was approximately 17 months. Most frequent toxicities observed were hand-foot syndrome $(50 \%, n=4)$ and diarrhea $(37.5 \%, n=3)$; two patients withdrawn treatment for adverse events and other two were subject to dose reductions. Median PFS was 24 months, with median OS at 5 yr of follow-up of $88 \%$

Conclusions: TKIs as sorafenib and vandetanib appear to be effective on ATC patients (median PFS of 24 months). Additionally, observed toxicities were acceptable in the majority of patients, with only two cases of drug discontinuation required, assuring the level of evidence of this therapeutic option.

\section{P3-03-136 \\ TREATMENT MODALITIES OF PATIENTS FOLLOWED-UP FOR RADIOIODINE (RAI) REFRACTORY DIFFERENTIATED THYROID CANCER (DTC) IN A REFERRAL CENTRE IN GREECE}

Elli Anagnostou ${ }^{1}$, Katerina Saltiki ${ }^{2}$, George Simeakis ${ }^{3}$, Alexios Travlos ${ }^{1}$,

Paraskevi Kazakou ${ }^{4}$, Maria Pissia $^{1}$, Maria Alevizaki ${ }^{5}$

${ }^{1}$ Endocrine Unit, Dept Medical Therapeutics, Alexandra Hospital,

Athens University School of Medicine, Athens, Greece, ${ }^{2}$ Clinical

Therapeutics, Athens, Athens, Greece, ${ }^{3}$ Endocrine Unit, Dept Medical

Therapeutics, Alexandra Hospital, Athens University School of

Medicine, Endocrinology Dept, Alexandra Hospital, Athens, Athens,

Greece, ${ }^{4}$ General Hospital of Alexandra, Athens, Greece, ${ }^{5}$ Endocrine

Unit, Alexandra Hospital, Endocrine Unit, Athens University, Athens,

Greece

Objectives:Few DTC cases do not respond to RAI and may develop metastatic disease. Additional treatments such as locoregional interventions or tyrosine-kinase inhibitors (TKIs) may then be required.

Methods:Of 1350 DTC patients followed-up in our department, $173(12.8 \%)$ presented disease persistence during follow-up. $49(28.3 \%)$ of them with RAI-refractory-DTC were analyzed.

Results:42/49 had distant metastases and 23/49 persistent cervical disease needing multiple surgeries. The patients were classified in two groups, G1: patients who underwent locoregional treatments $(n=38,77.5 \%)$ and $G 2$ : patients who were administered systemic therapy (sorafenib, lenvatinib) because of rapidly progressive disease $(n=11,22.4 \%)$. Distant metastases were present in 33/38 of G1 and 10/11 of G2; cervical disease persistence in 17/38 of G1 (12 underwent multiple surgeries, 11 additional external-radiation) and 6/11 of G2 (6 multiple surgeries, 4 additional external-radiation). During follow-up and before the initiation of systemic therapy, disease progression occurred in $89.5 \%$ of G1 and $100 \%$ of G2. The time to progression of structural and biochemical disease was for G1: $19.9 \pm 28.4$ and $17.1 \pm 10.8$, for G2: $7.6 \pm 2.5$ and $6.8 \pm 3.2$ months respectively. 12 of G1 patients died. Mean age of $\mathrm{G} 2$ at sorafenib initiation was $58.7 \pm 14.3$ years; metastases location was mediastinum $(n=2)$, lungs $(n=10)$, liver $(n=1)$, bones $(n=3)$. Mean follow-up period under sorafenib treatment was $15.3 \pm 9.3$ months $(0.5-30)$. Structural partialresponse occurred in 3 (monitoring-interval $22.3 \pm 7.5$ months), stabilization in 3 (monitoring-interval $10.6 \pm 6.8$ months), progression in 5 patients(monitoringinterval $13.9 \pm 10.5$ months). Two died, one of another malignancy. Adverseevents (hand-foot syndrome $n=8$ patients, arterial hypertension $n=4$, alopecia $\mathrm{n}=3$, gastrointenstinal events $\mathrm{n}=4$, anorexia $\mathrm{n}=1$ ) and dose modifications (dosereduction $n=2$, interruption $n=1$ ) were recorded. Three with resistance to sorafenib were treated with lenvantinib. One died (disease progression), one showed partial response and one stable disease (follow-up 3-6 months).

Conclusions: Locoregional treatments may offer disease stabilization in $35 \%$ of RAI-refractory-DTC patients. Treatment with TKIs (sorafenib or the newer-agent lenvatinib) may further result in stabilization in approximately $50 \%$ of patients with rapidly progressive disease.

\section{P3-03-137 \\ CENTRAL NECK DISSECTION CANNOT BE AVOIDED IN MEDULLARY THYROID CANCER (MTC) ON THE BASIS OF PRESURGICAL SERUM CALCITONIN (CT)}

Virginia Cappagli ${ }^{1}$, Sonia Meli ${ }^{2}$, Antonio Matrone ${ }^{1}$, Valeria Bottici', Liborio Torregrossa' ${ }^{2}$, Fulvio Basolo², Paolo Vitti', Gabriele Materazzi²,

Rossella Elise ${ }^{1}$

${ }^{1}$ Endocrine Unit of Clinical and Experimental Medicine, University of Pisa, Pisa, Italy, ${ }^{2}$ Department of Surgical, Medical, Molecular Pathology and Critical Area,University of Pisa, Pisa, Italy

Objectives: While it is well known that lymphnode metastases (LNFmets) have never found in RET gene carriers with a basal presurgical CT $<30-40 \mathrm{pg} / \mathrm{ml}$, to our knowledge there are no studies about this issue in sporadic adult MTC patients. The aim of the study was to look for a pre-operative serum CT value able to predict the absence of central nodes metastases, in order to avoid the lymphadenectomy of the central neck compartment (CCL) which is affected by a higher prevalence of complications.

Methods: We retrospectively analyzed the clinico-pathological and molecular features of MTC patients underwent surgery at our Department between 2005 and 2015. In this period we changed the laboratory assay for CT assessment: before September 2013 we used an immunometric assay [ELSAhCT; Cis-BioInternational, Gif sur Yvette, France, normal value $<10 \mathrm{pg} / \mathrm{ml}$ both in males and females], after September 2013 we used an immunoassay method [Immulite 2000, DPC, LA, USA, normal values $<11.5 \mathrm{pg} / \mathrm{ml}$ and $<18.2 \mathrm{pg} / \mathrm{ml}$ in females and males respectively].

Results: A total of 306 MTC patients underwent total thyroidectomy (TTX) and CCL in this study period. They were divided into 2 subgroups according to the assay used: group I $(\mathrm{n}=200)(\mathrm{CT}$ assay ELSA-hCT) and group II $(\mathrm{n}=50)$ (CT assay Immulite). Thirty-seven/200 patients of group I and 13/50 of group II had LFN-mets at histology. The highest value of CT able to identify all negative cases was $14 \mathrm{pg} / \mathrm{ml}$ in group I and $12.6 \mathrm{pg} / \mathrm{ml}$ (for female) and $18.6 \mathrm{pg} / \mathrm{ml}$ (for males) in group II which are, in both assays, very close to the upper limit of normal values.

Conclusions: We didn't find a preoperative CT cut-off able to identify MTC patients certainly not complicated by LNF-mets. According to our results all MTC adults patients should underwent at least TTX+CCL.

\section{P3-03-138 \\ LARYNX FUNCTIONAL PRESERVATION IN THE SURGICAL TREATMENT OF LOCALLY ADVANCED THYROID CANCER}

Min Sik Kim ${ }^{1}$, Young-Hoon Joo ${ }^{1}$, Sang-Yeon Kim ${ }^{1}, K_{\text {wang-Jae Cho }}{ }^{1}$

${ }^{1}$ The Catholic University of Korea, Department of Otolaryngology-Hns,

Seoul, Rep. of South Korea

Background: Locally advanced thyroid cancer usually invades into the larynx and requiring total laryngectomy for oncologic safety, although tumor infiltration is limited to the half of the larynx. We have performed VHLP(vertical hemilaryngopharyngectomy) in locally advanced laryngopharyngeal cancer to preserve the laryngeal function by the reconstruction of radial forearm free flap with tendon.

Subjects and Methods: We have applied VHLP in locally advanced thyroid cancer with laryngeal invasion in three cases which are needs to take a total laryngectomy, and we evaluate oncological and functional results.

Case report: All of three patients had advanced thyroid cancer with laryngeal invasion. The first case was spindle cell carcinoma of thyroid with paraglottic space invasion, the $2^{\text {nd }}$ case was papillary carcinoma with paraglottic space invasion, and the $3^{\text {rd }}$ case was anaplastic carcinoma with pyriform sinus invasion. In the case of anaplastic carcinoma, the cancer was recurred locally and distant metastasis, the patient died 2 months after surgery and other two cases were NED state. All three patients could preserve laryngeal function postoperative state.

Conclusion: Vertical hemilaryngopharyngectomy is one of the good surgical procedure to preserve the laryngeal function in the surgical treatment of locally advanced head and neck cancer especially invading into the larynx. In selected cases of thyroid cancer, this method could be a useful option for preserving the function of the larynx. 


\section{P3-04 Case Reports, Goitre and Nodules 3}

\section{P3-04-139 \\ THYROID CYSTS DISRIBUTION OBSERVED IN PEDIATRIC THYROID ULTRASONOGRAPHY FINDINGS}

Yo Kunii' ${ }^{1}$, Jeaduk Noh Yoshimua', Koji Mukasa', Hidemi Ohye' ${ }^{1}$, Miho

Suzuki', Masako Matsumoto ${ }^{1}$, Natsuko Watanabe ${ }^{1}$, Ai Yoshihara',

Nami Suzuki ${ }^{1}$, Kenji Iwaku ${ }^{1}$, Kiminori Sugino ${ }^{2}$, Koichi Ito ${ }^{3}$

${ }^{1}$ Ito Hospital, Department of Internal Medicine, Tokyo, Japan, ${ }^{2}$ Ito

Hospital, Department of Surgery, Tokyo, Japan, ${ }^{3}$ Ito Hospital, Tokyo

Background: Characteristic findings in children ultrasonography are multiple small cysts and ectopic intrathyroidal thymus. Thyroid cysts are found in up to $50 \%$ of cases depending on age. In this study, we examined the appearance site of thyroid cysts in children by ultrasonography, as there has been no report on this topic.

Methods: From January to December in 2010, there were 6846 patients of adenomatous nodules consulted at the initial visit in our hospital and 213 cases were children (18 years or younger) among them. Since it is difficult to distinguish colloid cysts from cysts associated with bleeding and degenerative changes by ultrasonography, cases without internal solid parts were extracted as "cysts". We divided thyroid gland into three parts; right, left and isthmus. Furthermore, we divided left and right thyroid glands into four parts each; the upper and lower sides, the dorsal and the ventral side. We examined the most frequent occurrence part of thyroid cyst among 9 sites retrospectively.

Results: The age distribution of children was 15 years in median (range 5-18 years), Sex: 29 males and 184 females. There were 165 cases $(77.5 \%)$ among 213 children who had only cystic change. Cysts were found more on the dorsal than the ventral; 133 out of 165 cases $(80.6 \%)$. Moreover, in the comparison of upper and lower site, the appearance (frequency) of cysts was significantly higher in the lower site (128 cases $(77.5 \%))$ than in the upper $(37$ cases $(22.4 \%))$. There was no appearance difference between left and right site. The cysts were small and the median of the maximum diameter was 3.3 $\mathrm{mm}$ (range $0.8-23.2 \mathrm{~mm}$ ).

Conclusion: We found that the thyroid cysts are often observed in the lower and dorsal side. We also report the pathological organization.

\section{P3-04-140 \\ HYDRODISSECTION OF PERITHYROIDAL SPACE TO OPTIMIZETHYROID RELATED PROCEDURE}

Hyundae Yoon ${ }^{1}$, Woo Jin Chang ${ }^{1}$, Jong Yup Bae ${ }^{1}$, Jong-Ryool Oh',

Inwook Song ${ }^{1}$, Hyeyeon Jeong ${ }^{1}$, Jin gu Bong ${ }^{1}$, Seung II Bae ${ }^{1}$, Ho Jin $\operatorname{Kim}^{1}$

${ }^{1}$ Raphael Hospital, Daegu, Rep. of South Korea

Introduction: Laser ablation has been accepted as a minimally invasive treatment in patients with symptomatic thyroid nodule. Although laser ablation has a low complication rate, unintended heat transferred to several critical organs may lead to a variety of complications. Several thermal protective measures such as hydrodissection, gas insufflation and body positioning are now used. Hydrodissection technique have been described during image guided thermal ablation of hepatoma or renal cell carcinoma. Hydrodissection technique is local injection of fluid to separate the target lesion and adjacent structures. It provides a thermal buffer, avoiding damage to critical organs and facilitating more complete ablation. It also reduce thermal-sink effects from adjacent vessels. Therefore, we applied this technique to thyroid field.

Case Report: We describe several cases in which hydrodissection technique was applied to avoid thermal damage during laser ablation of benign thyroid nodule and to facilitate fine needle aspiration of cervical lymph node located in inaccessible area. Hydrodissection was performed using normal saline solution mixed with lidocaine and epinephrine. And the solution was injected into the interstitial space between thyroid capsule and surrounding organs and it was injected until the distance between thyroid capsule and the surrounding organs was increased to more than $10 \mathrm{~mm}$. The average amount of fluid injected was less than $10 \mathrm{ml}$. As soon as separation was done, the procedure was implemented immediately. There was no complication related to hydrodissection. And there was no thermal damage of surrounding vital tissues after laser ablation and during the follow-up period.

Conclusion: Hydrodissection technique is safe and very useful adjuvant technique to avoid thermal damage during laser ablation of thyroid nodule. And this technique can also be very useful to make a passage when the target lesion is inaccessible.

\section{P3-04-141 \\ DIAGNOSTIC EVALUATION OF SCINTIGRAPHYCALLY ,WARM“ THYROID NODULES}

Miodrag Lacic ${ }^{1}$, Mirna Barisic-Smalcelj ${ }^{1}$

${ }^{1}$ Polyclinic Lacic; Thyroid Department, Zagreb, Croatia

Objectives: The purpose of this study was to evaluate different diagnostic techniques in evaluation of patients with scintigraphycally "warm" (indeterminable) thyroid nodules.

Methods: In this study we included 132 patients (pts) with scintigraphycally indeterminable ("warm") thyroid nodules. Further to clinical investigation in all patients we performed scintigraphy (Tc-99m pertechnetate thyroid scan), sonography, FNAB (fine needle aspiration biopsy) followed by cytological investigation, and thyroid blood test (TSH, T4, T3, anti-TPO and anti-TG).

Results: On clinical investigation $59 \%$ of pts had a palpable solitary nodule, $22 \%$ of investigated pts had multiple nodules, while in $19 \%$ of pts the existence of nodule was suspected. Equal incidence of solitary and multiple nodules $(42,5 \%)$ was determined by sonography, while in $15 \%$ of pts the nodule was not registered. $51 \%$ of nodules were isoechogenic on sonography, $36 \%$ hypoechogenic, $7 \%$ hyperechogenic and $6 \%$ of nodules had unechogenic echo structure. Based on sonography $75 \%$ of nodules were solid, $7 \%$ were pure cystic and $18 \%$ of nodules had both solid and cystic elements ("mixed" nodules). Calcifications were found in 4 nodules. The ratio between clearly and unclearly defined nodules was $1: 1,5.16 \%$ of nodules were as thick as the lobe itself. No malignant lesions were found on FNAB and pathology (2 pts). TSH was suppressed in 23 pts. Thirteen pts were hyperthyroid and only one pts was hypothyroid. There were no correlation between the age of pts and thyroid weight or the size and number of nodules in the thyroid. A positive correlation between the thyroid weight and the size of the nodules was found.

Conclusion: Applying this multi-modality diagnostic approach to the patients with scintigraphycally "warm" (indeterminable) thyroid nodules we were able to better understand the biological nature of these nodules and to achieve much better results in the management of these patients.

\section{P3-04-142 \\ EVALUATION OF VASCULARIZATION OF THYROID GLAND IN DIFFUSE GOITER} Elina Gasparyan ${ }^{1}$

${ }^{1}$ Medical Centre; Endocrinology; Medical Academy of Postgraduate Studies, St. Petersburg, Russian Federation

Aim: Evaluation of vascularization character with the use of Color Doppler regime (CD) and systolic velocity of blood flow (SVBF) in patients with diffuse toxic goiter (DTG) for assessment of thyroid (TH) function and tactics of their further treatment.

Materials and Methods: In our medical centre 68 patients with DTG and 66 patients with autoimmune thyroiditis (AIT, the group of comparison) aged 18-73 (average $42.6 \pm 12.9$ years old) were observed. All patients underwent usual clinical, laboratory, hormonal and instrumental tests. In ultrasound examinations with B- and CD regimes the thyroid sizes, outlines, echogenecity, structure and vascularization as well as SBF velocity in upper thyroid artery in $\mathrm{cm} / \mathrm{s}$ were estimated. Out of 68 patients with DTG 26 showed thyrotoxicosis and 42 patients showed euthyroidism (30 of them - medicamental and 12 - after the cancellation of therapy).Out of 66 patients with AIT 37 showed subclinical and overt hypothyroidism; 29 patients were in the state of euthyroidism (medicamental and without therapy).

Results: In the process of research in DTG we established the dependence of vascularization character and SVBF on TH function. All patients (100\%) with thyrotoxicosis showed considerable increase or hypervascularization of TH. At the same time, SVBF was in average $68.0 \pm 12.1 \mathrm{~cm} / \mathrm{s}$ (with the normal being $20.0-40.0 \mathrm{~cm} / \mathrm{s}$ ) and reached $80.0 \mathrm{~cm} / \mathrm{s}$. Euthyroidism in patients 
was characterized with normalization of thyroid vascularization irresrective of the presence or absence of antithyroid therapy. SVBF in them was in average $32.0 \pm 6.4 \mathrm{~cm} / \mathrm{s}$. We noticed the interrelation between $\mathrm{TH}$ vascularization character, SVBF, TSH level and TRAb values. In all patients with AIT in the state of hypothyroidism and euthyroidism with and without of therapy TH vascularization was normal and SVBF was in average $24.6 \pm 4.7 \mathrm{~cm} / \mathrm{s}$ which is considerably lower than in patients in the state of thyrotoxicosis.

\section{Conclusions:}

- Determination of the character of vascularization in CD regime and SVBF is noninvasive available study, permitting to assess thyroid function in patients with DTG within a few minutes

- Thyroid vascularization and high indexes of SVBF, observed in all patients on the stage of thyrotoxicosis, have diagnostic significance

- Normalization of vascularization and SVBF of TH in patients with DTG, together with normal TSH and TRAb, can serve as in indication for antithyroid therapy cancellation.

\section{P3-04-143 \\ THE ROLE OF THE HIGH FT4 LEVELS IN BIOPSY NEEDLE WASHOUT IN THE DIAGNOSE OF THE HYPERFUNCTIONING THYROID NODULES}

Nikolay Raykov ${ }^{1}$, Boyan Nonchev ${ }^{2}$, Borislav Chaushev ${ }^{3}$, Svetoslav Todorov 4 , Daniela Malcheva ${ }^{4}$, Snejanka Vicheva ${ }^{5}$, Asya Raykova ${ }^{6}$, Milena Avdzhiyska ${ }^{7}$, Miroslav Raykov ${ }^{7}$

${ }^{1}$ Medical Complex „plus“;varna, Bulgaria, Varna, Bulgaria, ${ }^{2}$ Clinic of Endocrinology, Medical University, Plovdiv, Bulgaria, Plovdiv, Bulgaria, ${ }^{3}$ Department of Nuclear Medicine "saint Marina", Varna, Bulgaria, Varna, ${ }^{4}$ Clinical Laboratory "status", Varna, Bulgaria, Varna, ${ }^{5}$ Department of Clinical Pathology, Oncological Dispensary "marko Markov", Varna, Bulgaria, Varna, ${ }^{6}$ First Department of Internal Medicine, Mbal Dobrich, Bulgaria, Varna, ${ }^{7}$ Medical University, Varna, Bulgaria, Varna

Thyroid US and USGFNA are golden standard in thyroid nodules evaluation. They provide an image and cytomorphological diagnose of the evaluated structures. The biopsy needle content consist of cellular material and biological substances.So FNA determinates the cytological and the probable functional diagnose of the node.

The evaluation of TGL,PTH and Calcitonin in the biopsy needle washout is applied during past 30 years.No literature data exist about the analysis of thyroid hormone levels in the needle WO from the nodules and their possible use for determination of functional active ones. The classic qualitative method is the thyroid scintigraphy.

Aim: To prove that the higher FT4 WO levels of analysed node/compared to that in the surrounding parenchyma/ can determine it as hyperfunctioning one.

Patients and methods:For the period 2012-2017 we performed 2650 FNA. We present 50 hyperfunctioning nodules in 45 patients. Nodes volume is $3.97 \pm 0,80 \mathrm{cc}$.

\section{Selection Criteria:}

1/Laboratory:suppressed TSH.

2/US:Nodules diameter bigger than one $\mathrm{cm}$. No US signs for ATD in surrounding parenchyma.

Cytological and FT4 WO samples were prepared from the nodule and parenchyma. ${ }^{99 \mathrm{~m}} \mathrm{Tc}$ thyroid scintigraphy was performed classifying nodes as compensated, subcompensated, decompensated.

Results: The nodules were cytologically benign.

FT4 WO in nodule: $104.96 \pm 35.46 / 10-25 \mathrm{pmol} / \mathrm{l} /$

FT4 WO in parenchyma: $6.34 \pm 2.41$.

Correlatioin FT4 WO node / FT4 WO parenchyma : 129,74 \pm 43.82

There was no clear correlation of FT4 WO results from the decompensated,subcompensated and compensated nodules.

We found a correlation between the nodules volume and interrelation FT4 WO nodule/ FT4 WO parenchyma $\mathrm{p}=0,002 ; \mathrm{r}=0.42$

Conclusion: The results show, that theFT4 WO levels from hyperfunctioning nodules were significantly higher than the parenchyma. Further studies are needed to prove the diagnostic value of this method. We consider the evaluation of FT4 WO as a potential diagnostic tool for the early diagnose of hypefunctioning thyroid nodules.

\section{P3-04-144}

ASSOCIATION OF AUTOIMMUNE THYROID DISEASE AND THROMBOTIC EVENTS: REPORT

\section{OF TWO CASES}

Claudia Camelia Podia Igna ${ }^{1}$, Mihaela Simescu²

${ }^{1}$ Astra Clinic, Sibiu, Romania, ${ }^{2}$ Sc Simedis Consult Srl, Sect 1,

Bucharest, Romania

Introduction: Thyroid dysfunction, hyperthyroidism and mild-moderate hypothyroidism, and autoimmunity may modify hemostasis leading to a hypercoagulability state.

There are several mechanisms involved in the pathogenesis of thrombosis in patients with thyroid diseases.

Hypothyroidism decreases fibrinolysis and determine endothelial dysfunction.

Hyperthyroidism induces a hypercoagulability state through an increase factor VIII activity.

On the other hand thyroid autoimmune process may be associated with other autoimmune syndrome such as antiphospholipid syndrome which can lead to thrombosis regardless of the thyroid function. The association of thyroid autoimmunity and thrombotic events may be due also to a genetic predisposition.

Case Report: We present two cases with associated autoimmune thyroid disease and thrombotic events.

The first case, a 34 y.o woman presented antiphospholipid syndrome with right axillary, brachial and subclavicular veins thrombosis and Graves' disease.

The onset of thrombosis preceded the onset of hyperthyroidism. During the onset of overt hyperthyroidism, associated with a high titer of anti-TSH receptor antibodies, the anticardiolipin antibodies were normalized under specific treatment.

The two diseases had an independent evolution under specific treatment.

The second one, a 40 y.o woman, presented with stroke at 36 y.o. After 4 years she developed autoimmune thyroiditis and hypothyroidism. In this case also the onset of thrombotic event preceded the onset of thyroid disease. The cause of hypercoagulability state in this second case is under investigation.

Both patients had high titers of specific disease antibodies.

Conclusion: The association of thyroid disease and thrombotic events in these two cases seems to be due to a genetic predisposition rather than to hypercoagulability state induced by thyroid dysfunction.

\section{P3-04-145 \\ HEART FAILURE AT THYREOTOXSICOSES DISEASE \\ Kristine Jojua \\ ${ }^{1}$ LTD David Metreveli Medical Centre, Tbilisi, Georgia}

It is known, that the disease of the heart is the most frequent cause of death of patient with thyreotoxicosis. A 49 years old women patient comes to consultation. 4 years ego she was diagnosed multinodular toxis goiter. The patient was treated with thyreostatic drugs periodically. When she fill herself good, she often stopped appointed treatment. At stage of diagnosed thyreotoxity were done ECG and echocardiography. they not find any disorders. After 2 years of diagnosed thyreotoxity was found tachisistolic palpitation. The patient was admitied in Emergency department with diagnosis of paroqsism of atrial fibrillation.After that this patient had normosistolyc atrial fibrillation and also diagnosed Heart failure stage 2 ( NYHA). We started thyreostatic therapy and when achieve remission of thyreotoxicoses, she was carried out radioiodtheraphy. In conclusion, it is necessary to note that thyreotoxicoses without any previous heart pathology can lead to the development of heart failure. 


\section{P3-04-146 \\ CHORDOMA AS A DISGUISE OF PAPILLARY THYROIDE CARCINOMA - A CASE REPORT}

Daniela Magalhães ${ }^{1}$, Cláudia Costa², Maria João Matos ${ }^{2}$, Ana Paula Santos $^{2}$, Isabel Azevedo ${ }^{3}$, Cristina Sanches ${ }^{4}$, Hugo Duarte ${ }^{5}$, Mariana Afonso $^{6}$, Manuel Jacome ${ }^{6}$, Isabel Torres ${ }^{2}$

${ }^{1}$ Department of Endocrinology, Diabetes and Metabolism, Centro Hospitalar São João, E.P.E., Faculty of Medicine, University of Porto, Instituto de Investigação e Inovação Em Saúde, University of Porto, Porto, Portugal, ${ }^{2}$ Endocrinology Department of Instituto Português de Oncologia Do Porto, Porto, Portugal, ${ }^{3}$ Medical Oncology Department of Instituto Português de Oncologia Do Porto, Porto, Portugal, ${ }^{4}$ General Surgery Department of Instituto Português de Oncologia Do Porto, Porto, Portugal, ${ }^{5}$ Nuclear Medicine Department of Instituto Português de Oncologia Do Porto, Porto, Portugal, ${ }^{6}$ Pathology Department of Instituto Português de Oncologia Do Porto, Porto, Portugal

Introduction: Bone is the second most common site of distant metastasis from thyroid cancer. The incidence of bone metastasis in papillary thyroid carcinoma is $1-7 \%$

Case Report: A 62-year-old male presented with a 9x7.5x9 cm bulky mass on the sacrum with soft tissue density on CT, leading to extensive lytic lesion of most of the sacred vertebrae and extending to the left iliac, suggestive of chordoma. His past medical history was significant for type- 2 diabetes, renal lithiasis, smoking and chronic alcoholism. He underwent total sacretectomy with partial excision and reconstruction of the left iliac. The anatomopathological examination revealed sacro-coccygeal involvement by predominantly papillary (follicular variant) thyroid carcinoma, with foci of non-differentiated (insular) carcinoma. Thyroid ultrasonography showed a solid nodule of 20 $\mathrm{mm}$ in the right lobe and two solid hypoechogenic nodules of 11 and $9 \mathrm{~mm}$ in the left lobe, the smallest with coarse calcifications. 18F-FDG-PET revealed a hypermetabolic focus in thyroid left lobe consistent with malignant neoplasia. He underwent total thyroidectomy. Histological examination revealed follicular and oxyphilic variants of multifocal papillary carcinoma, with dimensions ranging from 2 to $15 \mathrm{~mm}$, without signs of hematogenic, lymphatic or perineural permeation nor invasion of the capsule or extrathyroidal extension, with resection margins uninvolved by neoplasia (pT1b[m]NxM1R0). Radioactive iodine (131I) was then performed. Post-therapy scintigraphy showed hyperfixation in the remnant of the sacrum and lower lumbar spine, bilateral iliac and anterior cervical region. TSH-stimulated thyroglobulin was $24490 \mathrm{ng} / \mathrm{mL}$. Follow-up MRI shows recurrence and progression of the pelvic tumor lesion.

Conclusions: We present a rare case of a well differentiated thyroid carcinoma without other histological signs of unfavourable prognosis beyond multifocality presenting as osteolytic bone metastasis mimicking a chordoma. Bone metastasis are associated with a poor prognosis and, because overall survival is improved by complete resection, its early diagnosis and treatment is important.

\section{P3-05 Clinical Thyroidology}

\section{P3-05-147 \\ PLASMA LIPID METABOLISM IN WOMEN WITH SHORT TERM IATROGENIC SUBCLINICAL HYPERTHYROIDISM AFTER TOTAL THYROIDECTOMY AND RADIOIODINE THERAPY FOR THYROID CANCER TREATMENT: LIPID TRANSFERS TO HDL, CETP, LCAT, PON1, APO B48, LP(A) AND HDL PARTICLE SIZE Gilbert Sigal', Thauany Tavoni ${ }^{2}$, Fatima Freitas ${ }^{2}$, Bruna Silva², Raul Maranhão ${ }^{2}$, Lenine Brandão ${ }^{2}$ \\ ${ }^{1}$ Lipid Metabolism Laboratory, Heart Institute (Incor) of the Medical School Hospital, University of São Paulo, São Paulo, Brazil, Sao Paulo, Brazil, ${ }^{2}$ São-Paulo}

Objectives: Patients submitted to total or near total thyroidectomy and radioiodine therapy for thyroid cancer treatment are maintained in iatrogenic subclinical hyperthyroidism for long periods of time, eventually life-long. As thyroid hormones affect lipid metabolism it is important to explore the plasma lipids in this subclinical hyperthyroidism condition due to the implications of disturbed lipid metabolism and this study was aimed to determine the status of plasma lipids, apolipoproteins, enzymes with important role in lipid metabolism and lipid transfers to HDL, an important step in the metabolism of this anti-atherogenic lipoprotein.

Methods: 7 women (age: $42 \pm 12$; IMC: $27.4 \pm 6.8 \mathrm{~kg} / \mathrm{m}^{2}$ ), with thyroid cancer were included before thyroidectomy when they were euthyroid and again after total thyroidectomy and radioiodine therapy when they were in subclinical hyperthyroidism induced by levothyroxine treatment. Patients in use of levothyroxine before thyroidectomy or with diabetes,dyslipidemia on use of drugs that could interfere in lipid metabolism such as hypolipemiants, beta-bloquers, tiazidic diuretics were excluded. All patients included in the study show no methastases on whole body scan previous to radioiodine therapy. Blood samples were drawn on the day of thyroidectomy, in euthyroidism and again in subclinical hyperthyroidism, $2.8 \pm 0.6$ months after initiation of levothyroxine.

Results: Results are shown for each lipid parameter. First in euthyroidism followed by result in subclinical hyperthyroidism. TSH:1.96 \pm 2.04 vs $0.09 \pm 0.05 \mathrm{uUI} / \mathrm{mL}, \mathrm{p}=0.0486$; Free $\mathrm{T} 4: 1.01 \pm 0.18$ vs $1.35 \pm 0.20 \mathrm{ng} / \mathrm{dL}$, $\mathrm{p}=0.0020$; all others comparisons were non signicant: Total cholesterol: $208 \pm 27$ vs $202 \pm 25 \mathrm{mg} / \mathrm{dL}$; Triglycerides: $130 \pm 43$ vs $154 \pm 42 \mathrm{mg} / \mathrm{dL}$; nonHDL-cholesterol: $170 \pm 25$ vs $167 \pm 21 \mathrm{mg} / \mathrm{dL}$; LDL-C: $144 \pm 27$ vs $136 \pm 22 \mathrm{mg}$ / dL; HDL-C:38 \pm 7 vs $35 \pm 4 \mathrm{mg} / \mathrm{dL} ;$ ApoA-I:1.34 \pm 0.22 vs $1.23 \pm 0.10 \mathrm{~g} / \mathrm{L}$; ApoB: $90.8 \pm 37.9$ vs $78.4 \pm 20.5 \mathrm{~g} / \mathrm{L}$; Free cholesterol:53 \pm 6 vs $55 \pm 6 \mathrm{mg} / \mathrm{dL}$; ApoB48: $90.8 \pm 37.9$ vs $78.4 \pm 20.5 \mathrm{mg} / \mathrm{dL}$; Lp(a): $55.1 \pm 46.1$ vs $70.4 \pm 62.5 \mathrm{mg}$ / dL.

All others lipid parameters studied are shown in Table 1.

Conclusions: After the installation of the iatrogenic subclinical hyperthyroidism, the several parameters of the plasma lipid metabolism tested here were not changed. This outcome is also of interest for the understanding of the metabolic status of subclinical hyperthyroidism with other ethiologies.

Table 1. Lipid parameters in euthyroidism and subclinical hyperthyroidism

\begin{tabular}{llll}
\hline & $\begin{array}{l}\text { Euthyroidism } \\
(\mathrm{n}=7)\end{array}$ & $\begin{array}{l}\text { Subclinical } \\
\text { hyperthyroidism } \\
(\mathrm{n}=7)\end{array}$ & $p$ \\
& & $3.1 \pm 0.7$ & 0.9158 \\
\hline CETP $(\mu \mathrm{g} / \mathrm{mL})$ & $3.0 \pm 1.0$ & $9.3 \pm 2.1$ & 0.6372 \\
LCAT $(\mu \mathrm{g} / \mathrm{mL})$ & $8.9 \pm 2.3$ & $141 \pm 81$ & 0.7229 \\
PON1 $\left(\mathrm{nmol} / \mathrm{min}^{-1} . \mathrm{mL}^{-1}\right)$ & $129 \pm 58$ & $8.2 \pm 0.3$ & 0.0922 \\
HDL particle size $(\mathrm{nm})$ & $8.6 \pm 0.6$ & & \\
Lipids transfers to HDL $(\%)$ & & $6.6 \pm 0.7$ & 0.6962 \\
CE & $15.4 \pm 1.4$ & $15.7 \pm 1.1$ & 0.4995 \\
PL & $3.9 \pm 1.2$ & $3.4 \pm 0.8$ & 0.3446 \\
TG & $4.6 \pm 1.9$ & $3.9 \pm 1.1$ & 0.3718 \\
CL & & \\
\hline
\end{tabular}

CE: Cholesterol ester PL: Phospholipids TG: Triglycerides CL: Free cholesterol

\section{P3-05-148 \\ REFRACTORY HYPOTHYROIDISM DUE TO SELECTIVE MALABSORPTION OF ORAL THYROXINE}

Jana Brunova ${ }^{1}$, Simona Kratochvilova ${ }^{2}$

${ }^{1}$ Institute for Clinical and Experimental Medicine, Prague 4, Czech Republic, ${ }^{2}$ Diabetes Centre, Institute for Clinical and Experimental Medicine, Prague, Czech Republic

Case Report: We present young female (26y) with refractory hypothyroidism which developed after the thyroidectomy performed for papillary carcinoma. This patient suffers with selective malabsorption for oral L-thyroxin which was diagnosed after the exclusion of any other possible causes of oral therapy failure. She was treated with increased doses of levothyroxine and triiodothyronine including tablets from different pharmaceutical companies and administration of suppositories forms without effect on blood thyroxine level. Despite of huge doses of L-thyroxin (up to $1000 \mu \mathrm{g}$ per day) she became clini- 
cally severely hypothyroid with immeasurable fT4 level and TSH $>150 \mathrm{mU} / \mathrm{L}$. On examination, she was extremely tired, sleepy and she complained of dry skin, myalgia and arthralgia. Medicine intake under the physician supervision excluded a poor compliance. She was thoroughly investigated for refractory primary hypothyroidism. The examination of gastrointestinal tract was normal, including duodenal biopsy. Lactose intolerance and celiac disease was also excluded. She did not take any interfering medication, food or beverages. Because of her severe hypothyroidism it was decided to inject her with 500 $\mu \mathrm{g}$ levothyroxine (L-Thyroxin Henning inject) as intramuscular injections given on a once weekly basis. Her laboratory values improved significantly and TSH level gradually dropped to normal values. There was also sustained clinical improvement including her muscle strength and fatigue. Unfortunately after about one year of successful therapy she developed allergic reaction to this drug and similar products, which has manifested as the skin exanthema accompanied with a very severe itching (pruritus). At present she continues her therapy with L-Thyroxine Henning the $4^{\text {th }}$ year and skin symptoms are relieved with regular administration of diphenylhydramine I.V.

Conclusions: The diagnosis of the selective malabsorption of thyroxine can be established only after the exclusion of any other causes of the drug malabsorption. Intramuscular administration of levothyroxine is an effective alternative in refractory hypothyroidism.

\section{P3-05-149 \\ NO EVIDENCE OF INCREASE IN CALCITONIN CONCENTRATIONS OR DEVELOPMENT OF C-CELL MALIGNANCY IN RESPONSE TO LIRAGLUTIDE IN THE LEADER TRIAL \\ Laszlo Hegedüs ${ }^{1}$, Steven Sherman ${ }^{2}$, R Michael Tuttle ${ }^{3}$, \\ Bernt Johan von Scholten ${ }^{4}$, Søren Rasmussen ${ }^{4}$, \\ Julie Derving Karsbø/4, Gilbert Daniels ${ }^{5}$ \\ ${ }^{1}$ Department of Endocrinology and Metabolism, Odense University Hospital, Odense, Denmark, ${ }^{2}$ Department of Endocrine Neoplasia, The University of Texas MD Anderson Cancer Center, Houston, TX, USA, ${ }^{3}$ Endocrinology Service, Memorial Sloan Kettering Cancer Center, New York, NY, USA, ${ }^{4}$ Novo Nordisk A/S, Søborg, Denmark, ${ }^{5}$ Thyroid Unit, Cancer Center and Department of Medicine, Massachusetts General Hospital and Harvard Medical School, Boston, MA, USA}

Objectives: To date, there is no evidence that liraglutide stimulates calcitonin (CT) release in humans. This secondary analysis of the LEADER trial (NCT01179048) examined the long-term CT changes in patients exposed to liraglutide versus placebo.

Methods: Patients $(\mathrm{n}=9340)$ with type 2 diabetes and high risk for cardiovascular (CV) events were randomised 1:1 to liraglutide or placebo, and followed for up to 5 years. The study investigated a primary composite CV outcome. We analysed: the change in CT levels over time; CT outliers; and thyroid and C-cell neoplasms.

Results: Mean CT concentrations at baseline were similar between treatments, both in male and female patients. At 36 months, patients randomised to liraglutide showed no difference in mean $\mathrm{CT}$ concentrations versus patients randomised to placebo in males (estimated treatment ratio [ETR]: 1.03, 95\% CI: $1.00 ; 1.06 ; p=0.068$ ) and females (ETR: $1.00,95 \%$ CI: $0.97 ; 1.02$; $p=0.671$ ). In liraglutide versus placebo groups, a similar proportion of male

Table 1. Categorical summary of extreme post-baseline values for calcitonin

\begin{tabular}{|c|c|c|c|c|}
\hline \multirow{2}{*}{$\begin{array}{l}\text { Number of patients } \\
\text { Gender }\end{array}$} & \multicolumn{2}{|c|}{ Liraglutide, N (\%) } & \multicolumn{2}{|c|}{ Placebo, N (\%) } \\
\hline & Male & Female & Male & Female \\
\hline Number of patients & 3011 & 1657 & 2992 & 1680 \\
\hline Normal CT & $2146(75.4)$ & $1476(94.6)$ & $2149(77.0)$ & $1500(95.5$ \\
\hline High CT (>UNL) & $701(24.6)$ & $85(5.4)$ & $642(23.0)$ & $71(4.5)$ \\
\hline $\mathrm{CT}>20 \mathrm{ng} / \mathrm{L}$ & $139(4.9)$ & $5(0.3)$ & $133(4.8)$ & $5(0.3)$ \\
\hline $\mathrm{CT}>50 \mathrm{ng} / \mathrm{L}$ & $16(0.6)$ & - & $17(0.6)$ & - \\
\hline $\mathrm{CT}>100 \mathrm{ng} / \mathrm{L}$ & $3(0.1)$ & - & $2(0.1)$ & - \\
\hline
\end{tabular}

$\mathrm{CT}$, calcitonin; N, number of patients in the summary statistic; UNL, upper normal limit.

The minimum and maximum values from all scheduled and unscheduled post-baseline visits are used; these values are then categorised according to laboratory range values. These groups are not mutually exclusive. and female patients had a post-baseline CT concentration above the upper normal limit during the trial (Table). No episodes of C-cell hyperplasia or medullary thyroid carcinoma were reported in patients in the liraglutide group following randomisation

Conclusions: There was no indication of a difference in CT concentrations between treatments and no C-cell malignancies occurred in the liraglutide group following randomisation.

\section{P3-05-150 \\ THYROGLOBULIN ANTIBODIES BEFORE RADIOIODINE ABLATION PREDICT DIFFERENTIATED THYROID CANCER OUTCOME}

Pierpaolo Trimboli ${ }^{1}$, Mauro Imperiali ${ }^{2}$, Luca Giovanella ${ }^{3}$

${ }^{1}$ Thyroid Center, Oncology Institute of Southern Switzerland, Lugano, Switzerland, ${ }^{2}$ Department of Laboratory Medicine, Ente Ospedaliero Cantonale, Bellinzona, Switzerland, ${ }^{3}$ Department of Nuclear Medicine and Thyroid Centre, Oncology Institute of Southern Switzerland,

Bellinzona, Switzerland

Objective: Serum thyroglobulin (Tg) is essential to manage differentiated thyroid carcinoma (DTC). However, Tg determination is affected by circulating $\mathrm{Tg}$ antibodies $(\mathrm{TgAb})$ and a role of $\mathrm{TgAb}$ as surrogate biomarker has been proposed. Here we evaluated the role of $\mathrm{TgAb}$ measured before and after radioiodine ablation (RRA) as potential predictors of prognosis.

Methods: Patients treated since 2006 were screened. Cancers with structural relapse was defined as recurrent. Both $\mathrm{Tg}$ and $\mathrm{TgAb}$ were measured by immunoassays on the fully automated Kryptor ${ }^{\circledR}$ platform (BRAHMS Gmbh, Henningsdorf, Germany).

Results: A series of 215 DTC patients was finally enrolled, of whom $28.8 \%$ with positive preablation TgAb. Overall, $2.8 \%$ patients died by DTC and $11 \%$ recurred. Table 1 compares main features of the two groups. Highrisk class $(\mathrm{p}=0.004)$ and cancer relapse $(\mathrm{p}=0.007)$ occurred more frequently in positive $\operatorname{TgAb}$, while better disease-free survival was observed in negative group (Hazard Ratio 2.59, $\mathrm{p}=0.01$ ). Having positive preablation $\mathrm{TgAb}$ was significantly associated with risk to develop recurrence (Odds Ratio 3.57, $\mathrm{p}=0.004)$. Among positive TgAb subgroup, higher levels were recorded in recurrent cases $(\mathrm{p}=0.0001)$ and the most accurate preablation $\mathrm{TgAb}$ threshold was $107.5 \mathrm{IU} / \mathrm{mL}$. When TgAb were measured at first follow-up, recurrence rate was significantly $(\mathrm{p}<0.0001)$ higher in persistently positive $\mathrm{TgAb}$ patients $(75 \%)$ than normalized ones $(2.4 \%)$. At that time the highest negative predictive value could be obtained when considering $\operatorname{TgAb}$ normalization ( $<33 \mathrm{IU} /$ $\mathrm{mL}$ ) or reduction by $\geq 36.4 \%$.

Conclusions: Positive TgAb before RRA indicates higher risk of poor prognosis, but their significant drop 6-12 months later could be considered a favorable factor.

Table 1. Patients characteristics

\begin{tabular}{lll}
$\begin{array}{l}\text { TgAb-negative } \\
(\mathrm{n}=153)\end{array}$ & $\begin{array}{l}\text { TgAb-positive } \\
(\mathrm{n}=62)\end{array}$ & p-value \\
\hline
\end{tabular}

Clinical features

Females/Males

Age (years)

$111 / 42$

$43 / 19$

0.76

Histology

PTC/FTC

Risk categories

Low

Intermediate

53 (range 20-94)

51 (range 18-88)

0.67

High

$118 / 35$

$51 / 11$

0.51

Thyrotropin (TSH)

Preablation TSH (mUI/L) $77.8(35.9-158.2) \quad 84.5(44.6-137.2) \quad 0.65$

Radioiodine ablation

Iodine-131 activity (GBq) 3.7 (range 1.1-7.4) 4.4 (range 1.1-7.4) 0.09

Outcome

Recurrence $\quad 11(7.2 \%) \quad 13(20.9 \%)$

0.007

Disease related-death

$3(1.9 \%)$

$3(4.8 \%)$

0.48 


\section{P3-05-151 \\ PSYCHOSOCIAL DEVELOPMENTAL MILESTONES IN SURVIVORS OF CHILDHOOD DIFFERENTIATED THYROID CARCINOMA}

Marloes Nies ${ }^{1}$, Bernadette L. Dekker ${ }^{2}$, Gea A. Huizinga ${ }^{2}$, Esther Sulkers ${ }^{2}$, Mariëlle S Klein Hesselink ${ }^{2}$, Heleen Maurice-Stam ${ }^{3}$, Martha A. Grootenhuis ${ }^{3}$, Adrienne H. Brouwers ${ }^{2}$, Johannes G.M. Burgerhof ${ }^{2}$, Eveline W. van Dam ${ }^{3}$, Bas Havekes ${ }^{4}$, Marry M. van den HeuvelEibrink $^{5}$, Eleonora P.M. Corssmit ${ }^{6}$, Leontien C.M. Kremer ${ }^{3}$, Romana T. Netea-Maier ${ }^{7}$, Heleen J.H. van der Pa ${ }^{\beta}$, Robin P. Peeters ${ }^{5}$, John T.M. Plukker $^{2}$, Cécile M. Ronckers ${ }^{3}$, Hanneke M. van Santen ${ }^{8}$, Wim J.E. Tissing ${ }^{2}$, Gianni Bocca ${ }^{2}$, Thera Links ${ }^{9}$

${ }^{1}$ University of Groningen, University Medical Center Groningen, Department of Endocrinology, Groningen, Netherlands, ${ }^{2}$ Groningen, Netherlands, ${ }^{3} \mathrm{Amsterdam}$, Netherlands, ${ }^{4}$ Maastricht, Netherlands, ${ }^{5}$ Rotterdam, Netherlands, ${ }^{6}$ Leiden, Netherlands, ${ }^{7}$ Nijmegen, Netherlands, ${ }^{8}$ Utrecht, Netherlands, ${ }^{9}$ University Medical Center Groningen, Department of Endocrinology, Groningen, Netherlands

Objectives: The impact of childhood differentiated thyroid carcinoma [DTC] on achieving psychosocial developmental milestones (course of life $[\mathrm{CoL}]$ ) is unknown. The aim of this study was to assess whether CoL is influenced in young adult survivors of childhood DTC compared to peers and compared to other childhood cancer survivors [CCS].

Methods: Survivors of DTC, diagnosed between 1970-2013 at childhood age $(\leq 18 \mathrm{y})$ and treated in the Netherlands were included. Exclusion criteria were: follow-up $<5$ years after diagnosis, attained age $<18$ or $>35$ years, and/ or diagnosis of DTC as a second malignant neoplasm. Survivors were asked to gather peer controls. Thereby, 2 additional groups were used for comparison: peers from the general Dutch population and young adult CCS of other types of cancer: leukemia/lymphoma, solid tumors, and brain tumors. The Course of Life Questionnaire (CoLQ) was used to evaluate the achievement of milestones in psychosexual, autonomy, and social development. Mann-Whitney $U$ tests were used to compare scores of the CoLQ between survivors of childhood DTC and the 3 other groups separately.

Results: Thirty-nine survivors of childhood DTC (median age at evaluation 26.1 years, range 18.8-35.7) were included. Scores of survivors of childhood DTC on the CoLQ were compared to scores of 30 peer controls, 508 peers from the general Dutch population, and 351 CCS of other types of cancer. No differences were observed between scores on CoL between DTC survivors and peers. DTC survivors scored higher on social development than other CCS as a whole group $(\mathrm{P}=.008)$ and higher than leukemia/lymphoma ( $\mathrm{P}$ $=.004)$ and brain tumor survivors $(\mathrm{P}=.003)$ in sub-analyses. DTC survivors had higher psycho-sexual development-scores than brain tumor survivors $(\mathrm{P}$ $=.007$ ).

Conclusions: CoL in survivors of childhood DTC was comparable to peers and less affected than survivors of others types of childhood cancer.

\section{P3-05-152 \\ ASSOCIATION OF THYROID FUNCTION WITH FAT AND IRON CONTENTS OF LIVER}

$\underline{\text { Rehman Khattak }}{ }^{1}$, Jens-Peter Kühn ${ }^{2}$, Markus M. Lerch ${ }^{3}$, Henry

Völzke ${ }^{4}$, Till Ittermann ${ }^{5}$

${ }^{1}$ University Medicine Greifswald, Institute for Community Medicine Ship/Klinisch-Epidemiologische Forschung, Greifswald, Germany, Department of Zoology, Islamia College Peshawar (Cu), Pakistan, Greifswald, Germany, ${ }^{2}$ Greifswald, Germany, ${ }^{3}$ Department of Gastroenterology, University Medicine Greifswald, Germany, Greifswald, ${ }^{4}$ Ernst-Moritz-Arndt Universität Greifswald, Institut Für Community Medicine, Ship/Klinisch-Epidemiologische Forschung, Greifswald, Germany, ${ }^{5}$ University Medicine Greifswald, Institut für Community Medicine - Ship/Klinisch-Epidemiologische Forschung, Greifswald, Germany

Objective: Owing to the inconsistent findings regarding the association of thyroid dysfunction and liver dysfunction such as hepatic steatosis and liver iron overload, the purpose of this study was to investigate the relation between thyroid dysfunction and liver fat content, respectively iron content.

Methods: We obtained data from the Study of Health in Pomerania (SHIP). The final sample comprised 2734 subjects. Serum levels of thyroid-stimulat- ing hormones (TSH), free tri-iodothyronine (fT3), free thyroxine (fT4) were measured by immunochemiluminescent procedures. High and low TSH levels were defined using the cut-offs $0.40 \mathrm{mIU} / \mathrm{L}$ and $3.29 \mathrm{mIU} / \mathrm{L}$. Quantitative MRI was performed and liver fat content (proton-density fat fraction; PDFF) as well as liver iron content (transverse relaxation rate; R2*) was assessed using a 1.5-Tesla imaging system. Thyroid biomarkers were associated with PDFF and R2* by median regression analysis adjusted for confounders.

Results: There was no association between serum TSH levels and PDFF or R2*, but in females low TSH was inversely $[\beta=-0.60$; CI $(-0.80 ;-0.39)]$ associated with PDFF. High TSH $[\beta=-2.13$; CI $(-3.78 ;-0.49)]$ was inversely associated with PDFF content in men, but positively $[\beta=2.42$; CI $(0.68 ; 4.15)]$ in women. Except for liver iron content in males, we detected significant positive and inverse associations of fT3 and fT4 respectively with liver fat and iron content in the total population and in both sexes.

Conclusion: In our study we found inconsistent associations of thyroid dysfunction with hepatic steatosis. Likewise, results for iron deposits in the liver pointing towards different directions for males and females and for fT3 and fT4. Further studies are warranted to investigate this topic.

\section{P3-05-153 \\ BIGUANIDS AND THYROID RESISTENCE}

Armine Khroyan ${ }^{1}$, E. Toromanyan ${ }^{1}$, M. Badalyan ${ }^{1}, K$. Melikyan $^{1}$, A. Petrosyan ${ }^{1}$

${ }^{1}$ Yerevan State Medical University; Endocrinology, Yerevan, Armenia

Objectives: The objectives of our investigation are to find out the role of Metformin in thyroid resistance.

The research has been carried out at Reproductive Center «VitroMed» in 2015-2016.

100 women (ages $35 \pm 5$ ) with sings of hypothyroidism (weakness, dryness of skin, arterial hypotension, disturbance of menstrual cycle) and primary infertility have taken part in the clinical research.

They have been tested on TSH, FT4, anti-TPO, as well as undergone a thyroid ultrasound.

Results: TSH $32 \% \uparrow, 68 \%$ N FT 4 25\% $\uparrow, 75 \%$ N Anti-TPO 40\%,, $60 \%$ N

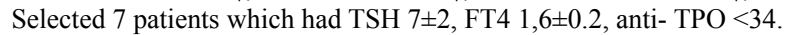

These 7 patients were suspected to have peripheral resistance against thyroid hormones and were prescribed $75-100 \mathrm{mkg}$ Levothyroxine during 3 months, but clinical signs and TSH were not improved. After patients were prescribed Levothyroxine (same dosage) and Metformin $500 \mathrm{mg}$ twice a day. And after 2 months tested TSH and results were -in 5 patient TSH- $3 \pm 0.7$, in 2 patients $5 \pm 0.9$, FT4 -in 7 patients were normal and they had no any symptoms of hypothyroidism.

Conclusion: The study revealed that the Metformin effect not only on insulin resistance, but also on thyroid resistance.

In cases when adequate amount of Levothyroxine was prescribed, but there was no improvement should be administered Metformin in place of increasing of Levothyroxine dosage.

\section{P3-05-154 \\ CORRELATION BETWEEN THYROID FUNCTION AND PSYCHOLOGICAL WELL-BEING \\ Radina Dimitrova $^{1}$, Mira Valentinova Siderova ${ }^{2}$, Kiril Hristozov ${ }^{3}$, Tatyana Radeva ${ }^{4}$, Hristo Kojuharov ${ }^{4}$ \\ ${ }^{1}$ Umhat St. Marina, Department of Endocrinology, Varna, Bulgaria, ${ }^{2}$ Univesity Hospital „St. Marina“, Medical University Varna, Department of Endocrinology, Varna, Bulgaria, 'University Hospital „St.Marina“, Department of Endocrinology, Varna, Bulgaria, ${ }^{4}$ University Hospital , St.Marina“, Department of Psychiatry, Varna, Bulgaria}

Introduction: Primary hypothyroidism (overt or subclinical) is found in a small percentage of depressive patients. On the other hand, depression is present more frequently in individuals with autoimmune thyroiditis, especially in those with hypothyroidism.

Objective: The aim of this study is to determine the prevalence of neuropsychiatric disorder among thyroxin treated hypothyroid patients after radioiodine therapy or thyroid surgery, among those with autoimmune thyroiditis, and to search a correlation between psychological distress, thyroid hormones and antibodies. 
Materials and Methods: 29 patients and 10 healthy controls participated in this study. Patients and controls were assessed with Depression Anxiety Stress Scales and with the 10 point scale of Montgomery and Asberg for the presence of depression. Thyroid function was evaluated by laboratory examination of TSH, FT4, FT3, TPO antibodies with Immulite 2000 and ultrasound with ALOKA $\alpha 7$.

Results: Psychological distress is observed in $72 \%$ in these patients and in $3 \%$ in controls ( $p$ 0.0244). Most common neuro-psychiatric disorder is self-established increased anxiety - in $66 \%$ in the patients' group and in $3 \%$ in healthy controls ( $\mathrm{p}$ 0.0604). Mean TSH level in patients with and without psychological distress is $2.04 \mu \mathrm{IU} / \mathrm{L}$ and $2.13 \mu \mathrm{IU} / \mathrm{L}$ respectively (p 0.6767). There was no significant difference in FT4 values (13,3 pmol/1 vs 14,3 pmol/1, p 0,2137) and TPO antibodies (189,9 U/ml vs $226 \mathrm{U} / \mathrm{ml}$, p 0,8932) between patient with and without neuro-psychiatric disorders. Mean FT3 level is 5.02 $\mathrm{pmol} / \mathrm{l}$ and $4.44 \mathrm{pmol} / \mathrm{l}$ respectively in these two groups of patients ( $\mathrm{p} 0.2004$ ).

Conclusion: Mood disorders screening is appropriate for individuals with hypothyroidism since mental well-being of patients may not be restored completely, regardless of the LT4 treatment. Our results indicate that mental wellbeing is independent of hormonal levels.

\section{P3-05-155}

\section{AN ATHYREOTIC PATIENT WITH T3 SECRETING} METASTASES AND NO CIRCULATING T4

Damiano Gullo ${ }^{1}$, Martina Tavarelli', Giulia Sapuppo ${ }^{1}$, Marco Russo ${ }^{1}$, Giacomo Lucio La Rosa ${ }^{1}$, Maria Luisa Arpi ${ }^{1}$, Sebastiano Squatrito ${ }^{1}$,

Gabriella Pellegriti ${ }^{1}$

${ }^{1}$ Endocrine Unit, Garibaldi-Nesima Hospital, University of Catania

Medical School, Catania, Italy

Introduction: The thyroid produces predominantly $\mathrm{T} 4$ and a small amount of T3, the active form of thyroid hormones (TH). T4 is considered a pro-hormone without intrinsec hormonal activity and is converted to T3 in peripheral target tissue cells by deiodinases. Athyreotic patients do not secrete endogenous $\mathrm{TH}$ and all circulating $\mathrm{T} 4$ and $\mathrm{T} 3$ originate from replacement treatment with L-T4. We describe a very rare case of an athyreotic patient with T3 secreting metastases that made the treatment with L-T4 unnecessary.

Case Report: A 78 years old male, presented with progressive dysphagia and dyspnea and a $6 \mathrm{~cm}$ palpable mass in the anterior region of the neck of increased thickness, with flushed skin and superficial venous reticules. He underwent total thyroidectomy at age 25 years, but since then did not perform any additional control. Under L-T4 $50 \mu \mathrm{g}$ /day $(0.7 \mu \mathrm{g} / \mathrm{Kg})$, his FT3 was 3.02 $\mathrm{pg} / \mathrm{ml}$ (2.6-4.4), FT4 $0.43 \mathrm{ng} / \mathrm{dl}(0.7-1.5)$ and TSH $1.8 \mu \mathrm{U} / \mathrm{ml}(0.4-4.5)$. CT scan showed a voluminous goiter with tracheal invasion and lung metastases. Biopsy confirmed an undifferentiated thyroid cancer. TG was 7,895 ng/ml with negative Ab Anti-TG. Considering the age and patient's clinical condition a tracheal prosthesis was placed and neck radiotherapy (30 Gray) was performed. The subsequent $\mathrm{CT}$ and bone scan showed a progressive reduction of the tracheal lumen by neoplastic infiltration and appearance of multiple bone and liver metastases. During a 2 year follow-up endogenous T3 production was suspected because of normal FT3 and low FT4 values. L-T4 was then reduced until a complete withdrawal. FT4 values gradually become undetectable while FT3 maintained within the normal limits $(2.88 \mathrm{pg} / \mathrm{ml})$, suggesting that the T3 sources were the metastatic sites. The patient had no sign or classical symptoms of hypothyroidism. The patient died 11 months later at the age of 81 for respiratory complications.

Conclusions: We describe the case of a patient without thyroid, no circulating T4, with plasma T3 in the normal range and apparent well-being relatively to the hypothyroid condition. This was likely due to the continuous release of $\mathrm{T} 3$ by the metastatic foci. This case is a unique natural in-vivo model that reinforces the general view that $\mathrm{T} 3$, not $\mathrm{T} 4$, is the only direct, biologically relevant agonist for nuclear $\mathrm{TH}$ receptor function.

\section{P3-05-156 \\ PREVALENCE OF AND FACTORS PREDICTING THYROID DYSFUNCTION AT THE TIME OF ST- AND NON-ST- ELEVATION MYOCARDIAL INFARCTION - THE THYRAMI 1 STUDY}

Avais Jabbar ${ }^{1}$, Lorna Ingoe ${ }^{2}$, Azfar Zaman ${ }^{3}$, Salman Razvi

${ }^{1}$ Institute of Genetic Medicine, Newcastle University, Newcastle University, UK, ${ }^{2}$ Queen Elizabeth Hospital, Gateshead, UK, ${ }^{3}$ Freeman Hospital and Newcastle University, Institute of Cellular Medicine, Newcastle, UK, ${ }^{4}$ Institute of Genetic Medicine, Newcastle Upon Tyne, UK

Introduction: Thyroid dysfunction is common affecting $5-10 \%$ of the adult population. Cardiovascular disease, including acute myocardial infarction (AMI), has been suggested as one of the adverse outcomes of thyroid disease. Furthermore, individuals with thyroid dysfunction at the time of AMI have worse outcomes. Despite this, there is scarce data on the prevalence of thyroid dysfunction in patients with AMI. We conducted a prospective observational multi-centre study to investigate the prevalence of and factors predicting thyroid dysfunction in patients with AMI.

Methods: Consecutive patients $(n=1970)$ from five hospitals within the North-East of England with AMI (both STEMI and NSTEMI) between January 2015 and December 2016 were recruited. Thyroid function tests were evaluated on the first available sample between 1-24 hours of admission. Patients on medications affecting thyroid function were excluded from the analysis. The prevalence of thyroid dysfunction was calculated and logistic regression analysis performed to assess relationship with demographic, clinical and biochemical variables.

Results: The thyroid status of the 1809 patients were: $78.6 \%(\mathrm{n}=1422)$ euthyroid, $17.3 \%(\mathrm{n}=314)$ subclinical hypothyroidism $(\mathrm{SCH}), 1.2 \%$ subclinical hyperthyroidism, $2.7 \%(\mathrm{n}=49)$ low T3 syndrome (LT3S), and overt hypothyroidism and hyperthyroidism $0.5 \%$ each. Predictors for $\mathrm{SCH}$ were increasing age with OR (Odds ratio $(95 \% \mathrm{CI})$ of 1.02 (1.01 - 1.04); $\mathrm{p}=0.001$, STEMI with OR 2.11 (1.52 - 2.93); $\mathrm{P}<0.001$ and troponin $\mathrm{T}$ with OR 1.04 $(1.03-1.05) ; \mathrm{p}=0.001$. The only significant predictor for LT3S was increasing age OR 1.05 (1.02 - 1.08); $\mathrm{p}=0.003$.

Conclusion: Thyroid dysfunction is common in patients presenting with AMI particularly SCH that is present in 1 in 6 . Furthermore, troponin rise is higher in SCH patients with AMI, independent of other factors, which may partly explain the increased morbidity and mortality observed in these patients. Interventional studies are necessary to evaluate if treatment postAMI improves outcomes.

\section{P3-05-157 \\ EVALUATION OF THE INTERRELATIONSHIPS BETWEEN THYROID FUNCTION, AUTOIMMUNITY, INSULIN RESISTANCE, LIPID PROFILE, HOMOCYSTEINE AND C-REACTIVE PROTEIN IN PATIENTS WITH AUTOIMMUNE THYROIDITIS}

Celestino Neves ${ }^{1}$, João Sérgio Neves ${ }^{1}$, Sofia Castro Oliveira ${ }^{1}$, Oksana Sokhatska ${ }^{2}$, César Esteves ${ }^{1}$, Miguel Pereira ${ }^{1}$, José Luís Medina ${ }^{3}$, Luís Delgado $^{2}$, Davide Carvalho 4

${ }^{1}$ Department of Endocrinology, Diabetes and Metabolism, São João Hospital Center. Faculty of Medicine of Porto University, Porto, Portugal, ${ }^{2}$ Immunology Department, São João Hospital, Faculty of Medicine, University of Porto, Porto, Portugal, ${ }^{3}$ Faculty of Medicine of Porto University, Porto, Portugal, ${ }^{4}$ Department of Endocrinology, Diabetes and Metabolism, São João Hospital Center. Faculty of Medicine of Porto University, Institute for Research and Innovation in Health (I3s), Porto, Portugal

Introduction: Since thyroid dysfunction has a major prevalence in the general population, it is essential to understand and establish the association between different levels of TSH, autoimmunity and an increased cardiovascular risk.

Objective: To evaluate the relationship between thyroid function, autoimmunity, insulin resistance and cardiovascular risk factors in patients with autoimmune thyroiditis (AIT). 
Methods: We defined three groups based on TSH levels: TSH $<2.5 \mu \mathrm{UI} /$ $\mathrm{ml}$, TSH 2.5 to $5.0 \mu \mathrm{UI} / \mathrm{ml}$ and TSH $>5.0 \mu \mathrm{UI} / \mathrm{ml}$. We analyzed the levels TSH, FT4, FT3, total cholesterol, HDL, LDL, apolipoprotein A1 (ApoA1), ApoB, Lipoprotein (a) [Lp(a)], homocysteine, anti-Tg, anti-TPO, CRP, folic acid and vitamin B12 levels, insulin resistance markers comprising HOMA-IR and WBISI (Whole-Body Insulin Sensitivity Index). For the statistical analysis we employed Spearman correlation, t-tests and Mann-Whitney tests. Data are presented as mean \pm standard deviation or median (25-75th percentile) according to distribution.

Results: Within our sample of 353 subjects with AIT 94\% were female. The mean age was $47.0 \pm 16.3$ years. Two hundred and fifty-three individuals belonged to the group TSH $<2.5$ while the group TSH 2.5-5.0 comprised 62 individuals, and the group TSH $>5.0$ comprised 27 individuals. There were no significant differences regarding the mean age or the mean BMI between groups. The group TSH $>5.0$ presented higher levels of HOMA-IR when compared to the other two groups [2.96 (1.76-4.59) in the group TSH $>5.0$ vs $1.86(0.97-2.56)$ in the group TSH 2.5-5.0 and $1.58(1.06-2.46)$ in the group $\mathrm{TSH}<2.5, \mathrm{p}=0.002]$. Regarding the total group, we found significant negative correlations between TSH and age $(\mathrm{r}=0.183, \mathrm{p}=0.001)$, FT3 and both HDL $(\mathrm{r}=0.118, \mathrm{p}=0.028)$ and ApoA1 $(\mathrm{r}=0.129, \mathrm{p}=0.024)$, and also between FT4 and IGI $(\mathrm{r}=0.112, \mathrm{p}=0.045)$. TSH was positively correlated with HOMA-IR $(\mathrm{r}=0.146, \mathrm{p}=0.018)$, and negatively correlated with WBISI $(\mathrm{r}=-0.146, \mathrm{p}=0.018)$ in the total group. Concerning the group TSH $<2.5$, positive correlations were observed between TSH and HDL ( $\mathrm{r}=0.136, \mathrm{p}=0.031)$, and between FT4 and both CRP $(r=0.136, p=0.037)$. Anti-TPO antibodies were positively correlated with total cholesterol $(\mathrm{r}=0.141, \mathrm{p}=0.026)$ while anti- $\mathrm{Tg}$ positively correlated with $\mathrm{ApoB}(\mathrm{r}=0.165, \mathrm{p}=0.013)$ and showed negative correlations with HDL $(\mathrm{r}=-0.139, \mathrm{p}=0.028)$ in the group $\mathrm{TSH}<2.5$. We found negative correlation in the group TSH 2.5-5.0 between anti-Tg and total cholesterol $(\mathrm{r}=0.371$, $\mathrm{p}=0.004, \mathrm{ApoB}(\mathrm{r}=-0.342, \mathrm{p}=0.022)$, and $\mathrm{Lp}(\mathrm{a})(\mathrm{r}=-0.470, \mathrm{p}=0.001)$, and positive correlations between FT3 and HDL $(r=0.358, p=0.030)$, and vitamin $\mathrm{B} 12$ $(\mathrm{r}=0.398, \mathrm{p}=0.024$.

Conclusions: Our results suggest that thyroid autoimmunity may affect lipid profile independent of thyroid function in patients with AIT.

\section{P3-06 Iodine and Reproduction}

\section{P3-06-158 \\ UNEXPECTED POSITIVE TSH-RECEPTOR ANTIBODIES (TR-AB) DURING THE SECOND TRIMESTER OF PREGNANCY: A THERAPEUTIC Challenge \\ Maria-Cristina Burlacu $^{14}$, G.J. Kahaly ${ }^{4}$, D. Gruson ${ }^{2}$, L. Baldeschi ${ }^{3}$, A. Boschi $^{3}$, C. Daumerie ${ }^{1}$ \\ ${ }^{1}$ Department of Endocrinology, University Hospital St-Luc, Brussels, Belgium; ' ${ }^{2}$ epartment of Clinical Biology, University Hospital St-Luc, Brussels, Belgium; ${ }^{3}$ Department of Neuroophtalmology, University Hospital St-Luc, Brussels, Belgium; ${ }^{4}$ Molecular Thyroid Research Laboratory, Johannes Gutenberg University Medical Center, Mainz, Germany}

Introduction: The recent 2017 Guidelines of the American Thyroid Association disease during pregnancy recommend maternal serum determination of TR-Ab during early pregnancy in patients with a past history of Graves' disease (GD) treated with ablation. If maternal TR-Ab is undetectable or low in early pregnancy, no further TR-Ab testing is needed (recommendation 52).

Case Report: We report the case of a 40-Year-old Caucasian female, with GD and active moderately severe Graves' Orbitopathy (GO) treated with intravenous high doses of steroids and subsequent orbital decompression surgery six years ago. A total thyroidectomy was performed two years later because of relapse of hyperthyroidism and wish of a second child. As TR-Ab was undetectable when measured by Elisa Medizym immunoassay, the pregnancy was authorized. Surprisingly, TRAb levels were positive at 20 weeks of pregnancy (TR-Ab $15.8 \mathrm{UI} / \mathrm{L}$, normal value $<2.5$ ) and were exclusively stimulatory when measured with the help of a FDA-cleared cell-based bioassay (SRR (specimen-to-reference ratio) $407 \%$, normal value $<140 \%$;). The pregnancy was prematurely terminated at 39.4 weeks for preeclampsia. The newborn was hyperthyroid due to the transplacental transmission of maternal TSAb. The newborn was put on thiamazole which led to biochemical euthyroidism and disappearance of TRAb within three months.

Conclusions: This case illustrates that undetectable TR-Ab during the first trimester does not preclude the seroconversion later in pregnancy. It also emphasizes the clinical relevance and utility of measuring functional TR-Ab in all patients with a history of autoimmune thyroid disease to timely diagnose potential fetal thyroidal complications. The unusual TRAb evolution in this patient raised the question of an abnormal immune tolerance of the pregnancy.

\section{P3-06-159 \\ IS URINARY IODINE IN EARLY PREGNANCY ASSOCIATED WITH GESTATIONAL DIABETES MELLITUS (GDM)?}

Seda Sancak ${ }^{1}$, Hasan Aydın², Ali Özdemir ${ }^{3}$, Mehmet Sargın ${ }^{4}$, Asuman Orçun $^{5}$, Ayhan Çelik ${ }^{6}$, Murat Erdogan ${ }^{7}$

${ }^{1}$ Ifatih Sultan Mehmet Training and Research Hospital, 2department of Endocrinology and Metabolism, Medical School of Marmara University, Istanbul, Turkey, ${ }^{2}$ Yeditepe University Hospital, Division of Endocrinology and Metabolism, İstanbul, Turkey, ${ }^{3}$ Fatih Sultan Mehmet Training and Educational Hospital, Departman of Internal Medicine, Istanbul, Turkey, Istanbul, Turkey, ${ }^{4}$ Kartal Lutfi Kirdar Education and Research Hospital, Diabetes Department, Istanbul, Turkey, Istanbul, Turkey, ${ }^{5}$ Kartal Lutfi Kirdar Training and Educational Hospital, Division of Biochemistry, Istanbul, Turkey, ${ }^{6}$ Fatih Sultan Mehmet Training and Educational Hospital, Division of Obstetrics and Gynecology, Istanbul, Turkey, ${ }^{7} \mathrm{lbni}$-I-Sina Hastanesi, Endokrinoloji Ve Met Hst B.D., Sihhiye, Ankara, Turkey

Background: I deficiency (ID) is recognised as a public health problem, and ID continue to be major public health problem in Turkey, affecting children and pregnant women.

The scientific evidence linking thyroid dysfunction with diabetes is growing large, but data investigating the relationship between iodine deficiency levels and GDM are inconsistent. However, it is not clear whether iodine deficiency status in early pregnancy is associated with the risk of GDM.

Methods: 346 pregnant women included in the study and were studied during early (11th-13th week of gestation) and at the beginnig of the 3rd trimester (24th-28th week of gestation). GDM was diagnosed based on 75-g oral glucose tolerance test.Serum concentration of TSH, fT3,fT4,insülin, urine iodine were measured using specific assays.

Results: 48 patients diagnosed as (GDM) included in the study. The TSH, FT4 and FT3 flevels were not significantly different when comparing 1st and beginning of the 3rd trimester. Maternal urinary iodine levels (UIC) in the 1 st trimester $110,06 \pm 51,4898 \mu \mathrm{g} / 1$ and $92,14 \pm 60,98 \mu \mathrm{g} / 1$ at the beginning of the 3 rd trimester. There was no significantly difference in the urinary iodine levels between 1 st trimester and at the beginning of the 3rd trimester.

Urinary iodine was not correlated with glucose, insülin concentrations, and insülin resistance (HOMA-IR). Among the TSH, fT3, fT4 levels in the 1st trimester or at the beginning of the 3rd trimester was not correlated with UIC in the 1 st trimester or at the beginning of the 3rd trimester.

Conclusions: Our study was not powered to detect associations between maternal iodine status and GDM. We have not identified urinary iodine deficiency in early pregnancy as a predictor of GDM.

\section{P3-06-160 \\ RELATIVES OF THE FIRST DEGREE WITH AUTOIMMUNE THYROID DISEASES LIVING IN THE REGION OF MILD IODINE DEFICIENCY}

Oksana Rymar' ${ }^{1}$, Anna Pyankova ${ }^{1}$, Svetlana Mustafina ${ }^{1}$

${ }^{1}$ Federal State Budgetary of Scientific Institution «institution of Internal and Preventive Medicine», Novosibirsk, Russian Federation

Objectives: The aim of this study was to determine the frequency Hashimoto`s thyroiditis (HT) or Grave`s disease (GD) in families with autoimmune thyroid disease (AITD).

Methods: The condition for inclusion in the study was the presence of a family member first-degree relatives with Hashimoto's thyroiditis (HT) or Grave's disease (GD), the study included 49 families. Family history of AITD was traced for two generations (parent-offspring) and for one generation (sib- 
lings). HT was defined as TAb positivity and hypothyroidism (subclinical/ overt).

Results: The estimate number of patients with GD and HT was 34\% and $66 \%$ persons, respectively. Family history of AITD was traced for two generations (parent-offspring, $57.4 \pm 1.6$ and $32.2 \pm 1.5$ years) in $77 \%$ (38 families), and for one generation in $23 \%$ ( 11 families) (siblings, whose average age was $33.6 \pm 3.5$ years). In one family $(2.5 \%)$ the disease was traced in both parents and their offspring. A hundred patients with AITD were examined. Family history of AITD was traced for parent-offspring with a diagnosis of HT 55\% (21 families), with a diagnosis of GD $26 \%$ (10 families) and with a diagnosis of HT and GD - 19\%. The prevalence of HT was $45 \%$ (5 families), of GD was $18 \%$ ( 2 families), and of HT and GD was 36\% (4 families) in siblings.

Conclusion: Among patients with a positive family history for AITD is dominated by a pair of parent-offspring with a diagnosis of HT (55\%). Among parents and offspring with AITD, the number of patients who reached compensation was $40 \%$ and $36 \%$.

\section{P3-06-161 \\ IS THERE ANY DIFFERENCE IN THYROID FUNCTION BETWEEN NORMAL PREGNANT WOMEN AND WOMEN WITH GESTATIONAL DIABETES MELLITUS (GDM)?}

George Simeakis ${ }^{1}$, Elli Anagnostou², Marina Mitropoulou ${ }^{3}$, Paraskevi Kontou $^{3}$, Katerina Saltiki ${ }^{2}$, Vassiliki Sarantopoulou ${ }^{3}$, Maria Alevizaki ${ }^{2}$, Eleni Anastasiou ${ }^{3}$

${ }^{1}$ Endocrine Unit, Dept Medical Therapeutics, Alexandra Hospital, Athens University School of Medicine, Endocrinology Dept, Alexandra Hospital, Athens, Athens, Greece, ${ }^{2}$ Endocrine Unit, Dept Medical Therapeutics, Alexandra Hospital, Athens University School of Medicine, Athens, Greece, ${ }^{3}$ Endocrinology Dept, Alexandra Hospital, Athens, Athens, Greece

Objectives: Thyroid function as well as glucose homeostasis are of great importance for a successful pregnancy outcome. The impact of thyroid hormone levels and thyroid autoimmunity in pregnancy has been widely studied, however results are controversial. The aim of this study was to examine aspects of thyroid function in normal pregnant women and those with GDM.

Methods: We studied 225 women during 24th-32nd gestation week. Demographic characteristics were recorded and a 75 gr OGTT was performed. Plasma glucose and insulin levels were measured at time $0^{\prime}-60^{\prime}-120^{\prime}$. HbA1c,TSH,FT4,AntiTPO and AntiTg were evaluated at time 0 '.

Results: $105 / 225(46.7 \%)$ were diagnosed with GDM according to IADSPG/WHO criteria. Women were classified in two groups: normal(N) and GDM. Women in the GDM group compared to $\mathrm{N}$ were significantly older(33.5 \pm 6.7 vs $29.4 \pm 7.6)$, had greater BMI( $30.5 \pm 5.6$ vs $27.8 \pm 4.7)$ and lower FT4 levels(12.1 \pm 2.6 vs $13.0 \pm 2.1),(\mathrm{p}<0.001)$. ROC analysis was performed and a cut off point for FT4 of $12.3 \mathrm{pmol} / \mathrm{lt}$ was identified. Women with FT4 $<12.3$ compared to those with FT4 $\geq 12.3$ were older, had greater BMI, higher HbAlc and higher insulin levels. Women in the GDM group had more often FT4 $<12.3$ compared to normal $(57.3 \%$ vs $34.4 \%, \mathrm{p}=0.003)$; FT4 $<12.3$ was associated with a higher risk of developing GDM (OR:2.6,CI95\%:1.4-4.5). Accordingly, women with FT4 $<12.3$ were more often obese $\left(\mathrm{BMI} \geq 30 \mathrm{kgr} / \mathrm{m}^{2}\right)$ compared to those with FT4 $\geq 12.3$ (46.3\% vs $26.2 \%$, p $<0.001$ ); women with FT4 $<12.3$ had higher risk to present obesity (OR:2.4,CI95\%:1.3-4.4). In multiple regression analysis model $\mathrm{BMI} \geq 30 \mathrm{kgr} / \mathrm{m}^{2}(\mathrm{p}<0.001)$ as well as FT4 $<12.3(\mathrm{p}=0.012)$ proved to be independent factors of developing GDM. TSH levels and autoimmunity didn't show any significant association with GDM.

Conclusions: This study reaffirms the crucial role of obesity in developing GDM. The correlation between low FT4, with both GDM and obesity, indicates possible FT4 involvement in the development of GDM among obese women. The pathogenic mechanisms of obesity-related thyroid dysfunction leading to GDM development need further investigation.

\section{P3-06-162 \\ REGISTRY BASED EVALUATION OF TREATMENTS RELATED TO THYROID DISORDERS AFTER 15 YEARS OF MANDATORY IODINE FORTIFICATION IN DENMARK}

Betina Thuesen ${ }^{1}$, Line Tang Moellehave ${ }^{2}$, Allan Linneberg ${ }^{3}$, Inge Bulow Pedersen ${ }^{4}$, Nils Knudsen ${ }^{5}$, Lars Ovesen ${ }^{6}$, Hans Perrild ${ }^{7}$, Lone B. Rasmussen ${ }^{8}$, Torben Jørgensen ${ }^{9}$

${ }^{1}$ Research Centre for Prevention and Health, Centre for Health, The Capital Region of Denmark, Glostrup, Denmark, Glostrup, Denmark, ${ }^{2}$ Research Centre for Prevention and Health, Centre for Health, The Capital Region of Denmark, Glostrup, Denmark, ${ }^{3}$ Research Centre for Prevention and Health, Centre for Health, The Capital Region of Denmark, Department of Clinical Experimental Research, Rigshospitalet, Glostrup, Denmark, Department of Clinical Medicine, Faculty of Health and Medical Sciences, University of Copenhagen, Denmark, Glostrup, Denmark, ${ }^{4}$ Dept. Of Medicine and Endocrinology, Medicinerhuset, Mølleparkvej Aalborg, Aalborg, Denmark, ${ }^{5}$ Department of Endocrinology, Bispebejrg University Hospital, Copenhagen, Denmark, Faculty of Health and Medical Sciences, University of Copenhagen, Denmark, Copenhagen, Denmark, ${ }^{6}$ Department of Gastroenterology, Slagelse Hospital, Slagelse, Denmark, Slagelse, Denmark, ${ }^{7}$ Department of Endocrinology, Bispebjerg University Hospital, Copenhagen, Denmark, Copenhagen, Denmark,

${ }^{8}$ Copenhagen, Denmark, ${ }^{9}$ Research Centre for Prevention and Health, The Capital Region of Denmark, Glostrup, Denmark, Faculty of Medicin, Aalborg University, Aalborg, Denmark, Faculty of Health Sciences, Copenhagen, Denmark, Glostrup, Denmark

Objectives: In Denmark, mandatory iodine fortification (IF) of salt was initiated in year 2000 because of mild/moderate iodine deficiency. The aim of this study was to evaluate the effect of IF on use of thyroid medication, prevalence of thyroid surgery and use of radio iodine during 15 years of follow-up.

Methods: Information from the Civil Registration register covering all Danish citizens were linked to data on use of thyroid medication from the Register of Medicinal Product Statistics and surgery and radio-iodine treatments for benign thyroid disorders from the Danish National Patient Registry. Results were standardized to the age- and sex-composition of the Danish population in 2000.

Results: Use of anti-thyroid medication increased prior to IF and continued to increase until the prevalence peaked in 2003 (458 users/100,000 person years (PY)). Since 2003 the prevalence has decreased to 341 users $/ 100,000$ PY in 2015 and the pre-fortification level was reached in 2011. Use of thyroid hormone therapy increased continually from 1995 to 2015 and nearly doubled from 1,200 users/100,000 PY in 2000 to 2,182 users/100,000 PY in 2015. Numbers of surgery procedures related to benign thyroid diseases were relatively constant between 1995 and 2008 (25.5-29.5 procedures/100,000 PY). Between 2009 and 2015 the numbers were slightly higher. Numbers of treatments with radio-iodine related to benign thyroid diseases were fairly constant.

Conclusions: A temporary increase in use of anti-thyroid medication was expected after introduction of IF. Also a slight increase in use of thyroid hormone therapy was expected. However, a constantly increasing use of thyroid hormone therapy from 1995 to 2015 cannot be explained alone by IF introduced in 2000 . Unexpectedly, IF had no significant effects on thyroid surgery procedures or use of radio-iodine indicating that other factors than intake of iodine influenced treatment of thyroid diseases in the public Danish health care system.

\section{P3-06-163 \\ TSH REFERENCE RANGES IN THE ASSESSMENT OF THYROID FUNCTION DURING PREGNANCY \\ Catarina Machado ${ }^{1}$, Patrícia Tavares ${ }^{1}$, Sara Monteiro ${ }^{1}$, Gustavo \\ Rocha $^{1}$, Marta Barbosa ${ }^{1}$, Maria João Oliveira ${ }^{1}$ \\ ${ }^{1}$ Centro Hospitalar de Vila Nova de Gaia/Espinho, Epe, Vila Nova de Gaia, Portugal}

Introduction: Pregnancy has an important impact on thyroid function and thyroid disorders in pregnancy are common. For women with no previous thyroid disease, new guidelines recommend using population and trimester- 
specific reference ranges for serum $\mathrm{TSH}$. If these reference ranges are not available, an upper reference limit of $4,0 \mathrm{uUI} / \mathrm{mL}$ may be used.

Objectives: To compare the prevalence of subclinical hypothyroidism in pregnancy using an upper reference of TSH of $4,0 \mathrm{uUI} / \mathrm{mL}$ versus previous cutoffs of $2,5 \mathrm{uUI} / \mathrm{mL}$ for the first trimester and $3,0 \mathrm{uUI} / \mathrm{mL}$ for the second and third trimesters.

Methods: Retrospective review of pregnant women with no previous thyroid disease, referred to endocrinology consultation following thyroid dysfunction detected during pregnancy in the last 4 years.

Results: 160 women were included, mean age $30,7 \pm 6,5$ years. $65(40,6$ $\%$ ) patients were referred in the first trimester (mean TSH 4,7 $\pm 2,8 \mathrm{uUI} / \mathrm{mL}$ ), 66 $(41,3 \%)$ in the second (mean TSH $5,0 \pm 1,6 \mathrm{uUI} / \mathrm{mL})$ and $29(18,1 \%)$ in the third (mean TSH 4,7 $1,6 \mathrm{uUI} / \mathrm{L}) .96$ patients $(60 \%)$ had TSH above $4,0 \mathrm{uUI} / \mathrm{mL}$. Iodine supplementation led to normalization of TSH in $27(16,9 \%)$ women. Levothyroxine was given to 106 patients (66,3\%): $38,7 \%, 51,9 \%$ and $9,4 \%$ in the first, second and third trimester, respectively. If an upper reference limit of $4,0 \mathrm{uUI} / \mathrm{mL}$ was used for TSH, 64 patients would have been characterized as euthyroid and treatment with levothyroxine would have been avoided in 28 patients $(26,4 \%): 12$ in the first trimester, 14 in the second and 2 in the third. Of these, $17,9 \%(\mathrm{n}=5)$ needed levothyroxine supplementation after pregnancy, the majority of which was diagnosed in the first trimester. There was no association between subclinical hypothyroidism and adverse pregnancy outcomes.

Conclusion: In this study, using a TSH cutoff of $4 \mathrm{uUI} / \mathrm{mL}$ following 2017 ATA guidelines would reduce the incidence of hypothyroidism in $40 \%$. Nonetheless, 5 women $(17,9 \%)$ who needed treatment with levothyroxine after pregnancy would have been missed.

\section{P3-06-164 \\ IODINE-RICH FOOD CONSUMPTION IN AGE GROUPS OF THE LATVIAN ADULT POPULATION FROM 1998 TO 2014}

leva Kalere $^{1}$, leva Strele ${ }^{1}$, Ilze Konrade ${ }^{1}$, Biruta Velika ${ }^{2}$, Daiga

Grinberga ${ }^{2}$, Inese Gobina ${ }^{1}$, Anita Villerusa ${ }^{1}$, Iveta Pudule ${ }^{2}$

${ }^{1}$ Riga Stradins University, Riga, Latvia, ${ }^{2}$ Centre for Disease Prevention and Control, Riga, Latvia

Objectives: Iodine is required for thyroid hormone synthesis, deficiency leading to thyroid structure changes, hypothyroidism. The main source of iodine is food: fish, dairy products and eggs. The most efficient way to prevent iodine deficiency is salt iodization. In Latvia a national program of iodization doesn't exist.

Methods: Data from the Health Behaviour among Latvian Adult Population survey for 1998-2014 were used. Study population was split into two age groups according to the median age of 39 years: $15-39$ and 40-64 years. Consumption prevalence, $\%$ and $95 \%$ confidence interval $(\mathrm{CI})$ and trends were calculated.

Results: The mean period prevalence of iodized salt consumption was higher in the younger group as compared to the older group: $10.0 \%(9.4,10.6)$ vs. $9.0 \%(8.4,9.6)$, respectively, $\mathrm{p}=0.021$. The prevalence increased 1998 2004 in both age groups but has decreased since then, $\mathrm{p}$ for trend $<0.001$.

A total of $57.5 \%(56.5,58.5)$ of participants in the younger group consumed fish during the previous week whereas it reached $71.7 \%(70.8,72.6)$ in the older group, $\mathrm{p}<0.001$
At least one glass of milk daily was consumed by $54.9 \%(53.9,55.9)$ in the younger group and a lower percentage of $49.0 \%(48.0,50.0)$ in the older group, $\mathrm{p}<0.001$. The prevalence of both fish and milk consumption followed a negative trend in both age groups during the study period, $\mathrm{p}$ for trend $<0.001$.

Egg consumption was higher in the older group than younger participants: $89.0 \%(88.4,89.6)$ vs. $83.1 \%(82.3,83.8), \mathrm{p}<0.001$. Egg consumption prevalence increased in both groups, $\mathrm{p}$ for trend 0.022 and 0.001 , respectively.

Conclusions: Iodized salt and milk consumption is higher in the younger age group, whereas fish and eggs are more consumed among respondents aged 40 and more. A similar negative trend in both age groups is observed regarding the iodine-rich food (iodized salt, milk, fish) consumption.

\section{P3-06-165 \\ A LONGITUDINAL STUDY OF MATERNAL THYROID HORMONES AND NEURODEVELOPMENTAL BEHAVIORAL CLINICAL MILESTONES IN OFFSPRING}

Nikku Yadav ${ }^{1}$, Atul Kathait ${ }^{2}$, Asha Chandola-Saklani ${ }^{3}$

${ }^{1}$ Centre for Biosciences and Clinical Research, School of Biosciences, Apeejay Stya University, School of Biosciences, Gurgaon, India,

${ }^{2}$ Centre for Biosciences and Clinical Research, School of Biosciences, Apeejay Stya University, Gurgaon, India, ${ }^{3}$ Dept Biosciences \& Clinical

Research, Sohna- Gurgaon, India

Background: During pregnancy, normal physiological changes occur concomitant with an enhanced metabolic demand for thyroxine/iodine. If dietary iodine intake is sufficient, normal equilibrium is achieved. With deficient iodine intake, pathological alterations are belived to occur leading to maternal thyroxineaemia and impaired development in the offsprings. However, these conclusions have been derived chiefly from cross sectional cohorts, there being very few longitudinal studies with follow up of the offspring permitting observation of developmental traits with respect to uterine history of thyroid hormones. Present study is an attempt in that direction.

Objective: To assess the role of iodine status and thyroid hormones in utero on the development of offspring.

Method: Present study is a follow up of offspring born to 438 young healthy women enrolled from 13 villages of Haryana after exclusion criteria with known hormonal history. Till date 129 offspring have been tracked. To monitor development neurodevelopmental behavioral clinical milestoneS(NDCM) prescribed WHO were used at 4,9 and 18months. Each milestone was given a score of 1 and correalted with maternalTSH,FT4. IECapproval \& informed consent were obtained. Data on on dietary-iodine intake, smoking habits, BMI,BP were recorded.

Results: All mothers used iodisided salt, had BMI and BP within normal range. None of then were smokers

Conclusion: It can be concluded that $7.7 \%$ offspring born to mothers with relatively high TSH,low FT4 indicated delayed development. Most striking is that all mothers used iodized salt throughout pregnancy. Obviously factors other than iodine deficiency may be involved.

Financial assistance from Apeejay Education Society is acknowledged.

Table 1. Maternal TSH and FT4 with neurodevelopemental behavioural clinical milestones (for abstract P3-06-165)

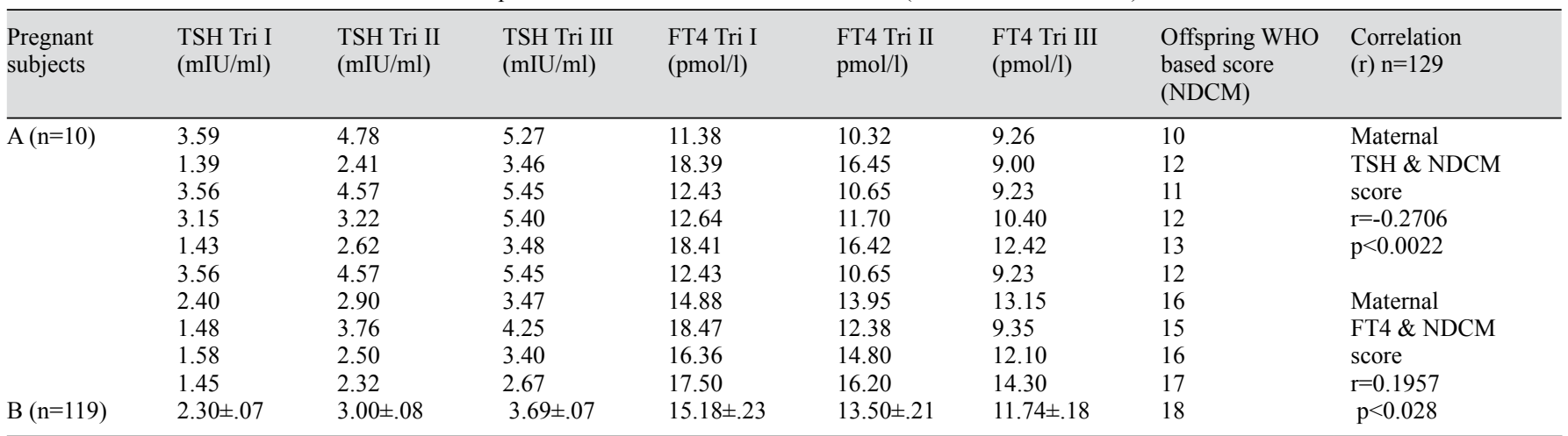

A: subjects with offspring showing delayed development, B: subjects with normal offspring 


\section{P3-06-166 \\ EVOLUTION OF THE IODINE STATUS IN PREGNANT WOMEN AFTER UNIVERSAL SALT IODIZATION IN ROMANIA}

Monica Livia Gheorghiu ${ }^{1}$, Horea Ursu' ${ }^{1}$, Irina Dumitrascu ${ }^{2}$, Ionela Pascanu $^{3}$, Carmen Georgescu ${ }^{4}$, Dan Mihu ${ }^{4}$, Delia Corina ${ }^{5}$, Geanina Mirela Toma ${ }^{5}$, Mihaela Stanciu 6 , Dragos Popescu ${ }^{6}$, Corina Raducanu Lichiardopol $^{7}$, Mihaela Vlad ${ }^{8}$, Ramona Aldea ${ }^{9}$, Stefania Tudorache ${ }^{7}$, Mihaela Vasile ${ }^{7}$, Mihail Popescu ${ }^{10}$, Claudia Camelia Podia Igna ${ }^{11}$, Mariana Purice ${ }^{12}$, Mihaela Nanu ${ }^{13}$, Alin Stanescu ${ }^{5}$, Mihaela Simescu ${ }^{12}$, Adina Mariana Ghemigian ${ }^{12}$

1"C.I.Parhon“ National Institute of Endocrinology, „C. Davila“ University of Medicine and Pharmacy, Bucharest, Romania, "2"Gr. T. Popa“ University of Medicine and Pharmacy, lasi, Romania, ${ }^{3}$ University of Medicine and Pharmacy Targu Mures, Targu Mures, Romania, 4"iuliu Hatieganu" University of Medicine and Pharmacy, Cluj-Napoca, Romania, "5"alessandrescu Rusescu" National Institute for Mother and Child Care, Bucharest, Romania, "'I. Blaga" University, Faculty of Medicine, Sibiu, Romania, ${ }^{7}$ Craiova University of Medicine and Pharmacy, Craiova, Romania, ${ }^{8 " V}$ Babes" University of Medicine and Pharmacy, Timisoara, Romania, ${ }^{9} \mathrm{Campulung}$ Hospital, Campulung, Romania, ${ }^{10}$ Ramnicu Sarat County Hospital, Ramnicu Sarat,

${ }^{11}$ Astra Clinic, Sibiu, Romania, ${ }^{12 " C}$.I.Parhon" National Institute of Endocrinology, Bucharest, Romania, 13"alessandrescu Rusescu“" National Institute for Mother and Child Care, Bucharest

Introduction: Before the implementation of Universal Salt Iodization in January 2003, Romania was known with mild iodine deficiency in more than $2 / 3$ of its counties. Pregnant women are more prone to iodine deficiency due to their higher iodine requirement.

Objective: To assess the evolution of the iodine status in Romanian pregnant women since 2001 to 2016, after implementation of the Universal Salt Iodization ( $25-40 \mathrm{mg}$ iodine $/ \mathrm{kg}$ salt).

Subjects and Methods: In 2016 median urinary iodine concentration (UIC) was evaluated by spectrophotometry (Sandell - Koltoff method) in the morning urine collected from 498 pregnant women in the $3^{\text {rd }}$ trimester of pregnancy from 15 counties). The study was approved by the local Ethics Committee. The results were compared with those of 4 previous studies using the same method: study 1 (1066 women from 11 counties in 2000-2001), study 2 (1387 women from 11 counties, $72 \%$ in the $1^{\text {st }}$ trimester, in Oct 2003 - Jan 2004), study 3 (1283 women from 11 counties in $2004-2005$ ), study 4 [1595 women from 21 counties (national representative sample), in the $3^{\text {rd }}$ trimester, in $2004-2005$ ).

Results: Median UIC in the 2016 study group was $121.3 \mathrm{mcg} / \mathrm{L}$ (range $5-623 \mathrm{mcg} / \mathrm{L}$ ), reflecting mild iodine deficiency during pregnancy (normal values $\geq 150 \mathrm{mcg} / \mathrm{L}$ in $61 \%$ of pregnant women). Median urinary iodine/creatinine ratio $=105.2 \mathrm{mcg} / \mathrm{L}$. Median UIC in the previously reported studies $1-4$ were $52.5 \mathrm{mcg} / \mathrm{L}, 51 \mathrm{mcg} / \mathrm{L}, 55 \mathrm{mcg} / \mathrm{L}, 73 \mathrm{mcg} / \mathrm{L})$, reflecting moderate iodine deficiency. Severe iodine deficiency (UIC $<50 \mathrm{mcg} / \mathrm{L}$ ) was recorded in $13.7 \%$ of women in 2016 , compared to $48.3 \%$ in study 2 and $33.7 \%$ in study 4. Iodized salt was consumed by $86 \%$ of women in 2016 , compared to $46 \%$ in study 2 and $76 \%$ in study 4 .

Conclusions: Median UIC has progressively improved in Romanian pregnant women after Universal Salt Iodization. However mild iodine deficiency is still prevalent in this population and efforts should be make to increase the use of iodine supplements during pregnancy.

\section{P3-06-167 \\ MONITORING OF ADVERSE PREGNANCY OUTCOME IN RURAL POPULATION FROM IODINE DEFICIENT ZONES: A MULTI-CENTRIC PILOT STUDY FROM NORTHERN INDIA}

Snehlata Tendi ${ }^{1}$, Nikku Yadav², Jayanti Semwaß ${ }^{3}$, Ruchi Juyaß ${ }^{3}$, Asha Chandola-Saklanit

${ }^{1}$ Centre for Biosciences and Clinical Research, School of Biosciences, Apeejaystya University, Sohna-Gurgaon, India, ${ }^{2}$ Centre for Biosciences and Clinical Research, School of Biosciences, Apeejay Stya University, School of Biosciences, Gurgaon, India, ${ }^{3}$ Department of Community

Medicine, Himalayan Institute of Medical Science, Swami Rama Himalayan University, Jolly Grant, Dehradun, India, ${ }^{4}$ Dept Biosciences \& Clinical Research, Sohna- Gurgaon, India

Background: The micro-nutrient Iodine is an integral part of the thyroid hormone molecule. Since thyroid hormones are known to have an impact on developmental and reproductive processes, deficiency of iodine in the environment can produce adverse obstetrical effects. In India despite Universal Salt Iodisation having been implemented large majority of population continue to inhabit zones declared iodine deficient and endemic by $\mathrm{WHO} /$ ICCIDD. The conspicuous lack of scientific studies on pregnancy outcomes in these areas prompted us to undertake a multi-centric study to monitor occurrence of miscarriages and still birth in young healthy women. This paper reports preliminary data from 29 villages under study from three states of indiaviz.,Uttarakhand, Haryana and Bihar.

Objective: To assess adverse pregnancy outcome in population inhabiting iodine-deficient zones.

Method: Two methods were followed for data collection 1. Current survey and 2. Data retrieval from Government health records on pregnancy outcomes viz miscarriage and still birth. Questionnaire based surveys were conducted in rural area of Bihar (4 villages), Haryana (13 villages) and Uttarakhand (12 villages). Data were also retrieved from annual and district health survey, census 2011 for Bihar and from health records available in primary health centers for Uttarakhand \& Haryana within last10 years.Study was approved by IEC

Results: Data on pregnancy outcome in populations inhabiting iodinedeficient zones from Bihar, Haryana and Utatrakhand are summarized in table 1.Our data are comparable to other reported studies irrespective of iodine status (Iodine deficient/ iodine sufficient).

Conclusion: Relatively low occurrence of adverse pregnancy outcomes is evident in these iodine deficient zones which is indeed surprising.

Financial assistance from Apeejay Education Society is gratefully acknowledged.

Table 1. (for abstract P3-06-167) Pregnancy outcome in iodine deficient area

\begin{tabular}{|c|c|c|c|c|c|c|}
\hline State & \multicolumn{3}{|c|}{ Data retrieval from records } & \multicolumn{3}{|c|}{ Current survey data } \\
\hline Bihar & AHS Census 2010 & 3.00 & NA & $\begin{array}{l}2016-17 \\
n=203\end{array}$ & $\begin{array}{l}11.81 \\
\pm 3.19 \%\end{array}$ & $\begin{array}{l}2.00 \\
\pm 1.15\end{array}$ \\
\hline Haryana & $\begin{array}{l}2010-16 \\
n=1689\end{array}$ & $\begin{array}{l}0.53 \\
\pm 0.16\end{array}$ & $\begin{array}{l}0.30 \\
\pm 0.12\end{array}$ & $\begin{array}{l}2012-16 \\
n=438\end{array}$ & $\begin{array}{l}0.73 \\
\pm 0.08\end{array}$ & $\begin{array}{l}0.40 \\
\pm 0.03\end{array}$ \\
\hline Uttarakhand & $\begin{array}{l}2012-17 \\
n=1024\end{array}$ & $\begin{array}{l}1.88 \\
\pm 0.56\end{array}$ & $\begin{array}{l}1.33 \\
\pm 0.67\end{array}$ & $\begin{array}{l}2016-17 \\
n=122\end{array}$ & $0.59 \%$ & $\begin{array}{l}0.98 \\
\pm 0.31\end{array}$ \\
\hline
\end{tabular}

NA: not available AHS: Annual Health Survey 


\section{European Thyroid Journal}

Abrahamsen, B. OP-10-74, OP-12-89

Accorroni, A. OP-07-53

Acuña, A. P2-02-68, OP-09-63, OP-11-79

Afonso, M. P3-04-146

Agapito, A.M. P2-05-103

Agate, L. P1-02-17, OP-06-45, OP-09-67

Águas, L. P2-04-92

Ahmed, S. P1-04-36

Ahn, Y.-C. P1-04-33

Ajdžanoviæ, V. OP-07-51

Akaishi, J. P1-03-22, P2-03-80, P3-03-131

Akirov, A. OP-02-12, OP-06-38

Albu, A. P2-03-74

Aldahri, F. P2-03-73

Aldahri, S.F. P2-03-73

Aldea, R. P3-06-166

Alekseev, D. P3-01-119

AlEssa, M. P2-03-73

Alevizaki, M. P1-01-02, P3-03-136, P3-06-161, OP-06-41

Alghammass, M. P2-03-73

Alibrandi, A. P2-01-57

Allegri, L. OP-03-15

Alshahrani, M. OP-03-20

Ambrosio, R. OP-05-33

Anagnostou, E. P3-03-136, P3-06-161, OP-06-41

Anastasiou, E. P3-06-161, OP-06-41

Andersen, C.L. OP-10-72

Andersen, S.L. OP-08-61, OP-10-70

Andersson, M. OP-08-55

Andrei, F. P2-03-74

Antonelli, A. P1-01-05, P1-01-10, P1-05-42, P2-06-104, P2-06-108, OP-09-64

Arpi, M.L. P3-05-155

Arvat, E. OP-04-28

Asanin, M. P1-05-44

Askitis, D. P2-03-79

Åsman, P. P2-01-56

Assanti, P. P2-05-101

Attard, M. P1-04-32

Avdzhiyska, M. P3-04-143

Aydın, H. P3-06-159

Aydoğan, B.İ. P2-03-78

Azevedo, I. P3-04-146

Azoulay, O. P2-05-96

Badalyan, M. P3-05-153

Badiu, C. P1-02-19, P2-05-102

Bae, D. P1-04-37, P2-05-95

Bae, D.S. P2-06-105

Bae, J.Y. P3-04-140

Bae, S.I. P3-04-140

Bajuk, N.B. P1-05-46

Bakalov, D. P3-01-116, P3-01-122

Baker, K. OP-03-20

Baldan, F. OP-03-15

Baldeschi, L. P3-06-158

Baldys-Waligorska, A. P1-03-26

Balkissoon, J. P3-03-132

Bano, A. OP-01-03

Barbato, F. P1-04-34, OP-04-23
Barbosa, M. P3-06-163

Barbu, C. P2-03-74

Barde, L. P2-02-66, OP-04-26

Bardet, S. P2-03-81

Barisic-Smalcelj, M. P3-04-141

Barjaktarovic, M. OP-08-59

Bartenstein, P. OP-03-17

Bartolome, A. P2-02-64

Baskal, N. P2-03-78

Basolo, F. P3-03-137, OP-09-67

Bath, S. OP-10-73

Batista, R. OP-03-19

Baudin, E. OP-06-42

Bauriaud, M. OP-04-22

Bednarczuk, T. OP-02-07

Beleslin-Nedeljkovic, B. P1-05-47, P1-06-53

Benbassat, C. OP-06-38

Berbel, P. OP-07-48

Berchner-Pfannschmidt, U. OP-07-52

Berdelou, A. OP-06-42, OP-10-71

Bergant, D. OP-04-29

Bermudez, G. P3-02-126

Bernal, J. OP-05-37

Bernardo, D.C. P2-04-83

Bertram, J. OP-03-17

Besic, N. P1-02-11

Bianchi, F. P1-01-01

Bianchini, A. P2-05-98

Bianco, L. P3-01-120

Bini, F. P2-05-100

Biscarini, F. P2-01-61

Bizzarri, G. P2-05-98

Blanchard, D. P2-03-81

Blay, K.L. OP-07-50

Bliddal, S. OP-08-56

Bocca, G. P3-05-151

Bocci, G. OP-09-64

Bocheva, Y. P1-05-38

Boelaert, K. OP-03-20, OP-09-66

Boelen, A. OP-11-77, OP-13-95

Bogavac, T. P1-02-21

Bogojevic, M. P2-06-112

Bolanowski, M. OP-02-07

Bonatti, A. P2-06-104

Bonelli, N. P2-05-101

Bong, J. P3-04-140

Bongiovanni, M. P2-02-63

Bonnema, S.J. P1-04-30, P2-02-70

Borget, I. OP-10-71

Borisov, R. P1-05-45

Borozan, S. P2-06-112, P3-02-128, P3-02-130

Borson-Chazot, F. OP-06-44

Boschi, A. P3-06-158

Boskovic, O. P2-06-112

Bosset, M. OP-06-44

Bossowska, A. P2-01-58

Bossowski, A. P2-01-58, P2-01-62, OP-02-07

Bottici, V. P1-02-17, P3-03-137, OP-06-43, OP-06-45

Bournaud, C. OP-06-44

Bowers, J. OP-13-95

Boyanov, M. P3-01-116, P3-01-122

Bozic, V. OP-09-62

Bozic-Antic, I. P1-02-21

Brandão, L. P3-05-147
Brandt Christensen, F. OP-02-09, P1-01-06

Braun, D. P1-06-51

Brenac, F. P2-03-81

Brix, T. OP-02-09, OP-02-13, OP-10-74, OP-12-89, P1-01-06

Brouwer, M.C. OP-11-77

Brouwers, A.H. P3-05-151

Brozzi, F. P1-01-01

Bruno, G. OP-12-83

Bruno, R. P1-04-32

Brunova, J. P3-05-148

Brusca, N. P1-01-10

Budianu, O. P2-03-74

Buffet, C. OP-04-26, P2-02-66, P2-03-81

Bulliard, Jean-L. P2-02-63

Bulotta, S. OP-11-78

Burgerhof, J.G.M. P3-05-151

Burlacu, M.-C. P2-01-61, P3-06-158

Busaidy, N.L. OP-06-39

Busiello, R.A. P1-06-54

Buziak-Bereza, M. P1-03-26

Cabanillas, M.E. P2-02-69, OP-06-39

Caeiro, C. P2-04-92

Cameselle-Teijeiro, J.M. OP-03-19

Campennì, A. OP-07-46, P1-02-14

Campi, I. P1-01-05, P1-05-39

Campo, S. OP-07-46

Campredon, P. P3-01-115

Canals, S. OP-07-48

Canas, L.R. OP-11-79

Canlar, S. P2-03-78

Cantara, S. OP-09-69

Canzi, C. P1-05-39

Caocci, G. P1-01-08

Cappagli, V. P3-03-137

Caragheorgheopol, A. P1-02-19, P2-05-102

Cardillo, G. OP-11-78

Cardinale, S. OP-09-69

Cardoso, L. P1-02-20

Carlé, A. OP-08-61, OP-12-86

Carles, Z. OP-01-02

Carmela, I. OP-01-02

Carnicelli, V. P1-06-52

Caron, P. P3-01-115, OP-04-22

Carra, S. OP-11-81

Carriço, M. P3-02-125

Carrilho, F. P1-02-20, P3-02-127, P3-02-129, P3-03-135, OP-03-19

Carvalho, D. P1-02-13, P2-04-92, P2-04-93, P3-01-123, P3-05-157

Casella, F. OP-06-43

Casini, G. P2-01-55

Castagna, M.G. P1-04-34, OP-04-23

Casti, A. P1-01-08

Catarino, D. P3-02-127, P3-03-135

Cavalcoli, F. P2-04-88

Čejović, S. P3-02-130

Celano, M. OP-11-78

Celik, A. P3-06-159

Cellini, M. P1-01-10
Centanni, M. P1-01-10, P2-06-108, OP-12-83

Cerdan, S. OP-05-37

Chabre, O. OP-10-71

Chaker, L. OP-01-03

Chalkiadakis, G. P2-03-76

Chandola-Saklani, A. P3-06-165, P3-06-167

Chang, W.J. P3-04-140

Chatterjee, K. OP-01-06

Chaushev, B. P3-04-143

Chella, A. OP-09-67

Cherau, N. OP-04-26

Chernenko, O. P2-03-82

Chittenden, S. P1-05-43

Chmielik, E. OP-09-68

Cho, K.-J. P1-03-27, P3-03-138

Chougnet, C. OP-10-71

Chowdhuri, S.R. P2-02-69

Christensen, H.M. OP-12-82

Christiansen, O.B. OP-08-56

Christinet, M.F. P2-04-90

Christoph, S. P1-06-49

Chrysoulaki, M. P2-03-76

Ciampi, R. OP-09-67

Ciappuccini, R. P2-03-81

Cinel, M. P2-03-78

Cioffi, F. OP-13-94, OP-13-97

Cirello, V. OP-06-40

Ciric, J. P1-05-47, P1-06-53, P2-06-107, P2-06-110, P3-01-118

Clarisse, B. P2-03-81

Clemmensen, C. OP-05-34

Clerget-Froidevaux, M.-S OP-13-95

Clevert, D.-A. OP-03-17

Cohen, O. P2-05-96

Colaci, M. P2-06-108

Colombo, C. P3-03-134, OP-03-18, OP-06-40

Colucci, G. P2-01-61

Combest, A. P3-03-132

Connell, M. P2-04-88

Coppola, M. P1-06-54

Çorapçıŏlu, D. P2-03-78

Corina, D. P3-06-166

Corneci, C. P1-02-19

Corre, R. P2-02-66

Corssmit, E.P.M. P3-05-151

Corti, A. OP-09-64

Corvilain, B. OP-09-65

Cosco, D. OP-11-78

Costa, C. P3-04-146

Costa, G. P1-02-20, P3-02-127, P3-02-129

Costagliola, S. OP-01-04

Costante, G. P1-04-32

Cour, J.L. OP-10-72, OP-12-82

Courbon, F. OP-04-22

Coutinho, J. P2-05-103

Couto, J. P1-02-18

Covelli, D. P1-01-05, P1-05-39, P2-01-61

Crespo, P. P2-02-68, OP-09-63

Criscuolo, C. OP-07-53

Cristani, M.T. P2-01-57

Cugini, C. P2-05-101 
Cunha, N. P1-02-18, P1-02-20, P3-03-135

Currò, N. P1-01-05, P1-05-39

Cvejic, D. P2-02-64, OP-09-62

Czarniecka, A. OP-04-25, OP-09-68

Czyżyk, A. P2-01-60

Dalmau-Bueno, A. OP-10-73

Damante, G. OP-03-15, OP-11-78

Damjanovic, S. P1-02-21, P1-05-44, P3-02-130

Danesi, R. OP-09-64

Daniels, G. P3-05-149

Daraki, V. P2-03-76

Daroszewski, J. OP-02-07

Darras, V. OP-07-47

D'Ascola, A. OP-07-46

Daumerie, C. P2-01-61, P3-06-158

Dayan, C. OP-01-05, OP-02-14

Dazzi, D. P1-01-05

De Cicco, E. OP-05-33

De Deken, X. OP-13-91

de Jong, M. OP-04-29

de Kock, L. P2-02-72

de Lange, P. OP-13-94, OP-13-97

de Leo, S. P3-03-134, OP-06-40

de Leval, L. P2-02-63

de Menezes, J.C. P2-02-66

De Raucourt, D. P2-03-81

de Rijke, Y. OP-08-59

De Santis, M. P2-05-101

De Siena, G. P1-06-48, P1-06-50

De Souza, E.C.L. OP-11-80

De Stefano, M.A. OP-05-33

De Vito, C. OP-12-83

de Vries, E. OP-13-95

Deandrea, M. P2-05-101, P3-01-120

Deandreis, D. OP-06-42

Decaussin, M. OP-06-44

Decherf, S. OP-13-95

Decristoforo, C. OP-04-29

Deiana, M.G. P2-05-101

Dekker, B.L. P1-02-16, P3-05-151

Delgado, L. P3-01-123, P3-05-157

Dell'Osso, L. P1-06-52

Demeneix, B. OP-07-50, OP-13-95

Demir, Ö. P2-03-78

Dencic, T.I. P2-02-64

Denis, R. OP-13-95

Dentice, M. OP-05-33

Deprez, M.-A. OP-07-47

Desidero, T.D. OP-09-64

Dhana, K. OP-01-03

Diana, T. P2-01-58

Dierckx, L.O. OP-04-22

Diker-Cohen, T. OP-02-12, OP-06-38

Dimitrova, R. P3-05-154

Djogo, A. P2-06-112

Djoric, I. P2-02-64

Djurdjevic, S.P. P1-05-44, P2-06-113

Djurovic, M.N. P1-05-44, P2-06-113

Dobrescu, R. P1-02-19, P2-05-102

Dohmann, M. OP-05-35

Doknic, M. P2-06-113

Dolapsaki, M. P2-03-76

Dold, S. OP-08-55

Dora, S. P1-01-07, P3-01-119

Dorigo, A. P2-05-101

Dorszewska, J. P2-01-60

Døssing, H. P1-04-30

Dottore, G.R. P2-01-55

Draman, M.S. P2-01-61, OP-01-05, OP-02-14

Duarte, H. P3-04-146

Duke, S. OP-04-27

Dumitrascu, I. P3-06-166
Dumont, J. OP-09-65

Dumont, J.E. OP-13-91

Duntas, L. P3-01-121

Dupuy, C. P2-02-66

Durante, C. P1-04-32, OP-04-28

Duskin-Bitan, H. OP-02-12

Duss, F.-R. P2-04-90

Dygun, O. P3-01-119

Eckstein, A. P2-01-61, OP-07-52

Eerdekens, A. OP-08-54

Efremidou, E.I. P2-03-79

Ehlers, L. OP-12-84

Elezovic-Kovacevic, V. P1-02-21

Elia, G. P1-05-42

Elisei, R. P1-01-01, P1-02-17, P3-03-137, OP-06-43, OP-06-45, OP-09-67

Eloy, C. OP-03-16

Emral, R. P2-03-78

Erba, P.A. OP-04-29

Erdogan, M. P2-03-78, P3-06-159

Esebidis, A. P2-03-79

Eskalli, Z. OP-13-91

Espada, M. OP-10-73

Esteves, C. P2-04-92, P3-01-123, P3-05-157

Evans, S. OP-01-05

Faber, J. OP-12-82

Fadiga, L. P3-02-129, P3-03-135

Fallahi, P. P1-01-05, P1-01-10, P1-05-42, P2-06-104, P2-06-108, OP-09-64

Faranda, A. P1-02-17

Farina, S. OP-11-80

Fatemi, S. P1-02-15

Fedak, D. OP-04-29

Feldt-Rasmussen, U. OP-08-56

Ferløv-Schwensen, C. OP-02-13

Fernandes, G. P2-04-92

Fernández, L.P. P2-02-65

Ferrari, S.M. P1-05-42, P2-06-104, P2-06-108, OP-09-64

Ferreira, L. OP-03-16

Ferri, C. P2-06-108

Ferru, A. OP-10-71

Fica, S. P2-03-74

Figueiredo, P. P1-02-18

Filetti, S. P1-04-32, OP-04-28

Finan, B. OP-05-34

Fine, N.H.F. OP-03-20

Flamant, F. OP-05-34

Fletcher, A. OP-03-20, OP-09-66, OP-11-79

Fliers, E. OP-11-77

Floroskoufi, P. P2-03-76

Flux, G. P1-05-43

Fogliaro, M.P. OP-07-49

Fontanini, G. OP-09-67

Forleo, R. P1-04-34, OP-04-23

Foulkes, W.D. P2-02-72, OP-08-60

Foy, C. OP-01-05

Fralassi, N. P1-04-34, OP-04-23

Franceschi, M. OP-08-55

Franco, O. OP-01-03

Françoise, M. OP-13-91

Franssen, G. OP-04-24

Frasca, F. P1-03-25, OP-07-46

Frascarelli, S. OP-13-90

Freitas, F. P3-05-147

Freitas, P. P2-04-93

Fresta, M. OP-11-78

Friesema, E.C.H. OP-11-80

Froberg, L. OP-04-29

Fugazzola, L. P1-05-39, P3-03-134, OP-03-18, OP-06-40

Führer, D. P1-06-49, OP-01-01

Fujiwara, A. OP-12-87

Furkert, J. OP-07-52
Gabat, J.A. P2-04-86

Gaberscek, S. P1-01-03, P1-05-46, OP-02-10, OP-12-85

Gabiache, E. OP-04-22

Gadawska-Juszczyk, K. P3-01-117

Gallowitsch, H.-J. P3-03-133

Gangemi, S. P2-01-57

Garberoglio, R. P2-05-101

Garcia, M-E. OP-10-71

Garcia-Verdugo, J.M. OP-07-48

Gargano, L. OP-12-83

Garino, F. P2-05-101, P3-01-120

Gasior-Perczak, D. P3-01-117

Gasińska, T. P2-01-60

Gasparyan, E. P2-05-99, P3-04-142

Gauthier, K. OP-05-34

Gawenda, P. OP-04-29

Gawlik, T. OP-09-68

Gedik, V.T. P2-03-78

Gentile, A. P1-06-54

Georgescu, C. P3-06-166

Ghander, C. OP-04-26

Ghelardoni, S. OP-07-49, OP-13-90, OP-13-92

Ghemigian, A.M. P3-06-166

Gheorghiu, C. P1-02-19

Gheorghiu, M.L. P3-06-166

Ghuzlan, A.A. P2-02-66

Giacco, A. OP-13-97

Giani, C. OP-09-67

Gianì, F. OP-07-46

Giannakou, M. P1-01-02

Giannini, G. OP-07-49

Giatromanolaki, A. P2-03-79

Gil, J. OP-01-02

Gimba, E. OP-03-16

Giorgadze, E. P1-05-41

Giovanella, L. P1-02-14, P2-05-100, P3-05-150

Girolamo, D.D. OP-05-33

Giuggioli, D. P2-06-108

Giulea, C. P2-03-74

Gobina, I. P3-06-164

Godballe, C. P1-04-30

Godinho, C. P2-05-103

Goglia, F. P1-06-54, OP-13-94, OP-13-97

Golmard, J.-L. OP-04-26

Gómez, R.G. P2-02-68

González-Granero, S. OP-07-48

Gorcheva, D. P1-05-45

Goscik, J. P2-01-62

Gothié, J.-D. OP-07-50

Gozdz, S. P3-01-117

Grabe, H. OP-12-88

Grand, M.K. OP-10-72

Grani, G. P1-04-32, OP-04-28

Graniæ, R. OP-08-55

Gray, M. P1-05-43

Graziano, F. P2-05-98

Greenfield, H. P3-01-123

Greenwood, R. OP-01-05

Grigore, A. P2-03-74

Grigoryeva, M. P3-01-114

Grimaldi, S. OP-06-42

Grinberga, D. P3-06-164

Groma, V. P2-01-59

Grootenhuis, M.A. P3-05-151

Grunenwald, S. P3-01-115

Gruson, D. P3-06-158

Guglielmi, R. P2-05-98

Guillén, M.A. OP-10-75

Guimarães, J.T. P1-02-13

Güiza, F. OP-08-54

Gullo, D. P3-05-155

Gullu, S. P2-03-78

Guxens, M. OP-10-73

Guyot, R. OP-05-34

Ha, E.J. P1-04-29, P1-04-35

Hadjieva, T. P3-02-124

Haerlingen, B. OP-01-04

Halperin, D. P2-05-96

Hamada, K. P2-05-94

Hames, K. P3-03-131

Han, M. P1-04-35

Handkiewicz-Junak, D. OP-04-25

Haridas, A. OP-01-05

Harmer, C. P1-05-43

Hartl, D. P2-02-66

Havekes, B. P3-05-151

Healy, S. OP-02-14

Hecht, F. P2-02-66

Hegedüs, L. P1-01-06, P1-04-30, P3-05-149, OP-02-09, OP-02-13, OP-10-74, OP-12-89

Heijboer, A. P1-02-16

Hella, Z. P2-04-84

Herman, W. P2-01-60

Hermanns, P. P2-04-89

Hernandez, A. OP-11-77

Hernández, M. OP-08-58

Hesselink, E.K. OP-01-02

Hesselink, M.S.K. P3-05-151

Heutte, N. P2-03-81

Higashi, K. P2-05-94

Hinz, K.M. OP-05-32

Hirawa, N. OP-12-87

Hirsch, D. OP-02-12, OP-06-38

Hodinka, L. P2-04-84

Hodson, D.J. OP-03-20

Hoenes, G.S. OP-01-01

Hofmann, M.-C. OP-06-39

Horiuchi, K. P1-03-24

Hoyer, I. OP-07-52

Hristozov, K. P1-05-38, P3-05-154

$\mathrm{Hu}$, M.I. OP-06-39

Huillard, O. OP-10-71

Huizinga, G.A. P3-05-151

Hyer, S. P1-05-43

Iannucci, L.F. OP-13-94

Ibarluzea, J. OP-10-73

Ignjatović, S. P1-06-53

Ikram, M. A. OP-01-03

Ilhéu, O. P1-02-18

Ilic, D. P1-02-21

Imbert, P. P3-01-115

Imperiali, M. P3-05-150

Imruetaicharoenchoke, W. OP-09-66

Ingoe, L. P3-05-156

Ingrisch, M. OP-03-17

Ion, O. P2-03-74 
Joo, Y.-H. P1-03-27, P3-03-138

Jorda, M. OP-01-02

Jørgensen, H.L. OP-10-74, OP-12-89

Jørgensen, T. P3-06-162, OP-12-84, OP-12-86

Jovanovic, L. P1-05-47

Jukic, T. OP-08-55

Jurecka-Lubieniecka, B. OP-02-07

Juyal, R. P3-06-167

Kahaly, G.J. P2-01-58, P3-06-158

Kaiser, U. P2-01-61

Kalere, I. P2-01-59, P3-06-164

Kalotinis, K. P2-03-76

Kam, B. OP-04-24

Kamashidze, K. P1-05-41

Karanikas, M.A. P2-03-79

Kardum, N. OP-07-51

Karsbøl, J.D. P3-05-149

Kassi, E. OP-06-41

Kast, M. P1-01-04

Kathait, A. P3-06-165

Kavaric, S. P2-06-112

Kavousi, M. OP-01-03

Kazakou, P. P3-03-136, OP-06-41

Keskin, Ç. P2-03-78

Khattak, R. P3-05-152

Khroyan, A. P3-05-153

Kim, H.J. P3-04-140

Kim, J.H. P1-03-23, P2-04-87

Kim, M.S. P1-03-27, P3-03-138

Kim, S.W. P1-04-33

Kim, S.-Y. P3-03-138

Kim, Y. P1-04-33

Kistorp, C. OP-12-82

Kitagawa, W. P1-03-22, P2-03-80, P3-03-131

Knudsen, N. P3-06-162, OP-08-61, OP-012-84, OP-012-86

Kobalava, Z. P2-04-91

Kobold, A.M. P1-02-16

Kohler, H. OP-01-01

Kohlfuerst, S. P3-03-133

Köhrle, J. OP-05-37, OP-12-82, OP-13-96

Kojuharov, H. P3-05-154

Koleva, V. P1-05-45

Kolodziej, M. P1-03-26

Kolte, A.M. OP-08-56

Kondo, T. P2-03-80

Konijnenberg, M. OP-04-29

Konrad, C. P1-01-04

Konrade, I. P2-01-59, P3-06-164

Kontou, P. P3-06-161

Koo, D.H. P1-04-37, P2-05-95

Korevaar, T. OP-08-59, OP-10-73

Kostova, B. P1-05-45

Kostovski, V. P2-06-110

Kovács, G.L. P2-04-84

Kovatcheva, R. P3-02-124

Kowalczyk, M. P2-01-60

Kowalska, A. P3-01-117

Kowalska, M. OP-09-68

Koziara, M. P1-03-26

Krajewska, J. OP-09-68

Kralj, B.V. P1-02-11

Kratochvilova, S. P3-05-148

Krause, G. OP-05-32, OP-07-52

Kremer, L.C.M. P3-05-151

Kretowski, A. P2-01-62

Krishnamurthy, S. P2-02-69

Krnjevic, D. P2-06-112, P3-02-128

Kropinska, A. OP-04-25

Kuś, A. OP-02-07

Kühn, J.-P. P3-05-152

Kula, D. OP-02-07

Kumorowicz-Czoch, M. P2-04-89

Kunii, Y. P3-04-139

Kuœ, J. OP-02-07

Kusiæ, Z. OP-08-55
Kyprianou, M. P1-01-02

Kyrilli, A. OP-09-65

La Rosa, G.L. P3-05-155

Lacic, M. P3-04-141

Łącka, K. P2-01-60

Lages, A. P1-02-20, P3-03-135

Lahav, Y. P2-05-96

Lamartina, L. P1-04-32, OP-04-28

Lamine, F. P2-04-90

Landucci, E. P1-06-48, P1-06-50

Lane, C. OP-01-05

Lang, B. P1-04-31

Langouche, L. OP-08-54

Lanni, A. OP-13-94, OP-13-97

Lantz, M. P2-01-56

Latsabidze, N. P2-06-111

Latteyer, S. P1-06-49, OP-01-01

Lauridsen, J.F. P1-04-30

Laurino, A. P1-06-48, P1-06-50

Lazic, M. OP-03-15

Leanza, C. OP-13-94

Leboulleux, S. P2-02-66, OP-06-42, OP-10-71

Leconte, A. P2-03-81

Ledwon, A. OP-04-25

Lee, H.S. P1-04-33

Lee, K.D. P1-04-33

Lee, S.K. P1-04-33

Leenen, P. OP-11-77

Leenhardt, L. P2-03-81, OP-04-26, OP-10-71

Lefort, M. P2-03-81

Lehmphul, I. OP-12-82

Lehnen, T. OP-05-31

Leite, V. OP-03-19

Lenasi, H. P1-05-46

Lentjes, E. P1-02-16

Leo, M. P1-01-01, OP-02-08

Lepore, S.M. OP-11-78

Lerch, M.M. P3-05-152

Levie, D. OP-10-73

Ležaiæ, L. OP-04-29

Liao, X.H. P1-06-49, OP-01-01

Liarigkovinos, T. P2-03-76

Lichiardopol, C.R. P3-06-166

Lifante, J-C. OP-06-44

Lillevang-Johansen, M. OP-10-74, OP-12-89

Lima, R.T. OP-03-16

Limbert, C. P3-02-125

Limone, P.P. P2-05-101, P3-01-120

Lind, B.S. OP-10-72

Lind, P. P3-03-133

Lindner, L.H. OP-03-98

Links, T. P1-02-16, P3-05-151, OP-01-02

Linneberg, A. P3-06-162, OP-12-84

Lokerse, W. OP-03-98

Lombardi, A. P1-06-54

Lombardi, C.P. OP-04-28

Lombardo, G.E. OP-11-78

Longari, V. P1-05-39

López, C. OP-08-58

López, C.C. P2-02-71

Lorenzini, L. OP-07-49, OP-13-90, OP-13-92

Lorusso, L. P1-02-17

Loukari, E. P1-01-02

Louzada, R.A.N. P2-02-66

Lozanov, B. P1-05-45

Lozanov, L. P1-05-45

Lucia-Curt, F. OP-07-48

Lucisano, G. P1-04-32, OP-04-28

Ludgate, M. P2-01-61, OP-01-05, OP-02-14

Luider, T.M. OP-05-36

Lumbroso, J. OP-06-42

Luongo, C. OP-07-50

Luquet, S. OP-13-95
Lussey-Lepoutre, C. OP-04-26

Lyratzopoulos, N. P2-03-79

Ma, E. P1-04-31

Machado, C. P3-06-163

Maciejewski, A. P2-01-60

Macut, D. P1-02-21

Madapusi, V.R. OP-05-32

Maecke, H. OP-04-29

Magalhães, D. P3-04-146

Maggisano, V. OP-11-78

Maia, A.L. OP-05-31

Maina, T. OP-04-29

Maino, F. P1-04-34, OP-04-23

Majewska, U. P3-01-117

Malandrino, P. P1-03-25

Malcheva, D. P3-04-143

Malej, A. P1-05-40

Males, L. OP-06-42

Mamalaki, E. P2-03-76

Mancino, G. OP-05-33

Manda, D. P2-05-102

Mangino, G. P1-01-10

Mannaioni, G. P1-06-50

Mantzou, E. P1-01-02

Maranhão, R. P3-05-147

Marcinkowski, P. OP-07-52

Marcocci, C. P1-01-01, P2-01-55, OP-02-08, OP-06-45

Marelli, F. OP-11-81

Marinò, M. P1-01-01, P2-01-55, OP-02-08

Marinozzi, F. P2-05-100

Mariotti, S. P1-01-08

Markosyan, R. P2-03-75

Marques, B. P1-02-18

Martin, C.S. P2-03-74

Martinelli, M. P1-04-34

Martins, D. P1-02-20, P3-03-135

Martins, R. P1-02-18

Martins, T. P1-02-18

Martirosian, N. P3-01-114

Maruta, T. P2-05-94

Marzocchi, C. OP-09-69

Masaki, C. P1-03-22, P2-03-80, P3-03-131

Masetti, G. P2-01-61

Masi, A. P1-06-50

Massolt, E. OP-04-24

Materazzi, G. P1-05-42, P2-06-104, P3-03-137, OP-09-64

Matos, MJ. P3-04-146

Matos, M.L.G. P2-05-103

Matrone, A. P1-02-17, P3-03-137, OP-06-43, OP-06-45

Matschnig, S. P3-03-133

Matsumoto, M. P3-04-139

Matsuzu, K. P1-03-22, P2-03-80, P3-03-131

Mattace-Raso, F. OP-01-03

Maurice-Stam, H. P3-05-151

Mauricio, D. OP-01-02, OP-08-58

Mazieres, J. P3-01-115

Mazzeo, S. OP-09-67

Mazzi, B. P1-01-01, OP-02-08

McCabe, C.J. OP-03-20, OP-09-66

Medici, B.B. OP-10-72

Medina, J.L. P3-01-123, P3-05-157

Meima, M.E. OP-01-06, OP-05-36

Mekjavić, P.J. OP-02-10, P1-01-03

Mekova, R. P3-01-116, P3-01-122

Meli, S. P3-03-137

Melikyan, K. P3-05-153

Mellano, M. P3-01-120

Melo, M. P1-02-20, P3-02-127, P3-02-129, P3-03-135, OP-03-19

Menconi, F, P1-01-01, OP-02-08

Méndez, C.F. OP-13-93

Ménégaux, F. OP-04-26, P2-03-81

Menicali, E. OP-03-21

Meringolo, D. P1-04-32

Meyer, F. OP-05-37

Mezyk, R. P3-01-117

Miccoli, P. P1-05-42, P2-06-104, OP-09-64

Michels, J.-J. P2-03-81

Micic, D. OP-02-11

Mignogna, C. OP-11-78

Mihu, D. P3-06-166

Mikina, E. P3-01-117

Mikolajczak, R. OP-04-29

Milasin, J. OP-03-15

Milenkoviæ, D. OP-07-51

Miler, M. OP-07-51

Miletic, M. P1-05-47, P2-06-107

Milic, N. OP-02-11

Milinković, N. P1-06-53

Miljic, D. P1-05-44, P2-06-113, OP-02-11

Miloševiæ, V. OP-07-51

Mio, C. OP-03-15

Miœkiewicz, P. OP-02-07

Miot, F. OP-09-65, OP-13-91

Miro, C. OP-05-33

Miron, A. P2-03-74

Mitropoulou, M. P3-06-161

Mizokami, T. P2-05-94

Mlynarski, W. P2-01-62

Modasia, B. OP-09-66

Moellehave, L.T. P3-06-162, OP-12-84

Möller, L.C. P1-06-49, OP-01-01

Molinario, E. OP-09-67

Molinaro, A. OP-01-04

Molinaro, E. P1-01-01, P1-02-17, OP-06-45

Mollsi, E. P2-06-109

Moniz, A.P. P1-02-18

Monk, R. OP-13-96

Monpeyssen, H. P2-03-8

Monteiro, S. P3-06-163

Montero, C. OP-01-02

Monti, S. P2-05-101

Moradpour, D. P2-04-90

Morante, M. P2-02-68

Morelli, S. OP-03-21

Moreno, M. P1-06-54, OP-13-97

Moretti, S. OP-03-21, OP-11-78

Morillo, J. P2-02-65 
Nedeljkovic-Beleslin, B P2-06-107, P2-06-110, P3-01-118

Nelson, P.J. OP-05-35, OP-03-98

Netea-Maier, R.T. P3-05-151

Neves, C. P2-04-93, P3-01-123, P3-05-157

Neves, J.S. P2-04-93, P3-01-123, P3-05-157

Nguyen, B. P3-03-132

Nguyen, M. OP-13-90, OP-13-92

Nguyen, N. P1-04-30

Nguyen, T. OP-10-71

Nicolucci, A. P1-04-32, OP-04-28

Niedziela, M. P2-02-72, OP-08-60

Nielsen, C.H. OP-08-56

Nielsen, H.S. OP-08-56

Nielsen, V.E. P2-02-70

Nies, M. P3-05-151

Nieto, H. OP-03-20, OP-09-66

Nisic, T. P1-05-47, P2-06-107

Nock, B. OP-04-29

Nonchev, B. P3-04-143

Novak, J. P1-02-11

Nucci, N. OP-03-21

Nygaard, B.I. OP-10-72, OP-12-82

Oak, C. P1-04-33

Obregón, M.J. OP-07-48

Oczko-Wojciechowska, M. OP-09-68

Ogimi, Y. P1-03-22, P2-03-80

Ognjanovic, S. P1-02-21

Oh, J.-R. P3-04-140

O'Heir, E. OP-04-23

Ohgimi, Y. P3-03-131

Ohkuwa, K. P1-03-22, P2-03-80, P3-03-131

Ohye, H. P3-04-139

Okamoto, T. P1-03-24

Okosieme, O. OP-01-05

Olczyk, T. OP-04-25

Oldani, B. P3-01-120

Olivarius, N.D.F. OP-10-72

Oliveira, A.I. P2-04-93

Oliveira, D. P1-02-20, P3-03-135

Oliveira, J. P2-04-92

Oliveira, M.J. P3-06-163

Oliveira, S.C. P2-04-93, P3-01-123, P3-05-157

Olsen, J. OP-10-70

Omi, Y. P1-03-24

Omrani, O. P1-04-36

Opitz, R. OP-01-04

Orçun, A. P3-06-159

Origlia, N. OP-07-53

Orlandi, F. P1-02-14, OP-04-28

Osaku, T. P1-03-22

Ovesen, L. P3-06-162, OP-08-61, OP-12-86

Özdemir, A. P3-06-159

Pacheco-Torres, J. OP-07-48

Paciaroni, A. P1-04-32

Pacini, F. P1-04-34, OP-04-23, OP-09-69

Paliczka-Cieslik, E. OP-04-25

Palyga, I. P3-01-117

Palyga, R. P3-01-117

Pandrc, M. P2-06-110

Pani, F. P1-01-08

Panteleeva, O. P3-01-114

Paoli, G. OP-13-97

Papadakis, G. P2-04-90

Paparo, S.R. P2-06-108

Papavasiliou, S. P2-03-76

Papini, E. P2-05-98

Park, C.W. P1-04-33

Park, H.K. P1-02-12

Park, J.-O. P1-03-27
Park, K.K. P1-03-23

Pascanu, I. P3-06-166

Paskas, S. OP-09-62

Paunovic, I. P2-02-64

Pavlovic, D. P1-05-44

Pawlaczek, A. OP-09-68

Pawlak-Adamska, E. OP-02-07

Payoux, P. OP-04-22

Pedersen, I.B. P3-06-162, OP-08-61, OP-12-86

Peeters, R. OP-01-03, OP-01-06, OP-04-24, OP-05-36, OP-08-59, OP-10-73, OP-11-80, P3-05-151

Peikrishvili, N. P1-01-07

Peitl, P.K. OP-04-29

Pell, J. OP-01-05

Pellegrini-Giampietro, D.E. P1-06-50

Pellegriti, G. P1-03-25, P3-05-155

Pellot-Barakat, C. P2-03-81

Pereira, A.M. P1-05-44

Pereira, M. P3-01-123, P3-05-157

Perrild, H. P3-06-162, OP-08-61, OP-12-86

Perrino, M. P3-03-134, OP-06-40

Perros, P. P2-01-61

Persani, L. P1-01-05, OP-11-81

Persichetti, A. P2-05-98

Pesenti, C. OP-03-18

Peshkova, T. P1-05-4

Pestana, A. OP-03-16

Petakov, M. P1-02-21, P1-05-44, P2-06-113

Petersen, M. OP-12-86

Petrosyan, A. P3-05-153

Petrovic, I. P1-02-15

Petunina, N. P3-01-114

Pevec, A.A.S. P1-02-11

Pfeifer, A. OP-09-68

Philippou, G. P1-01-02

Piaggi, P. P1-02-17

Piaggi, S. OP-09-64

Piccardo, A. P1-02-14

Picu, C. P2-05-102

Piekielko-Witkowska, A OP-05-30

Pieruzzi, L. OP-06-45

Pilli, T. OP-09-69

Pirnat, E. P1-01-03, OP-02-10, OP-12-85

Pissia, M. P3-03-136

Piwowar, M. P3-01-117

Planck, T. P2-01-56

Plukker, J.T.M. P3-05-151

Podia Igna, C.C. P3-04-144, P3-06-166

Podkrajsek, K.T. P1-05-40

Pohlenz, J. P2-04-89

Polychronidis, A. P2-03-79

Pontecorvi, A. OP-04-28

Poole, V. OP-09-66

Pooprasert, P. OP-01-05

Popawski, P. OP-05-30

Popescu, D. P3-06-166

Popescu, M. P3-06-166

Popovic, B. P1-02-21

Popovic, V. P2-06-113, OP-02-11

Poppe, K. P2-06-106

Porowska, B. OP-12-83

Poski, R. OP-02-07

Prabhakar, B.S. OP-09-69

Pratt, B. P1-05-43

Prezioso, C. OP-05-33

Prokop, J. P2-05-103

Prokopakis, E. P2-03-76

Proverbio, M.C. OP-03-18

Psaltopoulou, T. P1-01-02

Puch, Z. OP-04-25

Pudule, I. P3-06-164

Pugeat, M. OP-06-44
Puig-Domingo, M. OP-01-02, OP-08-58

Purice, M. P3-06-166

Puxeddu, E. P1-04-32, OP-03-21, OP-04-28, OP-11-78

Pyankova, A. P3-06-160

Queirós, J. P2-04-92

Racaru, L.V. OP-04-22

Radeva, T. P3-05-154

Rafael, L. P1-02-13

Ragusa, F. P1-05-42

Raimondi, L. P1-06-48, P1-06-50

Rajan, P. P1-04-36

Ramirez-Moya, J.A. OP-11-76

Ramos, E.F. P2-04-83

Rappai, S. P2-03-77

Rasmussen, Å.K. OP-08-56

Rasmussen, L.B. P3-06-162, OP-08-61, OP-12-86

Rasmussen, S. P3-05-149

Rathmann, D. OP-13-96

Raykov, M. P3-04-143

Raykov, N. P3-04-143

Raykova, A. P3-04-143

Rayman, M. OP-10-73

Razvi, S. P3-05-156

Read, M. OP-03-20, OP-09-66

Rebagliato, M. OP-10-73

Rebecchini, C. P2-02-63

Redda, M.G.R. P3-01-120

Refetoff, S. P1-06-49, OP-01-01

Reitsma, D. P3-03-132

Remaud, S. OP-07-50

Remmerie, M. OP-07-47

Rep, S. OP-04-29

Resta, F. P1-06-48, P1-06-50

Reverter, J.L. OP-01-02

Reznik, Y. P2-03-81

Ribeiro, C. P1-02-20, P3-02-127, P3-02-129, P3-03-135

Richard, S. OP-05-34

Richards, K.H. OP-13-96

Richell, G. P2-01-61

Riesco-Eizaguirre, G. P2-02-68, OP-09-63, OP-10-75, OP-11-79

Rieser, M. P3-03-133

Rios, E. OP-03-16

Ristić, A. P2-06-110

Robenshtok, E. OP-02-12, OP-06-38

Robertson, N. OP-02-14

Robledo, M. OP-01-02, OP-10-75

Rocha, A.G. OP-03-19

Rocha, G. P3-06-163

Rodic, G. P1-02-21

Rödl, W. OP-03-17

Rodrigues, E. P1-02-13

Rodrigues, F. P1-02-18

Rodrigues, I. P2-05-103

Rodríguez-Lloveras, H. OP-01-02

Romanov, I. P2-04-91

Romei, C. OP-06-43, OP-09-67

Ronckers, C.M. P3-05-151

Roque, C. P3-02-125

Roques, T. OP-04-27

Roskosz, J. OP-04-25

Rossetto, R. P2-05-101

Rossi, A. P3-01-120

Roumeliotaki, T. P2-03-76

Roussel, A. P2-03-81

Rrupulli, A. P2-06-109

Ruffilli, I. P2-06-104

Ruggeri, R. P2-01-57, OP-07-46

Rurale, G. OP-11-81

Rusinek, D. OP-09-68

Russo, D. OP-03-15, OP-11-78

Russo, M. P1-03-25, P3-05-155

Rutigliano, G. P1-06-52

Rybakova, M. P3-01-119

Rybicka, B. OP-05-30

Rymar, O. P3-06-160

Saakian, S. P3-01-114

Saavedra, A. P1-02-13

Saba, A. OP-13-90, OP-13-92

Sabatini, M. OP-07-53

Sabbaghian, N. P2-02-72, OP-08-60

Sabini, E. P1-01-01, OP-02-08

Sacripanti, G. OP-07-49, OP-13-90, OP-13-92

Saglietti, C. P2-02-63

Sagliocchi, S. OP-05-33

Sahlstrand-Johnson, P. P2-01-56

Saka, S. OP-12-87

Sakurai, M. P1-03-24

Salabert, A.-S. OP-04-22

Saltiki, K. P1-01-02, P3-03-136, P3-06-161, OP-06-41

Salvatore, D. OP-05-33

Salvi, M. P1-01-05, P1-05-39, P2-01-61

Samarut, J. OP-05-34

Sancak, S. P3-06-159

Sanches, C. P3-04-146

Sandholdt, H. OP-10-72

Santaguida, M.G. P1-01-10, OP-12-83

Santini, P. P1-01-01

Santisteban, P. P2-02-65, P2-02-68, P2-02-71, OP-09-63, OP-10-75, OP-11-76, OP-11-79, OP-13-93

Santos, A.P. P3-04-146

Santos, J. P1-02-18

Sapuppo, G. P1-03-25, P3-05-155

Saraiva, C. P3-02-125

Saraiva, J. P3-02-127, P3-02-129, P3-03-135

Sarantopoulou, V. P3-06-161

Sargin, M. P3-06-159

Savagner, F. OP-08-57

Savic, S. P1-05-47, P2-06-107, P3-01-118

Scarpa, L. OP-04-29

Scheffner, C. OP-05-32

Schipor, S. P2-05-102

Schlumberger, M. P2-02-66, OP-06-42, OP-10-71

Schmid, K.W. P1-06-49, OP-01-01

Schmohl, K. OP-03-17, OP-05-35, OP-03-98 
Siderova, M.V. P1-05-38, P3-05-154

Siersma, V. OP-10-72

Sigal, G. P3-05-147

Silva, A.M. OP-03-16

Silva, B. P3-05-147

Silva, R. P2-04-92

Silvestri, E. P1-06-54, OP-13-97

Simeakis, G. P3-03-136, P3-06-161, OP-06-41

Simescu, M. P3-04-144, P3-06-166

Sirbu, A. P2-03-74

Sivalingam, S. P1-01-06

Sjölander, S. P1-01-04

Skaaby, T. OP-12-84

Skuja, S. P2-01-59

Sluiter, W.J. P1-02-16

Smit, J. P1-05-44

Smith, A. P2-02-69

Smith, V. OP-09-66

Smith, V.E. OP-03-20

Soares, P. OP-03-16, OP-03-19

Sobrinho-Simões, M. OP-03-16, OP-03-19

Sokhatska, O. P3-01-123, P3-05-157

Sokolowski, A. P1-03-26

Solange, G. OP-04-22

Soldevila, B. OP-08-58

Solnica, B. OP-04-29

Song, H. P1-02-12

Song, I. P3-04-140

Sørensen, J.R. P1-04-30

Sorko, S. P3-03-133

Sorschag, M. P3-03-133

Šošiæ-Jurjeviæ, B. OP-07-51

Souteiro, P. P1-02-13, P2-04-93

Specker, E. OP-07-52

Spencer, C. P1-02-15

Spiezia, S. P2-05-101

Spitzweg, C. OP-03-17, OP-05-35, OP-03-98

Squatrito, S. P1-03-25, P3-05-155

Stanciu, M. P3-06-166

Stanescu, A. P3-06-166

Stanièiæ, J. OP-08-55

Stanley, E. P2-04-88

Stavrianos, C. P1-01-02

Stazi, M. OP-07-53

Steegers, E. OP-08-59

Stewart, J. P2-02-69

Stobiecka, E. OP-04-25

Stojanovic, M. P1-05-44, P1-05-47, P2-06-113

Stojilikovic, M. P3-01-118

Stojkovic, M. P1-05-47, P2-06-107, P3-01-118

Stoyanov, N. P3-03-132

Stoynova, M. P3-02-124

Stozek, K. P2-01-58

Stramazzo, I. P1-01-10

Strele, I. P3-06-164

Studen, K.B. OP-12-85

Sturgis, E. OP-06-39

Sugino, K. P1-03-22, P2-03-80, P3-03-131, P3-04-139

Sulaieva, O. P2-03-82

Sulkers, E. P3-05-151

Sunyer, J. OP-10-73

Surovtseva, O. OP-11-77
Słuszniak, A. P3-01-117

Suzuki, A. P1-03-22, P2-03-80, P3-03-131

Suzuki, M. P3-04-139

Suzuki, N. P3-04-139

Swan, K.Z. P2-02-70

Swierniak, M. OP-09-68

Sykiotis, G. P2-02-63, P2-04-90

Synoracki, S. P1-06-49, OP-01-01

Szpak-Ulczok, S. OP-09-68

Szymañski, K. OP-02-07

Szymonek, M. P3-01-117

Tabano, S. OP-03-18

Tacito, A. OP-06-43

Tajiri, J. P2-05-94

Takami, H. P1-03-22

Tallini, G. OP-04-28

Tamagno, G. P2-04-88

Tanaka, T. P1-03-22, P2-03-80

Tanteles, S. P3-01-121

Tatic, S. OP-09-62

Tavarelli, M. P1-03-25, P3-05-155

Tavares, C. OP-03-16

Tavares, P. P2-05-103, P3-06-163

Tavoni, T. P3-05-147

Taylor, P. OP-01-05, OP-02-14

Teles, L. P2-04-93

Tendi, S. P3-06-167

Terrien, J. OP-13-95

Terroir, M. OP-06-42

Terzidis, K. P1-01-02

Theocharous, P. P3-03-132

Thompson, R. OP-03-20, OP-09-66

Thuesen, B. P3-06-162, OP-12-84

Thvilum, M. OP-02-09, P1-01-06

Tiemeier, H. OP-10-73

Tissier, F. P2-03-81

Tissing, W.J.E. P3-05-151

Todorov, S. P3-04-143

Tokumitsu, H. P1-03-24

Toma, G.M. P3-06-166

Tomkins, M. P2-04-88

Tomoda, C. P1-03-22, P2-03-80, P3-03-131

Topaloviæ, Z. OP-08-55

Torlontano, M. P1-04-32, OP-04-28

Toromanyan, E. P3-05-153

Torregrossa, L. P3-03-137

Torres, I. P3-04-146

Toscano, V. P2-05-101

Tosheva, G. P1-05-38

Tran, R. P3-03-132

Travlos, A. P3-03-136

Trésallet, C. OP-04-26

Trimarchi, F. P2-01-57, OP-07-46

Trimboli, P. P1-02-14, P2-05-100, P3-05-150

Trofimiuk-Muldner, M. OP-04-29

Trubiroha, A. OP-01-04

Trukhina, L. P3-01-114

Trybek, T. P3-01-117

Tsakova, A. P3-01-116, P3-01-122

Tsakris, A. P3-01-121

Tsaousidou, S. P3-01-121

Tsvetov, G. OP-02-12

Tudorache, S. P3-06-166

Tumino, D. P1-03-25

Tumino, S. P1-04-32

Turbucz, P. P2-04-84
Turnell, A. OP-09-66

Tutter, M. OP-03-17, OP-03-98

Tuttle, R M. P3-05-149

Tyszkiewicz, T. OP-09-68

Tzifa, G. P2-03-76

Ugolini, C. OP-09-67

Urnauer, S. OP-03-17

Ursu, H. P3-06-166

Uruno, T. P1-03-22, P2-03-80, P3-03-131

Valenti, P. P2-05-101

Valerio, L. P1-02-17, OP-06-43

Valido, F. P1-02-18

Valle, D. P2-05-98

van Dam, EW. P3-05-151

Van de Beek, D. OP-11-77

Van den Berghe, G. OP-08-54

van den Heuvel-Eibrink, M.M. P3-05-151

van Der Deure, W.M. OP-11-80

van der Horst-Schrivers, A. P1-02-16

van der Pal, H.J.H. P3-05-151

van der Spek, A. OP-11-77

van Ginhoven, T. OP-04-24

van Gucht, A. OP-01-06, OP-011-80

van Hemel, B. OP-01-02

van Oudenaren, A. OP-11-77

van Santen, H.M. P3-05-151

Vancamp, P. OP-07-47

Vandernoot, I. OP-01-04

Vanhole, C. OP-08-54

Vannucchi, G. P1-01-05, P1-05-39

vanVelsen, E. OP-04-24

Varela, A. P2-04-93

Varzhapetyan, A. P2-05-97

Vasconcelos, C. P3-02-125

Vasile, M. P3-06-166

Vasovic, O. P1-05-44

Vasques, M. P2-05-103

Velari, L. P2-05-98

Velasco, I. OP-08-58

Velika, B. P3-06-164

Ventura, M. P1-02-20, P3-03-135

Verhaeghe, J. OP-08-54

Vicchio, T.M. P2-01-57, OP-07-46

Vicente, N. P1-02-20

Vicentini, L. P3-03-134, OP-06-40

Vicheva, S. P3-04-143

Vidranski, V. OP-08-55

Villalmanzo, N. OP-01-02

Villerusa, A. P3-06-164

Vinagre, J. OP-03-19

Viola, D. P1-02-17, OP-06-45, OP-09-67

Virgolini, I. OP-04-29

Virili, C. P1-01-10, P2-06-108, OP-12-83

Visser, T.J. OP-01-06, OP-05-36, OP-08-59, OP-11-80, OP-13-95

Visser, W.E. OP-01-06, OP-04-24, OP-05-36

Vitti, P. P1-01-01, P1-02-17, P2-01-55, P3-03-137, OP-02-08, OP-06-43, OP-06-45

Vlad, M. P3-06-166

Vlahov, J. P3-02-124
Volkova, A. P1-01-07, P3-01-119

Volpato, A. OP-05-32

Völzke, H. P3-05-152, OP-12-88

von Scholten, B.J. P3-05-149

Vrushi, L. P2-06-109

Vuckovic, L. P3-02-128

Vujosevic, S. P2-06-112, P3-02-130

Wagner, E. OP-03-17

Waguespack, S. OP-06-39

Wajner, S. OP-05-31

Walczyk, A. P3-01-117

Wang, W.-L. P2-02-69

Wassermann, J. OP-04-26

Watanabe, N. P3-04-139

Wawrusiewicz-Kurylonek, N. P2-01-62, OP-02-07

Wechselberger, A. OP-05-35

Wejaphikul, K. OP-01-06, OP-05-36

Wert-Carvajal, C. OP-11-79

Wert-Lamas, L. OP-10-75, OP-11-76, OP-11-79

Williams, M. P2-02-69

Willis, M. OP-02-14

Wiœniewski, J.R. OP-05-30

Wirth, E.K. OP-05-37

Wittfeld, K. OP-12-88

Wong, C. P1-04-31

Yadav, N. P3-06-165, P3-06-167

Yamada, K.H. P1-03-22, P2-03-80

Yang, S.I. P1-03-23, P2-04-87

Yann, G. OP-10-71

Yarkoni, T.R. P2-05-96

Yasuda, G. OP-12-87

Yehuda, M. P2-05-96

Yoo, J. P1-03-23, P2-04-87

Yoon, H. P3-04-140

Yoshida, Y. P1-03-24

Yoshihara, A. P3-04-139

Yoshimua, J.N. P3-04-139

Żaba, R. P2-01-60

Zaballos, M. P2-02-68, OP-09-63

Zafereo, M. OP-06-39

Žagar, I. P1-02-11

Zake, T. P2-01-59

Zaletel, K. P1-01-03, OP-02-10, OP-04-29, OP-12-85

Zaman, A. P3-05-156

Zarkovic, M. P1-05-47, P1-06-53, P2-06-107, P3-01-118

Zatelli, M.C. OP-04-28

Zerdoud, S. OP-04-22

Zevenbergen, C. OP-11-80, OP-13-95

Zhang, L. OP-01-05, OP-02-14

Zimmermann, M. OP-08-55

Ziora, K. P2-01-58

Zivaljevic, V. OP-09-62

Živanoviæ, J. OP-07-51

Zivojinovic, S. OP-02-11

Zmuc, J. P1-02-11

Zoras, O. P2-03-76

Zucchi, R. P1-06-52, OP-07-49, OP-07-53, OP-13-90, OP-13-92 